\title{
Text History of the Greek Numbers
}

Von

John William Wevers

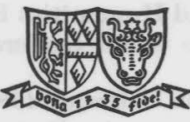

GÖTTINGEN・VANDENHOECK \& R UPRECHT・1982 


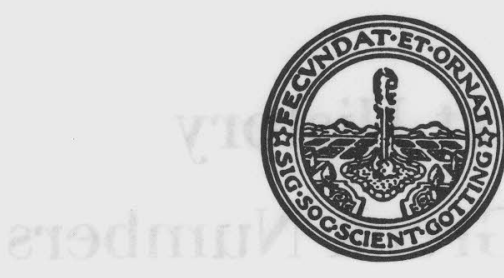

Vorgelegt in der Sitzung vom 3. Juli 1981

CIP-Kurztitelaufnahme der Deutschen Bibliothek

Wevers, John William:

Text History of the Greek Numbers / von John William

Wevers. - Göttingen : Vandenhoeck und Ruprecht, 1982.

(Mitteilungen des Septuaginta-Unternehmens 16) (Abhandlungen der Akademie der Wissenschaften in Göttingen, Philologisch-Historische Klasse ; Folge 3, Nr. 125)

ISBN 3-525-82411-4

NE: Septuaginta-Unternehmen: Mitteilungen des Septuaginta-Unternehmens; Akademie der Wissenschaften 〈Göttingen〉/ Philologisch-Historische Klasse: Abhandlungen der Akademie . . .

Research for this monograph was generously supported by grants from The Social Science and Humanities Research Council of Canada to whom the writer tenders his thanks.

(C) Vandenhoeck \& Ruprecht in Göttingen 1982. - Printed in Germany Ohne ausdrückliche Genehmigung des Verlages ist es nicht gestattet, das Buch oder Teile daraus auf foto- oder akustomechanischem Wege zu vervielfältigen. - Herstellung: Hubert \& Co., Göttingen 


\section{Table of Contents}

Chapter 1 The $x$ Group $\ldots \ldots \ldots \ldots \ldots \ldots \ldots \ldots \ldots \ldots \ldots \ldots \ldots \ldots$

Chapter 2 The Byzantine Text .................. 17

Chapter 3 The Hexaplaric Recension .............. 43

Chapter $4 \quad$ The Texts of B and A $\ldots \ldots \ldots \ldots \ldots \ldots \ldots \ldots$

Chapter $5 \quad$ Papyrus 963 as Textual Witness ............. 86

Chapter 6 The Critical Text (Num) $\ldots \ldots \ldots \ldots \ldots \ldots \ldots .94$

Index of Passages $\ldots \ldots \ldots \ldots \ldots \ldots \ldots \ldots \ldots \ldots \ldots \ldots \ldots \ldots \ldots \ldots$ 



\section{Chapter 1 The $x$ Group}

In THGG 152 it was stated that mss. 71 and 619 commonly go together in the second half of Genesis and contain a large number of unique readings. It was similarly noted in THGD 54 that $71^{\prime}-527$ often have readings at variance with 121-318-392 of the $y$ group. For Numbers it is abundantly clear that these form two distinct groups. To the former group, which I have arbitrarily designated the $x$-group one may with some hesitation add ms 509. That these constitute distinct groups should be clear from the following two lists. In List 1 are given instances of $y$-readings in which at least two out of the three remaining $y$ mss (with only random support from other mss) attest to the reading. List 2 consists of $x$-readings, i.e. readings supported by at least all but one of the extant $x$-mss. with no more than random support from other mss. By random support is meant ms support by individual rather than textual group support.

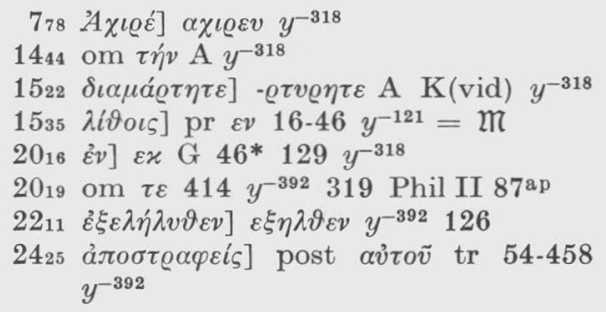

\section{List 1}

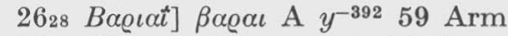

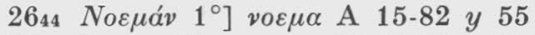

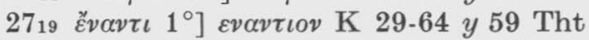
$\mathrm{Nm} 224$

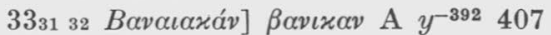

$\left.33_{47} \Gamma \varepsilon \lambda \mu \omega ́ v\right] \delta \varepsilon \lambda \mu \omega \nu y^{-392} 128^{\mathrm{mg}}{ }_{-669}$

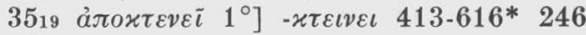
$y^{-121}$

The shortness of this list reemphasizes the colorless character of the $y$-group as a group as indicated for Genesis in THGG $139 \mathrm{ff}$. It is also clear that its text is related to codex $\mathrm{A}$.

\section{List 2}

12 om vi $\tilde{v} v$ B $x$ Bas II 145 Latcod 100

112 A $A \iota \sigma \alpha \delta \alpha i]$ $\mu \iota \sigma \alpha \delta \alpha \iota x^{-509} 59$

120 om $\tau \tilde{\eta} x^{-509}$

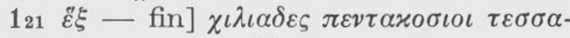
$\varrho \alpha \varkappa 0 \nu \tau \alpha \quad \varepsilon \xi \quad x^{-509}$

122 om $\alpha v i \tilde{\omega} v 1^{\circ} 82 x^{-509}$

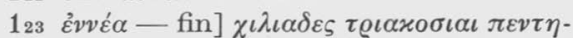
$\chi 0 v \tau \alpha \quad \varepsilon v v \varepsilon \alpha \quad x^{-509}$

124 om $\alpha \dot{\tau} \tau \tilde{\omega} v 1^{\circ} 458(\mid) x^{-509}$

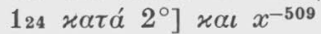

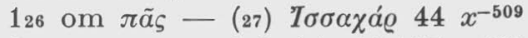

138 om $\pi \tilde{a} \varsigma-(39) \Delta a ́ v$ 44-107 $x^{-509}$

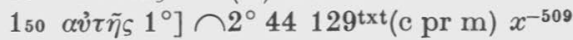

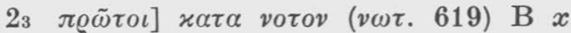
Latcod 100

$29 \quad$ om $\varkappa \alpha i \quad \varepsilon \xi \alpha \varkappa \iota \sigma i \lambda_{\iota} \iota \iota x^{-509}$

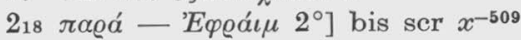

218 'E

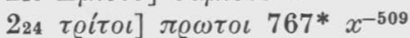

$\left.2{ }_{25} A \mu \iota \sigma \alpha \delta \alpha i\right] \sigma \alpha \delta \alpha \iota x^{-509} 407$; but ef $\sigma \alpha \delta \varepsilon$ $68^{\prime}-120$

232 om $\tau \tilde{\omega} \nu 1^{\circ} x^{-5 n 9}$

$232 \varepsilon \xi \alpha x o ́ \sigma \iota \alpha \iota]-\sigma \iota \iota \iota 707528 x^{-509}$

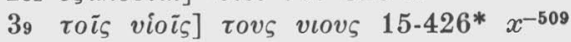
12059 Tht $N m$ 192 ap

$\begin{array}{lllllll}310 & \tau \tilde{\eta} \varsigma & \sigma \varkappa \eta \nu \tilde{\eta} \varsigma] & \tau \eta \nu & \sigma \varkappa \eta \nu \eta v & x^{-509} & 126\end{array}$ 


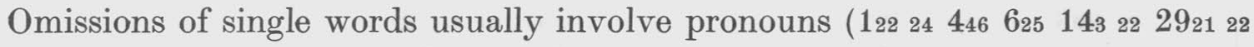
25283031333439 (twice) 3011357 ). The article is omitted at $1202323_{21} 9_{13} 1126$ 1619181031193412 ; xaí, at $231423132715_{24} 1812307$, and $\delta \varepsilon$, at 3227. Prepositions

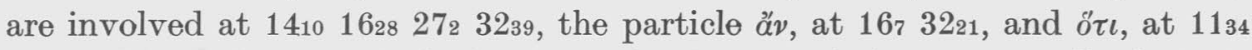

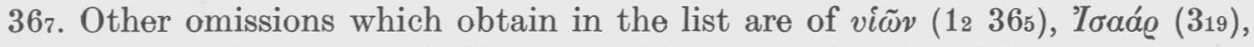

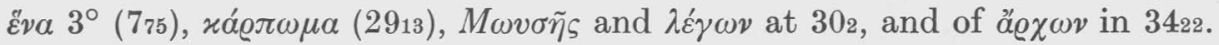

Only 12 instances of a longer text obtain in the above list and these involve but one word in all but one instance, viz. a dittograph of $\pi \alpha \varrho \alpha ́-' E \varphi \varrho \alpha ́ ı \mu 2^{\circ}$ in 218 . Of the remaining 11 three involve the articulation of a noun $\left(27_{4} 3152\right.$ 3212 ), one, the articulation of an infinitive (1541), two, the addition of the preposition $\varepsilon \iota \varsigma$ before a noun (1440 3512). The particle $\alpha \nu$ is inserted between $\tilde{\eta}$ and $\dot{\eta} \mu \varepsilon \varrho \alpha$ in 313 ; the conjunction $x \alpha \iota$ is added in 367 , the infinitive $\varepsilon \iota v \alpha \iota$, at $11_{29}$, and the adverb $\varepsilon \varkappa \varepsilon \iota$, in 1333. The addition of ov before $\pi \alpha \tau \alpha \xi_{\eta} \eta$ in 3516 substantially changes the meaning; it is, however, the result of dittography

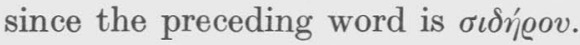

Change in nominal inflection may involve gender, case or number. Change in gender usually involves the gender of compound numbers: of 600 at 232,

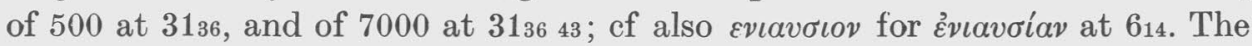
change of ós to $o$ at 1120 must be palaeographically based since the masculine is demanded by the context, the antecedent being rvoi $\omega$.

Change in case is also rare. Case after $\dot{\varepsilon} \pi \dot{i}$ is changed from genitive to accusative in 310 and from accusative to dative in 3530 . The dative pronoun $\mu \circ$ modifying $\zeta \eta \lambda o \tilde{\iota} \varsigma$ at $11_{29}$ is changed to $\varepsilon \mu \varepsilon$. The dative article $\tau \tilde{\omega}$ in the formulaic " $\tau \tilde{\omega} \mathrm{PN}, \delta \tilde{\eta} \mu \varsigma_{\varsigma}+$ genitive gentilic noun" is changed to the genitive at 26. The accusative becomes a nominative at 1510 . The occurrence of the accusative for $\delta \eta^{\prime} \mu o v 2^{\circ}$ in 3612 obtains under the influence of the immediately preceding $\tau \dot{\eta} \boldsymbol{v} \varphi v \lambda \dot{v} \boldsymbol{v}$. Change in number occurs only three times. Two of these involve an article before a proper noun. At 330 for $\tau o \tilde{v} K \alpha a \vartheta^{\vartheta}$ the article appears in the plural since $\tau \tilde{\omega} v \delta \dot{\eta} \mu \omega \nu$ precedes it. At 1634 the singular article

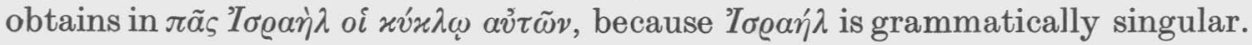
The only other change in number obtains at 154 where $\tau \dot{o} \delta \tilde{\omega} \varrho o v$ is changed to the plural.

Change in word order is equally rare. Two instances of change in order of compound numbers obtain in 12123 ; the only other change in word order occurs at 172 .

Change in verbal inflection often involves tense. Thus at 133 a future indicative is changed to aorist participle, a present tense is changed to aorist at $14{ }_{14}$ and to future at 3225. An original future indicative becomes present indicative in 1515 but present imperative at 326. An original aorist is changed to the present at 3515 and to the perfect at 167 .

Changes in mood occur three times: at 3013 a subjunctive is changed to the indicative, and the reverse obtains at 3215 ; for $16 z$ of the preceding paragraph. Change in voice is attested twice, at 1443 a passive is given a middle ending, 
and at 3230 a middle inflection obtains for a passive. Change in number obtains at 1431637315 . At 1627 the Hellenistic $\varepsilon \xi \eta \lambda \vartheta o \sigma \alpha v$ is found for the classical $\xi \xi \tilde{\xi} \lambda v_{0} 0 v$. And finally at 319 the augment of an aorist form obtains doubly, i.e. both before the stem and before the compound element in the variant $\varepsilon \pi \varrho o-$ $\varepsilon v о \mu \varepsilon v \sigma \alpha v$.

One of the more interesting variations in text involving change in lexical

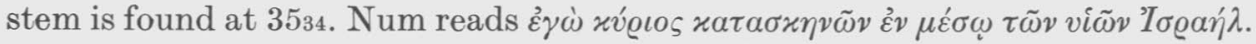
The $x$ variant changes the participle into $x \alpha \tau \alpha \sigma \varkappa \eta \nu \omega \sigma \varepsilon \omega \varsigma$ and omits $\dot{\varepsilon} v \mu \varepsilon \dot{\varepsilon} \sigma \omega$. Many lexical changes are simply errors palaeographically or phonetically con-

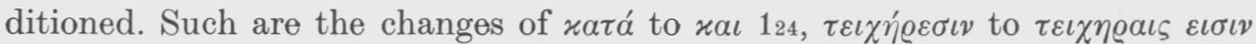

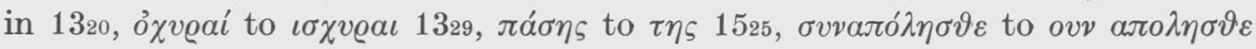
$16{ }_{26}$, $\pi \alpha \iota \sigma i v$ to $\pi \alpha \sigma \iota v 32_{4}$ and of $\sigma o v$ to ov in the same verse. Other changes are obviously due to the influence of the immediate context and illustrate scribal carelessness in copying the parent text. Such are the changes of $\pi \tau \varepsilon \varrho v \gamma i \omega \nu$ to

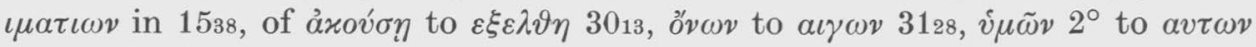
3354 and of $\tau \tilde{\omega} v v i \tilde{\omega} v$ to $\tau \eta \varsigma \varphi v \lambda \eta \varsigma 3423$.

Change of preposition is attested at $15_{10}$ of $\varepsilon i \zeta$ as $\varepsilon \pi \iota$ and at 1831 of $\dot{a} \nu \tau i$ as $\alpha \pi o$. Pronominal change occurs at 303 with $\tau o v \tau o$ for $\alpha \hat{v} \tau o \tilde{v} 2^{\circ}$, and at 309 with $\varepsilon a v \tau \eta v$ for $\alpha \dot{\tau} \tau \eta \dot{v}$. The substitution of synonyms or near synonyms occurs but rarely. The only ones attested are $\alpha \lambda \omega$ for $\ddot{\alpha} \lambda \omega \nu$ s at $15_{20} 18_{27}$, of $\overline{\chi_{\xi}}$ for $\delta \vartheta \varepsilon o ́ s$ at 165 and of $\varepsilon \lambda \alpha \lambda \eta \sigma \varepsilon(v)$ for $\varepsilon i \pi \varepsilon v$ at 1710 . Only wandering attention on the part of a scribal parent can explicate the substitution of $\pi \varrho \omega \tau o \iota$ for $\tau \varrho i$ i

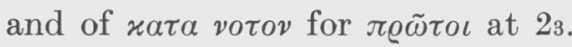

Change in compound element occurs at 1434 with $\propto \alpha \varepsilon \sigma \varkappa \varepsilon \dot{\psi} \alpha \sigma \vartheta \varepsilon$ to $\varepsilon \pi \varepsilon \sigma \varkappa$. and in 1638 with $\pi \varepsilon \operatorname{li}^{\prime} \varepsilon \mu \alpha$ to $\varepsilon \pi \iota \vartheta$. Change of simplex to compound obtains with

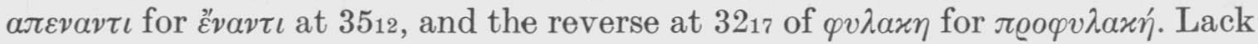
of assimilation produced $\varkappa \alpha \tau \varepsilon \sigma \tau \alpha \mu \varepsilon v o \iota$ at $31_{48}$ and $\varkappa \alpha \tau$ (for $\varkappa \alpha \vartheta^{3}$ ) at 153 ; ef also $\sigma v v \tau \varrho \iota \mu \alpha$ at 3214 .

That these four mss constitute an independent group is particularly evident from common errors in the tradition of the spelling of proper names. In the following list the Num spelling is given in parenthesis after the variant. ${ }_{112}$

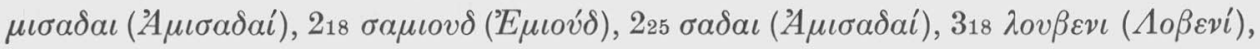

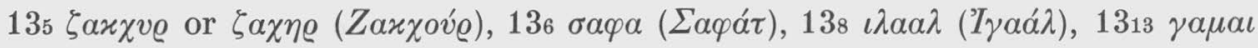

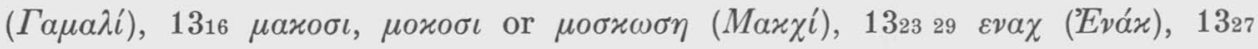

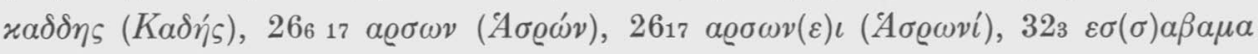

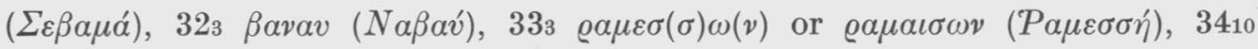

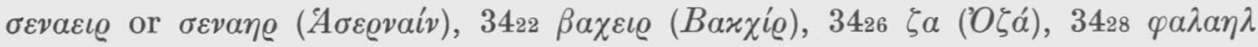

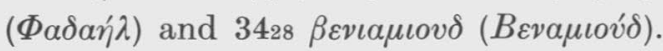

A major characteristic of the above list is the frequent occurrence of $\mathrm{B}$ or $\mathrm{B}^{\mathrm{c}}$ indicating random support of the $x$ variant text. The B text (or that of its corrector) supports the variant text 45 times whereas codex A occurs but twice. On the other hand the short list of 16 variants shown in list 1 as unique 
or almost unique variants of the $y$-group are supported by A five times and never by B.

This affinity of the $x$-group to the $\mathrm{B}$ tradition is further confirmed in the following list of variants in Codex $\mathrm{B}$ in which random support by one or more members of the $x$-group occur.

\section{List 3}
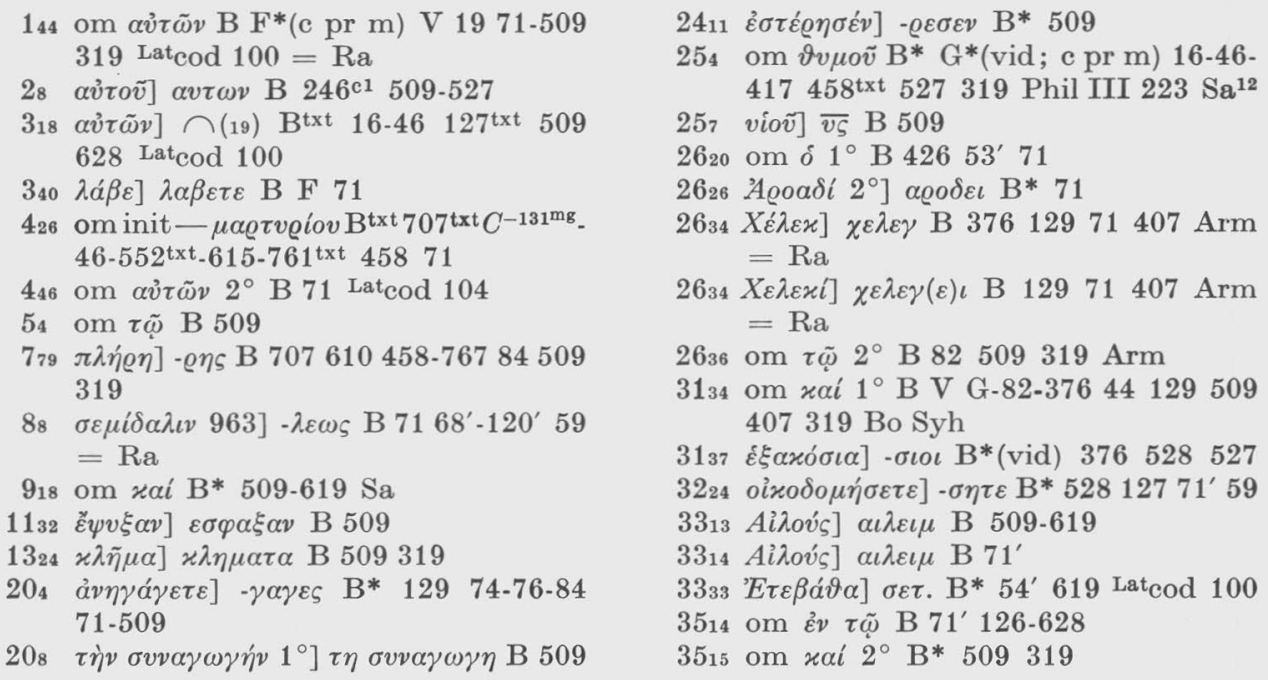

The $x$ group in its loyalty to the B text tradition is sometimes along with $\mathrm{B}$ a witness to Num over against almost all other witnesses. In the following list only $\mathrm{B}$ and $x$ together with no more than random support witness to $\mathrm{LXX}$.

List 4

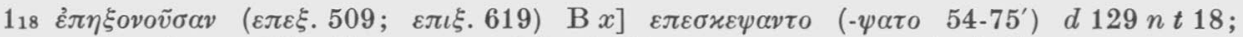
$\varepsilon \pi \varepsilon \sigma \varkappa \varepsilon \varphi \vartheta \eta \sigma \alpha v$ 53'; disposuerunt Latcod 100; recensuerunt eos Aeth Sa; $\varepsilon \pi \varepsilon \sigma \varkappa \varepsilon \pi \eta \sigma \alpha \nu$ (c var) rell

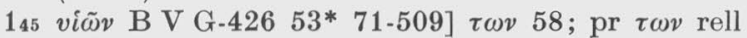

$337 \pi \alpha \sigma \sigma a ́ \lambda o v \varsigma$ (c var) B V 44-125 71-509 799 Latcodd 100104$]+\alpha v \tau \eta \varsigma z 646$; + $\alpha v \tau \omega \nu$ rell

$56 \tau \tilde{\omega} v 1^{\circ} \mathrm{A} \mathrm{B} \mathrm{G} x^{-619}$ Anast 376 Arab Sa] $\pi \alpha \sigma \omega v 12655$; pr $\pi \alpha \sigma \omega \nu$ ( $\left.\pi \alpha v \tau \omega v 29\right)$ rell

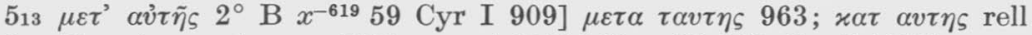

614 ó

$621 \ddot{\eta} v \mathrm{~B} x^{-619} \mathrm{Cyr}$ I 1053] $\eta[. . .963$; os 537; $\eta$ s rell

$7_{85} \tau \tilde{\omega} v$ áfi $\left.\omega v \mathrm{~B} 963458 x^{-619}\right] \tau \omega \alpha \gamma \iota \omega$ rell $=\mathrm{Ra}$

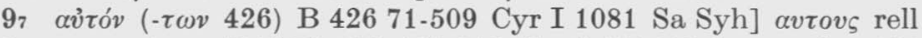

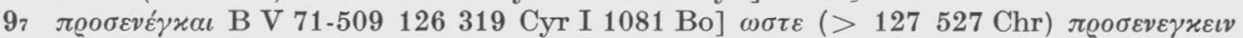

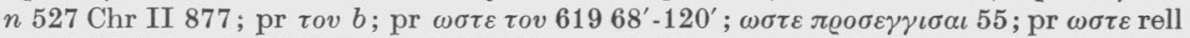

$1333 \pi \tilde{\alpha} \mathrm{B}_{\mathrm{B}} 426 x \mathrm{Bo}^{\left.\mathrm{AB}^{\mathrm{c}} \mathrm{Sa}\right]} x \alpha \iota \mathrm{G} C^{\prime \prime} 799 ;>\mathrm{Bo}^{\mathrm{B}^{*}}$; pr $x \alpha \iota$ rell

$143 \pi \alpha \iota \delta i \alpha \mathrm{B} \mathrm{M} 129 x$ Cyr I 373] $\tau \varepsilon \varkappa v \alpha \eta \mu \omega v b$; + $v \mu \omega v$ 44-107' 321; + $\eta \mu \omega v$ rell

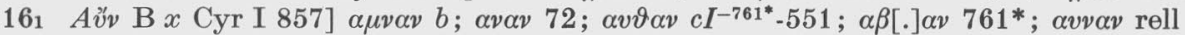

188 à $\alpha \varrho \chi \tilde{\omega} v$ B V $82129 x^{-619}$ Cyr I 837 Lat $\operatorname{cod} 100$ Sa] $\varepsilon v \tau o \lambda \omega v \mu o v$ Procop 844; $\alpha \pi \alpha \varrho \chi \iota \omega \nu$ $\mu o v$ 44; $+\mu o v$ rell

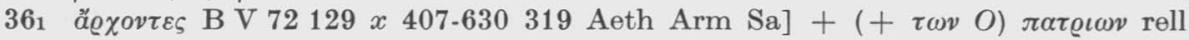


It remains to determine the place of the $x$ group within the text tradition of the book. In the final list variants are given support by $x$ and no more than three other text groups. As usual random support is disregarded. Text groups other than $x$ are indicated in parentheses.

\section{List 5}

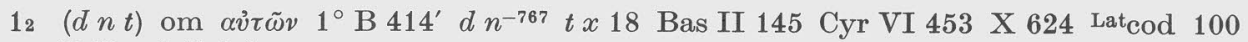
PsBas Is I 5 Arm

$12(d n t)$ om $\alpha v i \tilde{\omega} v 2^{\circ} \mathrm{B} \mathrm{V} d n^{-767} t x 18319$ Bas II $145 \mathrm{Cyr}$ VI $453 \times \mathrm{X}^{2} 4^{\text {Lat }}$ cod $100 \mathrm{Hi}$ Eph II 3 PsBas Is I 5 Arm

$12(d t)$ om $\alpha \dot{\tau} \tau \tilde{\omega} \nu 3^{\circ} \mathrm{B} 19 d 127 t x 18319$ Cyr VI $453 \times 624$ Latcod 100 Arm $=\prod$

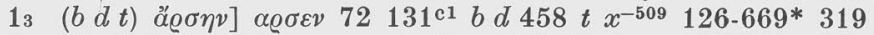

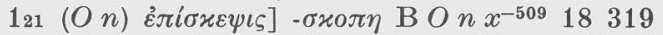

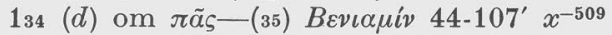

136 (d) om $\pi \tilde{\alpha} \varsigma-($ з

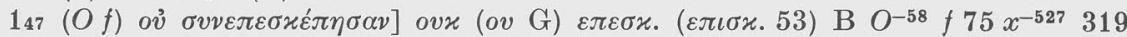

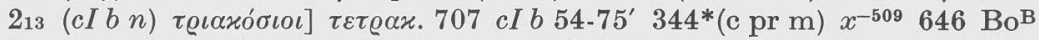

$\left.222(d t) \Gamma \alpha \delta \varepsilon \omega \nu \nu^{\prime}\right] \gamma \varepsilon \delta \varepsilon \omega \nu 77 d 458 t x^{-509} \mathrm{Bo}^{\mathrm{B}}$

$2{ }_{23}(d t) \tau \varepsilon \tau \varrho \alpha x o ́ \sigma \iota \iota ! ~ \tau \varrho \iota \varkappa . ~ 44-106 *-107^{\prime} t x^{-509} 799$

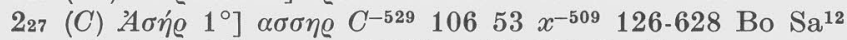

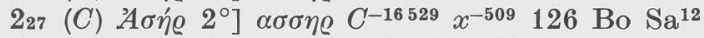

$31 \quad(f) \tilde{\eta} \dot{\eta} \mu \varepsilon \dot{\varrho} \varrho]$ tr $f 30 x^{-509} 12655$

$39 \quad$ (b) $\left.\mu \circ \iota^{\prime}\right] \mu o v 15 b x^{-509} 318$

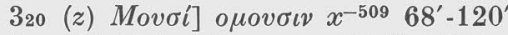

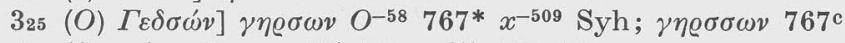

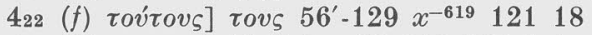

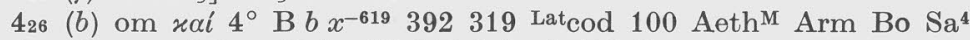

$435(f)$ om $\tau \dot{\alpha}$ है $\varrho \gamma \alpha$ B f $x^{-619} 319$ Latcod $100 \mathrm{Sa}$

65 (n) om $\tau \tilde{\eta} \varsigma$ $\varepsilon \dot{\chi} \chi \tilde{\eta} \varsigma$ B $96366454-75^{\prime} 28-85 x^{-619} 628799$ Cyr I 1041 Arm Sa ${ }^{4}$

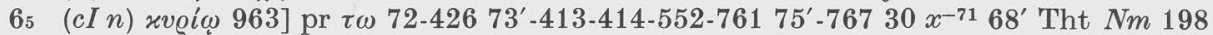

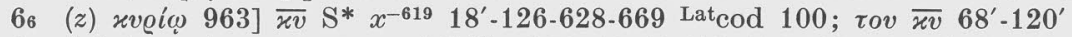

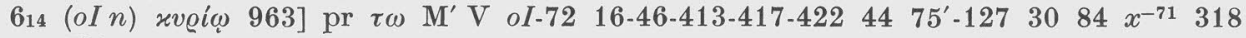
799

$72 \quad(z)$ om oi $2^{\circ} 82 x^{-619} 120^{\prime}-126-128-628-669319$

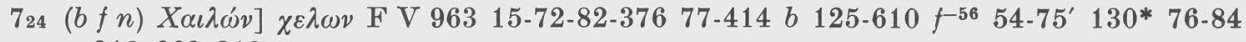
$x 318669319$

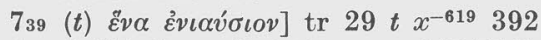

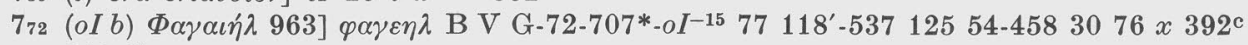
319 Co

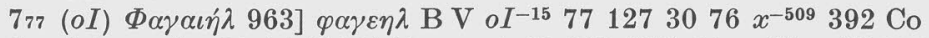

$7_{81}(n)$ om $\ddot{\varepsilon} v \alpha 3^{\circ} \mathrm{A}^{*} \mathrm{~V} 29^{\text {txt }}\left(\mathrm{c}\right.$ pr m)-82 $529107^{\prime} 56 n^{-767} x^{-619} 392120319$ Lat $_{\text {cod }} 100$

$814(O d t) \tau \tilde{\omega} \nu 963]>\mathrm{A} \mathrm{B} O^{-58} d 127-767 t x^{-619} 121=\mathrm{Ra}$

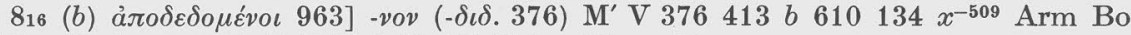

$10_{12}(O d)$ om $\tau$ ov $1^{\circ} \mathrm{B} \mathrm{V} \mathrm{O} O^{-58} 44^{\prime}-125 x^{-619}=\mathrm{Ra}$

$\left.11_{12}(O f n) \tau \dot{v} v \pi \dot{v} \nu \tau \alpha\right]$ tr B V $O^{-58} 422$ f $n x^{-619}$ Phil III 6 Chr I 476 Tht $N m$ 204ap $=$ Ra

$11_{35}(O n)$ om $\tau \tilde{\eta} \varsigma$ B F V O'-2958 $12954-75^{\prime} x 39259319799=\mathrm{Ra}$

124 (cI') om xaí $4^{\circ}$ - fin 376-381' 77-cI'-46 $5654 x^{-509} 18-68-126319$ Cyr II 600 Aeth Bo

$1210(d n t) \pi \varrho o ́ s] \varepsilon \pi \iota \mathrm{B} \mathrm{V} d 129 n^{-75} 321^{\prime} \mathrm{mg} t x 319$ Arab Arm Co $=\mathrm{Ra}$

1215 (n) $\varepsilon(\omega \varsigma]+$ ov $54-75^{\prime} 84 x^{-509} 319$ Cyr II 593

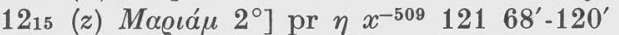

$\left.13_{25}\left(C^{\prime \prime}\right) \Phi_{\alpha} \varrho \alpha \gamma \xi\right]-\gamma \gamma \alpha C^{\prime \prime} x^{-509} 318^{\text {Lat }} \operatorname{cod} 100$ Aug Loc in hept IV 36

$1327(f) \varrho \tilde{\eta} \mu \alpha] \varrho \eta \mu \alpha \tau \alpha f x^{-509} \mathrm{Sa}$

$13_{29}(d n t)$ om ai B F*(c pr m) V $29 d n^{-767} t x$ Cyr I 373

$\left.14{ }_{13}(t) \dot{\varepsilon} v\right] \tau \eta \mathrm{B} 44^{\prime} 129127-767 t x^{-509}=\mathrm{Ra}$

$1414(n s)$ xú@ıc] $\overline{x \zeta} 58528 n^{-767} 28-30-85^{\prime}-346 x^{-509}$ 
$1420(o I) \varepsilon i \mu \iota] \varepsilon \sigma o \mu \alpha \iota$ oI-15-29 $x^{-509}$ Arm Co

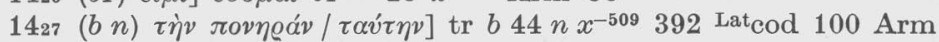

$1433(b f y)$ om $\alpha^{\prime} v \mathrm{~A} \mathrm{~B}^{*} \mathrm{~F}^{*}\left(\mathrm{c}\right.$ pr m) $70777 b 44 f^{-129} 458-767 x^{-509}$ y 12655319624

$\left.15_{1}(d t) \dot{\varepsilon} \lambda a ́ \lambda \eta \sigma \varepsilon v\right] \varepsilon \iota \pi \varepsilon(v) \mathrm{B} \mathrm{V} d 129 t x$ Cyr I $1029=\mathrm{Ra}$

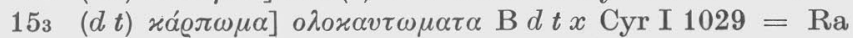

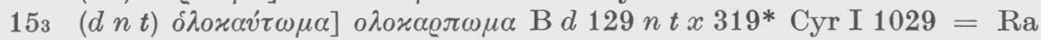

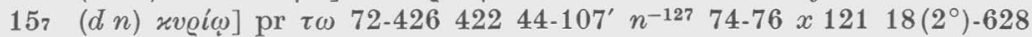

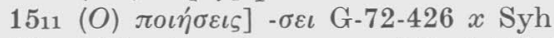

$1514(d t) \gamma \varepsilon ́ v \eta \tau \alpha \iota] \pi \varrho o \sigma \gamma \varepsilon v .551 d 127 t x$

1532 (n) $\tau \tilde{\eta} 2^{\circ}$ ] pr $\varepsilon v$ A $376 n^{-127} x^{-509} 318319$ Latcod 100 Syh

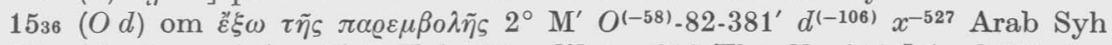

$15_{39}(d n t)$ om $\delta \pi i \sigma \omega 2^{\circ} \mathrm{B} \mathrm{V} d 129 n^{-767} t x 319$ Tht $N m 211$ Latcod 100 Arm Co

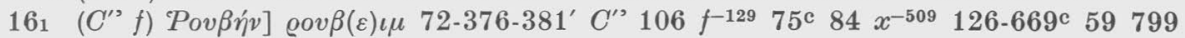

165 (d) Éavtóv $\left.1^{\circ}\right] \cap 2^{\circ} 551 d^{-44} 75^{\prime} 84-370 x^{-509}$ 126-628 Aeth

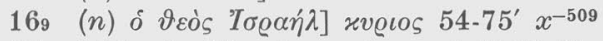

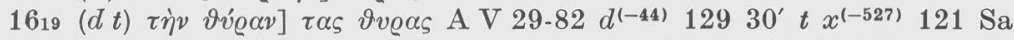

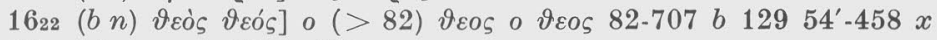

$1622(z)$ om $\tau \eta^{\prime} v \mathrm{~V} x z^{-628}$

$16_{29}(b) \varepsilon i$ $\left.x \alpha i\right]$ tr $b x^{-509}$ LatFac Def XII 3 Syh

1629 (b) $\left.x \alpha \tau^{2}\right] \quad x \alpha \tau \alpha \quad b \quad x^{-509}$

$\left.16_{45}(O) \varepsilon \tilde{\pi} \pi \varepsilon \sigma o v\right]-\sigma \alpha \nu \mathrm{B}^{\mathrm{c}} \mathrm{G}-29-426 x^{-527}$

189 $(d n t)$ om $\tau \tilde{\eta} \varsigma$ B $82 d n^{-767} t x^{-619} \mathrm{Cyr}$ I $837=\mathrm{Ra}$

$\left.2010(d f n) \varepsilon^{\prime} \xi \alpha^{\prime} \xi o \mu \varepsilon v\right] \varepsilon \xi \alpha \xi \omega \mu \varepsilon v$ 15-707 313-417-615* 19' 106-107' $f^{-246} 75^{\prime}-76784 x^{-619} 628$ $\begin{array}{lllll}55 & 59 & 319 & 799\end{array}$

264 $(O n) x \alpha i 2^{\circ}$ ] pr $\sigma v(\sigma o \iota 767)$ B VOn $x^{-619} 407$ Latcod 100 Arm Syh

$26{ }_{37}(O)$ om $\tau \alpha \tilde{v} \tau \alpha \mathrm{B}^{\mathrm{c}} \mathrm{F} O^{3}-58707129 x^{-619} 59$ Arm Sa Syh $=\mathfrak{m}$

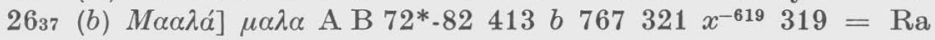

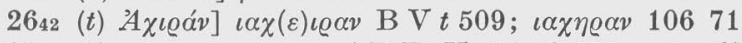

2650 (dnt) om $\pi \dot{v} v \tau \varepsilon$ xai B Fa V $963 d 129 n t x^{-619} 319$ Arm Bo

$27_{18}(d f t) \tau o ́ v$ 963] $>\mathrm{B}^{\mathrm{c}} \mathrm{F}$ K (vid) $\mathrm{M}^{\prime}$ 72-426 46 ${ }^{\mathrm{c}}-52^{\prime} d f^{-246} 767 t x^{-619} 18-12659646$

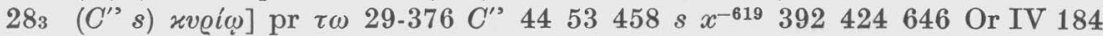

$\left.2810(O s) \sigma \alpha \beta \beta \alpha^{\prime} \tau o \iota s\right] \sigma \alpha \beta \beta \alpha \sigma \iota v\left(+\alpha v \tau o v O \mathrm{Syh}\right.$ ) $O 30^{\prime}-85^{\mathrm{mg}}-321^{\prime \mathrm{mg}} x^{-619} 68^{\prime}-120 \mathrm{Syh}$

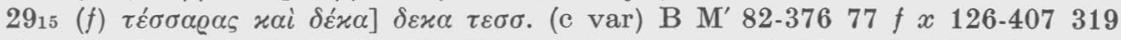

$2917(f t)$ idem B $\mathrm{M}^{\prime}$ V 963 82-376 $77-417 \quad 106$ f $t x$ 18-126-407-669 319

$29_{20}(d f t)$ idem B M' V 82-376 $77 d^{(-106 \text { txt })} f t x$ 18-126-407

$29_{23}(f)$ idem B V $963 \quad 72-82-376 \quad 77 \quad 44^{\prime} f x \quad \begin{array}{llll}126-407 & 416\end{array}$

$29_{24}(d)$ om $\alpha v i \tilde{\omega} v 1^{\circ} d^{-106} x^{-509}$

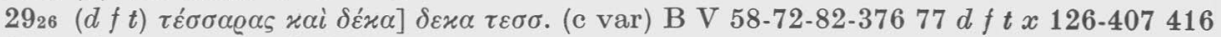

$29_{27}(d)$ om $\alpha \dot{\tau} \tau \tilde{\omega} v 1^{\circ} d^{-106} x^{-509}$

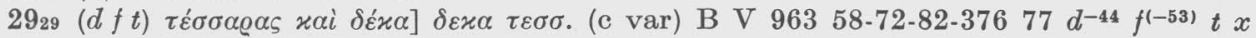
126-407 $416=\mathrm{Ra}$

$2932(d f t)$ idem B V 963 58-72-82-376 $77 d f t x \quad 126-407416$

$303(d n t) \ddot{\eta} \delta \varrho i \sigma \eta \tau \alpha \iota / \delta \varrho \iota \sigma \mu \tilde{e}] \operatorname{tr} \mathrm{B} \mathrm{F}^{\mathrm{a}} 96382 d 129 n t x 407319$ Or II 306 Latcod 100 Arm

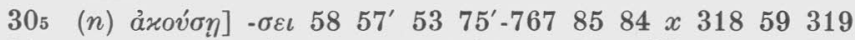

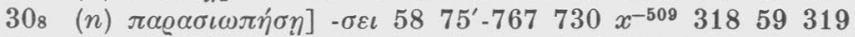

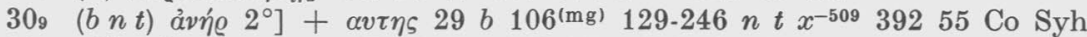

$\left.3014\left(C^{\prime \prime} d f\right) \alpha v \dot{n} \tilde{\eta}\right] \alpha v \tau \eta v$ F $72-376 C^{\prime \prime}(-417) 19 d^{-106} 53^{\prime}-12930^{\prime}-130^{\mathrm{c}}-343 \quad 134^{*}-370^{*} x^{-509}$ $318 \quad 126-407 \quad 624$

$30_{15}(o I y) \alpha v ่ \tau \tilde{\eta} \varsigma 2^{\circ}$ ] $\alpha v \tau \eta$ A $72-426-o I^{-15} 53^{\prime} 134 x^{-509}$ y 40755416

3015 (d) $\left.\eta^{2} \varkappa o v \sigma \varepsilon v\right] \frown(16) 72 d^{-106} x^{-509}$

$31_{10}\left(C^{\prime \prime} d t\right)$ om हैं $2^{\circ} \mathrm{B}^{\mathrm{c}} \mathrm{G}-82-426 C^{\prime \prime}-414417 d 53 \quad 127-767 t x^{-527} 40755319624$

$31_{29}(o I)$ xv@iov] $\overline{\varkappa \omega}$ G-72-oI $I^{-15} 46^{*}-4144453^{\prime} 30 x^{-527} 59$ Latcodd 100104

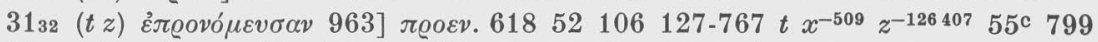

$\left.31_{47}(o I z) \varepsilon \tilde{\varepsilon}\right] \quad \varepsilon v \alpha \mathrm{V}$ oI $246 x^{-509} 18^{\prime}-126-407-628-630^{\prime} 55319624$

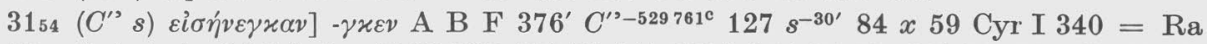

$323 \quad(b f n) \Delta \alpha \iota \beta \omega v] \delta \varepsilon \beta \omega \nu$ F $58-70746^{*}-77-414-529 b f 54-75-76730^{\prime}-34376 x^{-509} 1859319$ 


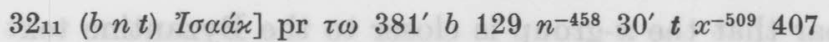

3211 (bnt) 'Т $\alpha \varkappa \omega ́ \beta]$ pr $\tau \omega 381^{\prime} b 129 n^{-458} 30^{\prime} t^{-84} x^{-509} 407$

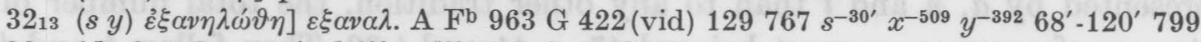

$3225(d) \dot{\eta} \mu \tilde{\omega} v] \eta \mu \nu v \mathrm{~A} d^{-106} x^{-509} 799$ Aeth Sa ${ }^{12}$

$3230 \quad(b)$ है $v$ in $\gamma \tilde{\eta}] \quad \varepsilon \iota \varsigma \quad \gamma \eta v 417 \quad b \quad x^{-509}$

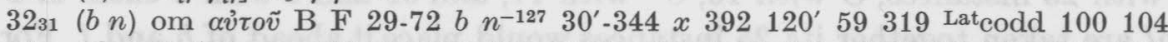

$\left.32{ }_{33}(d n) \Sigma \eta \omega^{\prime} v\right] \sigma \iota \omega \nu 58^{*} 528-739108 d 53^{\prime} n^{-127} 28-30370 x 318$ 120-122-630* 55319

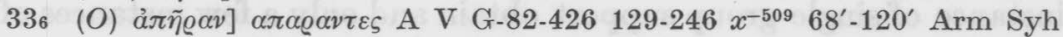

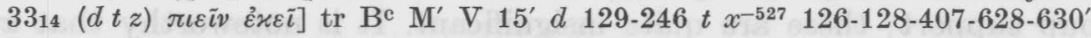

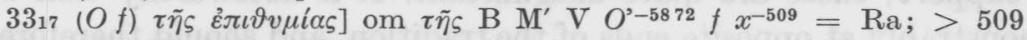

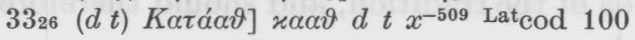

$33_{27}(d t)$ idem $d^{-125} t x^{-509}$

$\left.33_{29}(d n t) A \sigma \varepsilon \lambda \mu \omega \nu \alpha ́\right] \sigma \varepsilon \lambda \mu$. B $d^{-125} 129 n^{-54^{*} 767} t x 18799$ Arm Sa $=$ Ra

$\left.333_{35}(O) \varepsilon i \varsigma\right] \varepsilon v \mathrm{~B}^{\mathrm{e}} \mathrm{M}^{\prime} \mathrm{V} O^{-58}{ }_{-82} 129 x$

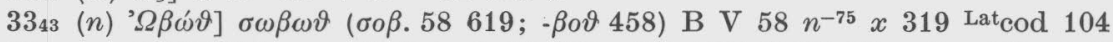

$33_{44}(n) \varepsilon^{\prime} \xi$ ' $\left.\Omega \beta \omega \vartheta\right] \varepsilon \varkappa \sigma \omega \beta \omega \vartheta(\sigma o \beta .58619 ;-\beta \circ \vartheta 458)$ B $\mathbf{M}^{\prime} 58 n^{-75} x$ Latcod $104 \mathrm{Sa}^{1}$

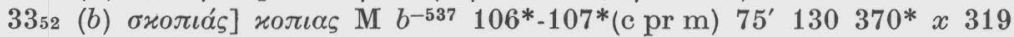

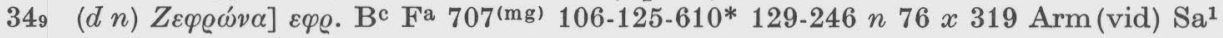

$\left.34_{13}(d f y) \tau \tilde{\omega} \dot{\eta} \mu i \sigma \varepsilon l\right] \tau o \quad \eta \mu \iota \sigma v$ (c var) 29-58-72-376 313-615 19' $44^{\prime}-125 \quad 53^{\prime}-246730 x^{-527}$ $y^{-121} 55319799$

$3426(d t) \Phi \alpha \lambda \tau \iota \dot{\eta} \lambda] \varphi \alpha \tau \iota \eta \lambda 37646-417 d 730 t x^{-509} 392416{ }^{\text {Lat }} \operatorname{cod} 104$

$356 \quad(b f)$ om $a{ }_{c} 1^{\circ} \mathrm{B} \mathrm{V} 96382 b f x 407319$ Cyr I $865=$ Ra

35 7 $(d)$ om $x \alpha i 1^{\circ} \quad 72-82 d^{-44} 458 x^{-509} 126 \quad 319$

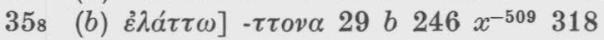

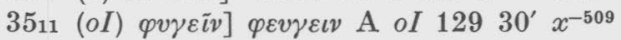

$\left.35_{21}(d n t) \vartheta \alpha v a ́ \tau \omega 1^{\circ}\right] \cap 2^{\circ} d 246 n^{-767} t x^{-527} 319$ AөthC Bo

$363 \quad(o I b z)$ om vi $\tilde{\omega} v$ oI $b$ 53-129 $75^{\prime} 76^{*} x^{-527} 18^{\prime}-126-628-669$ Arm

$364 \quad(d n t)$ om $\dot{\eta} 1^{\circ} 72-376^{\prime} d 54-75^{\prime} t x^{-509} 799$

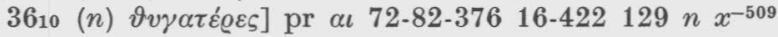

The following table indicates the approximate relationships of the $x$-group to the other textual groups. For these tables the subordinate groups of the Catena group have not been distinguished; thus $C^{\prime \prime}$ may stand for $C, c I, c I I$ or any combination of these. Column $A$ indicates the number of instances of support for an $x$-group variant by one group; column $B$, by two groups, and column $C$, by three groups. The total number is given in the last column.

$\begin{array}{lrrrr} & A & B & C & \text { Total } \\ O & 6 & 9 & 1 & 16 \\ o I & 4 & 4 & 1 & 9 \\ o I I & - & - & - & 0 \\ C^{\prime \prime} & 4 & 4 & 3 & 11 \\ b & 9 & 5 & 9 & 23 \\ d & 8 & 15 & 24 & 47 \\ f & 6 & 6 & 11 & 23 \\ n & 10 & 12 & 19 & 41 \\ s & - & 5 & - & 5 \\ t & 3 & 12 & 24 & 39 \\ y & - & 2 & 2 & 4 \\ z & 5 & 2 & 2 & 9\end{array}$


From this table it is clear that the $x$-group is closer to the Byzantine text represented by $d, n$ and $t$ than to any other tradition, the total number of instances of common support being $d 47, n 41$ and $t$ 39. Then follow $b$ and $f$ each with 23 instances, $O$ with $16, C^{\prime \prime}$ with 11 , and $o I$ and $z$ with 9 each. If $O$ and $o I$ are taken together its 25 instances would place it ahead of $b$ and $f$. For $s$ and $y$ no instance of single group support obtain and only a few instances of double group support; these are quite insignificant. It is noteworthy that $x$ and $y$ are demonstrably at opposite ends of the tradition, and should therefore be considered as quite distinct textual groups. 


\section{Chapter 2 The Byzantine text}

That the $d$ text group constitutes the Byzantine text family was already quite apparent from the study of the Lectionary texts in THGG $176 \mathrm{ff}$. Furthermore it was also clear that the $t$ group is intimately related with $d$ and could from Genesis ch. 21 onwards be regarded as a subgroup of $d$ (THGG 136f). The $n$ text for Genesis presented a somewhat complicated picture; for chh. $34-43$ the $n$ group was fully submerged in that of $d$, and outside these chh. showed closer relationship to $d$ than to other groups (cf THGG 106-111). In Deuteronomy where the $n$ group was subjected to further analysis (THGD $17 \mathrm{ff}$ ) its close relationship to the $d t$ tradition also shows that it can justifiably be called a second subordinate group in the Byzantine tradition.

For Numbers only two lections obtain in the texts edited by Høeg and Zuntz ${ }^{1}$, viz. 1116-17 24-29 and 242-35-9 17-18. Their collation demonstrates as in Genesis their witness to the Byzantine text. I present only the majority reading of the Lectionary texts in the following collation.

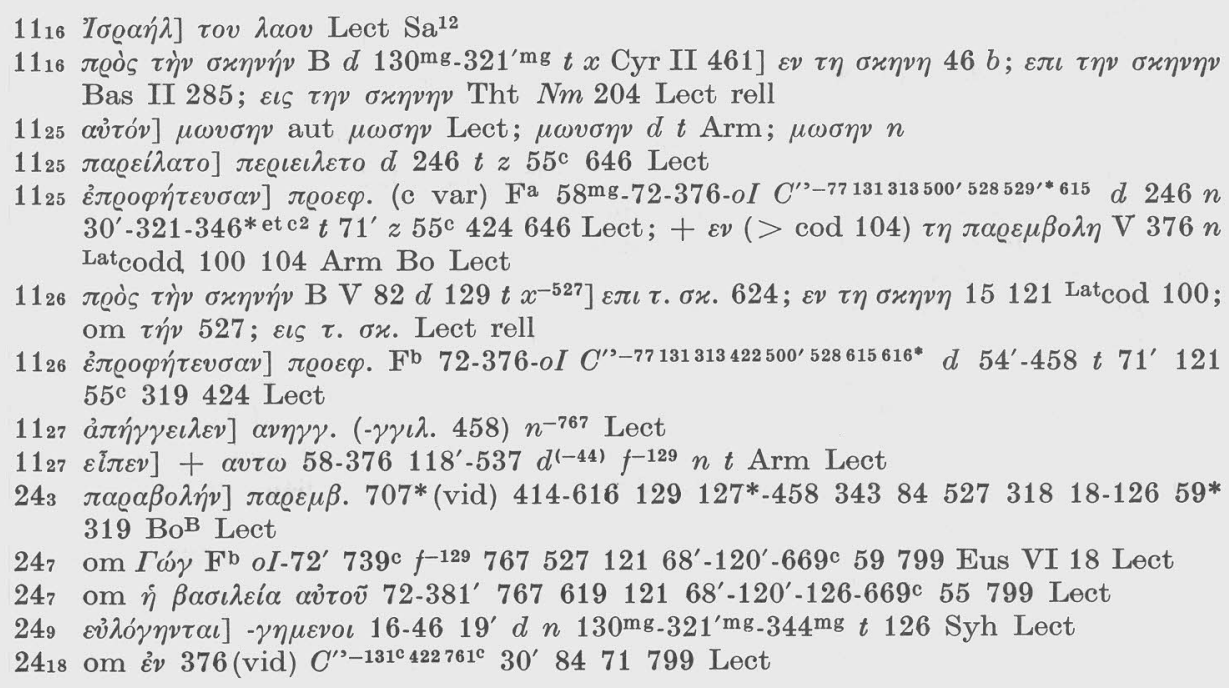

Except for $11_{16}\left(1^{\circ}\right)$ which is a unique reading, 243, 7 (twice) 18 , Lect supports the reading of $d n$ or $t$ text. When $d n t$ do not support a common variant Lect tends to support $n$.

1) Monumenta Musicae Byzantinae: Vol I Prophetologium ediderunt Carsten Høeg et Günther Zuntz. Hanniae, 1939-1970. 
It is proposed to subject this text type to somewhat closer scrutiny in this chapter, first of all, to determine whether or not it betrays recensional characteristics based on some immediate or mediate acquaintance with the Hebrew text tradition, and secondly to gain some insight into the general character of this divergent text as a whole.

A. Since the work of Origen strongly affected the subsequent text tradition throughout, the extent of hexaplaric influence on the Byzantine is first examined. In the list below are given the instances in which an asterisked variant is supported by the Byzantine group. Since these are understood to be $=\mathfrak{M}$, this fact is not recorded in the list.

\section{List 1}

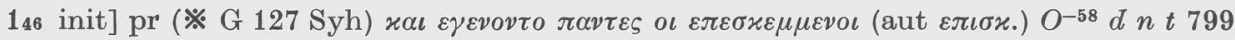
Arm Syh

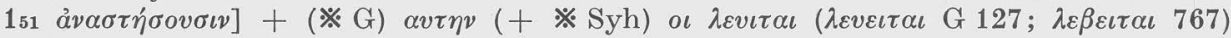
$0-70744 n t 55319799$ Arab Arm Syh; $+\alpha v \tau \eta v$ A $b$ Co

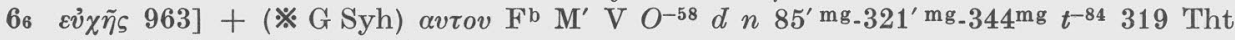
$\mathrm{Nm} 198$ Arm Bo $\mathrm{Sa}^{4} \mathrm{Syh}$

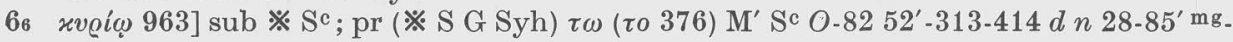
$321^{\prime} \mathrm{mg}_{-} 344^{\mathrm{mg}} t$ Tht $\mathrm{Nm} 198$ Bo Syh

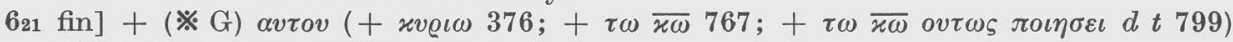
$\mathrm{V} O^{\prime} d 767 t 318799 \mathrm{Arab}^{\mathrm{S}} \mathrm{Sa}^{4}$

$\left.1110 \vartheta{ }^{\prime} \varrho \alpha \varsigma\right]+\left(※ \mathrm{Syh}^{\mathrm{L}}\right) \tau \eta \varsigma\left(>58^{*}\right) \sigma \varkappa \eta \nu \eta \varsigma \mathrm{O} d n t 527$ Arm Syh

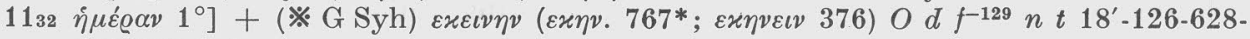
$669646 \mathrm{Syh}$

$\left.1327 \varepsilon \check{\varepsilon} \delta \varepsilon \xi_{\xi \alpha}\right]+(※ \mathrm{G} \mathrm{Syh}) \alpha v \tau o \iota \varsigma$ V O-29 $d t 121319$ Latcod 100 Aeth Bo Pal Syh

$\left.13{ }_{33} \varkappa \alpha \tau \alpha \sigma \varkappa \varepsilon \dot{\psi} \alpha \sigma \vartheta \alpha \iota\right]$ sub $※ \mathrm{Syh} ;+\left(※ \mathrm{G}\right.$ Syh) $\alpha v \tau \eta v O^{\prime-1558} n^{(-458)} 319$ Aeth Arm Pal $\mathrm{Sa}^{11} \mathrm{Syh}$

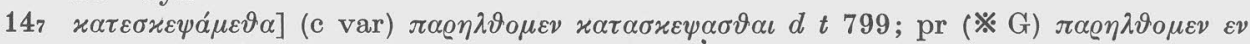
$\alpha v \tau \eta(+\ldots \mathrm{Syh})$ ע

The change of $\varkappa \alpha \tau \varepsilon \sigma \kappa \varepsilon \psi \alpha ́ \mu \varepsilon \vartheta \alpha$ to an infinitive in $d t$ seems at first blush to be based on the Hebrew, but is probably due to the influence of 1333 .

$1422 \sigma \eta \mu \varepsilon i \alpha]+(※ \mathrm{G}) \mu o v(+\measuredangle \mathrm{Syh}) \mathrm{V} O d t 799$ Arab Syh

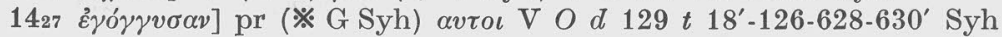

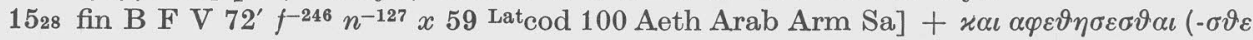
44) $\alpha v \tau \omega d t^{-84}$; $+\left(※ \mathrm{G}^{\mathrm{e}} \mathrm{Syh}^{\mathrm{L}} ; \div \mathrm{G}^{*}\right)$ ) $\alpha \iota \alpha \varphi \varepsilon \vartheta \eta \sigma \varepsilon \tau \alpha \iota$ (c var) $\alpha v \tau \omega(>82)$ rell $=\mathfrak{l}$

1627 Kó $\varepsilon$ B F M' V 72-707txt $f n x 39268^{\prime}-120^{\prime} 59319799$ Lateod 100 Aeth Arm Co] $+(\% \mathrm{G} \mathrm{Syh}) \varkappa \alpha \iota(>0125=\mathfrak{M}) \delta \alpha \vartheta \alpha v\left(\vartheta \alpha \nu 426^{*} \mathrm{c}\right.$ pr $\left.\mathrm{m}\right) \varkappa \alpha \iota \alpha \beta \iota \omega \nu$ (c var) rell

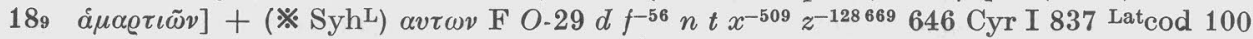
Arm $\mathrm{Sa}^{11} \mathrm{Syh}=$ edd

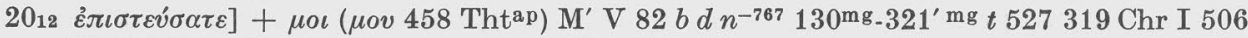

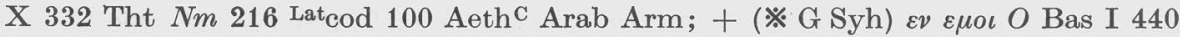
Syh

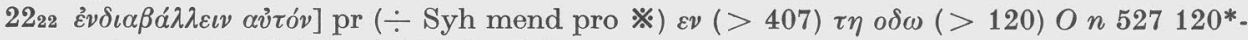
407 Or IV 409 Latcod 100 Bo Syh

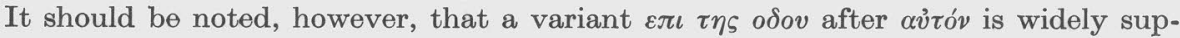
ported as well.

$\left.22{ }_{23} \dot{\varepsilon} \pi \alpha \dot{\tau} \tau \xi \xi \varepsilon v\right]+(※ \mathrm{Syh}) \beta \alpha \lambda \alpha \alpha \mu \mathrm{M}^{\prime} \mathrm{mg}$ V O $d n t 527$ Or IV 409 Lat $\operatorname{cod} 100$ Arab Syh

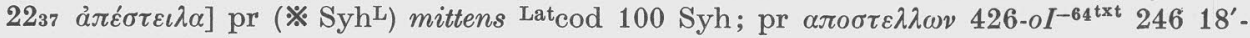
628-630'; pr $\alpha \pi 0 \sigma \tau \varepsilon i \lambda \alpha \varsigma 376$ b d $d^{-125}$ n $t 319$

$23{ }_{23} \operatorname{T\alpha \varkappa \omega }_{\beta} 2^{\circ}$ ] pr (※ SyhL) $\tau \omega \mathrm{F}^{\mathrm{b}} O^{-376} 414 d n^{(-458)} t 527$ Or III 223 Cels II 420 Tht $N m 220 \mathrm{Syh} ; \tau \omega \iota \alpha \varkappa \alpha \varkappa \omega \beta 376$ 


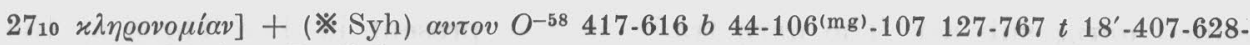
$630^{\prime} 799$ Arm Bo Syh

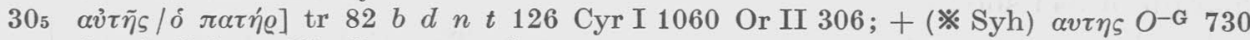

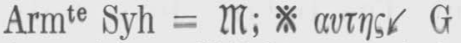

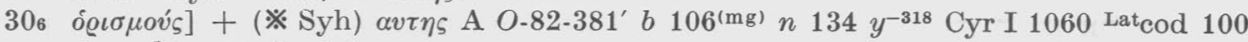
Aug Num 57 Co Syh

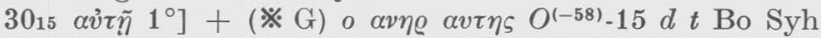

$\left.32{ }_{37}{ }^{'} E \lambda \varepsilon \alpha \lambda \eta^{\prime}\right]$ pr (※ G Syh) $\tau \eta v O^{-426} 422 b f^{-129} n 799 \mathrm{Syh}$

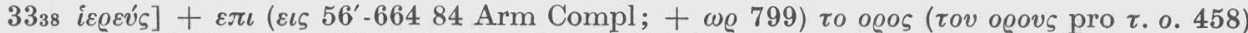

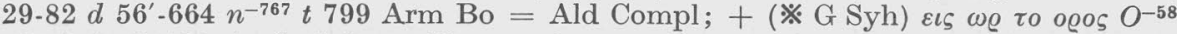

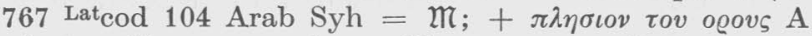

$342 \alpha v i \tau]+※ \eta \gamma \eta \prec \eta \mathrm{G} ;+(※ 85) \eta \gamma \eta(+※ 344) \eta \tau \iota \varsigma\left(>\mathrm{M}^{\prime} 58.426799 \mathrm{Syh}\right) \mathrm{M}^{\prime}$

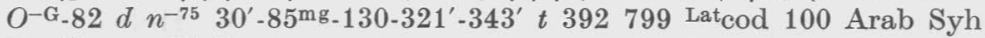

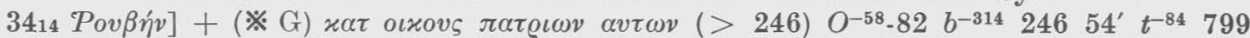
Latcodd 100104 (vid) Arab Syh

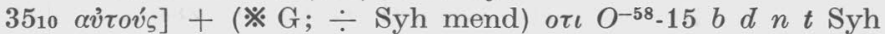

$\left.36{ }_{3} \quad(\tau \tilde{\omega} v \quad \varphi v \lambda \tilde{\omega} v) v i \tilde{\omega} v\right]$ pr (※ Syh) $\tau \omega \nu \quad 29-82-376 \quad 551 \quad 44-125^{\prime}-610^{c} \quad 54^{\prime} t^{-76^{*}} 55 \quad 319799$ Syh $=$ Ald: contra $\mathfrak{K}$, but ef $\tau \tilde{\omega} \nu]$ pr $(※ \mathrm{G}) \tau \omega \nu v \iota \omega \nu \mathrm{G}-426=\mathfrak{M}$

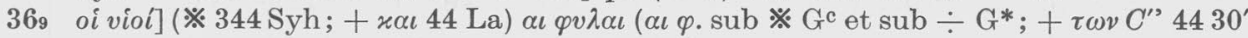
-85-344 392646 ) $v \iota \omega v$ (bis scr 82 ) $O-82 C^{\prime \prime} d n 30^{\prime}-85^{\mathrm{mg}}-344^{\mathrm{mg}} t 392646799$ Lat $^{\prime}$ codd 100104 Syh

That the Byzantine text form was somewhat influenced by the hex recension is apparent from the above list. It is, however, not a primary witness to $O$ as the chapter on The Hexaplaric Recension (pp. 43ss) clearly shows.

A few instances in the above list need special comment. At 621 the final

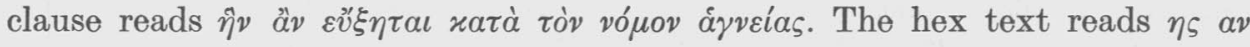

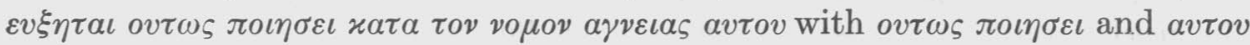
sub ast; this corresponds to the Hebrew אשר ידר כן יעשה על תורת נזרו. The

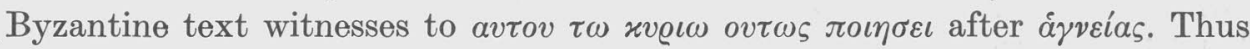

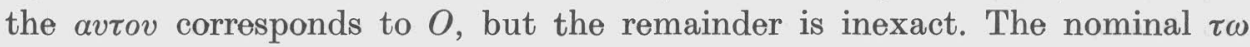

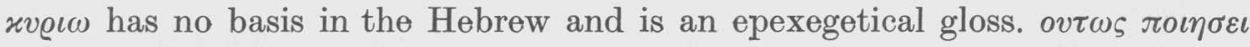
may be due to $O$ influence but it is in the wrong place.

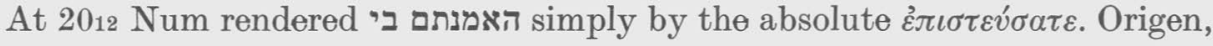
as might be expected, added $\varepsilon v \varepsilon \mu o$, an exact equivalent for 2 . The Byzantine text adds $\mu o \iota$ to the verb. This need not actually be dependent on the $O$ tradition, however, since this text tends to render explicit that which is implicit.

At 2237 Num does not render the free infinitive שלח שלח in the expression שלחתי This deficiency is filled by Origen by the addition of the present participle $\alpha \pi 0 \sigma \tau \varepsilon \lambda \lambda \omega \nu$ (ả $\pi \dot{\varepsilon} \sigma \tau \varepsilon \iota \lambda \alpha$ ), whereas the Byzantine text adds the aorist form a one a hint as to possible independent recensional activity.

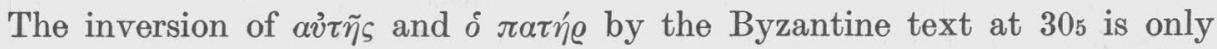
formally similar to the $O$ tradition; it is probably only a stylistic change. The

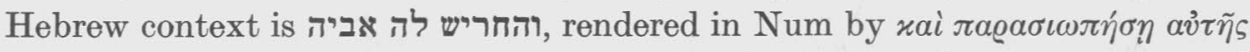

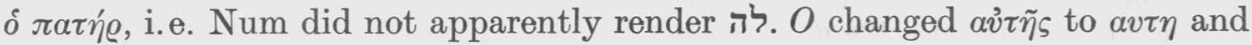


added $\alpha v \tau \eta \varsigma$ sub ast. The $\alpha v \tau \eta$ tradition was present in The Three as the margin of 344 shows.

Finally the variants at 3338 need comment. The Hebrew text reads

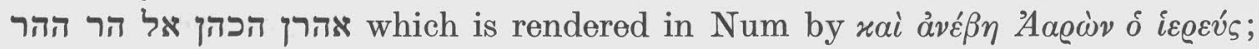
i. e. the prepositional phrase is omitted. This was "corrected" by Origen through the addition of $\varepsilon \iota \varsigma \omega \varrho \tau o$ oøos. The Byzantine text, on the other hand, has

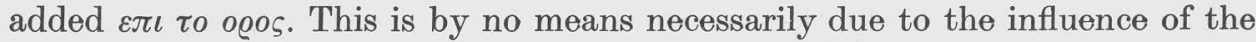
$O$ text, however, since $\varepsilon \pi \iota \tau o$ o@os may simply represent an independent amplification of the text. The use of the preposition $\varepsilon \pi \iota$ seems to me to indicate the fact that the plus is not based on any acquaintance with the Hebrew text or Hebraizing recensions/translations.

Other instances of possible hex plusses also attested by the Byzantine text tradition but without an asterisk are given in list 2. Only variants supported by at least one member of the primary $O$ witnesses (including Syh) are listed as probably hex in character. Since these additions are understood to be $=\mathfrak{n}$, this fact is not indicated.

\section{List 2}

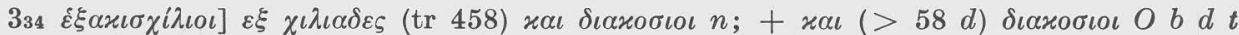
Latcod 100 Arab Arm (but cf List 4)

$\left.349 \tau \tilde{\omega} \nu 1^{\circ}\right]$ pr $\pi \alpha \varrho \alpha$ G-426 $d^{-106} f 54^{\prime} 343^{\prime} t 646799$ Syh

$435 \tilde{\varepsilon} \omega \varsigma]$ pr $\varkappa \alpha \iota 58-426 n^{-75}$

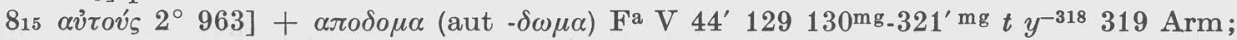
$+\delta o \mu \alpha\left(\right.$ aut $\left.\delta \omega \mu \alpha ;-\mu \alpha \tau \alpha 1^{\prime}\right)$ 58-376 b f $f^{-129} n$ Lat $\operatorname{cod} 100$ (datum)

$\left.13_{21} \pi i \omega v\right]+\varepsilon \sigma \tau \iota(v) 426 d 246 n t 319$ Arm(vid) Bo Pal

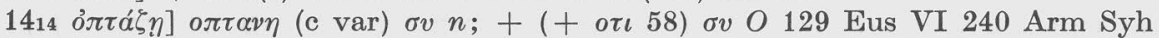

$1532 \tau \tilde{\eta} 2^{\circ}$ ] pr $\varepsilon v$ A $376 n^{-127} x^{-509} 318319$ Latcod 100 Syh

$\left.21_{33} \alpha v i \tau o \tilde{\zeta}\right]+\alpha v \tau \circ \varsigma \mathrm{V} O^{-58} d n t 527 \mathrm{Sa}^{12} \mathrm{Syh}$

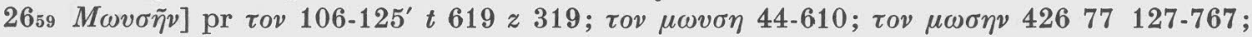
lmwšs Syh

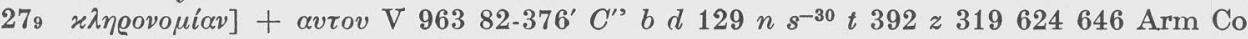
Syh

The addition of the pronoun is also attested in 963 and was therefore already in the tradition before Origen's time. If his LXX parent text did not have it, he reintroduced it as is clear from the support by $376^{\prime} \mathrm{Syh}$.

$27_{19} \sigma v v \alpha \gamma \omega \gamma \tilde{\eta} \varsigma$ 963] pr $\tau \eta \varsigma 426 d 53^{\prime}-56^{\mathrm{c}}-129 n t^{-76} 619 y^{-392} 68^{\prime} 319$ Tht $N m 224$

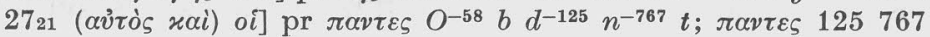

This is certainly hex since Syh attests to $\pi \alpha v \tau \varepsilon \varsigma$ sub ast before avizós.

$\left.2722 \sigma v v \alpha \gamma \omega \gamma \tilde{\eta}_{\varsigma}\right]$ pr $\tau \eta \varsigma^{2} \quad 381^{\prime}-426422 \quad 125 \quad 53^{\prime}$ n $28-85^{(\mathrm{mg})} 61955319$

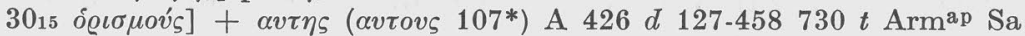

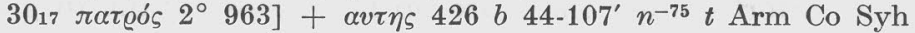

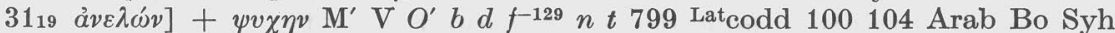

$\left.31_{27} \sigma v v \alpha \gamma \omega \gamma \tilde{\eta} \varsigma\right]$ pr $\tau \eta \varsigma$ ( $\tau \eta$ 75) A $O^{-426} \cdot 381^{\prime} 414106^{(\mathrm{mg})} 129 n t^{(-370)} 527$ Cyr I 333 bis

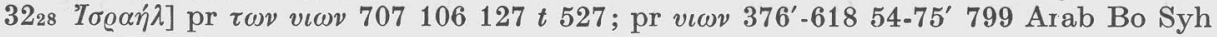
$=\mathrm{Compl}$

$\left.32{ }_{33} A \mu \rho \varrho \propto \alpha i ́ \omega \nu\right]$ pr $\tau \omega \nu$ A $58-37673^{\prime} b d f^{-129} n^{-458} t 55799$

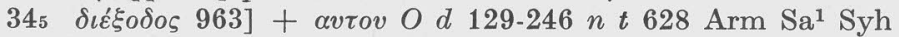

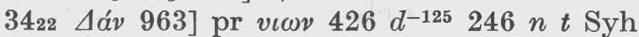


Also considered hex are changes in word order to fit that of $\mathfrak{k}$. The following list is limited to those supported by at least one of the primary witnesses to the $O$ text.

\section{List 3}

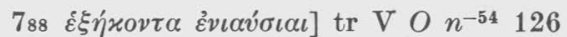

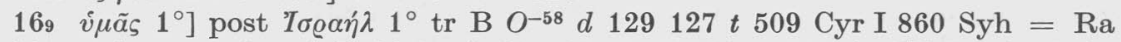

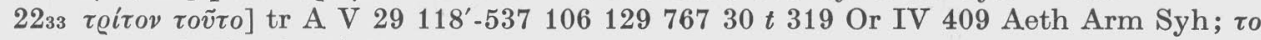

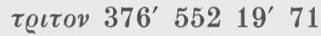

$\left.2240 \pi \varrho o^{\prime} \beta \alpha \tau \alpha\right]$ et $\mu o ́ \sigma \chi o v \varsigma$ tr $376^{\prime} n 527$ Arm Syh

$\left.233_{3} \mu \circ \iota \varepsilon i \xi \eta\right] \delta \varepsilon \iota \xi \eta \mu \circ \iota$ o $\vartheta \varepsilon \circ \varepsilon d ; \operatorname{tr} 42659$ Arm Syh $=\mathfrak{m}$

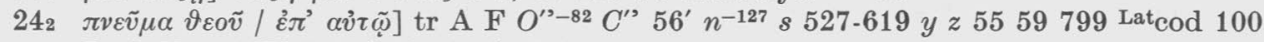
Ruf Num XVII 2 Aeth Arab Syh

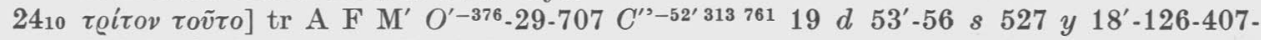
628-630' 59799 Arm Bo Syh

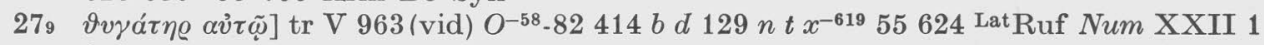
Syh

That 963 had the transposed order is practically certain. The line in question reads $[\eta \alpha v \tau \omega \vartheta v \gamma \alpha \tau] \eta \varrho \delta \omega \sigma \varepsilon$, and 10 letters is precisely what is lacking. Thus the variant order was already in the tradition by the time of Origen.

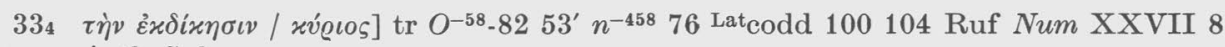
Aeth Syh

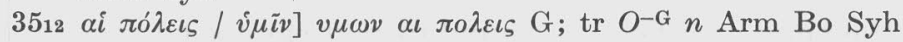

Post-hexaplaric activity resulting in the omission of materials which Origen had placed under the obelus is examined in the chapter on the Hexaplaric Recension. This is also represented in list 4 . That the omission is $=\mathfrak{W}$ is not noted.

\section{List 4}

$12 \alpha v \dot{\omega} \tilde{v} 3^{\circ}$ ] sub $\div \mathrm{G}$; > B $19 d 127 t \times 18319$ Cyr V1 453 X 624 Latcod 100 Arm

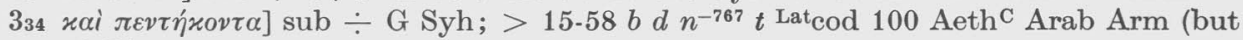
ef List 2)

63 àjò oľvov $2^{\circ}$ ] sub $\div \mathrm{G} \mathrm{Syh;} \mathrm{>} \mathrm{58-72-381'} d f n^{(-458)} t 61959319 \mathrm{Cyr}$ I 1041 Eus

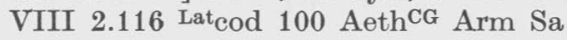

66 $\pi a ́ \sigma \eta]$ sub $\div \mathrm{G} \mathrm{Syh;}>58 n^{-767}$ Arm

$104 \pi a ́ v \tau \varepsilon \zeta] \mathrm{pr} \div \mathrm{Syh} ;>58 n^{-767} 527319$ Arm

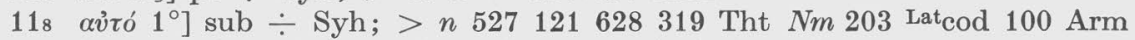

$\left.11_{27} \lambda \varepsilon \dot{\gamma} \gamma \omega \nu\right]$ sub $\div$ Syh; $>b d^{(-44)} n t 126$ Aeth Arm Sa

2016 xं́oı๐] sub $\div \mathrm{G}$; $>58552 d 53^{\prime} 126$ Arab

318 ov́v-fin] sub $\div \mathrm{G}$; > 58-426 $d^{-106} 527$ Arab

$333_{2}$ av̉นá] sub $\div$ G Syh; $>72-381^{\prime} d 66455799$ Latcod 104 Spec 44 Aeth Arm

It is of course possible that the obelus was lost in the tradition. In the following list are given omissions in the Byzantine text group which are equal to $\mathfrak{T}$ but for which no obelus tradition is extant.

\section{List 5}

58 om $\delta$ A oI $n 13068^{\prime} 55$ Tht $N m 195$

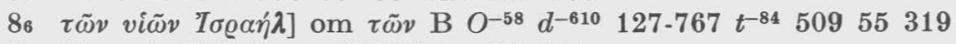

814 idem A B $O^{-58} d 127-767 t x^{-619} 121$ 


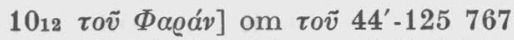

$10{ }_{17}$ oi vioi $\left.\Gamma \varepsilon \delta \sigma \omega ́ v\right]$ om oi $O^{-58}-707 C^{-16}-417 d^{-44} 12975321 * 509 * 392 * 319$

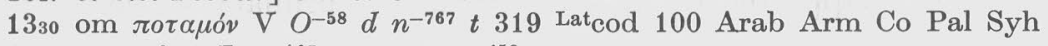

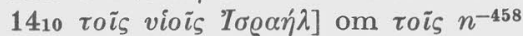

1536 om $\pi \tilde{\alpha} \sigma \alpha$ j $\sigma v v \alpha \gamma \omega \gamma \eta^{\circ} 2^{\circ} O^{(-58)}-82-381^{\prime} d^{-106} f^{-129}$ 509-619 Arab Syh

1536 om $\varepsilon^{\prime} \xi \omega \tau \tilde{\eta} \varsigma \tau \alpha \varrho \varepsilon \mu \beta 0 \lambda \eta \tilde{\zeta} 2^{\circ} \mathrm{M}^{\prime} O^{(-58)}-82-381^{\prime} d^{(-106)} x^{-527}$ Arab Syh

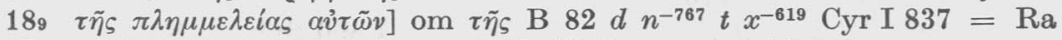

$\left.18{ }_{23} \tau \dot{\alpha} \alpha \dot{\alpha} \mu \varrho \tau \dot{\eta} \mu \alpha \tau \alpha \alpha \dot{v} \tau \tilde{\omega} v\right]$ om $\tau \alpha \dot{~ 15-618 *(c ~ p r ~ m) ~} d^{-106} 509$

1830 om ảsó $1^{\circ} n 319$ Bo

1830 om ả $\pi o^{\circ} 2^{\circ}$ n 319 Lat $\mathrm{Lod} 100$ Bo

1912 om «ai $2^{\circ} 15^{*}-82-376550^{\prime} 118^{\prime}-53753^{\prime}-129 n^{-127} 30619126416799$ Eus VI 12 Bo

217 om ö $\tau \iota 1^{\circ} \mathrm{V} \mathrm{O} \mathrm{O}^{-58} 44 n^{-127} 30619 z 646$ Latcod 100 Aeth Arm Syh

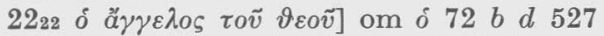

$248 \tau \alpha \tilde{\imath} \varsigma \beta$

2658 om xai $1^{\circ} 58-72-82 n 76392126$ Bo

3011 om ह่v $528 d^{-106} 53^{\prime} 509128$

$31_{22}$ om $x \alpha i 2^{\circ} 3^{\circ} 4^{\circ} d 71^{\prime}$

336 om $\tau \iota O^{-58}-29-82739^{*} d^{-106} f^{-129} 54-75^{\prime} 84527 \quad 18^{\prime}-126-630^{\prime}$ LatPsAmbr Mans 3 Co Syh

3531 om $\pi$ a@á $d^{-106} 84$

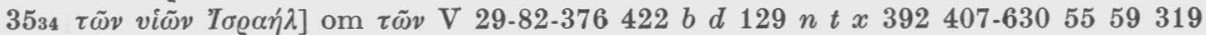

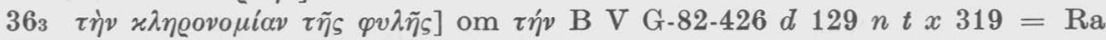

$3612 \tau o \tilde{v} \pi \alpha \tau \varrho o ̀ \varsigma ~ \alpha v ่ \tau \tilde{\omega} \nu]$ om $\tau$ ov $82 \quad b \quad n^{-767} 126319$

The above list includes the omission of articles which only formally correspond to $\mathrm{T}$; since the noun modified is a bound form it cannot be articulated in Hebrew, and these instances are to be discounted in any evaluation of possible hex influence on the text tradition (these are $86141017 \quad 1410189232222248$ $353436312)$.

A proper evaluation of the extent of possible hex influence on the Byzantine text must involve a comparison of the above lists with the corresponding lists in ch. 3. Such a comparison shows that hex influence is comparatively minimal.

It remains to discover whether or not the Byzantine text shows direct or indirect Hebrew influence which is nonhexaplaric in origin. In the list below are given all instances of formal correspondences to the text of $\mathfrak{k}$ which seem not to stem from Origen; the reading of $\mathfrak{k}$ is given in each instance.

\section{List 6}

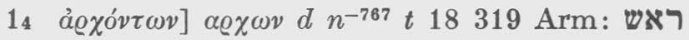

The variant singular need not be due to Hebrew influence since it could be created by syncopation as well.

$14 \pi \alpha \tau \varrho \tilde{\omega} v]+\alpha v \tau \omega \nu$ 16-46 106-107't 392319 Co: ef

It is unlikely that the variant is due to the influence of $\mathfrak{m}$, but rather to

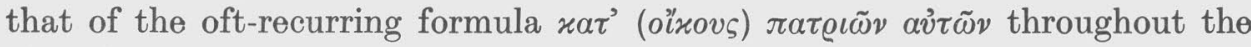
ch. (cf. ө.g. vv. 202224 )

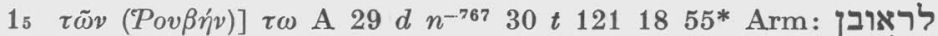

16 $\tau \tilde{\omega} v(\Sigma v \mu \varepsilon \dot{v} v)] \tau \omega$ A 528-551 $d n^{-767} t 12118$ Arm: לשמעון
} 


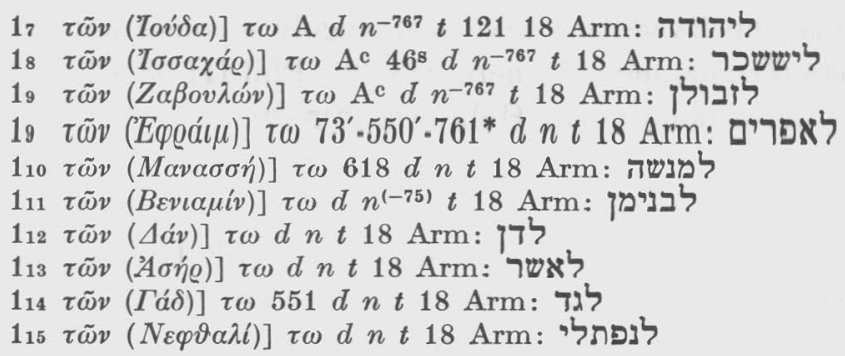

In each of the above instances (vv. 5-15) the dative article of the variant text correctly renders the Hebrew preposition whereas the genitive plural of Num constitutes a free interpretation.

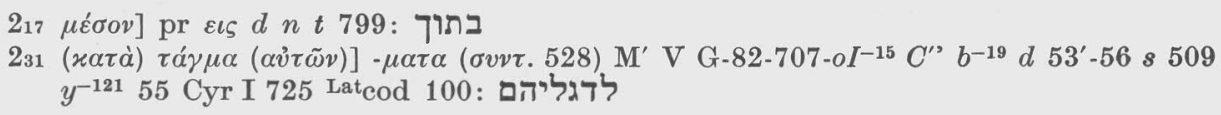

The origin of the variant is uncertain as its mixed support indicates.

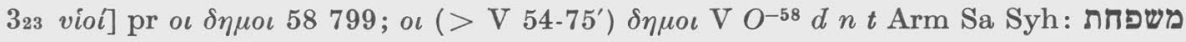

This may well represent a prehexaplaric revision already present in Origin's parent text.

427 (

The command is to Moses alone (cf. v. 21), and Num is consistently singular from vv. $21-28$, but then becomes plural in v. 29 (singular in $\mathfrak{K}$, and v. 32 (as $\mathfrak{M}))$. The number of the verbs is too mixed in the tradition to posit Hebrew influence in v. 27.

448 ó

The tradition of compound numbers is complicated by the fact that numbers are often represented in the mss by short forms. It would be dangerous to posit Hebrew influence in the presence (or absence) of a conjunction.

787 ai $\vartheta v \sigma i \alpha \iota \alpha v i \tilde{\omega} v]$ pr $\varkappa \alpha \iota \mathrm{B}^{*} 707 d f^{-129} n t 319$ Arm Sa: ומנחתם

The correspondence of the variant text and $\mathfrak{M}$ is by no means evidence of Hebrew influence. The $x \alpha \iota$ of the variant text comes between $\delta \omega \dot{\delta \varepsilon \kappa \alpha}$ and $\alpha i$ and may be palaeographically conditioned. For the secondary nature of xal ef p. 100.

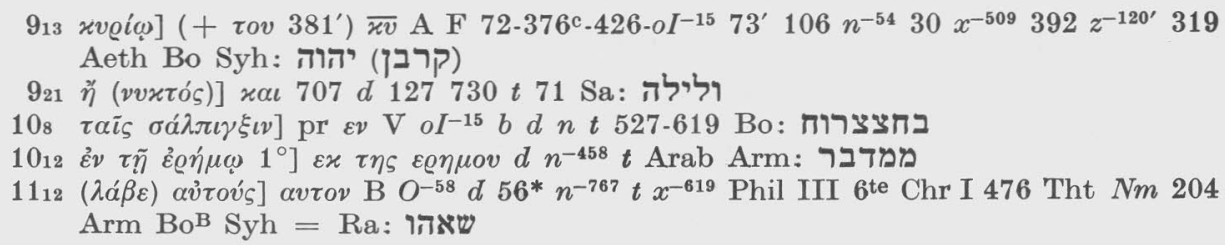


It should be noted that the Byzantine text is strangely inconsistent, since

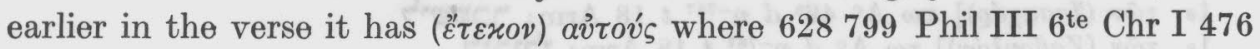
Tht Nm 204 have avtov corresponding to Hebrew's ילדתיהו.

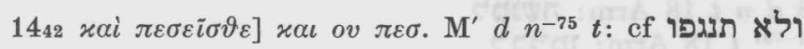

The introduction of the negative particle corresponds to $\mathfrak{m}$. It could have been introduced from the context however without Hebrew influence, since

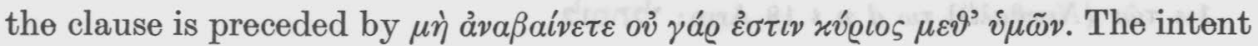
of the Hebrew is "lest you be smitten" which is hardly met by the variant text and it is more likely that the simple negative was thoughtlessly introduced under the influence of the context.

$1815 \lambda v \tau \varrho \omega \vartheta \eta \dot{\sigma} \sigma \varepsilon \tau \alpha l] \lambda v \tau \varrho \omega \sigma \eta d^{-125} n^{-767} t 319$ Arm; redimis Latcod $100:$ תפדה

The variant text is not necessarily due to Hebrew influence, since $\lambda v \tau \varrho \omega ́ \sigma \eta$ occurs in the next clause.

209 $\sigma v v \varepsilon \dot{\tau} \alpha \xi \varepsilon v]+\alpha v \tau \omega$ V $72 b d n t 527319$ Aeth $^{\text {F Arm }}$

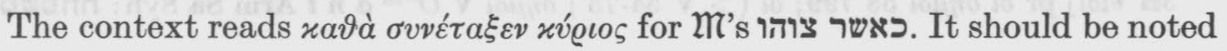
that V 319 also omit xíøıs. The introduction of the pronoun may well be ex par, since the formula is a common one.

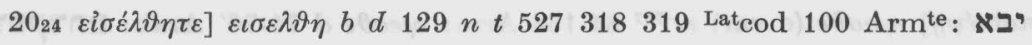

The context reads "let Aaron be gathered to his people, for not will ye (he) enter the land." The LXX "corrected" the Hebrew text since both Moses and

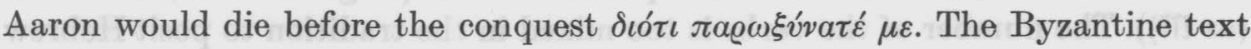
simplifies the text and its correspondence with $\mathfrak{k}$ may be a coincidence.

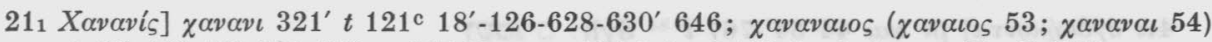
A 72-426 53'-56*-129 $n 527$ Procop $856^{\text {Lat }}$ cod 100 Arab Arm ${ }^{\text {ap }} \mathrm{Bo} \mathrm{Sa}^{1012} ; \chi \alpha v \alpha \alpha v$ הכנעני : Aeth Syh

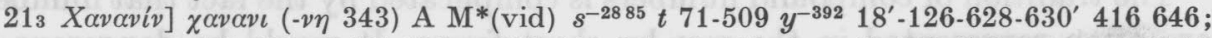
$\chi \alpha \nu \alpha v \alpha i o v$ 72-426-oI 53'-129 n 527-619 Lateod 100 Arm Sa ${ }^{10} 12$ Syh; $\chi \alpha \nu \alpha \alpha v d^{(-44)}$ Aeth: הכנעני

$\left.215 \varepsilon^{\xi} \xi \dot{\eta} \gamma \alpha \gamma \varepsilon \varsigma(\hat{\eta} \mu \tilde{\alpha} \varsigma)\right]-\gamma \alpha \gamma \varepsilon \tau \varepsilon$ (c var; $\varepsilon \xi \alpha \gamma$. 509) A $\mathrm{M}^{\prime} \mathrm{V} 82 d 129 n 321 *$ (vid) $t x^{-619} 121$ $55 \mathrm{Sa}^{412}:$ העליתנו

$\mathfrak{T}$ is vocalized as a plural verb. The context requires a plural verb : xai $x a \tau \varepsilon$ -

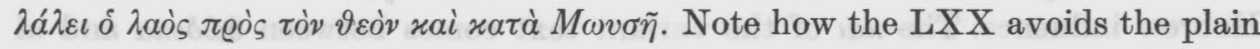
speech of the Hebrew by using two different prepositions in the context as well as using the singular $\dot{\varepsilon}^{\xi} \xi \gamma \gamma \alpha \gamma \varepsilon \xi$. The rebellious challenge is thereby mitigated and only Moses is said to have brought the people from Egypt in order to die in the desert. The variant text is probably simply due to the plural context rather than to Hebrew influence.

$2222 \tau o \tilde{v} \vartheta \varepsilon \circ \tilde{v}] \overline{x v} 376314 d t 527$ Aeth Bo:

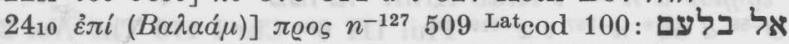

הנני : 2414 idov́ $]+\varepsilon \gamma \omega 64-381 d f^{-56^{*}} 127 t$ t3 


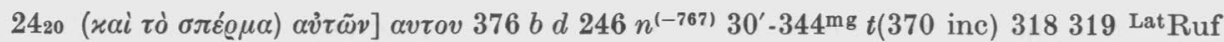
Num XIX 1ap: ואחריתו

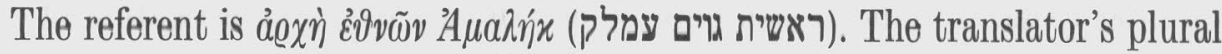

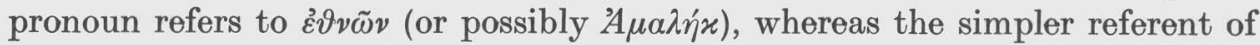
the variant must be $A \mu \alpha \lambda \eta \dot{x}$.

$254 \quad \tau \tilde{\varphi} M \omega v \sigma \tilde{\eta}] \pi \varrho \circ \varsigma \mu \omega v \sigma \eta v\left(-\sigma \eta \mathrm{M}^{\prime} ; \mu \omega \sigma \varepsilon \iota 72\right) \mathrm{A} \mathrm{M}^{\prime}$ 58-72-oI d s t 619 y 55 319: $2619 x \alpha i(v i o i)]>n 126319$ Lat $\operatorname{lod} 100$ :

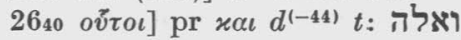

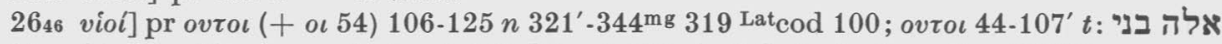

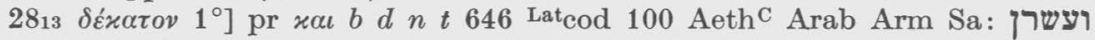

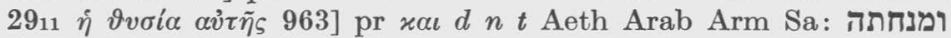

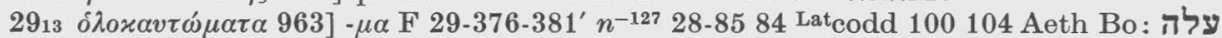

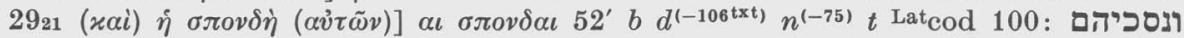

The variant happens to correspond to $\mathfrak{M}$ but this is meaningless. $\dot{\eta} \vartheta v \sigma i \alpha$ and $\dot{\eta} \sigma \pi \circ \delta \delta \dot{\eta}$ in $\mathrm{v} .18$ and $\hat{\eta} \vartheta v \sigma i \alpha$ in v. 21 are also in the plural in the Byzantine text.

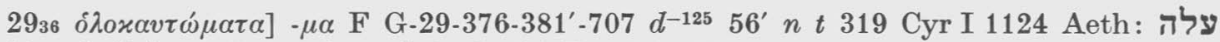

$304 \eta \ddot{\eta}(\delta \varrho i \sigma \eta \tau \alpha \iota)] x \alpha \iota d n t$ Lat $\operatorname{cod} 100 \mathrm{Bo}$ : ואסרה

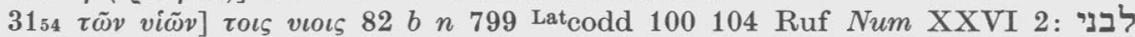

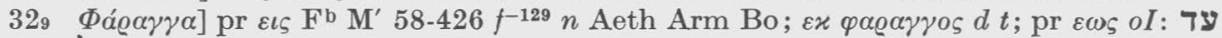
נחול

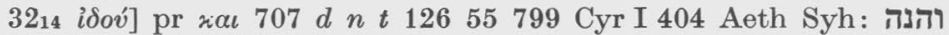

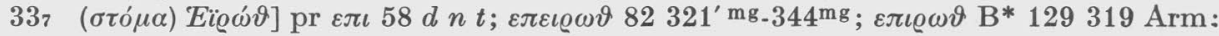
פי החירת

At first blush the introduction of $\varepsilon \pi \iota$ might seem related to פי hardly be correct since the translator rendered it by $\sigma \tau{ }^{\prime} \mu \alpha$. The reading of $\mathrm{B}$ shows that it was palaeographically conditioned; the initial $\varepsilon \iota$ was carelessly copied as $\varepsilon \pi \iota$ yielding $\varepsilon \pi \iota \omega \vartheta$, or as seems more likely $\varepsilon \pi \varepsilon \iota \varrho \omega \vartheta$. This in turn produced either $\varepsilon \pi \iota \omega \vartheta$ or $\varepsilon \pi \varepsilon \iota \omega \omega \vartheta$ (or $\varepsilon \pi \iota \varepsilon \iota \varrho \omega \vartheta)$. Since $\sigma \tau o ́ \mu \alpha$ is preceded by $\dot{\varepsilon} \pi i$ as well, the process of corruption was made easier.

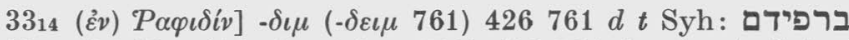

3315 (

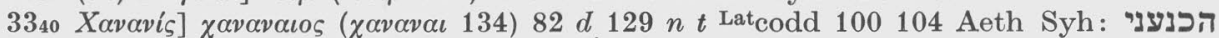

$\left.34{ }_{14} \tilde{\varepsilon} \lambda \alpha \beta \varepsilon v\right] \varepsilon \lambda \alpha \beta o v$ d $t 799$ Aeth Bo: לקחו

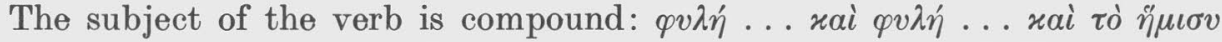
$\varphi v \lambda \tilde{\eta} \varsigma$ in which case the translator normally uses a singular verb. The Hebrew usually does so as well. The plural verb is equally justifiable from a Greek point of view and the change need not be due to the Hebrew text.

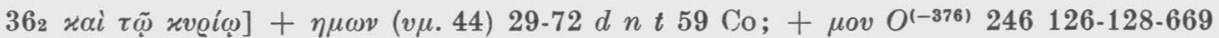
ואדני :

The addition of $\eta \mu \omega v$ is hardly due to the Hebrew text but to the occurrence of $\tau \tilde{\omega}$ zvoi $\omega \dot{\eta} \mu \tilde{\omega} v$ earlier in the verse.

$\left.364 \pi \alpha \tau \varrho \iota \tilde{\alpha}_{\zeta}(\dot{\eta} \mu \tilde{\omega} \nu)\right] \pi \alpha \tau \varrho \iota \omega \nu \mathbf{M}^{\mathrm{txt}} d^{(-106)} n^{-458} t$ 646: 
B. Whether or not the Byzantine text was recensionally conditioned by the Hebrew text either immediately or mediately as the above instances might be interpreted to indicate can only be determined by an investigation of the general character of the text type. Thus if the text has many omissions an occasional omission which corresponds to $\mathfrak{W}$ is probably mere coincidence. Or if a verbal inflection is commonly changed to fit the context an occasional correspondence to $\mathfrak{W}$ has little meaning.

In list 6 above omissions involve xai or the article, as well as one of a pronoun. The addition of xa occurs a number of times as does the change of conjunc-

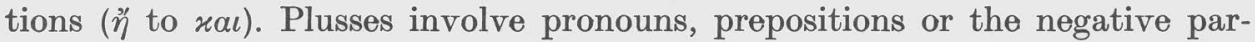
ticle. Changes may involve number, case, preposition, lexical stem or the spelling of proper names. Each of these will be investigated as to whether it is a common tendency in the Byzantine tradition. All instances are contra $\mathfrak{K}$.

1. Variants involving the addition or omission of $x a \iota$ are common in this text type. As in list 6 the addition of $\varkappa$ o occurs more often than its omission (39 times over against 24). Since these are all contra $\mathfrak{m}$ there is little point to detailing all the evidence. The interested reader is referred to the apparatus

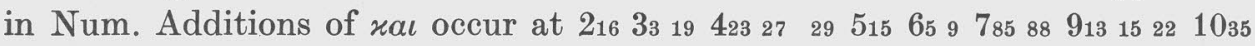

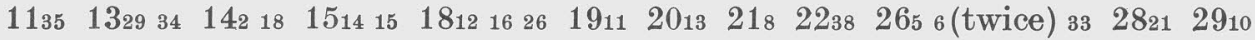

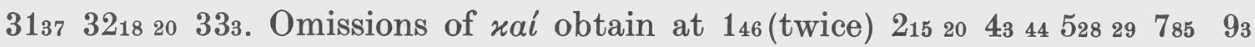

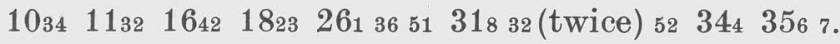

It is interesting to observe that $\varkappa a \iota$ is substituted for $\eta$ in only one instance

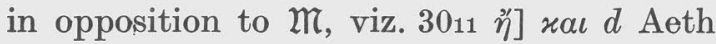

2. The Byzantine group is characterized by much more nominal articulation than is Num. Nouns are articulated in 78 instances where Num has the noun unarticulated. Over against this the article is omitted only 22 times. Furthermore the article (used as relative pronoun) is added before $\varepsilon \dot{v} \tau o \tilde{l}_{\zeta} \varkappa \tau \dot{\eta} v \varepsilon \sigma \iota v$ in

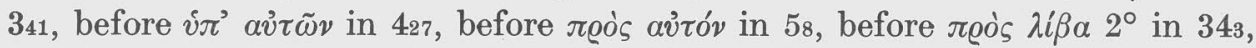

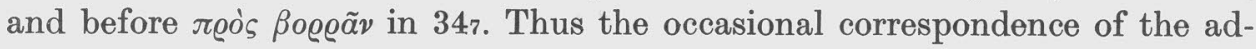
dition or omission of an article to the Hebrew text is probably mere coincidence.

3. Addition or omission of pronouns.

In the following list all instances are contra $\mathfrak{m}$.

List 7

12 om $\alpha \dot{\tau} \tau \tilde{\omega} \nu 1^{\circ} \mathrm{B} 414^{\prime} d n^{-767} t x 18$ Bas II 145 Cyr VI $453 \times 624{ }^{\text {Lat }}$ Cod 100 PsBas Is I 5 Arm

12 om $\alpha \dot{\tau} \tau \tilde{\omega} v 2^{\circ} \mathrm{B} \mathrm{V} d n^{-767} t x 18319$ Bas II 145 Cyr VI 453 X 624 Latcod $100 \mathrm{Hi}$ Eph II 3 PsBas Is I 5 Arm

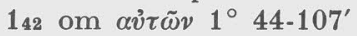

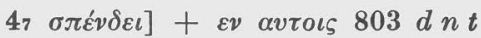

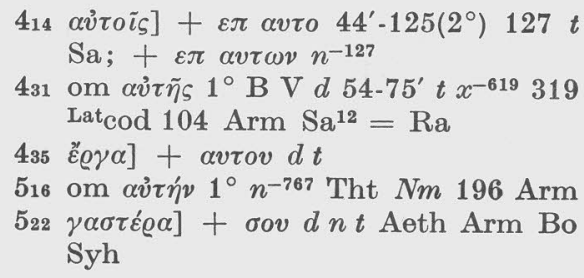
Syh 
787 om $\alpha v i \tilde{\omega} v 1^{\circ} d$ Bo

$788 \alpha v ่$ óv 963] $+\alpha v \tau \alpha d n t 799$

$9_{10}$ om ös $d t 126319$

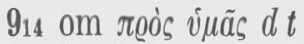

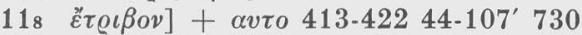
$t 509392799$ Aeth Bo SyhL; + $\alpha v \tau o v 125$

$1111 \varepsilon \dot{\pi} \tau \vartheta \varepsilon \tilde{\imath} \nu \alpha \iota]+\mu o \iota\left(\mu \varepsilon 107\right.$ Tht $\left.^{\mathrm{ap}}\right) d 767$ $t 527319$ Tht $N m 204$ Arm

$\left.11_{17} \lambda \alpha o v\right]+\tau o v \tau o v 58 d n^{-75} t$ Bas II 285 Tht Nm 204 Aeth Arab Arm Co

$11_{27} \varepsilon[\pi \varepsilon v]+\alpha v \tau \omega 58-376118^{\prime}-537 d^{(-44)}$ $f^{-129} n t$ Arm

$1415 \lambda \alpha o ́ v]+\sigma o v 376 d^{-125} 54^{\prime} t$ Arm

1427 om å $\mathrm{B}^{*} \mathrm{~V} d^{-44} 75 t 318319$ Lat $^{-40}$ $100 \mathrm{Sa}$

$1437 \not ̋ \nu \vartheta \varrho \omega \pi \circ \iota]+\varepsilon x \varepsilon \iota v \circ \iota b d^{(-125)} n t 799$ Aeth-CG Arm Syh

1539 om $v \mu \tilde{\omega} v 2^{\circ} \mathrm{B} 15-82 c I-551 d 129 n^{-767}$ $t^{-84} x^{-509} z 319$ Tht $N m 211^{\text {Lat }}$ cod 100

1611 om $\sigma o v 15 d 120 *(\mathrm{c}$ pr m)

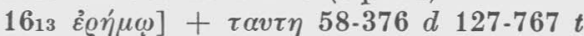
799 Lat cod $100 \mathrm{Co}$

$\left.16{ }_{13} \not ้ \varrho \chi \omega v\right] \mathrm{pr} \sigma v b d 127 t$ Arm Bo

$1615 \varepsilon i \lambda \eta \varphi \alpha]+\tau \iota d t$

1634 om $\alpha v \tilde{\omega} v 1^{\circ} d$

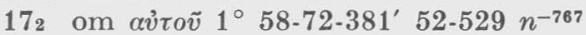
527799 Lat $^{c o d} 100$ Arm Bo Sa ${ }^{1}$

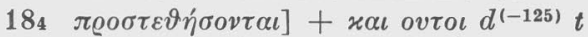

189 $\propto \alpha \varrho \pi \omega \mu a ́ \tau \omega \nu]+\alpha v \tau \omega \nu d t \mathrm{Arm}^{a p}$

$\left.18_{16} \sigma v v \tau i \mu \eta \sigma \iota \varsigma\right]+\alpha v \tau o v 29-72-376131^{\mathrm{c}}$ 54-75' 59 Arab Arm Sa ${ }^{12}$ Syh

$\left.18_{19} \delta \varepsilon \delta \omega \varkappa \alpha\right]+\alpha v \tau \alpha d 54-75^{\prime} t{ }^{\text {Lat }} \operatorname{cod} 100$ Co

192 om $\eta \tilde{V} d 53^{\prime}-12954-75^{\prime} t 7131859^{\mathrm{c}}$ 319 Arm Bo

195 om $\alpha \dot{\tau} \tilde{\eta} \varsigma 2^{\circ} d^{-106} 458319$ LatPsAug Serm Cai II 38.2

$19_{14}$ oixía $\left.2^{\circ}\right]+\varepsilon x \varepsilon \iota v \eta \mathrm{V} d t 799$

$19_{15}$ om ह่ंत' $\alpha \dot{\tau} \tau \tilde{\omega} b d t 126799$ Phil I 281 II 261 Eus VI 12

$\left.19_{21} \varepsilon ้ \sigma \tau \alpha \iota 1^{\circ}\right]+\tau o v \tau o d^{(-125)} n^{-75} t 121$ 799 Latcod 100

$19_{22}$ om $\alpha v ่ \tau o \tilde{v} 82 d 54^{\prime} t$ Lat $c o d 100$ $\left.19_{22} \psi v \chi \eta^{\prime}\right]+\varepsilon \varkappa \varepsilon \iota v \eta d 54^{\prime}-767 t 799$

$2017 \alpha \dot{\alpha} \gamma \varrho \tilde{\omega} v]+\sigma o v d 53^{\prime}-129127-767 t 527$ 319 Arm

$228 v \dot{x} \tau \alpha]+\tau \alpha u \tau \eta v b d n t 527$ Latcod 100 Aeth Arm Co Syh

$2_{25}$ om $\varepsilon \alpha v \tau \eta \dot{v} n^{-127} 527$

2232 om $\alpha \dot{\tau} \tilde{\omega} 246 n^{-127} 126^{\text {txt }}$

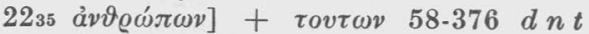
Aeth Arm Co

$2238 \beta a ́ \lambda \eta]+\mu o \iota n^{(-767)} 28-85^{\prime m g}-321^{\prime m g}$. $344^{\mathrm{mg}} 527319$ Latcod $100^{2}$

$23{ }_{3}$ om oov $d 53 t$

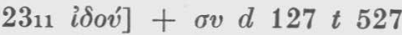

$2326 \vartheta \varepsilon o ́ \varsigma]+\pi \varrho \circ \mu \varepsilon 106 t$

2410 om $\mu o v 414 d t$ Latcod 100

264 $x \alpha i 2^{\circ}$ ] pr $\sigma v$ B V $O n$ 71.509 407 Latcod 100 Arm Syh

2637 om $\alpha \dot{\tau} \tilde{\omega} 552 n^{-127}$ Lat $\operatorname{cod} 100$ Arm

2655 ỏvó $\mu \alpha \sigma \iota v]+\alpha v \tau \omega v n^{-127} \mathrm{Arm}^{a p}$

271 om $\alpha v \tilde{\omega} v d^{-106}$

$\left.2711 \omega^{\sigma} \sigma v\right]+\alpha v \tau \omega 16-4644-106^{(\mathrm{mg})}-107$ $54^{\prime}-767 t 318799$ Bo

2924 om $\alpha v i \tilde{\omega} \nu 3^{\circ} d^{-106}$

$29_{27}$ om $\alpha v ่ \tau \tilde{\omega} v 1^{\circ} d^{-106} x^{-509}$

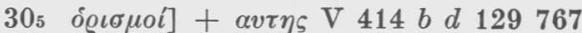
$t 40755319$ Cyr I $1060 \mathrm{Sa}$

309 ảví@ $\left.2^{\circ}\right]+\alpha v \tau \eta \varsigma 29 b 106^{(\mathrm{mg})} 129$. 246 n $t x^{-509} 39255$ Co Syh

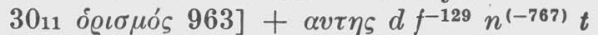
$x^{-509} 628$

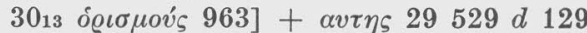
54-458 $t 509318 z^{-407} 319646$ Arm Co

31 fin] $+\varepsilon v \alpha v \tau \eta \mathrm{V} d f^{-56^{*}} t$

3110 om $\alpha v i \tilde{\omega} v 1^{\circ} 72 d$

$32_{26}$ om $\dot{\eta} \mu \tilde{\omega} \nu 1^{\circ} d^{-106}$ Lat $\operatorname{cod} 104$

3226 om $\dot{\eta} \mu \tilde{\omega} v 2^{\circ} d^{-106}$

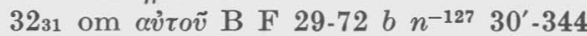

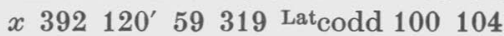

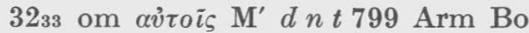

3352 om $\alpha \dot{v} \tau \tilde{\omega} v 3^{\circ} d^{-106}$

$3519 \alpha i \mu \alpha]+\alpha v \tau o v d t$

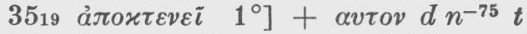

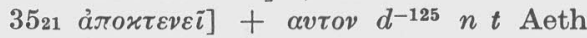
Arm

In the above list of 69 instances, 39 constitute additions of pronouns or pronominal phrases, and 30 , of omissions. There seems to be a greater tendency towards amplification than towards the reverse in this text group. In list 6 there were four instances of pronouns added and only one omission; whether these were due to Hebrew influence rather than part of the general character of this text type must remain uncertain. In general the Byzantine text seems to betray a fair amount of freedom with respect to the pronominal tradition.

4. Addition, omission or change of preposition. 


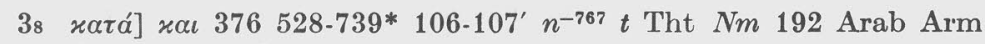

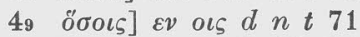

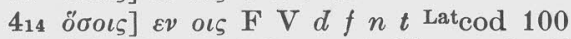

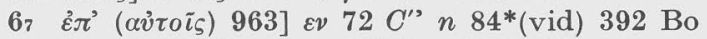

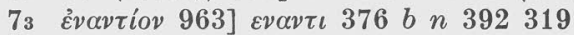

$\left.9_{11} \dot{\varepsilon} v\left(\tau \tilde{\omega} \mu \eta v^{\prime}\right)\right]>n^{-767} 527$

$911 \pi \iota x \varrho i \delta \omega N]$ pr $\varepsilon \pi \iota 376-707 b d 246 n t 527319$ Latcod 100 AethCG

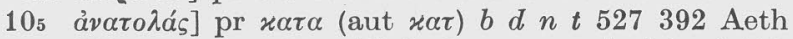

106 $\lambda i \beta \alpha]$ pr $x \alpha \tau \alpha 537 n^{-127}$ Aeth; pr $\pi \varrho \circ \varsigma d t$ Latcod 100 Ambr Sat II 107 Or Matth 52

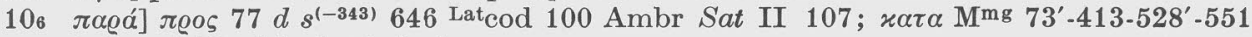
$b^{-19} n$ LatOr Matth 52 Aeth

$10_{35} \chi\left\llcorner\lambda \iota a^{\prime} \delta \alpha \varsigma\right]$ pr $\varepsilon \iota \varsigma C^{\prime \prime} d n 28-30^{\prime}-85^{\prime} \mathrm{mg} t$ Latcod 100 Aeth Arm

111 है่v $\alpha v \tau \iota]-\tau \iota 0 \nu$ C $-46 d^{-44} 54^{\prime} t 527$

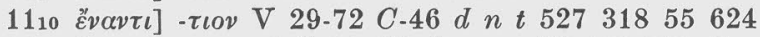

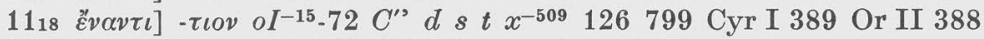

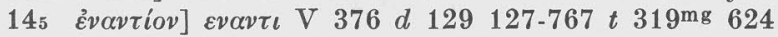

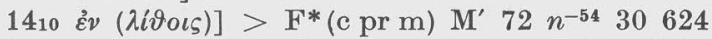

$\left.1431 \eta^{2}\right] \alpha \varphi \eta \varsigma \mathrm{M}^{\mathrm{mg}} d n^{-767} t$ Lat $^{\text {cod } 100 \mathrm{GregIl}} \operatorname{Tr} 11$

1437 है

155 om éri $1^{\circ} d^{-106}$

$155 \delta \sigma \mu \eta v]$ pr $\varepsilon \iota \varsigma 414 d t 39259$ Aeth Arm

$\left.1514 \delta \sigma \mu \eta^{\prime}\right]$ pr $\varepsilon l \varsigma d t 509$ Latcod 100 Arm $\mathrm{Bo}^{\mathrm{B}}$

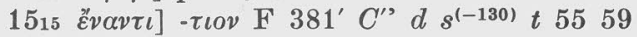

1539 om ỏ $\pi i \sigma \omega 2^{\circ} \mathrm{B} \mathrm{V} d 129 n^{-767} t x 319$ Tht $N m 211$ Latcod 100 Arm Co

162 है้ $\alpha \nu \tau \iota]-\tau \iota O \nu 16.46 d n t$

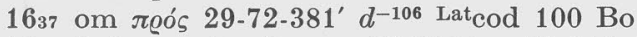

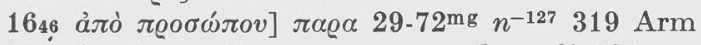

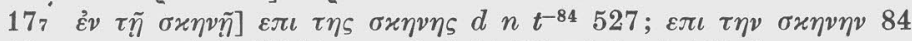

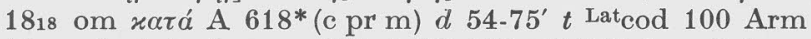

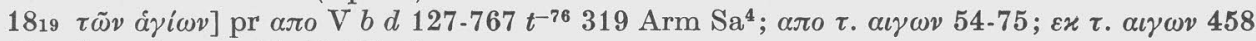

1819 E้v $\alpha v \tau$ ] $-\tau \iota$ - $16-46-422 d t$

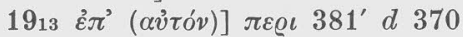

$\left.19_{20} \varepsilon \dot{\pi} \pi^{\prime}(\alpha \dot{v} \tau o ́ v)\right] \pi \varepsilon \varrho \iota d^{(-125)} 370$

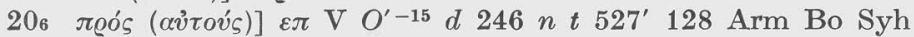

215 om हैं $2^{\circ} C^{\prime \prime} n^{-767} \& 527646$

2123 om $\varepsilon i \varsigma 2^{\circ} 82 d 370$

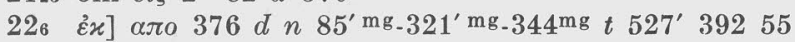

$233 \varepsilon v ̉ \vartheta \varepsilon i \alpha \nu]$ pr $\varepsilon \iota \varsigma 58 d t$; pr $\varepsilon \pi \mathrm{F}^{\mathrm{b}} n 527^{\prime}$ Tht $N m 219$

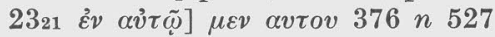

$\left.23{ }_{26} \tau \tilde{\omega}(B \alpha \lambda \alpha \dot{\alpha})\right] \pi \varrho \circ \varsigma 376 C^{\prime \prime}-52^{\prime 313417} 10654 t$

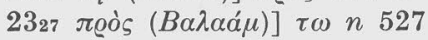

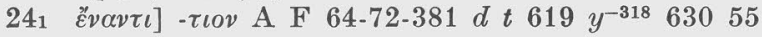

$\left.24{ }_{13} \pi \alpha \varrho^{\prime}(\dot{\varepsilon} \mu \alpha v \tau o \tilde{v})\right] \alpha \pi 376 d n t$

$\left.25{ }_{13} \delta \iota \alpha \vartheta \dot{\eta} \varkappa \eta\right] \varepsilon \iota \varsigma \delta \iota \alpha \vartheta \eta \varkappa \eta \nu(-\varkappa \eta \varsigma ~ 75)$ 58-376 $d n^{-458} t 407$ LatLuc Parc 1 Bo

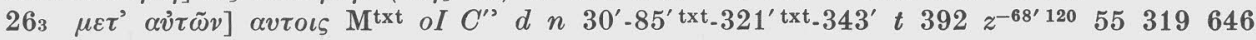
Lat $\operatorname{cod} 100$ Bo

2719 है $\left.v \alpha v \tau \iota 2^{\circ} 963\right]-\tau \iota \circ v$ A 29-58-oI $d n t 619 y^{-392} 68^{\prime} 55319$ Tht $N m 224$

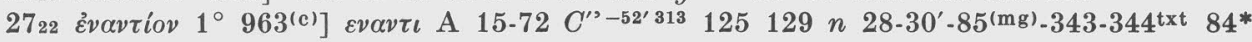
12155646

289 है่ $\left(\dot{\varepsilon} \lambda \alpha \hat{i}^{\prime} \omega\right)$ 963] $>73^{\prime} d^{-106} 53^{\prime}$

2813 ö $\mu \dot{\eta} v]$ pr $\varepsilon \iota \varsigma$ 376-707*(vid) $b d n t$ Aeth Arm ${ }^{\text {te }}$

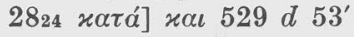

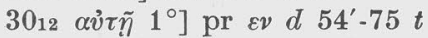

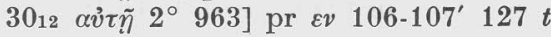

313 Eै $\alpha \alpha \tau \iota]-\tau \iota O \nu d t 630$

$3110 \stackrel{\varepsilon}{\varepsilon v}(\pi v \varrho i)]>\mathrm{B}^{c} \mathrm{G}-82-426 C^{\prime \prime}-414417 d 53127-767 t x^{-527} 40755319624$ 
3112 om $\pi \varrho o ́ s ~ 2^{\circ} \mathrm{V} 414 d 129 t 624$ Latcod 100 Bo

3143 ảió 963] > V 16-46 $d n^{-767} t$

$31_{47}$ ảंò $\tau \tilde{\omega} \nu 3^{\circ}$ ] om ảंó 422799 Lat $_{\text {codd }} 100104 ;>d^{-106}$

3149 ảं $(\alpha \dot{\tau} \tilde{\omega} v)]>d^{-106}$ Phil II 240 (sed hab 192)

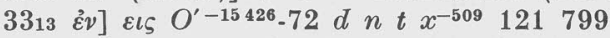

$3314 \varepsilon v] \quad \varepsilon \iota \varsigma \quad 58-72 d 129$ n $t 527121319$

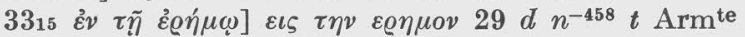

$3317 \dot{\varepsilon} v] \varepsilon \iota \varsigma 72 d f^{-129} n t 318$

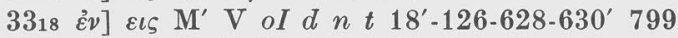

$3319 \varepsilon \dot{v}]$ eı५ $d n^{(-458)} 30^{\prime} t 799$

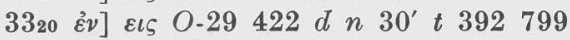

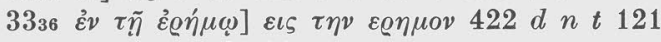

$33_{44}$ ह่v $\left.1^{\circ}\right]$ Els $d 129 n t$

$3347 \varepsilon \dot{\pi} i] \varepsilon \iota \varsigma$ d $246 t$ Aeth Arm

$33_{49} \pi \alpha \varrho a ́$ ] $\varepsilon \pi \iota$ oI-72-82 52'-417-422-761*(c pr m) $b d^{-106} 246 n^{(-458)} 321509-527 y^{-121} z$

$\left.344 \pi \alpha \varrho \varepsilon \lambda \varepsilon v ́ \sigma \varepsilon \tau \alpha \iota 2^{\circ}\right] \varepsilon \iota \varsigma d^{-106} ;+\varepsilon \iota \varsigma 37652106 n 730^{*} t 318799$ Bo

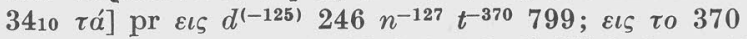

$\left.34_{10} \dot{a} v \alpha \tau o \lambda \tilde{\omega} v\right]$ pr $\alpha \pi(o) \mathrm{V} \mathrm{G}-376 b d^{(-125)} 246 n t 126$ Arm Bo

$35_{1} \pi \alpha \varrho \alpha$ 963] $\varepsilon \pi \iota n^{-127}$

3512 Eैv $\alpha v \tau \iota]-\tau \iota 0 \nu d 129 n^{-127} t$

3515 om $\varepsilon i s 82 n^{-767} 40755$ Aeth Arm

$367 \varepsilon \dot{\pi} \pi i^{963}$ ] $\varepsilon \iota$ G-707 $b d n t 12659799$ Latcodd 100104 Aeth Arm $=\mathfrak{m}$

This list shows that the Byzantine text betrays a certain amount of freedom in the matter of prepositions over against the Num text. In list 6 there are seven instances involving prepositions in which the variant text equals $\mathbb{2}$. Four involve the addition of a preposition: of $\varepsilon \iota \varsigma$ in 217329 , of $\varepsilon v$ in 108 and of $\varepsilon \pi \iota$ in 337 . In list 8 , wherever such additions do not equal $\mathfrak{m}$, a similar pattern emerges, that is $\varepsilon \pi \iota$ is added at 911 and $\varepsilon \iota \varsigma$ in $1035 \quad 1551423325132813 \quad 34910$. It is quite possible that the correspondence to $\mathfrak{K}$ in the instances from list 6 is a coincidence. Change of preposition in list 6 is indicated at $10_{12}$ ( $\dot{\varepsilon} v$ to $\varepsilon x$ ) and 2410 ( $\dot{\varepsilon} \pi i$ to $\pi \varrho \circ \varsigma$ ). The above list shows numerous instances of change of preposition, and too much importance must not be attached to the occasional correspondence of such a change to $\mathfrak{M}$. Similarly the change of $\tau \tilde{\omega} M \omega v \sigma \tilde{\eta}$ to a $\pi \varrho \circ$ construction which is $=\mathfrak{M}$ is paralleled in the above list in 2326 (and exactly the reverse in 2327 ).

5. At 1442 a positive statement was changed into a negative statement thereby formally corresponding to $\mathfrak{K}$. It was there (List 6) argued that this was due to the context rather than to Hebrew influence.

It is interesting to note that such changes occur in the Byzantine text type in places where it is contrary to $\mathfrak{K}$.

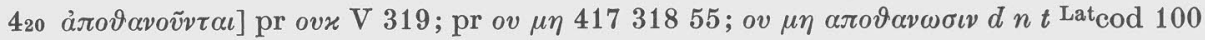
Arm Bo

79 $\left.\tilde{E}^{\prime} \chi 0 v \sigma \iota v\right] \mathrm{pr}$ ovx $d t$

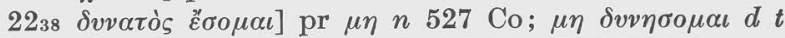

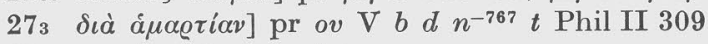

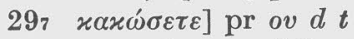

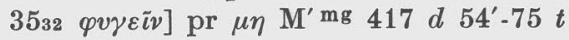


In all these cases as at 1442 a negative particle in the context obtains which may have influenced the tradition.

6. Change in number in list 6 is a common phenomenon and may involve verbs, nouns or pronouns. It is, however, a characteristic of the Byzantine text group also against the Hebrew text as the following list amply demonstrates.

\section{List 9}

$144 \dot{\varepsilon} \pi \varepsilon \sigma \varkappa \varepsilon ́ \psi \alpha \tau o]-\psi \alpha \nu \tau o\left(\varepsilon \pi \iota \sigma \varkappa .107^{\prime}\right)$ B Feprm $\mathrm{M}^{\prime} d 127^{\mathrm{c}} 74^{\mathrm{c}}-76^{\prime}$ Aeth Arm $\mathrm{Bo}^{\mathrm{AB}^{\mathrm{c}}} \mathrm{Sa}^{1}$ $\mathrm{Syh}=\mathrm{Ra}$

A compound subject obtains here.

$\left.144 \pi \alpha \tau \varrho \tilde{\alpha}_{\zeta}\right]-\varrho \iota \omega \nu$ F V 29-376 $d 53^{\prime} n 130^{\mathrm{mg}} 346^{\mathrm{mg}} t 31868^{\prime} 59$ Lat $\operatorname{cod} 100$ Arm Co Syh

$\left.210 \tau \alpha^{\prime} \gamma \mu \alpha\right]-\mu \alpha \tau \alpha C^{\prime}-761-414^{\prime}-422 d 53^{\prime}-56$ 75' $x$ 68'-120'-126-628 646 BoA

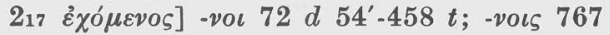

$\left.218 \tau \alpha^{\prime} \gamma \mu \alpha\right]-\mu \alpha \tau \alpha 131 d f^{-246} 7573074-76^{\prime} 68^{\prime}-126 \mathrm{Bo}^{\mathrm{A}}$

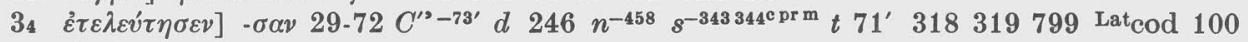
Aeth

With compound subject.

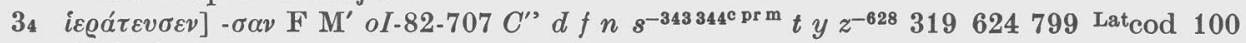
Aeth Arm

With compound subject.

$\left.3_{24} \pi \alpha \tau \varrho \iota \tilde{c}_{\zeta}\right]-\varrho \iota \omega \nu 417 d n t 799$ Latcod 100 AethCG Arm Co

$44 \quad \ddot{\gamma} \gamma\llcorner O \nu] \alpha \gamma \iota \alpha d n^{-127^{*}} t 646 \quad$ Armap $^{\text {ap }}$

$\left.4_{13} \varepsilon \dot{\pi} \iota \vartheta \dot{\eta} \sigma \varepsilon \iota\right]-\sigma o v \sigma \iota(v) \mathrm{M}^{\mathrm{mg}} C^{\prime \prime} d n 85^{\mathrm{mg}} \cdot 346^{\mathrm{mg}} .730 t 319416$ Cyr I 852 Lat $c o d 100$ Aeth Arm Bo

$442 \delta \tilde{\eta} \mu \circ]] \delta \eta \mu \circ \iota d$ 127-767 $t$ Lat $\operatorname{cod} 104$ Arm

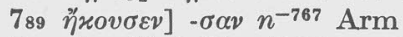

789 ö] $\alpha \mathrm{V} d^{-125} 54^{\prime}-767 t 799$ Arm Bo

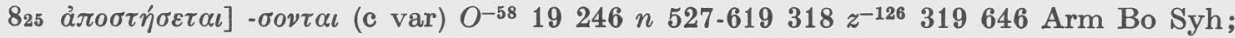
$-\sigma \tau \eta^{\sigma \tau} 126$

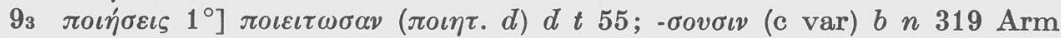

$93 \pi \circ \eta \dot{\sigma \varepsilon \iota \varsigma} 2^{\circ}$ ] - $\sigma o v \sigma \iota v$ (c var) $d n t$ Arab Arm

$105 \sigma \alpha \lambda \pi \iota \varepsilon i \tau \varepsilon] \sigma \alpha \lambda \pi \iota \varepsilon \iota d$

$114 \varepsilon \dot{\varepsilon} \pi \varepsilon \vartheta \dot{v} \mu \eta \sigma \alpha v]-\sigma \varepsilon v$ (c var) $\mathrm{B} O^{-58} 313^{*} 106-125^{\prime} n^{-458} t x^{-619} 624$ Phil II 298 Cyr I 389 II $461 \mathrm{Arm}^{\mathrm{ap}}$

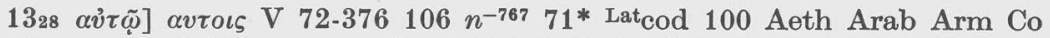

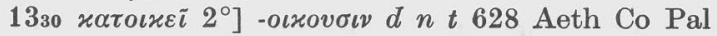

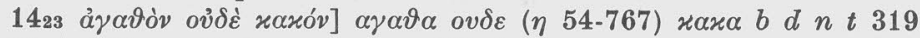

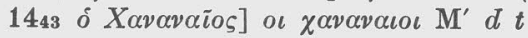

15. $\pi \circ\left\llcorner\eta^{\sigma} \varepsilon \iota \varsigma\right]-\sigma \varepsilon \tau \varepsilon d^{(-125)} t$

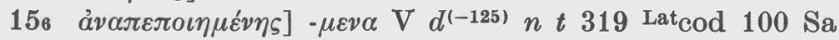

159 idem $d n t$ Lat cod 100

$1539 \tau \tilde{\omega} \nu \delta \iota \alpha \nu 0 \iota \tilde{\omega} v] \tau \eta \delta \iota \alpha \nu \circ \iota \alpha d^{-106}$

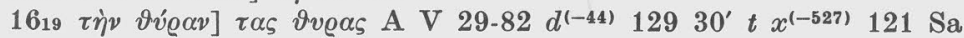

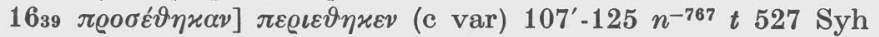

$173 \pi \alpha \tau \varrho \iota \tilde{\omega} v]-\varrho \iota \alpha, d 54^{\prime}-458$ t $527 \mathrm{corm} 319$

175 ä] ov d 129 n $t 527$ Arm Bo

$\left.179 \varepsilon \varepsilon^{2} \alpha \beta o v\right]-\beta \varepsilon v$ V 58-72 $C^{\prime 3}{ }^{-46^{5}} 5^{\prime} 31319 d^{-125} f^{-129} n 30-85^{*} t x y^{-318} z^{-126407} 646799$ Aeth Arm; of $\varepsilon \beta \alpha \lambda \varepsilon v$ B

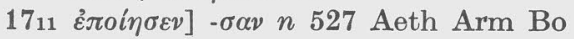

With compound subject.

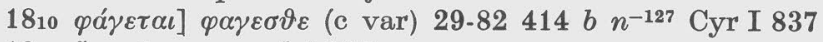

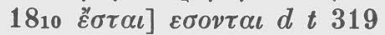

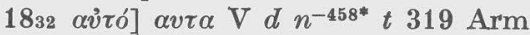

$1918 \lambda \eta \dot{\mu} \mu \varepsilon \tau \alpha \iota] \lambda \eta \psi o v \tau \alpha \iota$ (c var) $72 d n t$ Eus VI 12 Aeth Arm Bo 
208 $\pi \circ \tau \iota \varepsilon i \tau \varepsilon] ~ \pi о \tau \iota \varepsilon \iota ~(\pi o \tau \varepsilon \varepsilon \iota ~ 44) ~ V d 319$ Lat $\operatorname{Vod} 100$

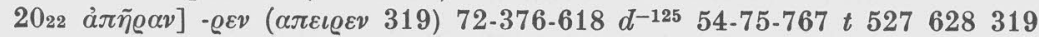

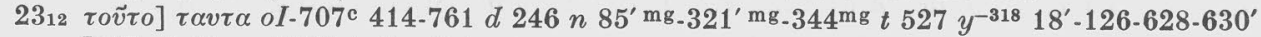
LatRuf Num XVI 1 Bo Syh

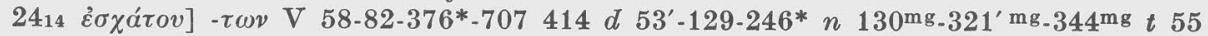

$2419 \sigma \omega \zeta o ́ \mu \varepsilon v o v]$-vovs (-vov 120*) $58 b d t 120$ Aeth Arm

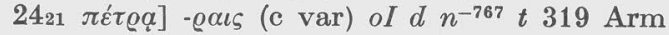

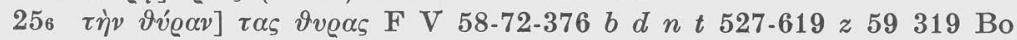

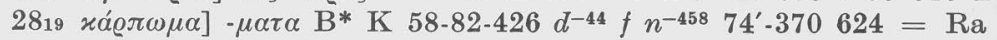

$2824 \delta \tilde{\omega} \varrho \circ v] \delta \omega \varrho \alpha d^{-106}$

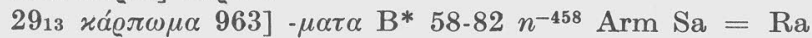

$2918 \dot{\eta} \vartheta v \sigma i \alpha] \alpha \iota \vartheta v \sigma \iota \alpha \iota$ V 618*(vid) 52' b $106 n t 407$ Lat codd 100104 (vid) Arm

$\left.29_{21} \quad \dot{\eta} \quad \vartheta v \sigma i \alpha\right] \alpha \iota \vartheta v \sigma \iota \alpha \iota 52^{\prime} b d^{(-106 \mathrm{txt})} n^{(-75)} t 509$ Lat codd 100104

$303 \quad \tau \dot{o} \varrho \tilde{\eta} \mu \alpha] \tau \alpha \varrho \eta \mu \alpha \tau \alpha d t$

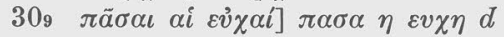

$3013 \mu \varepsilon v \varepsilon \tilde{l}] \mu \varepsilon v 0 v \sigma \iota(v) d n^{(-767)} t$ Bo Syh

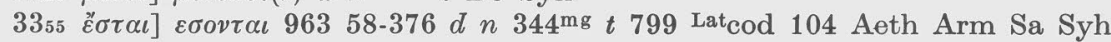

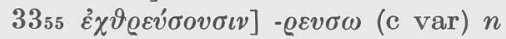

$368 \alpha v \dot{\eta} \tilde{\zeta}] \alpha v \tau \omega v$ oII-707 $16^{*}-77-131-422-500-550-551^{\mathrm{c}}-739^{*} d 246 n 8^{\mathrm{mg}}-344^{\mathrm{mg}} t x^{-509}$ $392^{*} 18^{\prime}-126-407-628-630^{\prime} 799$ Aeth $^{-\mathrm{C}}$ Co

From this list it appears that change in number occurs commonly in this text group both for verbs and nouns but only once (368) for pronouns.

7. Change in case was attested in list 6 outside of the consistent change of

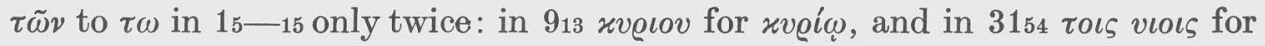

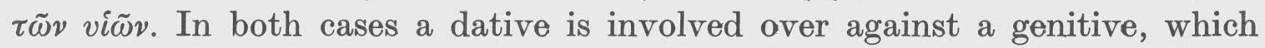
corresponds respectively in Hebrew to a $ל$ phrase over against the second element in a bound phrase.

The Byzantine text attests a large number of variants involving case which in view of the fact that case inflection does not exist in Masoretic Hebrew can have nothing to do with Hebrew influence. In the following list only such variants are given that could conceivably reflect the Hebrew distinction mentioned above, i.e. genitive/dative variants. In each case the relevant Hebrew text is given as well.

\section{List 10}

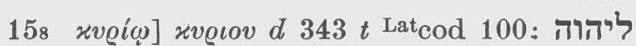

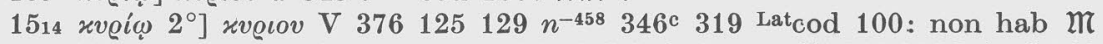

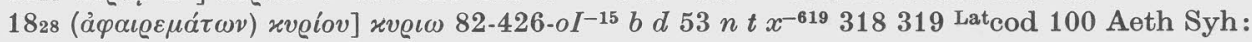
תרופת

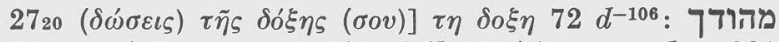

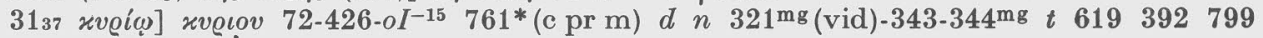
Aeth: :

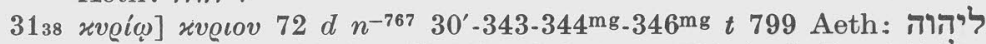

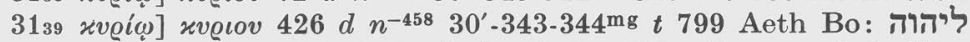

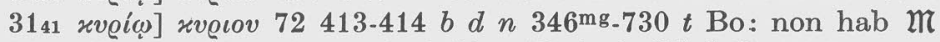

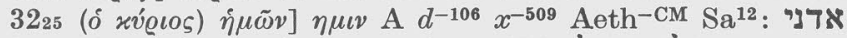

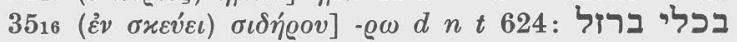

Again the text group under discussion exercises a certain freedom within the text tradition which has nothing to do with immediate or mediate Hebrew influence. 
8. Change in lexeme under possible Hebrew influence was attested in list 6

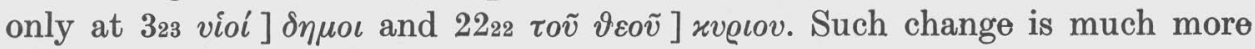
common in contrast to $\mathfrak{N}$ as the following list indicates.

List 11

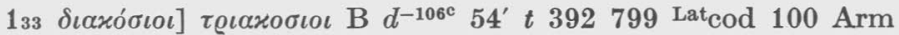

$135 \tau \varepsilon \tau \varrho \alpha x o ́ \sigma \iota \iota \iota] \tau \varrho \iota \alpha x o \sigma \iota \iota \iota d^{-106 c} 85^{*}$ (vid) $t 392799$

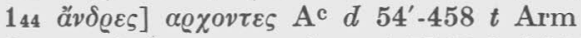

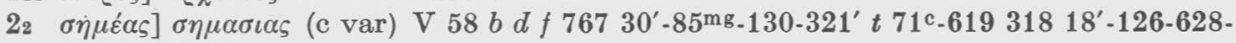
669319799

$216 \pi \alpha \varrho \varepsilon \mu \beta \circ \lambda \tilde{\eta} \varsigma] \varphi v \lambda \eta \varsigma 107^{\prime}-125 n$ Arm

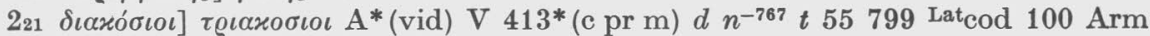

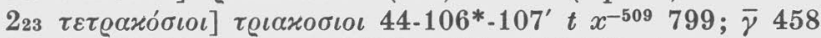

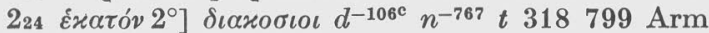

$232 \pi \varepsilon v \tau \alpha \varkappa o ́ \sigma \iota \iota !] ~ \varkappa \alpha \iota\left(>d^{-44}\right) \varepsilon \xi \alpha x o \sigma \iota 0 \iota d^{-125^{*}} n^{-767} t 799$ Arm Bo

$328 \varepsilon \xi \xi \alpha \varkappa o ́ \sigma \iota \iota \iota] \tau \varrho \iota \alpha \varkappa o \sigma \iota \iota \iota d n^{-767} t 799$ Arm

$336 \quad \alpha v \dot{\omega} v 1^{\circ}$ ] $\tau 0 v \tau \omega v d^{-125} n^{-767} t$ Arm

$336 \quad \alpha v \dot{\tau} \tilde{\omega} \nu 2^{\circ}$ ] $\tau 0 v \tau \omega \nu d^{(-44)} n^{-767} t$ Arm

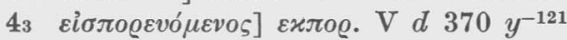

$49 \quad$ ả $\gamma \delta \varepsilon i \alpha] \alpha \gamma \iota \alpha$ A $58-72 d 53^{\prime}-56-129^{\left(\mathrm{mg}^{\mathrm{cl}}\right)} 54-767 t 71318$

$4_{12} \delta \varepsilon \varrho \mu \alpha \tau i \nu \omega$ 803] $\delta \varepsilon \varrho \mu \alpha \tau \iota$ A $b$ n Aeth

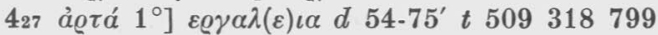

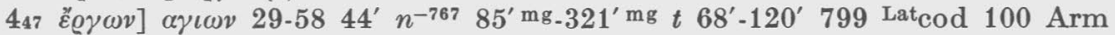

$522 \pi \varrho \tilde{\eta} \sigma \alpha \iota] \pi \varrho \iota \sigma \alpha \iota(-\sigma \varepsilon \quad 799) 29 \quad 46^{\text {s.414-529c }} b d^{-44} \quad 127-458343 t^{-84} 71319799 \mathrm{Chr}$ II 917

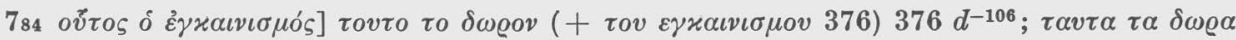
106

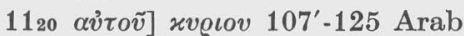

$1125 \alpha \dot{\tau} \tau \dot{v}] \mu \omega(v) \sigma \eta v d n t$ Arm

$\left.11_{25} \pi \alpha \varrho \varepsilon i \lambda \alpha \tau o\right] \pi \varepsilon \varrho \iota \varepsilon 1 \lambda \varepsilon \tau o\left(-\lambda \alpha \tau o 55^{*}\right) d 246 t z 55646$

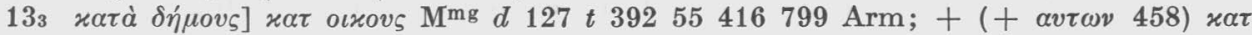
oเxovৎ $\mathrm{V} n^{-127}$

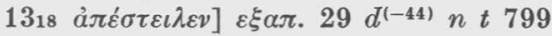

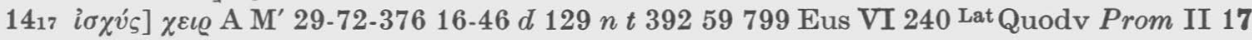
Arab Arm

$1437 \pi \lambda \eta \gamma \tilde{\eta}] \quad \gamma \eta 107^{\prime} t$

$14_{43}$ ov $\left.\varepsilon i v \varepsilon x \varepsilon v\right] \delta \iota \tau^{2} d^{-106}$

$\left.151 \dot{\varepsilon} \lambda a^{\prime} \lambda \eta \sigma \varepsilon v\right] \varepsilon \iota \pi \varepsilon(v) \mathrm{B} \mathrm{V} d 129 t x$ Cyr I $1029=\mathrm{Ra}$

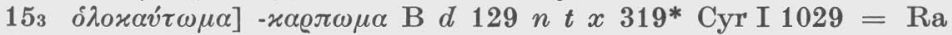

$159 \pi \varrho \circ \sigma \circ i \sigma \varepsilon l] \pi \circ \iota \eta \sigma \varepsilon \iota \varsigma \mathrm{V} 29 d t$

$1514 \gamma \varepsilon \dot{v} \eta \tau \alpha \iota] \pi \varrho o \sigma \gamma \varepsilon v .551 d 127 t x$

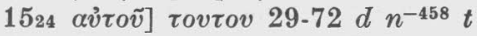

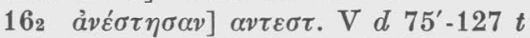

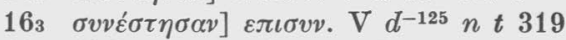

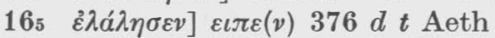

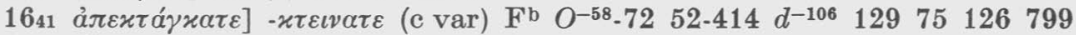

$1643 \varepsilon i \sigma \tilde{\eta} \lambda \vartheta \varepsilon v] \varepsilon \sigma \tau \eta d n t$; steterunt Arm

$181 \alpha \mu \alpha \varrho \tau i \alpha \varsigma 1^{\circ}$ ] $\alpha \pi \alpha \varrho \chi \alpha \varsigma$ B $M^{\text {txt }}$ oI $d t x^{-527} 68^{\prime}-120^{\prime}-126416799$ Cyr I 837

$182 \varphi v \lambda \dot{\eta} v]$ vıovৎ $d t 319$

1820 ö $\tau \iota] \varepsilon \tau \iota d t$

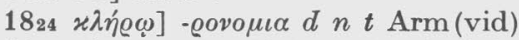

$1832 v i \tilde{\omega} v] \alpha \gamma \iota \omega v \quad 72-618 \quad 414 d^{-106} \quad 28 \quad 669$

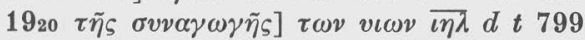

$\left.20{ }_{6} \tilde{\lambda} \lambda \vartheta \varepsilon v\right] \varepsilon \iota \sigma \eta \lambda \vartheta \varepsilon v$ (c var) $\mathrm{V} C^{\prime \prime} d n 30^{\prime}-85^{\prime} \mathrm{mg} .321^{\prime} \mathrm{mg} t 527646{ }^{\text {Lat }} \mathrm{cod} 100$

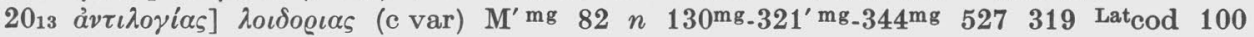
Arm 
$2014 \sigma v ́] \varepsilon v d t$

$218 M \omega v \sigma \tilde{\eta} v] \alpha v \tau o v d \mathrm{Sa}^{10}$

219 है $\sigma \tau \eta \sigma \varepsilon v] \varepsilon \pi \varepsilon \sigma \tau$. $d$ n $t 527$

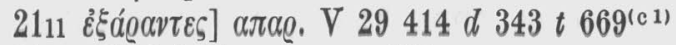

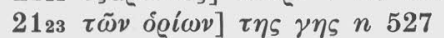

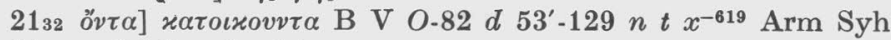

$\left.21_{34} x \alpha \vartheta \hat{\omega} \varsigma\right] x \alpha \vartheta \alpha d t$

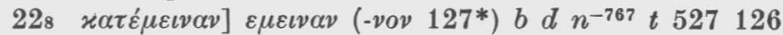

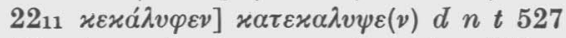

$236 \quad \dot{\varepsilon} \varphi \varepsilon \iota \sigma \tau \eta \dot{r} \varkappa \varepsilon \iota] \pi \alpha \varrho \iota \sigma \tau$. (c var) $n 527$

$2324 a \dot{v} \alpha \sigma \tau \eta \dot{v} \sigma \varepsilon \tau \alpha \iota] \alpha \nu \alpha \beta \eta \sigma . d t 630$

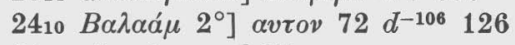

$2423 \varepsilon i \pi \varepsilon v] \varepsilon \varphi \eta d^{-106}$

$256 \quad a \delta \varepsilon \lambda \varphi o ́ v] \lambda \alpha o v d^{-106}$

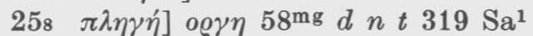

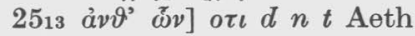

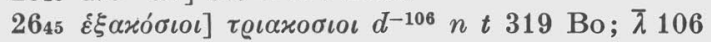

$2654 \varkappa \alpha \vartheta \omega \varsigma$ ] $\varkappa \alpha \vartheta \alpha n$

$278 \pi \varepsilon \varrho \vartheta \eta \dot{\sigma \varepsilon \varepsilon \varepsilon]}\left(+\vartheta v \gamma \alpha \tau \eta \varrho d^{-106}\right) \delta \omega \sigma \varepsilon \tau \varepsilon d^{-106}$ LatRuf Num XXII 1 Aeth Bo

$278 \quad \tau \tilde{\eta} \quad \vartheta v \gamma \alpha \tau \varrho i] \quad \tau \omega \alpha \delta \varepsilon \lambda \varphi \omega d^{-106}$

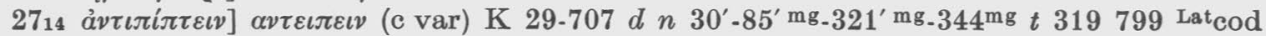
100 Aeth Bo

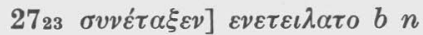

$\left.289 \pi \varrho \circ \sigma \alpha^{\prime} \xi \varepsilon \tau \varepsilon\right] \pi \varrho \circ \sigma \varepsilon \tau \alpha \xi \varepsilon \mathbf{7 3}^{\prime} d$

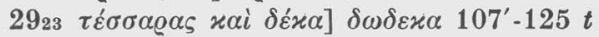

312 है $\sigma \chi \alpha \tau \sigma \nu] v \sigma \tau \varepsilon \varrho o \nu \mathrm{V} d t$

$3111 \sigma x \tilde{\nu} \lambda \alpha$ 963] $\sigma x \varepsilon v \eta d^{-106} t 407$ Bo

$31_{18}$ oi $\left.\delta \varepsilon v\right] \varepsilon \gamma v \omega\left(-v \omega \sigma \varepsilon v\right.$ 85) A F 15-29-72 107'-125 $129 n 85^{\prime} \mathrm{mg}_{-} 321^{\prime} \mathrm{mg} y^{-121}$

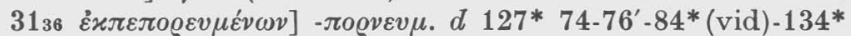

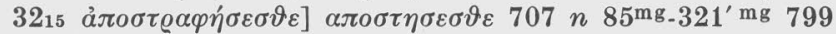

$\left.3241 \varepsilon \dot{\varepsilon} \pi \alpha{ }^{\prime} \lambda \varepsilon \iota 1^{\circ}\right] \pi 0 \lambda \varepsilon \iota \varsigma d^{(-44)} 129 n^{-127} t 319$ Arab Arm Sa

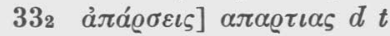

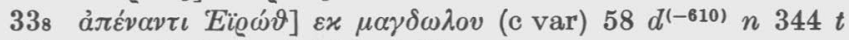

$\left.33_{42} \pi \alpha \varrho \varepsilon v \varepsilon \dot{\beta} \alpha \lambda o v\right] \alpha \pi \eta \lambda \vartheta o v d^{-106}$

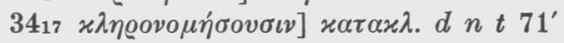

Many of these variants are due to the influence of the context; others are palaeographically conditioned; still others are stylistic changes. A substantial number of instances involve change in numbers. These are probably due to the common use of letters used to indicate numbers, and confusion in the text tradition easily results from this practice.

9. Particularly characteristic of the Byzantine text type is the variant tradition in the transcription of proper names. Little purpose would be served in presenting a list of all such variants. Since many of these show great divergence from the original transcription the following list is presented to illustrate this divergence by placing the Num transcription and the text of $\mathfrak{M}$ in parentheses after the variant. For the ms support the reader is referred to the apparatus of Num. The list is merely illustrative.

\section{List 12}

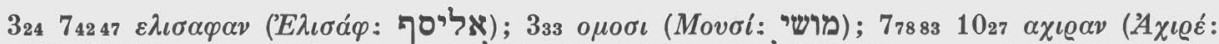

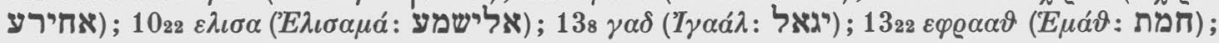




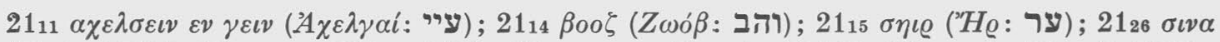

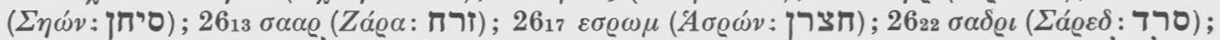

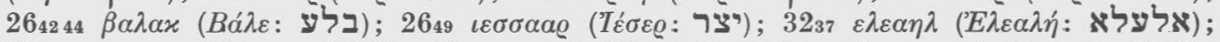

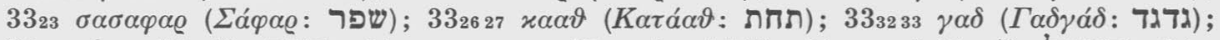

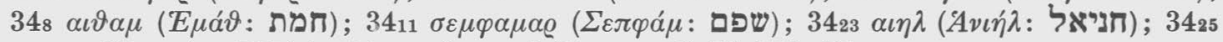

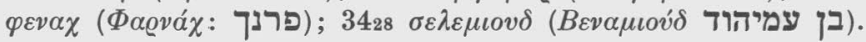

The above list represents various types of errors which are to be found in the Byzantine text type. This tradition concerning proper names is on the whole quite untrustworthy; it represents a late and often corrupt textual tradition. Should the $d n t$ text on occasions actually be closer to $\pi$ than Num, this is probably accidental, since there is no compelling evidence in this tradition of a revision based on acquaintance with the Hebrew text.

C. Since both in Genesis and Deuteronomy the Biblical text used by Chrysostom and Theodoret seems to represent a late and mixed text (cf THGG ch. 10 and THGD 25-30) it remains to investigate whether the Byzantine text group might have been their text.

Since the book of Numbers was not quoted by these fathers extensively the results can hardly be conclusive, and in order that one may receive a complete picture of the problems posed by their text a full list of their variant readings is given in the following list.

List 13

$35 \quad \dot{\varepsilon} \lambda \alpha \dot{\lambda} \eta \sigma \varepsilon v] \varepsilon ı \pi \varepsilon 125$ Tht $N m 192$

35 om $\lambda \varepsilon ́ \gamma \omega \nu 125$ Tht $N m 192$

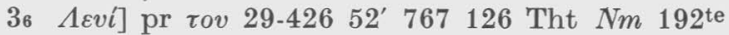

37 है $v \alpha \nu \tau \iota]-\tau \iota \circ v 646$ Tht $\mathrm{Nm} 192$

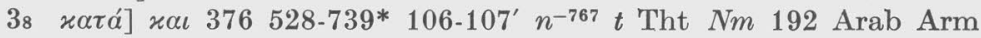

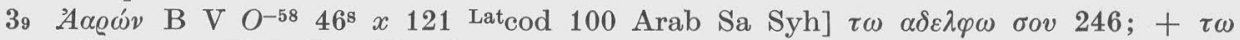
$\alpha \delta \varepsilon \lambda \varphi \omega$ oov Tht $\mathrm{Nm} 192$ rell

$39 \delta \varepsilon \delta o \mu \varepsilon ́ v o l]-\nu o v$ V 64-381'-618 $C^{\prime \prime} 53^{\prime} 30^{\prime}-85509319$ Tht $N m 192^{\text {te }}$

39 oṽ App I

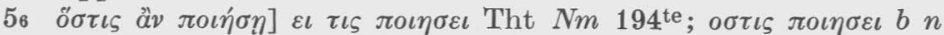

56 $\tau \tilde{\omega} \nu \alpha \mu \alpha \varrho \tau \iota \tilde{\omega} v$ A B G $x^{-619}$ Anast 376 Arab Sa] pr $\pi \alpha v \tau \omega \nu 29 ; \pi \alpha \sigma \omega \nu \alpha \mu \alpha \varrho \tau \iota \omega \nu 126$; $\pi \alpha \sigma \omega \nu 55 ;$ pr $\pi \alpha \sigma \omega \nu$ Tht $N m 194$ rell $=\mathfrak{m}$

58 om $\delta$ A oI $n 13068^{\prime} 55$ Tht $N m 195=\mathfrak{m}$

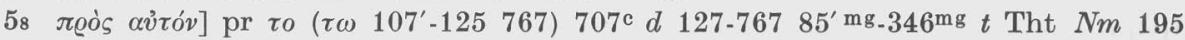

58 xveí $\omega$ B $b$ 509] $\tau o v \overline{x v} 72$; > 16-46 53'; pr $\tau \omega$ Tht $N m 195$ rell

$58 \quad \dot{\varepsilon} v \quad \alpha \dot{v} \tau \tilde{\omega}]$ ad fin tr $414 b d f n t$ Tht $N m 195$ Bo

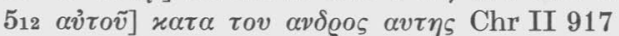

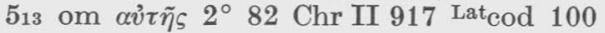

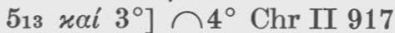

513 om $\alpha \dot{\imath} \eta \dot{~} 2^{\circ} \mathrm{Chr}$ II 917 Arm

$\left.514 \alpha v i \tilde{\omega} 1^{\circ} 963\right] \mathrm{pr} \varepsilon \pi \quad 72-82-376761 b 50968^{*}-12255799 \mathrm{Chr}$ II $917=2 \pi ; \tau \omega \alpha \nu \delta \varrho \iota$ $\alpha v \tau \eta \varsigma d n t$ Arm Bo

514 om $\varkappa \alpha i 2^{\circ}-\alpha v i \tau o \tilde{v} 2^{\circ} \mathrm{Chr}$ II 917

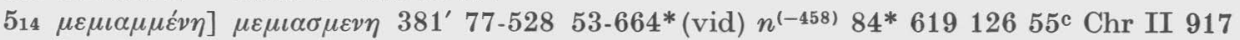
Cyr I 909

$515 \delta \tilde{\omega} \varrho o v]+(※ \mathrm{G}$ Syh) $\alpha v \tau \eta \varsigma O \mathrm{Chr}$ II $917 \mathrm{Syh}=\mathfrak{m}$

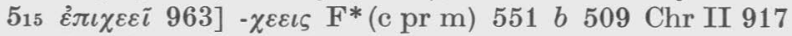

$515 \varepsilon \dot{\varepsilon} \tau \vartheta \vartheta \dot{\eta} \sigma \varepsilon \iota]-\sigma \varepsilon \iota \varsigma$ F $b 509 \mathrm{Chr}$ II 917

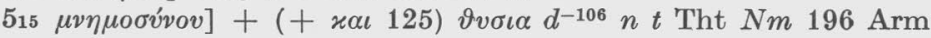


516 om $\alpha \dot{\tau} \tau \dot{\eta} v 1^{\circ} n^{-767}$ Tht $N m 196$ Arm

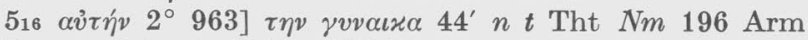

517 om ó i

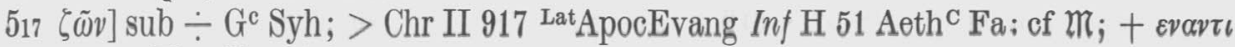
xveıv Tht $\mathrm{Nm} 196^{\text {te }}$

517 in $\gamma \tilde{\eta} \varsigma]$ pr $\alpha \pi \circ \mathrm{K} \mathrm{V} O C^{\prime \prime} 76730^{\prime}-130^{\mathrm{mg}}-321^{\prime} \mathrm{mg} z \mathrm{Chr} I I 917 \mathrm{Syh}=\mathrm{m}$

517 om $\tau \tilde{\eta} \varsigma 3^{\circ}$ - $\alpha i^{\prime}$ ult $\mathrm{Chr}$ II 917

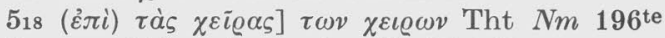

$519 \pi \alpha \varrho \alpha \beta \varepsilon \dot{\beta} \eta \varkappa \alpha \varsigma] \pi \alpha \varrho \varepsilon \beta \eta \varsigma$ Chr II 917

519 vंл́́] $\pi \varrho \circ$ A $\mathrm{Chr}$ II 917

$\left.5_{19} \alpha \dot{\vartheta} \hat{\omega} \alpha\right] \alpha \vartheta \omega \circ \mathrm{F}^{\mathrm{b}} 551 n$ Tht $\mathrm{Nm} 197^{\mathrm{te}} ; \sigma \omega \alpha \mathrm{Chr}$ II 917

$\left.520 \sigma v^{-}-0 \tilde{z}^{z} \sigma \alpha\right] \pi \alpha \varrho \varepsilon \beta \eta \varsigma \mathrm{Chr}$ II 917

$\left.520 \eta^{\prime}\right] \varkappa \alpha \iota$ V $76730^{\prime}-321^{\prime} \mathrm{mg} 319 \mathrm{Chr}$ II 917 Aeth

520 $\mu \varepsilon \mu i \alpha v \sigma \alpha \iota]$ pr $\sigma v\left(\sigma o \iota\right.$ G) $O b d n^{-767} t$ Tht $N m 197$ Lat cod 100 Syh

520 жai] $\eta$ Tht $\mathrm{Nm} 197$

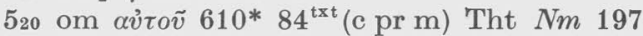

521 om init- yvvauxi $d t 619$ Chr II 917 Tht $N m 197$

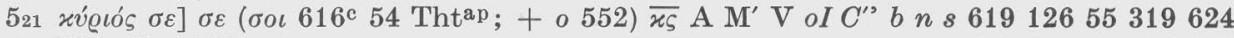

Tht $N m 197$

$5_{21}$ om $\tau o ́ v-\varkappa \alpha i$ ult Chr II 917

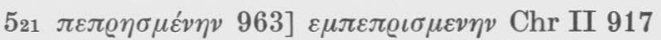

$522 \varepsilon \dot{\pi} \pi \varkappa \alpha \tau \alpha \varrho \omega \mu \varepsilon v o v]$ - $\tau \eta \varrho \alpha \mu \varepsilon v o v$ Chr II 917

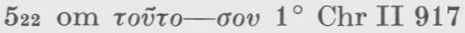

$\left.5_{22} \pi \varrho \tilde{\eta} \sigma \alpha \iota\right] \pi \varrho \iota \sigma \alpha \iota 29 \quad 46^{\mathrm{s}}-414-529^{\mathrm{c}} b d^{-44} \quad 127-458 \quad 343 t^{-84} \quad 71319799 \mathrm{Chr}$ II 917

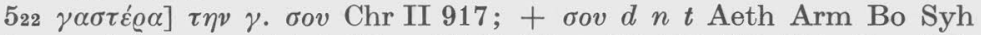

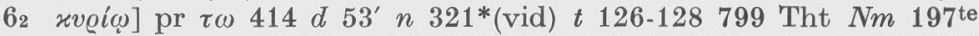

$65 x v \varrho i \omega]$ pr $\tau \omega 72-426 \quad 73^{\prime}-413-414-552-761 \quad 75^{\prime}-767 \quad 30 x^{-71} 68^{\prime}$ Tht $N m 198$

$\left.6_{6} \varepsilon v \hat{\chi} \tilde{\eta} s 963\right]+\left(※ \mathrm{G}\right.$ Syh) $\alpha v \tau o v \mathrm{~F}^{\mathrm{b}} \mathrm{M}^{\prime} \mathrm{V} \mathrm{O}^{-58} d n{ }^{-5} 5^{\prime} \mathrm{mg}_{-} 321^{\prime} \mathrm{mg}_{-} 344^{\mathrm{mg}} t^{-84} 319$ Tht $\mathrm{Nm} 198 \mathrm{Arm} \mathrm{Bo} \mathrm{Sa}^{4} \mathrm{Syh}=\mathfrak{m}$

66 жvoí $\omega$ 963] sub ※ $\mathrm{S}^{\mathrm{c}}$; pr (※ S G Syh) $\tau \omega(\tau o ~ 376) \mathrm{M}^{\prime} \mathrm{S}^{\mathrm{c}} 0.8252^{\prime}-313-414 d n 28$ $85^{\prime} \mathrm{mg}_{-} 321^{\prime} \mathrm{mg}_{-} 344^{\mathrm{mg}} t$ Tht $N m 198$ Bo Syh

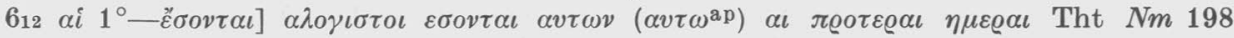
Cf $\alpha \lambda o \gamma \iota \sigma \tau o \iota$ pro $\alpha^{\prime} \lambda o \gamma o \iota$ in $d n^{-767} t$

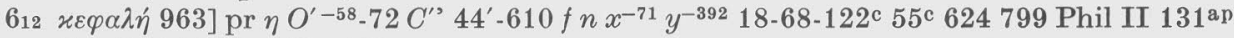
Cyr I 1041 Tht $N m 198$

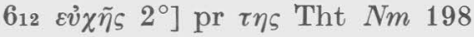

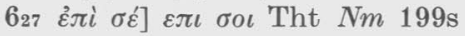

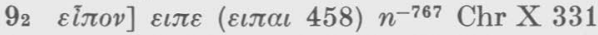

92 om oi $376 * 458319 \mathrm{Chr}$ X 331

$97 \quad \dot{x} \alpha \dot{\vartheta} \alpha \varrho \tau o l]+\varepsilon \sigma \mu \varepsilon v 56^{\mathrm{mg}}{ }_{2} 246 \mathrm{Chr}$ II $877^{\mathrm{Lat}} \operatorname{cod} 100$; cf pr (※ Syh) $\varepsilon \sigma \mu \varepsilon v O$ Syh

97 om oṽv 319 Chr II 877 Latcod 100

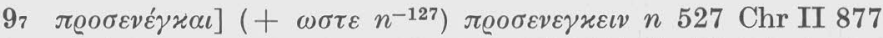

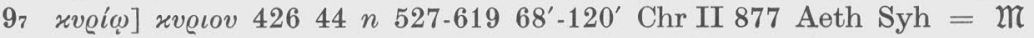

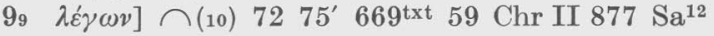

910 om äv $\varrho \omega \pi o \varsigma 2^{\circ} \mathrm{F}^{\mathrm{b}} 72 d 5^{\prime} 126319 \mathrm{Chr}$ II 877 Cyr I 1081 Latcod 100 Bo Sa ${ }^{12}$

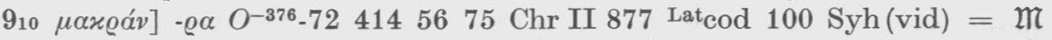

910 om $\eta^{\prime} 2^{\circ} 37610654^{\prime}-458527$ Chr II 877 Arm Syh

910 om xai Chr II 877 Aeth Arm Sa ${ }^{12}$

910 om $x v \varrho i \omega 72528455 \mathrm{Chr}$ II 877

$9_{14}$ om $v \mu \tilde{i} v$ xoi Tht $\operatorname{Ios} 277$

$\left.10_{2} \dot{\alpha} \varrho \gamma v \varrho \tilde{\alpha} \varsigma \varepsilon^{3} \lambda \alpha \tau \alpha^{\prime} \varsigma\right]$ tr B V b $d 129 n t^{-84^{\text {txt }}} x^{-619} 319 \mathrm{Chr}$ II 881 Cyr I 397 V 773 X 837

Latcod 100 Ambr Sat II 107

106 $\sigma \alpha \lambda \pi \iota \sigma \tilde{v} \sigma \iota v]-\pi \iota \varepsilon \iota \tau \varepsilon$ Tht $N m 201$

$\left.10_{6} \alpha \hat{\tau} \tau \tilde{\omega} \nu\right] v \mu \omega \nu$ Tht $N m 201$

109 om $\delta \varepsilon$ Tht $N m 202$

$\left.10_{10} \sigma \alpha \lambda \pi \iota \varepsilon i \tau \varepsilon\right]$ pr $\varkappa \alpha \iota \mathrm{Chr}$ II $881 \mathrm{A \theta th}^{-\mathrm{C}}=\mathfrak{l}$ 


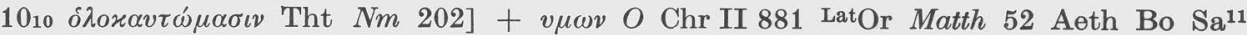
$\mathrm{Syh}=\mathfrak{m}$

1010 om ėंi $2^{\circ}$ Chr II 881 LatAmbr Sat II 107 Bo

1010 Ěv $\alpha v \tau \iota]-\tau \iota ० v \mathrm{M}^{\prime} C^{s}-414^{\prime}-57$ \& 619 z 646 Cyr X 580 Tht $N m 202$

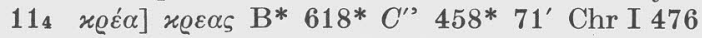

$115 \dot{\varepsilon} \mu \nu \eta \dot{\sigma} \eta \eta \varepsilon v]+\gamma \alpha \varrho \mathrm{F}^{\mathrm{a}} d n^{-127} t 527 \mathrm{Chr}$ I 476 Lat Quodv Prom II 14 Arm

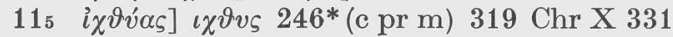

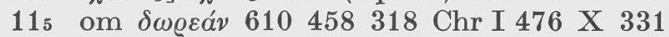

115 om $x \alpha i 1^{\circ}$ Phil III 19ap Chr X 331 Lat Quodv Prom II $14=\mathfrak{m}$

115 om xai $3^{\circ} 799 \mathrm{Chr}$ X 331 Lat Quodv Prom II 14

115 om xai $4^{\circ} 730799 \mathrm{Chr}$ X 331 Latcod 100 Quodv Prom II 14

115 om xai $5^{\circ} \mathrm{Chr}$ X 331

$115 \sigma \varkappa o ́ \varrho \delta \alpha]-\varrho o \delta \alpha \mathrm{F}^{\mathrm{b}} 376-707^{\mathrm{c}}-o I^{-15^{*}} 16-46-77-414^{\prime}-529^{\prime} \mathrm{c}_{-} 761^{\mathrm{c}} b d^{-610} 767^{\mathrm{c}}$ 28-85-343 84 71 126-128-407-628-630'55c prm 59416646 Phil III 19ap Chr I 476 X 331 Cyr I 389 II 461 Or II 388

116 vvví] vvv F $72 \quad 422-529 \quad 54-75^{\prime} 343^{\prime} 59$ Chr I $476 \quad \mathrm{X} 331$

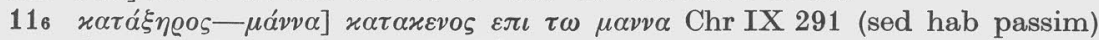

$118 \eta \eta \lambda \eta \vartheta 0 v] \eta \lambda \varepsilon \vartheta$ ov $58^{\mathrm{mg}} 77 d t^{-76^{*}} 39268^{\prime}-120^{\prime}$ Tht $N m 203^{\text {te }}$

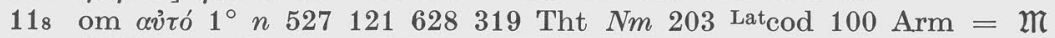

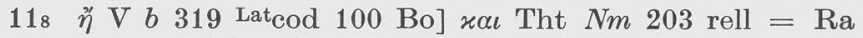

118 om av่có $2^{\circ} b$ Tht $N m 203$ Latcodd 94-96 $100 \mathrm{Arm}=\mathfrak{m}$

118 om av่ró $3^{\circ}$ Tht $N m 203$ Arm

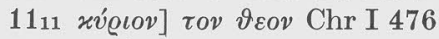

$1111 \dot{\varepsilon} \pi \iota \vartheta \varepsilon \tilde{\imath} \nu \alpha \iota]+\mu o \iota\left(\mu \varepsilon 107\right.$ Tht $\left.^{\mathrm{ap}}\right) d 767 t 527319$ Tht $N m 204$ Arm

$\left.1112 \tau \dot{v} v \pi \alpha^{\prime} \tau \alpha\right]$ om $\pi \alpha^{\prime} \tau \alpha$ Tht $N m$ 204te Lat $\operatorname{cod} 100$; tr B V $O^{-58} 422 f n x^{-619}$ Phil III 6 Chr I 476 Tht $N m$ 204ap $=$ Ra

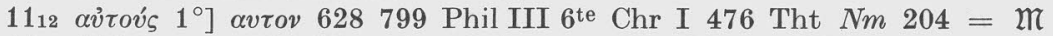

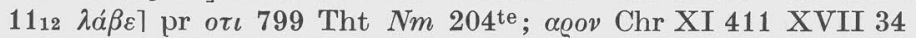

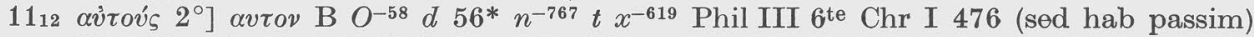
Tht $\mathrm{Nm} 204 \mathrm{Arm} \mathrm{Bo}$ Syh $=\mathrm{Ra} \mathfrak{m}$

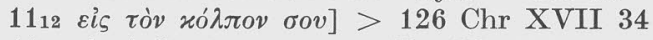

$1112 \omega \sigma \varepsilon i$ ả $\varrho \iota] \omega \varsigma \alpha v \alpha \varrho \eta \mathrm{Chr}$ XI $411 ; \omega \sigma \varepsilon \iota\left(+\alpha v^{\mathrm{ap}}\right) \lambda \alpha \beta o \iota$ Tht $N m 204$

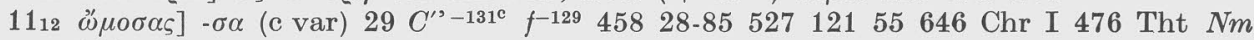
$204^{\text {ap }}$ Bo

$\left.11_{15} \varepsilon \ddot{\lambda} \lambda \varepsilon \circ \varsigma\right] \chi \propto \varrho \iota v \mathrm{~F} \mathrm{O}^{-426} 55176 \mathrm{Chr}$ I $476 \mathrm{Co}: \operatorname{ef~App~I~}$

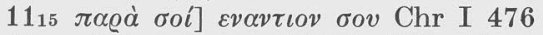

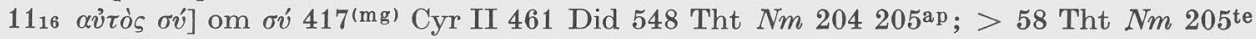
$=\mathfrak{M}$

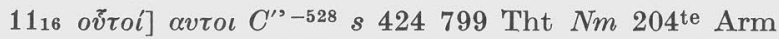

$1116 \pi \varrho o ́ \varsigma 2^{\circ}$ ] $\varepsilon \iota \varsigma$ A F M' $\mathrm{M}^{\prime} O^{\prime \prime} C^{\prime \prime}-46 f n s^{-130^{\mathrm{mg}} 321^{\prime \mathrm{mg}}}$ y $z 5559319624$ Tht $\mathrm{Nm} 204$

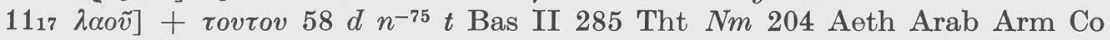

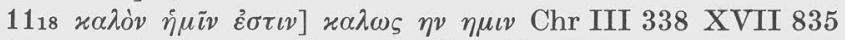

1120 om $\dot{\eta} \mu \varepsilon \varrho \tilde{\omega} \nu 64^{\mathrm{txt}}$ (c pr m) Tht $\mathrm{Nm} 205$

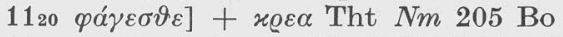

$\left.11_{21} \chi \iota \lambda \iota \alpha ́ \delta \varepsilon \zeta\right]+\varepsilon \iota \sigma \iota$ Chr I 506 Isid 1488

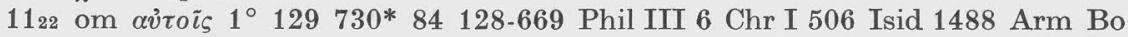

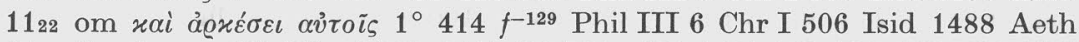

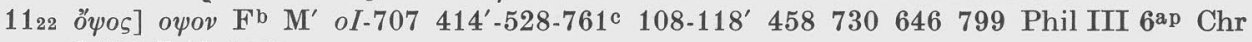
I 506 Isid 1488

1122 om av่roĩs $3^{\circ} \mathrm{A}$ oI 16-46 7555 Phil III 6 Chr I 506 Isid 1488 Latcod 100 Arm Bo

$\left.11_{29} \mu \circ \iota\right] \varepsilon \mu o \iota$ Chr XVI 520 Cyr VII 720

$\left.11_{29} \tau i \xi\right]+\alpha v$ Tht $N m 206^{\text {te }}$

$\left.11_{29} \delta \omega \dot{\eta}\right] \delta \omega \mathrm{V}$ G $75319 \mathrm{Chr}$ X 331 Tht $N m$ 206ap

$\left.11_{29} \pi \alpha \dot{v} \tau \alpha\right] \alpha \pi \alpha v \tau \alpha$ Chr X 331

$11_{29} \pi \varrho \circ \varphi \eta \dot{\tau} \alpha \varsigma+\varepsilon \iota v \alpha \iota x^{-509} \mathrm{Chr}$ IX $192 \mathrm{XVI} 520$ Sev 513 Tht $\mathrm{Nm} 206^{\text {ap }}$

122 $\lambda \varepsilon \lambda \dot{\alpha} \lambda \eta \varkappa \varepsilon(v)$ B 7244 74-76 630] $\varepsilon \lambda \alpha \lambda \eta \sigma \varepsilon(v)$ Chr X 331 Cyr II 592593 rell

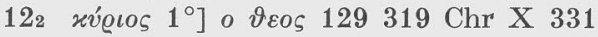




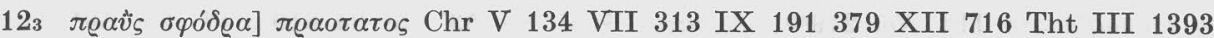

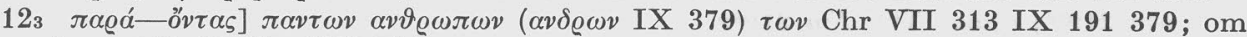
öv $\alpha$ ऽ $24655 \mathrm{Chr}$ XII 716

123 om rov่ $1^{\circ} 417 n^{-54} 84126$ Cyr II 592597 Tht III 1393

$126 \quad \dot{v} \mu \tilde{\omega} v] v \mu \nu$ Cyr II 600 Tht $\mathrm{Nm} 208^{\text {te }}$

126 om xveí Cyr VI 172 Tht $N m 208$

$\left.12_{6} \lambda \alpha \lambda \eta \dot{\sigma} \sigma \alpha \hat{\tau} \tau \tilde{\omega}\right]$ tr $n$ Tht $N m 208^{\text {te }}$

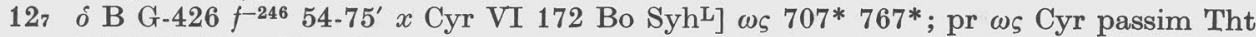
III $700 \mathrm{Nm} 208$ rell

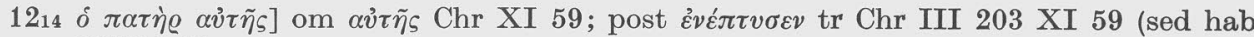
XIV 248)

$\left.12{ }_{14} \pi \tau v^{\prime} \omega \nu\right] \varepsilon \mu \pi \tau .376 f^{-129}$ Chr III 203 XI 59 Tht $N m$ 209; > Chr XIV 248 LatRuf Num VII 4 Bo

1214 om $\alpha \dot{\tau} \tilde{\eta} \varsigma 2^{\circ}$ Chr III 203 (sed hab XI 59)

$12_{14} \dot{\varepsilon} v \tau \varrho \alpha \pi \eta \dot{\sigma \varepsilon \tau \alpha l]}$ ( $\left.+\alpha \nu \mathrm{Chr}\right) \varepsilon v \varepsilon \tau \varrho \alpha \pi \eta$ Chr III 203 Tht $N m$ 209ap

$1214 \alpha \dot{\varphi} \circ \varrho \iota \sigma \vartheta \eta ं \tau \omega]-\vartheta \eta \sigma \varepsilon \tau \alpha \iota$ A 54-75' 799 Cyr II 592 Tht $N m$ 209te; $\mu \varepsilon \iota v \alpha \tau \omega$ Chr III 203

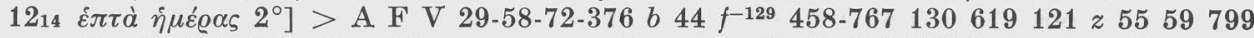

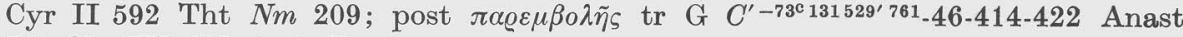

384 Chr III 203 Aeth Bo

$\left.1416 \delta v^{\prime} \alpha \sigma \vartheta \alpha \iota\right] \delta v v \eta \vartheta \vartheta v \alpha \iota$ Tht II 993

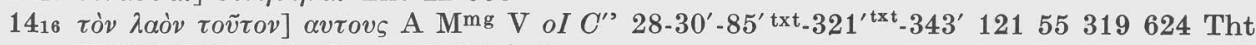
II 993 Lat Quodv Prom II 17 Aeth

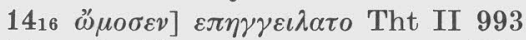

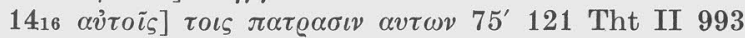

$1416 \varkappa \alpha \tau \varepsilon \sigma \tau \varrho \omega \sigma \varepsilon v] \varkappa \alpha \tau \eta v \alpha \lambda \omega \sigma \varepsilon v$ Tht II 993

$\left.1421 \dot{\alpha} \lambda \lambda \alpha^{\prime}\right] \alpha \lambda \lambda \eta n^{-127} 509319$ Tht II 41

$1421 \zeta \tilde{\omega} v] \zeta \eta \mathrm{V} 72414 d^{-125} 129 *$ (c pr m) $t$ 319*(c pr m) Cyr III 545 Procop 1936 Tht II $41 \mathrm{Nm} 210^{\text {te }}$ Lat cod 100 Bo

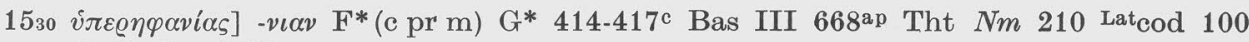
Aeth Armte Bo

$\left.15_{31} \dot{\eta} \dot{\alpha} \mu \alpha \varrho \tau i \alpha\right] \alpha \mu . \gamma \alpha \varrho d n t$ Tht $N m 211$ Arm

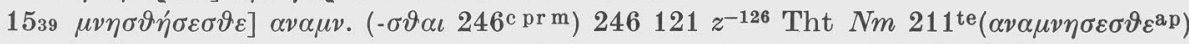

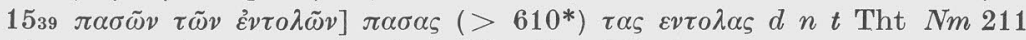

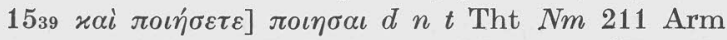

$15{ }_{39}$ om óxí⿴囗⿱一一 $2^{\circ} \mathrm{B} \mathrm{V} d 129 n^{-767} t \times 319$ Tht $N m 211$ Latcod 100 Arm Co

1539 om $\dot{v} \mu \tilde{\omega} \nu 2^{\circ} \mathrm{B} 15-82 c I-551 d 129 n^{-767} t^{-84} x^{-509} z 319$ Tht $N m 211$ Latcod 100

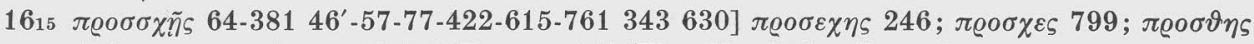

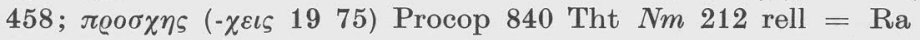

1622 ๖ำ B F 15-29-58-72-426 528* $129767 x 62855$ 59] pr $\eta$ Tht $N m 213$ rell

$\left.1640 \mu \eta \vartheta \varepsilon i_{\zeta}\right] \mu \eta \delta \varepsilon \iota \varsigma$ A F M' V 58-oI' $C^{\prime \prime} b 56^{\prime} n^{-127} s 76$ y $z^{-126} 5559$ Tht $N m 213$

$\left.17_{12} \alpha \dot{\pi} \alpha \hat{\alpha} \dot{\lambda} \alpha \mu \varepsilon \nu\right]+\varepsilon \xi o \lambda \omega \lambda \alpha \mu \varepsilon \nu$ Chr XVII 858

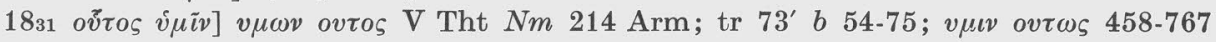

$1920 \alpha$ a $] \varepsilon \alpha v$ B $376^{\prime} 413 d n t 71799$ Eus VI 12 Tht $N m 215^{\text {te }}=\mathrm{Ra}$

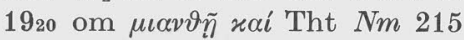

$2010 \varepsilon \dot{\xi} \dot{\alpha} \xi o \mu \varepsilon v] \varepsilon \xi \alpha \xi \omega 125246126$ Tht I $1732 \mathrm{Nm} 216^{\text {te }}$

2010 v $\mu \tilde{i} v \ddot{\delta} \delta \omega \varrho] \operatorname{tr} \mathrm{Chr} \mathrm{X} 332$

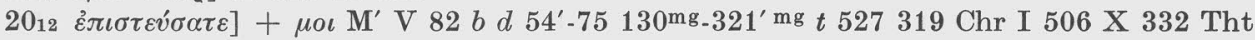
$\mathrm{Nm} 216^{\text {te }}$ Lat $_{\text {cod }} 100$ AethC Arab Arm

2012 vi $\tilde{\omega} v]$ pr $\tau \omega v$ A M $\mathrm{M}^{\prime} \mathrm{V}$ oI 413-422 $b d n 30^{\prime} t$ 527-619 $392319 \mathrm{Chr}$ I 506 X 332 Cyr II 489492 VI 452 Tht $\mathrm{Nm} 216$

2012 om $v \mu \varepsilon i \bar{\zeta} 58 \mathrm{Chr} \mathrm{I} 506 \mathrm{Bo}=\mathfrak{m}$

$2012 \delta \varepsilon \delta \omega \varkappa \alpha] \varepsilon \delta \omega \varkappa \alpha$ A M' V G-oI-15 $C^{\prime \prime-413} 53^{\prime}-129 n^{-458}$ s $527 y^{-392} 18-12655319 \mathrm{Chr}$

I $506 \mathrm{X} 332 \mathrm{Cyr}$ II passim Tht $\mathrm{Nm} 216$

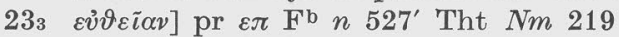

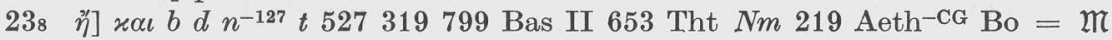

238 om $\delta C^{\prime s}-414$ Tht $N m 219^{\text {te }}$ 


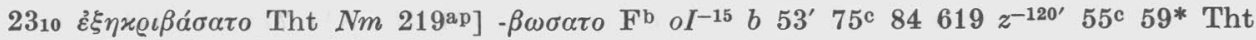
$N m 219^{\text {te }} ; \varepsilon \xi \alpha x \rho$. Tht $N m$ 219ap

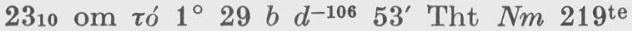

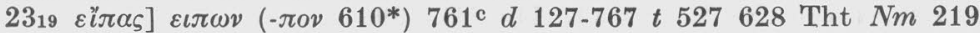

2319 ovंxi $2^{\circ}$ ] ovx F V 82-376' d 56' n $t 527624799$ Tht $N m 219$

$23{ }_{20}$ àं

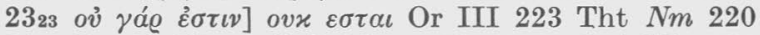

$23{ }_{23} \operatorname{T}_{\alpha} \alpha \omega \beta 2^{\circ}$ ] pr (※ Syh $\left.{ }^{\mathrm{L}}\right) \tau \omega \mathrm{F}^{\mathrm{b}} O 414 d n^{(-458)} t 527$ Or III 223 Cels II 420 Tht $\mathrm{Nm} 220$ Syh

$\left.242 \dot{\varepsilon}^{\prime}\right] \varepsilon v$ B $b^{-19}$ Tht $N m 221=\mathrm{Ra}$

$2424 K \iota \tau \iota \alpha i \omega v] \chi \varepsilon \tau \iota \eta \mu$ Tht $N m 221^{\text {te }} ; \chi \varepsilon \tau(\tau) \iota \varepsilon \iota \mu$ (c var) $761^{\text {txt }} d 127^{\text {c }}-458^{\text {txt }} t 319$ Tht $N m$ $221^{\text {ap }}$

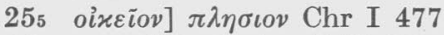

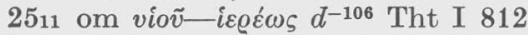

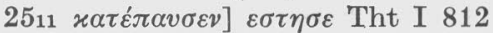

$2511 \zeta \eta \lambda \tilde{\omega} \sigma \alpha i]+(\div \mathrm{G}) \alpha v \tau o \nu$ V $\mathrm{O}^{-58}$ Tht I 812 Bo $=\mathfrak{m}$

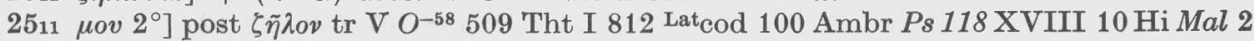

Hil Ps CXVIII 3 Arm Syh $=\mathfrak{m}$

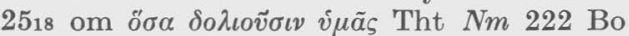

2719 om av̉นóv Tht $\mathrm{Nm} 224$

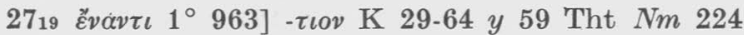

2719 है $v \alpha v \tau \iota 2^{\circ} 963$ ] - $\tau \iota$ A A 29-58-oI $d n t 619 y^{-392} 68^{\prime} 55319$ Tht $N m 224$

$2719 \sigma v v \alpha \gamma \omega \gamma \tilde{\eta} \varsigma$ 963] pr $\tau \eta \varsigma 426 d 53^{\prime}-56^{\mathrm{c}}-129 \mathrm{nt}^{-76} 619 y^{-392} 68^{\prime} 319$ Tht $N m 224$

2719 om $x \alpha i{ }^{\circ} 3^{\circ}$-fin 44-125 71319 Tht $N m 224$

$27_{20}$ ăv 963] > F 767 Procop 877 Tht $N m 224$ Syh

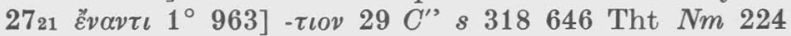

$318 \varepsilon \dot{v}$ @o

$318 \sigma v ́ v-\mathrm{fin}] \mu \varepsilon \tau \alpha \tau \omega v \tau \varrho \alpha v \mu \alpha \tau \iota \omega \nu$ Anast $573 \mathrm{Chr} \mathrm{XV} 193$

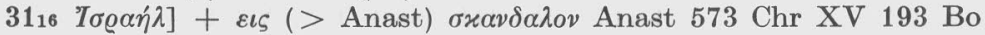

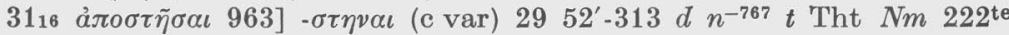

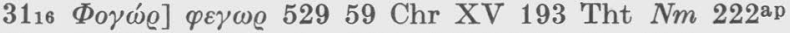

$\left.31_{16} \dot{\eta} 963\right]>376-o I n^{-767} 71^{\prime} 799$ Tht $\mathrm{Nm} 222$

Though Chr and Tht do not quote Numbers extensively certain interesting conclusions may be drawn. It is obvious, first of all, that a large number of unique or almost unique readings obtain in the above list. These need not be taken seriously since in most instances they do not reflect a true textual tradition but rather the individualistic freedom of the church father over against the Biblical text.

Secondly the text of $\mathrm{Chr} / \mathrm{Tht}$ has been influenced by the work of Origen. In at least ten instances from the list additions to the text almost certainly are due to the hexapla. These are $\left.56 \tau \tilde{\omega} v \alpha \mu \alpha \varrho \tau i \tilde{\omega} v] \mathrm{pr} \pi \alpha \sigma \omega v ; 514 \alpha \dot{v} \tau \tilde{\omega} 1^{\circ}\right] \mathrm{pr}$ $\varepsilon \pi ; 515 \delta \tilde{\omega} \varrho o \nu]+\alpha v \tau \eta \varsigma ; 517 \tau \tilde{\eta} \varsigma \gamma \tilde{\eta} \varsigma]$ pr $\alpha \pi \circ ; 66 \varepsilon v \dot{v} \chi \tilde{\eta} \varsigma]+\alpha v \tau o v ; 66 \varkappa v \varrho i \omega]$ pr $\tau \omega ;$

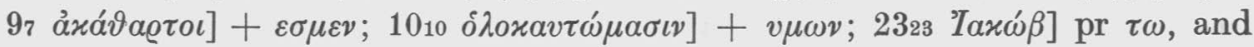
$2511 \zeta \eta \lambda \tilde{\omega} \sigma \alpha i]]+\alpha v \tau o \nu$.

Furthermore the Chr/Tht citations betray the strong influence of the Byzantine text type. If one analyzes the above list quantitatively it appears that of variants supported by no more than three text families in the list the $d n t$ families are best represented. The following table makes this clear. Column $A$ gives the number of instances in which one group supports the variant; $B$, two groups, and $C$, three groups. 


$\begin{array}{lrrrr} & A & B & C & \text { Total } \\ O & 7 & - & - & 7 \\ 0 I & 1 & 4 & 1 & 6 \\ C^{\prime \prime} & 3 & 4 & 2 & 9 \\ b & 5 & 3 & 2 & 10 \\ d & 2 & 7 & 13 & 22 \\ f & 2 & 1 & 1 & 4 \\ n & 14 & 5 & 11 & 30 \\ s & - & 2 & 3 & 5 \\ t & - & 7 & 13 & 20 \\ x & 1 & 1 & - & 2 \\ y & 1 & - & - & 1 \\ z & 1 & - & 2 & 3\end{array}$

It thus appears that $\mathrm{Chr} / \mathrm{Tht}$ variants are supported by the families to the following extent: $n 30, d 22, t 20, b 10, C^{\prime \prime} 9, O 7, o I 6, s 5, f 4, z 3, x 2$ and $y$. That the $\mathrm{Chr} / \mathrm{Tht}$ were strongly influenced by the Byzantine type text is clear.

It must be borne in mind, however, that this does not thereby fully identify their text. Equally important are the instances in which the Byzantine text

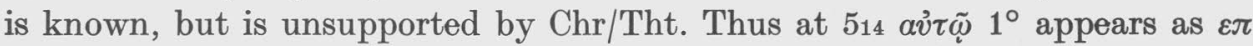

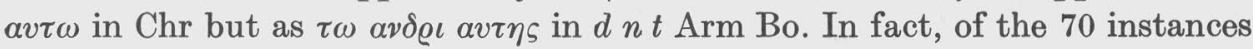
involved in this analysis 28 are not supported by representatives of the Byzantine group, i.e. have a text opposed to the group.

One question remains: is this Byzantine text type to be equated with the Lucianic recension? It has already been noted that this text shows little if any influence from the Hebrew text apart from the influence of hex.

On the other hand, the question remains whether the text contains doublet traditions, i.e. is it characterized by the commonly accepted mark of Lucianic work, the presence of doublets. To examine this possibility the plusses in the $d n t$ tradition are given in the following list. It should be mentioned that none of these plusses comes from the Hebrew tradition.

List 14

$120 \delta v v a ́ \mu \varepsilon \iota]+\overline{\imath \eta \lambda}$ 58-376-707 $d n t 18$ Arm Syh

$153 \varepsilon \dot{\varepsilon} \alpha v \tau i o l] \varepsilon v \alpha v \tau \iota$ (

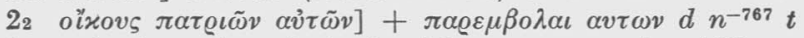

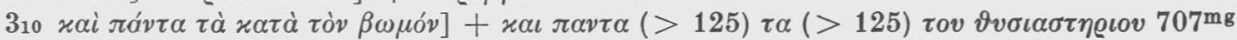
d $t 799$

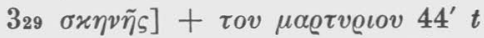

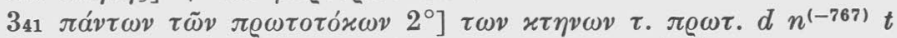

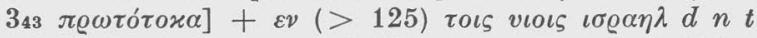

$420 \ddot{\alpha} \iota \iota \alpha]+\tau \omega \nu \alpha \gamma \iota \omega \nu d^{-610} n t$; pr $\tau \omega \nu \alpha \gamma \iota \omega \nu 610$

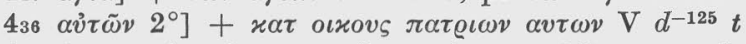

$\left.4_{48} \varepsilon \dot{\pi} \iota \sigma \varkappa \varepsilon \pi \varepsilon \dot{\nu} \tau \varepsilon \varsigma\right] \varepsilon \pi \iota \sigma \varkappa \varepsilon \varphi \vartheta \varepsilon \nu \tau \varepsilon \varsigma(\varepsilon \pi \varepsilon \sigma \varkappa .84) \pi \alpha \nu \tau \varepsilon \varsigma d t ;+\pi \alpha \nu \tau \varepsilon \varsigma 71$ y 799 
$515 \vartheta v \sigma i \alpha \mu \nu \eta \mu o \sigma v ́ v o v]+(+x \alpha \iota 125) \vartheta v \sigma \iota \alpha d^{-106} n t$ Tht $N m 196$ Arm

$520 \mu \varepsilon \mu i \alpha v \sigma \alpha \iota]$ pr $\sigma v(\sigma o \iota \mathrm{G}) O b d n^{-767} t$ Tht $N m 197$ Lat $^{2} \operatorname{Ood} 100$ Syh

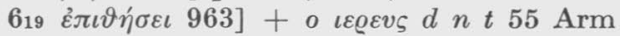

$73 \quad \approx \mu \alpha \xi \alpha \nu$ 963] $\alpha \mu \alpha \xi \alpha \mu \iota \alpha d 370 ;+\mu \iota \alpha v$ 58-376 $n t^{-370}$ Aeth Arm

710 ắ $\left.\chi 0 \nu \tau \varepsilon \varsigma 1^{\circ} 963\right]+\overline{\imath \eta \lambda} \mathrm{V} b 4^{\prime} t$ Lat $\operatorname{cod} 100$

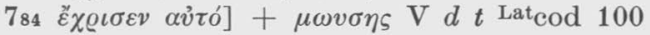

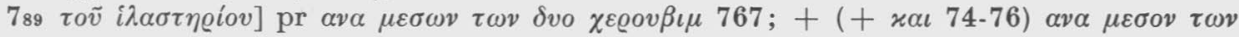
$\delta v o \quad \chi \varepsilon \varrho o v \beta \iota \mu$ (c var) $d^{-125} 54^{\prime} t 799$ Arm

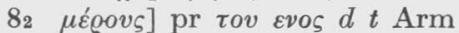

$811 \varepsilon \tilde{\varepsilon} \sigma \alpha]+\tau \eta \varsigma(\tau \eta 54-458) \sigma \varkappa \eta v \eta \varsigma d n^{-767} t$ Arm

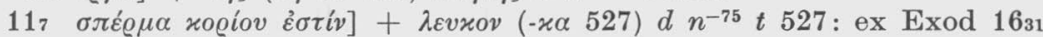

$119 \pi \alpha \varrho \varepsilon \mu \beta \circ \lambda \eta \dot{v}] \gamma \eta v \varepsilon \pi \iota(>16-46) \tau \eta \varsigma \pi \alpha \varrho \varepsilon \mu \beta \circ \lambda \eta \varsigma$ 16-46 $d t$

$1132 x \alpha i$ है $\psi v \xi \alpha v]$ pr $\varkappa \alpha \iota \varepsilon \sigma \varphi \alpha \xi \alpha v(-\xi \varepsilon v 767) 58-376 d f^{-129} n t 55$ Arm

$1317 \gamma \tilde{\eta} \nu]+\chi \alpha \nu \alpha \alpha \nu\left(-\nu \alpha v 54^{*}\right) \mathrm{V} d^{-44} n 130^{\mathrm{mg}}-321^{\prime} \mathrm{mg} t 39255319799$ Arab

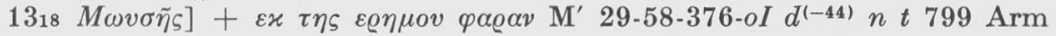

$\left.14{ }_{12} \pi \alpha \tau \alpha^{\prime} \xi \omega\right] \operatorname{pr} \alpha \varphi \varepsilon \varsigma \mu \varepsilon(>458) \varkappa \alpha \iota(>799) \mathrm{M}^{\prime}$ 58-376 d nt 55799 Arm

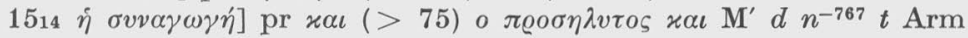

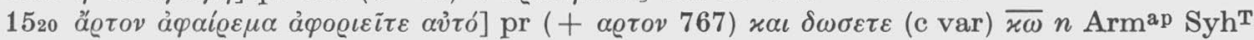

$1531 \alpha \mu \alpha \varrho \tau i \alpha]+\gamma \alpha \varrho d n t$ Tht $N m 211$ Arm

$\left.15{ }_{38} \tau \tilde{\omega} \nu \pi \tau \varepsilon \varrho v \gamma i \omega \nu\right]$ pr $\tau \omega \nu \iota \mu \alpha \tau \iota \omega \nu$ $\varkappa \alpha \iota d^{-106}$

169 $\quad \dot{\varepsilon} \varkappa(\sigma v \nu \alpha \gamma \omega \gamma \tilde{\eta} \varsigma)]+\mu \varepsilon \sigma o v d 127 t$ Arab Sa

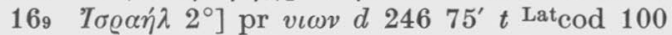

$\left.16{ }_{13} \dot{\alpha} \nu \eta \dot{\gamma} \gamma \alpha \gamma \varepsilon \varsigma \dot{\eta} \mu \tilde{a} \varsigma\right]+\varepsilon \xi \alpha \iota \gamma v \pi \tau o v$ V $58 b n^{-127}$

$173 \quad x \alpha \tau \dot{\alpha} \varphi v \lambda \dot{\eta} v]$ bis ser $127 t 527$

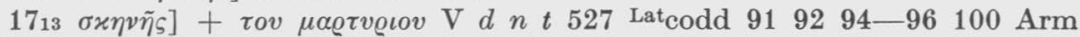

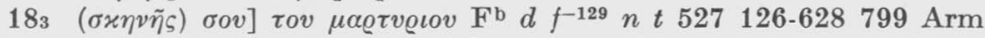

$1819 \delta \varepsilon \delta \omega \kappa \alpha]+\alpha v \tau \alpha d 54-75^{\prime} t$ Lat $\operatorname{cod} 100 \mathrm{Co}$

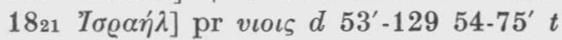

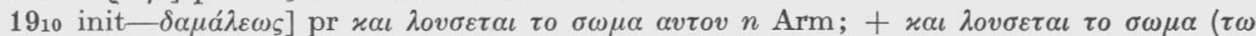
$\sigma \omega \mu \alpha \tau \iota$ pro $\tau . \sigma .610) \alpha v \tau o v d t$ Aeth $^{\mathrm{C}}: \operatorname{ex} 8$

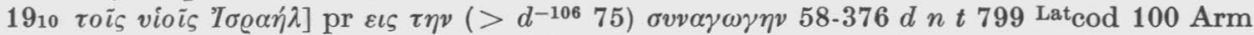

$19_{14}$ oixi $\left.\alpha v\right]+\alpha \varkappa \alpha \vartheta \alpha \varrho \tau \sigma \varsigma \varepsilon \sigma \tau \alpha \iota d t$ 799: ex sq; + $\varepsilon x \varepsilon \iota v \eta v \alpha x \alpha \vartheta \alpha \rho \tau \sigma \varsigma \varepsilon \sigma \tau \alpha \iota \mathrm{V}$

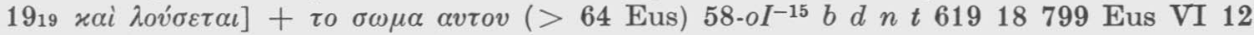
Aeth Arm

$202 \quad \tilde{\delta} \delta \omega \varrho]+\varepsilon x \varepsilon \iota d^{-610} n^{-767} t 527 \mathrm{Arm}^{\text {ap }} \mathrm{Sa}^{12}$

$202 \pi v v \alpha \gamma \omega \gamma \tilde{\eta}]+\pi \iota \varepsilon \imath(\pi \circ \varepsilon \varepsilon v 458) 58 d n t 527$ Lat cod 100 Arab Arm Sa ${ }^{12}$

$205 \sigma v \varkappa \alpha i]$ pr $\varepsilon \iota \sigma \iota(v)$ n 527; $+\varepsilon \iota \sigma \iota v d t$

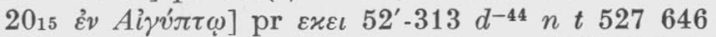

$2025 \sigma v v \alpha \gamma \omega \gamma \tilde{\eta} \varsigma]+(+\tau \omega v 527) v \iota \omega v \overline{\imath \eta \lambda} 82-376 b d^{(-125)} n t 527$ Latcod 100

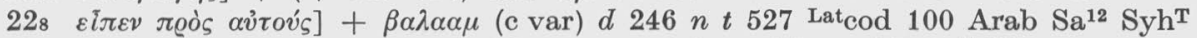

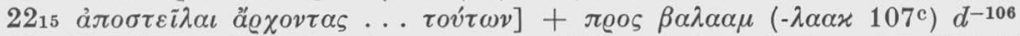

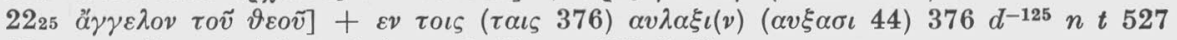

$\left.22{ }_{25} \mu \alpha \sigma \tau i \xi \alpha \iota \alpha \hat{\tau} \tau \dot{\eta} \nu\right]+\beta \alpha \lambda \alpha \alpha \mu O^{-426}$ nt $527 \mathrm{Sa}^{12}$

$\left.222_{38} \tau \dot{\partial} \varrho \tilde{\eta} \mu \alpha\right]$ pr $\varrho \eta \mu \alpha \pi \lambda \eta v d t$ Bo

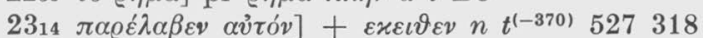

$2318 \alpha \dot{\alpha} \alpha \lambda \alpha \beta \omega \dot{ } v]+\beta \alpha \lambda \alpha \alpha \mu$ (c var) $\mathrm{M}^{\prime} \mathrm{mg} d^{-125} n^{-458} t 52755$

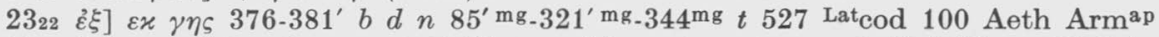

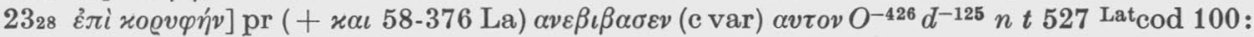
ex 2241

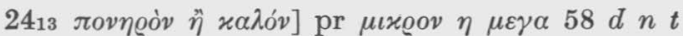

$2415 \grave{a} v \alpha \lambda \alpha \beta \omega ́ v]+\beta \alpha \lambda \alpha \alpha \mu d 127 t 55$

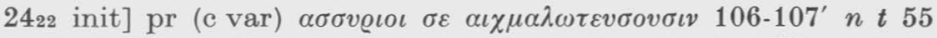

$2423 \mathrm{fin}]+(\sim \mathrm{Syh}) \varepsilon \pi \iota \tau \eta \varsigma \gamma \eta \varsigma \mathrm{M}^{\mathrm{mg}} d n t 416 \mathrm{Syh}^{\mathrm{T}^{\mathrm{mg}}}$

264 xai $\left.2^{\circ}\right]+\eta \sigma \alpha v d t$

$264 \mathrm{fin}]+\left(\mathrm{c}\right.$ var) $\tau \omega \alpha \varrho \alpha \delta \iota \delta \eta \mu о \varsigma$ o $\alpha \varrho \alpha \delta \iota \mathrm{V} 44-107^{\prime} t$ : cf $44 ;+\tau \omega \alpha \varrho \alpha \delta \iota v 125 ;+\tau \omega \alpha \varrho \alpha \delta \iota$

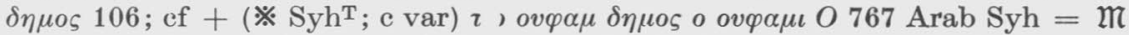

$2718 \pi v \varepsilon \tilde{v} \mu \alpha]+\vartheta \varepsilon o \tilde{v} \mathrm{~F}^{a} \mathrm{M}^{\prime} \mathrm{V} 707 b d n t 55319$ Latcod 100 Ruf Num XXII 4 Syh 
$287 \tau$ ขov iv $]+(\sim \mathrm{Syh})$ oเvov $\mathrm{M}^{\mathrm{mg}} 37644^{\prime}-10785^{\mathrm{mg}}-344^{\mathrm{mg}} t \mathrm{Syh} ;+\mu \varepsilon \tau \varrho o v(>127) \tau o v$ oivov $n$

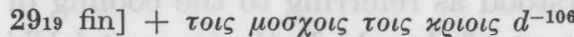

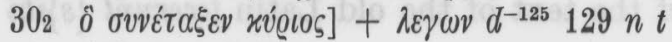

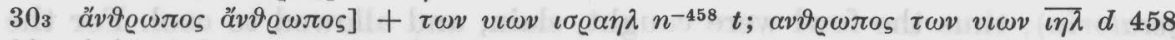

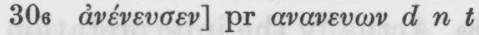

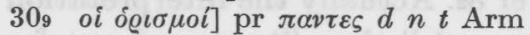

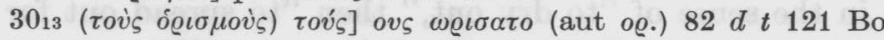

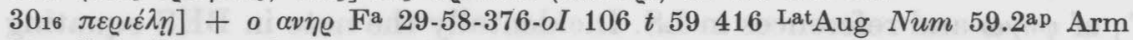

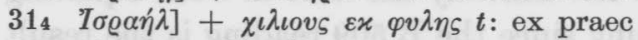

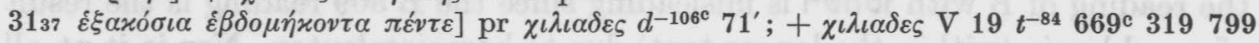
Armap Bo

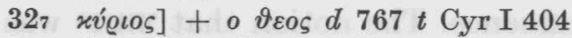

$3210 \ddot{\omega} \mu \sigma \sigma \varepsilon v]+\overline{x \zeta} n t$

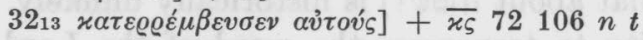

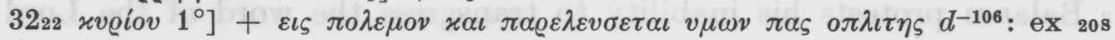

$\left.32_{32} \delta \iota \alpha \beta \eta \sigma o ́ \mu \varepsilon \vartheta \alpha\right]+\tau o v \iota 0 \varrho \delta \alpha v \eta v d n t$ Syh

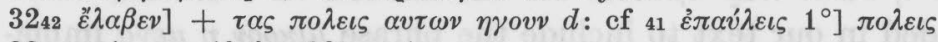

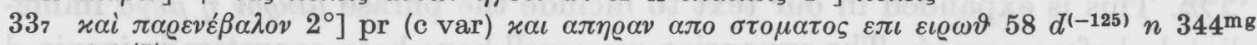
$t^{(-84 \mathrm{txt})} 121$ : ef 8

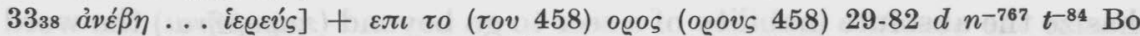

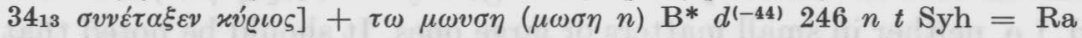

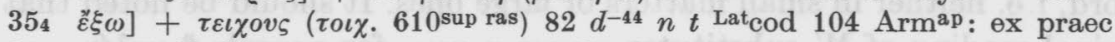

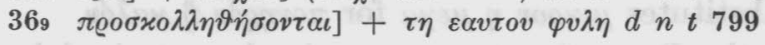

From the above list it would appear that the Byzantine text type is an expansionist type text. Over against this, however, it must be said that this text, particularly in the $d$ family, has a large number of omissions so that it would be quite incorrect simply to designate this text as expansionist.

An examination of the above list shows that most of the expansions are easily explained. They often constitute importations from the context, clarifying glosses such as the addition of subject or object or the rendering explicit what is implicit, or are due to familiar phrases such as "(tent) of testimony," "(spirit) of God," "(before) the Lord," "upon the land," "among the sons of Israel," "(holy) of holies," or "(land) of Canaan."

There are, however, surprisingly few genuine doublets in the list. At 310 it is said that Aaron and his sons are to guard their priesthood $\varkappa \alpha i \pi \alpha$ $\alpha \tau \alpha \tau \dot{\alpha} \varkappa \alpha \tau \dot{\alpha}$

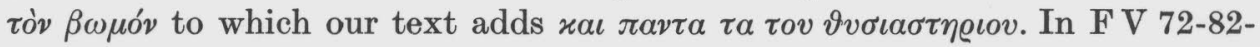
$707^{\text {txt }} b 767392 z 59646$ Aeth Arm Bo $\mathrm{Sa}^{4}$ this text also appears but as

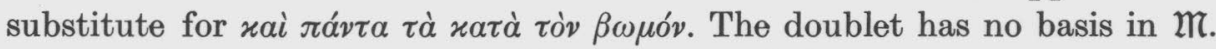

At 436 the census of the sons of Kaath is recorded $x \alpha \tau \dot{\alpha} \delta \eta \mu o v \varsigma \alpha v \tau \tilde{\omega} \nu$ to which

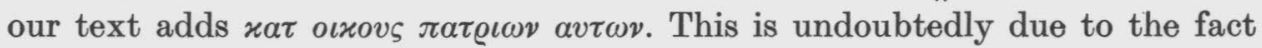
that the longer text occurs in the parallel verses $34,38,40,42,44$ and 46 .

An apparent doublet appears in 1132 in the account of the gathering of the quails. The people spent, it is said, an entire day and night as well as the following day in gathering quails to which $\mathfrak{N}$ adds "and they spread them out throughout around the camp." This was

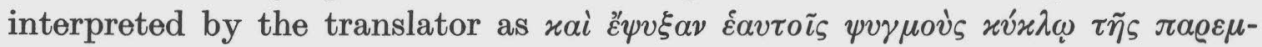
$\beta о \lambda \tilde{\eta} \varsigma$. The Byzantine text adds $\varkappa \alpha \iota \varepsilon \sigma \varphi \alpha \xi \alpha \nu$ before this clause, whereas B 509 
have $\varepsilon \sigma \varphi \alpha \xi \alpha v$ for $\ddot{\varepsilon} \psi v \xi \alpha v$. I suggest that the gloss originated in an attempt to clarify a difficult text. The text was understood as referring to the cooling of the bodies of the gathered quails (cf the text of the old Latin fecerunt $\{s\} i b i$ refrigeratoria); thus the fowl were caught, slain, and allowed to cool. For the equivalence of $\psi v \dot{\chi} \chi /$ ש 6 ef also Sam II $17_{19}$ Jer 82. Actually the interpretation was probably intended in the sense of "to dry out," then "to spread out for drying"; cf $\psi v \gamma \omega$ and references in LS. Incidentally the note in BHS equating the reading of B with רישחו is misleading since the B 509 reading is the result of parablepsis (within the longer text) due to homoioteleuton. Nor is it at all likely that the gloss was due to Hebrew influence. The notion that שט was misread as ישחטר by a revisor (then what about שטח ? is historically unlikely.

At $24_{13}$ Balaam protests his inability to transgress the word of the Lord

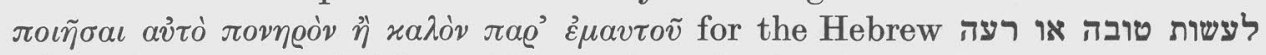

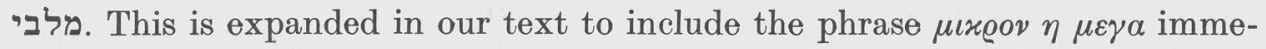
diately before лovnoóv. The expansion may well have been exegetically inspired to emphasize the absolute inability of a seer to go beyond ( $\pi \alpha \varrho \alpha \beta \tilde{\eta} v \alpha \iota)$ the word of the Lord, i.e. neither in small matters or large ones. It should be noted that

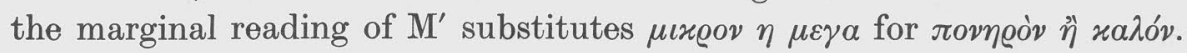

It can hardly be said that the Byzantine text group is characterized by doublets. It represents a text development which can be explained on the whole as an inner Greek one; that it is the result of a thoroughgoing recension does not appear to be correct. That this text type constitutes the Lucianic recension is possible but remains unproven. 


\section{Chapter 3 The Hexaplaric Recension}

The principal witnesses to hex are mss G-58-376-426 and Syh; of these ms 58 is the most aberrant. G, a IV. to V. Century uncial ms, has many hex signs as does Syh; it is unfortunately incomplete. Folios which contained 785-1118 $18_{2}-30 \quad 20_{22}-25_{2}$ and $26_{3}-29_{12}$ are no longer extant. Syh is almost entirely extant in two Bible mss, $\mathrm{Syh}^{\mathrm{L}}$ and $\mathrm{Syh}^{\mathrm{T}}$; only the following texts are lacking: $11-3$ and $67-77$.

Primary evidence for hex activity is to be found in the additions to Num which are sub ast. Though the asterisk tradition is not consistently correct in the mss., all of it is presented in the following list. If the possible source of the hex reading is known it is given in parentheses at the end of the citation.

\section{List 1}

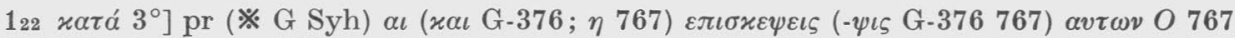
Syh $=$ Sam: ef $\mathfrak{m}$

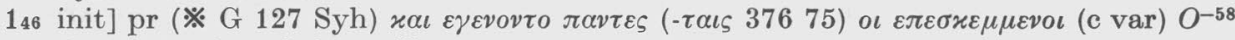
$d n t 799$ Arm Syh $=\mathbb{2}$

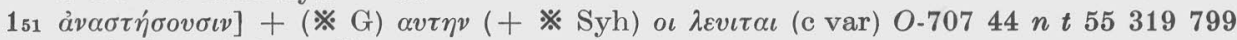
Arab Arm Syh $=\mathfrak{m}$

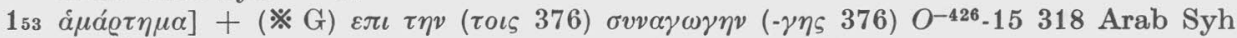
$=\mathfrak{m}$

$29 \quad \dot{\varepsilon} \varkappa \alpha \tau o ́ v]+(※ \mathrm{G} \mathrm{Syh}) \chi \iota \lambda \iota \alpha \delta \varepsilon \varsigma(\chi \varepsilon \iota \lambda . \mathrm{G}) O^{-376} \mathrm{Syh}=\mathfrak{m}$

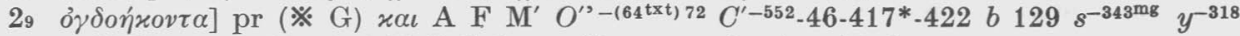
$z^{-126628} 5559624646 \mathrm{Bo}^{\mathrm{B}} \mathrm{Syh}=\mathfrak{m}$; et quadraginta $\mathrm{Bo}^{\mathrm{A}}$

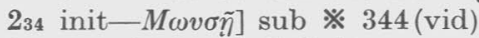

$\left.3_{25} \sigma \varkappa \eta v \eta^{\prime}\right]+(※ \mathrm{G} \mathrm{Syh}) \varkappa \alpha \iota \eta \sigma x \varepsilon \pi \eta \quad 767 \mathrm{Syh}=\mathfrak{m}$

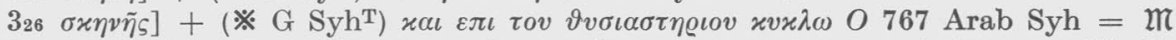

$\left.3_{38} \varkappa \alpha \tau \alpha \dot{-}-\sigma \varkappa \eta \nu \tilde{\eta} s\right]$ pr $※$ contra tabernaculum contra orientem $\measuredangle \mathrm{Syh}^{\mathrm{mg}} ;+(※ \mathrm{G}) \alpha \pi 0$

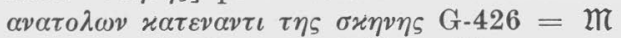

$\left.3_{47} \pi \varepsilon \dot{v} \tau \varepsilon\right]+(※ \mathrm{G}) \pi \varepsilon v \tau \varepsilon \mathrm{O}^{-58}=\mathfrak{m}$

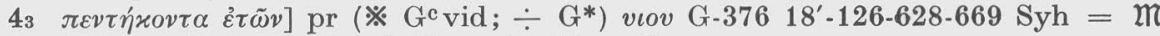

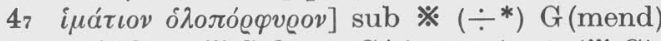

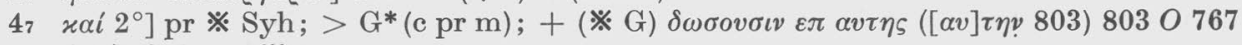
Arab $\mathrm{Syh}=\mathfrak{m}$

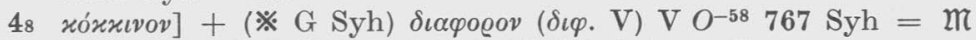

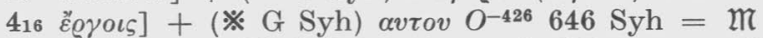

$419 \varepsilon \ddot{x} \alpha \sigma \tau o v]+(※ \mathrm{G} \mathrm{Syh}) \varepsilon \pi \iota \tau \eta v$ $\delta o v \lambda(\varepsilon) \iota \alpha v \alpha v \tau o v O 767$ Arab $\mathrm{Syh}=\mathfrak{m}$

$423 \varepsilon \dot{\varepsilon} \pi \dot{\alpha} v \omega]+(※ \mathrm{G}) \varepsilon x \varepsilon \iota \mathrm{O}^{-426}$ : contra $\mathfrak{k}$

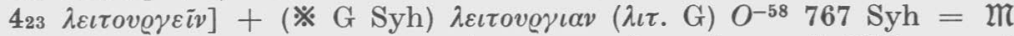

426 avinjs] +

58-72 13161959646 Aeth) $\tau \eta \varsigma$ $\alpha v \lambda \eta \varsigma\left(\tau \eta \varsigma \quad 1^{\circ}\right.$ - $\alpha v \lambda \eta \varsigma$ sub $\%$ G Syh) V 0-29-72

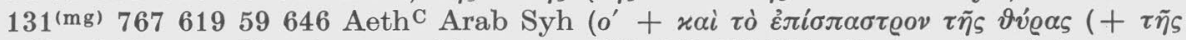

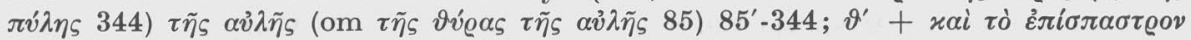

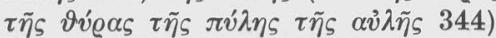

$426 \delta \sigma \sigma \alpha 1^{\circ}$ - $\left.\sigma \varkappa \eta \nu \tilde{\eta} \varsigma\right]$ sub $※ \mathrm{G}$ (mend) 


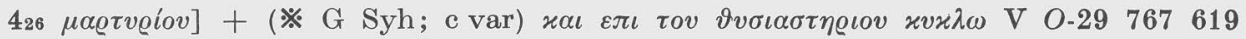
$\mathrm{Syh}=\mathfrak{m}$

$427 \dot{\eta} \lambda \varepsilon \imath \tau o v \varrho \gamma[\alpha]$ pr (※ G Syh) $\pi \alpha \sigma \alpha 0.29619 \mathrm{Sa}^{4} \mathrm{Syh}=\mathfrak{m}$

$430 \pi \varepsilon v \tau \eta \varkappa o \nu \tau \alpha \varepsilon \tau o \tilde{v} \varsigma] \mathrm{pr}(※ \mathrm{G} \mathrm{Syh})$ vเov $\mathrm{O}^{-426} \mathrm{Syh}=\mathfrak{m}$

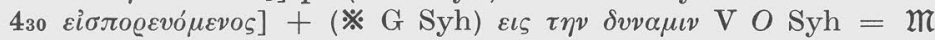

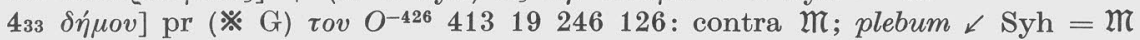

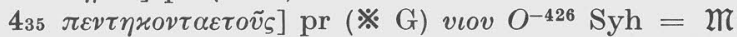

$439 \varepsilon \tilde{\omega} \omega]$ pr (※ Syh) $x \alpha \iota$ A $376^{\prime} b$ Latcod $100 \mathrm{Syh}=\mathfrak{m} ; * x \alpha \iota \varepsilon \omega \varsigma \ltimes \mathrm{G}$

$\left.4_{39} \pi \varepsilon v \tau \eta \varkappa 0 \nu \tau \alpha \varepsilon \tau o \tilde{v}_{\zeta}\right] \mathrm{pr}(※ \mathrm{Syh})$ vıov 58-376 $\mathrm{Syh}=\mathfrak{M}$

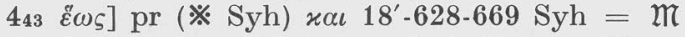

$4_{47} \varepsilon \tilde{\varepsilon} \omega \varsigma$ pr $(※ \mathrm{G} \mathrm{Syh}) x_{\alpha \iota} \mathrm{O}^{-426} \mathrm{Syh}=\mathfrak{m}$

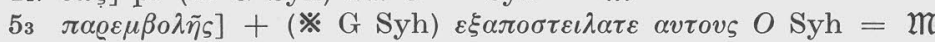

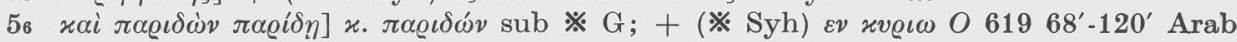
$\mathrm{Syh}=\mathfrak{m}$

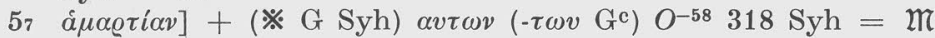

$57 \pi \lambda \eta \mu \mu \varepsilon \dot{\lambda \varepsilon} \varepsilon \alpha v]+(※ \mathrm{G} \mathrm{Syh}) \alpha v \tau o v O 767 \mathrm{Syh}=\mathfrak{m}$

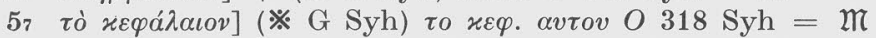

$510 \alpha v i \tau \tilde{v}]+(※ \mathrm{G}$ Syh) $\alpha v \tau \omega$ V O $767319 \mathrm{Syh}=\mathfrak{m}$

510 xai $2^{\circ}$ - fin] sub $※ \mathrm{G}$ Syh mend

$\left.5_{15} \delta \tilde{\omega} \varrho o v\right]+(※ \mathrm{G}$ Syh $) \alpha v \tau \eta \varsigma O \mathrm{Chr}$ II $917 \mathrm{Syh}=\mathfrak{k}$

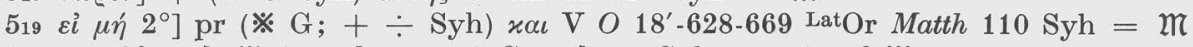

522 бov $2^{\circ}$ ] sub $※$ (mend pro $\div$ ) $\mathrm{G}$; sub $\div$ Syh (recte): of $\mathfrak{M}$

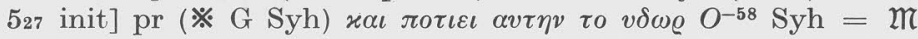

$\left.5_{27} x_{i \iota \lambda i \alpha}\right]+(※$ G Syh) $\alpha v \tau \eta \varsigma$ O $767 \mathrm{Chr}$ II 917 Latcod $100 \mathrm{Aug}$ Loc in hept IV 12 Arm $\mathrm{Co} \mathrm{Syh}=\mathfrak{m}$

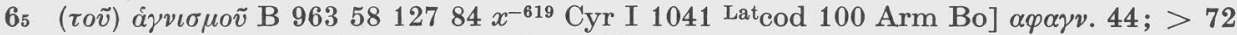
$319 ;+(※ \mathrm{G} ; \div$ Syh $) \alpha v \tau o v$ rell $=\mathfrak{m}$

$65 x \varepsilon \varphi \alpha \lambda \tilde{\eta} s]+\left(※\right.$ G Syh vid) $\alpha v \tau o v\left(\alpha v \tau o v \quad 56^{\mathrm{c}}\right)$ V $0.29 f^{-129} 767628319$ Arm Co $\mathrm{Syh}=\mathfrak{m}$

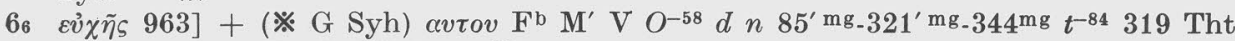
$\mathrm{Nm} 198$ Arm Bo $\mathrm{Sa}^{4} \mathrm{Syh}=\mathfrak{m}$

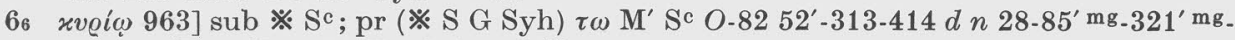
$344^{\mathrm{mg}} t$ Tht $N m 198$ Bo Syh: ef $\mathfrak{m}$

$\left.67 \pi \alpha \tau \iota^{\prime} 963\right]+(※ \mathrm{G} \mathrm{Syh}) \alpha v \tau o v O^{-58} 767 \mathrm{Syh}=\mathfrak{m}$

$67 \mu \eta \tau \varrho i$ 963] $+(※ \mathrm{G} \mathrm{Syh}) \alpha v \tau o v \mathrm{O}^{-58} 767 \mathrm{Syh}=\mathfrak{m}$

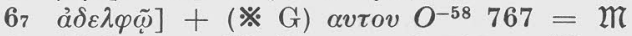

$67 \quad a j \delta \varepsilon \phi \tilde{\eta}]+(※ \mathrm{G}) \alpha v \tau o v O 767=\mathfrak{K}$

69 $\xi$

612 init] $\mathrm{pr}(※ \mathrm{G}) x_{\iota \iota} O^{(-58)}$ Aeth $=\mathfrak{m}$

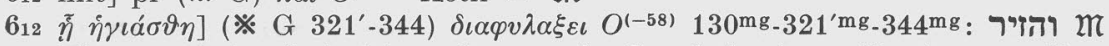

The metobolus is lacking after $x \alpha_{\iota}$ in $G$; ef the immediately preceding citation. Possibly the first part of v. 12 in Origen's hexapla read $\div \eta \eta \gamma \iota \alpha \sigma \vartheta \eta \nVdash \varkappa \alpha \iota \delta \iota \alpha \varphi v \lambda \alpha \xi \varepsilon \iota$ $\tau \omega \measuredangle \varkappa v \varrho \iota$; ef the texts of $\mathrm{ms} 58$ and 767 in App. I.

612 xveí 963$] \mathrm{pr}$ (※ G) $\tau \omega \mathrm{F} \mathrm{S} O^{3}-(58) 707619 z 59$ : cf $\mathfrak{n}$

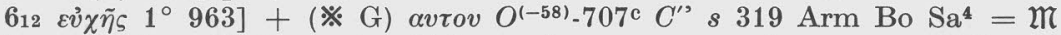

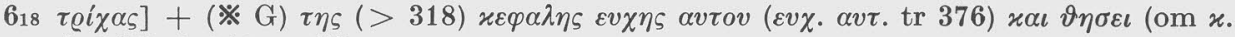

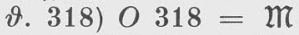

$\left.621 \varepsilon v ं x \tilde{\eta}_{\varsigma} 1^{\circ} 963\right]+\left(※ \mathrm{G}^{\mathrm{c}} ; \div \mathrm{G}^{*}\right) \alpha v \tau o v O C^{\prime \prime} b 610 s 318 \mathrm{Bo} \mathrm{Sa}^{4}=\mathrm{m}$

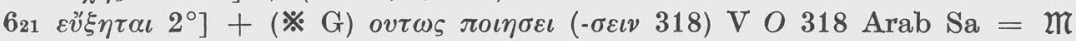

$\left.621 \quad \alpha \gamma v \varepsilon i \alpha{ }_{5} 963\right]+(※ \mathrm{G}) \alpha v \tau o v$ V $O^{\prime} d 767 t 318799$ Arab $\mathrm{Sa}^{4}=\mathfrak{m}$

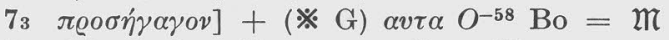

$\left.7_{72} \varepsilon v \delta \varepsilon \varkappa \alpha ́ \tau \eta\right]+※ \eta \mu \varepsilon \varrho \alpha \prec \mathrm{G}=\mathfrak{m}$

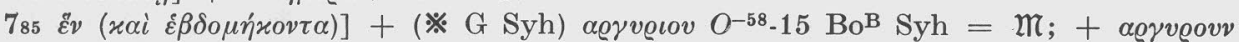
$85^{\prime} \mathrm{mg}_{-} 321^{\prime} \mathrm{mg} 319$; $x \alpha \iota \varepsilon \beta \delta$. sub $※ \mathrm{G}$

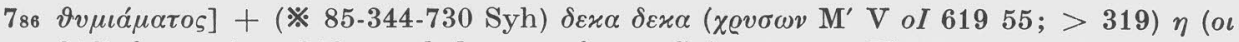

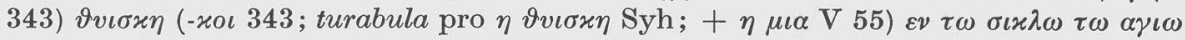
$(\alpha \gamma \iota \omega \sigma \iota \varkappa \lambda \omega$ pro $\sigma . \tau$. $\alpha .30) \mathrm{M}^{\prime} \mathrm{V} O^{\prime-58} s^{-321} 619 y^{-392} 18-126-62855319 \mathrm{Syh}=\mathfrak{m}$ 
$812 \chi \varepsilon i \varrho \alpha \varsigma 963]+\left(\right.$ Syh) $\alpha v \tau \omega \nu$ A $O^{-58} b$ 18'-126-628-669 Latcod 100 Arm Co Syh $=\mathfrak{m}$

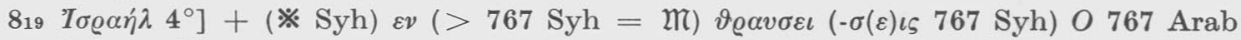
Syh $=\mathfrak{m}$

$\left.8_{25} \tau \tilde{\eta} \varsigma\right] \mathrm{pr}$ (※ Syh) $\tau \eta \varsigma \delta v v \alpha \mu \varepsilon \omega \varsigma$ (c var) $O 767 \mathrm{Syh}=\mathfrak{m}$

$\left.9_{3} \quad \tau o ́ v\right]$ pr (※ Syh) $\pi \alpha v \tau \alpha O^{-58} \mathrm{Syh}=\mathfrak{m}$

$\left.9_{3} \quad \tau \eta^{\prime}\right]$ pr (※ Syh) $\pi \alpha \sigma \alpha \nu O^{-58} \mathrm{Syh}=\mathfrak{m}$

95 init] pr (※ Syh) $\varkappa \alpha \iota \varepsilon \pi \circ \imath \eta \sigma \alpha v \tau o \pi \alpha \sigma \chi \alpha \mathrm{V} \mathrm{O} 106^{\mathrm{mg}} \mathrm{Syh}=\mathfrak{m}$

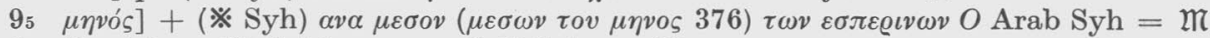

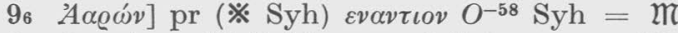

$97 \quad \bar{\eta} \mu \varepsilon i \bar{\zeta}]+(※ \mathrm{Syh}) \varepsilon \sigma \mu \varepsilon \nu \mathrm{O}$ Syh: contra $\mathfrak{m}$

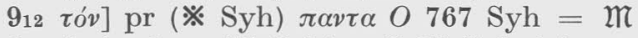

$\left.9_{21} \quad \bar{\eta} \mu \varepsilon \dot{\varrho} \propto \varsigma\right]$ pr (※ Syh) $\eta O^{-376}$ Syh ( $\sigma^{\prime}$ aut die Syh)

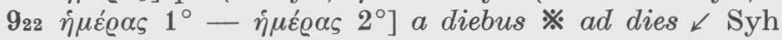

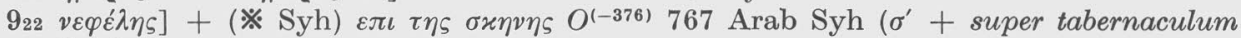
Syh)

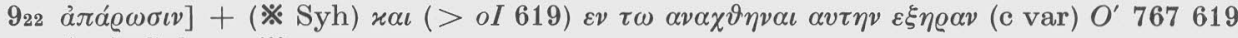
Arab $\mathrm{Syh}=\mathfrak{m}$

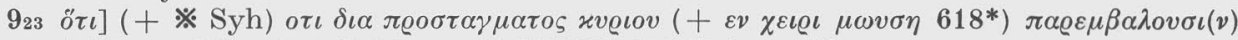

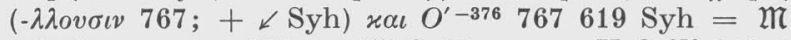

$\left.10_{3} \pi \tilde{\alpha} \sigma \alpha\right]$ pr $\varepsilon \pi \iota \sigma \alpha \iota 376$; pr (※ Syh) $\pi \varrho \circ \varsigma \sigma \varepsilon \mathrm{V} \mathrm{O} O^{-376} 246619 z 646$ LatOr Matth 52 Syh $=\mathfrak{m}$

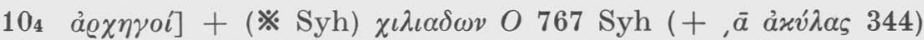

109 xv@iov] $+($ Syh) $\vartheta \varepsilon o v v \mu \omega v O$ Arab Syh $=\mathfrak{m}$

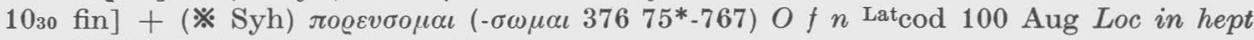
IV 25 Aeth Arm Syh $=\mathbb{m}$

$\left.10_{31} \mu \varepsilon \vartheta^{\prime} \eta \tilde{\eta} \tilde{\omega} v\right] \mathrm{pr}(※ \mathrm{Syh}) \varepsilon v \tau \eta \pi \alpha \varrho \varepsilon \mu \beta o \lambda \eta 426 \mathrm{Syh}:$ ef $\mathfrak{M} ;+x \alpha \iota \varepsilon v \tau \eta \pi \alpha \varrho \varepsilon \mu \beta o \lambda \eta O^{-426}$

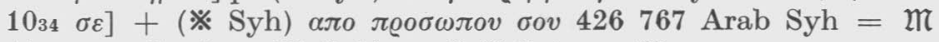

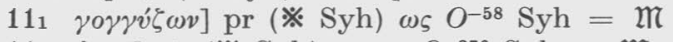

$1110 \varrho \gamma \tilde{\eta}]+(※ \mathrm{Syh}) \alpha v \tau o v O^{-376} \mathrm{Syh}=\mathfrak{m}$

$\left.114 \quad \delta 2^{\circ}\right]$ sub $※ \mathrm{Syh}^{\mathrm{T}}$

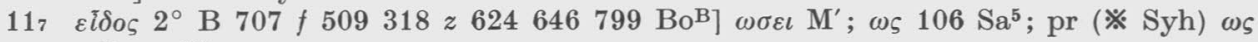
rell $=\mathfrak{m}$

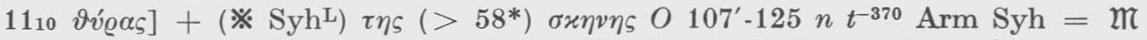

$\left.11_{11} \tau o \tilde{v} \lambda \alpha o \tilde{v}\right]$ pr (※ Syh) $\pi \alpha v \tau o \varsigma O^{-58} 24618^{\prime}-126-628-630^{\prime} \mathrm{Syh}=\mathfrak{m}$

$1114 \tau \dot{v} \nu \lambda \alpha o ́ v] \mathrm{pr}(※ \mathrm{Syh}) \pi \alpha v \tau \alpha O^{-58} \mathrm{Syh}=\mathfrak{m}$

$\left.11_{19} \delta \dot{v} o\right]+(※ \mathrm{G}$ Syh) $\eta \mu \varepsilon \varrho \alpha \varsigma O 246 \mathrm{Syh}=\mathfrak{m}$

$\left.11_{32} \dot{\eta} \mu \varepsilon \dot{\varrho} \alpha v 1^{\circ}\right]+(※ \mathrm{G} \mathrm{Syh}) \varepsilon x \varepsilon \imath v \eta v O d^{-44} f^{-129} n t$ 18'-126-628-669 $646 \mathrm{Syh}=\mathfrak{m}$

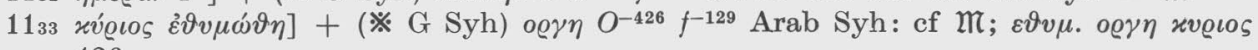
426

$126 a \dot{x} \alpha \hat{v} \sigma \alpha \tau \varepsilon]+(※ \mathrm{G} \mathrm{Syh}) \delta \eta O f^{-129} \mathrm{Syh}=\mathfrak{m}$

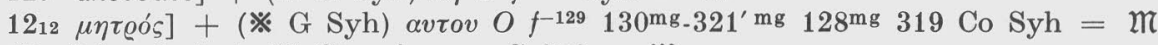

$\left.133_{3} \not ̋ \nu \delta \varrho \alpha \tilde{v} \alpha\right]+(※ \mathrm{G}) \alpha v \delta \varrho \alpha \varepsilon v \alpha \mathrm{G}-376=\mathfrak{T}$

$\left.13_{24} \dot{\alpha} v \alpha \varphi \circ \varrho \varepsilon \tilde{v} \sigma \iota v\right]+(※ \mathrm{G} \mathrm{Syh}) \delta v \sigma \iota(v) \mathrm{V} O 767 \mathrm{Syh}=\mathfrak{m}$

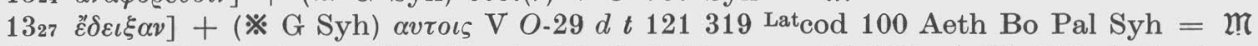

$\left.13_{33} \varkappa \alpha \tau \alpha \sigma \varkappa \varepsilon \dot{\psi} \alpha \sigma \vartheta \alpha \iota\right]$ sub $※ \mathrm{Syh} ;+(※ \mathrm{G} \mathrm{Syh}) \alpha v \tau \eta \nu O^{\prime-1558} n^{(-458)} 319$ Aeth Arm Pal Sa ${ }^{11}$ Syh (+ $\left.\alpha v i \eta \dot{v} 85-321^{\prime}-344\right)$

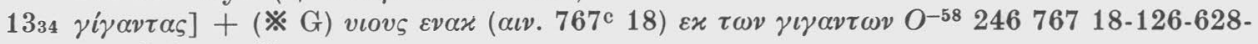
$630 \mathrm{Syh}=\mathfrak{m}$

$\left.141 \varphi \omega \nu \eta^{\prime}\right]$ pr (※ Gc Syh) $\tau \eta v O^{-58} \mathrm{Syh}=\mathfrak{k} ;+(※ \mathrm{G} \mathrm{Syh}) \alpha v \tau \omega \nu O b$ Arab $\mathrm{Syh}=\mathfrak{k}$

$145 \pi \varrho o ́ \sigma \omega \pi o v]+(※ \mathrm{G} \mathrm{Syh}) \alpha v \tau \omega \nu O$ Arab Arm Co Syh $=\mathfrak{m}$

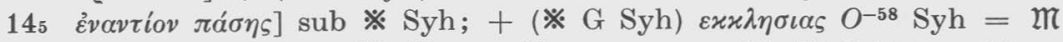

$147 \varkappa \alpha \tau \varepsilon \sigma \varkappa \varepsilon \psi a ́ \mu \varepsilon \vartheta \alpha]$ pr (※ G) $\pi \alpha \varrho \eta \lambda \vartheta \circ \mu \varepsilon v \varepsilon v \alpha v \tau \eta(+$ Syh) $\varkappa \alpha \iota$ (bis scr G) $O$ Arab Syh: of $\mathfrak{M}$

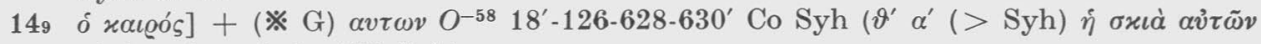
$\left.\sigma^{\prime} \dot{\eta} \sigma x \dot{\pi} \pi \eta \alpha \dot{\tau} \tilde{\omega} \boldsymbol{v} 108 \mathrm{Syh}\right)$

$1422 \sigma \eta \mu \varepsilon i \alpha]+(※ \mathrm{G}) \mu o v(+\measuredangle \mathrm{Syh}) \mathrm{VO} d t 799 \mathrm{Arab} \mathrm{Syh}=\mathfrak{m}$ 


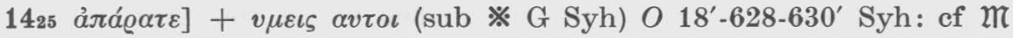

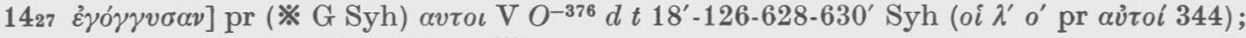

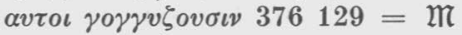

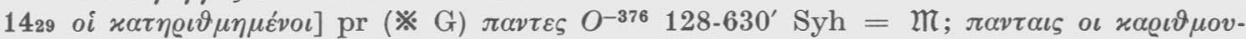
$\mu \varepsilon v o \iota 376$

$1431 \pi \alpha \iota \delta i \alpha]+(※ \mathrm{G}) v \mu \omega \nu$ O 767 18'-126-628-630' Sa Syh $=\mathbb{2}$

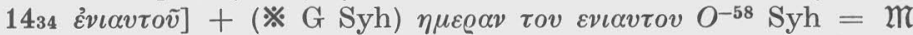

$1435 \tau \tilde{\eta} 1^{\circ}$ ] pr (※ G Syh) $\pi \alpha \sigma \eta O 24618^{\prime}-126-628-630^{\prime}$ Syh $=\prod$

$1436 \tau \dot{v} 2^{\circ}$ ] pr (※ G Syh) $\pi \alpha \sigma \alpha v O^{-376}$ Aeth Syh $=\mathfrak{K} ; \pi \alpha \sigma \alpha v 376$

$\left.1439 \alpha^{\prime}\right]$ pr (※ G Syh) $\pi \alpha \nu \tau \alpha O b$ Arab Syh $=\mathfrak{l i m s s}^{\mathrm{mss}}$

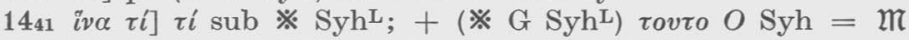

1441 v $\mu i v$ ] sub $※ \operatorname{Syh}^{\mathrm{L}}$ (mend pro $\div$ )

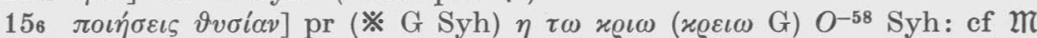

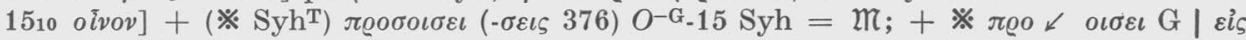
$\sigma \pi o v \delta \dot{\eta} v]$ sub $※ \operatorname{Syh}^{\mathrm{L}}$ (mend)

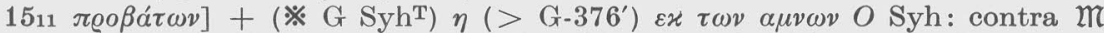

This hex addition is puzzling. Possibly Origen's Hebrew text had a gloss in it; in any event $2 \pi$ does not support the plus.

$1518 \varepsilon \dot{\varepsilon} \gamma \omega \dot{0}]$ sub $※ \mathrm{G} \operatorname{Syh}^{\mathrm{T}}$ (mend)

$\left.15_{23} \varkappa \alpha \vartheta \alpha^{\prime}\right] \varkappa \alpha \tau \alpha \pi \alpha \nu \tau \alpha\left(\operatorname{sub} \% \mathrm{G} \mathrm{Syh}^{\mathrm{T}}\right) \alpha(\omega \varsigma 58) O \mathrm{Syh}=\mathfrak{m}$

1528 fin B F V 72' $f^{-246} n^{-127} x 59$ Latcod 100 Aeth Arab Arm Sa] $+\left(※ \mathrm{G}^{\mathrm{c}} \mathrm{Syh}^{\mathrm{L}} ; \div \mathrm{G} *\right.$ )

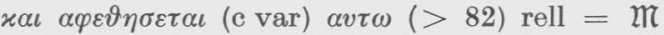

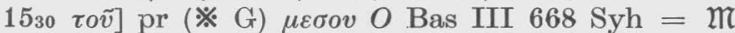

$\left.15_{36} \lambda i \vartheta o \iota \zeta\right]$ pr (※ Syh) $\varepsilon v$ A $O^{\prime(-58)} C^{\prime \prime}-550^{\prime} 767 s^{(-30)} 619 y^{(-392)} 18-68^{\prime}-62855319624$ Bo $\mathrm{Syh}=\mathfrak{m}$

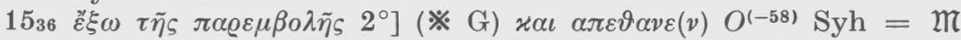

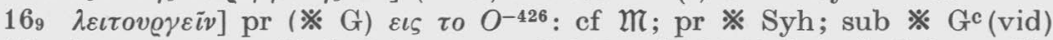

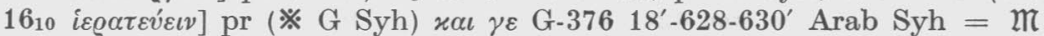

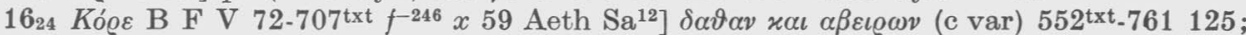

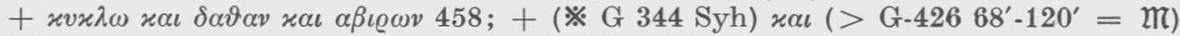

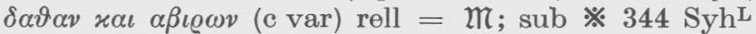

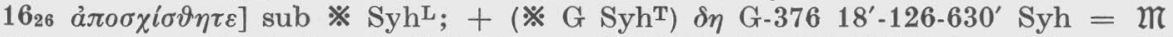

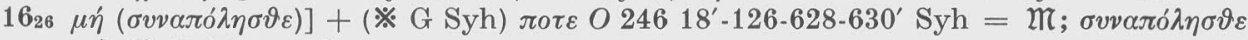
sub $※ \operatorname{Syh}^{\mathrm{L}}$ (mend)

16 Kó Kó $(x \dot{u} x \lambda \omega)$ B F M' V 72-707txt $f n x 39268^{\prime}-120^{\prime} 59319799$ Latcod 100 Aeth Arm Co] + (※ G Syh) $x \alpha \iota(>O 125=\mathfrak{m}) \delta \alpha \vartheta \alpha v(\mathrm{c}$ var) $\varkappa \alpha \iota \alpha \beta \iota \omega \nu$ (c var) rell $=\mathfrak{m}$; $x v \dot{x \lambda \omega} \operatorname{sub} ※ \operatorname{Syh}^{\mathrm{L}}$ (mend)

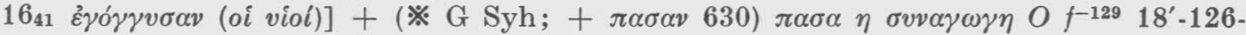
628-630' 646 Arab $\operatorname{Syh}(\mathrm{s}$ metob $\mathrm{T})=\mathfrak{M}$; oi vioi $\mathrm{sub} ※ \mathrm{Syh}^{\mathrm{L}}(\mathrm{mend})$

$1647 \varepsilon i \xi]+\left(※ \mathrm{G} \mathrm{Syh}^{\mathrm{T}}\right) \mu \varepsilon \sigma \eta v O f^{-129} \mathrm{Syh}=\mathfrak{m}$

$\left.16_{48} \tau \varepsilon \vartheta \nu \eta x o ́ \tau \omega \nu\right]$ sub $※ \operatorname{Syh}^{\mathrm{L}}($ mend)

$16_{48} \tau \tilde{\omega} \nu 2^{\circ}$ ] pr (※ G) $\alpha v \alpha \mu \varepsilon \sigma o v 0.15 f^{-129}$ Aeth Arab Bo Syh $=\mathfrak{m}$

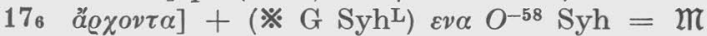

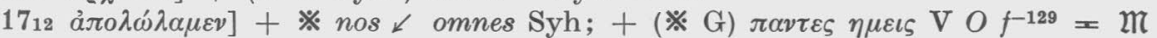

181 бov $2^{\circ} \mathrm{B}$ V $58529129 x^{-527} 126 \mathrm{Cyr}$ I $\left.837 \mathrm{Arab} \mathrm{Co}\right]+($ Syh) $\mu \varepsilon \tau \alpha$ (sup ras 75), $\sigma o v$ $(>628)$ rell $=\mathfrak{M}$; sub $※ \mathrm{G}$ (mend)

$181 \sigma o v$ ult $]+\left(※\right.$ G Syh) $\mu \varepsilon \tau \alpha \sigma o v\left(>56^{*}\right) O^{-58} f^{-129} 121 \mathrm{Syh}=\mathfrak{2}$

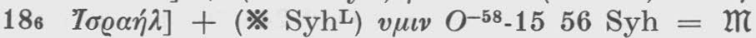

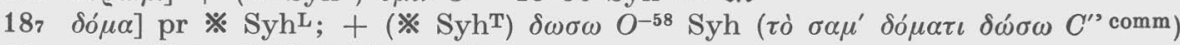

$188 \tau \tilde{\omega} \nu$ vi $\tilde{\omega} v]$ sub $※ \operatorname{Syh}^{\mathrm{L}}(\mathrm{mend})$

189 $\alpha \mu \alpha \varrho \tau(\tilde{\omega} v]+\left(※ \mathrm{Syh}^{\mathrm{L}}\right) \alpha v \tau \omega \nu$ F $O-29 d f^{-56} n t x^{-509} z^{-128669} 646$ Cyr I 837 Latcod $^{100}$ Arm $\mathrm{Sa}^{11} \mathrm{Syh}=\mathfrak{m}$

$1816 \mu \eta v \iota \alpha i o v]+(※ \mathrm{Syh}) \lambda v \tau \varrho \omega \sigma \eta O \mathrm{Syh}=\mathfrak{m}$

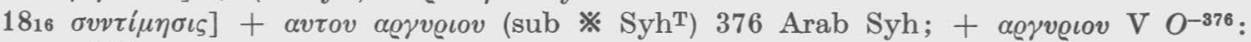
ef $\mathfrak{i n}$

$1817 \sigma \tau \varepsilon \dot{\varepsilon} \alpha]+\left(※ \mathrm{Syh}^{\mathrm{L}}\right) \alpha v \tau \omega \nu \mathrm{O}^{-58}-15 \mathrm{Bo} \mathrm{Syh}=\mathfrak{m}$

$1818 \varkappa \varrho \varepsilon ́ \alpha]+\left(※ \mathrm{Syh}^{\mathrm{L}}\right) \alpha v \tau \omega \nu \mathrm{O}^{-58} \mathrm{Sa}^{12} \mathrm{Syh}=\mathfrak{\eta}$ 
$1826 \dot{\varepsilon} v x \lambda \dot{\eta} \varrho \omega]+\left(+※ \mathrm{Syh}^{\mathrm{L}}\right) v \mu \omega \nu \mathrm{O}^{-58} 767 \mathrm{Syh}=\mathfrak{m}$

$\left.18_{29} \alpha \dot{\alpha} \propto \alpha i \varrho \varepsilon \mu \alpha\right]$ pr (※ Syh) $\pi \alpha \nu O^{-58} 130^{\mathrm{mg}}-321^{\prime} \mathrm{mg} 18^{\prime}-126-630^{\prime} 319$ Latcod $100 \mathrm{Syh}=\mathfrak{m}$

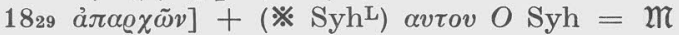

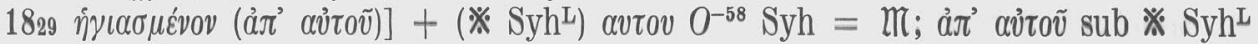
(mend)

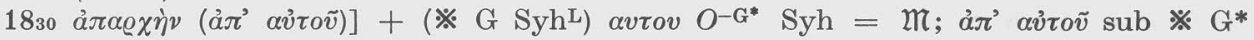
(mend)

1832 à $\pi \propto \varrho \eta \dot{\eta} v]+(※ \mathrm{G} \mathrm{Syh}) \alpha v \tau o v O 767 \mathrm{Sa} \mathrm{Syh}=\mathfrak{m}$

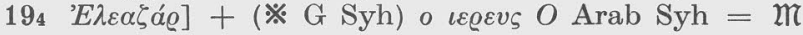

$\left.194 \alpha v i \tilde{\eta} \varsigma 1^{\circ}\right]+\left(\% \mathrm{G}\right.$ Syh) $\tau \omega \delta \alpha \varkappa \tau v \lambda \omega \alpha v \tau o v \mathrm{~V} O^{-376} 108^{\mathrm{mg}} 767$ 18'-126-628-630' 646 Aeth' Arab Syh $=\mathfrak{M}$

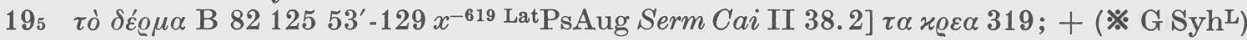

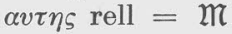

$\left.198 \alpha v i \tau o \tilde{v} 1^{\circ}\right]+\left(※ \mathrm{G} ;+\ldots \mathrm{Syh}^{\mathrm{L}}\right) \varepsilon v v \delta \alpha \tau \iota \mathrm{O}^{(-376)}$ Aeth $\mathrm{C}$ Syh $=\mathfrak{m}$

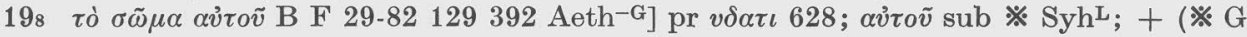

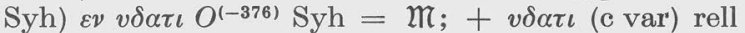

$198 \quad \varepsilon \sigma \pi \varepsilon \dot{\varrho} \alpha \varsigma$ pr $※ \tau \eta \varsigma \ltimes G=\mathbb{G}$

$1912 \alpha \gamma \nu \iota \sigma \vartheta \eta \dot{\sigma \varepsilon \tau \alpha l]}+(※ \mathrm{G}) \varepsilon v \alpha v \tau \omega 0.15 \mathrm{Syh}=\mathfrak{k}$

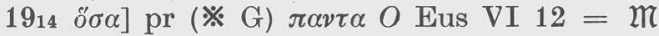

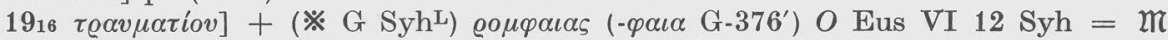

$\left.19_{18} \tau \alpha^{\prime}\right]$ pr $(※ \mathrm{G} ;+\ldots \mathrm{Syh}) \pi \alpha \nu \tau \alpha O \mathrm{Syh}=\mathfrak{m}$

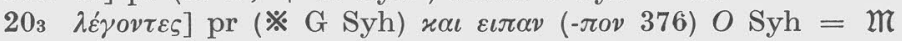

$\left.204 \pi \tilde{\alpha}_{s}\right]$ pr $\left(※ \mathrm{G} ;+\ldots \mathrm{Syh}^{\mathrm{L}}\right) \varepsilon x \varepsilon \iota O^{-376} \mathrm{Syh}=\mathfrak{m}$

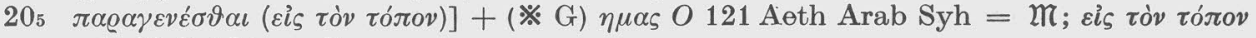
sub $※ \operatorname{Syh}^{\mathrm{L}}$ (mend)

$206 \pi \varrho o ́ \sigma \omega \pi \circ v]+\left(※ \mathrm{G} \mathrm{Syh}^{\mathrm{L}}\right) \alpha v \tau \omega \nu$ O Arab Arm Co Syh $=\mathfrak{m}$

$2011 \tau \tilde{\eta} \varrho a ́ \beta \delta \omega]$ sub $※ \mathrm{Syh}^{\mathrm{L}} ;+(※ \mathrm{G}) \alpha v \tau o v \mathrm{~V} O \mathrm{Syh}=\mathfrak{m}$

$2012 \varepsilon \dot{\pi} \iota \sigma \tau \varepsilon v \dot{\sigma} \alpha \tau \varepsilon]+\mu o \iota \mathrm{M}^{\prime} \mathrm{V} 82 b d 54^{\prime}-75130^{\mathrm{mg}}-321^{\prime} \mathrm{mg} t 527319 \mathrm{Chr}$ I 506 X 332 Tht $N m 216^{\text {te Lat }}$ cod 100 Aethc Arab Arm; + (※ G Syh) $\varepsilon v \varepsilon \mu o \iota O$ Bas I 440 Syh $=\mathfrak{m}$

$2023 \not \alpha \varrho \omega ́ v]$ pr $※ a d \measuredangle \mathrm{Syh}=\mathfrak{M}$

$\left.20_{26} \varepsilon \ddot{v} \delta v \sigma o v\right]+(※ \mathrm{Syh}) \alpha v \tau \eta v$ O 121 Co $\mathrm{Syh}=\mathfrak{m}$

$\left.2028 \varepsilon \varepsilon^{\xi} \xi \varepsilon \dot{\varepsilon} \delta \sigma \varepsilon v\right]+(※ \mathrm{Syh}) \mu \omega v \sigma \eta \varsigma(\mu \omega \sigma .58-426) \mathrm{V}$ O-82 $\mathrm{Syh}^{-\mathrm{G}}=\mathfrak{M}$

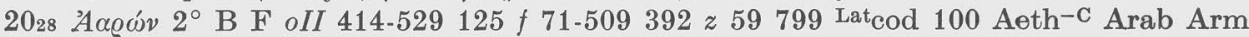
Co] pr $\varepsilon \varkappa \varepsilon \iota 551 b^{-19} ;+(※ \mathrm{Syh}) \varepsilon \varkappa \varepsilon \iota$ rell $=\mathfrak{m}$

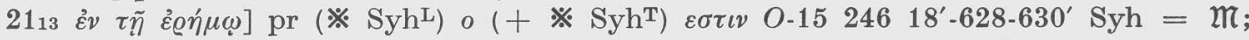
$+\swarrow$ Syh

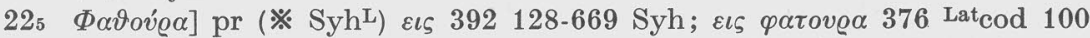

The correctness of the ast is highly questionable. Though $\varepsilon \iota \varsigma$ is present in one hex Greek ms, there is no preposition in $\mathfrak{m}$.

$2217 \sigma \varepsilon]+(※ \mathrm{Syh}) \sigma \varphi \circ \delta \varrho \alpha O^{-58} 24676718^{\prime}-126-628-630^{\prime}$ Bo Syh $=\mathfrak{m}$

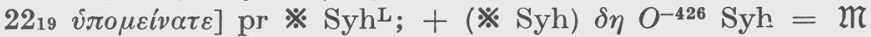

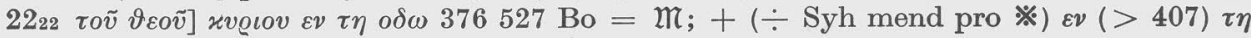

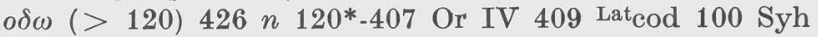

$\left.22{ }_{23} \varrho о \mu \varphi \alpha i \alpha v\right]+\left(※ \mathrm{Syh}^{\mathrm{L}}\right) \alpha v \tau o v \mathrm{O}^{-58}$ Or IV 409 Co $\mathrm{Syh}=\mathfrak{n}$

$\left.2223 \varepsilon \dot{\pi} \alpha^{\prime} \tau \alpha_{\xi \varepsilon}\right]+(※ \mathrm{Syh}) \beta \alpha \lambda \alpha \alpha \mu$ (balam La) M'mg V Odnt 527 Or IV 409 Latcod 100 Arab Syh $=\mathfrak{m}$

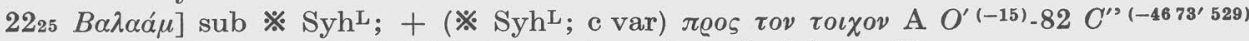
$246 s^{(-343)} 619 y^{-392} z^{(-628)}$ LatAug Num 50 Arab Syh $=\mathfrak{m}$

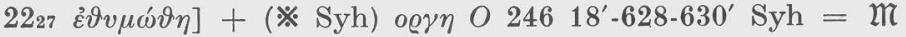

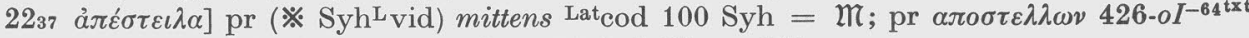
246 18'-628-630'; pr $\alpha \pi \circ \sigma \tau \varepsilon i \lambda \alpha \varsigma 376 b d^{-125} n t 319$

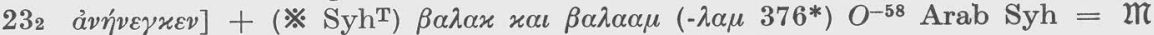

$236 \alpha \dot{v} \tau o \tilde{v} 1^{\circ}$ ] sub $※ \operatorname{Syh}(\mathrm{mend}) ;+\alpha v \tau o s O^{-58}=\mathfrak{m}$

Obviously it is $\alpha v \tau o 5$, not $\alpha v \tau o \tilde{v}$ which belongs sub ast.

$2315 \pi \alpha \varrho \alpha ́ \sigma \tau \eta \vartheta \iota]+\left(※ \mathrm{Syh}^{\mathrm{L}}\right) \alpha v \tau \sigma v \mathrm{~V} 426 \mathrm{Syh}=\mathfrak{k}$

$2320 \alpha \dot{\pi} \tau \sigma \tau \varrho \varepsilon ́ \psi \omega]+(※ \mathrm{Syh}) \alpha v \tau \eta v$ O 767 LatRuf Num XVI 2 Syh $=\mathfrak{m}$ 


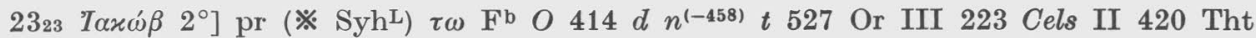
$N m 220$ Syh: ef $\mathfrak{m}$

242 qviás] $+\left(※ \mathrm{Syh}^{\mathrm{L}}\right) \alpha v \tau o v O \mathrm{Syh}=\mathfrak{m}$

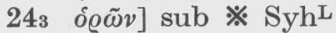

244 init $-\vartheta \varepsilon o \tilde{v} 1^{\circ}$ ] sub $※$ M 344 Syh

$248 \dot{\varepsilon} \chi \vartheta \varrho \tilde{\omega} v]$ pr $※ \mathrm{Syh}^{\mathrm{L}}$

The tradition of the asterisk must be faulty for the last three citations.

$2410 \varepsilon \dot{\varepsilon} \vartheta \mu \omega \hat{\vartheta} \eta]+(※ \mathrm{Syh}) \circ \varrho \gamma \eta O^{-58} 767 \mathrm{Syh}=\mathfrak{m}$

$2411 \tau \iota \mu \eta \dot{\sigma} \sigma \omega]$ pr $\left(※ \mathrm{Syh}^{\mathrm{L}}\right) \tau \iota \mu \omega \nu \mathrm{O}^{-58} \mathrm{Syh}=\mathfrak{k}$

2416 a่xov́ $\omega v]$ pr (※ Syh) $\varphi \eta \sigma \iota O^{-58}-15106^{c}$ Arab Syh $=\mathfrak{M}$

$254 x v \varrho i \omega$ B V $82 d 53^{\prime} n^{-75^{*}} t$ 71-509 319 Cyr I 908 IV 300] > 75*; pr (※ G) $\tau \omega$ rell: ef $\mathfrak{n}$

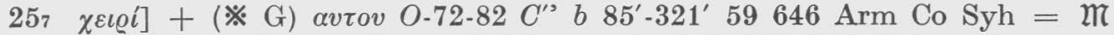

$2511 \zeta \eta \lambda \tilde{\omega} \sigma \alpha i]+(\div$ mend pro $※ \mathrm{G}) \alpha v \tau o v$ V $\mathrm{O}^{-58}$ Tht I 812 Bo $=\mathfrak{m}$

$\left.2512 \delta \iota \alpha \vartheta \eta^{\prime} \varkappa \eta v\right]$ pr (※ G Syh $\left.{ }^{\mathrm{L}}\right) \tau \eta v$ G-426 Syh; +

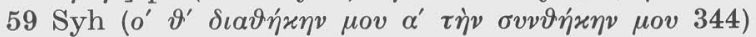

The hex must have had $\mu o v$ rather than $\tau \eta v$ sub ast.

$\left.2518 \delta 0 \lambda \iota \tau^{\prime} \eta \tau \iota\right]+\left(※ \mathrm{G} \mathrm{Syh}^{\mathrm{L}}\right) \alpha v \tau \omega \nu \mathrm{O}-15 \mathrm{Syh}=\mathfrak{m}$

2690 o $\tau o \iota]+\left(※ \mathrm{Syh}^{\mathrm{T}}\right) \delta \alpha \vartheta \alpha v x \alpha \iota \alpha \beta \iota \omega \nu\left(\mathrm{c}\right.$ var) $O^{-58}$ Lat $\mathrm{cod} 100 \mathrm{Syh}=\mathfrak{m}$

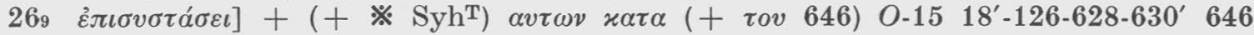
$\mathrm{Syh}=\mathfrak{m}$

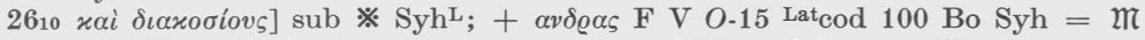

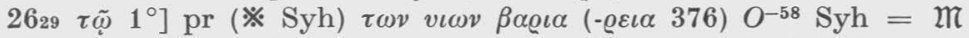

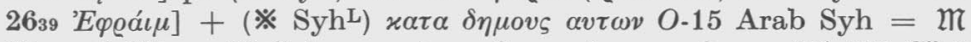

$2639 \tau \tilde{\omega} 2^{\circ}$ ] pr (※ Syh; c var) $\tau \omega \beta \alpha \chi \alpha \varrho \delta \eta \mu о \varsigma$ o $\beta \alpha \chi \alpha \varrho \iota \mathrm{M}^{\prime} 426 C^{\prime \prime} 246 s 392$ 18-126-628 646 Arab Syh $=\mathfrak{m}$

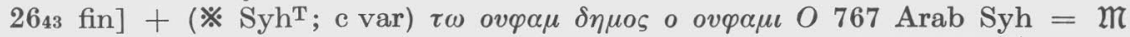

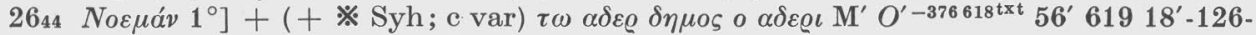
628-630' $\mathrm{Bo}^{\mathrm{B}} \mathrm{Sa} \mathrm{Syh}=\mathfrak{K}$

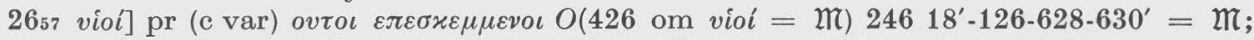
$\mathrm{pr} ※ \varepsilon \pi \varepsilon \sigma \varkappa \varepsilon \mu \mu \varepsilon v \omega \nu \swarrow 85^{\mathrm{mg}} ;+\varepsilon \pi \varepsilon \sigma \varkappa \varepsilon \mu \mu \varepsilon v \omega \nu 130^{\mathrm{mg}}-321^{\prime} \mathrm{mg} ;+※$ visitati $\swarrow \mathrm{Syh}$

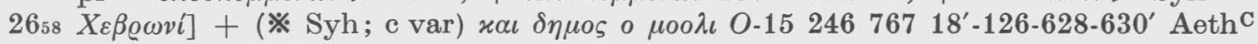
Arab Syh $=\mathfrak{m}$

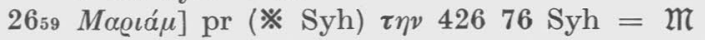

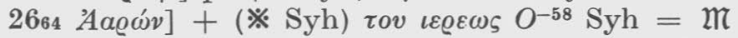

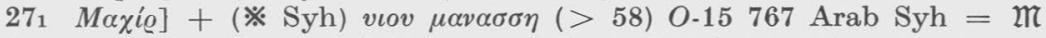

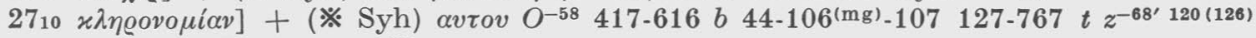
799 Arm Bo Syh $=\mathfrak{m}$

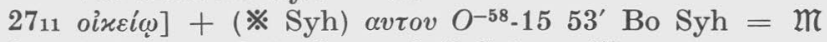

$\left.27_{15} \mathrm{fin}\right]+(※ \mathrm{Syh}) \lambda \varepsilon \gamma \omega \nu \mathrm{O}^{-58} \mathrm{Syh}=\mathfrak{m}$

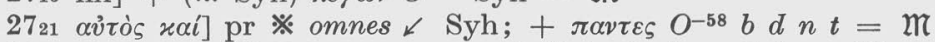

$285 \dot{\varepsilon} \lambda \alpha i \omega]+\left(※\right.$ Syh) $\varkappa \varepsilon \varkappa o \mu \mu \varepsilon v \omega O^{-58}$ Arab Arm Syh $=\mathfrak{m}$

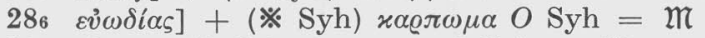

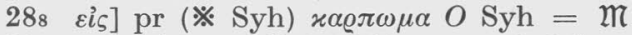

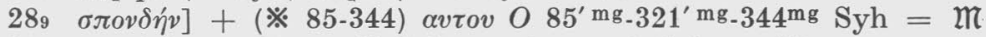

$\left.2812 \sigma \varepsilon \mu \iota \delta \alpha^{\prime} \lambda \varepsilon \omega \varsigma 1^{\circ}\right]+(※ \mathrm{Syh}) \varepsilon \iota \varsigma \vartheta v \sigma \iota \alpha \nu O \mathrm{Syh}=\mathfrak{M}$

$\left.2812 \sigma \varepsilon \mu \iota \delta a ́ \lambda \varepsilon \omega \varsigma 2^{\circ}\right]+(※ \mathrm{Syh}) \varepsilon \iota \varsigma \vartheta v \sigma \iota \alpha v \mathrm{M}^{\prime} O \mathrm{Syh}=\mathfrak{m}$

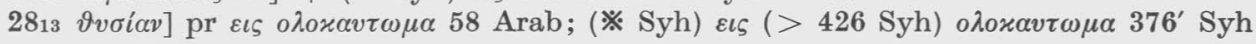
$=\mathfrak{m}$

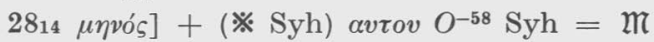

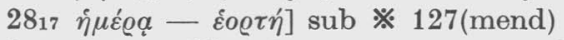

$\left.28_{23} \mathrm{fin}\right]+(※ \mathrm{Syh}) \pi \circ \imath \eta \sigma \tau \varepsilon\left(\mathrm{c}\right.$ var) $O 619121^{\mathrm{mg}} z^{-120} 646 \mathrm{Syh}=\mathfrak{m}$

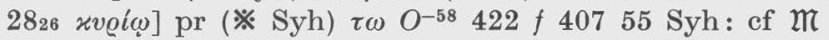

$2826 \varepsilon \beta \delta o \mu a ́ \delta \omega \nu]+(※ \mathrm{Syh}) v \mu \omega \nu O^{-58}-15 \mathrm{Arab} \mathrm{Syh}=\mathfrak{m}$

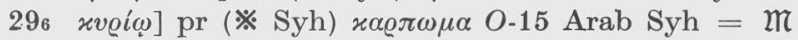

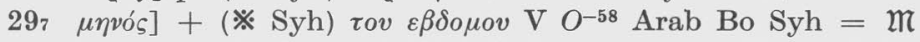

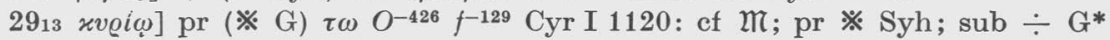




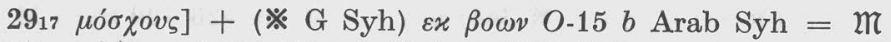

$29_{39} \pi \lambda \dot{v} v-\tilde{v} \mu \tilde{\omega} v 2^{\circ}$ ] sub ※ (mend) G Syh

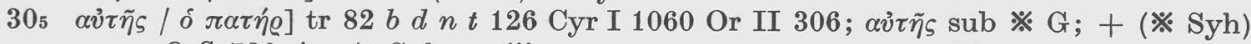
avins $0^{-\mathrm{G}} 730$ Armte Syh $=\mathbb{M}$

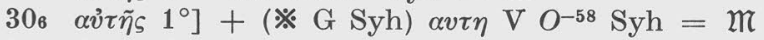

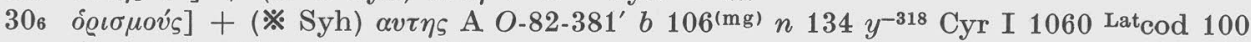
Aug Num 57 Co Syh $=\mathfrak{m}$

306 fin] $+(※$ G Syh $) \alpha v \tau \eta O^{-58} \mathrm{Syh}=\mathfrak{m}$

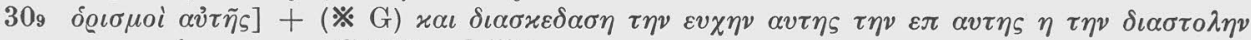

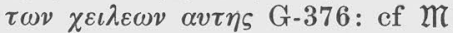

3010 ö $\sigma \alpha]$ pr (※ G Syh) $\pi \alpha v \tau \alpha O \mathrm{Syh}=\mathfrak{k}$

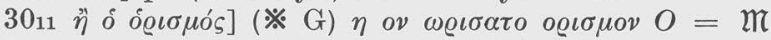

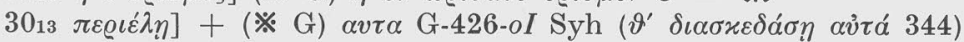

$3013 \pi \varepsilon \varrho \iota \varepsilon i \lambda \varepsilon v]+(※ \mathrm{G}) \alpha v \tau \alpha(+\ltimes \mathrm{Syh}) \mathrm{O}^{(-58) \operatorname{Lat}} \operatorname{cod} 100 \mathrm{Syh}=\mathfrak{m}$

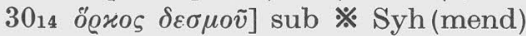

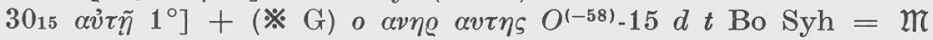

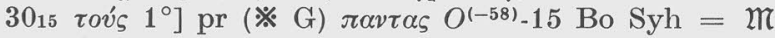

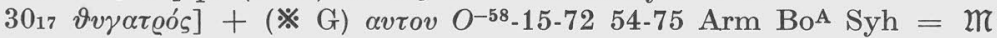

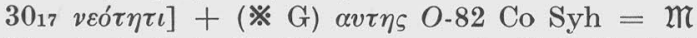

$\left.31_{6} i \varepsilon \varrho \varepsilon ́ \omega \varsigma\right]+(※ \mathrm{G}) \varepsilon \iota \varsigma \pi \alpha \varrho \alpha \tau \alpha \xi \xi \nu \mathrm{V} O^{-58}-15 \mathrm{Syh}=\mathfrak{m}$

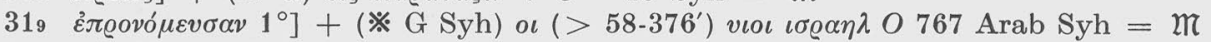

$\left.319 \tau \eta^{\prime} 2^{\circ}\right]$ pr (※ G Syh) $\pi \alpha \sigma \alpha \nu$ O $767 \mathrm{Syh}=\mathfrak{m}$

$31{ }_{10} \tau \alpha \dot{s} 3^{\circ}$ ] pr (※ G) $\pi \alpha \sigma \alpha \varsigma O \mathrm{Syh}=\mathfrak{m}$

3117 है $\gamma v \omega \varkappa \varepsilon v]+(※ \mathrm{Syh}) \alpha \nu \delta \varrho \alpha \varepsilon \iota \varsigma O^{-58} f^{-129} \mathrm{Syh}=\mathfrak{m}$

$\left.31_{23} \pi v \varrho i\right]+\left(※\right.$ Syh; c var) $\delta \iota \alpha \xi \varepsilon \tau \varepsilon \varepsilon v \pi v \varrho \iota 15-376^{\prime} f^{-129}$ LatRuf Num XXV 6 Arab Syh $=\mathfrak{m} ;+\circledast x \alpha \iota(\sup \operatorname{ras}) \pi v \varrho \iota \iota \varepsilon \xi \varepsilon \tau \alpha \iota\llcorner\mathrm{G}$

$3124 i \mu \alpha ́ \tau \iota \alpha]+(※ \mathrm{G}) v \mu \omega \nu O^{-58} f^{-129} \mathrm{Cyr}$ I 329 Latcod 100 Ruf Num XXV 6 Arab Sa $\mathrm{Syh}=\mathfrak{m}$

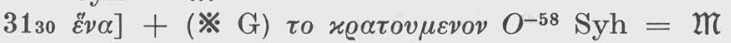

$\left.31_{32} \varepsilon \dot{\gamma} \varepsilon v \eta^{\prime} \vartheta\right]+(※ \mathrm{G}) \tau \alpha \sigma \varkappa v \lambda \alpha O^{-58} 56^{\prime} \operatorname{Syh}(+\tau \dot{\alpha} \sigma \varkappa v \tilde{\lambda} \alpha$ (c var) 130-321')

$\left.31_{32} \varepsilon \beta \delta \circ \mu \eta^{\prime} \varkappa o \nu \tau \alpha\right]+(※ \mathrm{G}) \chi \iota \lambda_{\iota \alpha \delta \varepsilon \varsigma}$ (c var) A Feprm $\mathrm{M}^{\prime}$ G-29-426-707-oI $C^{\prime \prime} b^{-19} 246 s$ $y^{-392} z^{-126407669^{*}} 55624$ Syh $=\mathfrak{n}$

$\left.31_{36} \tau \varrho \iota \alpha \varkappa o ́ \sigma \iota \alpha \iota\right], \bar{\gamma} 77$; + (※ G) $\chi \iota \lambda \iota \alpha \delta \varepsilon \varsigma$ (c var) A F M' $O^{\prime \prime}-7282 C^{\prime \prime}-7756^{\prime} s^{-343344^{\circ}} 509 y$ $z^{-126407} 5559624799$ Syh $=\mathfrak{M}$

$\left.31_{38} \tau \varepsilon \dot{\lambda} \lambda o \varsigma\right]+(※ \mathrm{G}) \alpha v \tau \omega \nu O^{-58}-15 \quad 53^{\prime}-56-246^{\mathrm{mg}} 767 \mathrm{Syh}=\mathfrak{m}$

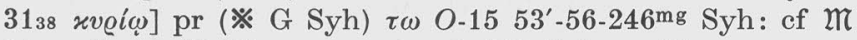

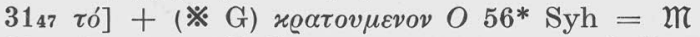

$3150 \eta \mu \tilde{\omega} v]$ pr $\left(※ \mathrm{G} ;+\tau \omega \nu 669^{c}\right) \psi v \chi \omega \nu O^{-58}-15$ 128-630' Arab Syh $=\mathfrak{n}$

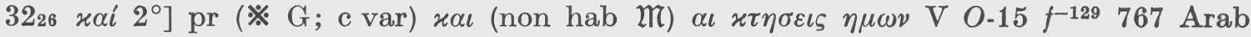
$\mathrm{Syh}=\mathfrak{m}$

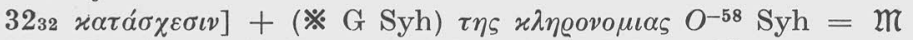

$\left.3235 \Sigma \omega \varphi a^{\circ}\right]$ pr (※ G) $\alpha \tau \alpha \varrho \omega \vartheta O^{-58}$ Arab Syh $=\mathfrak{n}$

3237 xai $2^{\circ} 7+\left(※ \mathrm{G}\right.$ Syh) $\tau \eta v O^{-426} 422 b f^{-129} n 799$ Syh $=\mathfrak{n}$

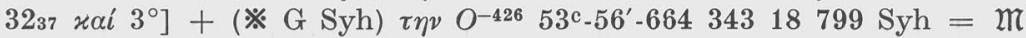

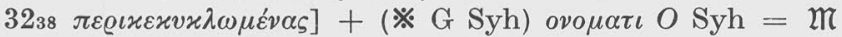

3239 Eis] sub $\% G$ (mend)

$332 \sigma \tau \alpha \vartheta \mu o i] ~+(※ \mathrm{G}) \alpha v \tau \omega \nu \varkappa \alpha \iota(>82-707=\mathfrak{m}) \mathrm{O}^{-58}-15-82-707$ Arab $\mathrm{Syh}=\mathfrak{m}$

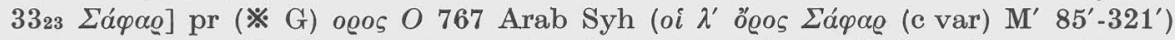

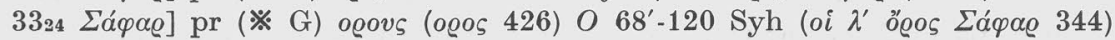

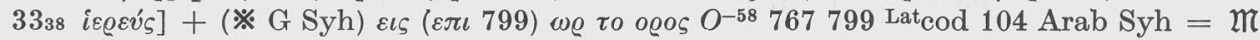

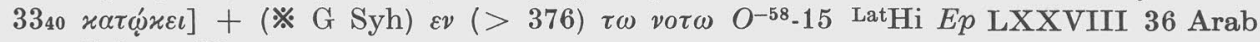
$\mathrm{Syh}=\mathfrak{m}$

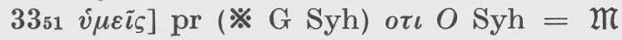

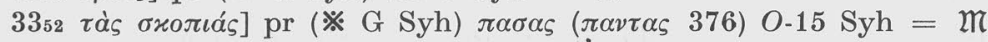

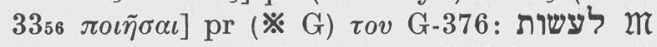

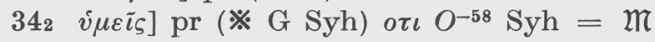


$342 \alpha v i \eta]+※ \eta \gamma \eta \ltimes \eta \mathrm{G}$; $+($ ( 85$) \eta \gamma \eta(+※ 344) \eta \tau \iota \varsigma\left(>\mathrm{M}^{\prime}\right.$ 58-426 $\left.799 \mathrm{Syh}\right) \mathrm{M}^{\prime}$ $O^{-\mathrm{G}-82} d n^{-75} 30^{\prime}-85^{\mathrm{mg}}-130-321^{\prime}-343^{\prime} t 392799$ Latcod $^{\prime} 100$ Arab Syh $=\mathfrak{2 m}$

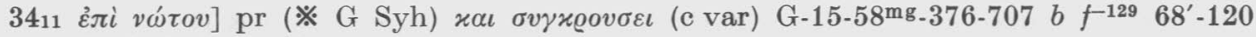
Arab $\mathrm{Syh}=\mathfrak{m}$

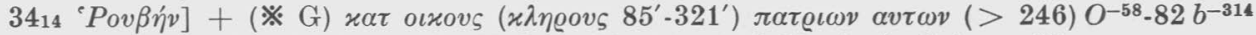
$24654^{\prime} 85^{\prime} \mathrm{mg}_{-} 321^{\prime} \mathrm{mg} t^{-84} 799$ Latcodd $^{-100} 104$ (vid) Arab Syh $=\mathfrak{m}$

$\left.34{ }_{18} \tilde{\varepsilon} \nu \alpha\right]+(※ \mathrm{G}) \alpha \varrho \chi \sigma \nu \tau \alpha(\alpha \varrho \chi o v \mathrm{G}) \varepsilon v \alpha \mathrm{G}-426130^{\mathrm{mg}}-321^{\prime} \mathrm{mg}$ Syh $=\mathfrak{2}$

$\left.353 \quad \alpha v i \tilde{\omega} v 2^{\circ}\right]+(※ \mathrm{G}) x \alpha \iota \tau \eta v \pi \alpha \varrho \xi \varepsilon \iota \alpha v \tau \omega v O \mathrm{Syh}=\mathfrak{m} \mid x \alpha i 3^{\circ}$ - fin] sub $※ \mathrm{G}$ (mend)

$355 \delta \iota \sigma \chi \imath$ iovs $1^{\circ}$ ] pr (※ G) $\varepsilon \pi \iota$ G-376 Syh: ef $2 \pi$

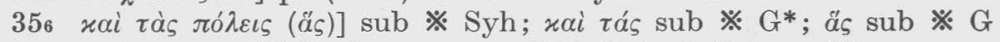

$356 \tau \alpha v i \tau \alpha \iota]+(※ \mathrm{G}) \delta \omega \sigma \varepsilon \tau \varepsilon(-\tau \alpha \iota 376) O$ Aeth Syh $=\mathfrak{M}$

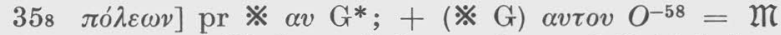

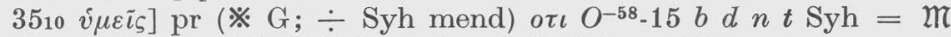

$3511 \pi o ́ \lambda \varepsilon \iota \varsigma]+\left(※ \mathrm{G}\right.$ Syh) $\pi 0 \lambda \varepsilon \iota \varsigma O^{-58} \mathrm{Syh}=\mathfrak{M}$

3515 init] pr (※ G) $\pi 0 \lambda \varepsilon \iota \varsigma\left(-\lambda \iota \varsigma\right.$ G) $O^{-58}$ Arab Syh $=\mathfrak{n}$

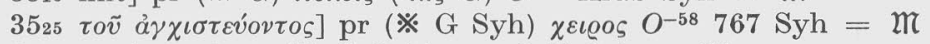

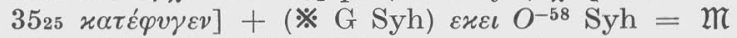

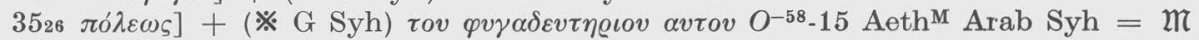

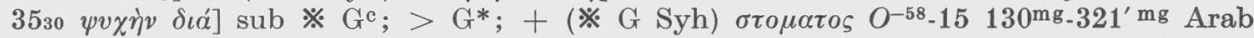
$\mathrm{Syh}=\mathfrak{m}$

$355_{32}$ ov̉] pr (※ G Syh) $x \alpha \iota O^{-426} 739 b 75^{\prime}$ Aeth Arm Bo Syh $=\mathbb{2}$; sub $※$ G*

$363 \quad \tau \tilde{\omega} v \quad \varphi v \lambda \tilde{\omega} v]$ pr $(※ \mathrm{G}) \tau \omega v \quad v \iota \omega v \mathrm{G}-426=\mathfrak{l}$

$363 v i \tilde{\omega} v]$ pr (※ Syh) $\tau \omega \nu$ 29-82-376 551 44-125'-610 $54^{\prime} t^{-76^{*}} 55319799 \mathrm{Syh}$ : contra $\mathfrak{n}$

$366 \delta \dot{\mu} \mu \nu v]+(※ \mathrm{G} \mathrm{Syh}) \tau \eta \varsigma \varphi v \lambda \eta \varsigma$ O 246 126-128-669 Syh $=\mathbb{2}$

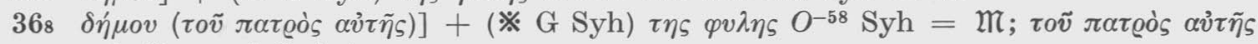
sub ※ mend G Syh

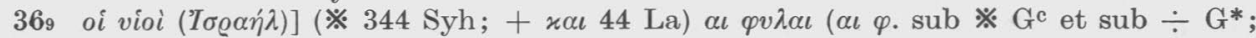
$+\tau \omega v C^{\prime \prime} 4430^{\prime}-85-344392$ 646) $v \iota \omega v$ (bis ser 82) O-82 $C^{\prime \prime} d n 30^{\prime}-85^{\mathrm{mg}}-344^{\mathrm{mg}} t 392$

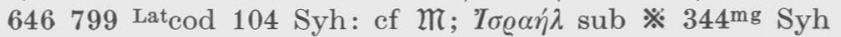

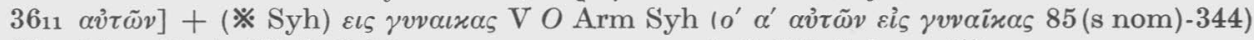
3613 M

All of the above citations except one are attested in G or Syh or in both with a hex sign. Occasionally the sign is at the wrong place, or the sign is the wrong one, that is an obelus instead of the asterisk, but the original intent of Origen's work can be reconstructed from the tradition. Of the 305 citations listed above 22 are clearly wrong; these are $23447\left(1^{\circ}\right) 23\left(1^{\circ}\right) 26\left(2^{\circ}\right) 335102214411511181648$ $\begin{array}{lllllllll}188 & 225 & 2434 & 2817 & 2939 & 3014 & 3239 & 356 & 363 .\end{array}$

In the remaining $283 \mathrm{Syh}$ attests the hex sign 216 times, whereas $\mathrm{G}$ has the sign 183 times. Other witnesses are insignificant; 344 has it six times; 85 , four times, and S, $127321^{\prime}$ and 730 each has one.

In the course of transmission many of the signs were omitted by copyists. The following list details additions in the text tradition which correspond to $\mathfrak{m}$ but without the sign tradition. Citations which seem to be hex in origin are marked with a star. As in List 1 the possible source as given in the second apparatus is given in parentheses at the end. Since all instances compared equal $\mathfrak{k}$, that fact is not noted.

In order not to weigh down the list with insignificant material, instances in which Aeth Arm or Arab add the conjunction uniquely, i.e. without support in the Greek tradition are not given. These are given in the apparatus, but it is most unlikely that such instances are based on a Greek parent text. 


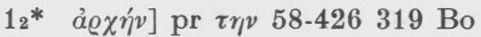

$\left.14 * \varepsilon x \alpha \sigma \tau o \varsigma \mathrm{F}^{\mathrm{a}}\right]+\varepsilon \varkappa \alpha \sigma \tau \circ \varsigma$ A F G-29-426 $56 y^{-318} z^{-18} 59624 \mathrm{Syh}\left(o^{\prime}+\varepsilon^{*} x \alpha \sigma \tau o \varsigma\right.$ 344)

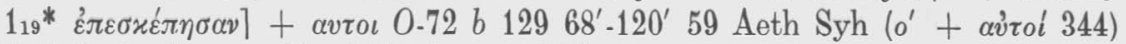

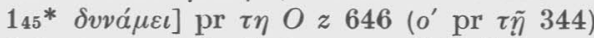

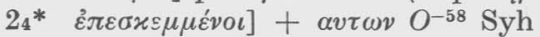

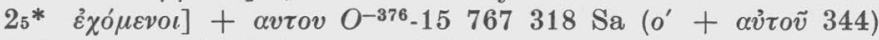

$\left.2{ }_{6}^{*} \dot{\varepsilon} \pi \varepsilon \sigma \varkappa \varepsilon \mu \mu \varepsilon \dot{v o \iota}\right]+\alpha v \tau o v$ O Syh

$\left.28^{*} \varepsilon \dot{\varepsilon} \varepsilon \varepsilon \sigma \varkappa \varepsilon \mu \mu \varepsilon \dot{v o l}\right]+\alpha v \tau \circ v O \mathrm{Syh}$

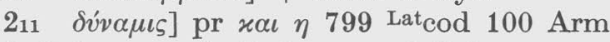

$\left.2{ }_{11}^{*} \varepsilon \dot{\pi} \pi \varepsilon \sigma \varkappa \varepsilon \mu \mu \varepsilon \dot{v o \iota}\right]+\alpha v \tau o v$ O Syh

213* $\left.{ }^{*} \pi \varepsilon \sigma \varkappa \varepsilon \mu \mu \varepsilon ́ v o \iota\right]+\alpha v \tau \omega \nu \mathrm{G}-426=\mathfrak{T} ;+\alpha v \tau o v$ 58-376 Syh $=$ Sam

215 init] pr $x \alpha \iota \eta \quad b 319$ Latcod 100 Arm

$\left.2{ }_{15}^{*} \dot{\varepsilon} \pi \varepsilon \sigma \varkappa \varepsilon \mu \mu \varepsilon \dot{v} v \iota\right]+\alpha v \tau \omega \nu O \mathrm{Syh}$

$\left.216^{*} \varepsilon \varkappa \alpha \tau o ́ v\right]+\varkappa \alpha \iota \mu \iota \alpha \mathrm{V} O^{-58} 344^{\mathrm{mg}}$ Arab Syh

$216 * \delta \varepsilon v i \varepsilon \varrho o \iota]$ pr $x \alpha \iota O^{-58} f^{-246}$ Aeth Syh

$217 \quad \mu \varepsilon \dot{\sigma} \sigma o v] \mathrm{pr} \varepsilon \iota \varsigma d$ n $t 799$

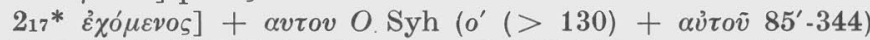

$27^{*}$ fin B V $\left.707 d f^{-246} n t x 39259799 \mathrm{Arm} \mathrm{Sa}^{412}\right]+\alpha v \tau o v 376 C^{-16}-46-417 \mathrm{Bo}$;

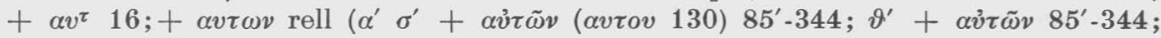
$\left.o^{\prime}+\alpha v \dot{\tau} 0 \tilde{v}(-\tau \omega \nu 344) 85^{\prime}-344\right)$

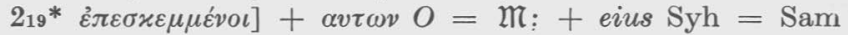

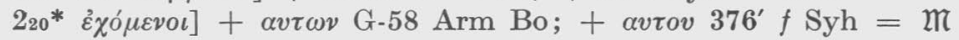

221 init] pr $x \alpha \iota \quad \eta 799$ Lat cod 100 Arm

$\left.22_{21}^{*} \dot{\varepsilon} \pi \varepsilon \sigma \varkappa \varepsilon \mu \mu \varepsilon \dot{v} \sigma \iota\right]+\alpha v \tau \omega \nu O^{-58} \mathrm{Syh}$

$\left.22_{23}^{*} \dot{\varepsilon} \pi \varepsilon \sigma \varkappa \varepsilon \mu \mu \varepsilon \dot{v o \iota}\right]+\alpha v \tau \omega \nu O^{-58} \mathrm{Syh}$

224* $\tau \varrho i \tau o \iota]$ pr $x \alpha \iota O^{-376} 68^{\prime}-120^{\prime}$ Aeth Syh

$\left.2{ }_{26}^{*} \dot{\varepsilon} \pi \varepsilon \sigma \varkappa \varepsilon \mu \mu \varepsilon \dot{v} v \iota\right]+\alpha v \tau \omega \nu \mathrm{O} \mathrm{Syh}$

$\left.22^{*} \dot{\varepsilon} \pi \varepsilon \sigma x \varepsilon \mu \mu \varepsilon \dot{v o l}\right]+\alpha v \tau \omega \nu O^{-58} \mathrm{Syh}$

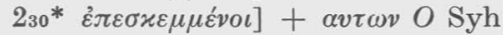

$\left.2{ }_{31}^{*} \dot{\varepsilon} \varkappa \alpha \tau \sigma^{\prime} \nu\right]+\chi\left\llcorner\lambda \iota \alpha \delta \varepsilon \varsigma\left(\chi \varepsilon\llcorner\lambda . \mathrm{G}) \mathrm{O}^{-58} \mathrm{Syh}\right.\right.$

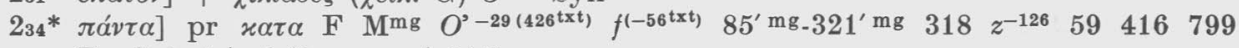
Bo Syh (ó oi $\lambda^{\prime}$ pr xatá 344)

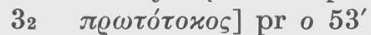

$\left.37^{*} \tau \tilde{\omega} \nu v i \tilde{\omega} \nu\right]$ pr $\pi \alpha v \tau \omega \nu$ G-426 18'-126-628-669 646 Syh (o' pr $\pi \alpha^{\prime} v \tau \omega \nu$ 344)

$\left.3_{21} * \delta \tilde{\eta} \mu \circ \iota\right]$ pr $\varepsilon \iota \sigma \iota(v) O^{-58}$ Arm Syh

$\left.3{ }_{25}^{*} \varkappa \alpha \dot{\lambda} v \mu \mu \alpha\right]+\alpha v \tau \eta \varsigma$ O 68'-120' Lat $\operatorname{cod} 100 \mathrm{Syh}$

$\left.334 * \varepsilon \xi \alpha x \iota \sigma \chi i \lambda_{\iota \circ \iota}\right]+x \alpha \iota \delta \iota \alpha$ ${ }^{2}$

Note also that for $x \alpha i \pi \varepsilon v \tau \eta \dot{r} r o v \tau \alpha$ which follows immediately and has no counter-

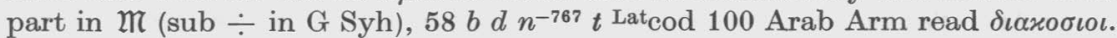

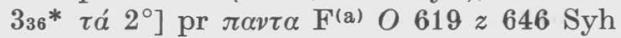

$3{ }_{37} * \pi \alpha \sigma \sigma a ́ \lambda o v \varsigma$ B V 44-125 $x^{-619} 799$ Latcodd 100 104] $\pi \alpha \lambda o v \varsigma \alpha v \tau \eta \varsigma 120 *$; + $\alpha v \tau \eta \varsigma$ $z^{-120^{*}} 646 ;+\alpha v \tau \omega \nu$ rell $=\mathfrak{m}$

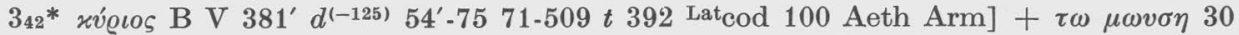
Latcod 104; $+\tau \omega \mu \omega \sigma \eta 458 ;+\alpha v \tau o \iota \varsigma b ;+\alpha v \tau \omega$ rell $=\mathfrak{2}$

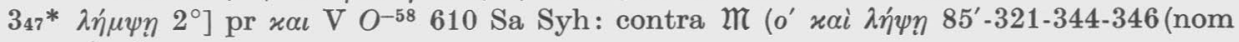
abse)

$\left.3{ }_{49}^{*} \tau \tilde{\omega} v \pi \lambda \varepsilon o v \alpha \zeta o ́ v \tau \omega \nu\right]$ pr $\pi \alpha \varrho \alpha$ G-426 $d^{-106} f 54^{\prime} 343^{\prime} t 646799$ Syh

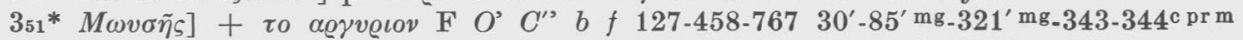
$619 y^{-121} z 59319646799$ Lat $^{2}$ codd 100 104(vid) Aeth Arab Bo Syh

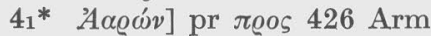

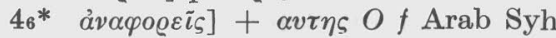

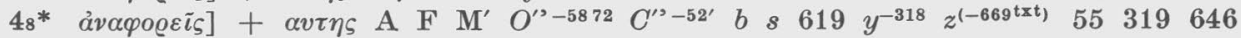
799 Syh

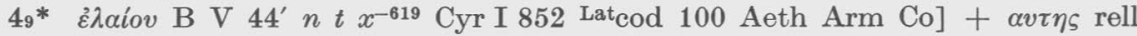

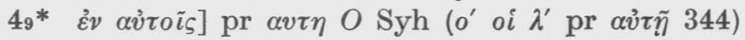

$411 \quad \varkappa \alpha \lambda v \mu \mu \alpha \tau l]$ pr $\varepsilon v 321^{\prime} \mathrm{mg}$ 


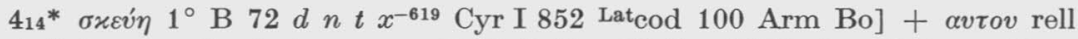

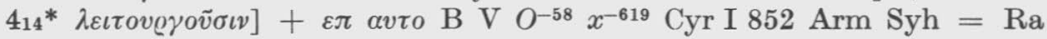

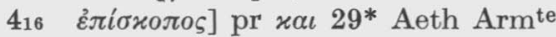

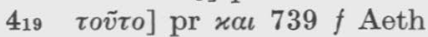

419* $\varepsilon \varkappa \alpha \sigma \tau o v]$ pr $\varepsilon v \alpha O$ Arab Syh

$\left.4_{23}^{*} \varepsilon \tilde{\varepsilon} \omega\right]$ pr $x \alpha \iota \quad 58-426 \quad b^{-537} 319$ Lat $\operatorname{cod} 100=27^{\mathrm{mss}}$

426* $\tau \dot{a} \pi \varepsilon \varrho \iota \sigma \sigma a ́$ B $82 d n^{-767} t x^{-619} 319$ Latcod 100 Aeth Arm Bo Sa ${ }^{12}$ ] abundantiam eius omnem $\mathrm{Sa}^{4} ;+\tau o v \tau \omega \nu b ;+\alpha v \tau \omega \nu$ rell $=\mathfrak{M}$

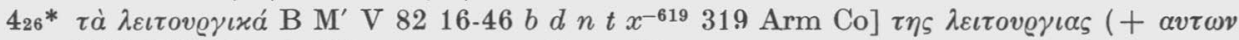
392) 392 Lat cod $100 ;+\alpha v \tau \omega v$ rell $=\mathfrak{M}$

$4{ }_{26}^{*}$ ö $\sigma \alpha 2^{\circ}$ ] pr $\pi \alpha \nu \tau \alpha\left(\pi \alpha \nu \tau\right.$ V) V O ${ }^{-58}-29619$ Aeth Syh

$428 * \tau \tilde{\omega} v v i \tilde{\omega} v]$ pr $\tau o v(>376) \delta \eta \mu o v(-\mu o v \varsigma 767)$ O-29 $767619 \mathrm{Syh}$

$\left.434 x \alpha \tau^{\prime}\right]$ pr $x \alpha \iota 125$ Aeth

$\left.435^{*} \varepsilon \bar{\varepsilon} \omega \varsigma\right]$ pr $x \alpha \iota$ 58-426 $n^{-75}$

$\left.4_{36}{ }^{*} \pi \varepsilon v \tau \eta^{\prime} \varkappa o v \tau \alpha\right]$ pr $\varkappa \propto \iota$ F $426 \quad 16-46-529509318$ 18-68 $\left(2^{\circ}\right)-120\left(2^{\circ}\right)-628$

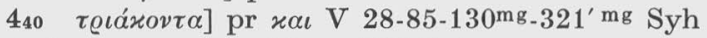

$\left.4_{43} * \pi \varepsilon v \tau \eta \varkappa о \nu \tau \alpha \varepsilon \tau \sigma \tilde{v} \varsigma\right]$ pr filii Syh

$\left.4_{47} * \pi \varepsilon v \tau \eta \varkappa о \nu \tau \alpha \varepsilon \tau \sigma \tilde{v} \varsigma\right]$ pr filii Syh

$\left.4_{48} * \dot{\varepsilon} \pi \iota \sigma \varkappa \varepsilon \pi \varepsilon \dot{v} \tau \varepsilon \varsigma\right]+\alpha v \tau \omega \nu O \mathrm{Syh}$

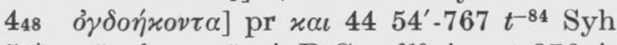

$5_{6} * \quad \tau \tilde{\omega} v \alpha \mu \alpha \varrho \tau \iota \tilde{\omega} v$ A B G $x^{-619}$ Anast 376 Arab Sa] pr $\pi \alpha v \tau \omega \nu 29$; $\pi \alpha \sigma \omega v \alpha \mu$. 126; $\pi \alpha \sigma \omega v$ $55 ;$ pr $\pi \alpha \sigma \omega v$ rell $=\mathfrak{n}$

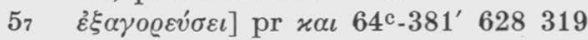

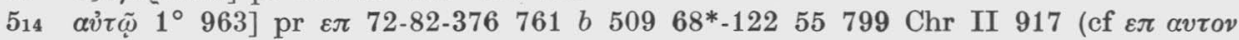
$\mathrm{V} 319)$

$\left.514 \alpha v i \tau \tilde{\omega} 2^{\circ} 963\right]$ pr $\varepsilon \pi 761 b^{-19} 56^{\prime}-12950959$ Lat codd $919294-96$ Syh (ef $\varepsilon \pi \alpha v \tau o v$ $\mathrm{V} 106^{\mathrm{c}} t 319$; $\varepsilon \pi \alpha v \tau \eta 19$; $\left.\varepsilon \pi \alpha v \tau \eta v 106^{*}\right)$

517* $\tau \tilde{\eta}_{\varsigma} \gamma \tilde{\eta} \varsigma$ ] pr $\alpha \pi \circ \mathrm{K} \mathrm{V} \mathrm{O} C^{\prime \prime} 76730^{\prime}-130^{\mathrm{mg}}-321^{\prime} \mathrm{mg} z \mathrm{Chr}$ II 917 Syh

$\left.5_{20} * \mu \varepsilon \mu i \alpha \nu \sigma \alpha \iota\right] \operatorname{pr} \sigma v(\sigma o \iota \mathrm{G}) \mathrm{O} b d n^{-767} t$ Tht $N m 197 \mathrm{Lat}^{\circ} \operatorname{cod} 100 \mathrm{Syh}\left(o^{\prime} \mathrm{pr} \sigma v^{\prime} 344\right)$

64* $\left.\varepsilon^{\prime \prime \omega}\right]$ pr $x \alpha \iota O^{-426} 24618^{\prime}-628-669$ Lat cod $100 \mathrm{Syh}$

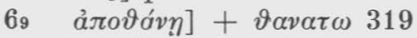

$711^{*}$ ă@ $\left.\omega \nu 2^{\circ}\right]+\varepsilon \iota \varsigma 426313^{(*)}-41730$ Eus VI 353 Syh

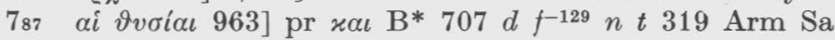

$\left.814^{*} \varepsilon \dot{\varepsilon} \mu \iota^{\prime}\right]+o \iota \lambda \varepsilon v \iota \tau \alpha \iota O 24618^{\prime}-126-628-669 \mathrm{Syh}$

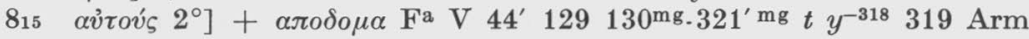

817 [ె] $\mathrm{pr} \varepsilon v 376$

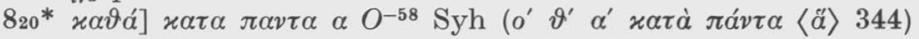

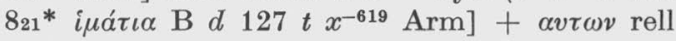

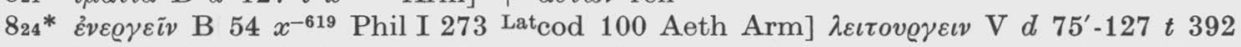

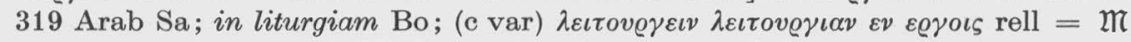

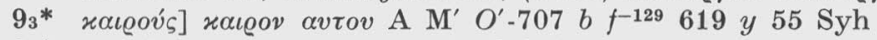

95* $\left.{ }^{*} \alpha \vartheta \alpha^{\prime}\right] \quad x \alpha \tau \alpha \pi \alpha \nu \tau \alpha \alpha(\omega \varsigma$ 58) $O \mathrm{Syh}$

$\left.9_{13} \quad \bar{\varepsilon} \xi \circ \lambda \varepsilon \vartheta \varrho \varepsilon v \vartheta \dot{\eta} \sigma \varepsilon \tau \alpha \iota\right]$ pr $\varkappa \alpha \iota n^{-767} 527$ Lat $\operatorname{cod} 100$

$\left.9_{21} * \alpha \pi \alpha \varrho \circ \tilde{\sigma} \sigma \iota 2^{\circ}\right]$ pr $x \alpha \iota c I^{-57(528)}{ }_{-551}=\mathfrak{M} ; x \alpha \iota \alpha \pi \varepsilon \varrho .246 ; x \alpha \iota \varepsilon \xi \eta \varrho \alpha \nu O^{-58}$

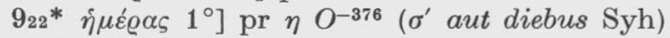

922* $\dot{\eta} \mu \varepsilon \alpha_{\varrho} 2^{\circ}$ ] pr $\eta \mathrm{V}$ 58-72 ( $\sigma^{\prime}$ aut tempore $\left.\mathrm{Syh}\right)$

$108 \tau \alpha \tilde{\imath} \varsigma]$ pr $\varepsilon v \mathrm{~V}$ ol ${ }^{-15} b d n t 527-619 \mathrm{Bo}$

109 $\tau \alpha \tilde{\iota} \varsigma \sigma a ́ \lambda \pi \iota \gamma \xi \iota v]$ pr $\varepsilon v \mathrm{M}^{\prime} d t 71799$ Cyr X passim

$\left.10_{10} \tau \alpha \tilde{i} \zeta \quad \sigma a \dot{\lambda} \pi \iota \gamma \xi \iota v\right]$ pr $\varepsilon v \quad b \quad 319$

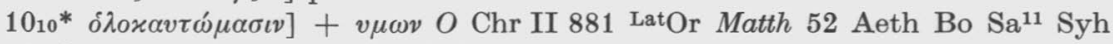

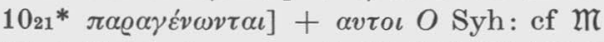

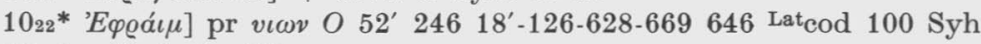

$\left.10{ }_{31} * \mu \eta^{\prime}\right]+\delta \eta 426$

$\left.10_{34} \delta \iota \alpha \sigma \varkappa o \varrho \tau \iota \sigma \vartheta \dot{\eta} \tau \omega \sigma \alpha \nu\right]$ pr $x \alpha \iota$ oI-15-72 $C^{\prime \prime} 19^{\prime} s^{-321} x^{-509}$ LatSpec 33 Aeth Arm Bo $\mathrm{Sa}^{11}$

$\left.10_{34} \varphi v \gamma \varepsilon \dot{\tau} \omega \sigma \alpha v\right]$ pr $\varkappa \alpha \iota 7252^{\prime} 76730126$ LatSpec 33 Aeth

$11{ }_{28} \varepsilon[\pi \varepsilon v]$ pr xal 18 Tht $\mathrm{Nm}$ 207ap $\mathrm{Syh}^{\mathrm{T}}$ 


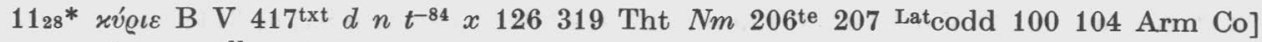
$+\mu o v$ rell

$\left.12_{2} \quad j \mu \tilde{i} v\right]$ pr $\varepsilon v$ A

$1211^{*}$ xं $\varrho\llcorner\varepsilon]+\mu 0 v 0^{-\mathrm{G}} f^{-129}$ Arab Syh

$\left.1211^{*} \mu \eta^{\prime}\right]+\delta \eta 426$

$\left.1212^{*} \mu \eta^{\prime}\right]+\varkappa \alpha \iota O f^{-129} \operatorname{Syh}\left(o^{\prime}+\varkappa \alpha i^{\prime} 344\right)$

$1213 i \alpha \sigma \alpha l]+\delta \eta 767$

1321* $\pi i \omega \nu]+\varepsilon \sigma \tau \iota(v) 426 d 246 n t 319$ Arm(vid) Bo Pal

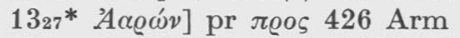

$\left.133_{3} * \pi \tilde{\alpha} \varsigma \mathrm{B} \mathrm{V} 426 x \mathrm{Bo}^{\mathrm{AB}} \mathrm{B}^{\mathrm{S}} \mathrm{Sa}\right] \varkappa \alpha \iota \mathrm{G} C^{\prime \prime} 799 ;>\mathrm{Bo}^{\mathrm{B}^{*}} ; \mathrm{pr} x \alpha \iota$ rell $=\mathfrak{m}$

$14{ }^{*}{ }^{*} \pi \alpha \iota \delta i \alpha \mathrm{B} \mathrm{M} \mathrm{M}^{\prime} 129 x$ Cyr I 373] $\tau \varepsilon \varkappa v \alpha \eta \mu \omega \nu b ;+\eta \mu \omega \nu(v \mu \omega \nu$ 44-107' 321) rell $=\mathfrak{m}$

$\left.\left.144^{*} \varepsilon \tau \dot{\varepsilon} \varrho \omega\right)\right]+\alpha v \tau o v O \mathrm{Syh}\left(o^{\prime}+\alpha v i \tau o \tilde{v} 344\right)$

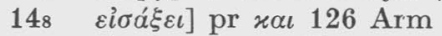

$\left.141_{12} \vartheta \alpha \nu a ́ \tau \omega\right]$ pr $\varepsilon v \mathrm{~V}$

$1416 * \varkappa \alpha \tau \varepsilon \dot{\sigma} \varrho \varrho \omega \sigma \varepsilon v]$ pr $\varkappa \alpha \iota$ G-426 Arm ${ }^{\text {ap }}$ Syh

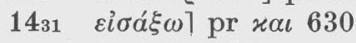

1530* $\varepsilon_{\xi} \xi_{0 \lambda \varepsilon \vartheta \varrho \varepsilon v \vartheta} \dot{\sigma} \sigma \varepsilon \tau \alpha \iota$ (c var) B V $58 x 5559$ Aeth Arm ${ }^{\text {te }}$ Bo] pr $\varkappa \alpha \iota$ rell

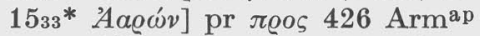

$15_{35} \lambda i \vartheta \circ \varsigma$ ] $\mathrm{pr} \varepsilon v 16-46 y^{-121}$

$\left.15{ }_{35}^{*} \sigma v v \alpha \gamma \omega \gamma \eta^{\prime}\right]+\varepsilon \xi \omega \tau \eta \varsigma \pi \alpha \varrho \varepsilon \beta \rho \lambda \eta \varsigma$ G-376 74'-84 128-630' LatPsAmbr Mans 27 Arab Syh

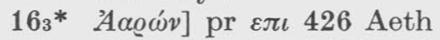

$16{ }^{*} \varepsilon i \pi \alpha \nu$ (c var) B V $72 d n t x$ Latcod $\left.100 \mathrm{Arm} \mathrm{BoA}\right] \lambda \varepsilon \gamma o v \sigma \iota v 319$; + $\pi \varrho \circ{ }^{\mathrm{A}}\left(>376^{*}\right)$

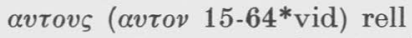

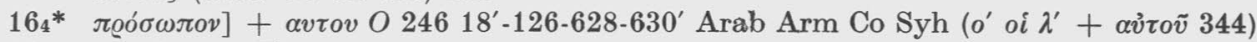

$1631 \quad \dot{\eta} \gamma \tilde{\eta}]+\eta \mathrm{V} t$

$\left.16_{32} \tau{ }^{\prime}\right]$ pr $\pi \alpha \nu \tau \alpha b$

1633* ö $\alpha \alpha$ B G-426 b $129 n^{-767} 509319$ Latcod 100 Arm Co] pr $\pi \alpha v \tau \alpha$ rell

$\left.16{ }_{36} * M \omega v \sigma \tilde{\eta} \nu\right]+\lambda \varepsilon \gamma \omega \nu 376106^{\mathrm{c}}$ Arab Syh

$\left.1640^{*} M \omega v \sigma \tilde{\eta}\right]+\alpha v \tau \omega \mathrm{B} \mathrm{O}-5812971^{\prime} \mathrm{Syh}\left(o^{\prime} \vartheta^{\prime} \alpha^{\prime}+\alpha v \tau \tilde{\omega} 344\right)$

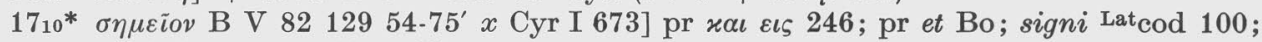
$>319 ;$ pr $\varepsilon \iota \varsigma$ rell $=\mathfrak{m}$

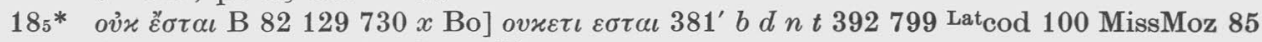
Arm; $+\varepsilon \tau \iota$ (c var) rell $=\mathfrak{m}$

$\left.186^{*} \dot{\varepsilon}^{\prime} \gamma \omega^{\prime}\right]+\iota \delta o v$ V $O 5618^{\prime}-628-630^{\prime} 646$ Sa Syh $\left(\alpha^{\prime} o^{\prime} \vartheta^{\prime}+i \delta o v ́ 344\right)$

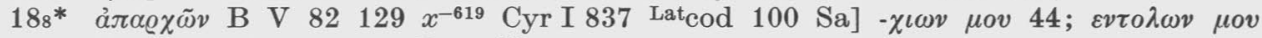
Procop 844; $+\mu o v$ rell $=\mathfrak{m}$

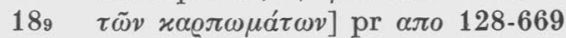

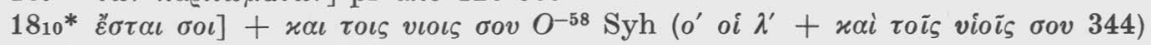

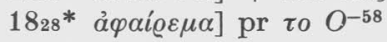

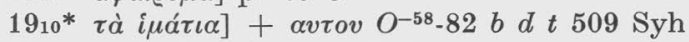

$\left.19_{12} \tau \tilde{\eta} \dot{\eta} \mu \varepsilon \in Q \alpha 1^{\circ}\right]$ pr $\varepsilon \nu 376$

$\begin{array}{lllllll}1913 & \dot{\varepsilon} x \tau \varrho\llcorner\dot{\eta} \sigma \varepsilon \tau \alpha \iota\end{array}$ pr $\varkappa \alpha \iota$ V $72 \quad b \quad 767319$ Lat $\operatorname{cod} 100$

$\left.19_{19} \quad \tau \tilde{\eta} 5^{\circ}\right]$ pr $\varepsilon v$ A 82 414-550' $54^{\prime}-7531855$

$2012 *$ A A $\left.\omega^{\prime} v\right]$ pr $\pi \varrho \circ \varsigma 426$ Arm

$2019 * \tau \dot{\alpha} x \tau \dot{\eta} v \eta$ B V $82 d n^{-767} t x^{-619}$ Phil II $87^{\text {te }} \mathrm{Sa}^{12}$ ] pecora nostra Lat cod 100 Aeth; + rell $=\mathfrak{m}$

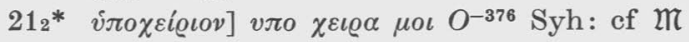

217 है $\lambda \varepsilon \gamma o \nu]$ pr $\varkappa \alpha \iota 392$

$\left.218 i \delta \omega^{\prime} v\right]$ pr $x \alpha \iota$ V $15-376 \quad b \quad n \quad 527319$

219 öøıs] pr ó Fe prm $\mathrm{M}^{\prime} 72-376-618$ b 53' $127-76771 y^{-121} 18^{\prime}-120^{\prime}-628-630^{\prime} 59319 \mathrm{Cyr}$ II 637

$2112 \dot{\varepsilon} \varkappa \varepsilon \bar{\imath} \varepsilon v]$ pr $\varkappa \alpha \iota \mathrm{Fb}^{\mathrm{b}} 61968^{\prime}-120^{\prime}$ (o' oi $\lambda^{\prime}$ pr $\left.\varkappa \alpha i i^{344}\right)$

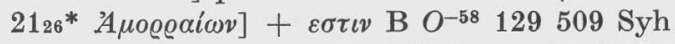

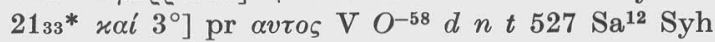

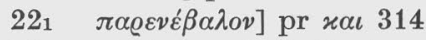

$22_{13}$ ov่x] pr quia Aeth Arm 
2217* ö $\sigma \alpha$ (aut $\alpha$ ) B* $\mathrm{V} \mathrm{b}^{-314} d 53^{\prime}-129$ nt 527319 Latcod $100 \mathrm{Arm} \mathrm{Sa]} \mathrm{os} 314$; o B $130^{\mathrm{mg}}$. $321^{\prime} \mathrm{mg}_{-} 344^{\mathrm{mg}}$ 71-509; $\pi \alpha \nu \tau \alpha \mathrm{F} 29 C^{-16}-417^{*} 392^{*}$; pr $\pi \alpha \nu \tau \alpha \mathrm{F}^{\mathrm{a}}$ rell $=\mathfrak{m}$

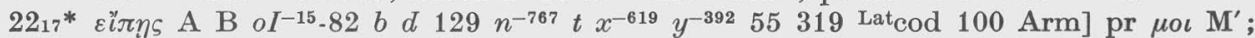
$+\mu o \iota$ rell $=\mathfrak{m}$

2218* $\tau \circ \tilde{v} \vartheta \varepsilon o \tilde{v}]+\mu o v O 414343$ Arab Syh $\left(o^{\prime}\right.$ oi $\left.\lambda^{\prime}+\mu o v 344\right)$

$\left.22{ }_{26} * \tilde{\eta} \nu\right]+$ odos $O^{-58} \mathrm{Syh}^{\mathrm{T}}$

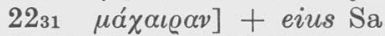

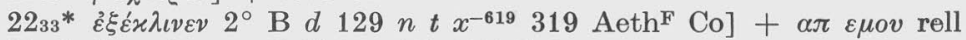

$22{ }_{37} *$ ö $\left.v \tau \omega \varsigma\right]$ pr $\eta \mathrm{O}^{-58}-15^{\mathrm{c}} 68^{\prime}-120^{\prime}$ Syh

$\left.23{ }^{*} \varepsilon^{\prime \prime}\right]+\pi \omega_{\varsigma} O 619 z \operatorname{Syh}\left(\sigma^{\prime} \varepsilon \dot{l} \pi \tilde{\omega} \varsigma\right.$ 344)

235 oṽ $\left.\omega_{\xi}\right]$ pr $\varkappa \alpha \iota 66955$ Lat cod 100 Aeth Arm Pal

$\left.23{ }_{19} * \dot{\varepsilon} \mu \mu \varepsilon v \varepsilon i\right]+\alpha v \tau \omega O$

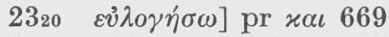

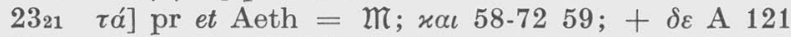

241 ovंx] pr $x \alpha \iota \mathrm{F}$ Latcod 100

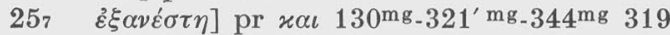

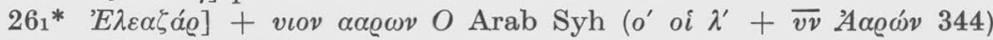

$264 \quad \dot{\varepsilon} \xi] \varepsilon \varkappa \quad \gamma \eta \varsigma$ V Arab

$2610 \varkappa \alpha \tau \dot{\varepsilon} \pi \iota \varepsilon v$ pr $\varkappa \alpha \iota 246$

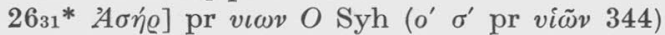

2640 init] $\operatorname{pr} x \alpha \iota d^{(-44)} t$

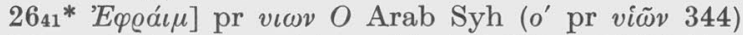

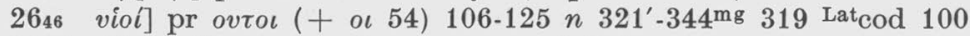

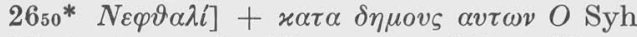

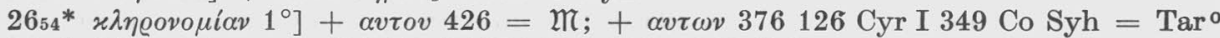

$\left.2659^{*} M \omega v \sigma \tilde{\eta} v\right]$ pr $\tau o v 42677 d 127-767 t 619 z 319$ Syh

279 xinoovouiav] $+\alpha v \tau o v$ V $963 O^{-58}-82 C^{\prime \prime} b d 129 n s^{-30} t 392 z 319624646$ Arm Co Syh

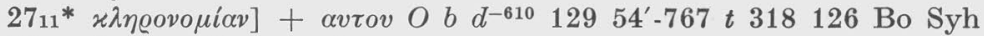

$\left.27_{11} \delta \iota x \alpha i \omega \mu \alpha\right]$ pr $\varepsilon \iota \varsigma d t$

$\left.2717^{*} \varepsilon \tilde{\varepsilon} \sigma \tau \iota v\right]+\alpha v \tau 0 \iota \varsigma 426$ Phil II 104UF

$\left.2719^{*} \varepsilon \dot{\varepsilon} \tau \varepsilon \lambda \tilde{\eta} 2^{\circ}\right]+\alpha v \tau \omega O$

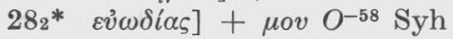

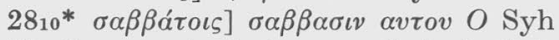

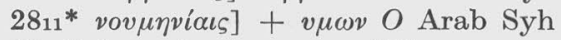

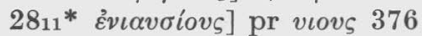

$2812 *$ init] pr xaı $O$ Arm Syh

2813 init] $\operatorname{pr} x \alpha \iota b d n t 646$ Lat $^{2} \operatorname{cod} 100$ Aeth $^{\mathrm{C}}$ Arab Arm Sa ( $\sigma^{\prime}$ pr $x \alpha i$ 344)

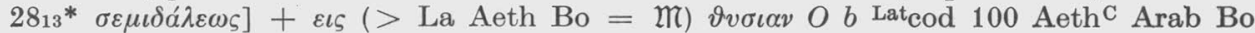
Syh $\left(o^{\prime} \vartheta^{\prime} \alpha^{\prime}+\right.$ Eis $\left.\vartheta v \sigma i \alpha v 344\right)$

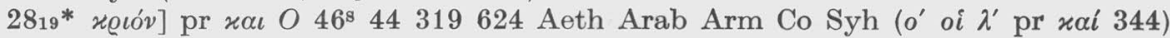

$\left.28{ }_{19} \varepsilon \pi \tau \alpha \dot{~}\right]$ pr $x \alpha \iota b$ Aeth BoA Sa

294 init] pr $x \alpha \iota d 246 n t$ Aeth Arm

298* $\left.\delta \lambda o x \alpha v \tau \omega^{\prime} \mu \alpha \tau \alpha\right]+\tau \omega(>426) \overline{\varkappa \omega} O$ Syh $\left(o^{\prime}\right.$ oi $\lambda^{\prime} \delta \lambda o x \alpha v i \omega \mu \alpha \tau \tilde{\varphi} \overline{\varkappa \omega}$ 344)

$\left.29_{11} \quad \eta \quad \vartheta v \sigma i \alpha\right]$ pr $x \alpha \iota d n t$ Aeth Arab Sa

2915 init B M $\mathrm{M}^{\prime}$ V $963 O^{\prime-58}-82 f^{-246} x 407319$ Cyr I 1120 Aeth Syh] pr $x \alpha \iota$ rell

$2917 *$ init $963 \quad b 509]$ pr $x \alpha \iota$ rell $=\mathrm{Ra}$

2919 ai $\left.1^{\circ}\right]$ pr $\varkappa \alpha \iota 458$ Aeth Arm Sa

$29_{20}$ init] pr $x \alpha \iota$ A 1295450939218646 Aeth Arab Arm Bo

$29_{29}$ init] pr $x \alpha \iota$ V $b 458$ Aeth Arm Co

2932 init] pr $x \alpha \iota b$ Aeth Arab Arm Co

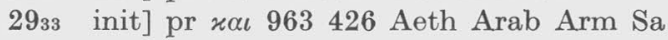

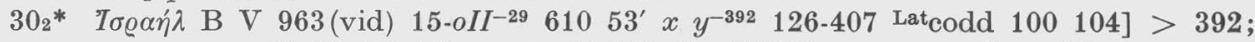

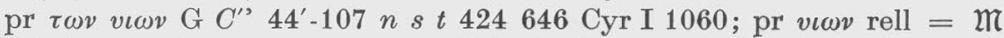

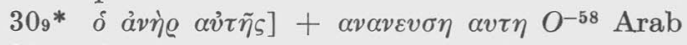

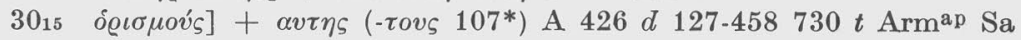

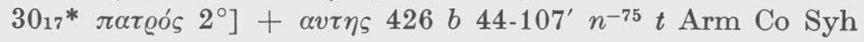


$\left.319 \tau \alpha^{\prime} 1^{\circ}\right]$ pr $\pi \alpha \nu \tau \alpha 30^{\prime}$

3118* $\pi \tilde{\alpha} \sigma \alpha \nu$ B $\left.82129 x^{-527} 407319\right] \varkappa \alpha \iota \pi \alpha \sigma \alpha \nu \delta \varepsilon f^{-129} ;+\delta \varepsilon O^{-376} \mathrm{Syh} ; \mathrm{pr} x \alpha \iota$ rell $=\mathfrak{m}$

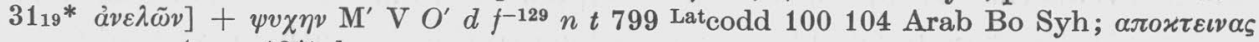
$\psi v \chi \eta v\left(-\chi \eta \varsigma 19^{*}\right) b$

$3119 *$ o $\left.2^{\circ}\right]$ pr $\pi \alpha \varsigma O f^{-129}$ Syh

$\left.31{ }_{19}^{*} \tau \tilde{\eta} \tilde{\eta} \mu \varepsilon \dot{\varepsilon} \varrho\right]$ pr $\varepsilon v O^{-376} 53^{\prime}-56$

$\left.3119 \quad \tau \tilde{\eta} 3^{\circ}\right]$ pr $\varepsilon v \quad 58 \quad 53-56^{\prime}-664^{\mathrm{c}}$

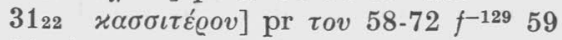

$\left.31{ }_{27}^{*} \sigma v v \alpha \gamma \omega \gamma \tilde{\eta} \varsigma\right]$ pr $\tau \eta \varsigma(\tau \eta 75)$ A $O^{-426}-381^{\prime} 414106^{(\mathrm{mg})} 129 n t^{(-370)} 527$ Cyr I 333

$\left.311^{*} * \tau \dot{\lambda} \lambda o \varsigma\right]+\alpha v \tau \omega \nu 15-376 \quad b \quad 767$

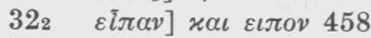

$329 \quad \varphi \alpha ́ \varrho \alpha \gamma \gamma \alpha]$ pr $\varepsilon \omega \varsigma$ oI

3214 init] pr $x \alpha \iota 707 d n t 12655799$ Cyr I 404 Aeth Syh

$\left.32{ }_{26} * \tilde{\varepsilon} \sigma o \nu \tau \alpha \iota\right]+\varepsilon x \varepsilon \iota O f^{-129}$ Syh $\left(o^{\prime}\right.$ oi $\left.\lambda^{\prime}+\varepsilon \dot{\varepsilon} \varepsilon \tilde{\imath} 344\right)$

$32{ }_{27} *$ xv́@ı६ $]+\mu o v O^{-58} 128-630^{\prime}$

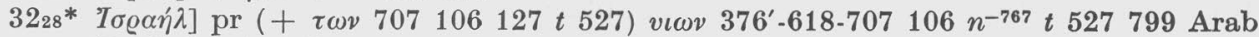

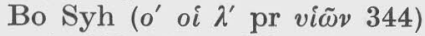

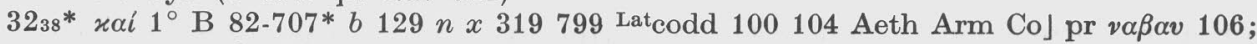
$+\tau \eta v \nu \alpha \beta \omega 707^{\mathrm{c}}$; pr (c var) $x \alpha \iota \tau \eta v \nu \alpha \beta \omega$ rell $=\mathfrak{m}$

$333^{*}$ init] pr $x \alpha \iota$ A $O^{\prime} y^{-318} 18^{\prime}-126-628-630^{\prime} 55799$ Aeth Sa Syh (ó oi $\lambda^{\prime}$ pr $x \alpha i$ 344)

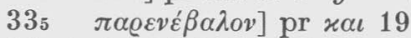

$33_{14} * \tilde{\eta} v \mathrm{~B} \mathrm{M}^{\prime} \mathrm{V}$ oI $d$ 129-246 n $30 t x 318$ 126-128-407-628-630'319 799 Arm Bo] pr $i b i$ Aug Loc in hept IV $120 ;+\varepsilon x \varepsilon \iota$ rell $=\mathfrak{m}$

$\left.33_{33} \Gamma \alpha \delta \gamma \alpha^{\prime} \delta\right]$ pr $\tau 0 v 707 C^{\prime-57^{\prime} 529-46^{\prime} s^{-321}}$

$\left.33_{38} \quad \mu \iota \tilde{\alpha}\right]$ pr $\varepsilon v 82$

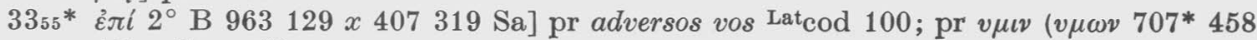
18) rell $=\mathfrak{m}$

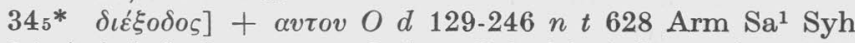

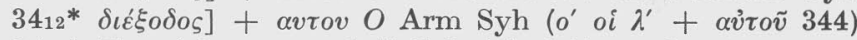

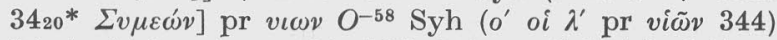

$\left.3422 * \Delta \alpha^{2}\right] \operatorname{pr} v \iota \omega v 426 d^{-125} 246 n t \operatorname{Syh}\left(o^{\prime}\right.$ oi $\lambda^{\prime} \operatorname{pr} v i \tilde{\omega} v 344$ )

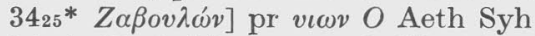

$3428 * N \varepsilon \varphi \vartheta \alpha \lambda i]$ pr $v \iota \omega \nu O C^{\prime \prime} 106 s 392319$ Aeth Sa Syh

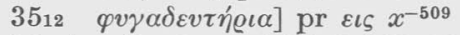

$3515 * \pi o ́ \lambda \varepsilon \iota \varsigma]$ pr $\varepsilon \xi$ V $O 767$ 126-128-630' Syh

$35_{21} * \chi \varepsilon \iota \varrho$ i $+\alpha v \tau o v O^{-58} 767$ Arm Co Syh

$\left.35_{21} * \sigma v v \alpha v \tau \tilde{\eta} \sigma \alpha \iota\right]+\alpha v \tau o v O^{-58}$

35.3* $\pi \alpha v \tau i]$ pr $\varepsilon v O^{-58}$ b 407-630 Cyr VII 625 (o' oi $\lambda^{\prime} \mathrm{pr} \dot{\varepsilon} v 344$ )

$\left.35_{25} \tau \tilde{\omega} 1^{\circ}\right] \mathrm{pr} \varepsilon v 29$

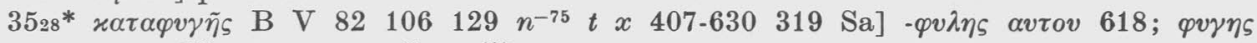
$\alpha v \tau o v \mathrm{M}^{\prime} ;+\alpha v \tau o v$ rell $=\mathfrak{m}$

$355_{32} *$ v $\left.\gamma \alpha \delta \varepsilon v \tau \eta \varrho i \omega v\right]+\alpha v \tau o v \mathrm{O} \mathrm{Syh}$

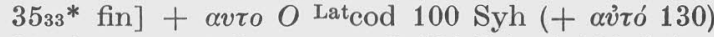

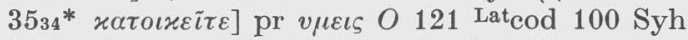

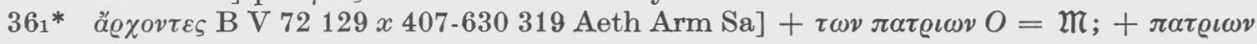
rell

$362^{*}$ xv@í $\left.2^{\circ}\right]+\mu o v O^{(-376)} 246$ 126-128-669 Syh

List 2 contains a further group of 237 correspondences in the tradition to the text of $\mathfrak{m}$. Of these 161 have been starred, that is to say have been judged to have their origin in Origen's work. Each of these is supported by at least one or more of the main hex witnesses in the tradition; in fact, most of them are supported in the commonly recurring pattern found in List 1, i.e. by $O$ Syh or by $O^{-58} \mathrm{Syh}$. These must be taken along with those of List 1 as being primary evidence for the hex recension. 
An analysis of the remaining 76 instances which are probably not hex in origin reveals that 34 find only random support and may be dismissed as coincidence. Of the remainder, 14 variants belong to the Byzantine text tradition; ef the discussion of its character on pp. 18-34. Nine are supported by the $b$ group; four are found on the margins of $s \mathrm{mss}$, for which of THGG $67 \mathrm{f}$, and four are $f$ readings. Five are supported by four or more groups but not by $O$ ( 787108342813 2915). One variant (279) is supported by at least four groups including $O$; it ean hardly be hex in origin since the reading is also found in 963. The support for the remaining variants in List 2 are scattered singly among the other text groups ( $o I C^{\prime \prime} s x y$ and $z$ ). It would appear that the only identifiable recensional activity occasioned by "correction" towards the Hebrew in the Numbers text tradition is the hexaplaric.

Further evidence for hexaplaric activity is to be found in changes in word order to correspond to that of Origen's Hebrew text, as was argued in THGG 59f. This evidence is given in List 3. Since these correspond in each case to $\mathfrak{T}$, this fact is not noted.

List 3

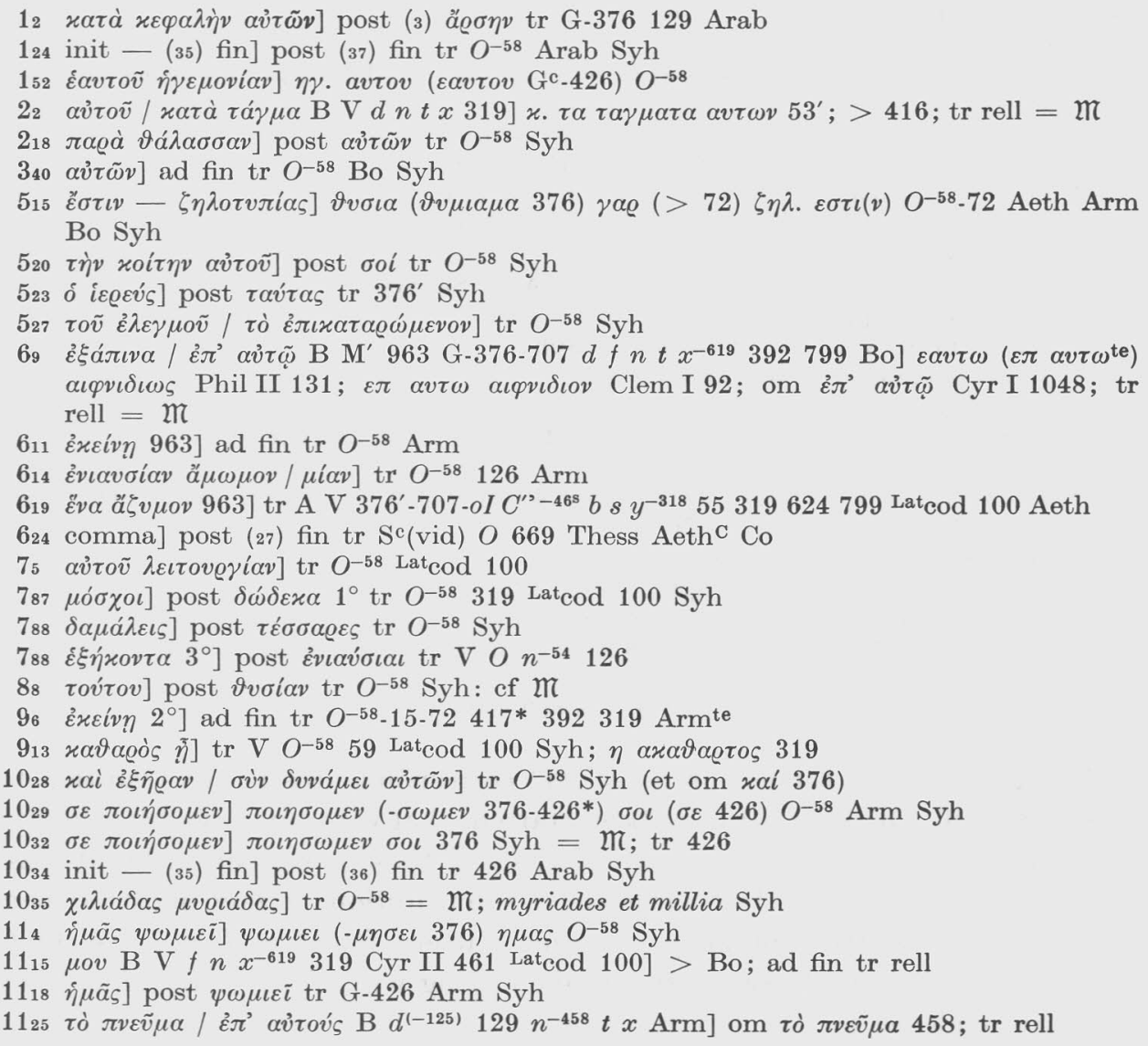


$122 \quad M \omega v \sigma \tilde{\eta} \mu o ́ v \omega] \mu o v \omega \mu \omega v \sigma \eta(\mu \omega \sigma \varepsilon \iota 426) 426422$ LatRuf Num VI 6

124 v $\mu \varepsilon \tilde{i} \zeta$ / oi $\tau 0 \varepsilon \tilde{\zeta} \zeta]$ tr $O^{-58}$

$1422 \mu o v / \tau \tilde{\eta}_{S} \varphi \omega \nu \tilde{\eta}_{S} \mathrm{~B} \mathrm{M}^{\prime} \mathrm{V} d 129 t x$ Cyr II 609] tr rell

158 ảjò $\tau \tilde{\omega} v \beta 0 \tilde{\omega} v$ / $\pi 0 เ \tilde{\eta} \tau \varepsilon]$ tr A F M' O $^{\prime \prime} C^{\prime \prime} b f^{-129}$ s y z 5559319624 Cyr I 1029 Latcod 100 Arab Bo Syh

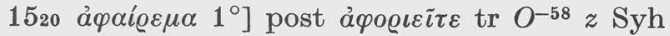

$165 \alpha v 3 \tau o \tilde{v} 1^{\circ}$ ] post $\sigma v v \alpha \gamma \omega \gamma \eta \dot{v} \operatorname{tr} O^{-58} 417^{*} b \quad 54-75799$ Latcod 100 Arm Syh

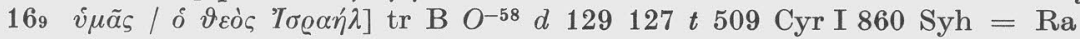

1640 Ë $\sigma \tau \iota v]$ post $A \alpha \varrho \omega ́ v$ tr $O^{-58}$ Syh (vid)

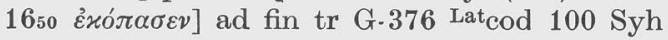

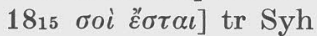

$1819 \sigma o i \delta \varepsilon ́ \delta \omega \varkappa \alpha$ B $\mathrm{M}^{\prime}$ V $\left.82 b 53^{\prime}-129127-767 x^{-619} \mathrm{Arm}\right] \sigma v \varepsilon \sigma \tau \iota\left(-\tau \alpha \iota^{*}\right) 319$; + $\alpha v \tau \alpha d$ $54-75^{\prime} t$ Lat $c o d 100$ Co; tr rell $=\mathfrak{M}$

$1910 \tau \dot{\alpha} i \mu \alpha ́ \tau \iota \alpha]+\alpha v \tau o v$ et post $\delta \alpha \mu \alpha \dot{\lambda} \varepsilon \omega \varsigma \operatorname{tr} O^{-58} b$ Syh

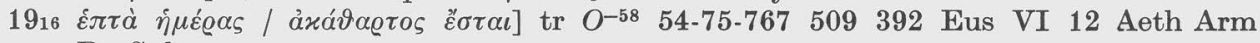
Bo Syh

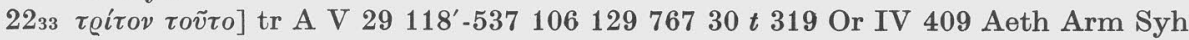

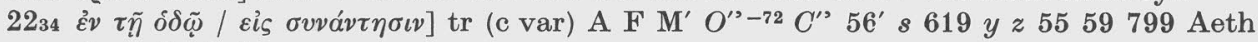
Arab Sa Syh

$2240 \pi \varrho o ́ \beta \alpha \tau \alpha]$ et $\mu o ́ \sigma \chi 0 v \varsigma \operatorname{tr} O^{-58} n 527$ Arm Syh

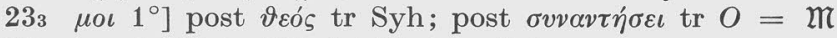

$233 \mu \circ \iota \delta \varepsilon i \xi \eta]$ tr $426 d t 59$ Arm Syh

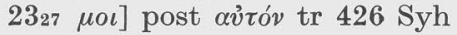

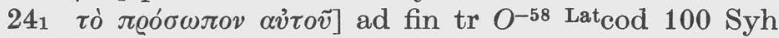

$242 \pi v \varepsilon \tilde{v} \mu \alpha \vartheta \varepsilon \sigma \tilde{v}]$ ad fin tr A F $O^{\prime \prime}-82 C^{\prime \prime} 56^{\prime} n^{-127} s$ 527-619 y z 5559799 Latcod 100 Ruf Num XVII 2 Aeth Arab Syh

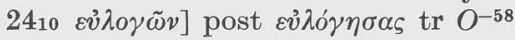

$2410 \tau \varrho i \tau o \nu]$ ad fin tr A F M $\mathrm{M}^{\prime} O^{\prime-376-29-707 C^{\prime \prime}-52^{\prime} 313761} 19 d 53^{\prime}-56$ s 527 y $z^{-68^{\prime} 120} 59799$ Arm Bo Syh

$2413 \mu \circ \iota]$ post $\delta \tilde{\omega}$ tr F V O'-58-707 Latcod 100 Arm Syh

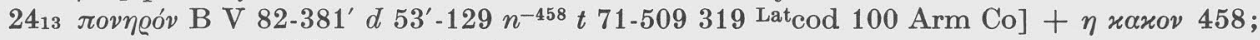
$\mu \iota \varkappa \varrho о \nu \eta \mu \varepsilon \gamma \alpha \mathrm{M}^{\prime \mathrm{mg}}$; et $\varkappa \alpha \lambda o ́ v$ tr rell

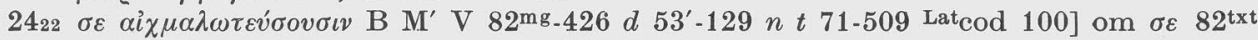
319 Arm; tr rell $=\mathfrak{m}$

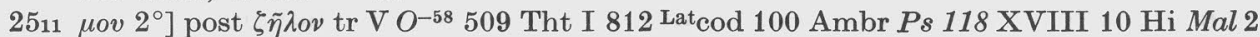
Hil Ps CXVIII 3 Arm Syh

$2515 \tau \tilde{\eta} M \alpha \delta \iota \alpha \nu i \tau \iota \delta \iota / \tau \tilde{\eta} \pi \varepsilon \pi \lambda \eta \gamma v i \alpha]$ tr $O^{-58}$

$2515 \dot{\varepsilon} \sigma \tau \iota v]$ ad fin tr $O^{-58}$ Syh

2615 init - (23) fin] post (27) fin tr $O^{-58}$ Arab Syh

2628 init - (31) fin] post (47) fin tr $O^{-58}$ Arab Syh

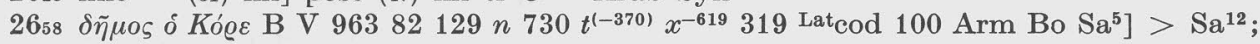
post Movoí tr rell $=\mathfrak{m}$

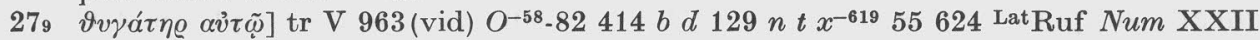
1 Syh

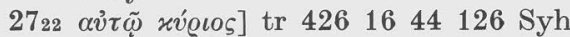

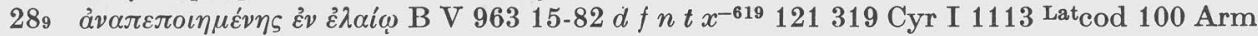
Bo $\left.\mathrm{Sa}^{1}\right]$ post $\vartheta v \sigma i \alpha v$ tr rell

$\left.28_{22} \quad \check{\varepsilon} \nu \alpha\right]$ post $\alpha \mu \alpha \varrho \tau i \alpha \varsigma$ tr $426 \mathrm{Syh}$

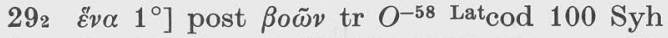

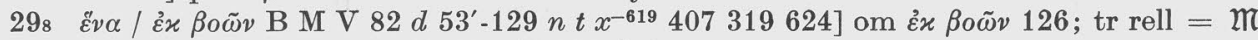

$\left.29_{22} \quad \check{v} v \alpha\right]$ post $\alpha \mu \alpha \varrho \tau i \alpha \varsigma \operatorname{tr} 42644$ 126-128 Syh

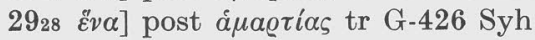

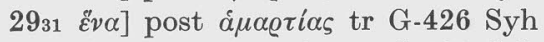

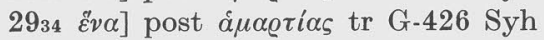

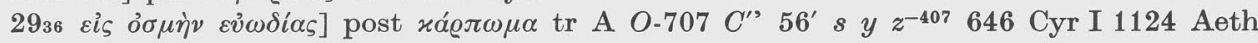
Arab Syh

$2938 \tilde{\varepsilon} \nu \alpha]$ post $\alpha \mu \alpha \varrho \tau i \alpha \varsigma$ tr G-426 Syh 


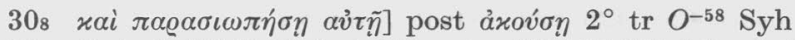

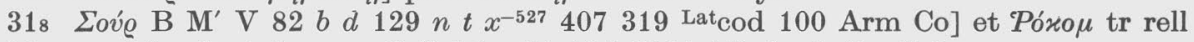

$3130 \pi \varrho \circ \beta a ́ \tau \omega \nu]$ et oै $v \omega v \operatorname{tr} O^{-58}$ Latcodd 100 104(vid) Arab Bo Syh

322 Pov $\beta \eta \dot{v}]$ et $\Gamma a ́ \delta$ tr $376^{\prime}$ Syh

327 xv́@ı૬] ad fin tr $O^{-58} 799$ Aeth Syh

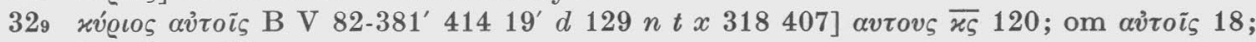
tr rell $=\mathfrak{m}$

$\left.32_{16} \tilde{\omega} \delta \varepsilon\right]$ post $\dot{\eta} \mu \tilde{\omega} v 1^{\circ} \operatorname{tr} O^{-58} \mathrm{Syh}$

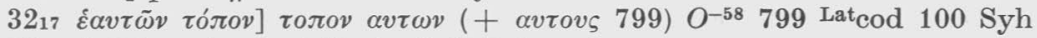

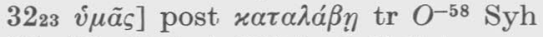

$32{ }_{25}$ Pov $\left.\beta \eta^{\prime} v\right]$ et $\Gamma \alpha ́ \delta \operatorname{tr} O^{-58} \mathrm{Syh}$

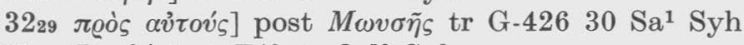

$32_{29}$ Pov $\left.\beta \dot{\eta} v\right]$ et $\Gamma a ́ \delta$ tr $O^{-58} \mathrm{Syh}$

$32_{31}$ Pov $\left.\beta \eta^{\prime} v\right]$ et $\Gamma a^{\prime} \delta \operatorname{tr} O^{-58} 16-46 \mathrm{Syh}$

3231 o xó@ı६] post $\lambda \dot{\varepsilon} \gamma \varepsilon \iota \operatorname{tr} O^{-58}$ Latcod 100 Arm Syh

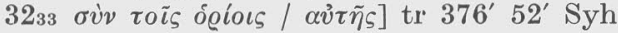

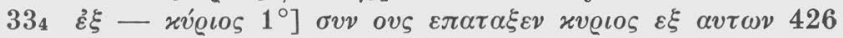

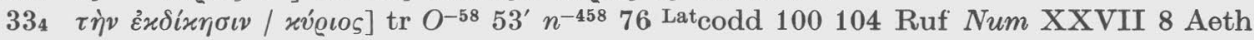
Syh

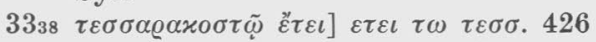

$3353 \alpha v i \tilde{\omega} v]$ ad fin tr G-426: ef $\mathfrak{M}$

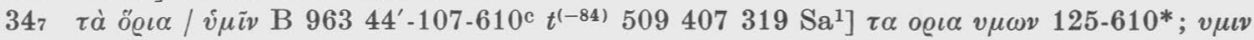

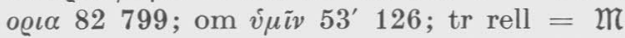

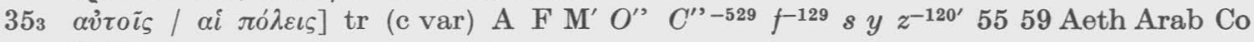
Syh

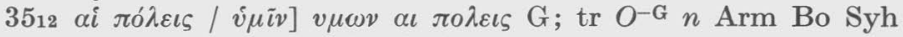

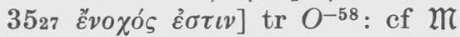

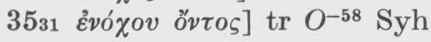

$355_{33}$ ov่x $\left.\dot{\varepsilon} \xi \iota \lambda \alpha \sigma \vartheta \dot{\eta} \sigma \varepsilon \tau \alpha \iota\right]$ post $\gamma \tilde{\eta} \operatorname{tr} O^{-58} \mathrm{Syh}$

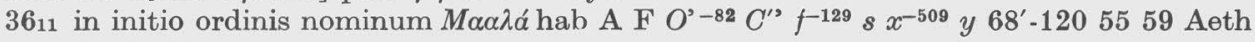
Arab Syh

The changes in word order are also examples of hex activity. In each case one or more of the major hex witnesses attests to the change.

The above lists have also been analyzed in order to discover the spread of hex recensional materials within the text tradition. Instances where more than three text groups (i.e. beyond the $O$ witnesses) support the reading have been disregarded in the table below, as well as random support. Included in $s$ are readings found on the margins of $s \mathrm{mss}, 85^{\prime}-321^{\prime}-344$. Support by a text group means support by the majority of mss within the group except for $z$ where support is identified as meaning at least four mss.

In the table below Column A gives the number of instances where the text group is the only group (outside of $O$ ) supporting the reading; Column $\mathrm{B}$, where the text group is one of two groups supporting, and Column C, one of three groups. The last column gives the total number of readings involved.

\begin{tabular}{|c|c|c|c|c|}
\hline Column & $A$ & $B$ & $C$ & Total \\
\hline$C^{\prime}$ & - & 3 & 4 & 7 \\
\hline$c I$ & 1 & - & - & 1 \\
\hline$b$ & 11 & 4 & 7 & 22 \\
\hline$d$ & - & 7 & 11 & 18 \\
\hline
\end{tabular}




$\begin{array}{lrrrr}f & 23 & 3 & 4 & 30 \\ n & 8 & 4 & 9 & 21 \\ s & 4 & 6 & 5 & 15 \\ t & 1 & 11 & 12 & 24 \\ x & 1 & - & - & 1 \\ y & - & 3 & 3 & 6 \\ z & 37 & 7 & 5 & 49\end{array}$

From this table it is clear that the $z$ group is somewhat more hexaplaric than all others with a total of 49 instances of support (out of a total of 566 readings) followed by $f$ with $30, t$ with $24, b$ with $22, n$ with $21, d$ with $18, s$ with 15 , $C^{\prime \prime}$ together with $c I, 8 ; y$ with 6 , and $x$ with only 1 .

Within the $n$ group $\mathrm{ms} 767$ is often aberrant. When its text differs from the $n$ group it shows hexaplaric influence. In the above lists $n^{-767}$ has the hex reading only twice, whereas 767 supports the hex reading over against $n 59$ times. It is, however, an $n$ text in its major allegiance.

In the following list the possibility of Origenian revision of proper names is explored. The evidence of Syh is not given since Paul of Tella commonly used the Peshitta form rather than his own transcription from the Greek. The list is limited to those names which appear to have been influenced by the Hebrew within the text tradition.

\section{List 4}

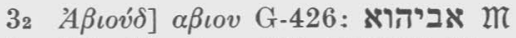

34 2 A

$34 \quad \Sigma_{\iota v a ́]} \sigma \iota v \alpha \iota 58-426 n^{-767}:$ סיני

$314 \Sigma(v \alpha ́] \sigma \iota v \alpha \iota 426 n^{-767}:$ סיני $\mathfrak{m}$

$317 \Gamma \varepsilon \delta \sigma \omega \dot{v}] \gamma \eta \varrho \sigma \omega \nu O^{-58}$ LatRuf Num IV 1: ג ג ג

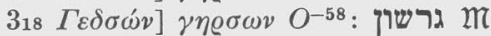

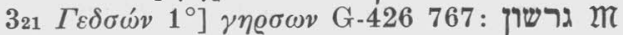

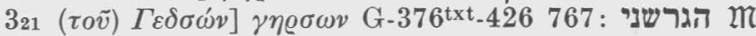

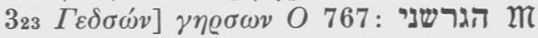

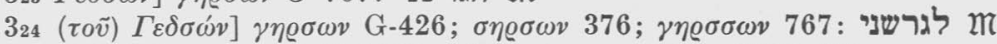

$\left.3{ }_{25} \Gamma \varepsilon \delta \sigma \omega^{\prime} v\right] \gamma \eta \varrho \sigma \omega \nu O^{-58} 767 * x^{-509} ; \gamma \eta \varrho \sigma \sigma \omega \nu 767 c$ : גר שרון 2

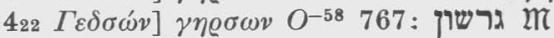

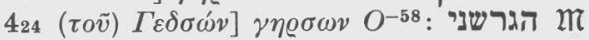

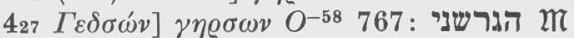

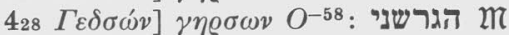

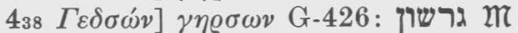

$\left.{ }_{441} \Gamma \varepsilon \delta \sigma \omega v\right] \gamma \eta \varrho \sigma \omega v$ G-426 767 : גרשון

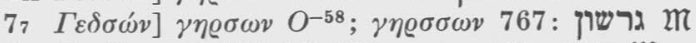

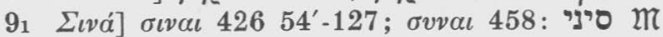

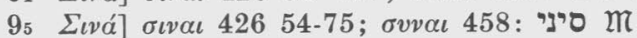

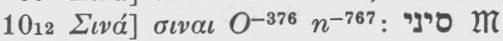

$1017 \Gamma \varepsilon \delta \sigma \omega \nu] \gamma \eta \varrho \sigma \omega \nu 426 ; \gamma \eta \varrho \sigma \sigma \omega \nu 767$ : גרשי

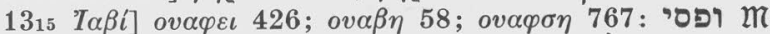

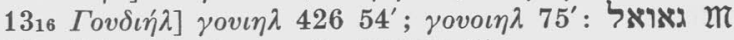

$161 A \beta \iota \omega v] \alpha \beta \varepsilon \iota \omega \mu \mathrm{G}:$ : אבירם

$\left.16{ }_{12} A \beta \iota \varrho \omega \nu\right] \alpha \beta \varepsilon \varrho \omega \mu$ G: אבירם : $2 \pi$

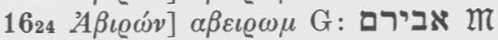

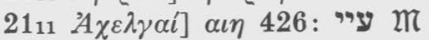


$2118 M \alpha v \vartheta \alpha v \alpha i v] \mu \alpha \vartheta \vartheta \alpha v \alpha$ 426:

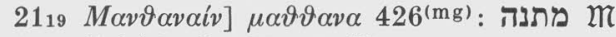

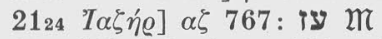

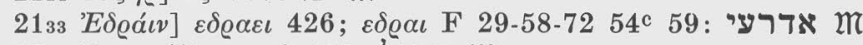

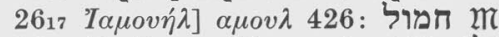

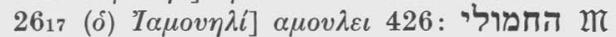

$2620 \Sigma \alpha \mu \varrho \alpha ́ \mu] \sigma \alpha \mu \varrho \alpha v$ 82-426 767: שמרי $2 \pi$

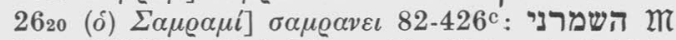

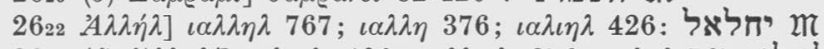

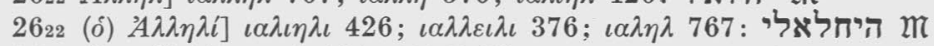

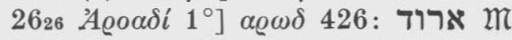

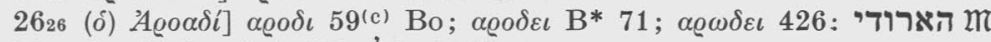

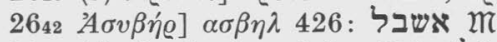

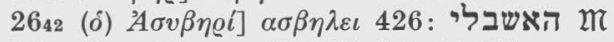

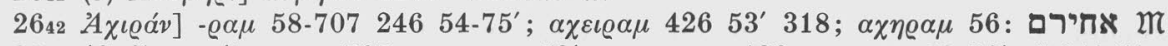

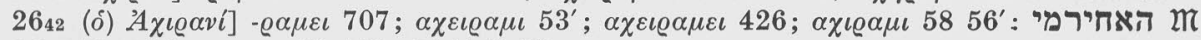

$2643 \Sigma \omega \varphi \alpha ́ v] \sigma \omega \varphi \alpha \mu$ 58-426: שפופם $\mathfrak{k}$

2643 (o) $\Sigma \omega \varphi \alpha v i] \sigma \omega \varphi \alpha \mu \iota 58 ; \sigma \omega \varphi \alpha \mu \varepsilon \iota 426:$ השופמי $2 \pi$

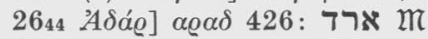

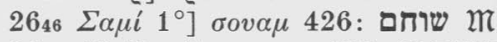

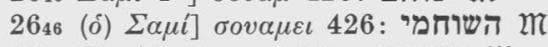

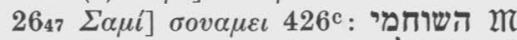

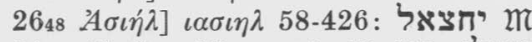

2648 (o) $2 A \sigma \iota \eta \lambda i] \iota \alpha \sigma \iota \eta \lambda \iota 58-426$ : היחצאל $2 \pi$

$2657 \Gamma \varepsilon \delta \sigma \omega v] \gamma \eta \varrho \sigma \omega v 426767$ : גרשון $2 \pi$

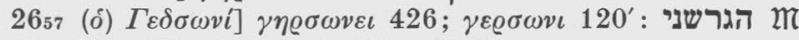

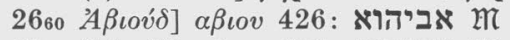

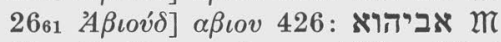

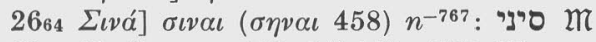

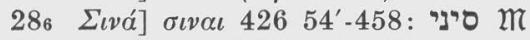

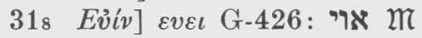

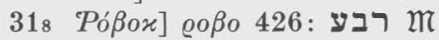

$\left.32_{3} \quad \Sigma \varepsilon \beta \dot{\mu} \mu \alpha\right] \sigma \varepsilon \beta \alpha \mu$ 426: שבם $\mathfrak{m}$

$\left.32{ }_{35} \Sigma \omega \varphi \alpha ́ \varrho\right] ~ \sigma \omega \varphi \alpha v$ F 15-29-426 $s^{-2885} y^{-392}$ Aeth; $\sigma o \varphi \alpha \nu C^{\prime \prime} 19$ 28-85 68'-120 59; $\omega \varphi \alpha v 82$;

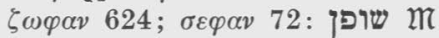

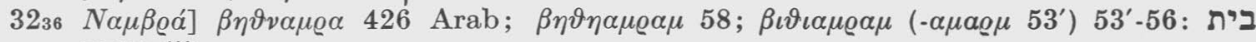
נמרה $2 \pi$

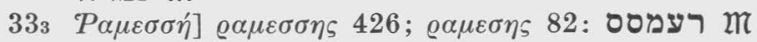

335 P $\left.\alpha \mu \varepsilon \sigma \sigma \eta^{\prime}\right]-\sigma \sigma \eta \varsigma \mathrm{B}^{\mathrm{c}} \mathrm{G}-426$ 509:

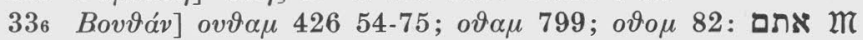

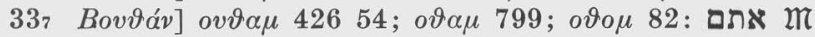

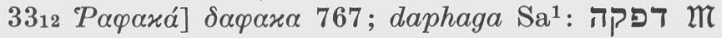

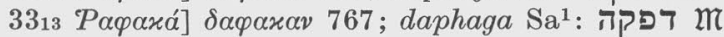

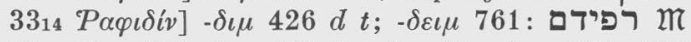

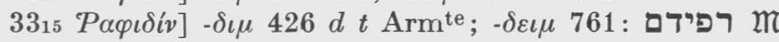

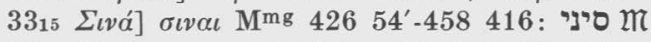

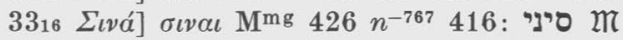

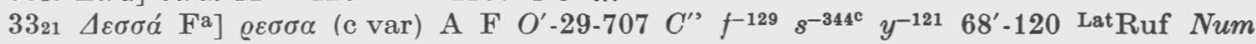
XXVII 12: רסה $\mathfrak{m}$

$33_{22} \Delta \varepsilon \sigma \sigma a ́$ Fa] $\varrho \varepsilon \sigma \sigma \alpha$ (c var) A F $O^{\prime}-29 C^{\prime \prime} f^{-129} s^{-344 c} y^{-121} 68^{\prime}-120$ :

$\left.33_{24} X \alpha \varrho \alpha \delta \alpha^{\prime} \vartheta\right]-\delta \alpha \quad 82-426-707 \quad 53^{\prime}-56 \quad 68^{\prime}-120:$ ח 2 חרדה

$\left.33{ }_{25} X \alpha \varrho \alpha \delta \alpha^{\prime}\right]-\delta \alpha$ 82-426-707 53'-56 68'-120 Latcod 104:

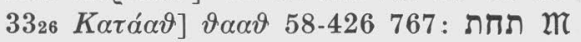

$33_{27} K \alpha \tau \alpha \dot{\alpha \vartheta]} \vartheta \alpha \alpha \vartheta 58-426$ 767; $\tau \alpha \alpha \vartheta 318$ : $5 \pi$ ת m

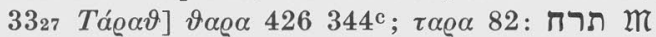

$33{ }_{28}$ Tá $\left.\alpha \vartheta\right] \vartheta \alpha \varrho \alpha 426344^{\mathrm{c}}$; $\tau \alpha \varrho \alpha$ 82;

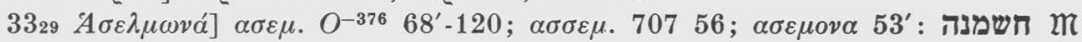

60 


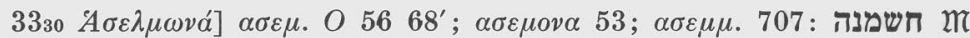

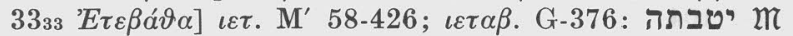

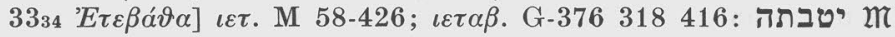

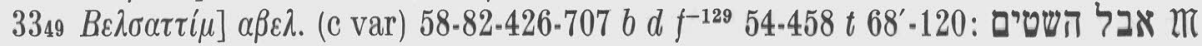

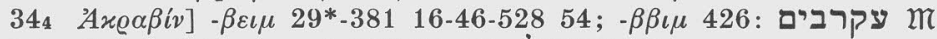

$3420 \Sigma \alpha \lambda \alpha \mu \iota \dot{\eta} \lambda] \sigma \alpha \mu o v \eta \lambda 346^{\mathrm{mg}}$ (vid): שמואל $\mathrm{m}$

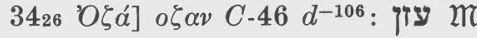

Out of the 90 instances in List 4 all but six are attested by $O$ witnesses, particularly by 426 and by 58 to a lesser extent. From this fact it would appear that Origen did "correct" a number of proper names, probably on the basis of the text of The Three, even though he does not state this as a principle underlying his hexaplaric activity.

The six instances not attested by $O$ witnesses are to be found at 21242664

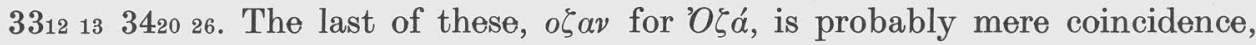

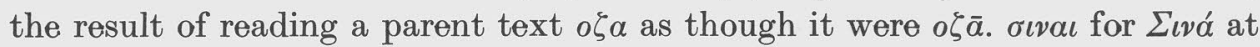
2664 is not a hex reading at all, since its support by $n^{-767}$ is meaningless. Most of the $n$ mss read $\sigma \iota v a \iota$ consistently throughout Numbers for $\sum \iota v a$, but do not show particular hex activity otherwise.

It remains to test whether post hexaplaric activity can be demonstrated in the text tradition with respect to the obelus tradition. In the following list are detailed instances in which the tradition omits text under the obelus. Since all of the instances are equivalent to $\mathfrak{N}$, that fact is not noted.

\section{List 5}

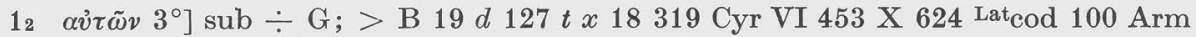

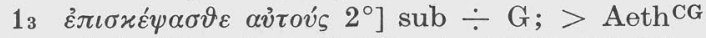

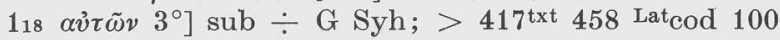

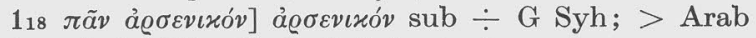

$120 \alpha v i \tilde{\omega} \boldsymbol{v} 4^{\circ}$ ] sub $\div \mathrm{G} \mathrm{Syh;}>b$

1 зо $\left.\alpha \dot{\tau} \tau \tilde{\omega} \nu 4^{\circ}-\dot{\alpha} \varrho \sigma \varepsilon v \iota x a ́\right]$ sub $\div \mathrm{G}$ Syh; $>107^{\prime}$

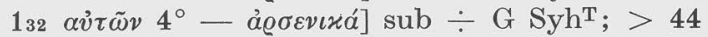

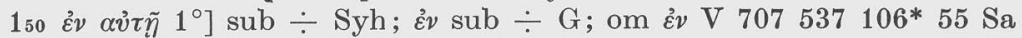

153 Ėv $\alpha v \tau i ́ o l]$ sub $\div \mathrm{G} \mathrm{Syh}^{\mathrm{T}}$; > 58-376 Arm Bo

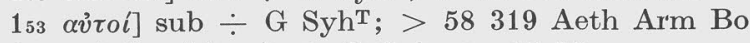

154 xai Aa@óv] sub $\div$ G Syh; > 1575

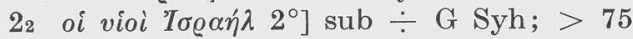

214 oi $-\alpha v \dot{\tau}$ ov ] sub $\div \mathrm{G}$ Syh; $>$ Arab

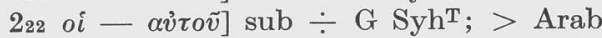

229 oi $-\alpha \hat{v} \tau o \tilde{v}]$ sub $\div \mathrm{G} \operatorname{Syh}^{\mathrm{T}} ;>\mathrm{Arab}$

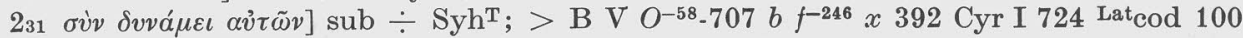
Aeth Arab Co $\mathrm{Syh}^{\mathrm{L}}=\mathrm{Ra}$

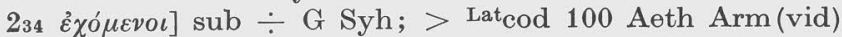

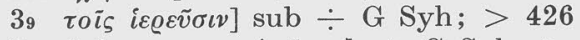

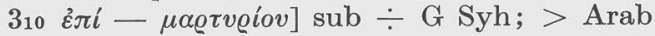

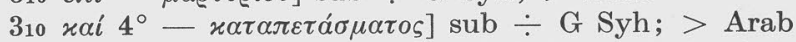

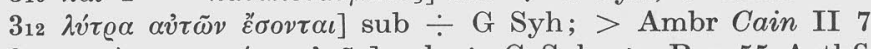

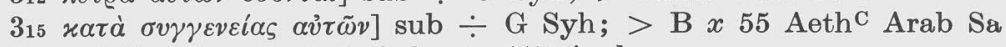

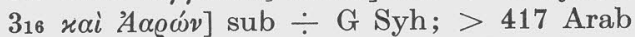

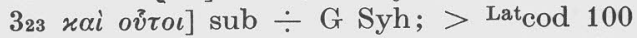

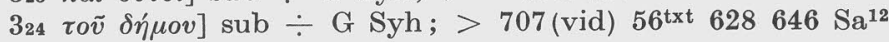




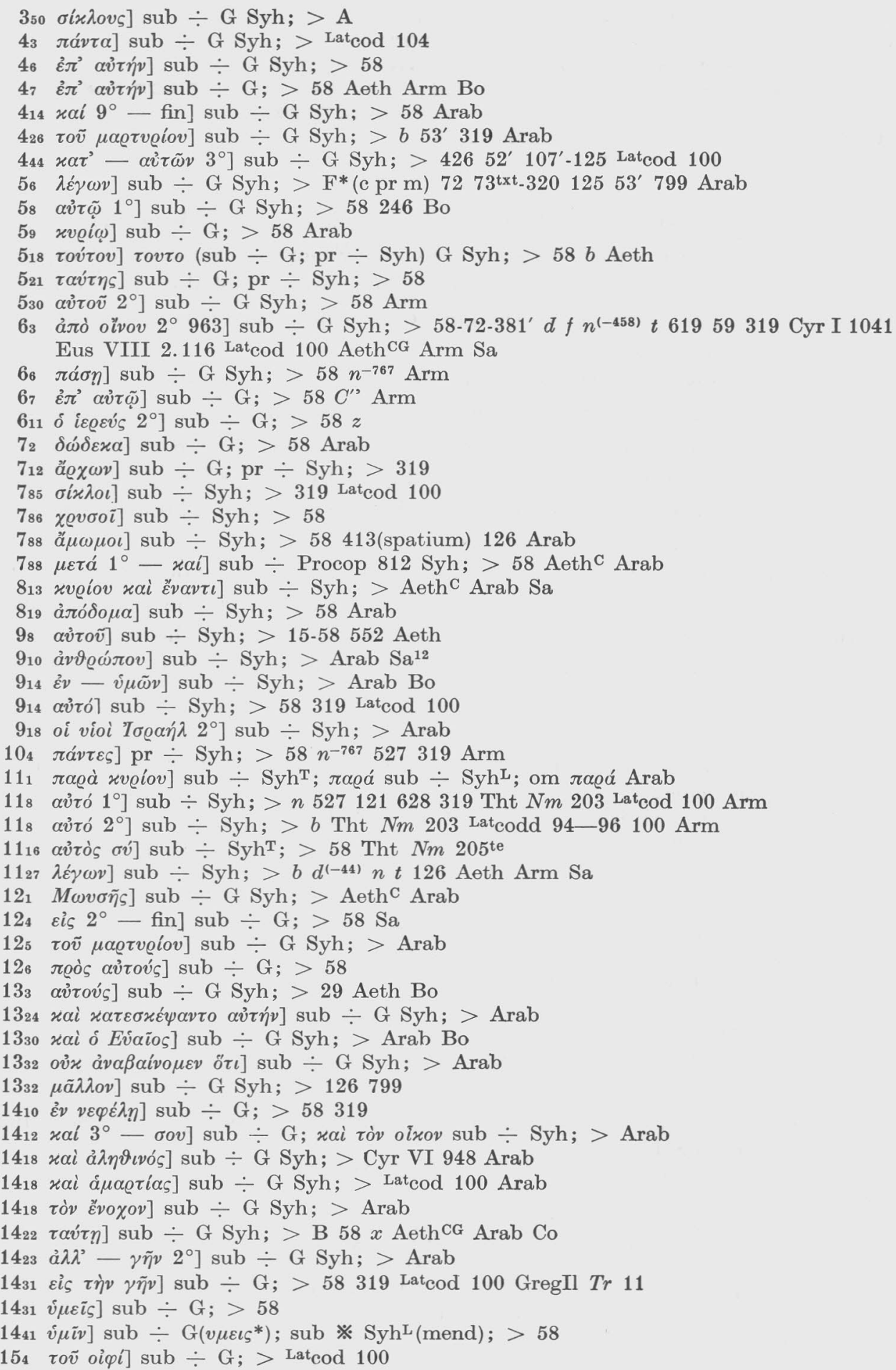




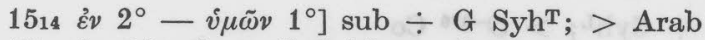

1514 v $\left.\mu \varepsilon \tilde{I}_{5}\right] \mathrm{sub} \div \mathrm{G} \mathrm{Syh}^{\mathrm{L}} ;>\mathrm{Sa}$

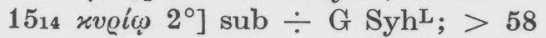

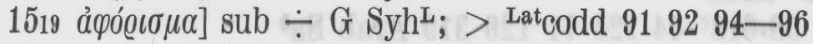

$15_{20}$ av่tó] sub $\div$ Syh; $>58$

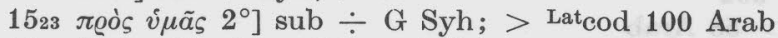

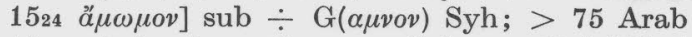

$\left.15_{27} \mu i \alpha v\right]$ sub $\div \mathrm{G} ; \mathrm{pr} \div \mathrm{Syh} ;>58$ LatHi $C$ Pel I 35

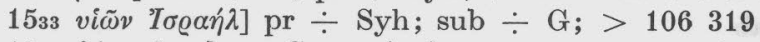

$\left.1535 \lambda \varepsilon^{\prime} \gamma \omega v\right]$ sub $\div \mathrm{G} ;>$ Arab

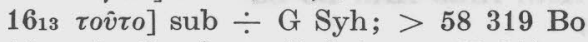

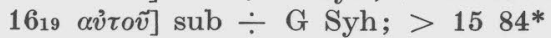

$1625 \pi a ́ v \tau \varepsilon \varsigma]$ sub $\div$ G Syh' ${ }^{\mathrm{T}}$; $>58319$

1630 xi $2^{\circ}-\alpha v i \tilde{\omega} v 2^{\circ}$ ] sub $\div \mathrm{G} \mathrm{Syh}$; om $x \alpha i 2^{\circ}-\alpha v i \tilde{\omega} v 1^{\circ} 58 ;>A r a b=\mathfrak{m}$

$1637 \tau \dot{\alpha} \chi \alpha \lambda x \tilde{\alpha}]$ pr $\div$ Syh; sub $\div \mathrm{G} ;>58$ Arab

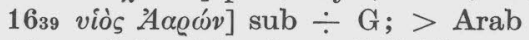

1644 rai $A \alpha \varrho \omega ́ v]$ sub $\div \mathrm{G} \mathrm{Syh}^{\mathrm{T}} ;>\mathrm{A}^{*} 551 \mathrm{Sa}^{12}$

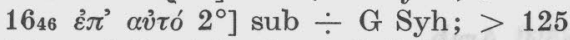

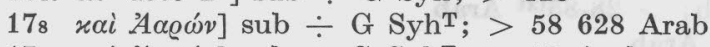

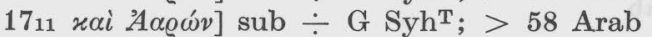

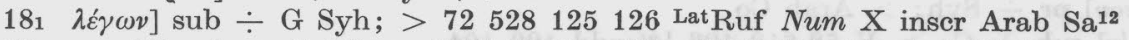

183 бov $2^{\circ}$ ] sub $\div \mathrm{Syh}^{\mathrm{T}}$; > B V 58-82 $x^{-527} z^{-126628} 319646$ AethCG $\mathrm{Sa}=\mathrm{Ra}$

$188 \mu \circ \iota] \mathrm{pr} \div \mathrm{Syh}^{\mathrm{T}} ;>58120$

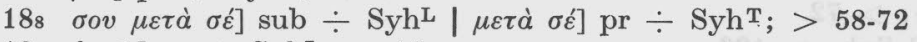

1826 v $\mu \varepsilon \tilde{i} \varsigma] \mathrm{pr} \div \mathrm{Syh}^{\mathrm{L}} ;>44$

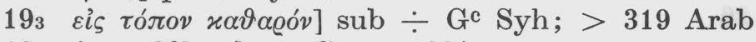

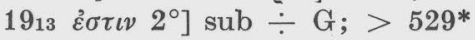

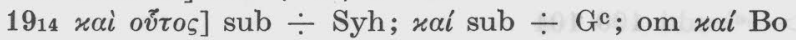

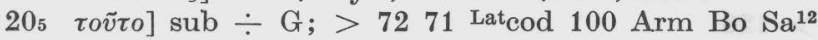

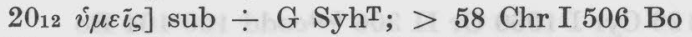

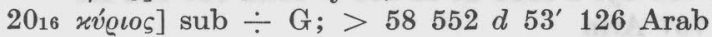

2017 бov $2^{\circ}$ ] sub $\div \mathrm{Syh}^{\mathrm{L}}$; > 16-46 Phil II 87UF Aeth

$\left.2020 \delta \iota^{\prime} \varepsilon \dot{\varepsilon} \mu \tilde{v}\right]$ sub $\div$ G Syh; $>$ Latcod 100

2025 है $v \nu v \iota$ - fin] sub $\div$ Syh; $>$ Arab

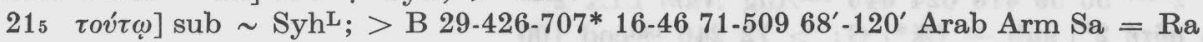

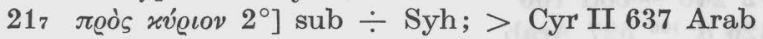

$2116 \pi \iota \varepsilon i v]$ sub $\div$ Syh; $>58551$

2121 hó

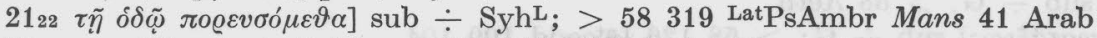

2122 oov $2^{\circ}$ ] sub $\div$ Syh $^{\mathrm{L}} ;>128319$ LatPsAmbr Mans 41

$\left.21_{24} \dot{\varepsilon} \sigma \tau \iota v\right]$ pr $\div \operatorname{Syh}^{\mathrm{L}} ;>58$

$226 \sigma v^{\prime} 1^{\circ}$ ] sub $\div$ Syh; $>C^{\prime \prime} 53^{\prime} 75$ 28-85'-321' 527-619 318 z 5559319 Latcod 100 Caes Serm CXIII 2 Ruf Num XIII 5 Bo

$226 \sigma^{\prime} 2^{\circ}$ ] sub $\div$ Syh; > $7244527392^{\text {Lat }}$ cod 100 Caes Serm CXIII 2 Ruf Num XIII 5 Arm Bo

2210 av่นó́c],sub $\div \mathrm{Syh}^{\mathrm{L}}$; $>58767319$ Bo

$22{ }_{18} \dot{\varepsilon} v$ - fin] sub $\div \mathrm{Syh}^{\mathrm{T}} ;>58319 \mathrm{Arab}$

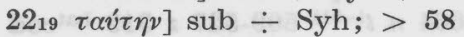

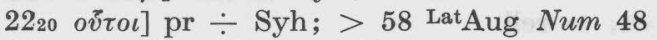

2241 il] sub $\div$ Syh; $>82 z$

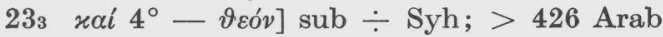

$\left.237 \mu \circ 2^{\circ}\right] \mathrm{pr} \div \mathrm{Syh} ;>\mathrm{Co}$

$\left.23_{17} \pi a ́ v \tau \varepsilon \varsigma\right]$ sub $\div \mathrm{Syh}^{\mathrm{T}} ;>58$

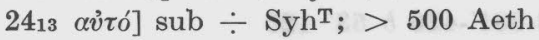

$\left.25_{2} \tau \tilde{\omega} \nu \vartheta v \sigma \iota \tilde{\omega} \nu \alpha \hat{v} \tau \tilde{\omega} \nu\right]$ sub $\div \mathrm{Syh} ;>58 \mathrm{Arab}$

2515 O

$2516 \lambda a ́ \lambda \eta \sigma o v-$ fin] sub $\div \mathrm{G}$; > 58-426 $41784^{\text {txt }}(\mathrm{c}$ pr m) 319 Arab 


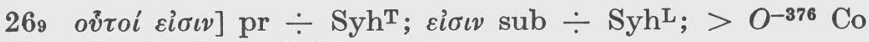

$2610 \alpha v i \tau o \tilde{v}]$ sub $\div \mathrm{Syh}^{\mathrm{L}}$; > Sa

2661 है $v 2^{\circ}$ - fin] pr $\div$ Syh; > Arab

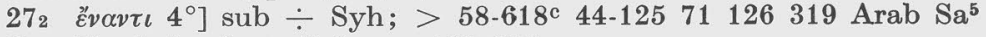

2712 Xaváav] sub $\div$ Syh; > 129392

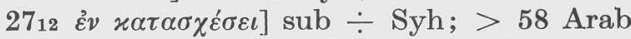

$27_{13} \dot{\varepsilon} v$ - fin] sub $\div$ Syh; $>$ Arab

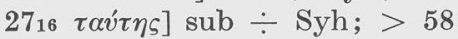

$2718 \lambda \dot{\varepsilon} \gamma \omega \nu]$ sub $\div$ Syh; $>58$ Arab

$282 \lambda \varepsilon \dot{\gamma} \gamma \omega v]$ sub $\div$ Syh; $>58-72-82125509$ Aeth Arab Arm Bo Sa ${ }^{12}$

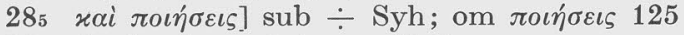

2818 i $\mu$ iv $]$ sub $\div$ Syh; $>58 \mathrm{Sa}$

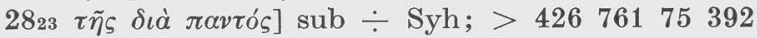

$2825 \dot{\varepsilon} v \alpha \dot{v} \tau \tilde{\eta}]$ sub $\div$ Syh; $>125$ Latcod 100

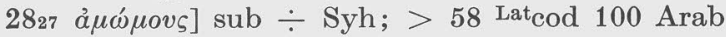

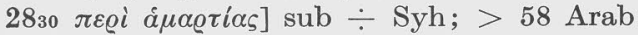

$2831 \mu o \iota]$ sub $\div$ Syh; $>58$ Latcod 100 Arm

$2911 \quad \dot{\varepsilon} \xi \iota \lambda \alpha \dot{\sigma} \sigma \alpha \sigma \vartheta \alpha \iota \pi \varepsilon \varrho i$ v $\mu \tilde{\omega} v]$ sub $\div$ Syh; $>28-85^{\text {txt }}$ Arab

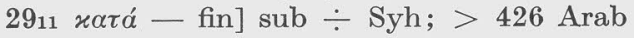

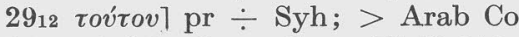

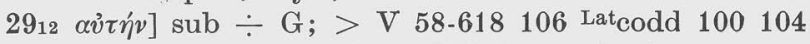

$\left.2913 \tau \tilde{\eta} 1^{\circ}-\pi \varrho \omega ́ \tau \eta\right]$ sub $\div \mathrm{G} \mathrm{Syh;}>$ Arab

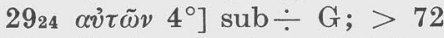

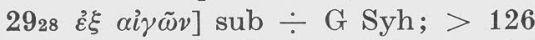

$2931 \varepsilon \dot{\varepsilon} \xi \alpha i \gamma \tilde{\omega} \nu]$ sub $\div$ G Syh; $>126$

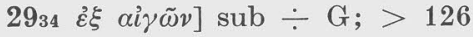

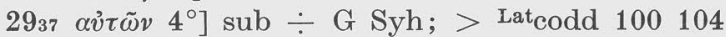

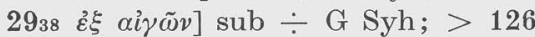

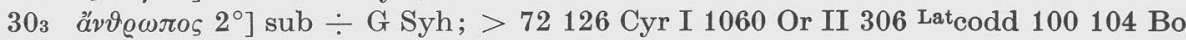

$30_{6}$ ảv $\left.\alpha \varepsilon \varepsilon v ́ \omega v\right]$ sub $\div \mathrm{G} \mathrm{Syh;} \mathrm{>} 125767$ Arm

$30 \mathrm{~g}$ ov่ $\left.-\alpha \hat{\imath} \tau \tilde{\eta} \varsigma 5^{\circ}\right]$ sub $\div \mathrm{G}$ Syh; $>58-426$

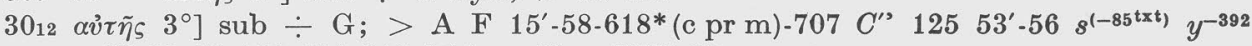

$z^{-407} 5559416624646$ LatAug Num LIX $2^{\text {te }}$

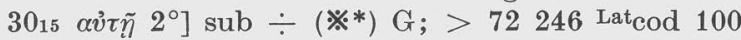

318 ov́v — fin] sub $\div \mathrm{G}$; > 58-426 $d^{-106} 527$ Arab

$3111 \alpha \dot{\tau} \tau \tilde{\omega} v]$ sub $\div \mathrm{G} ;>29-5852718$

$3117 \pi \alpha \dot{\sigma} \eta]$ sub $\div \mathrm{G} ;>58$ Aeth

$\left.3121 \tau \tilde{\eta} \varsigma \pi \alpha \varrho \alpha \tau \alpha^{\prime} \xi \varepsilon \omega \varsigma\right]$ sub $\div \mathrm{G} ;>58126$ Latcodd 100104

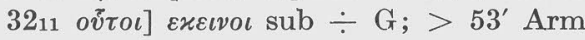

3211 of $\left.3^{\circ}-a \dot{\gamma} \alpha \vartheta \hat{o} v\right]$ sub $\div \mathrm{G} ;>58 \operatorname{Aeth}^{\mathrm{C}}$ Arab

3230 Eis $1^{\circ}-X \alpha v \alpha ́ \alpha v 1^{\circ}$ ] sub $\div \mathrm{G}$; $>426$ Arab

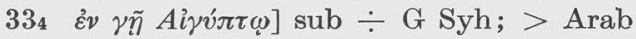

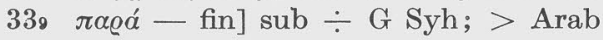

$33_{36}$ xaí $\left.3^{\circ}-\Phi \alpha \varrho a ́ v\right]$ sub $\div \mathrm{G}$; $>426$ Arab

3352 av่ á] sub $\div$ G Syh; > 72-381' $d 66455799$ Latcod 104 Spec 44 Aeth Arm

$3354 \alpha v i \tilde{\omega} v 1^{\circ} \mathrm{B}^{(\mathrm{mg})} \mathrm{M}^{\prime} \mathrm{V} 963$ (vid) 58-376'-oI $56^{\mathrm{mg}}-129-664 n t^{(-84)} 509-527 z 319$ Lat $^{\prime}$ cod

$\left.100 \mathrm{Bo} \mathrm{Sa}^{1}\right\rceil \tau \alpha v \tau \eta \nu 799$; sub $\div \mathrm{G} \mathrm{Syh;} \mathrm{>} \mathrm{rell}$

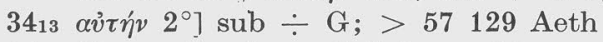

$\left.34{ }_{13} M \alpha v \alpha \sigma \sigma \eta^{\prime}\right]$ sub $\div \mathrm{G}$ Syh; $>82$

$34_{18}$ v $\mu \tilde{\imath}$ ] $]$ sub $\div \mathrm{G}\left({ }^{*}\right) \mathrm{Syh} ;>\mathrm{V} 552$

$3512 \tau \dot{\alpha} \alpha \tilde{l} \mu \alpha]$ sub $\div$ G Syh; $>72$

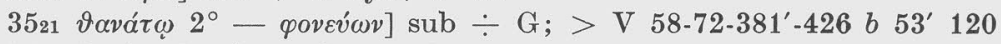

3532 o $\mu \varepsilon \dot{\gamma} \alpha \varsigma$ sub $\div \mathrm{G}$; $>58$

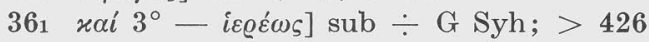

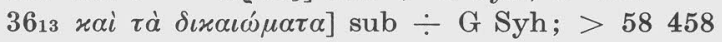

64 
Most of the above instances of omissions agreeing with $\mathfrak{k}$ are undoubtedly coincidences and should not be taken seriously. It is, however, not without significance that of witnesses to such omissions ms 58 heads the list with 73 followed by Arab with 66 instances. Next in order is Latin codex 100 with 25; 319 , with 22, and 426, with 15. For Arab and the influence of Hebrew on it ef $J$ W Wevers, The Textual Affinities of the Arabic Genesis of Bib. Nat.Arab 9, Studies of the Ancient Palestinian World, ed. by J. W. Wevers and D. B. Redford, Toronto 1971. That ms 58 gave evidence of post-hexaplaric activity was shown in THGD $43-47$ for Deuteronomy.

From lists 1 and 2 it was evident that hex additions in the text tradition were to be found in $O^{-58}$ more often than in the common witness of $O$. From List 5 it would seem that the copyist of 58 often omitted materials under the asterisk. It is interesting to speculate whether this copyist tended to omit textual materials under hex signs without distinguishing asterisks and obeluses. On the whole, ms 58 seems to be the result of further hex activity beyond that of Origen. On the other hand, it also presents over against the text tradition as a whole an at times curiously expanded text; thus at $527 \mathrm{~ms} 58$ has introduced an interpretative gloss taken from Josephus Antiq III 273. 


\section{Chapter 4 The Texts of $B$ and $A$}

It is the purpose of this study to examine the character of our two oldest complete texts of Numbers, to determine their place within the text history of the book, and to explore whether one can validly speak of a B vs an A text type. This last point is particularly important in the light of the tendency of scholars of former generations to limit Septuagint citations largely to these two codices. Thus Hatch-Redpath's well-known Concordance to the Septuagint is based principally on these two mss. Or if one reads the apparatus to the text of Numbers in $\mathrm{Ra}$ it is clear that except for an occasional hex reading Rahlfs limited himself to variants in A \& B including the text of their correctors.

It should be said that this last-named practice is a most unfortunate one, particularly with reference to readings from $\mathrm{B}$. The corrections in $\mathrm{B}$ are much later than $\mathrm{B}^{*}$, and only first hand corrections deserve to be cited. $\mathrm{B}^{\mathrm{c}}$ should be regarded as an independent witness within the text history and not as an adjunct to $\mathrm{B}$. Accordingly readings of $\mathrm{A}^{c}$ and $\mathrm{B}^{\mathrm{c}}$ are disregarded in this essay. I have discussed the text of the B correctors in Numbers elsewhere (in a Festschrift for R. J. Williams).

A. The text of Numbers has been copied far more carefully by the B scribe than was the case in Deuteronomy (cf THGD 48f). The following list presents a list of errors in B caused by homoioteleuton or homoiarchon.

List 1

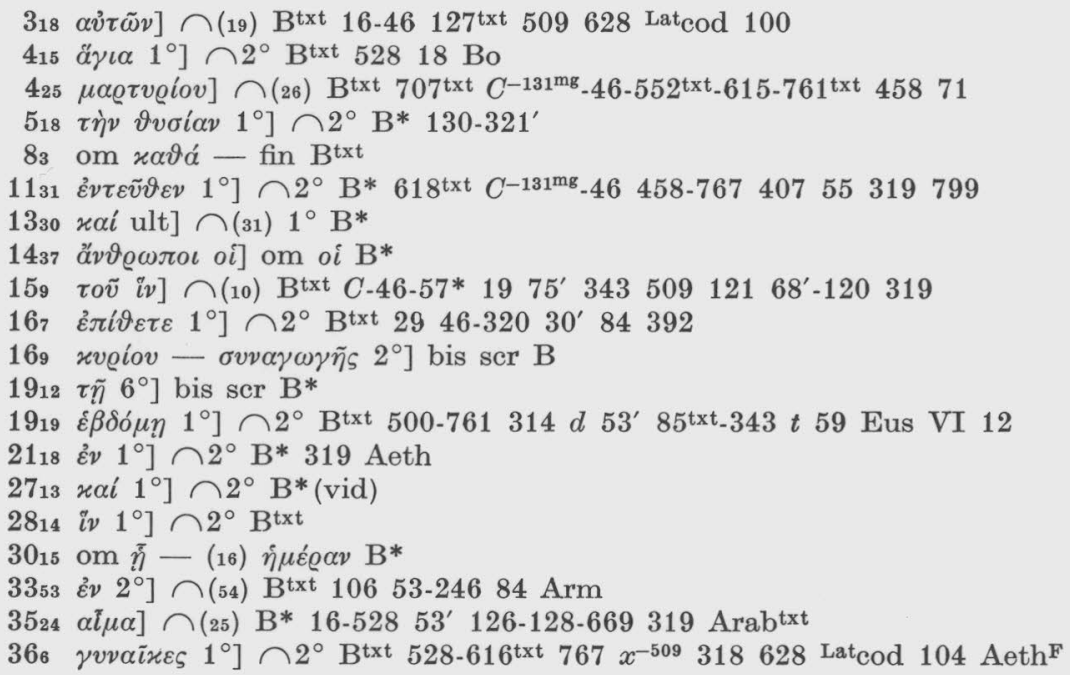


Compared to Deuteronomy the B text of Numbers is carefully copied; copyist errors due to homoioteleuton or homoiarchon are infrequent.

This conclusion is also confirmed by the fact that there obtain relatively few unique readings in $\mathrm{B}$. The following list presents these readings for Numbers. By unique reading is meant a reading found only in B as far as the Greek evidence is concerned.

List 2

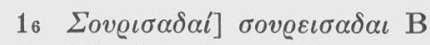

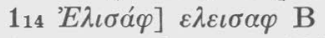

$147 \pi \alpha \tau \varrho \iota \tilde{\alpha} \zeta]$ pr $\tau \eta \varsigma \mathrm{B}^{*}$

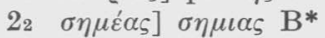

216 iñ $]$ pr $\varepsilon \varkappa \mathrm{B}^{*}$

218 om viós $\mathrm{B}^{*}$ Aeth $^{\mathrm{M}}$

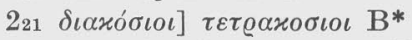

$226 \varepsilon \dot{\pi} \tau \alpha x o ́ \sigma \iota \iota \iota] \pi \varepsilon \nu \tau \alpha \varkappa o \sigma \iota \iota \iota \mathrm{B}^{*}$

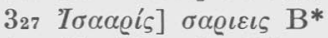

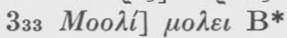

$3_{35}$ A $\left.A \beta \iota \chi \alpha \dot{\iota} \iota\right] \alpha \beta \varepsilon \iota \chi \alpha \iota \lambda \mathrm{B}$

$418 \Lambda \varepsilon v \iota \tau \tilde{\omega} v] \pi 0 \lambda \varepsilon \iota \tau \omega \nu$ B*

$\left.431 \alpha v i \tau \tilde{\omega} \nu 2^{\circ}\right] \tau \omega \nu \mathrm{B}^{*}$

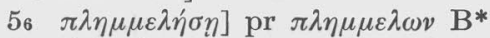

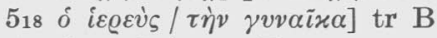

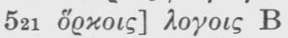

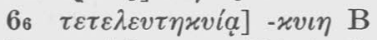

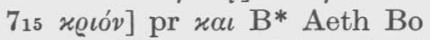

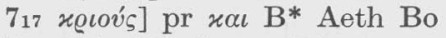

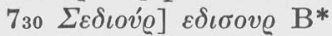

$7_{60}[\alpha \alpha \delta \varepsilon \omega v i]$ j $\gamma \alpha \delta \alpha \iota \omega v \varepsilon \iota$ B

$788 \tau \varrho a ́ \gamma o \iota ~ \varepsilon \xi \xi \dot{\eta} x o v \tau \alpha]$ post $\varepsilon \xi \eta \dot{\eta} \varkappa o \nu \tau \alpha 3^{\circ}$ tr B*

813 om xal $2^{\circ} \mathrm{B}^{*}$

819 om $\varepsilon^{2} v 2^{\circ} \mathrm{B}$

$\left.8_{26} \dot{\varepsilon} \varrho \gamma \tilde{\alpha} \tau \alpha \iota\right] \varepsilon \varrho \gamma \alpha \zeta \varepsilon \tau \alpha \iota \mathrm{B}^{*}$

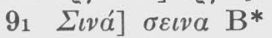

$\left.95 \quad \Sigma_{\imath} a^{\prime}\right] \sigma \varepsilon \imath v \alpha \mathrm{B}^{*}$

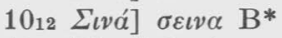

$\left.10_{20}{ }^{~} E \lambda \iota \sigma \alpha ́ \varphi\right] \varepsilon \lambda \varepsilon \iota \sigma \alpha \varphi \mathrm{B}$

$\left.10_{36} \quad \dot{\varepsilon} v\right]$ pr $x \alpha \iota \mathrm{B}^{*}$

116 om ov̉oźv $\mathrm{B}^{*}$

1116 o $[\delta \alpha \varsigma]$ o $\delta \varepsilon \varsigma \mathrm{B}^{*}$

$\left.11_{20} \dot{\mu} \tilde{\omega} v\right]+\varkappa \varrho \varepsilon \alpha \mathrm{B}^{*}$

$\left.11_{21} M \omega v \sigma \tilde{\eta} s\right]+\pi \varrho \circ \overline{x \nu} \mathrm{B}^{*}$ Lat $\operatorname{cod} 100$ Arab

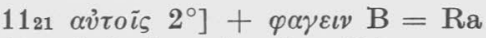

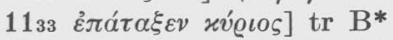

$135 \Sigma \alpha \mu o v ́] \sigma \alpha \mu o v \eta \lambda$ B Syh

$\left.13_{13} A \mu \iota \dot{\eta} \lambda\right] \alpha \mu \varepsilon \imath \eta \lambda \mathrm{B}$

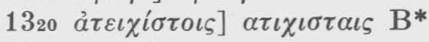

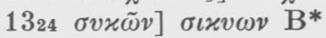

$\left.141 \varphi \omega v \eta^{\prime}\right] \varphi \omega v \eta$ B Latcod 100 Aeth

$14{ }_{13}$ aेvท́ $\left.\gamma \alpha \gamma \varepsilon \varsigma\right] \eta \gamma \alpha \gamma \varepsilon \varsigma \mathrm{B}^{*}$

$15_{16}$ om $\dot{v} \mu \tilde{i} \nu 1^{\circ} \mathrm{B}^{*}$

$\left.15_{31} \tau \grave{o} \varrho \tilde{\eta} \mu \alpha\right] \tau \alpha \varrho \eta \mu \alpha \tau \alpha \mathrm{B}^{*}$

$1533 \sigma v \lambda \lambda \varepsilon \dot{\gamma} \gamma o v \tau \alpha]-v \tau \varepsilon \varsigma \mathrm{B}^{*}$
1539 om vi $\mu \varepsilon i \tilde{\zeta}_{5} \mathrm{~B}^{*}$

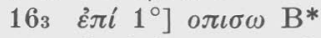

$16_{7}$ ăv $\left.\dot{\varepsilon} x \lambda \varepsilon^{\prime} \xi \eta \tau \alpha \iota\right] \varepsilon x \lambda \varepsilon \gamma \varepsilon \tau \alpha \iota \mathrm{B}^{*}$ (vid)

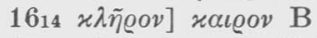

$\left.16{ }_{33} \alpha v i \tau \tilde{\iota}_{5}\right] \alpha v \tau \omega \nu \mathrm{B}$ Lat $\operatorname{cod} 100=\mathrm{Ra}$

$\left.16{ }_{38} \lambda \varepsilon \pi i \delta \alpha \varsigma\right]-\delta \varepsilon \varsigma \mathrm{B}^{*}$

$1640 \pi \varrho \circ \sigma \varepsilon \dot{\lambda} \vartheta \eta]-\vartheta \eta \tau \varepsilon \mathrm{B}^{*}$

$1648 \vartheta \varrho \alpha \bar{v} \sigma \iota \varsigma]+\varepsilon v \tau \omega \lambda \alpha \omega \mathrm{B}^{*}$

$179 \varepsilon \ddot{\varepsilon} \lambda \alpha \beta o v] \varepsilon \beta \alpha \lambda \varepsilon v \mathrm{~B}$

$\left.188 \tau \tilde{\omega} v 1^{\circ}\right] \alpha v \tau \omega v \mathrm{~B}^{*}$

$19_{14}$ oixiá $\left.1^{\circ}\right]$ pr $\tau \eta \mathrm{B}^{*}$

1918 v $\sigma \sigma \omega \pi \sigma \nu\rceil-\pi \iota v$ B*

2022 om $\dot{\eta} \mathrm{B}^{*}$

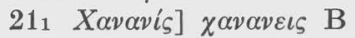

$211 \quad 2 A \vartheta \propto \varrho i \mu]-\varrho \varepsilon \iota \nu \mathrm{B} \quad \mathrm{Sa}^{4}$

$215 i v \alpha \tau i]+\tau o v \tau o \mathrm{~B}$

217 $\left.\dot{a} \varphi^{\prime}-\mathrm{fin}\right] \sup \operatorname{ras} B$

$\left.21_{11} A^{A} \chi \varepsilon \lambda_{\gamma} \gamma \alpha i\right] \quad \chi \alpha \lambda \gamma \lambda \varepsilon \iota \mathrm{B}$

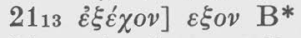

$2120 \nu \alpha ́ \pi \eta v] \iota \alpha \nu \eta v \mathrm{~B}$

$\left.21_{23} I \alpha \dot{\alpha} \sigma \sigma \alpha\right] \varepsilon \iota \sigma \sigma \alpha \mathrm{B}^{*}$

$\left.229 \pi \varrho \sigma^{2}\right] \pi \alpha \varrho \alpha \mathrm{B}^{*}$

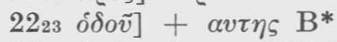

$\left.22_{36} \ddot{\eta} \dot{\varepsilon} \sigma \tau \iota v\right] \eta \tau \iota \varsigma \mathrm{B}^{*}$ (vid)

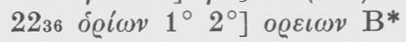

$22_{37}$ o้ $\left.v \tau \omega \varsigma / o v ่ \delta v v \eta ́ \sigma o \mu \alpha \iota\right] \operatorname{tr} \mathrm{B}$

241 om $\alpha \dot{\tau} \tau o \tilde{v} \mathrm{~B}^{*}$

$244 \quad \forall \pi v \omega]+\operatorname{ras} 1-2 \operatorname{litt} \mathrm{B}$

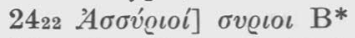

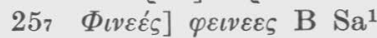

2511 vioṽ] vios B* Latcod 100

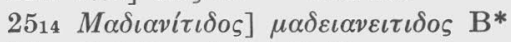

$\left.2514 \Sigma \alpha \lambda \omega^{\prime}\right] \sigma \alpha \lambda \mu \omega \nu$ B Bo

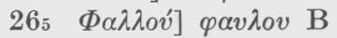

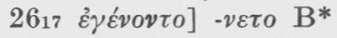

$2618 \delta \tilde{\eta} \mu \circ \iota] \delta \eta \mu \omega \mathrm{B}^{*}$

$26{ }_{19}$ Фovat]

$\left.26{ }_{20} \Sigma \alpha \mu \varrho \alpha ́ \mu\right] \sigma \alpha \mu \alpha \varrho \alpha \mu \mathrm{B}^{*}$

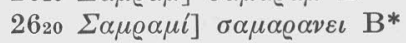

$2629 M \varepsilon \lambda \chi\left\llcorner\eta^{\prime} \lambda\right] \mu \varepsilon \lambda \lambda \iota \eta \lambda \mathrm{B}^{*}$

$\left.26{ }_{29} M \varepsilon \lambda \chi \imath \eta \lambda i\right] \mu \varepsilon \lambda \lambda \iota \eta \lambda \varepsilon \iota \mathrm{B}^{*}$

$\left.2634{ }^{2} A \chi \iota \varepsilon \zeta \varepsilon \varrho i\right]$ $\alpha \chi \iota \varepsilon \zeta \varepsilon \iota \varrho \varepsilon \iota \mathrm{B}$

2648 A $\left.A \iota \eta^{\prime} \lambda\right] \sigma \alpha \eta \lambda \mathrm{B}^{*}$

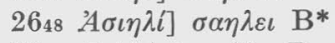

$2649 \quad \Sigma \varepsilon \lambda \lambda \eta \dot{\eta} \mu] \sigma \varepsilon \lambda \lambda \eta \mathrm{B}$

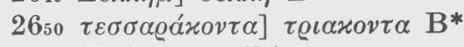

$2661 \Sigma \imath v a ́$ ] $\sigma \varepsilon \imath v \alpha \mathrm{B}^{*}$ 
$\left.27{ }_{14} K \alpha \delta \eta_{\zeta}\right]$ pr $\varepsilon v$ B Aeth Bo

$286 \quad \Sigma \iota v \alpha ́] \quad \sigma \varepsilon \iota v \alpha$ B; $\sigma \varepsilon \iota[\ldots 963$

$288 x v \varrho i \omega] \overline{x v} \mathrm{~B}^{*}$

$2815 x v \varrho i \omega 963] \overline{x v} \mathrm{~B}^{*}$

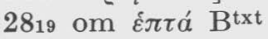

$\left.28_{24} x v \varrho i \omega\right] \overline{x v} \mathrm{~B}^{*}$

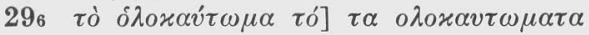
$\mathrm{B}^{*}$

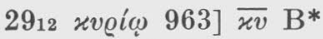

2913 xvoí $\overline{x v}$ B*

$\left.29_{36} x v \varrho i \omega\right] \overline{x v} \mathrm{~B}^{*}$

309 åv] $\varepsilon \alpha v$ B*

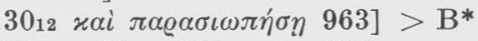

$31_{4} \chi\left\llcorner\lambda i o v \varsigma 1^{\circ}\right.$ ] $\chi \varepsilon \iota \lambda \iota \circ \mathrm{B}^{*}$

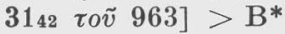

$321 \pi \lambda \tilde{\eta} \vartheta_{\circ} 1^{\circ}$ ] pr ras 2 litt B

$324 \pi \alpha \varrho \varepsilon \delta \omega x \varepsilon v] \pi \alpha \varrho \alpha \delta \varepsilon \delta \omega x \varepsilon v \mathrm{~B}$

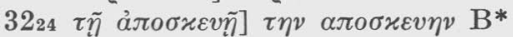

$3224 \pi \circ \imath \dot{\sigma} \sigma \varepsilon \tau \varepsilon]$ pr $\tau o v \tau o$ B*

$\left.32{ }_{36} N \alpha \mu \beta \varrho \alpha ́\right]$ $v \alpha \mu \varrho \alpha \mu \mathrm{B}$

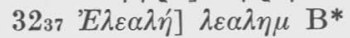

3238 om $\tau \dot{\eta} v 2^{\circ} \mathrm{B}^{*}$

$33_{20}$ 'P $\left.\varepsilon \mu \mu \omega \dot{v}\right] \varrho \alpha \mu \mu \omega \nu \mathrm{B}$

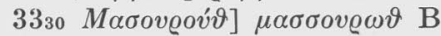

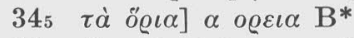

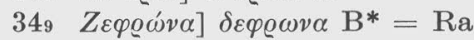

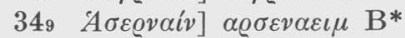

3412 है $\sigma \tau \alpha \iota$ v $\mu \tilde{\imath} v]$ tr $\mathrm{B}$

3425 'E

$\left.34{ }_{26} \Phi \alpha \lambda \tau \iota \eta^{\prime} \lambda\right]$ $\varphi \alpha \lambda \tau \varepsilon \iota \eta \lambda \mathrm{B}$

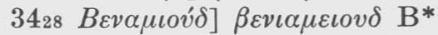

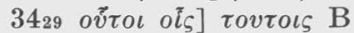

$3514 \gamma \tilde{\eta}]$ pr $\tau \eta \mathbf{B}$

3528 om $x \alpha i$ B*

$\left.355_{30} \psi v \chi \eta \dot{v} 2^{\circ}\right] \psi v \chi \eta \varsigma \mathrm{B}$

It is clear from the brevity of this list that the text of Numbers has been much more carefully copied than that of Deuteronomy. Many of the variants obtain in the spelling of proper names in which B is notoriously inexact elsewhere as well. It should be noted that the B copyist commonly spelled $\iota$ as $\varepsilon \iota$ in proper names, e.g. $\sigma \varepsilon \iota v \alpha, \varepsilon \lambda \varepsilon \iota \sigma \alpha \varphi, \alpha \mu \varepsilon \iota \eta \lambda, \varphi \alpha \lambda \tau \varepsilon i \eta \lambda$. This particular itacism will not be recorded in the next list, which is an expansion of list 2 through variants which are almost unique to $\mathrm{B}$. By almost unique is meant support by no more than three Greek witnesses beyond that of $\mathrm{B}$. The purpose of this list is to discover whether there are any close relatives to B in the text tradition. It will of course also give some further insight into the character of B's textual aberrancy.

List 3

$14 x \alpha \tau^{\prime}$ oĭxovऽ] $\mathrm{pr} x \alpha \iota \mathrm{B}^{*} 128$

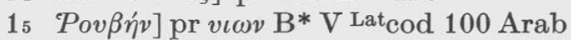

$\left.1_{45} \sigma v^{v}\right] \varepsilon v$ B* 58-72 59

$28 \quad \alpha v \dot{\tau} \tau \tilde{v}] \alpha v \tau \omega \nu$ B $246^{\mathrm{c} 1}$ 509-527

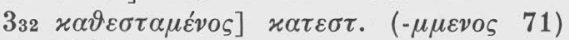
B G 71

$3_{40} \lambda \alpha^{\prime} \beta \varepsilon$ 803] $\lambda \alpha \beta \varepsilon \tau \varepsilon$ B F 71

$3_{41}$ om èv $\tau o i \varsigma$ $\varkappa \tau \eta^{\prime} v \varepsilon \sigma \iota v \mathrm{~B}^{\text {txt }} 669\left(1^{\circ}\right)$

$343 \delta \iota \alpha x o ́ \sigma \iota \iota \iota]-\sigma \iota \alpha \iota$ B 414

$\left.3_{45} \lambda \dot{\alpha} \beta \varepsilon\right] \lambda \alpha \beta \varepsilon \tau \varepsilon \mathrm{B}^{*} 127$

$\left.3_{46} \tau \varrho \iota \tilde{\omega} \nu\right] \tau \varrho \varepsilon \iota \varsigma \mathrm{B}^{*} 376$

$\left.3_{46} \delta \iota \alpha x \circ \sigma i \omega \nu\right]-\sigma \iota \iota \iota \mathrm{B}^{*} 376$

415 ov่x] ovx $\mathrm{B}^{*} 5830318$

446 om xai $2^{\circ} \mathrm{B}^{*}(\mid) 73^{*}(\mathrm{c}$ pr m)

$4_{46}$ om $\alpha v i \tilde{\omega} v 1^{\circ}$ B $x^{-619}$ Lat $^{\circ} \operatorname{cod} 104$ (vid) $=\mathrm{Ra}$

446 om $\alpha \dot{\tau} \tau \tilde{\omega} v 2^{\circ}$ B 71 Lat $\operatorname{cod} 104$

$\left.\begin{array}{ll}5 & o v]\end{array}\right]+\mu \eta \mathrm{B}$ Cyr I $977=\mathrm{Ra}$

54 om $\tau \tilde{\omega}$ B 509

$5132^{\circ}$ 963] $\eta v$ B Cyr I $909=$ Ra

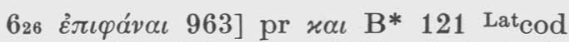
100 FirmMat Consult II 5 Aeth Arab Arm

$720 \pi \lambda \dot{n} \varrho$ 963] - ڤクs B S 8459

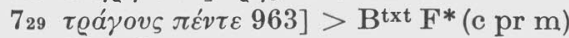
30-343

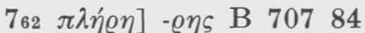

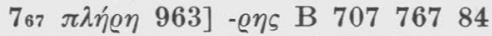

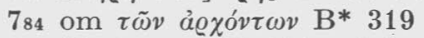

$\left.787 \beta \varepsilon_{\varepsilon}\right]+\alpha \iota$ B 318; pf $\beta$ oo $\alpha \iota 799$

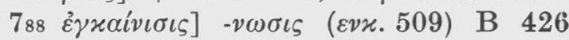
$509=\mathrm{Ra}$

$822 \varkappa \alpha \vartheta a ́] \quad \varkappa \alpha \vartheta \omega \varsigma \mathrm{B}^{*} 58-7259=\mathrm{Ra}$

$825 \dot{\varepsilon} \varrho \gamma \tilde{a} \tau \alpha \iota] \varepsilon \varrho \gamma \alpha \zeta \varepsilon \tau \alpha \iota$ B 130

918 om $x \alpha i$ B* 509-619 Sa

$9_{21}$ om $x \alpha i 4^{\circ}-(22)$ भ̆ B 129 71-509 Sa $=\mathrm{Ra}$

$10_{29}$ ' $\left.\Omega \beta \alpha^{\prime} \beta\right]$ o $\beta \alpha \beta$ B 34371799 Procop 817

$\left.11_{32} \varepsilon ै \psi v \xi \alpha \nu\right] \varepsilon \sigma \varphi \alpha \xi \alpha \nu$ B 509

$\left.11_{33} \lambda \alpha o^{\prime}\right]+\alpha v \tau o v$ B* 44 
124 om $\pi \alpha \varrho \alpha \chi \varrho \tilde{\eta} \mu \alpha \mathrm{B}^{*} 618^{\text {txt }} 126$

124 om Eis $1^{\circ}$ B 7259

1212 om xai B* 799 Bo

135 Zaxxov́@] [axxve B 509

$\left.13_{22} E \mu \alpha \dot{\vartheta}\right] \varepsilon \varphi \alpha \alpha \vartheta$ B 376509 Lat $\operatorname{lod} 100$ $\mathrm{Sa}^{1}=\mathrm{Ra}$

$\left.13_{24} \varkappa \lambda \tilde{\eta} \mu \alpha\right] x \lambda \eta \mu \alpha \tau \alpha$ B 509319

$\left.13_{33} \tau \tilde{\eta} \varsigma \quad \gamma \tilde{\eta} \varsigma\right]$ pr $\varepsilon \pi \iota \mathrm{B}^{*} 610$

$141 \varepsilon ้ \delta \omega \varkappa \varepsilon v] \varepsilon v \varepsilon \delta \omega \varkappa \varepsilon v$ B $130^{\mathrm{c}}-346^{\mathrm{mg}} 319$

1410 om $\varepsilon v \nu i \vartheta o \iota \varsigma ~ B^{*} 126$

$1414 \sigma v ่ \pi \circ \varrho \varepsilon v \dot{\eta}]$ ]

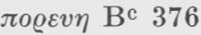

1515 om $\tau \alpha ́ s$ B $129509=\mathrm{Ra}$

$\left.15{ }_{25} \varepsilon \dot{\xi} \xi \imath \alpha \dot{\sigma} \sigma \varepsilon \tau \alpha \iota\right]+\pi \varepsilon \varrho \iota \alpha v \tau o v \mathrm{~B}^{*} 19$; $\mathrm{cf}+$ $\pi \varepsilon \varrho \iota ~ \alpha v \tau \omega 458$

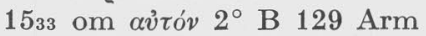

$\left.15_{36} \dot{\varepsilon} \lambda_{\imath} \vartheta \circ \beta \delta \hat{\lambda} \eta \sigma \alpha v\right]-\sigma \varepsilon \nu$ B $767527^{\prime}$

$1617 \vartheta v \mu i \alpha \mu \alpha] \vartheta v \mu \iota \alpha \mu \alpha \tau \alpha \mathrm{B}^{*} 72^{*}$

$1646 \varepsilon \dot{\varepsilon} \xi i \lambda \alpha \sigma \alpha \iota] \varepsilon \xi(\varepsilon) \iota \lambda \alpha \sigma \varepsilon \tau \alpha \iota \mathrm{B}^{*} 126$

$1647 \varepsilon \dot{\varepsilon} \eta \tilde{\varrho} \varkappa \tau o]-\varkappa \tau \alpha \iota \mathrm{B}^{*} 29106^{\mathrm{c}}$

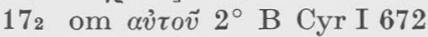

$175 a ̊ k v] \varepsilon \alpha v \mathrm{~B} 129=\mathrm{Ra}$

181 om xai $4^{\circ} \mathrm{B}^{*} \mathrm{~V} 246458$

188 A A $\varrho \omega ́ v] \alpha \alpha \alpha \varrho \omega \nu \mathrm{B}(\mid) 610^{*}$

189 om $\alpha v \tilde{\omega} v 2^{\circ} \mathrm{B}^{*} 72$

1818 om $\varkappa \alpha \vartheta a ́$ B* 318

1828 om rvoi $\omega$ B 319 Latcod 100 Arab

$1830 \ddot{\alpha} \lambda \omega \nu \circ \varsigma] \alpha \lambda \omega \mathrm{B}$ G 71 Cyr I $844=\mathrm{Ra}$

$201 \pi \varrho \omega \tau \omega] \tau \varrho \iota \tau \omega \mathrm{B}^{*} 106$

205 åv $\eta \dot{\alpha} \gamma \varepsilon \tau \varepsilon]-\gamma \alpha \gamma \varepsilon \varsigma \mathrm{B}^{*} 129 \mathrm{Lat}_{\mathrm{C}} \mathrm{cod} 100$

$\left.205 \tau \dot{v} v \pi 0 \nu \eta \varrho o ́ v ~ \mathrm{Fa}^{\mathrm{a}}\right]>\mathrm{B}^{*} \mathrm{~F} 59$

$208 \tau \dot{\eta} v \sigma v \nu \alpha \gamma \omega \gamma \eta \dot{v} 1^{\circ}$ ] $\tau \eta \sigma v v \alpha \gamma \omega \gamma \eta$ B 509

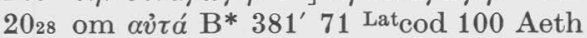

214 om $\tau \dot{\eta} v$ B 82 54-75 = Ra

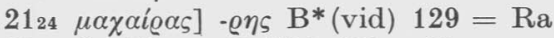

$\left.226 \quad \not{\alpha} v 1^{\circ}\right] \varepsilon \alpha v$ B $376458=\mathrm{Ra}$

$22_{6} \quad \ddot{v} 2^{\circ}{ }^{\circ} \varepsilon \alpha v \mathrm{~B} 376458=\mathrm{Ra}$

$228 \not ̋ \nu] \varepsilon \alpha \nu$ B 376 Cyr I $440=\mathrm{Ra}$

$22_{15}$ हैं $\left.\iota\right]$ post $B \alpha \lambda \alpha \dot{\alpha} \varkappa$ tr B $29=\mathrm{Ra}$

$22_{29}$ om $\mu$ ov B 426

238 ả@á $\sigma \circ \mu \iota]-\sigma \omega \mu \alpha \iota$ B 75-767 = Ra

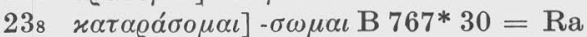

$23_{22}$ om $\delta 1^{\circ} \mathrm{B}(\mid) 509=\mathrm{Ra}$

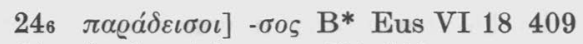

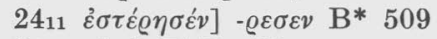

257 vio ṽ] $\overline{v \zeta}$ B 509

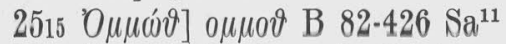

2626 A@o $\alpha \delta^{\circ} 1^{\circ} 2^{\circ}$ ] $\alpha \varrho o \delta \varepsilon \iota \mathrm{B}^{*} 71$

$\left.26{ }_{34} X \varepsilon \lambda \varepsilon x i\right] \chi \varepsilon \varepsilon \lambda \varepsilon \gamma \varepsilon \iota$ B 129407 Arm; of $\chi \varepsilon \lambda \varepsilon \gamma \iota 71=\mathrm{Ra}$

$\left.26{ }_{35} \Sigma v \chi \varepsilon \mu i\right] \quad \sigma v \chi \varepsilon \mu \varepsilon \varepsilon \iota \mathrm{B}^{*} 82$

2636 om $\tau \tilde{\omega} 2^{\circ}$ B 82509

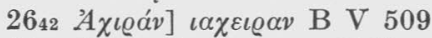

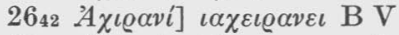

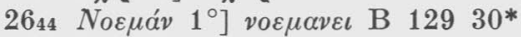

$\left.266_{60} \varepsilon \dot{\gamma} \gamma \varepsilon v \nu \eta \dot{\eta} \eta \sigma \alpha \nu\right] \varepsilon \gamma \varepsilon v \eta \vartheta \eta \sigma \alpha \nu$ B 5871

$278 \eta \tilde{~}] \quad \eta v \mathrm{~B} 71$

$2712 \pi \varepsilon \varrho \alpha \nu] \pi \varepsilon \varrho \alpha$ B* 799

$27_{14}$ ov่ $]$ ovx $\mathrm{B}^{*} 76730392$

$31_{23}$ om ó $\mathrm{B}^{*} 730$

$\left.31_{23} \quad \ddot{\alpha} \nu\right] \varepsilon \alpha \nu \mathrm{B}^{*} \mathrm{G}=\mathrm{Ra}$

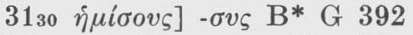

$31_{30} \tau o \tilde{v} 2^{\circ}$ ] $\tau 0 v \tau \omega \nu \mathrm{B}^{*} 53^{\prime}$

$\left.31_{36} \dot{\varepsilon} \pi \tau \alpha \varkappa \iota \sigma \chi i \lambda_{\iota} \alpha\right] \pi \varepsilon v \tau \alpha \varkappa \iota \sigma \chi(\varepsilon) \iota \lambda \iota \alpha \iota \mathrm{B}^{*} 407$

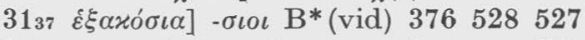

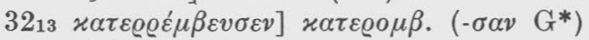
B G

$3217 \pi \varrho \circ \varphi v \lambda \alpha x]^{3}-x \eta v$ B V 82 Cyr I 404

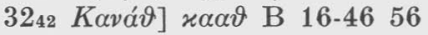

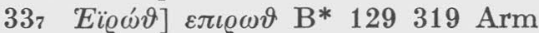

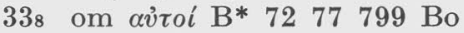

$33{ }_{13}$ Aiגov́c] $\alpha \iota \lambda \varepsilon \iota \mu$ B 509-619

$33{ }_{14}$ Aiגov́c] $\alpha \iota \lambda \varepsilon \iota \mu$ B 71'

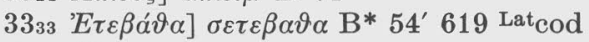
100

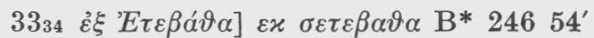
Latcod 100

$\left.33_{44} \pi \varepsilon \varrho \alpha \nu\right] \pi \varepsilon \varrho \alpha$ B* 799

$33_{50}$ om $\lambda \dot{\varepsilon} \gamma \omega \nu$ B* 44 Arab

$\left.34{ }_{4} \Sigma^{\prime} v v \alpha\right]$ cvvax B 68'-120

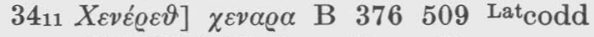
100104 (vid) Arm Bo = Ra

3514 om $\tau$ ác $1^{\circ} \mathrm{B}^{*} 528$

3515 xai $\left.2^{\circ} 963\right]>\mathrm{B}^{*} 509319$

$\begin{array}{llll}3610 & \tau \tilde{\omega} & M \omega v \sigma \tilde{\eta}] \text { om } \tau \tilde{\omega} \mathrm{B} 527=\mathrm{Ra}\end{array}$

As might well be expected an examination of list 3 yields no clear results. If one disregards all correspondence occurring five times or less, the following results obtain: $\mathrm{B}$ and 509 agree 20 times; $\mathrm{B}$ and 71,13 times; further agreements in descending order of frequency are: 129, ten times; 376 , eight times; 72 and 319, six times, and Cyril, six times. That two members of the $x$ group should head the group is not surprising in view of what was said about that group in chapter 1. Ms 129 belongs to the $f$ group but it frequently diverges from it; when it does it often goes with B. That Cyril's text should be a B type text is particularly interesting since Cyril was an Alexandrian. 
In Deuteronomy it was noted that there were traces of recensional activity present in the B text. In the list below I give the instances where the text of $B$ corresponds to $\mathfrak{W}$ over against Num. Should the reading of $B$ be attested in Apparatus II that equation is given together with the source(s). Otherwise the text of $\mathfrak{k}$ is given for comparison.

\section{List 4}

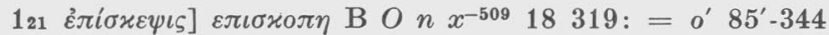

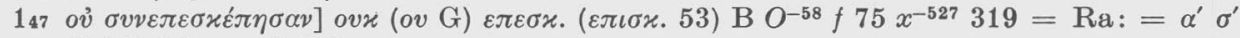
$\vartheta^{\prime} 85^{\prime}-344$ et $o^{\prime} 344$

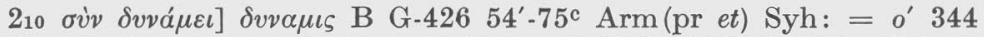

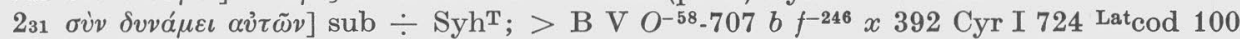
Aeth Arab Co Syh ${ }^{\mathrm{L}}=\mathrm{Ra} \mathfrak{m}$

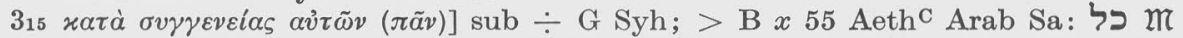

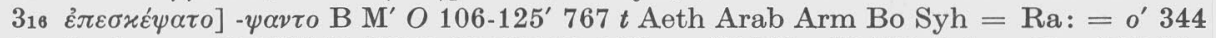

$\left.43 \varepsilon \varepsilon \varepsilon^{\prime \prime}\right]$ pr $x \alpha \iota$ B F M' V 833 O-29-707 $19 d^{-125} t x^{-619} 392 z 59799$ Syh = Ra: 7צו $\mathfrak{M}$

$414 \lambda \varepsilon \iota \tau o v \varrho \gamma o \tilde{\sigma} \sigma v]+\varepsilon \pi \alpha v \tau o(\alpha v \tau \omega \mathrm{Cyr}) \mathrm{B}$ V $O^{-58} x^{-619}$ Cyr I 852 Arm Syh $=$ Ra: זישרות

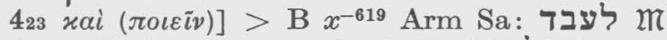

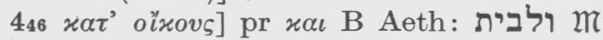

$510 x \alpha i(\alpha \dot{v} \eta \dot{\varrho})]>\mathrm{B}^{*}=\mathrm{Ra}: \boldsymbol{w}^{*} \mathfrak{n}^{\mathrm{L}}$

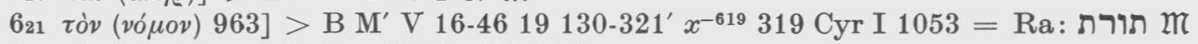

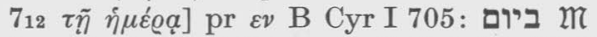

${ }_{8} \tau \tilde{\omega} \nu(v i \tilde{\omega} v)$ 963] $>\mathrm{B} O^{-58} d^{-610} 127-767 t^{-84} 50955319=\mathrm{Ra}$ : בני $\mathfrak{m}$

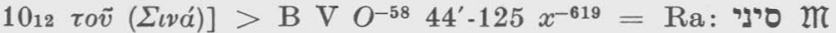

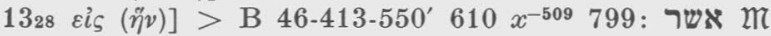

1422 (

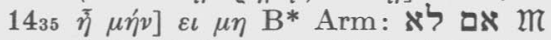

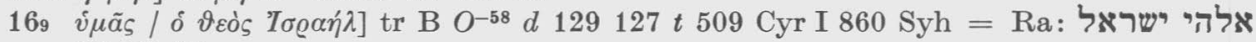
ロ ภก $\mathfrak{M}$

$183(\tau \tilde{\eta} \varsigma \sigma \varkappa \eta \nu \tilde{\eta} \varsigma) \sigma o v] \mathrm{sub} \div \mathrm{Syh}^{\mathrm{T}} ;>\mathrm{B} \mathrm{V} 58-82 x^{-527} z^{-126628} 319646$ Aeth CG Sa $=$ $\mathrm{Ra}:$ : $1 \pi$

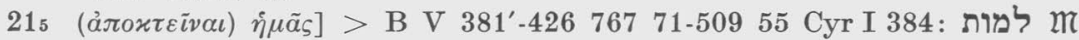

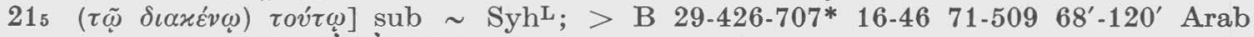

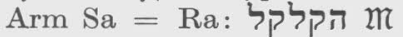

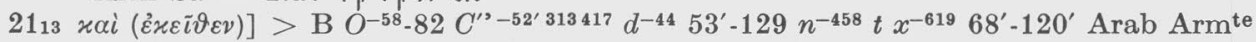
SyhT: משם $\mathfrak{M}$

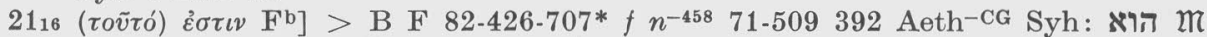

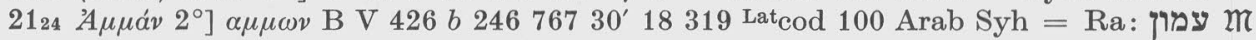

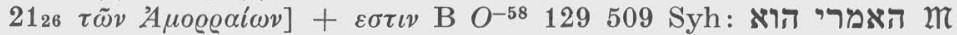

$2228 \pi \varepsilon \pi \circ i \eta$ xás] $\varepsilon \pi \circ$ in $\sigma \alpha \mathrm{B} \mathrm{O}^{-58} 106 n t 527=\mathrm{Ra}:=o^{\prime}$ oi $\lambda^{\prime} 344$

$248 \delta$ ó $(\vartheta \varepsilon o ́ s)]>B$ V 82-426 $d^{-44} t 71-509319$ Eus VI 409 Or IV $250=$ Ra: לx $\mathfrak{N}$

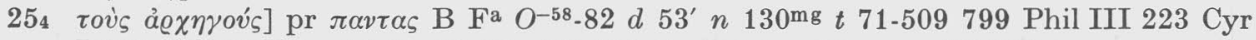
I 908 IV 300 Latcodd $919294-96100$ Co Syh $=\mathrm{Ra}:=o^{\prime} \vartheta^{\prime} 344$

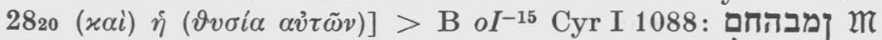

$3532 \tau \tilde{\omega} v(\varphi v \gamma \alpha \delta \varepsilon v \tau \eta \varrho i \omega v)$ 963] > B* V 53'-129:

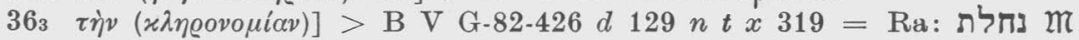

Not all of the instances in the above list are due to Hebrew influence. Particularly omissions which happen to correspond to $\mathfrak{T}$ may well be due to coincidence. Thus the omission of an article at 62186363 only formally corresponds to $\mathfrak{M}$ and the correspondence has no significance. On the other hand, a number of instances in the list are clearly hex in origin. This certainly applies to the 
additions in 43142126254 as well as to the change in word order at 169 . The text of $B$ is not entirely free from hex influence, but it is not an important factor; the text of B remains indeed the best witness to Num that we have.

B. Before comparing the text of $\mathrm{B}$ to that of $\mathrm{A}$ an investigation into the peculiarities of the A text would seem appropriate.

It should be noted that the ms contains a large number of readings sup ras. These readings are not included in the subsequent discussion.

List 5 presents unique readings of $\mathrm{A}$; as in the case of list 2 uniqueness applies here only to the primary Greek tradition.

\section{List 5}

121 om $\dot{\varepsilon} x-P o v \beta \eta \dot{v} \mathrm{~A}^{*}$

132 M $\alpha v \alpha \sigma \sigma \eta ́]$ ] $\mu \alpha v v$. A Latcod 100

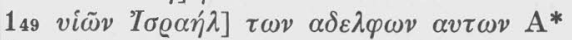

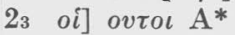

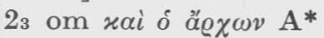

$26 \tau \varepsilon \dot{\varepsilon} \sigma \alpha \propto \varrho \varepsilon \varsigma] \tau \varepsilon \sigma \sigma \varepsilon \varrho \varepsilon \varsigma \mathrm{A}$

$\left.214 \varphi v \lambda \eta^{\prime}\right] \varphi v \mathbf{A}$

$217 \mu \varepsilon \dot{\sigma} \sigma o \nu]$ pr $\alpha \nu \alpha \mathbf{A}$

$2{ }_{29}$ Aiváv] $\alpha \iota \mu \alpha v$ A

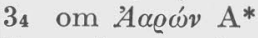

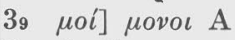

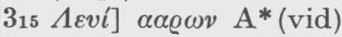

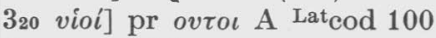

330 om $\delta$ A*

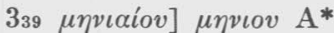

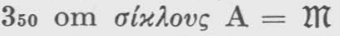

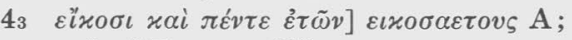
sed of $\bar{x} \varepsilon \tau o v \varsigma 458$

$4_{13}$ init - $\left.\varepsilon \pi \imath \vartheta \eta^{\prime} \sigma \varepsilon l\right] \varkappa \alpha \iota \varepsilon \pi \imath \vartheta \eta \sigma \varepsilon \iota \varsigma$ $\tau o \nu ~ \varkappa \alpha-$ $\lambda v \pi \tau \eta \circ \alpha \mathbf{A}$

$\left.4{ }_{18}{ }^{3} \lambda \varepsilon \vartheta \varrho \varepsilon v \dot{\sigma} \sigma \tau \varepsilon\right] \varepsilon \xi \circ \lambda \varepsilon \vartheta \varrho$. A

$431 \mathrm{fin}]+\tau \eta \varsigma \sigma \varkappa \eta \nu \eta \varsigma \mathrm{A}$

$4_{40}$ om init - $\alpha \dot{v} \tau \tilde{\omega} v 1^{\circ} \mathrm{A}$

$\left.4_{40} \delta \iota \sigma \chi i \lambda \iota 0 \iota\right]$ bis ser $A^{*}$

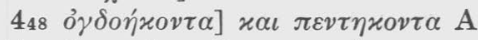

$449 \varepsilon \dot{\tau} \pi \varepsilon \sigma x \varepsilon \dot{\varepsilon} \psi \alpha \tau o]$ pr ovऽ A

$\left.5_{2} \pi \propto \varrho \varepsilon \mu \beta 0 \lambda \tilde{\eta} \varsigma\right] \sigma v v \alpha \gamma \omega \gamma \eta \varsigma \mathbf{A}$

54 om av่นov́s A

$64 \pi \alpha \dot{v} \tau \omega v] \pi \alpha \sigma \omega v \mathrm{~A}$

$650 \% \sigma \alpha \varsigma$ 963] $\alpha \varsigma \mathrm{A}$

$6120 \ddot{\tau} \iota]$ o

$\left.7_{2} \pi \alpha \varrho \varepsilon \sigma \tau \eta x o ́ \tau \varepsilon \varsigma\right] \pi \alpha \varrho \alpha \sigma \tau \eta \varkappa o \nu \tau \varepsilon \varsigma \mathrm{A}$

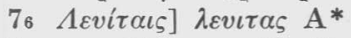

$7_{20}$ om $\delta \varepsilon \dot{x \alpha} \chi \varrho v \sigma \tilde{\omega} v \mathrm{~A}^{\text {txt }}$

$\left.7_{62} \chi \varrho v \sigma \tilde{\omega} v\right] \chi \varrho v \sigma \omega \mathrm{A}^{*}$

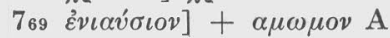

777 om $\tau o ́$ A*

$\left.7_{86} \vartheta v \mu \iota \alpha ́ \mu \alpha \tau \sigma \varsigma\right]+\varphi \iota \alpha \lambda \alpha \iota \alpha \varrho \gamma v \varrho \alpha \iota \delta \omega \delta \varepsilon x \alpha$

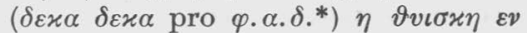
$\tau \omega \sigma \iota x \lambda \omega \tau \omega \nu$ a $\tau \iota \omega \nu \mathrm{A}$

84 om $\alpha \dot{v} \tau \tilde{\eta} \varsigma 1^{\circ} \mathbf{A}$

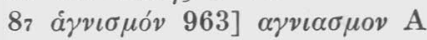

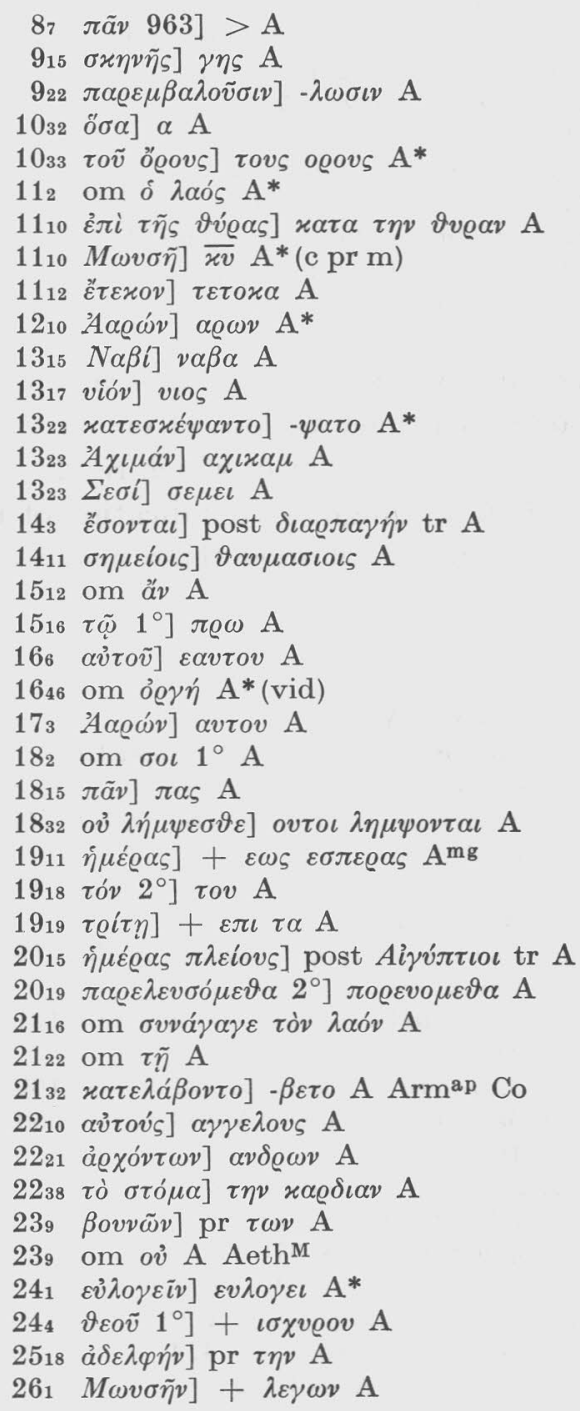


$2617 A \sigma \varrho \omega \dot{~} \alpha \sigma \tau \varrho \omega \nu \mathrm{A}$

$\left.26{ }_{38} \varepsilon \dot{\pi} \tau \alpha \varkappa o ́ \sigma \iota \iota \iota\right] \pi \varepsilon v \tau \alpha \varkappa o \sigma \iota \iota ~ A$

$\left.26{ }_{39} \Sigma o v \tau \alpha \dot{\lambda} \alpha\right]$ ] $\omega \sigma o v \sigma \alpha \lambda \alpha$ A

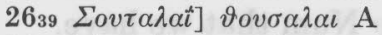

$\left.2646 \Sigma \alpha \mu i 1^{\circ}\right] \sigma \alpha \mu \varepsilon i \delta \eta \mathrm{A}$

$\left.26_{46} \quad \Sigma \alpha \mu i 2^{\circ}\right] \sigma \alpha \mu \varepsilon \iota \delta \eta \iota \mathrm{A}$

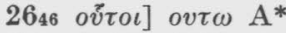

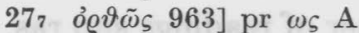

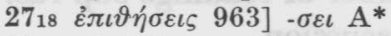

$2814 \alpha v ं \tau \tilde{\omega} \nu] \alpha v \tau \omega \mathrm{A}$

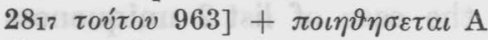

2819 om $\ddot{\alpha} \mu \omega \mu \circ \iota \mathrm{A}$

2822 om $\pi \varepsilon \varrho i$ a $\mu \alpha \varrho \tau i \alpha \varsigma$ A

$\left.29_{21} \alpha \dot{v} \tau \tilde{\omega} v 1^{\circ}\right] \cap 2^{\circ} \mathrm{A}$

308 àvं்] $\overline{\pi \eta \varrho} \mathrm{A}^{*}$

3014 $v \chi \eta^{\prime} v 963$ ] $\alpha v \tau \eta v \mathrm{~A}$

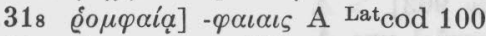

$31_{17}$ ả $\pi \circ \varkappa \tau \varepsilon i \nu \alpha \tau \varepsilon 2^{\circ}$ ] $-\varkappa \tau \varepsilon v \varepsilon เ \tau \varepsilon \mathrm{A}$

$3122 \chi \alpha \lambda x o \tilde{v}] x \alpha \lambda$ xov A

$\left.31_{23} \delta \iota \varepsilon \lambda \varepsilon v ́ \sigma \varepsilon \tau \alpha \iota 2^{\circ}\right] \mathrm{pr}$ ov $\mathrm{A}$

$324 \quad \gamma \tilde{\eta} \varkappa \tau \eta v o \tau \varrho o ́ \varphi \circ \varsigma] ~ \operatorname{tr} \mathrm{A}$

$327 \quad \delta \iota \alpha \beta \tilde{\eta} v \alpha \iota$ 963] $\alpha v \alpha \beta \eta v \alpha \iota \mathrm{A}$

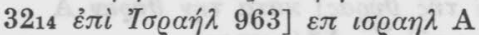

3216 ảं

$3217 \varepsilon \alpha v \tau \tilde{\omega} v]-\tau \omega \mathrm{A}$
$3221 \varepsilon \tilde{\varepsilon} \omega \varsigma \omega \varsigma \mathbf{A}$

$\left.3226 x \alpha i 1^{\circ}-\tilde{\eta} \mu \tilde{\omega} v 2^{\circ}\right]$ post $\tilde{\eta} \mu \tilde{\omega} \nu 3^{\circ}$ tr A

3229 Гá $\delta$ 963] $\gamma \alpha \lambda \alpha \alpha \delta$ A

$\left.32{ }_{35} \Sigma \omega \varphi \alpha \dot{\varrho}\right]$ pr $\gamma \eta v$ A

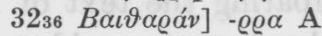

3236 om $x \alpha i$ ult A

3238 xai $1^{\circ}$ ] pr $\varkappa \alpha \iota \tau \eta v \beta \alpha \mu \omega$ A

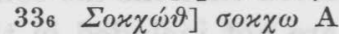

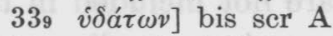

3311 om $\Sigma i v$ A(l)

$33_{12}$ om Liv A (|)

$\left.33{ }_{56} \pi \circ \iota \tilde{\eta} \sigma \alpha \iota\right] \pi \circ \iota \eta \sigma \varepsilon \tau \alpha \iota$ A

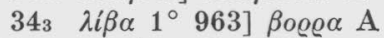

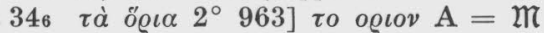

3417 o $\tau 0 \tilde{v}]$ vıos $\mathrm{A}$

$3424 \Sigma \alpha \beta \alpha \vartheta a ́]$ - $-\vartheta \alpha \nu$ A

3521 à

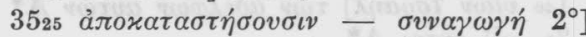
post $\varphi v \gamma \alpha \delta \varepsilon v \tau \eta \varrho i ́ o v$ tr $\mathrm{A}$

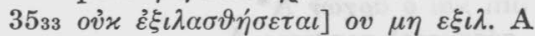

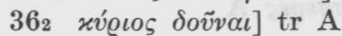

36 r xai 963$]>\mathrm{A}$

369 om ov $\mathrm{A}$

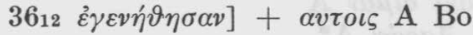

The list of unique readings is not particularly large. Many of the readings are easily identified as copyist errors and are of no significance. Of more interest is an expanded list in which random support up to three Greek witnesses join A. This list might give indication of possible close textual relatives within the tradition. List 6 gives these instances.

List 6

$110 M \alpha v \alpha \sigma \sigma \eta ́] ~ \mu \alpha v v$. A 458121

118 om $\tau \eta^{\prime} v$ A 72

$\left.1_{33} M \alpha v \alpha \sigma \sigma \eta^{\prime}\right] \mu \alpha v v$. A 121 Lat $\operatorname{cod} 100$

150 om $x \alpha i$ ult A 59319 Bo

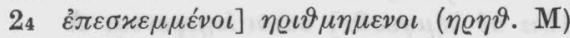
A $\mathrm{M}^{\mathrm{mg}} 121$

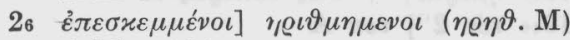
A $\mathrm{M}^{\mathrm{mg}} 121$

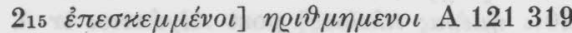

$\left.217 \tau \tilde{\omega} \nu 2^{\circ} \mathrm{Fa}^{\mathrm{a}}\right]>\mathrm{A}^{*} \mathrm{~F}$

$\left.217 \varepsilon^{2} \xi \alpha \varrho \circ \tilde{} \sigma \iota v\right] \alpha v \alpha \zeta \varepsilon v \xi \xi v \sigma \iota(v)$ A $118^{\mathrm{mg}} 121$ 319

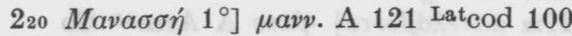

$\left.2_{20} M \alpha v \alpha \sigma \sigma \eta^{\prime} 2^{\circ}\right] \mu \alpha v v$. A $458121{ }^{\text {Lat }} c o d$ 100

$225 \AA \mu \iota \sigma \alpha \delta \alpha i] \quad \sigma \alpha \mu \iota \sigma \alpha \delta \alpha \iota$ A 59

33 oi $2^{\circ}$ ] pr $x \alpha \iota$ A 121

$3_{13} \quad\{\gamma i \alpha \sigma \alpha]-\sigma \alpha \varsigma$ A 376121

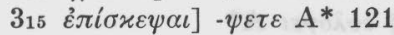

$3_{27}$ A

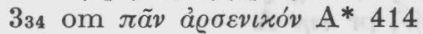

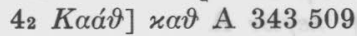

$414 \delta \iota \varepsilon \mu \beta \alpha \lambda \circ \tilde{\sigma} \sigma \iota v] \varepsilon \mu \beta \alpha \lambda o v \sigma \iota(v)$ A 121126 59

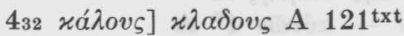

$\left.5_{13} \eta^{j} 1^{\circ} \mathrm{F}^{\mathrm{b}}\right]$ pr $\mu \eta$ A F 55799

519 vंлó] $\pi \varrho \circ \varsigma$ A Chr II 917

$\left.7_{12} \tau \tilde{\eta} 1^{\circ}-\pi \varrho \omega \tilde{\tau} \eta\right]$ post $\alpha \dot{v} \tau o \tilde{v} \operatorname{tr} \mathbf{A} 126$ Arm Bo

$\left.7_{54} M \alpha v \alpha \sigma \sigma \eta ́\right] ~ \mu \alpha v v$. A 127121

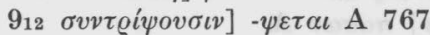

$\left.9_{13} \pi \circ \iota \tilde{\eta} \sigma \alpha \iota\right]$ pr $\tau o v$ A 318

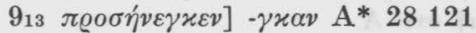

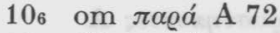

$\left.10_{23} M \alpha v \alpha \sigma \sigma \dot{]}\right] \mu \alpha v v$. A 121

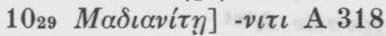

128 xv@iov] $\mu \circ v$ A Cyr II 592

$1210 \alpha \dot{\alpha} \varepsilon \dot{\sigma \tau \eta]} \alpha \pi \eta \lambda \vartheta \varepsilon v$ A 376

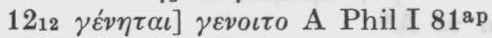

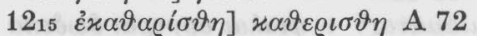

$13_{4} \dot{\varepsilon} \xi \alpha \pi \varepsilon ́ \sigma \tau \varepsilon\llcorner\lambda \varepsilon \nu] \alpha \pi \varepsilon \sigma \tau$. A 126

$\left.13{ }_{5} \Sigma \alpha \mu v^{\prime}\right] \sigma \alpha \mu \alpha \lambda \iota \eta \lambda$ A 72-618 19

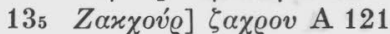

$\left.133_{20} \alpha v i \tilde{\eta} \varsigma\right]$ ] $\alpha v \tau \eta v$ A 121 


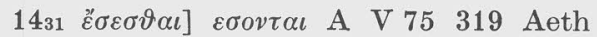
Arm Syh

$\left.1433 \dot{v} \mu \tilde{\omega} v 2^{\circ}\right] \alpha v \tau \omega \nu$ A 121

$1436 \pi \varepsilon \varrho$ 门 $\varepsilon \pi \iota$ A 125527

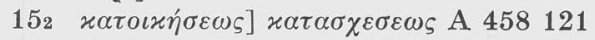

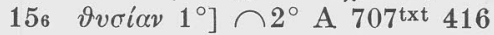

$15_{20}$ ả $\left.\alpha \varrho \varrho \chi \eta \dot{\eta}\right]-\chi \omega v$ A 121

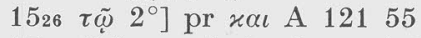

$178 \dot{\varepsilon} \xi \dot{\eta} v \varepsilon \gamma \varkappa \varepsilon v] \varepsilon \xi \eta \nu \vartheta \eta \eta \sigma \varepsilon v$ A 55

1912 Ě $\sigma \tau \alpha \iota 1^{\circ}$ ] $\varepsilon \sigma \tau \iota \nu$ A 12159

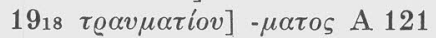

$205 \sigma \pi \varepsilon i \varrho \varepsilon \tau \alpha \iota] \sigma \pi \varepsilon \varrho \varepsilon \iota \tau \alpha \iota$ (- $\tau \varepsilon 56)$ A 56* 121

$2019 \tau \varepsilon] \delta \varepsilon$ A 55

$\left.21_{14} \lambda \varepsilon \dot{\gamma} \varepsilon \tau \alpha \iota\right] \lambda \varepsilon \gamma \varepsilon \tau \varepsilon$ A 120

$\left.21_{18} M \alpha v \vartheta \alpha v \alpha i v\right]-v \imath v$ A 52-414 55* Arm

$2122 \dot{\alpha} \mu \pi \varepsilon \lambda \tilde{\omega} v \alpha]+\sigma o v$ A 121

$\left.21_{30} \dot{\alpha} \pi \circ \lambda \varepsilon \check{\tau} \tau \alpha \iota\right]-\lambda \varepsilon \iota \tau \varepsilon$ A 376509

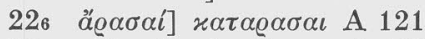

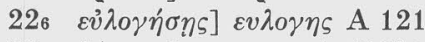

228 @ं $\mu \alpha \tau \alpha] \pi \varrho \alpha \gamma \mu \alpha$ A $426^{*}$ (c pr m) Cyr I 440

$2218 B \alpha \lambda \alpha \alpha \dot{\mu}] \beta \alpha \lambda \alpha \mu$ A 118' Lat $\operatorname{cod} 100$

2218 om xai $2^{\circ}-B \alpha \lambda a ́ x 1^{\circ} A^{*} 125$

2218 жаi $\left.3^{\circ}\right] \eta$ A 55

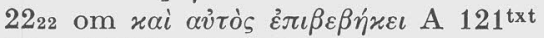

$\left.22_{29} \varepsilon i \mathrm{~F}^{\mathrm{b}}\right] \eta \mathrm{A} \mathrm{F} 376$

2230 om $\sigma o v 1^{\circ}$ A 29319

$233 \varepsilon \ddot{l} \mu \circ \iota] \varepsilon \mu \circ \iota \mathrm{A}^{*} 59^{*}$

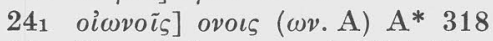

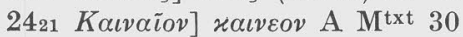

$\left.26{ }_{20} \Sigma \alpha \mu \varrho \alpha ́ \mu\right] \alpha \mu \beta \varrho \alpha \nu$ A 75619

2626 om $\tau \tilde{\omega} 2^{\circ}$ — fin A 707509121

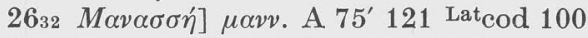

2633 idem A V 458121 Lat $_{\text {cod } 100}$

2638 idem A 75121 Lat $_{\text {cod }} 100$

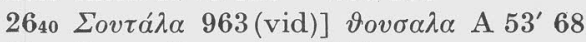

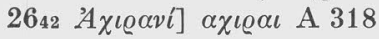

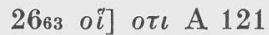

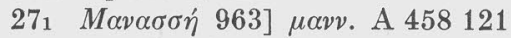

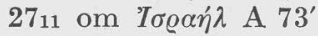

$\left.287 \sigma \pi 0 \nu \delta \dot{\eta} v 1^{\circ}\right] \sigma \pi 0 v \delta \eta$ A 55

$28_{23}$ om $\tau \tilde{\eta} s 2^{\circ}$ A 44121

$298 \quad \ddot{\alpha} \mu \omega \mu о \iota]$ a $\alpha \omega \mu о v \varsigma$ A 129121

303 xv@i $(\omega]$ pr $\tau \omega$ A 739767

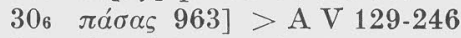

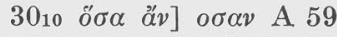

3015 om $\tau$ ov́s $2^{\circ}$ A 730

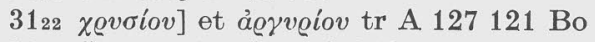

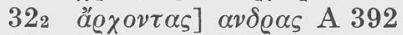

3211 om $\tau \tilde{\omega}$ A 121

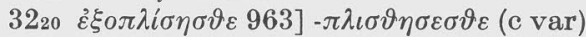
A 59319

322 om xaí $4^{\circ}$ A $321^{\prime} 628$ Aeth

$\left.322_{32} \gamma \tilde{\eta} v\right]$ pr $\tau \eta v$ A 392 18-628

$\left.32{ }_{33} M \alpha v \alpha \sigma \sigma \eta^{\prime}\right] \mu \alpha v v$. A 458121 Latcod $100^{2}$

32394041 idem A 458121

$3242 K \alpha v \alpha \dot{\vartheta}] \varkappa \alpha \alpha v \alpha \vartheta$ A 121

$333_{32}$ om $\tau$ ó A 121

$348 \Sigma \alpha \varrho \alpha \delta \alpha ́] \quad \sigma \alpha \delta \alpha \delta \alpha \varkappa$ A 29-58 55

$\left.34{ }_{13} M \alpha v \alpha \sigma \sigma \eta^{\prime}\right] \mu \alpha v v$. A $246458121 \mathrm{Lat}_{\mathrm{c}} \mathrm{Cod}$ 100

3414 idem A 246458121

3417 om vi $\mu$ iv A 126 Sa

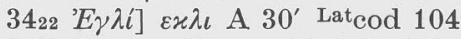

3423 M $\alpha \nu \alpha \sigma \sigma \dot{\eta}] \mu \alpha \nu v$. A 246121

3427 'A $\chi \iota \omega ́$ ] $\alpha \chi \iota \omega \beta$ A $12168^{\prime}$

$355_{3}$ om $x \alpha i^{\prime} 3^{\circ}$ - fin A 7212659 Arab: homoiot

3525 «ง่นจขี 963] > A 70784 Cyr I 581 VII 625 Aeth Arm

361 M $\left.2 v \alpha \sigma \sigma \eta^{\prime}\right] \mu \alpha v v$. A 75121

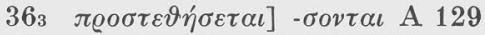

368 om $\varepsilon \dot{\varepsilon} x \quad \tau \tilde{\omega} v \quad \varphi v \lambda \tilde{\omega} v$ A 761

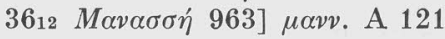

An analysis of the above list shows that one manuscript stands out as closely related to codex A; ms 121 joins $A$ in the list 51 times, whereas the next in line is ms. 458 with only 12 , followed by 55 and 59 with eight each, and by 126 and 319 with six each. No other witness appears more than five times. The high number of agreements between $A$ and 121 is in part due to the fact that the name Manasseh is commonly spelled with a double " $n$ " in both witnesses. If these instances were to be disregarded there would still remain 32 agreements between A and 121, though only one would still obtain between $\mathrm{A}$ and 458. It is obvious that ms 121 is closely related to A.

In the next list possible recensional influence on the text of codex A is examined. All instances of correspondence between the text of A and that of $\mathfrak{m}$ over against $\mathrm{Num}$ are given in list 7 . Since all instances equal $\mathfrak{K}$, that fact is not noted, but the $\mathfrak{M}$ text is given except where the variant text is attested in Apparatus II in which case that equation is recorded. 


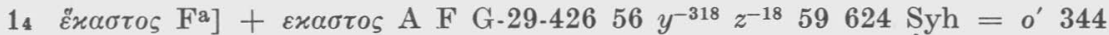

$15 \tau \tilde{\omega}($ Pov $\beta \eta \dot{v})] \tau \omega$ A $29 d n^{-767} 30 t 12118$ 55* Arm: :לראובן $\mathfrak{m}$

$16 \tau \tilde{\omega} v(\Sigma v \mu \varepsilon \omega \dot{v})] \tau \omega$ A 528-551 $d n^{-767} t 12118$ Arm: לשמעון $\mathfrak{k}$

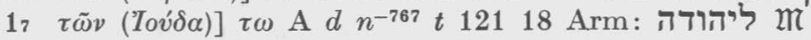

$\left.118 \sigma v v \eta_{\gamma} \alpha \gamma o v\right] \varepsilon \xi \varepsilon x \varkappa \lambda \eta \sigma \iota \alpha \sigma \alpha \nu$ (c var) A $\mathrm{M}^{\prime}$ txt $o I-29-707^{\mathrm{mg}}$ (vid) $C^{\prime \prime}$ bs $31855624=o i$ $\lambda^{\prime} 344^{\mathrm{txt}}$

$149 \tau \dot{\eta} v(\Lambda \varepsilon v i)]>A 72 b$ 106-125 $127-458 x^{-527} 392319$ Cyr I 845: לוי

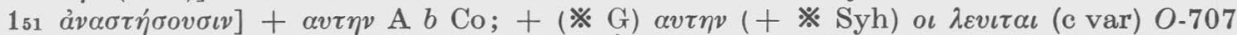
$44 n t 55319799$ Arab Arm Syh: יקימו אתו הלוים

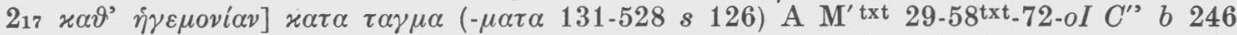

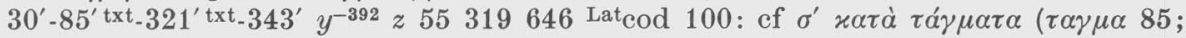
- $\tau$ - 5 130) $85^{\prime}-344$

$2{ }_{17}$ fin B V $\left.707 d f^{-246} n t x 39259799 \mathrm{Arm} \mathrm{Sa}^{412}\right]+\alpha v \tau o v 376 C^{-16}-46-417 \mathrm{Bo}$; $+\alpha v^{\tau} 16$; $+\alpha v \tau \omega \nu$ rell: $=\alpha^{\prime} \sigma^{\prime} \vartheta^{\prime} \alpha v i \tilde{\omega} \nu$ (aut $\alpha v \tau o v$ ) 85'-344

317 oi (vioí)] > A M' G-64-426 $C^{-16}-528 \quad 44-125246 \quad 130-321^{\prime} x^{-509} z^{-18126} 55624799$ : בני $2 \pi$

338 áлт́́ $30^{\prime}-85^{\prime}$ txt $321^{\prime}$ txt_343' $61912155:=\vartheta^{\prime} 344^{\text {txt }}$

350 бixגovs] sub $\div \mathrm{G}$ Syh; $>\mathrm{A}:$ non hab $\mathfrak{k}$

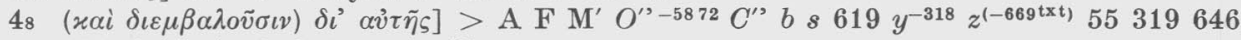
799 Aeth Arab Syh: ושמו

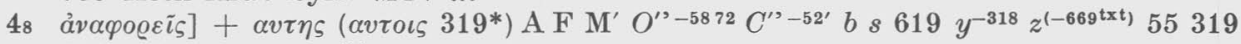
646799 Syh : בדיו $2 \pi$

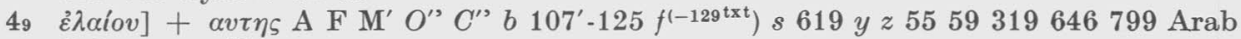
Syh: שמנה 2 ש

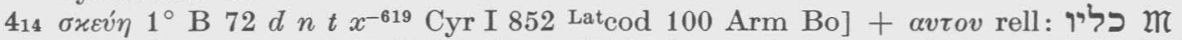

$426 \pi \varepsilon \varrho \iota \sigma \sigma \alpha ́]+\alpha v \tau \omega v$ A F M $M^{\prime}$ V $O^{\prime \prime}-82 C^{\prime \prime} f 767 s 619$ y z 5559646799 Arab Syh: מיתריהם

$426 \lambda \varepsilon เ \tau o v \varrho \gamma(x a ́]+\alpha v \tau \omega \nu$ A F $O^{\prime \prime}-(72) 82 C^{\prime \prime}-1646 f s 619$ y z 5559646799 Aeth Arab Syh: צבדתם

$439 \quad \varepsilon \omega \varsigma]$ pr (※ G Syh) $x \alpha \iota$ A $O^{-58} b$ Latcod 100 Syh: ועד $2 \pi$

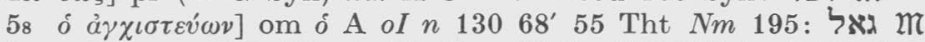

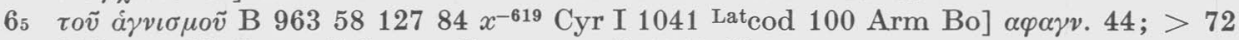

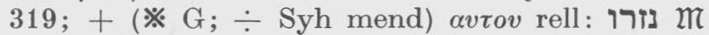

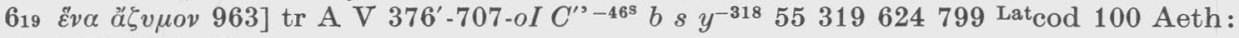

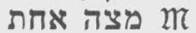

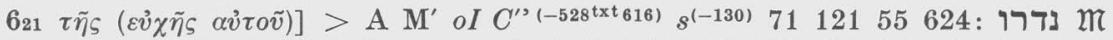

$\left.812 \chi \varepsilon \tilde{\imath} \varrho \alpha_{\zeta} 963\right]+(※ \mathrm{Syh}) \alpha v \tau \omega \nu \mathrm{A} \mathrm{O}^{-58} b$ 18 $^{\prime}$-126-628-669 Latcod 100 Arm Co Syh: ידיהם

821 ( $2 \dot{\alpha}) i \mu \alpha \dot{\tau} \iota \alpha$ B $d 127 t x^{-619}$ Arm] + $2 v \tau \omega \nu$ rell: $\mathfrak{m}$

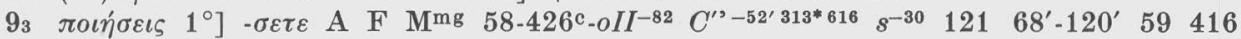
Aeth Arab Syh:

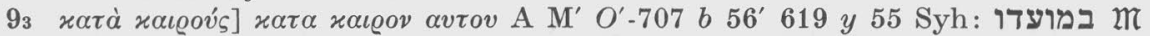

$\left.117 \varepsilon i \delta{ }^{2} 2^{\circ} \mathrm{B} 707 f 509318 z 624646799 \mathrm{Bo}^{\mathrm{B}}\right] \omega \sigma \varepsilon \iota \mathrm{M}^{\prime}$; $\omega_{\varsigma} 106 \mathrm{Sa}^{5}$; pr (※ Syh) $\omega \varsigma$ rell: כעין

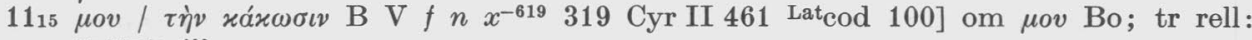
ח ברעתי

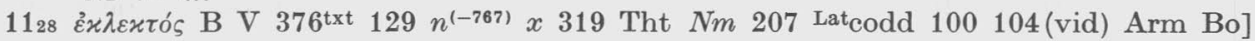
+ aviov rell: מבחריו

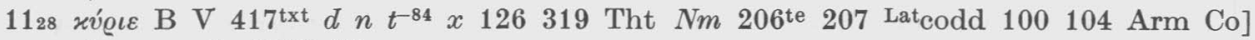
$+\mu o v$ rell: אדני

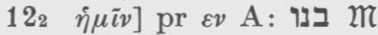

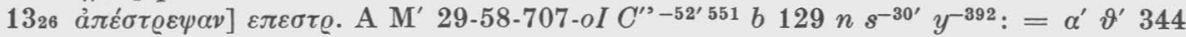

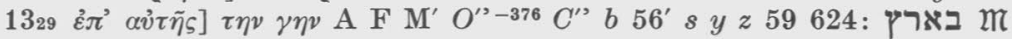

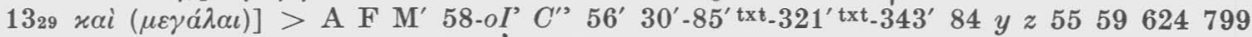
Latcod 100 Aeth Arab Syh: גדלת m 
$\left.13{ }_{33} \pi \tilde{\alpha} \varsigma \mathrm{B} \mathrm{V} 426 x \mathrm{Bo}^{\mathrm{AB}^{\mathrm{c}}} \mathrm{Sa}\right] x \alpha \iota \mathrm{G} C^{\prime \prime} 799 ;>\mathrm{Bo}^{\mathrm{B}^{*}}$; pr $x \alpha \iota$ rell: וכל $\mathrm{m}$

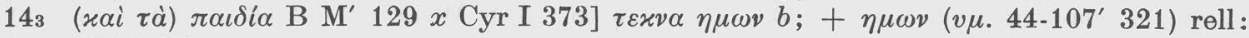
ו ו $2 \pi$

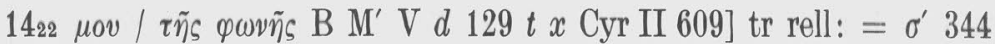

$\left.1428 \tilde{\eta} \mu \dot{\eta}^{2}\right] \varepsilon \iota \mu \eta$ A G $41756^{*} 54321318$ 59c 799 Latcod 100 Arm:

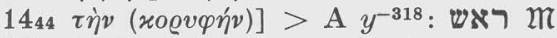

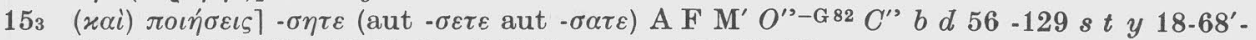
128-407-630-669c 55 59 Cyr I 1029 verss: ועיתם $\mathrm{k}$

$154 \dot{\varepsilon} v 1^{\circ}$ - fin] (c var) $\varepsilon v \tau \varepsilon \tau \alpha \varrho \tau \omega$ $\tau o v \iota v \varepsilon \lambda \alpha \iota \omega$ A F M $\mathrm{M}^{\prime} \mathrm{V} O^{\prime \prime}-29426 C^{\prime \prime}-414 b^{-19} s 3925559$

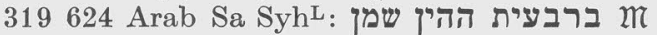

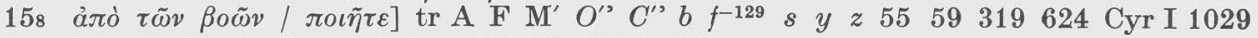

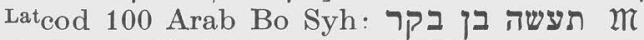

1528 fin B F V 72' $f^{-246} n^{-767} x 59$ Latcod 100 Aeth Arab Arm Sa] + (※ Gc SyhL; $\div$ G*)

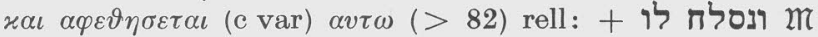

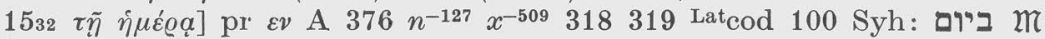

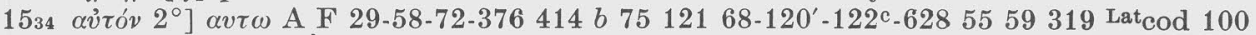
Armte Bo Syh: לו $2 \pi$

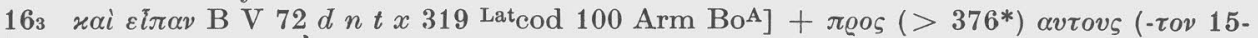
64*vid) rell: ויאמרו אלהם

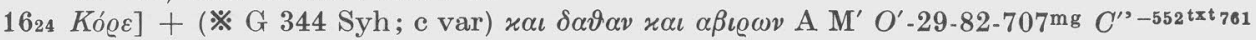
קרח דתן: ואבירם $2 \mathfrak{k}$

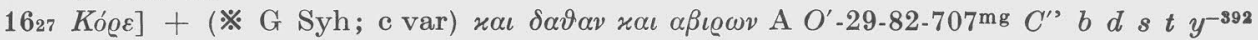
18'-126-628-630'55 624 Arab Syh: קרח דתן ואבירם

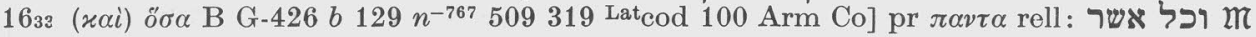

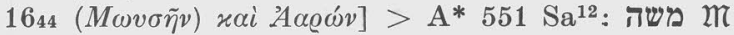

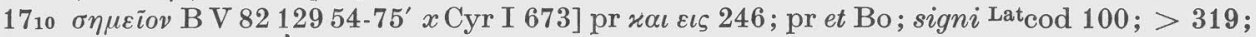
pr $\varepsilon \iota \varsigma$ rell: :

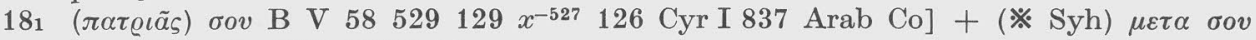
(> 628) rell: איר איר

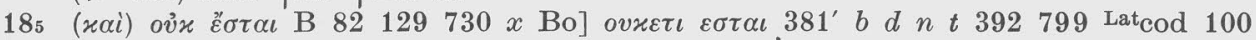
MissMoz 85 Arm; + $\varepsilon \tau \iota$ (c var) rell: ולא יהיה צוד

$188 \alpha \dot{\pi} \alpha \varrho \chi \tilde{\omega} v$ B V $82129 x^{-619}$ Cyr I 837 Latcod $\left.100 \mathrm{Sa}\right]+\mu o v$ rell:

1819 боi $\delta \varepsilon \delta \omega \varkappa \alpha]$ tr A F $O^{\prime \prime}-82 C^{\prime \prime} 56^{\prime} s 619$ y z 5559624646799 Cyr I 840 Aeth Arab Syh: נתתי ל $\mathrm{m}$

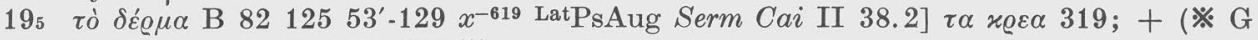

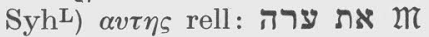

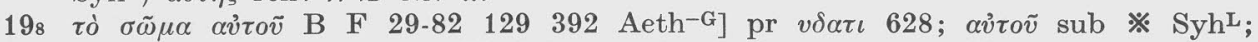
$+(※ \mathrm{G} \mathrm{Syh}) \varepsilon v v \delta \alpha \tau \iota O^{(-376)} \mathrm{Syh} ;+v \delta \alpha \tau \iota$ rell:

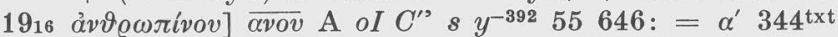

$\left.19_{19} \tau \tilde{\eta} \dot{\eta} \mu \varepsilon^{\circ} a 3^{\circ}\right]$ pr $\varepsilon v$ A 82 414-550' 54'-75 318 55: ביום 2

2019 ( $\varkappa \alpha i) \tau \dot{\alpha} x \tau \eta \dot{v \eta}$ B V $82 d n^{-767} t x^{-619}$ Phil II 87te Sa $^{12}$ ] pecora nostra Latcod 100 Aeth; $+\mu o v$ rell: "מקומ $1 \pi$

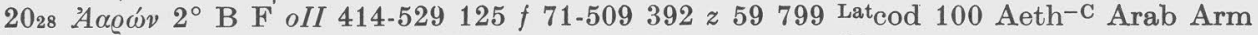
Co] pr $\varepsilon \varkappa \varepsilon \iota 551 b^{-19}$; + (※ Syh) $\varepsilon x \varepsilon \iota$ rell: אהרץ שם $2 \pi$

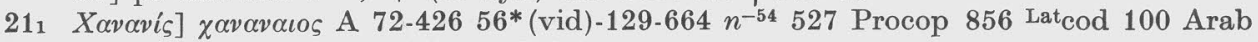
Armap Bo $\mathrm{Sa}^{1012}:=$ oi $\lambda^{\prime} 108 \mathrm{Syh}$

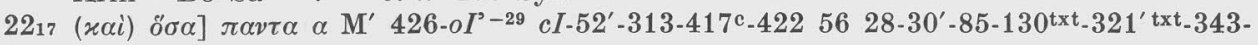
$344^{\text {txt }} y^{-392^{*}} 799$; pr $\pi \alpha v \tau \alpha$ A Fa $O^{-426} 16-46-414^{\prime} 246619 z 5559$ Aeth Arab Bo Syh: אש

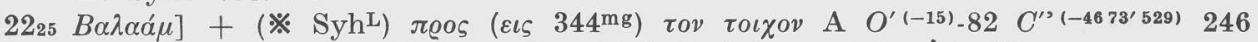
$s^{(-343)} 619 y^{-392} z^{(-628)}$ LatAug Num 50 Arab Syh: + 27

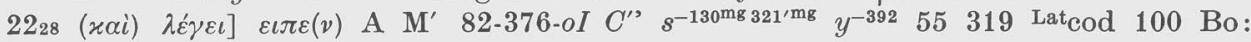
ראת $m$

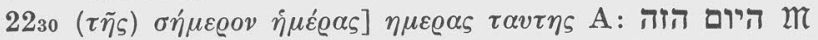

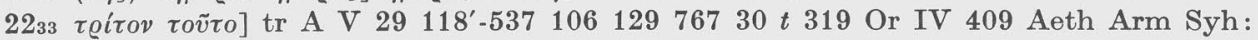
ז של של 


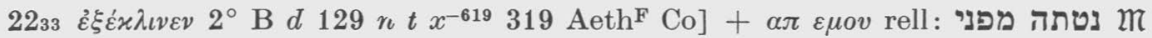

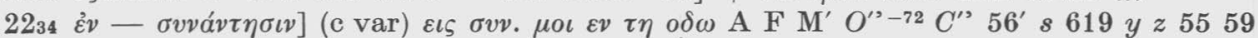
624799 Aeth Arab Sa Syh: לקראתי בדרך

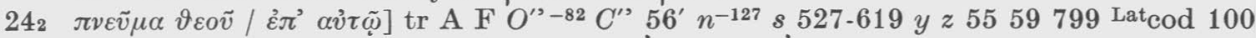
Ruf Num XVII 2 Aeth Arab Syh: עליו רוח אלהים $2 \pi$

$245 \sigma o v$ / oi oixol] tr A F M' $O^{\prime \prime}-82381^{\prime} C^{\prime \prime}-552761 s 619 y^{-318} z^{-126} 55319799$ : אהליך

$2410 \tau \varrho i \tau o v$

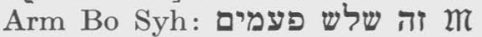

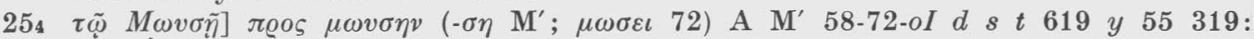
אל משה

254 xveí $\omega$ B V $82 d 53^{\prime} n^{-75^{*}} t$ 71-509 319 Cyr I 908 IV 300] $>75^{*}$; pr (※ G) $\tau \omega$ rell: ליהוה

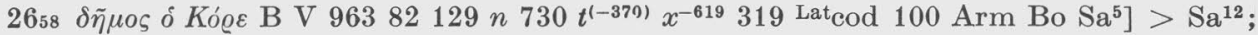
post Movoi tr rell: משפחת המושי משפחת הקרחי 27

272 $\quad \sigma v v \alpha \gamma \omega \gamma \tilde{\eta} \varsigma]$ pr $\tau \eta \varsigma$ A V 58-72-426 $551 b d^{-106}$ 129-246 458121 126-128-628-630' 59 : M

2720 oi (vioí) 963$]>A^{*} 707 b^{-19} 75 \quad 30-343 \quad 126319$ : 20

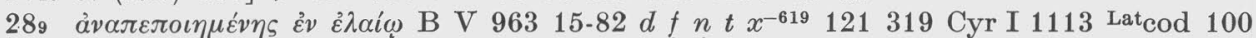
Arm Bo Sa ${ }^{1}$ post $\vartheta v \sigma i \alpha v$ tr rell : מנחה בלולה בשמן $\mathfrak{m}$

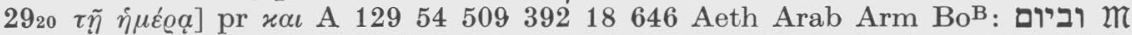

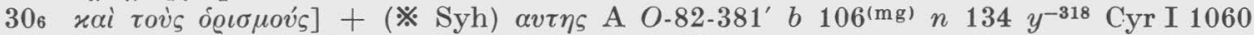
Latcod 100 Aug Num 57 Co Syh: ואסריה $2 \pi$

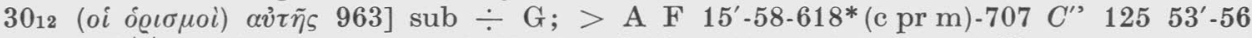
$s^{\left(-85^{\text {txt }}\right)} y^{-392} z^{-407} 5559416624646$ LatAug Num 59.2 2 te $:$ רס

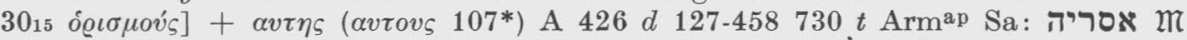

$\left.31_{18} \pi \tilde{\alpha} \sigma \alpha v \mathrm{~B} O^{-376}-82129 x^{-527} 407319 \mathrm{Syh}\right] \mathrm{pr} x \alpha \iota$ rell: וכל

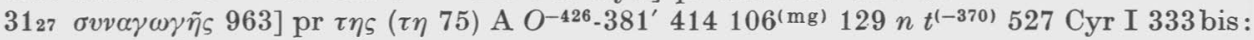
חעדה

$\left.31_{32}(\varkappa \alpha i) \varepsilon \beta \delta \delta \mu \eta \dot{n} x o v \tau \alpha\right]+(※ \mathrm{G}) \chi \iota \lambda \iota \alpha \delta \varepsilon \varsigma$ (c var) A Fc prm M' G-29-426-707-oI C' $b^{-19} 246 s$ $y^{-392} z^{-126407669^{*}} 55624$ Syh: ושבעים אלף $2 \pi$

$3136 \tau \varrho \iota \alpha x o ́ \sigma \iota \alpha \iota 963]+(※ \mathrm{G}) \chi \iota \lambda \iota \alpha \delta \varepsilon \varsigma$ (c var) A F M' $O^{\prime \prime}-7282 C^{\prime \prime} 56^{\prime}$ 28-85'-321'-344* 509 $y z^{-126407} 5559624799$ Aeth Arab Bo Syh: שלש מאות אלף $\mathfrak{k}$

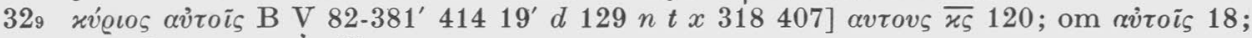
tr rell: להם יהוה $2 \pi$

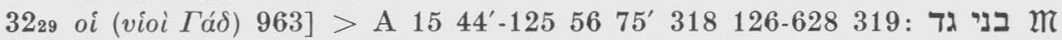

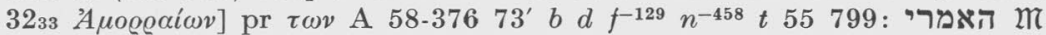

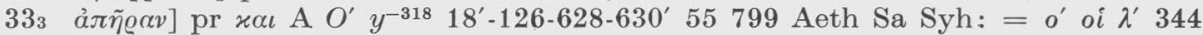

$\left.3314 \eta^{j} v\right]+\varepsilon x \varepsilon \iota$ A F $O^{\prime} C^{\prime \prime} b 53^{\prime}-56 s^{-30} y^{-318} 18-68^{\prime}-12059$ Lat $^{\prime}$ cod 100 Aeth Arab Sa Syh: שיה שמש $2 \pi$

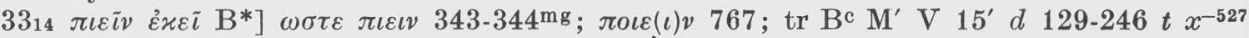

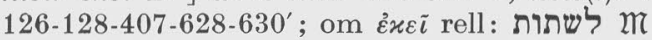

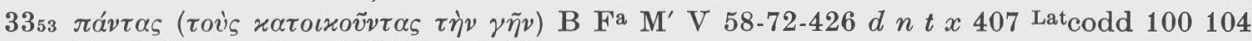
Bo $\left.\mathrm{Sa}^{1}\right]>$ rell: ef את הארץ

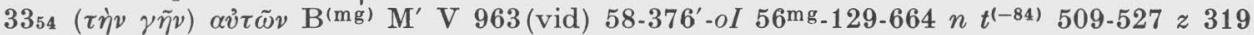
Latcod $\left.100 \mathrm{Bo} \mathrm{Sa}{ }^{1}\right] \tau \alpha v \tau \eta v 799$; sub $\div \mathrm{G} \mathrm{Syh;} \mathrm{>} \mathrm{rell:}$

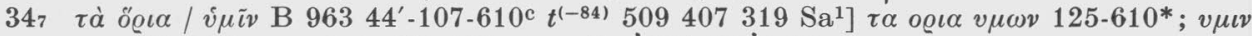

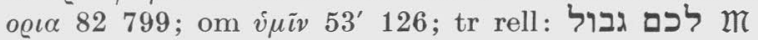

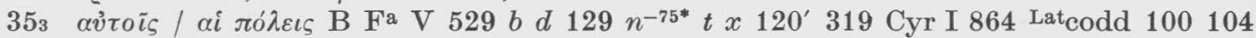

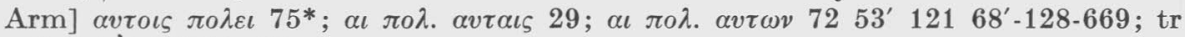
rell: הערים להם 2 ה

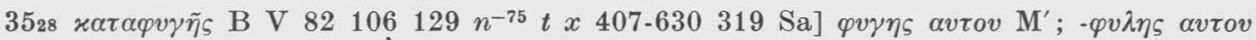

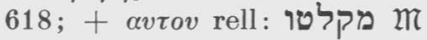

It is obviously not suggested that all the variants in list 7 are recensional in origin, since some of the correspondences are only formal. On the other hand, it is quite apparent that the $\mathrm{A}$ text has been substantially influenced by hex. 
Many popular hex readings are present in A, whereas B has only a few. In the case of widespread hex readings B usually supports the non-recensional text, whereas A supports the variant text. The contrast between this list and list 4 is indeed noteworthy in this respect.

C. It remains to be determined whether $B$ and $A$ represent different text types within the text tradition. List 8 details instances in which A witnesses to Num, and B along with no more than four text groups (random support being disregarded) supports a variant tradition. Support of a reading by a group means support by at least half the members of the group. The group support is in each case summarized in parentheses.

\section{List 8}

$12(x)$ om viw̃v B $x$ Bas II 145 Latcod 100

$12(d n t x)$ om $\alpha v \tilde{\omega} v 1^{\circ} \mathrm{B} 414^{\prime} d n^{-767} t x 18$ Bas II 145 Cyr VI 453 X 624 Latcod 100 PsBas Is I 5 Arm

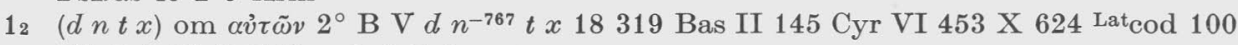
$\mathrm{Hi}$ Eph II 3 PsBas Is I 5 Arm

$12(d t x)$ om $\alpha v \tilde{\omega} v 3^{\circ}$ B $19 d 127 t x 18319$ Cyr VI 453 X 624 Lat $^{\circ}$ cod 100 Arm

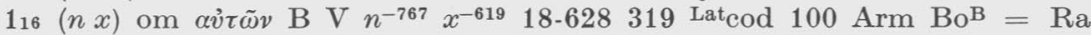

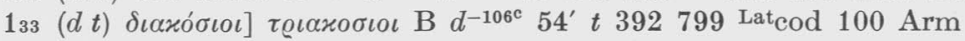

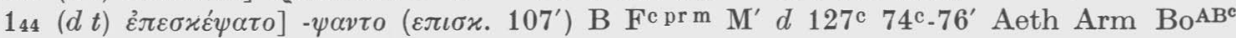
$\mathrm{Sa}^{1} \mathrm{Syh}=\mathrm{Ra}$

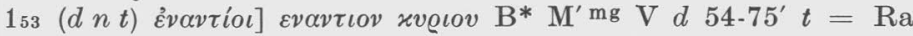

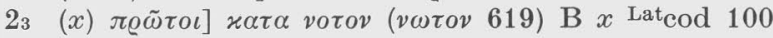

$332(d t)$ viós] pr o B V $19 d^{-125} t 126646 \mathrm{Bo}=\mathrm{Ra}$

$340 \quad(n x)$ om $\alpha v i \tilde{\omega} v$ B 54-75' $x^{-619}$ Lat codd $100104=$ Ra

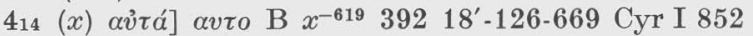

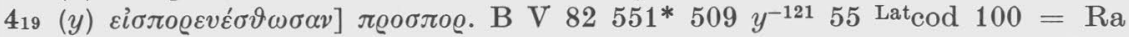

425 (b) $\varkappa \alpha \tau \alpha \varkappa a ́ \lambda v \mu \mu \alpha] ~ \varkappa \alpha \lambda v \mu \mu \alpha$ B M V 707 b 84txt(c pr m) $121126319=\mathrm{Ra}$

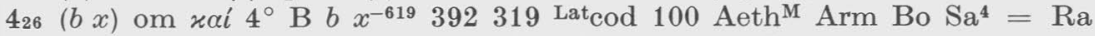

$435(f x)$ om $\tau \dot{\alpha}$ है $\varrho \gamma \alpha$ B $f x^{-619} 319$ Lat $\operatorname{cod} 100 \mathrm{Sa}=\mathrm{Ra}$

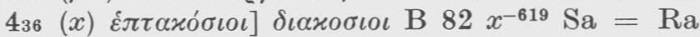

$446(x)$ om $\alpha v i \tilde{\omega} v 1^{\circ} \mathrm{B} x^{-619}$ Lat cod 104 (vid) $=\mathrm{Ra}$

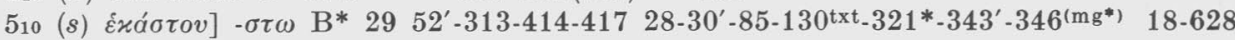
319646

$\left.715(f n x) \varepsilon_{\varepsilon} v \alpha 3^{\circ} 963\right]>\mathrm{B} \mathrm{F} \mathrm{F}^{*}\left(\mathrm{c}\right.$ pr m) V 29-82-707txt $f n^{-767} 730 x^{-619} 126-628319$ Lateod 100 Aeth-C $^{-C}$

$777(o I x) \Phi \alpha \gamma \alpha \iota \eta^{\prime} \lambda$ 963] $\varphi \alpha \gamma \varepsilon \eta \lambda \mathrm{B} \mathrm{V}$ oI ${ }^{-15} 771273076 x^{-509} 392$ Co

$\left.786(o I I b f) \pi \lambda \eta^{\prime} \varrho \varepsilon \iota \varsigma\right]-\varrho \eta \varsigma$ B F 381'-oII $I^{(-72)} b^{-537} 44 f^{-56} 767 \quad 30-85^{*}-3438471628$

$816(f) \pi a ́ v \tau \omega \nu]+\tau \omega v \mathrm{~B}^{*} f^{-129}$

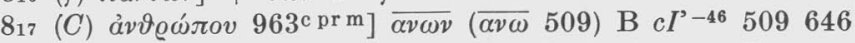

$\left.8_{21}(d t) \varepsilon ै ं \pi \lambda v v \alpha v\right]-v \alpha \nu \tau o \mathrm{~B} \mathrm{M}^{\prime} 15-376 d 56127 t=\mathrm{Ra}$

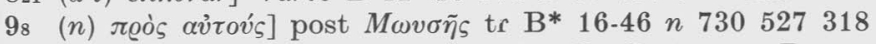

$9_{13}(n z)$ ăv] $\varepsilon \alpha \nu$ B $29129 n$ 527-619 z Cyr I $1081=\mathrm{Ra}$

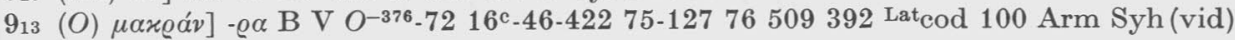
$=\mathrm{Ra}$

$914(n)$ om ovi $\omega_{\varsigma}$ B $129 n^{-767} 71-509$ Aeth Arab Arm Co $=$ Ra

103 (z) $\sigma \alpha \lambda \pi \iota \varepsilon i \varsigma] \sigma \alpha \lambda \pi \iota \sigma \varepsilon \iota \varsigma \mathrm{B} * 619 z=\mathrm{Ra}$

$105(b d) \pi \alpha \varrho \varepsilon \mu \beta a ́ \lambda \lambda o v \sigma \alpha \iota]-\beta \alpha \lambda o v \sigma \alpha \iota \mathrm{B}^{*} \mathrm{~V}$ 72-618 52'-73'-551-616*-739 $b$ d $5654-767 \quad 370$ $71 \quad 18-126 \quad 55 \quad 59646$

$1032(C s) \stackrel{\alpha}{2}] \varepsilon \alpha \nu \mathrm{B}^{*} \mathrm{~F} 58-82-707 C^{\prime \prime-528} s 39259=\mathrm{Ra}$

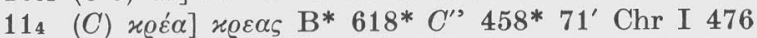

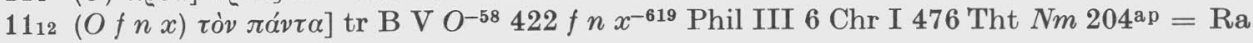


$11_{26}(x)$ om $\tau o ́$ B $x^{-619}$

$\left.11_{29}(x) \mu \circ \iota\right] \varepsilon \mu \varepsilon$ B $x^{-527} 392$

$11_{34}(x)$ om ó $\tau \iota \mathrm{B} x$

$11_{35}(O n x)$ om $\tau \tilde{\eta} \varsigma$ B F V $O^{\prime}-295812954-75^{\prime} x 39259319799=\mathrm{Ra}$

$1210(d n t x) \pi \varrho o ́ c] \varepsilon \pi \iota \mathrm{B} \mathrm{V} d 129 n^{-75} 321^{\prime m g} t x 319$ Arab Arm Co $=$ Ra

$133 \quad(x)$ à $\pi \circ \sigma \tau \varepsilon \lambda \varepsilon \bar{\imath} \varsigma]-\sigma \tau \varepsilon \iota \lambda \alpha \varsigma$ B $x^{-509}$

$138 \quad(x)$ I $\gamma \alpha \alpha \dot{\lambda} \lambda] \iota \lambda \alpha \alpha \lambda$ B $x$

$1313(x) \Gamma \alpha \mu \alpha \lambda i]$ j $\alpha \mu \alpha \iota$ B $x 319$ Arm

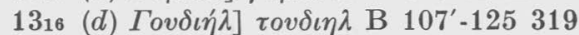

$\left.13{ }_{20}(f n) \varepsilon i 1^{\circ}\right] \eta$ B F $29528^{(m g)}-529^{*} f^{-129} 54^{*}-458-7673084^{\text {c vid }} 509^{*} 392^{*} 68^{\prime}-12055^{*}$

$\left.13_{20}(d f n) \varepsilon i 2^{\circ}\right] \eta$ B $15-29-707 d^{-44} f n^{-127} 50955319799$ Lat $_{\text {cod }} 100$

$\left.13_{21}(f x) \varepsilon i 2^{\circ}\right] \quad \eta$ B F 29-82-381' $313^{*} f$ 458-767 $x^{-527} 68^{\prime}-120^{\prime} 319799$

$\left.13_{23}(x)^{\prime} E v a ́ x \mathrm{~F}^{\mathrm{b}}\right] \varepsilon v \alpha \chi \mathrm{B}$ F $129 x \mathrm{Sa}=\mathrm{Ra}$

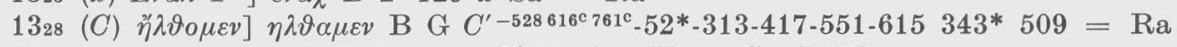

$13{ }_{29}(d n t x)$ om $\alpha i \mathrm{~B} \mathrm{~F} \mathrm{~F}^{*}\left(\mathrm{c}\right.$ pr m) V $29 d n^{-767} t x$ Cyr I 373

$13_{29}(x)$ 'Eváx] $\varepsilon v \alpha \chi$ B $129 x$ Sa $=\mathrm{Ra}$

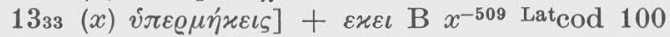

$1410(x)$ om $\varepsilon \dot{v} 3^{\circ} \mathrm{B} x$ Aeth

$1413(t x) \varepsilon \dot{\varepsilon} v] \tau \eta \mathrm{B} 44^{\prime} 129127-767 t x^{-509}=\mathrm{Ra}$

$1423(C)$ ov่ $3^{\circ}$ ] ov $\chi \mathrm{B}^{*} C^{-77}-52-73^{\prime}-313-615^{*}-761^{*} 392126319799$

$1424(x)$ om xaí $2^{\circ}$ B V $72106 x 55$ Cyr II 609 Latcod 100 Aeth Arm Co = Ra

$1427(d t)$ om $a ̊ \mathrm{~B}^{*} \mathrm{~V} d^{-44} 75 t 318319$ Latcod $100 \mathrm{Sa}$

1427 (b) $\mu o v] \varepsilon \mu o v$ B V 108-118-314*30'-321' c $121319=\mathrm{Ra}$

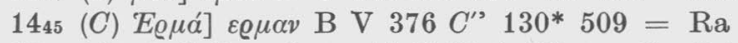

$\left.15_{1}(d t x) \varepsilon \dot{\lambda} a \dot{\lambda} \eta \sigma \varepsilon v\right] \varepsilon \iota \pi \varepsilon(v) \mathrm{B}$ V $d 129 t x$ Cyr I $1029=\mathrm{Ra}$

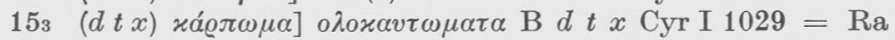

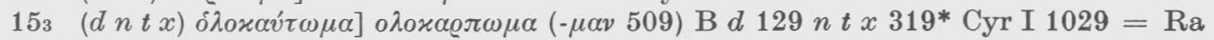

156 (O) $\left.\varepsilon i \varsigma 1^{\circ}\right]$ pr $\eta \mathrm{B} O^{-58}$ 509-527 $\mathrm{Syh}=\mathrm{Ra}$

$15_{12}(d t)$ ovi $\left.\omega \varsigma\right]$ ov $\tau \omega \mathrm{B} \mathrm{M} d^{(-610)} 54 t 509$ 128-628-630' $=\mathrm{Ra}$

$15_{20}(x)$ å $\left.\lambda \omega v o \varsigma\right] \alpha \lambda \omega \mathrm{B} x^{-509}$ Cyr VI $568=\mathrm{Ra}$

$15_{24}(o I I f) \pi \circ\left\llcorner\eta \sigma^{\prime} \varepsilon l\right]-\sigma \eta \mathrm{B}^{*} \mathrm{~F} \mathrm{~K} \mathrm{M}^{\prime} 15^{\prime}-o I I^{-82} 46-422-500^{*}-528-616^{\mathrm{c}} f^{-129} 127527669^{*}$

$\left.15_{33}(f n t) \xi v \dot{\lambda} \alpha\right]+(+\varepsilon v 54-458) \tau \eta \eta \mu \varepsilon \varrho \alpha \tau \omega v \sigma \alpha \beta \beta \alpha \tau \omega v \mathrm{~B} f n^{-75} t 527799=\mathrm{Ra}$

$1539(d n t x)$ om ỏjíw $2^{\circ} \mathrm{B} \mathrm{V} d 129 n^{-767} t x 319$ Tht $N m 211$ Latcod 100 Arm Co

163 (C) $M \omega v \sigma \tilde{\eta} v]-\sigma \eta \mathrm{B}^{*} 707 C^{\prime \prime}-464221928-30^{\prime}-8555$

$\left.169(x z) \sigma v v \alpha \gamma \omega \gamma \eta \tilde{\eta} \varsigma 2^{\circ}\right] \sigma \varkappa \eta \nu \eta \varsigma \mathrm{B} \mathrm{F}^{\mathrm{a}} 56^{\prime} x z 799 \mathrm{Cyr} \mathrm{I} 860 \mathrm{Sa}$

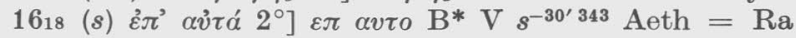

$1626(d t x) \delta \sigma \alpha c] \omega v$ B M txt V $376 d 129 t x=$ Ra

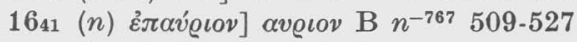

189 $(d n t x)$ om $\tau \tilde{\eta} s$ B $82 d n^{-767} t x^{-619}$ Cyr I $837=$ Ra

$\left.1810(c I I) \varphi \alpha^{\prime} \gamma \varepsilon \tau \alpha l\right]$ $\varphi \alpha \gamma \varepsilon \tau \varepsilon$ B $58 \quad 46-52^{\prime}-417-616^{c} 130^{*} 71630$ Bo

$1812(x)$ om $x \alpha i$ $2^{\circ}$ B $426 x^{-619}$

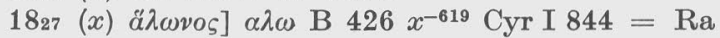

$18_{27}(x)$ om $\omega_{\varsigma} 2^{\circ}$ B $129 x^{-619} 319 \mathrm{Cyr}$ I $844 \mathrm{Bo} \mathrm{Sa}^{1}=\mathrm{Ra}$

$1828(o I I b s z)$ äv] $\varepsilon \alpha \nu$ B F $58-o I I^{-82} b 56^{\prime} s 619392 z 59^{c} 319$ Cyr I $844=\mathrm{Ra}$

$1829(x)$ ả $\pi$ ć $^{\circ}$ ] pr $\eta \mathrm{B} \mathrm{V} 129 x^{-619} 392 \mathrm{Cyr}$ I $844 \mathrm{Sa}^{1}=\mathrm{Ra}$

$\left.19_{20}(d n t) \alpha^{\prime} v\right] \varepsilon \alpha \nu$ B $376^{\prime} 413 d n t 71799$ Eus VI 12 Tht $N m 215^{\text {te }}=$ Ra

204 (t) à $\nu \gamma \hat{\alpha} \gamma \varepsilon \tau \varepsilon]-\gamma \alpha \gamma \varepsilon \varsigma \mathrm{B}^{*} 129$ 74-76-84 71-509

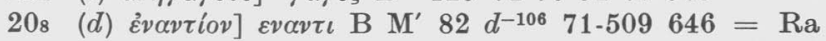

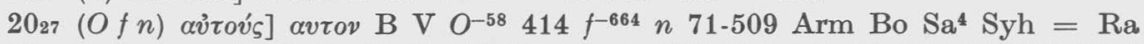

2028 (bd) om $\tau$ óv $1^{\circ}$ B $16-46-414-552-761 \quad b \quad 107^{\prime}-125 \quad 12984 \quad 71-509 \quad 392 \quad 55^{\text {txt }}$ (c pr m) $319=\mathrm{Ra}$

219 (oIn) ह̌ $\delta \alpha x \varepsilon v] \varepsilon \delta \alpha x \nu \varepsilon v \mathrm{~B} o I^{-15}-29537 n^{-767} 71^{\prime} 392$ Cyr II 637 Arm Syh = Ra

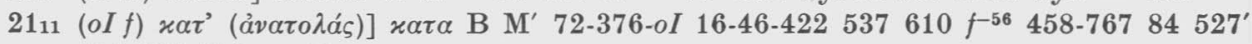
126-669 $799=\mathrm{Ra}$

$\left.21_{12}(d t) Z \alpha \varrho \varepsilon \delta\right] \zeta \alpha \varrho \varepsilon \tau \mathrm{B} 52^{*} d t 318^{*}(\mathrm{vid})=\mathrm{Ra}$

$\left.21_{13}(f) \pi \varepsilon ́ \varrho \alpha v\right] \pi \varepsilon \varrho \alpha \mathrm{B}^{*} 53^{\prime}-129$

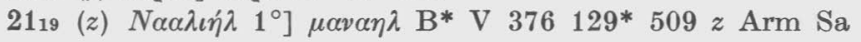




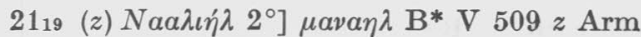

$21_{22}(f)$ om åv B 53'-129 71-509 55= Ra

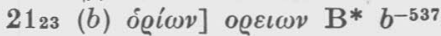

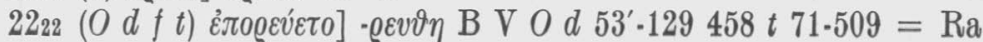

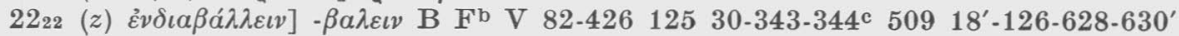

$\left.22_{24}(f) \alpha \mu \pi \varepsilon \lambda \omega \nu \omega \nu\right]-\lambda \omega \nu$ B V 53'-129 71(vid)-509 Or IV $409=\mathrm{Ra}$

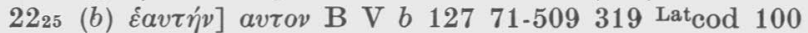

$2233(b x) v \tilde{v} v]+o v v$ B $b x^{-619}$ Latcod $100=\mathrm{Ra}$

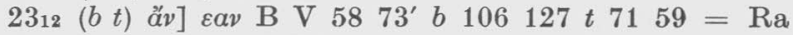

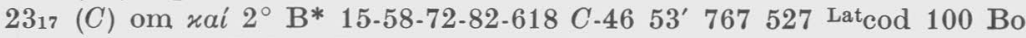

242 (b) $\left.\dot{\varepsilon} \pi^{\prime}\right] \varepsilon v$ B $b^{-19}$ Tht $N m 221=\mathrm{Ra}$

247 $(t z)$ om aข่นõ $2^{\circ}$ B $107^{\prime} 129$ 76-84-134 71-509 18'-126-628-630-669* Eus VI 409 Or IV 250 Arab $\mathrm{Bo}^{\mathrm{A}} \mathrm{Sa}$

$\left.24{ }_{16}(f n t) v \psi i \sigma \tau o v\right]$ pr $\pi \alpha \varrho \alpha\left(\pi \alpha \varrho 664 ;+\tau o v\right.$ 84*) B V 82-376 $106 f^{-56^{*}} n t$ 71-509 392319 Phil III 191 Lat codd $919294-96100$ Bo $=$ Ra

2421 (b y) Kaıvaiov] xevaıov B 72 b 85 527-619 $y^{-318} 68^{\prime}$

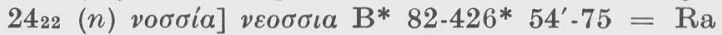

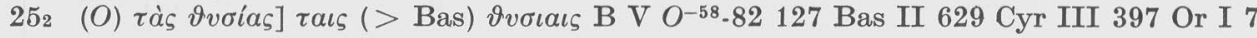
$=\mathrm{Ra}$

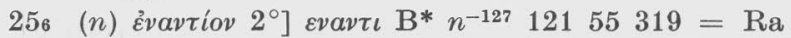

$264(O n x)$ rai $2^{\circ}$ pr $\sigma v(\sigma o \iota 767)$ B V O $n x^{-619} 407$ Latcod 100 Arm Syh

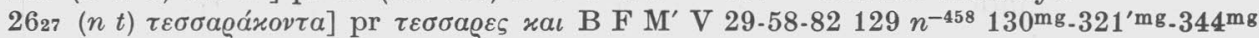
$t 50931840759$ Arm

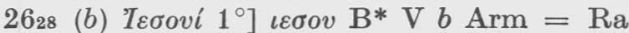

$2651(d)$ om xai $3^{\circ} \mathrm{B}^{*} 417$ d $76784128-669319$

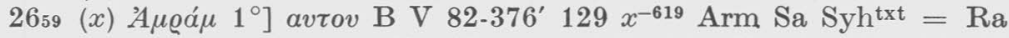

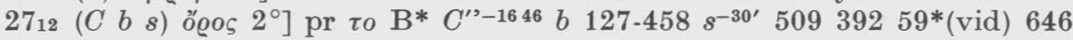

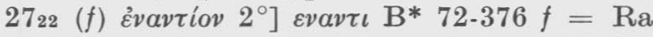

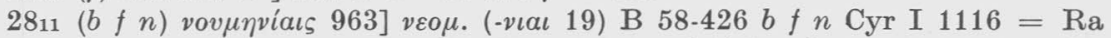

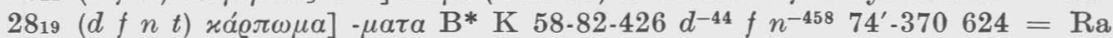

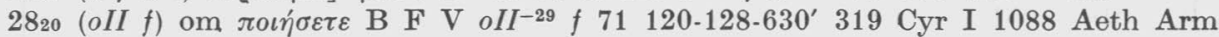
$\mathrm{Co}=\mathrm{Ra}$

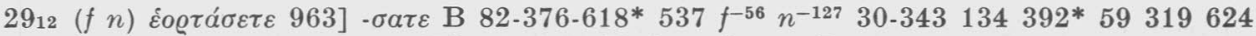

$299_{13}(n)$ xá $\pi \omega \mu \alpha$ 963] - $\mu \alpha \tau \alpha \mathrm{B}^{*} 58-82 n^{-458}$ Arm Sa $=$ Ra

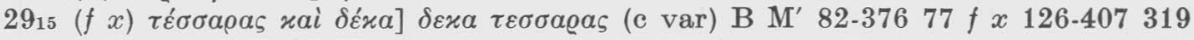

$29_{17}(f t x)$ idem B $\mathbf{M}^{\prime}$ V 963 82-376' 77-417 106 f $t x$ 18-407-669

$2920(d f t x)$ idem B $\mathrm{M}^{\prime} \mathrm{V}$ 82-376 $77 d^{\left(-10 \text { txt }^{\text {tx }}\right.} f t x$ 18-126-407

$29_{26}(d f t x)$ idem B V 58-72-82-376 $77 d f t x 407416$

$\left.303 \quad(b) \stackrel{\alpha}{a} v 2^{\circ}\right] \varepsilon a v$ B G $b 12954121=\mathrm{Ra}$

3013 (z) äv $\left.2^{\circ} 963\right]$ eav B G 509 18'-68'-120-630' $646=\mathrm{Ra}$

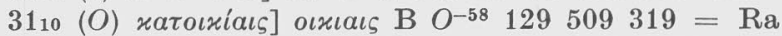

$31_{28}(x)$ oै $\left.v \omega v\right] \alpha \iota \gamma \omega v$ B Fa V $82129 x^{-527} 407319$ Arm Sa = Ra

$\left.31_{36}(x) \pi \varepsilon v \tau \alpha x o ́ \sigma \iota \alpha\right]-\sigma \iota \alpha \iota$ B 127 (vid) $x^{-527} 407$

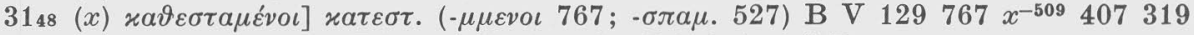

$\left.31_{50}(f) \chi \lambda \iota \delta \tilde{\omega} v \alpha\right]$ pr $\varkappa \alpha \iota \mathrm{B}^{*} 58 f^{-129} 319$ Cyr I 340 Aeth C Bo

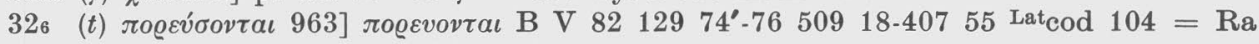

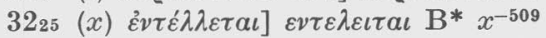

$3230(f) \delta \iota \alpha \beta \iota \beta a ́ \sigma \varepsilon \tau \varepsilon]-\sigma \alpha \tau \varepsilon$ В $422-529 f \quad 130-344^{*} 52739212055$

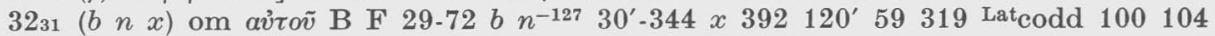

$3233(o I z)$ om $\tau \tilde{\eta} \varsigma 1^{\circ} \mathrm{B}^{*}$ oI-29 413 18'-126-628-630' 799

$32{ }_{39}(x)$ om eis B $x$

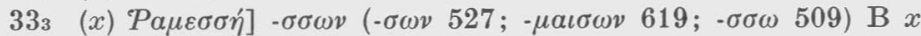

335 (f $t$ ) $\left.\Sigma o \chi \chi \omega_{\vartheta}\right] \sigma o \chi \omega \vartheta \mathrm{B}^{*} \mathrm{M}^{\prime} 82-376^{\mathrm{c}} 53^{\prime}-12954730 \mathbf{7 4}^{\prime}-76-84^{*}$ (c pr m) $\mathrm{Sa}^{12}$

$33_{17}(O f x)$ om $\tau \tilde{\eta} \varsigma$ B $\mathrm{M}^{\prime} \mathrm{V} O^{\prime-5872} f x^{-509}=\mathrm{Ra}$

$\left.33_{20}(x) \Lambda \varepsilon \beta \omega v a ́\right]$ $\lambda \varepsilon \mu \omega v \alpha(-v v \alpha$ 509) B $767 \times 407=\mathrm{Ra}$

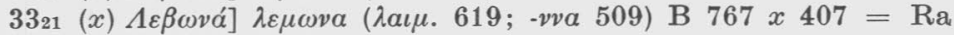

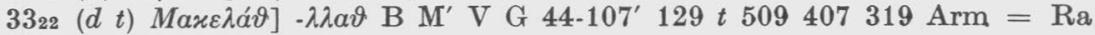


$33_{23}(t)$ idem B M' Vc G 107' $129 t 509407$ Arm $=$ Ra

$33_{29}(d n t x)$ A $(\alpha \varepsilon \lambda \mu \omega \nu \alpha ́] \sigma \varepsilon \lambda \mu$. B $d^{-125} 129 n^{-54^{*} 767} t x 18799$ Arm Sa $=\mathrm{Ra}$

$33_{30}(d n t)$ idem B Fa V 44-107' $129 n t 799$ Arm Sa $=\mathrm{Ra}$

$33_{31}(n x)$ Bavaıaxáv] Bavaıa B V 376 54'-75 $x 319$ Sa $^{1}=\mathbf{R a}$

$333_{32}(n)$ idem B V $54^{\prime}-7571^{\prime} \mathrm{Sa}^{1}=\mathrm{Ra}$

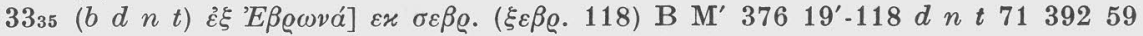

$333_{35}$ (n) $\left.\Gamma \varepsilon \sigma \iota \omega \nu\right] \gamma \varepsilon \sigma \sigma . \mathrm{B}^{*} \mathrm{~F}^{\mathrm{a}}$ 54-75' 84392 Lat $\operatorname{cod} 104$

$33_{43}(n)$ ' $\left.\Omega \beta \omega \dot{\vartheta}\right] \sigma \omega \beta \omega \vartheta$ B V 54'-767 71-509 319

$\left.33_{44}(n x) \varepsilon^{\prime} \xi ' \Omega \beta \omega \vartheta\right] \varepsilon \varkappa \sigma \omega \beta \omega \vartheta$ (c var) $\mathrm{B} \mathbf{M}^{\prime} 58-72 n x 59 \mathrm{Sa}^{1}$

$\left.333_{54}(n) \not ̈ \nu\right] \varepsilon \alpha v \mathrm{~B}^{*} n^{-75}=\mathrm{Ra}$

$3355(n t) \stackrel{\alpha}{a}] \varepsilon a v \mathrm{~B}^{*} \mathrm{~V} 376106 n t 61968^{\prime}-120319=\mathrm{Ra}$

$34{ }_{13}(d n t)$ xv́øเos] $+\tau \omega \mu \omega v \sigma \eta(\mu \omega \sigma \eta n) \mathrm{B}^{*} d^{(-44)} 246 n t \mathrm{Syh}=\mathrm{Ra}$

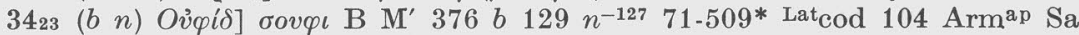

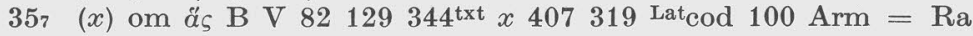

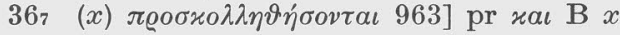

Many of the variants in the above list are noted as "= Ra." For a discussion of their secondary nature ef chapter 6 .

A more precise statement on the relations of the B text to the text tradition can now be made by noting the number of instances in which the $\mathrm{B}$ variant is supported by the individual text groups. To make the statement more complete those instances from list 4 in which no more than four text groups support a $\mathrm{B}$ reading are added to the statistics from list 8 .

In the table below the vertical column designated $A$ shows the number of instances a single text group supports a $B$ reading; column $B$ shows the number of instances in which a B reading is also supported by two text groups; column $C$, by three text groups, and column $D$, by four such. The last column gives the total number of these instances. Throughout this table random support is disregarded. No distinction has been made among the catena groups, i.e. among sub groups and/or $C$. Among the hex witnesses support by one or both sub groups but not by $O$ is given separately.

$\begin{array}{lrrrrr} & A & B & C & D & \text { Total } \\ O & 4 & 2 & 8 & 5 & 19 \\ o I \text { or oII } & 2 & 6 & 1 & 1 & 10 \\ C & 7 & 1 & 1 & - & 9 \\ b & 8 & 7 & 4 & 3 & 22 \\ d & 3 & 12 & 11 & 16 & 42 \\ f & 8 & 10 & 10 & 6 & 34 \\ n & 11 & 13 & 14 & 13 & 51 \\ s & 2 & 1 & 1 & 1 & 5 \\ t & 3 & 17 & 13 & 16 & 49 \\ x & 37 & 15 & 12 & 14 & 78 \\ y & 1 & 1 & - & - & 2 \\ z & 5 & 5 & - & 1 & 11\end{array}$

From this table it is obvious that $o I / o I I C^{\prime \prime} s y$ and $z$ are only tangentially influenced by a B type text. As has already been mentioned in chapter 1 above, 
$x$ is closely related to $\mathrm{B}$; it is clear from the above table that the relationship is closer than that of any other group. Thus out of 91 instances in which the B reading is supported by only one group it is $x 37$ times, whereas the next in order is $n$ with only 11 . From the total number in the final column it appears that the Byzantine groups are also significantly related to B in the text tradition, $n$ being represented 51 times, $t, 49$, and $d 42$ times. Group $f$ comes next with 34 , followed by $b$ with 22 , and $O$ with 19 . The remainder, as indicated above, is insignificant.

In the final list the same conditions imposed on the B variants in list 8 are here imposed on variants found in codex A. As for list 8 the support by groups is summarized in parentheses. For the $s$ group a majority of $85^{\prime} \mathrm{mg}_{-} 321^{\prime} \mathrm{mg}-344^{\mathrm{mg}}$ is included as an $s$ reading as well.

\section{List 9}

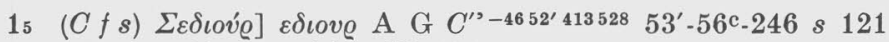

$130(x)$ viois $2^{\circ}$ ] vıos $A^{*} x^{-509} 12155$

$147(o I C s)$ om $\varphi v \lambda \tilde{\eta} s$ A $o I-29 C^{\prime \prime-131^{(}(414 \prime)} s 12155424624$

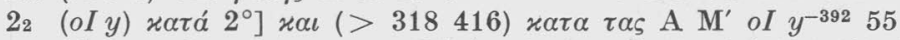

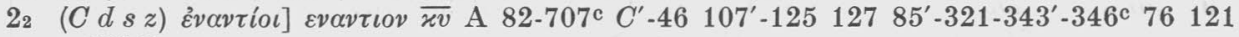
$z^{-122 * 126} 646$

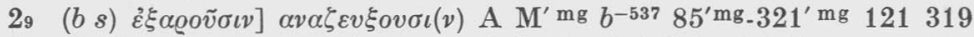

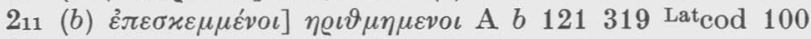

213 (b) idem A $b 121319$ Latcod 100

$216(o I C b s)$ idem A oI-29 $C^{\prime \prime} b 30^{\prime}-85^{\prime}$ txt-321-343'-346 txt 12155319646 Lat $^{\text {tod }} 100$

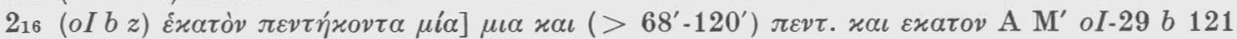
$z^{-18126} 55$

$216(b s) \varepsilon \xi \xi \alpha \varrho o \tilde{v} \sigma \iota v] \alpha \nu \alpha \zeta \varepsilon v \xi o v \sigma \iota v$ A $\mathrm{M}^{\prime} \mathrm{mg} \quad b \quad 85^{\prime} \mathrm{mg}_{-} 321^{\prime} \mathrm{mg} 121319$

$218(O C) \pi \alpha \varrho a ́] ~ \varkappa \alpha \tau \alpha$ A $\mathrm{F}^{\mathrm{a}} \mathrm{M}^{\prime} \mathrm{mg}$ V $O C^{\prime \prime}-131^{\mathrm{c}} 767 \quad 121$ 126-128-628-669 55319646

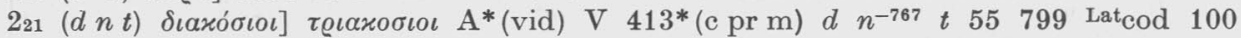
Arm

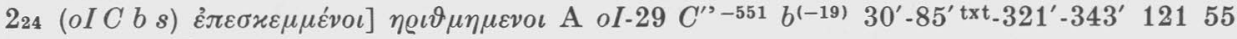

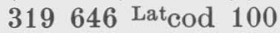

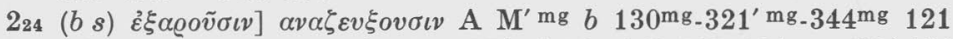

$\left.2{ }_{26}(d t x) \delta v^{\prime} \alpha \mu \iota \varsigma\right]-\mu \varepsilon \iota \varsigma$ A $376-707^{*} d 5^{\mathrm{c}} 458-767321 t x$ 68'-120' 55

226 (b) $\varepsilon \dot{\pi} \varepsilon \sigma \varkappa \varepsilon \mu \mu \varepsilon ́ v o \iota] ~ \eta \varrho \iota \vartheta \mu \eta \mu \varepsilon v o \iota$ A $b 319$ Latcod 100

$228(d t x) \delta v ́ v \alpha \mu \iota \varsigma]-\mu \varepsilon \iota \varsigma A^{*} 376 d^{-44} 56^{\mathrm{c}} 458-767 t x \quad 68^{\prime}-120^{\prime} 55$

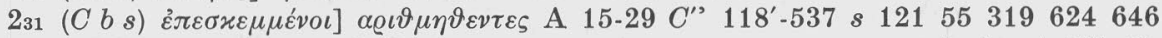

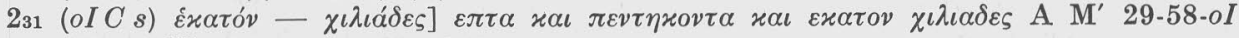
$C^{\prime s} s^{-343^{\mathrm{mg}}} 12155319624646$

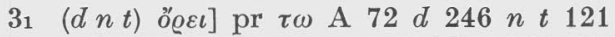

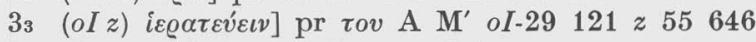

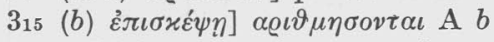

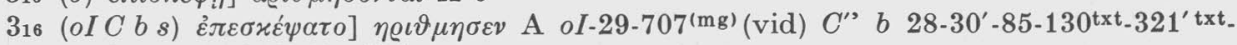
$343^{\prime} 12155319624 \mathrm{Sa}^{4}$

$316(o I b)$ ôv $\tau \varrho o ́ \pi o v] ~ \varkappa \alpha \vartheta \alpha$ A $o I-29 b 5412155624$

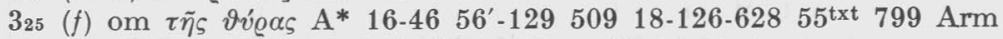

$3_{27}(d t y)$ ' $\left.\sigma \alpha \alpha \varrho i \varsigma\right] \sigma \alpha \alpha \varrho \varepsilon \iota \varsigma \mathrm{A}$ G-426 $d t y^{-318} 5559 \mathrm{Arm}$

$\left.3_{36}(d t) \alpha v i \tilde{\eta} \varsigma 3^{\circ}\right] \alpha v \tau \omega v \mathrm{~A}^{*}$ (vid) $d t$ Aeth

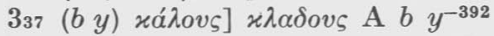

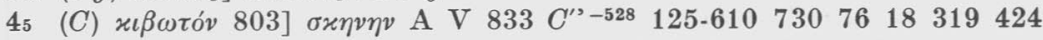

$411(b n) \delta \varepsilon \varrho \mu r \tau i \nu \omega 803]-\mu \alpha \tau \iota$ A $707 b n^{(-767)}$ Aeth

$412(b n) \delta \varepsilon \varrho \mu \alpha \tau i v \omega$ 803] $-\mu \alpha \tau \iota$ (bis scr 118') A $b n$ Aeth 


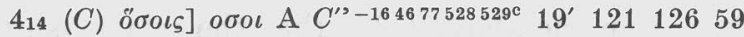

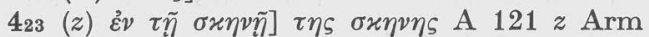

$424(b f n)$ om $\tau o \tilde{v} 2^{\circ}$ A G-376 $52-417-552 b 125 f^{(-129 \text { txt })} n^{-767} 12155$

$\left.425(C f n s) \alpha^{\prime} \lambda v \mu \mu \alpha 1^{\circ}\right]$ ] $\alpha \tau \alpha \varkappa \alpha \lambda$. A Bc F M' 58-64-381 $C^{\prime \prime}-5277^{* 320414528} 56-129-246^{\mathrm{c}}$ $n^{-75} s^{-321^{*}(343)} 31859$

$425(o I f y) \alpha v i \tilde{\eta} \varsigma 2^{\circ}$ ] $\alpha v \tau \eta v$ A $\mathrm{M}^{\prime} 29-58-72-o I f 71 y^{-392} 55 \quad 59646$

433 (oIf $y z$ ) vioṽ] pr $\tau$ ov A 426 -oI $f 619 y^{-392} z 646$

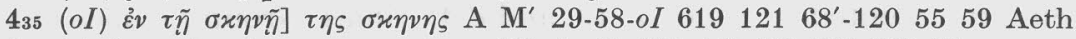

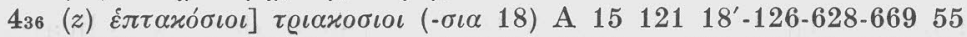

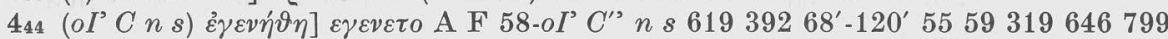

$444(O I)$ om $\alpha v j \tau \tilde{\omega} \nu 1^{\circ}$ A $58-o I^{-64^{\text {txt }}} 414125767619318 \quad 5559$

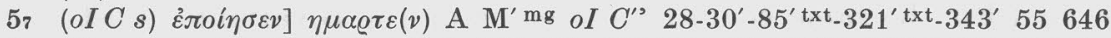

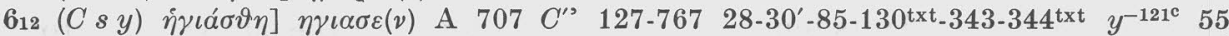
319624 Cyr I 1041

$\left.751(n) \stackrel{\varepsilon v \alpha}{ } 3^{\circ} 963\right]>A$ V 82-707 54-75' 28-130-343' 509 68*-120-122 Latcodd 100104 Aeth $^{-\mathrm{CH}}$

$757(f n)$ E̊va $\left.3^{\circ} 963\right]>$ A $707 f^{-246} 54-75^{\prime} 343-730509120^{\prime}-122$ Latcodd $^{\circ} 100104$ AethM

763 (n y) om $\tilde{\varepsilon} v \alpha 3^{\circ}$ A V 82-707 $413 \quad 54-75^{\prime} 343509 y^{-318}$ Latcodd 100104

$\left.775(x) \& v v \alpha 3^{\circ} 963\right]>A$ V 82 16-46-528 $767 x^{-619} 18-126$

$\left.781(n x) \quad \check{\varepsilon} v \alpha 3^{\circ} 963\right]>A^{*}$ V $29^{\text {txt }}\left(\mathrm{c}\right.$ pr m)-82 $529 \quad 107^{\prime} 56 \quad n^{-767} x^{-619} 392120319$ Lat cod 100

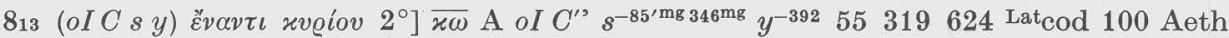

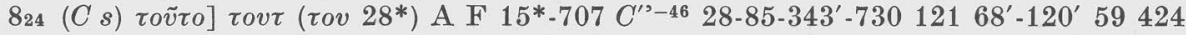

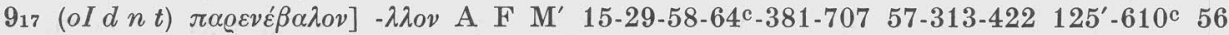
$54^{\prime}-458321^{\prime}-344-730 t 121$ Aeth Arm Sa

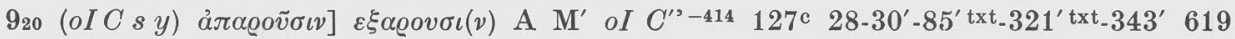
$y^{-392} 55319646$

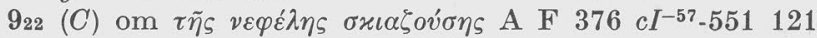

$109(o I C s y) \sigma \eta \mu \alpha \nu \varepsilon i \tau \varepsilon] \sigma \alpha \lambda \pi \iota \varepsilon \iota \tau \varepsilon$ A $M^{\mathrm{mg}} \mathrm{V} \quad o I C^{\prime \prime} 44 s^{-130 \mathrm{mg} 321^{\prime m g}} y^{-392} 126 \quad 55 \quad 319$ 416 LatOr Matth 52

$10{ }_{14}(o I C s y)$ vi $\left.\tilde{\omega} v\right]$ pr $\tau \omega \nu$ A $58-o I C^{\prime \prime}-(57) 529 s 619 y^{-392}$

$\left.10_{25}(o I C s) A^{\prime} A \mu \sigma \alpha \delta \alpha i\right] \quad \mu \iota \sigma \alpha \delta \alpha \iota \mathrm{A} o I^{-64} C^{\prime \prime}{ }^{-73^{*}} s^{-30} 12155$

$\left.1030(O C s y) \dot{a} \lambda \lambda a^{\prime}\right] \alpha \lambda \lambda \mathrm{A} \mathrm{V} \mathrm{O-376-15-72^{ \prime }} C^{\prime \prime-422} 52975 s^{-321^{*}} 509 y^{-392} 185559319$

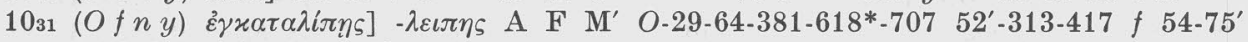
$30-343^{\prime} 134^{*} 509 y^{-392} 55319$

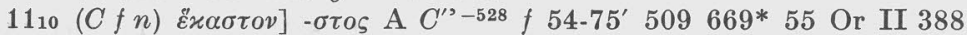

$11_{12}(o I y)$ om $\tau \dot{\eta} v$ A oI $y^{-392} 55624$

$11_{13}(o I y)$ om $\mu \circ \iota$ A $\mathbf{M}^{\prime}$ ol $y^{-392} 55624$

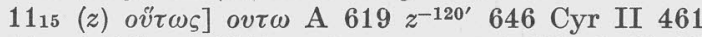

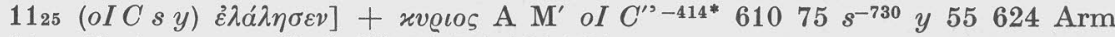

$11_{31}(C n)$ om $\tau \tilde{\eta} \varsigma 1^{\circ}$ A $C^{\prime \prime} 54-75^{\prime} 121799$

$\left.11_{32}(b f) \tau \eta^{\prime} 4^{\circ}\right] \tau \eta$ A Fc 58-72-381' $529 b^{-19} f^{-129} 54-7512159$

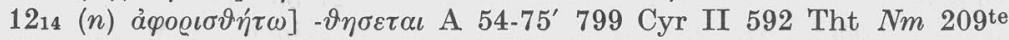

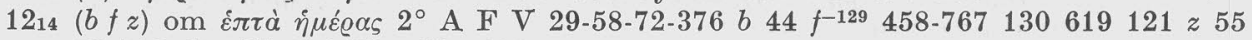
59799 Cyr II 592 Tht $N m 209$

$\left.1319(f n) \varepsilon i 1^{\circ}\right] \eta$ A F $\mathbf{M}^{\prime} 29 f 54-75^{*}-45871^{\prime} 319799$

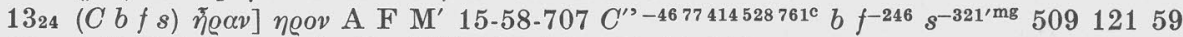

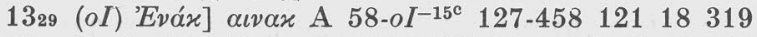

$\left.1330(b y) \tau \tilde{\eta} 2^{\circ} \mathrm{F}^{\mathrm{a}}\right]>$ A F $58-72-82417$ b $56 \quad 30509-527 \quad y^{-318} 59799$ LatBeda Sam 273 Arm

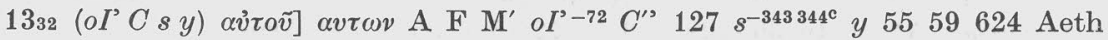

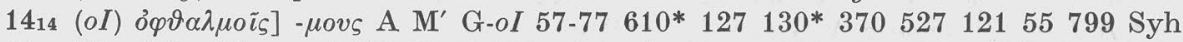

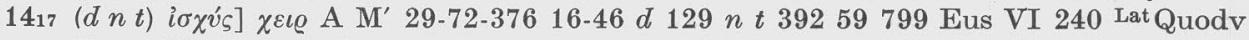
Prom II 17 Arab Arm

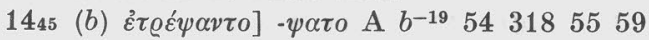

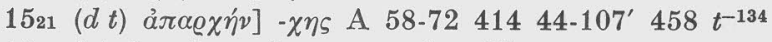

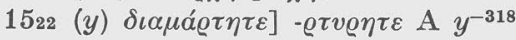




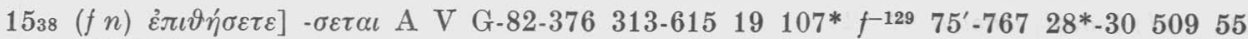
319799

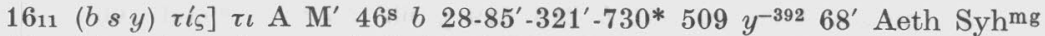

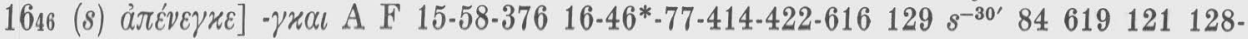
$6695559319^{*}$

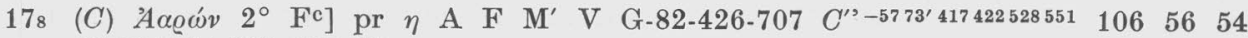
85-321' 74-370 $527 \quad 55$

185 (oI \& y) $\left.p v \lambda \alpha^{\prime} \xi \varepsilon \sigma \vartheta \varepsilon\right]-\xi \varepsilon \tau \varepsilon$ A oI 16-46-414-616-761 30'-130-343'-346 $y^{-392}$

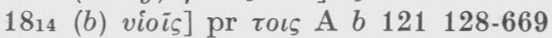

$1815(C s y) \mu \eta \dot{\tau} \varrho \alpha \nu] \mathrm{pr} \pi \alpha \sigma \alpha \nu \mathrm{A} \mathrm{M}^{\prime} 15^{\prime}-58 C^{\prime 3}-1646552^{\text {txt }} s 619 y^{-121} 55$ Procop 845

$1818(d n t)$ om $x \alpha \tau \alpha^{\prime} \mathrm{A} 618^{*}\left(\mathrm{c}\right.$ pr m) $d 54-75^{\prime} t$ Latcod 100 Arm

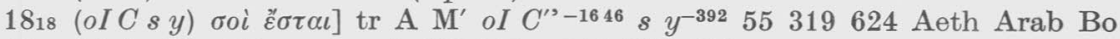

$18_{24}(C s)$ vi $\left.\tilde{\omega} v 2^{\circ}\right] \mathrm{pr} \tau \omega v$ A $C^{\prime \prime} 246 s 318319624$

1828 ( $f n) \delta \omega ́ \sigma \varepsilon \tau \varepsilon]-\sigma \varepsilon \tau \alpha \iota$ A V 72*-82-376 $19106 \quad 56^{\prime}-664 \quad 75^{\prime}-76750955319$

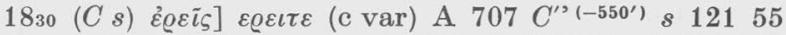

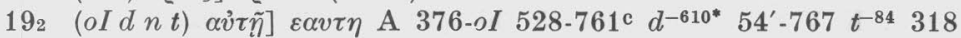

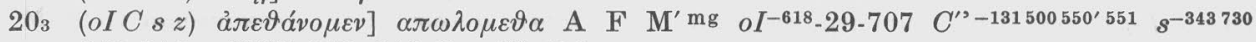
$619318 z^{-126628} 319646$ Cyr II 488

$2024(O b f n) \delta \varepsilon \delta \omega \varkappa \alpha] \varepsilon \delta \omega \varkappa \alpha$ A V $O^{\prime-15376-72} 52^{\prime}-313-414^{\prime}-422 b 53^{\prime}-129 n^{-458} \quad 74-76527$ 1215559646

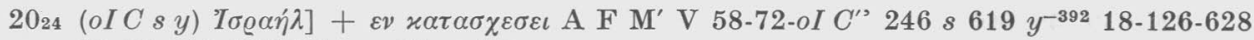
5559319646 LatAug Num 39

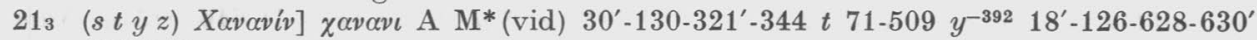
416646

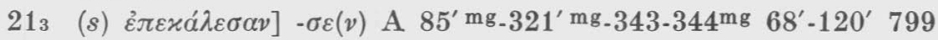

$\left.21_{12}(o I) Z \alpha \varrho \varepsilon ́ \delta\right] \zeta \alpha \varrho \varepsilon$ A oI 121

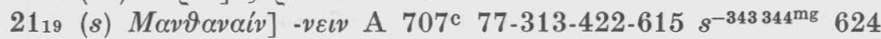

$2125(o I C s y) \alpha v i \tilde{\eta}] \alpha v \tau \alpha \iota \varsigma$ A $\mathrm{M}^{\prime} 58-o I C^{\prime \prime} s 619$ y 55624

$\left.21_{27}(n) \varepsilon \ddot{\imath} \lambda \vartheta \varepsilon \tau \varepsilon\right] \varepsilon \lambda \vartheta \alpha \tau \varepsilon$ A V 29-58 $129 n 121\left(2^{\circ}\right)-392 \quad 55^{*}(\mathrm{c}$ pr m) 624799

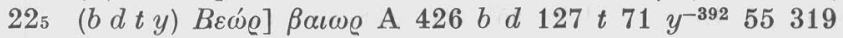

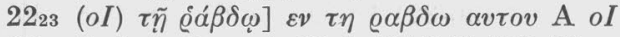

$2230(b f)$ àró] $\varepsilon \varkappa$ A $29 b \quad 53^{\prime}-246 \quad 458 \quad 55$

$\left.22_{31}(C s z) B \alpha \lambda \alpha \alpha \dot{\mu}\right]$ pr $\tau$ ov A $C^{\prime 3}-52 s 619121 z 319$

$\left.22_{32}(o I C s y) \varepsilon i \pi \varepsilon v\right] \lambda \varepsilon \gamma \varepsilon \iota \mathrm{A} \mathrm{M}^{\prime}$ oI-82 $C^{\prime \prime} s^{-85^{\prime} \mathrm{mg}_{321^{\prime} \mathrm{mg}}} y^{-392} 55319624$

$\left.22_{38}(b f s) \lambda \alpha \lambda \eta^{\prime} \sigma \omega\right] \quad \varphi v \lambda \alpha \xi \omega \lambda \alpha \lambda \eta \sigma \alpha \iota$ A $82-707 \quad b \quad f^{-129} 85^{\prime m_{-}} 321^{\prime m g}-344^{\text {mg }} 121319624^{\mathrm{mg}}$ 799 Aeth

238 (y) xv́@ı६] pr o A $\mathbf{M}^{\prime} 15 \quad 30^{\prime}-343^{\prime} y^{-392}$

$23{ }_{19}\left(o I^{\prime} n y z\right)$ ov̉oć] ov $\delta$ A F $58-o I^{\prime-82} 56^{\prime} 54-75^{\prime} 619 y^{(-392)} z 55624799$

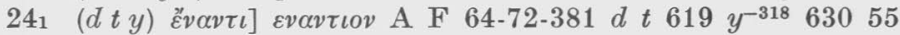

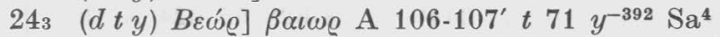

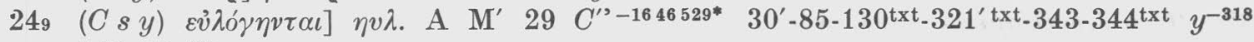
6695559

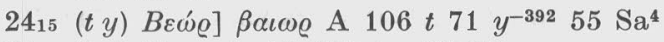

$2422(t y)$ idem A $15125 t y^{-392} 55$

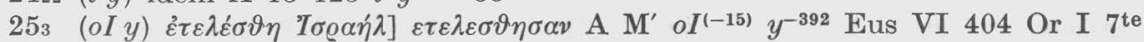

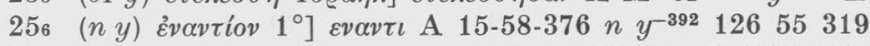

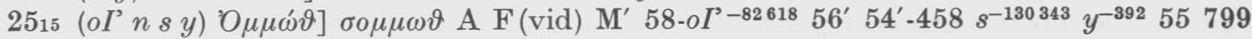
Lat cod 100

262 (dt $t z) \sigma v v \alpha \gamma \omega \gamma \tilde{\eta} \varsigma]$ pr $\tau \eta \varsigma$ A $d$ 458-767 $t^{-84^{*}} 18^{\prime}-120-628-630^{\prime}$

$263 \quad(o I d y)$ om $\delta$ iعovús A oI $I^{-15} d^{-106} y^{-392} 55319$ Aeth

$2618(x) \tau \tilde{\omega}]$ zov A $85 x^{-71} 12168^{\prime}-120$ Bo Syh

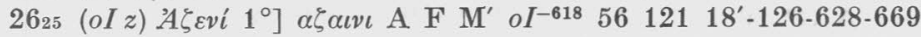

$2625(o I z){ }^{\prime} A \zeta \varepsilon v i 2^{\circ}$ ] $\alpha \zeta \alpha \iota v \iota$ A F $\mathrm{M}^{\prime}$ oI ${ }^{-618} 1211^{\prime}-126-628-630^{\prime}$

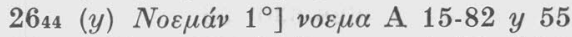

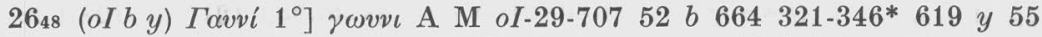

2648 (oI y) $\Gamma a v v i 2^{\circ}$ ] $\gamma \omega v v \iota$ A F M' oI-707 52 130'-321' 619 y 55

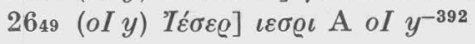


$26_{49}(o I)$ '

2657 (oI) $\Gamma \varepsilon \delta \sigma \omega v] \gamma \varepsilon \delta \sigma \omega \nu \iota$ A $o I 318$

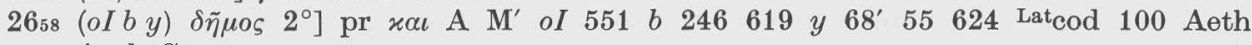
Arab Co

$2658(n t) X \varepsilon \beta \varrho \omega v i] \quad \chi \varepsilon \beta \varrho \omega \nu \mathrm{A} n^{-767} t^{(-370)} 121624 \mathrm{Co}$

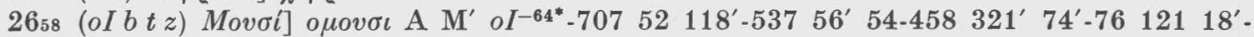
126-628-630' $55624 \mathrm{Bo}^{\mathrm{B}}$

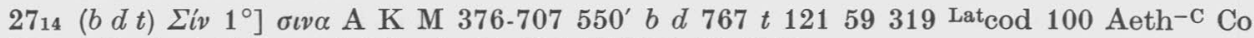

2717 (oI) $\omega \sigma \varepsilon i$ 963] $\omega \varsigma$ A $\mathrm{M}^{\prime}$ oI 12112655 Phil II $104^{\mathrm{F}}$

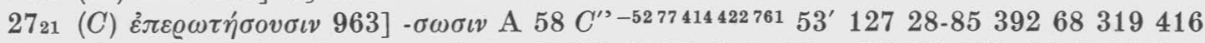

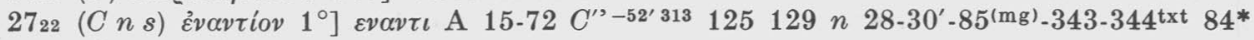
12155646

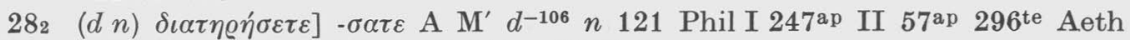

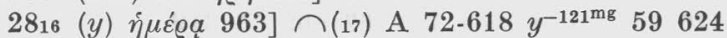

$2817(y)$ om $\dot{\varepsilon} \pi \tau \alpha^{\prime}-$ fin A 15 121 txt_318 624

$2818(f) \pi \circ\llcorner\eta \dot{\sigma} \sigma \varepsilon \tau \varepsilon]-\sigma \varepsilon \tau \alpha \iota$ A K V 82-376 56'-664 75' 30-130* 50912055624

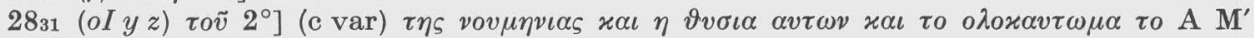
$58-o I 127619$ y $z^{-407} 55624646$

298 (OC s y) om xv@í $\omega$ A F M $\mathrm{M}^{\prime} O^{\prime} C^{\prime \prime}-52^{\prime 131^{\circ} 414} s$ y $55624 \mathrm{Syh}$

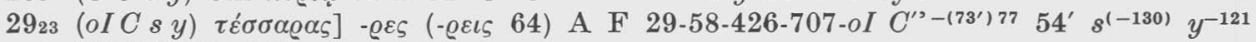
$18^{\prime}-630^{\prime} 59646$

$2926(o I C s z)$ idem A F 29-426-707-oI $C^{\prime \prime}-77 s 392$ 18-68'-120-630 59646

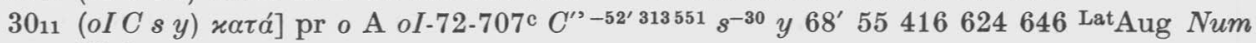
59.2

$\left.3015(o I x y)\left(\dot{\varepsilon} \pi^{\prime}\right) \alpha v i \tilde{\eta} \varsigma\right] \alpha v \tau \eta$ A $72-426-o I^{-15} 53^{\prime} 134 x^{-509}$ y 40755416

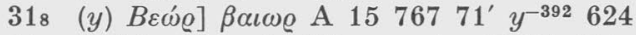

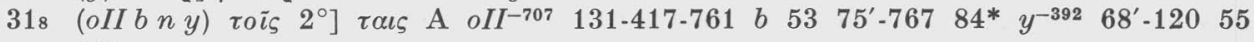
416

3118 (d n s y) oi $\delta \varepsilon v] \varepsilon \gamma v \omega$ A F 15-29-72 107'-125 $129 n 130^{\mathrm{mg}_{-} 321^{\prime} \mathrm{mg}} y^{-121}$

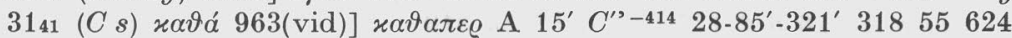

$\left.31_{43}(o I s y z) \tau \tilde{\eta} s\right]$ pr $\tau o$ A $\mathrm{M}^{\prime}$ oI ${ }^{-618} 52^{\prime}-31328-85^{\text {txt }}-130-321^{\prime}$ txt_-343' $y^{-392^{*}} 18^{\prime}-628-630^{\prime}$ $624=\mathrm{Ra}$

$32{ }_{3} \quad(C s y)$ 'A

$324 \quad(o I y z)$ om $\tau \tilde{\omega} v$ A $\mathrm{M}^{\prime}$ oI-82 $y^{-392} 18^{\prime}-126-628-630^{\prime}$

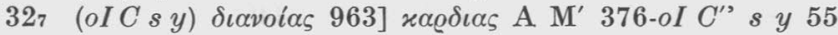

3216 (f) $\left.\dot{\eta} \mu \tilde{\omega} v 1^{\circ}\right]$ v $\mu \omega \nu$ A $417-422-528-551^{*} 53^{\prime}-246527$

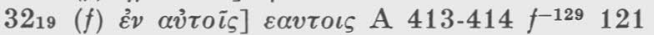

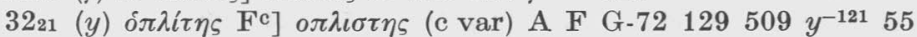

$3225(d x) \dot{\eta} \mu \tilde{\omega} v] \eta \mu \iota v \mathrm{~A} d^{-106} x^{-509} \mathrm{Sa}^{12}$

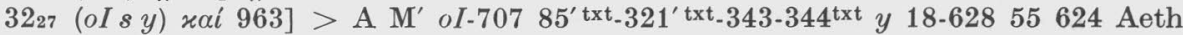

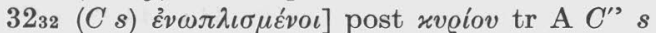

$32{ }_{36}(b y) \quad N \alpha \mu \beta \varrho \alpha ́$ ] $\alpha \mu \beta \varrho \alpha \nu$ A 381' $52^{\prime}-414^{\prime}-528-529 b 54 \quad y^{-392} 55$

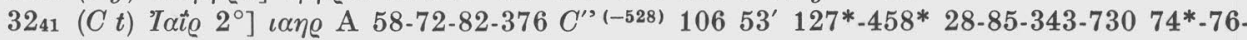
8459319799

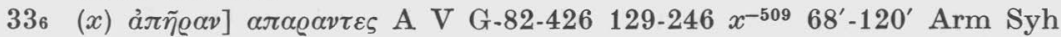

$\left.33_{15}(C \& y) \Sigma_{\iota v a ́}\right]$ pr $\tau \eta$ A F $58 C^{\prime \prime}-422529761^{*} 118^{\prime} 129 s y^{-318} 68^{\prime}-12059$

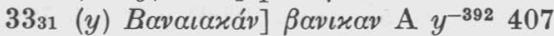

3332 (y) idem A $y^{-392} 407$

$\left.33_{41}(O f) \varepsilon i \zeta\right] \varepsilon v$ A Bc $\mathbf{M}^{\prime}$ O-29-707 $56^{\prime}-129509 \quad 121 \quad 68^{\prime}-120^{\prime} 59$

$\left.33_{47}(C s y) \Delta \varepsilon \beta \lambda \alpha \vartheta a ́ l \mu\right] \delta \alpha \iota \beta \lambda$. A G $C^{\prime \prime}-4657414529(551) 552127$ 28-30-85-321'-344 84* $y^{-318}$ 407

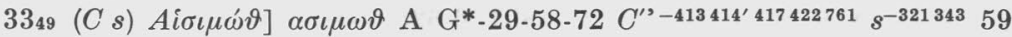

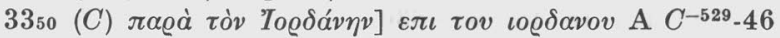

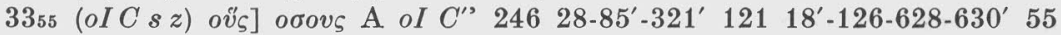

$\left.34{ }^{2}(o I C f t) \sum_{\varepsilon} v v \alpha\right] \sigma \varepsilon \varepsilon v v \alpha \varkappa \mathrm{A} o I^{-618} C^{\prime \prime}-52320413414417528 f^{-129}$ 28-85-343 121 126-128-628669

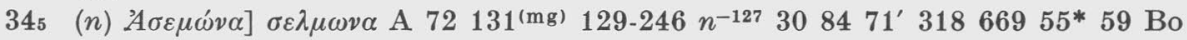


$\begin{array}{lllll}346 & (b) & i & 1^{\circ} & 963]\end{array}>$ A $82 \quad b \quad 121$

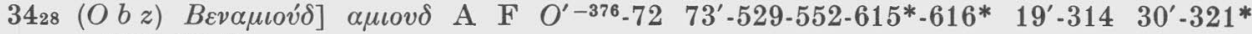
$z^{-1868^{\prime}} 5559$

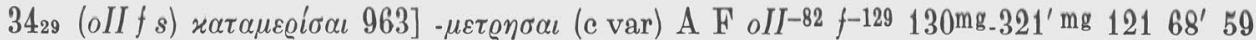
Latcodd 100104 Aeth

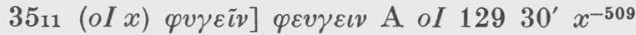

$3515(x)$ idem A $29129 * x^{-509}$

$\left.355_{31}(o I z) \pi \varepsilon \varrho i\right]$ e $\varepsilon \iota$ A V oI 414246121 18'-126-628-669 55

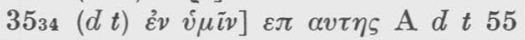

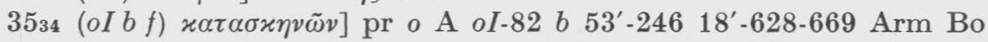

In the following table variants from list 7 in which no more than four text groups support an A variant are also included. Vertical column $A$ gives the number of instances in which one text group supports an A variant; column $B$, two text groups; column $C$, three, and column $D$, four text groups. The final column gives the total number of instances of a text group supporting a variant also supported by codex A. The sub-groups $o I / o I I$ have been differentiated only when $O$ is not involved. The $C$ groups have not been kept apart. The $s$ readings supported by the majority of $85^{\prime} \mathrm{mg}_{-} 321^{\prime} \mathrm{mg}_{-} 344^{\mathrm{mg}}$ have been included.

$\begin{array}{lrrrrrrr} & A & B & C & D & \text { Total } & & \\ O & - & 3 & 5 & 7 & 15 & 11 & \\ \text { oI/oII } & 9 & 14 & 18 & 33 & 74 & 1 & - \\ C & 6 & 9 & 18 & 29 & 62 & 4 & - \\ b & 8 & 14 & 15 & 12 & 49 & 5 & - \\ d & 1 & 6 & 16 & 5 & 28 & 9 & \\ f & 4 & 8 & 8 & 6 & 26 & 18 & \\ n & 5 & 15 & 11 & 12 & 43 & 6 & \\ s & 3 & 9 & 21 & 34 & 67 & 3 & - \\ t & - & 9 & 15 & 7 & 31 & 7 & \\ x & 5 & 5 & 4 & - & 14 & 12 & \\ y & 10 & 14 & 20 & 28 & 72 & 2 & - \\ z & 3 & 6 & 11 & 9 & 29 & 8 & \end{array}$

The affiliations of $\mathrm{A}$ are quite different from those of $\mathrm{B}$. The clearest picture of A's affinities is found in the totals of the last column. At the head of the list stands $o I$ (and $o I I$ ) with 74 instances of agreement, followed by $y$ with 72, $s$ with 67 and $C^{\prime \prime}$ with 62 . These are then followed by $b$ with $49, n$ with 43 , $t$ with $31, z$ with $29, d$ with 28 and $f$ with 26 . At the end of the list are $O$ (as distinct from its sub groups with 15 and $x$ with 14 .

Over against this agreements with $\mathrm{B}$ found $y$ as farthest removed from $\mathrm{B}$ with only 2 agreements. This was followed by $s$ with $5, C^{\prime \prime}$ with 9 and oI/oII with 10 ; i. e. the four which head the list in agreeing with $A$ are at the bottom of the list of agreements with $\mathrm{B}$. On the other hand, $x$ stood closest to $\mathrm{B}$, but is farthest removed from the A type text.

It would then appear that it is not entirely false to speak of an A type text over against a B type text as far as the text history of Numbers is concerned. 


\section{Chapter 5 Papyrus 963 As Textual Witness}

963 is our oldest substantial Greek witness for Numbers; its outstanding importance is illustrated by the fact that it was copied before the time of Origen, i.e. it must represent a so-called prehexaplaric text. Because of its age its text has on occasions been taken as determinative for the text of Num, e.g.

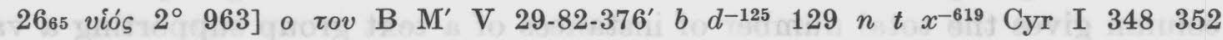
$\mathrm{Syh}=\mathrm{Ra}$

In designating the immediate ancestry of Caleb and Joshua Num fluctuates

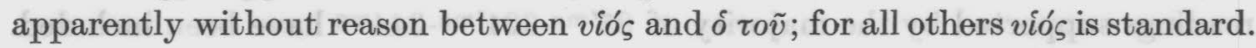
Since no pattern of translation is discernible it seemed best to follow the oldest witness, particularly since codex $\mathrm{A}$ also supports the reading.

Not that one should exhibit undue reverence for 963 just because of its age. The copyist was not overly careful as the following list of unique readings shows.

\section{List 1}

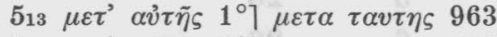

$\left.513 \mu \varepsilon \tau^{\prime} \alpha v i \tilde{\eta} \varsigma 2^{\circ}\right] \mu \varepsilon \tau \alpha$ $\tau \alpha v \tau \eta \varsigma 963$

519 ảंò $\tau o \tilde{v}]$ [...] 963

Probably 963 omitted $\tau o \tilde{v}$ in view of the next variant.

519 om $\tau o \tilde{v} 2^{\circ} 963$

$5_{21}$ om $\varepsilon^{\prime} v 1^{\circ}-\gamma v v \alpha \iota x i 9^{\text {txt }}$

This was simply the result of carelessness. The word before $\dot{\varepsilon} v$ is $\gamma v$ vaix $\alpha$.

$\left.5_{27} \pi \varrho \eta \sigma \vartheta \dot{\eta} \sigma \varepsilon \tau \alpha \iota\right] \pi \varrho \eta \vartheta \eta \sigma \varepsilon \tau \alpha \iota 963$

$64 \alpha \dot{\alpha} \mu \varepsilon \dot{\lambda} \lambda o v] \alpha \pi \varepsilon \lambda o v 963$

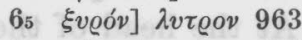

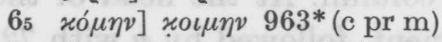

611 á $\gamma \iota \alpha ́ \sigma \varepsilon l] \alpha[\gamma \iota] \eta \sigma \eta 963^{*}$ (c pr m)

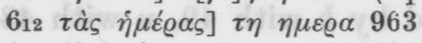

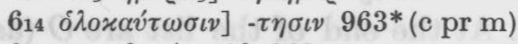

617 om $\vartheta v \sigma i \alpha v 1^{\circ} 963$

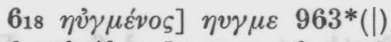

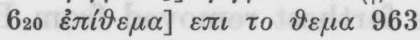

$621 \alpha v ่ \tau \circ \tilde{v} 3^{\circ}$ ] $\alpha v \tau \eta \varsigma 963$

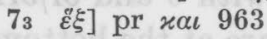

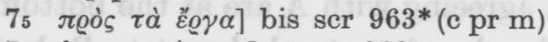

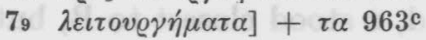

$\left.7_{12} \AA^{A} A \iota v \alpha \delta \alpha ́ \beta\right] \alpha \mu \varepsilon \iota v \alpha \delta \varepsilon \beta 963$

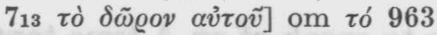

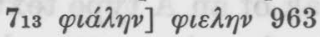

963 consistently spells $\varphi \iota a ́ \lambda \eta v$ with an $\varepsilon$ in this chapter. The reading is also extant in vv. $19,25,37,43,49$, $55,61,67,73$ and 79 ; in each instance the spelling is unique.

$\left.718 N \alpha \vartheta \alpha \nu \alpha \eta^{\prime} \lambda\right] \quad \nu \alpha \nu \alpha \vartheta \alpha \eta \lambda \quad 963^{\mathrm{c}}$ (vid)

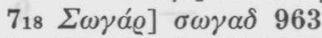

$\left.7_{29}{ }^{2} E \lambda_{\iota \alpha} \beta\right] \quad \varepsilon \lambda_{\varepsilon \iota \alpha} \beta \quad 963$

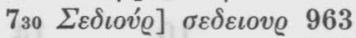

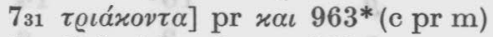

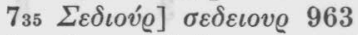

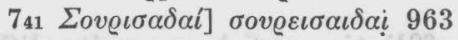

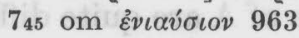

748 om $\tau \tilde{\omega} \nu 963$

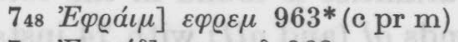

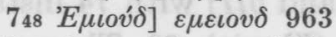

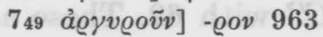

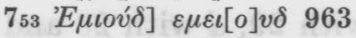

754-59 bis ser $963^{*}$

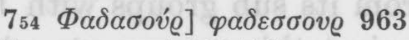

758 om $\pi \varepsilon \varrho i$ $963\left(1^{\circ}\right)$

759 om èvı

$\left.{ }_{759} \Gamma \alpha \mu \alpha \lambda \iota \eta^{\prime} \lambda 963\left(2^{\circ}\right)\right] \varepsilon \lambda \varepsilon \iota \sigma \alpha \mu \alpha 963\left(1^{\circ}\right)$

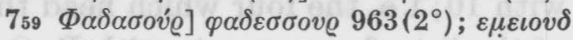
$963\left(1^{\circ}\right)$

760 A $A \beta \iota \delta a ́ v] \alpha \beta \varepsilon \iota v \alpha \delta \alpha v 963$

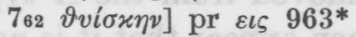

$7_{63}$ om हैंx - (64) xaí 963

$\left.7_{70} \varepsilon \tilde{v} \nu \alpha\right]$ Ev $963^{*}(\mathrm{c} \mathrm{pr} \mathrm{m})$ 


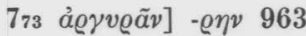

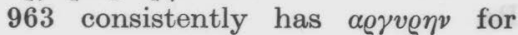

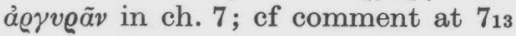

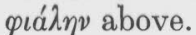

$\left.7_{77}{ }^{\circledR} E \chi \varrho \alpha ́ v\right][\varepsilon] \chi \vartheta \varrho \alpha v 963$

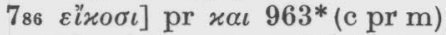

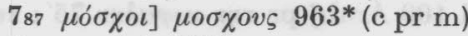

83 om Evvós 963

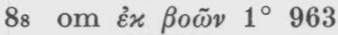

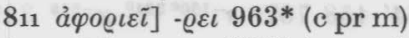

$812 \tau \tilde{\omega} v] \alpha v \tau \omega \nu \quad 963^{*}$ (c pr m)

813 है $v \alpha \nu \tau \iota 4^{\circ}$ ] $-v \tau \iota 0 v 963^{*}(\mathrm{c}$ pr m)

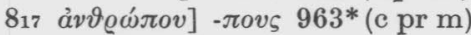

$817 \gamma \tilde{\eta}] \tau \eta 963$

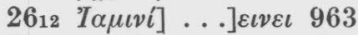

2633 $M \alpha \chi i \varrho 1^{\circ}$ ] $\cap 2^{\circ} 963$

$2664 \tau \tilde{\omega} \nu] \tau \omega$ 963*

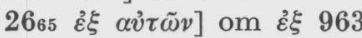

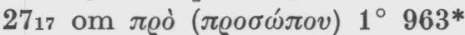

$2717 \pi$ $^{\circ} 2^{\circ}$ ] $\alpha \pi \circ 963^{*}$

$27_{22}$ Év $\left.v v i i^{\prime} v 1^{\circ}\right] \cap 2^{\circ} 963^{*}$ (vid)

$\left.29_{12} \dot{\eta} \mu \varepsilon \dot{\varepsilon} \varrho\right] \tau \eta \mu \varepsilon \varrho \alpha 963$
2914 om rai $1^{\circ} 963^{*}$ (vid)

$29_{23}$ om $\tau \tilde{\eta} \dot{\eta} \mu \dot{\varepsilon} \varrho a$ 963*

$29_{35}$ om ov $963^{*}$

$29_{36}$ Évıavoíovs] pr $\vec{\varepsilon} 963$

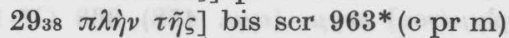

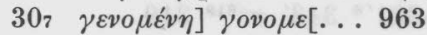

$\left.308 \quad \sigma \tau \eta \dot{\sigma o v \tau \alpha \iota} 2^{\circ}\right]$ $\sigma v \sigma \tau \eta[. .963$

$\left.3014 \varepsilon \dot{\chi} \chi \dot{\eta}^{\prime}\right] \varepsilon v \chi \eta v$ 963*(c pr m)

$3016 \mu \varepsilon \tau \dot{\alpha} \tau \eta \dot{v} v] \mu \varepsilon \tau \quad \alpha v[\tau \eta] \nu 963$

3016 om $\eta^{v} 963^{*}$

$\left.31_{12} M \omega \alpha ́ \beta\right] \mu о \alpha[\beta] 963$

$\left.31_{26} \alpha \dot{v} \vartheta \varrho \omega ́ \pi o v\right]$ pr $\tau o v 963 *$ (c pr m)

$\left.31_{40} \delta v \dot{o}\right]$ pr жu 963

$\left.3148 \chi \iota \lambda i c^{\prime} \varrho \chi \circ \iota\right]$ pr $\varkappa \alpha \iota 963$

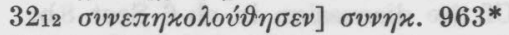

$32{ }_{13}$ ह่ $\left.\pi i\right]$ pr $x \alpha \iota 963^{*}$ (c pr m)

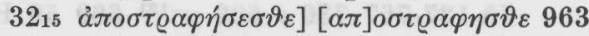

$3229 \varepsilon i \zeta]$ Ev 963*

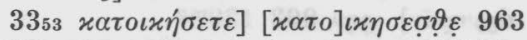

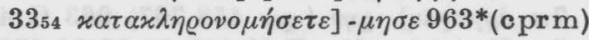

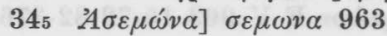

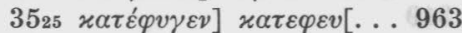

None of these unique readings needs to be taken seriously. Most of them represent careless errors on the part of the copyist, often resulting in an impossible text. Nonetheless in view of the age of the $\mathrm{ms}$ they have all been recorded in the apparatus.

It may be noted that 963 like B has a preference for the itacistic $\varepsilon \iota$ for $\iota$. This is particularly obvious for proper names; ef 72930354148535960 . Since the variant is valueless for text criticism it will be disregarded in subsequent lists.

List 2 gives all the remaining secondary readings in 963 (except for the itacistic $\varepsilon \iota$ spelling for $\iota$ ) with the exception of those which could have been the result of mediate or immediate Hebrew influence.

List 2

$\left.5_{13} \lambda \alpha \dot{\vartheta} \eta\right] \lambda \alpha \beta \eta 9637768^{\prime}$

$\left.5_{13} \eta^{\circ} 1^{\circ}\right] \eta v 96382 \quad b \quad 68^{\prime}-120^{\prime}$

514 光] $x_{\alpha \iota} 963319$

$\left.5_{17} \lambda \alpha \beta \omega v\right] \alpha v \alpha \lambda \alpha \beta \omega v 963$ (vid) $b$

$5_{24}$ xai $\left.1^{\circ}\right] \cap 2^{\circ} 96352^{\prime}-413-414-528 d 767370$ 126-669 Aeth

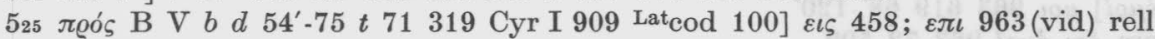

$\left.5_{29} \tilde{\omega}\right]$ o 963707

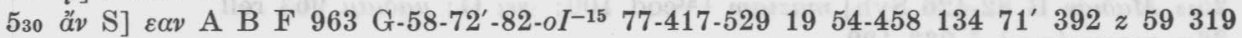

$=\mathrm{Ra}$

64 o[vov] oเvov $963 *$ (c pr m) 319

65 om $\tau \tilde{\eta} \varsigma \varepsilon \dot{v} \chi \tilde{\eta} \varsigma$ B 963664 54-75' 28-85 $x^{-619} 628799$ Cyr I 1041 Arm Sa ${ }^{4}$

67 દ̇лi $2^{\circ} \mathrm{A} \mathrm{B}^{*} \mathrm{~V}$ G-29-72-376-oI $I^{-15} b d^{-125} n 130^{\mathrm{mg}-321^{\prime} \mathrm{mg}} t 61931855319 \mathrm{Aeth}^{-\mathrm{CG}}$

Arm] $>963$ rell

67 om av่นoṽ $1^{\circ} 9637252^{\prime} 53^{\prime} 7571628$ Lat $^{\circ}$ cod 100 Arm

$68 x v \varrho i \omega$ A B V G-29-426 $761 n^{-767} 50955$ Cyr I 1041] pr $\tau \omega 963$ rell

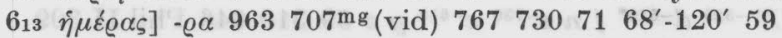

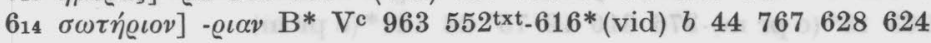


$615 \vartheta v \sigma i \alpha v] \vartheta v \sigma \iota \alpha\left(-\sigma \iota 963^{*}\right)$ B $963 n^{-458} x^{-619}=$ Ra

$615 \sigma \pi \circ \nu \delta \dot{\eta} v] \sigma \pi 0 \nu \delta \eta$ (c var) B $963 n x^{-619}=\mathrm{Ra}$

616 om $\tau o ́$ $1^{\circ} 963$ Latcod 100

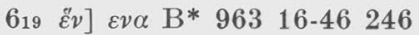

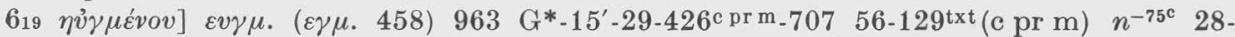
$30-85^{\prime}-321^{\prime} *-343^{\prime} x^{-619} 319$

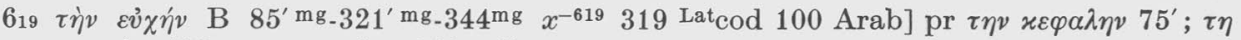

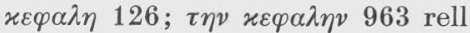

$\left.6{ }_{20} \alpha \tilde{\gamma} \iota \circ \nu\right] \alpha \gamma \iota \alpha, 963 \mathrm{Sa}^{4}$

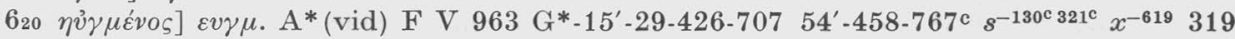

625 om $\sigma \varepsilon 2^{\circ}$ B $963 x^{-619}$

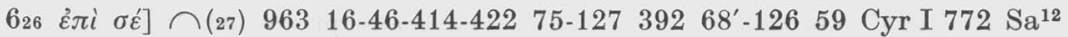

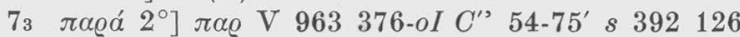

$710 \tau \dot{\alpha} \delta \tilde{\omega} \varrho \circ \nu$ Aeth] $\tau \alpha(>19) \delta \omega \varrho \alpha 963$ rell $=\mathrm{Ra}$ Cf the discussion in ch 6 Sect $8 \mathrm{~b}$.

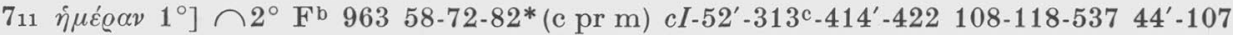
75-127-767 $730 t 509 y^{-121} 66959$ Bo

$712 \quad N \alpha \alpha \sigma \sigma \omega v] v \alpha \sigma \sigma \omega \nu 963$ Bo

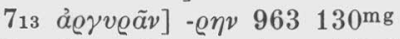

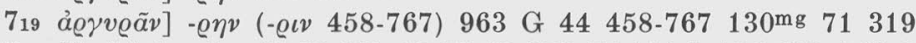

$\left.7_{24} X \alpha \iota \lambda \omega \omega\right] \quad \chi \varepsilon \lambda \omega \nu$ F V 963 15-72-82-376 $77-414 b \quad 125-610 f^{-56} \quad 54-75^{\prime} \quad 130^{*} \quad 76-84 x 318$ 669319

$725 \alpha \varrho \varrho \gamma v \varrho \tilde{\nu}]]-\varrho \eta v(-\varrho \iota v 458) 963458 \quad 509319$

$7_{31}$ idem $963130^{\mathrm{mg}} 319$

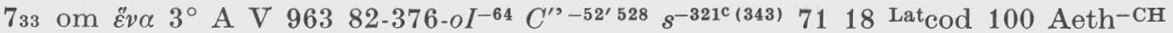

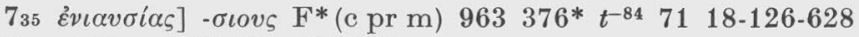

735 vioũ] vios $\mathrm{V} 963$ 458-767

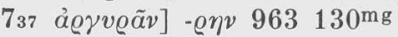

$741 \quad$ Èv $\iota \alpha v \sigma i \alpha \varsigma]-\sigma \iota 0 v \varsigma 9635256 \quad 619 \quad 18-126-628$

741 vioṽ] vıos $96329-707^{*} 30-343^{\prime} 74^{\prime} 392$

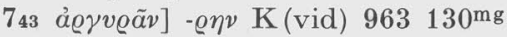

747 om $\tau$ tó 963413

755 å

$7_{61}$ idem $963130^{\mathrm{mg}}$

$\left.7_{65} \Gamma \alpha \delta \varepsilon \omega \nu i\right]$

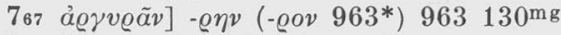

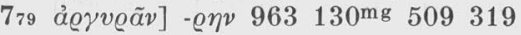

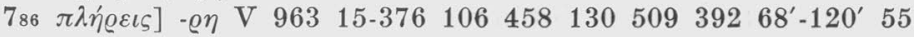

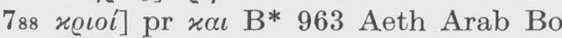

$788 \alpha ⿱ \mu \nu \alpha \alpha \delta \varepsilon \varsigma]-\delta \alpha \varsigma$ 963* 72509121

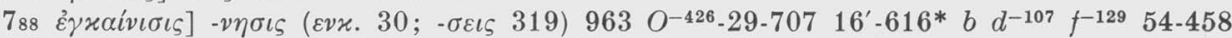
$30-130$ 84-134 71 y $68^{\prime}-120 *-128-628319$

789 xv@íov] pr vov 96345884

$\left.789 \chi \varepsilon \varrho \circ \beta_{i} \mu\right]-\beta \varepsilon เ \nu$ F 963 15*-64-707 30-85'-343'

$81 M \omega v \sigma \tilde{\eta} v] \mu \omega \sigma \eta v 963426 n$ Cyr I 608

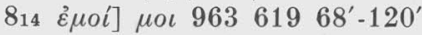

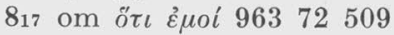

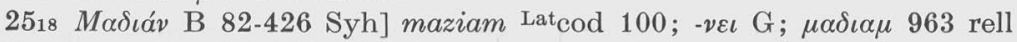

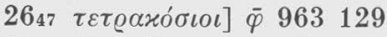

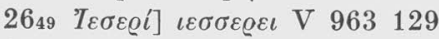

$\left.26_{49} \Sigma \varepsilon \lambda \lambda \eta^{\prime} \mu\right] \sigma \varepsilon \lambda \eta \mu \quad 963 \quad 58-376-381 *_{-618} 77 b^{-19} 129307131859$

$2649 \Sigma \varepsilon \lambda \lambda \eta \mu i ́] \quad \sigma \varepsilon \lambda \eta \mu \varepsilon \iota 9637712971$

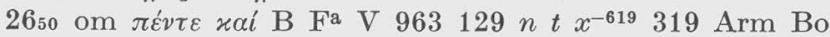

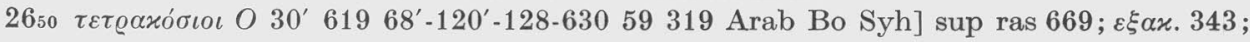
$\tau \varrho \iota \alpha \varkappa .963$ rell

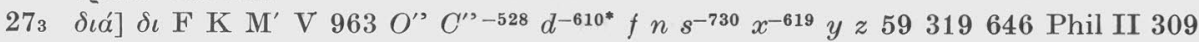

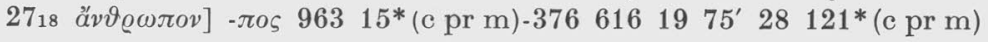


$2718 \alpha v ่ \tau o ́ v] \cap(19) 963 * 130-321^{\prime}$

2721 oi (vioí)] >963 $24661968^{\prime}-126319$

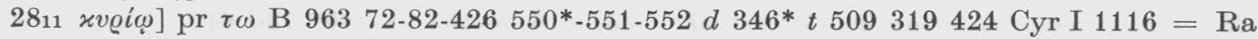

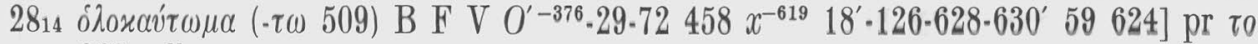
963 rell

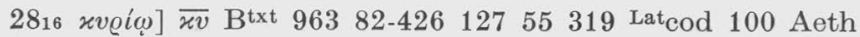

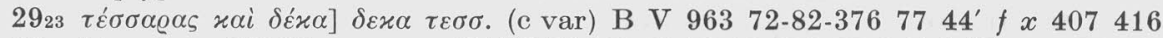

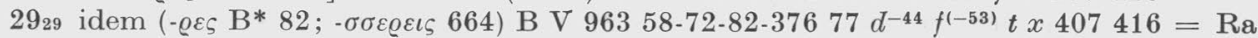

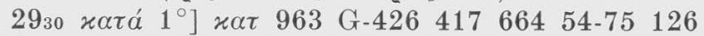

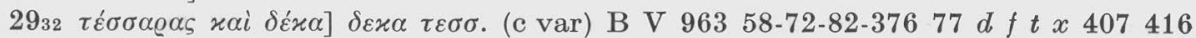

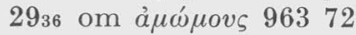

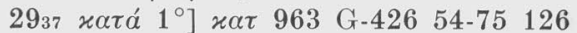

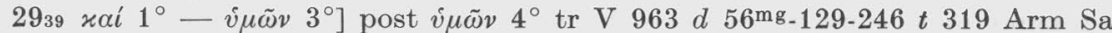

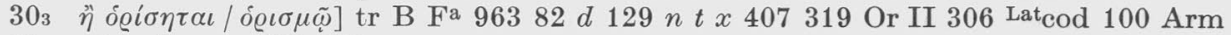

3013 xं́øı૬] pr o 963458

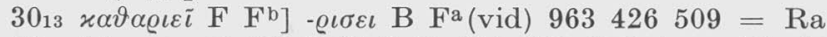

$3016 \lambda \eta \dot{\mu} \mu \varepsilon \tau \alpha \iota$ A B* F V G-82 56* 509624$] \lambda \eta \psi \eta 46^{\mathrm{s}} ; \lambda \eta \psi . \mathrm{F}^{\mathrm{b}} 963$ rell

$316 \quad \sigma \eta \mu \alpha \sigma \iota \tilde{\omega} v]$ ] $\sigma \eta \mu \varepsilon \iota \omega \nu 963 \quad 72-426$

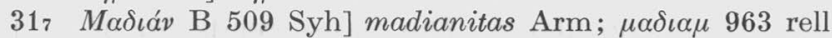

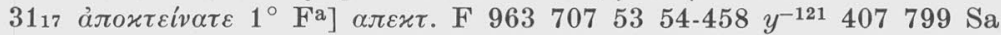

$3121 \quad \delta$ 963*(vid)] ov $\mathrm{V} 963^{\mathrm{e}} 129 z^{-407}$

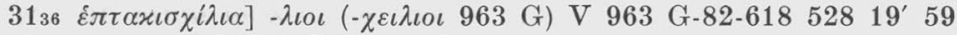

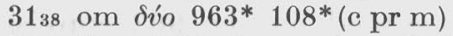

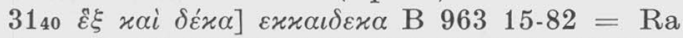

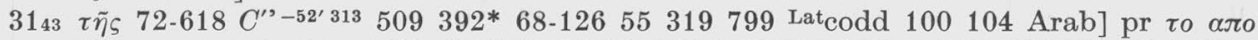

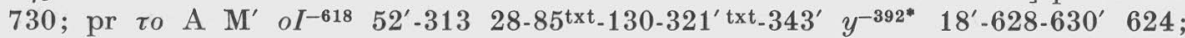
pr ало 963 rell

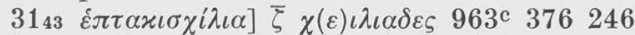

$\left.31_{49}(\pi \circ \lambda \varepsilon \mu \iota \sigma \tau \tilde{\omega} v) \tau \tilde{\omega} \nu\right]>963$ (vid) 15417319

$3211 \ddot{\omega} \mu о \sigma \alpha]-\sigma \varepsilon(v)$ B 963 G-82-426 414 129-246* $x 407$ Aeth

3212 viós] pr o $963^{*}\left(\mathrm{c}\right.$ pr m) $7252^{\prime}-313 x^{-509}$

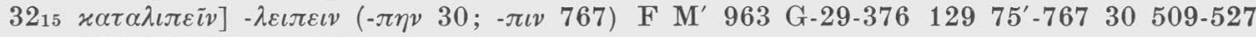
$392 \quad 407-628 \quad 55319$

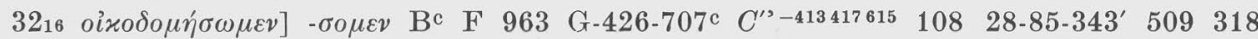
$18^{\prime}-68^{\prime}-120^{\mathrm{c}}-126-630^{\prime} 624$ Cyr I $404{ }^{\text {Lat }}$ cod 100 Co

$322_{21}$ oл $\left.\lambda i \tau \eta \varsigma\right]$ o $\pi \circ \lambda \iota \tau \eta \varsigma 963624$

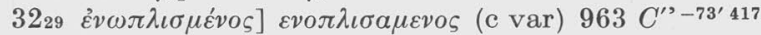

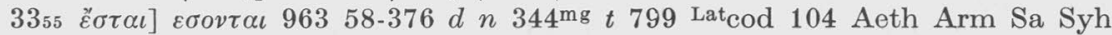

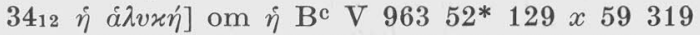

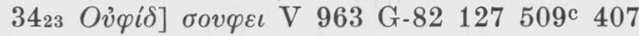

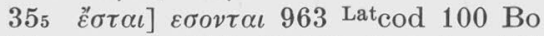

356 om ås $1^{\circ} \mathrm{B} \mathrm{V} 96382 b f x 407319$ Cyr I $865=\mathrm{Ra}$

Many of these are obviously secondary, and can be dismissed out of hand. In a number of cases 963 does support a popular variant. Thus 963 proves the

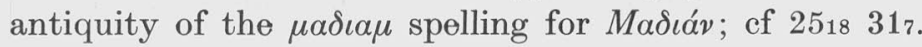

More problematic are a few readings which seem to show recensional influence even though the papyrus is pre-Origen in date. In the following list all instances of variant readings witnessed by 963 which either equal $\mathfrak{m}$ or equal a known hexaplaric reading are given.

\section{List 3}

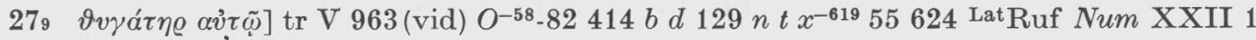
Syh: לו בו 


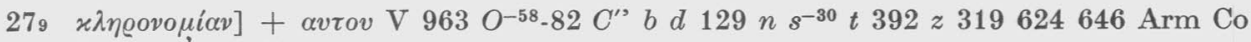
Syh: נחות $2 \pi$

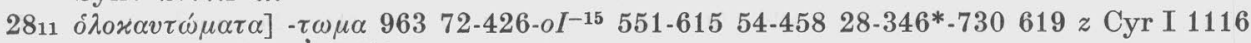
Aeth Sa Syh: עלה $2 \pi$

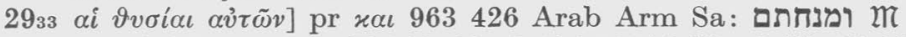

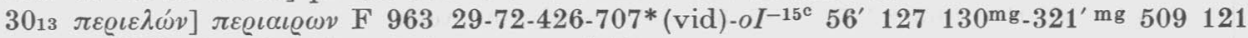
$z^{-126407} 59416$ 646: = o' $\pi \varepsilon \varrho \iota \iota \varrho \tilde{\omega} v 344$

3016 idem A F $96329-426-o I^{-15^{\mathrm{c}}} b^{-19} 56^{\prime} 127 \quad 130^{\mathrm{mg}}-321^{\prime} \mathrm{mg} 121 z^{-126407} 416624646$ : $=o^{\prime} \pi \varepsilon \varrho \iota \iota \varrho \tilde{\omega} \nu 344$

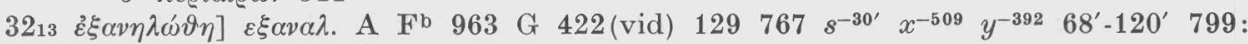
$=\sigma^{\prime} \dot{\varepsilon}^{\prime} \xi \alpha \nu \alpha \lambda \omega \vartheta \tilde{\eta} 344^{\mathrm{txt}}$

$3515 \tau \tilde{\omega} \quad \dot{\varepsilon} v \dot{v} \mu \tilde{i v}]$ om $\tau \tilde{\omega} 963129$ Arm: בתוכם

This is probably mere coincidence since the $\tau \tilde{\omega}$ follows $\pi \alpha \varrho \circ i x \omega$ and might easily be omitted because of homoioteleuton.

Only the first two instances in the list need in my opinion be taken seriously. The change in word order in 279 could be coincidence since it makes no difference as far as the Greek context is considered. The clause reads $\varepsilon \dot{\alpha} v \delta \dot{\varepsilon} \mu \dot{\eta} \eta \tilde{\eta}$

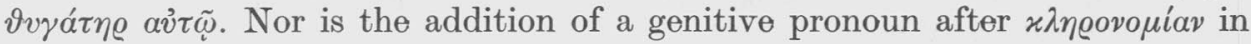

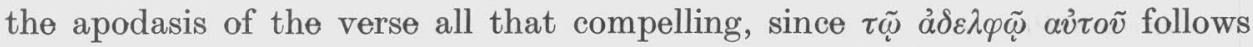
$x \lambda \eta \varrho o v o \mu i \alpha v$ in the Num text. That an $\alpha v \tau o v$ was added by the 963 copyist may be due to the fact that av่

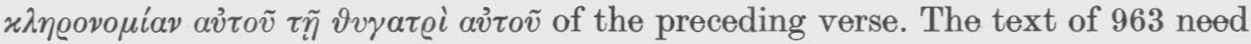
not be due to Hebrew influence at all.

It is, however, in its positive witness to Num that the real value of 963 lies. In list 4 are presented all instances in which 963 has the Num text, but A and $\mathrm{B}$ do not.

\section{List 4}

62 åv 963] $\varepsilon \alpha v$ A B $n 318$ Or II 316 Tht $N m$ 197ap $=$ Ra

$64 \quad \sigma \tau \varepsilon \mu \varphi v ่ \lambda \omega v$ 963] $-\lambda \lambda \omega v$ A B $707 \quad 56^{\prime}-12954-75^{\prime} s^{-130 m g} 343$ y 126646

$814 \tau \tilde{\omega} v 963]>\mathrm{A} \mathrm{B} O^{-58} d 127-767 t x^{-619} 121=\mathrm{Ra}$

2917 init $963 \quad b \quad 509]$ pr $x \alpha \iota$ rell $=$ Ra $\mathfrak{k}$

2935 init V $96346129-24612163059$ Latcodd 100104$] \mathrm{pr} \varkappa \alpha \iota$ rell $=\mathrm{Ra}$

$\left.29_{36} x \alpha ́ \varrho \pi \omega \mu \alpha 9^{*} 3^{*}\right]-\mu \alpha \tau \alpha$ A B $963^{c} 58-82129509 y^{-392} z 646$ Sa $=\mathrm{Ra}$

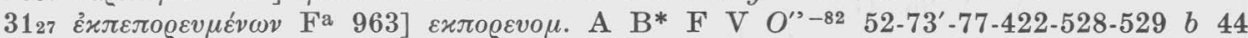
$f^{-129} 54-75^{\prime} x^{-527}$ y $68^{\prime}-126-6695559319624799=\mathrm{Ra}$

3145 xai $1^{\circ} 963 O^{\prime-58-72} C-46118^{c} n^{(-75)} t$ Latcodd 100104 Aeth ${ }^{C}$ Arab Arm Bo Syh] $>$ rell $=\mathrm{Ra}$

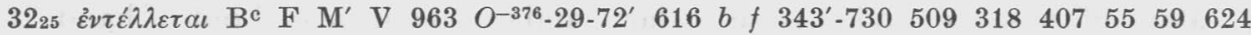
Latcodd 100104 (vid)] $\varepsilon v \tau \varepsilon \lambda \varepsilon \iota \tau \alpha \iota \mathrm{B}^{*} x^{-509}$; $\varepsilon v \tau \varepsilon \tau \alpha \lambda \tau \alpha \iota$ (c var) rell

$347 \varkappa \alpha \tau \alpha \mu \varepsilon \tau \varrho \eta ́ \sigma \varepsilon \tau \varepsilon$ 963] - $\sigma \varepsilon \tau \alpha \iota$ (c var) A B M $\mathrm{M}^{\prime} \mathrm{V} 376$ 57-73'-313-615-761c $53^{*}-56^{\prime} 75^{\prime} 509$ 55319799 Aeth

$367 \pi \alpha \tau \varrho \iota x \tilde{\eta} \varsigma$ 963] $\pi \alpha \tau \varrho \iota \alpha \varsigma$ A B F $\mathrm{F}^{\mathrm{a}} \mathrm{F}^{\mathrm{b}} \mathrm{M}^{\prime}$ oI ${ }^{\prime-82707} C^{\prime \prime} 246 s^{-85^{\mathrm{mg}}{ }_{344^{\mathrm{mg}}}} x y z^{-120} 5559319$ Cyr IX $900=\mathrm{Ra}$

Most of the instances in this list represent improvements to the Ra text and are discussed in chapter 6 below. The other instances are fairly obviously early errors already found in the old uncial witnesses.

List 5 represents instances where 963 confirms the reading of $\mathrm{A}+$ over against the secondary reading of $\mathrm{B}+$. 
$\left.5_{13} \eta^{\circ} 2^{\circ} 963\right] \eta v$ B Cyr I $909=$ Ra

$527 \dot{\varepsilon} v 963]>$ B S* 68' 799 Cyr I 909 Armap $^{a}$

529 $\stackrel{\alpha}{2}$ 963] $\varepsilon \alpha v$ B G $19458319=\mathrm{Ra}$

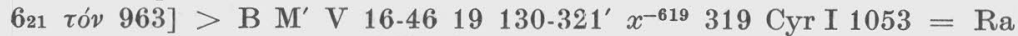

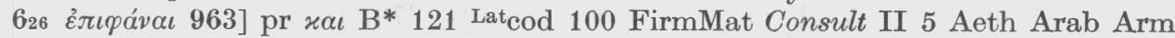

$715 \varepsilon v \alpha 3^{\circ} 963$ ] $>\mathrm{B} \mathrm{F}^{*}\left(\mathrm{c}\right.$ pr m) V 29-82-707txt $f n^{-767} 730 x^{-619} 126-628319$ Lat $^{\circ}$ cod 100 Aeth $^{-\mathrm{C}}$

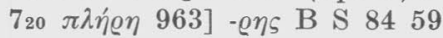

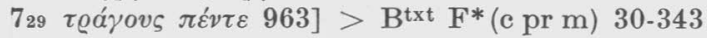

$\left.7_{41} \tau \varrho \alpha ́ \gamma o v \varsigma \pi \varepsilon ́ v \tau \varepsilon 963\right]>\mathrm{B}^{\text {txt }} \mathrm{F}^{*}(\mathrm{c}$ pr m) 2931818 Latcod 104

767 $\pi \lambda \eta \dot{\varrho} \eta 963]-\varrho \eta \varsigma$ B 70776784

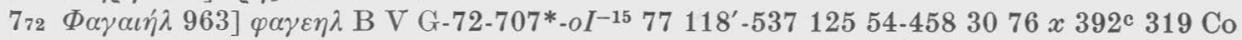

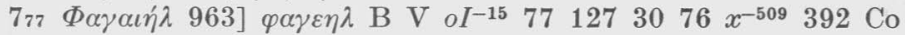

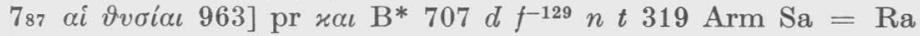

$86 \tau \tilde{\omega} \nu 963]>\mathrm{B} O^{-58} d^{-610} 127-767 t^{-84} 50955319=\mathrm{Ra}$

$\left.88 \sigma \varepsilon \mu i \delta \alpha \lambda_{l v} 963\right]-\lambda \varepsilon \omega \varsigma$ B $7168^{\prime}-120^{\prime} 59=\mathrm{Ra}$

2622 o $\left.3^{\circ} 963\right]>\mathrm{B} 413-414 * 246 * 767$

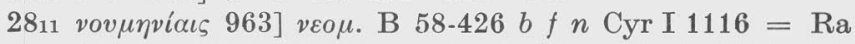

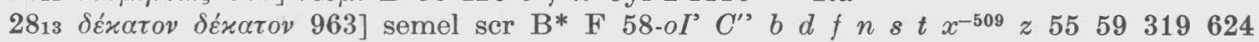
Aeth $^{-\mathrm{C}}$ Bo $=$ Ra

2815 xv@i $\omega 963] \overline{x v} \mathrm{~B}^{*}$

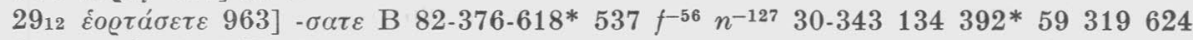

2912 xv@i $\omega 963] \overline{x v} \mathrm{~B}^{*}$

$29{ }_{13} \varkappa \alpha ́ \varrho \pi \omega \mu \alpha$ 963] - $\mu \alpha \tau \alpha \mathrm{B}^{*} 58-82 n^{-458} \mathrm{Arm} \mathrm{Sa}=\mathrm{Ra}$.

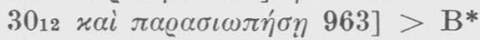

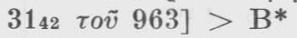

$3354 v \mu \tilde{\omega} v 1^{\circ}$ 963] $\alpha v \tau \omega v$ B $381^{*}$ (vid) 16-46 458 84* 392319

3515 xаi $\left.\tau \tilde{\varphi} 2^{\circ} 963\right]$ om xai $\mathrm{B}^{*} 509319$

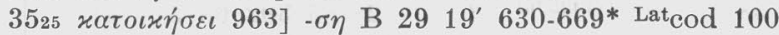

$3532 \tau \tilde{\omega} v 963]>\mathrm{B}^{*} \mathrm{~V} 53^{\prime}-129$

$367 \pi \varrho \circ \sigma \circ \lambda \lambda \eta \vartheta \dot{\eta} \sigma o v \tau \alpha \iota$ 963] pr $\varkappa \alpha \iota \mathrm{B} x$

In this list 963 and codex A both support Num, whereas B represents a variant text. For those variant readings in the list which Rahlfs adopted as his text ef the discussions in ch. 6 .

In the final list 963 confirms the B text as Num, whereas A constitutes a secondary text.

\section{List 6}

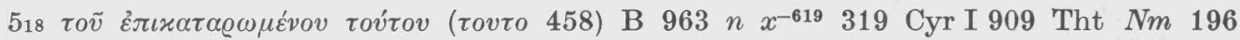

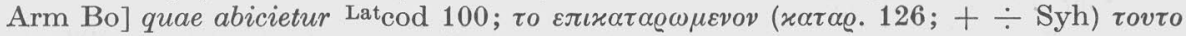
$(\mathrm{sub} \div \mathrm{G} ;>58 \mathrm{~b}$ Aeth $=\mathfrak{W})$ rell

521 xv́@ı́s $\sigma \varepsilon$ B F $963 O^{\prime-376} d f t x^{-619} y z^{-68^{\prime}} 12659799$ Chr II 917 Latcod 100] $\sigma \varepsilon$ o $\overline{x \varsigma} 552$; $\sigma o \iota \overline{x \varsigma} 616^{\mathrm{c}} 54$ Tht $N m$ 197ap; om $\sigma \varepsilon 3766^{\prime}$; tr rell

$527 \varepsilon e^{a} \nu$ B S $963 O^{-58} 417^{\text {txt }} f^{-246} x^{-619} 319$ Chr II 917 Cyr I 909 Sa Syh] $\varepsilon \iota(\varepsilon v$ 707) $\mu \varepsilon v$ 72-707*; $+\mu \eta 528 ;+\mu \varepsilon v$ rell

$65 \tau$ นิ

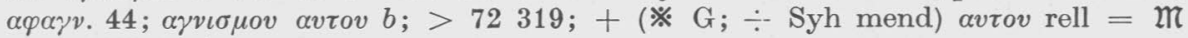

65 ö $\left.\sigma \alpha_{\varsigma} 963\right] \alpha \varsigma \mathrm{A}$

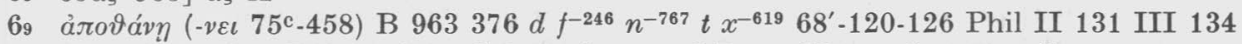
Clem I 92 Cyr I 1048 Arm Co] $+\vartheta \alpha \nu \alpha \tau \omega 319=\mathfrak{m}$; pr $\vartheta \alpha v \alpha \tau \omega$ rell

$610 x \alpha i \tau \tilde{\eta} \mathrm{B} \mathrm{V} 963 O^{-58} d f n t x^{(-619)}$ Cyr I $\left.10411048 \mathrm{Bo} \mathrm{Sa}^{4}\right]$ om $x \alpha i$ 392; $\tau \eta \delta \varepsilon$ rell

610 voббoús (voбovs G-707*) B F V 963 G-15'-426-707 127 30-130-321'-343' 134 55]

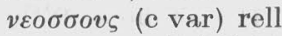

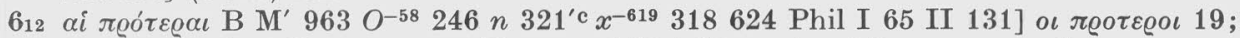
$\alpha \iota \pi \varrho \circ \tau \varepsilon \varrho \circ \iota \mathrm{F} b^{-19} ; \alpha \iota \pi \varrho o^{\tau} \varrho 126 ;$ om $\alpha i \mathrm{~V} f^{-246} ; \alpha \iota(>64) \pi \varrho \circ \tau \varepsilon \varrho o v$ rell 
$\left.614 \varepsilon v \propto \alpha 2^{\circ} 963\right]>$ A 725294412155319624

618 vंлó B $96344^{\prime} 74-76^{\prime}-134^{c} 509392^{c}$ Cyr I 1053 Latcod 100 Sa] $\varepsilon \pi \iota$ rell

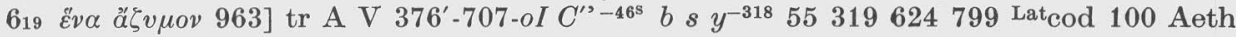
$=\mathfrak{m}$

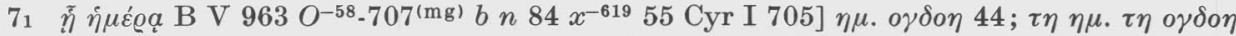

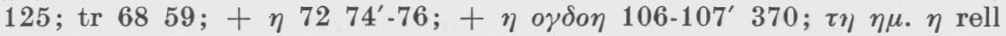

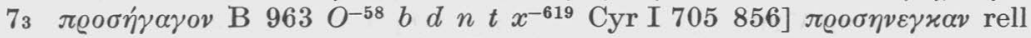

78 viô $\mathrm{B} 963 \quad 376509126 \mathrm{Cyr}$ I 856] pr $\tau$ $90 v$ rell

$\left.751 \varepsilon \tilde{\varepsilon} \alpha \alpha 3^{\circ} 963\right]>A$ V $82-70754-75^{\prime}$ 28-130-343' 509 68*-120-122 Latcodd 100104 Aeth $^{-\mathrm{CH}}$

757 Ëv $\left.3^{\circ} 963\right]>$ A $707 f^{-246} 54-75^{\prime} 343-730509$ 120'-122 Latcodd 100104 Aeth $^{\mathrm{M}}$

$\left.7_{75} \quad \tilde{\varepsilon} v \alpha 3^{\circ} 963\right]>A$ V 82 16-46-528 $767 x^{-619} 18-126$

$785 \tau \tilde{\omega} \nu$ á $\gamma i \omega v$ B $\left.963458 x^{-619}\right] \tau \omega \alpha \gamma i \omega$ rell $=\mathrm{Ra}$

$87 \quad \alpha \gamma \nu \imath \sigma \mu o ́ v$ 963] $\alpha \gamma \nu \iota \alpha \sigma \mu o v \mathrm{~A}$

$87 \pi \tilde{\alpha} v 963]>\mathrm{A}$

$812 \chi \varepsilon \tilde{\varrho} \alpha \varsigma$ 963] + (※ Syh) $\alpha v \tau \omega \nu$ A $O^{-58} b$ 18'-126-628-669 Latcod 100 Arm Co Syh $=\mathfrak{m}$

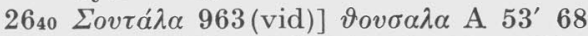

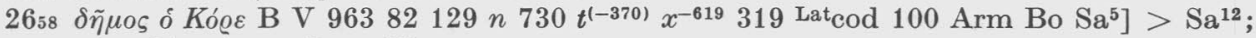
post Movoí tr rell $=\mathfrak{m}$

$2658 \delta \tilde{\eta} \mu \circ \varsigma 3^{\circ} \mathrm{B} \mathrm{V} 963$ (vid) $82129 n t^{(-370)} x^{-619} 319$ Lat $_{\text {cod }} 100 \mathrm{Bo}$ ] pr $x \alpha \iota$ rell $=$ Sam

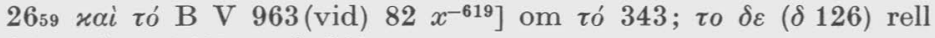

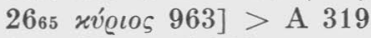

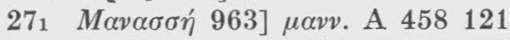

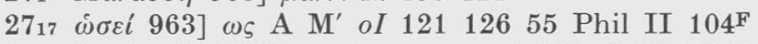

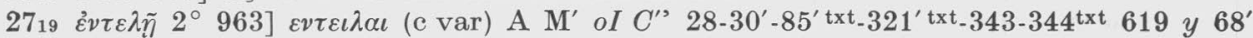
55 LatRuf Num XXII 4 AethC

2720 oi 963$]>A^{*} 707 \quad b^{-19} 75 \quad 30-343 \quad 126319$

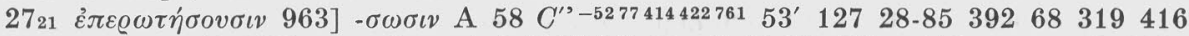

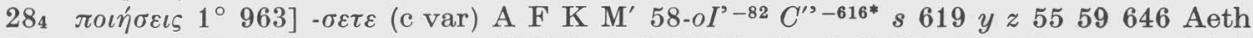

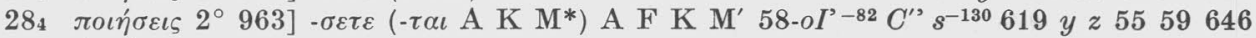
Aeth

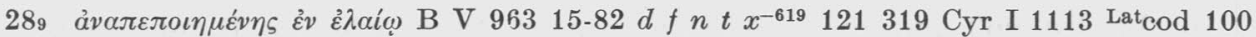
Arm Bo $\left.\mathrm{Sa}^{1}\right]$ post $\vartheta v \sigma i \alpha v$ tr rell $=\mathfrak{m}$

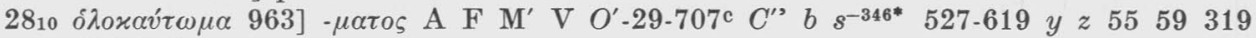
624646

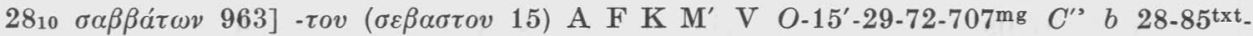
$130-321^{\prime}-343-344^{\text {txt }} 619$ y $z 5559319624646$

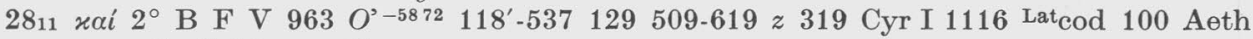
Arm Co $\mathrm{Syh}]>$ rell

$\left.28{ }_{16} \dot{\eta} \mu \varepsilon \dot{\varrho} q 963\right] \cap(17)$ A $72-618 y^{-121^{\mathrm{mg}}} 59624$

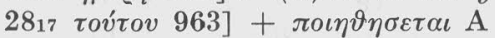

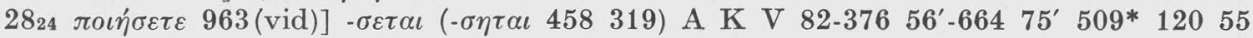
319

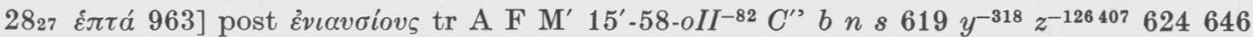
Latcod 100 Aeth Arab Co

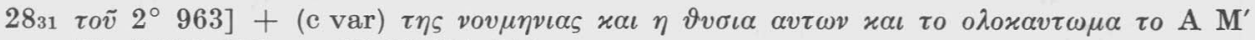
58-oI 127619 y $z^{-407} 55624646$

296 xai $4^{\circ}$ B V $963426131^{(\mathrm{mg})} 129 n^{(-458)} t 407$ Aeth ${ }^{\mathrm{CM}}$ Bo Syh] $>$ rell

2915 init B M' V $963 O^{\prime-58}-82 f^{-246} x 407319$ Cyr I 1120 Aeth Syh] pr $x \alpha \iota$ rell $=\mathfrak{m}$

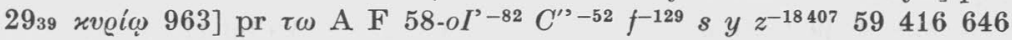

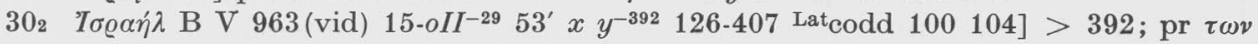
$v \iota \omega \nu(>610)$ G $C^{\prime \prime} d^{-125} n s t 424646 \mathrm{Cyr}$ I 1060; pr $v \iota \omega v$ rell $=\mathfrak{m}$

306 rá $\sigma \alpha \varsigma$ 963] $>$ A V 129-246

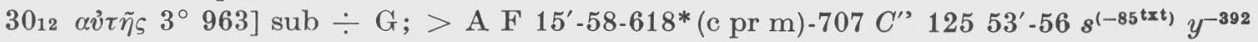
$z^{-407} 5559416624646$ LatAug Num 59.2 ${ }^{\text {te }}=\mathfrak{M}$

$3014 \psi v \chi \eta \dot{v}$ 963] $\alpha v \tau \eta v \mathrm{~A}$ 


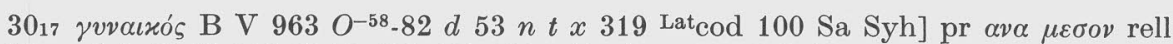

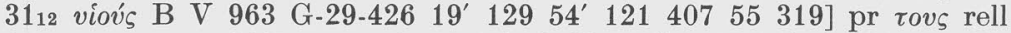

$31_{27} \sigma v v \alpha \gamma \omega \gamma \tilde{\eta}_{\varsigma}$ 963] pr $\tau \eta \varsigma$ ( $\tau \eta$ 75) A $O^{-426}-381^{\prime} 414106^{(\mathrm{mg})} 129 n t^{(-370)} 527 \mathrm{Cyr}$ I 333 bis $=\mathfrak{m}$

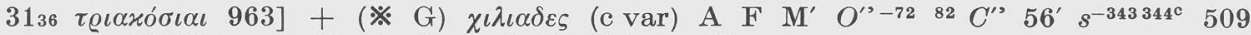
$y z^{-126407} 5559624$ Aeth Arab Bo Syh $=\mathfrak{m}$

$31_{41} \varkappa \alpha \vartheta \alpha^{\prime} 963$ (vid)] $\varkappa \alpha \vartheta \alpha \pi \varepsilon \varrho$ A $15^{\prime} C^{\prime \prime}-41428-85^{\prime}-321^{\prime} 31855624$

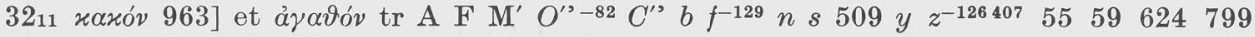
Aeth Arab Bo Syh

$3213 \varepsilon \tilde{\varepsilon} \omega \varsigma 963]+\alpha v \mathrm{~A}$ oI $C^{\prime \prime} b s x^{-509} y^{-121} 18-407-628$

$3220 \dot{\varepsilon} \xi \circ \pi \lambda i \sigma \eta \sigma \vartheta \varepsilon$ 963] $-\pi \lambda \iota \sigma \vartheta \eta \sigma \varepsilon \sigma \vartheta \varepsilon$ (c var) A 59319

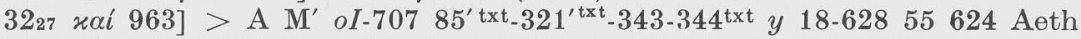

$322_{29}$ oi vioi $\left.2^{\circ} 963\right]$ om oi A $15 \quad 44^{\prime}-125 \quad 56 \quad 75^{\prime} \quad 318$ 126-628 319

$\left.32{ }_{29} \Gamma{ }^{\prime} \dot{\delta} 963\right] \gamma \alpha \lambda \alpha \alpha \delta$ A

$3354 \alpha v i \tilde{\omega} v 1^{\circ} \mathrm{B}^{(\mathrm{mg})} \mathrm{M}^{\prime} \mathrm{V} 963$ (vid) 58-376'-oI 56 $6^{\mathrm{mg}}$-129-664 $n t^{(-84)} 509-527 z 319$ Lat cod 100 $\left.\mathrm{Bo} \mathrm{Sa}{ }^{1}\right] \tau \alpha v \tau \eta v 799$; sub $\div \mathrm{G} \mathrm{Syh;}>$ rell $=\mathfrak{m}$

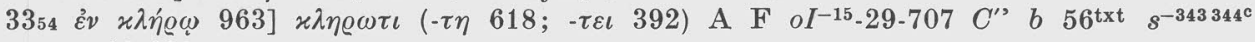
y 18.628

$3355 \varepsilon \dot{\varepsilon} \pi \grave{i} 2^{\circ}$ B $\left.963129 \times 407319 \mathrm{Sa}\right] \mathrm{pr} v \mu \omega \nu 707 * 458$ 18; pr adversos vos Latcod 100; pr $v \mu \iota v$ rell $=\mathfrak{n}$

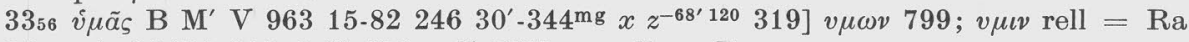

$\left.34_{2} \tau \dot{\eta} v 2^{\circ} \mathrm{B}^{*} 963 \mathrm{O}^{-58} 129 n^{-75} 799\right]>$ rell $=\mathrm{Ra}$

$\left.34{ }_{3} \quad \lambda i \beta \alpha 1^{\circ} 963\right] \beta о \varrho \varrho \alpha \mathrm{A} ; \beta о \varrho \varrho \alpha \nu 55$

344 А $s t y^{-318} \quad 18^{\prime}-126-628-630^{\prime} 55$

$\left.346 \quad \dot{\eta} 1^{\circ} 963\right]>$ A $82 \quad b \quad 121$

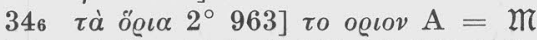

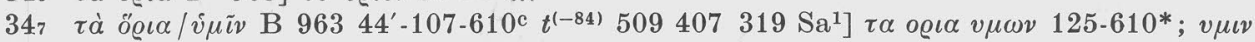

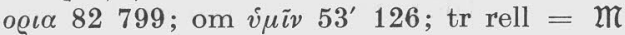

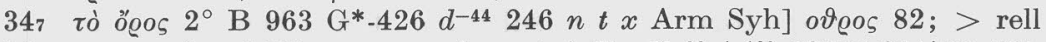

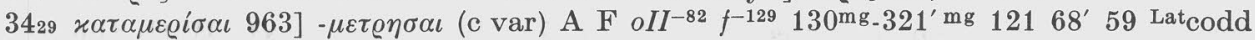
100104 Aeth

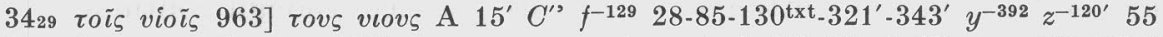

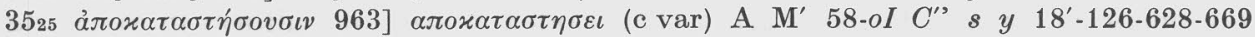
Latcod 100 Arm

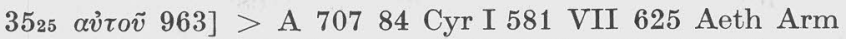

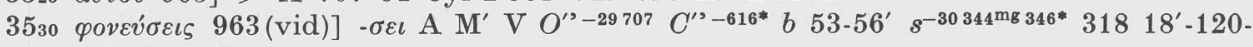
628-669c 55

367 x $\alpha i$ 963] $>\mathrm{A}$

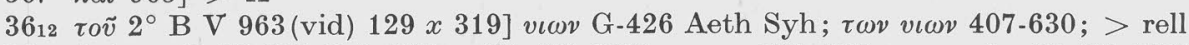

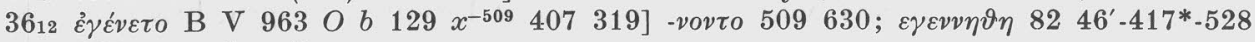
$767 ; \varepsilon \gamma \varepsilon v \eta \vartheta \eta$ rell

The length of this list compared to that of the preceding confirms the conclusion of chapter 4 that the B type text is a much better witness to Num than is the A type text.

The value of 963 for the Numbers text lies more in its confirmation of a Num text than in its establishment. It is unfortunate that no more of its text is extant since it is almost two centuries older than B. Furthermore its text is much more closely related to $B$ than to $A$, which fact would also accent its value as an ancient and superior text, and of the highest importance for recovering the earliest from of the Numbers text. 


\section{Chapter 6 The Critical Text (Num)}

As in earlier volumes of the Gottingen Septuagint certain general rules of thumb have been followed. Since the old uncial texts normally add the $v \tilde{v}$

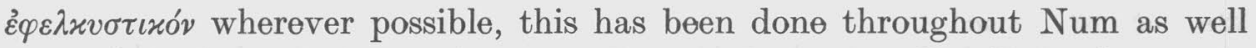
regardless of the practice of later scribes (and occasionally those of papyri).

A further general practice followed concerns the transcription of the gentilic ending. This has always been transcribed by $-\iota$ in spite of the fact that the oldest uncials commonly have $-\varepsilon \iota$. It has been argued elsewhere (cf Gen $489 \mathrm{ff}$ ) that the correct transcription of the Hebrew /i/ and /i/ is "iota" in Hellenistic Greek and this system has been followed throughout. Accordingly the masculine plural gentilic is regularly transcribed by $-\iota \mu$ and a masculine plural noun form by $-\iota \mu$.

The classical future stem for $\lambda \alpha \mu \beta \dot{\alpha} v \varepsilon \iota v$ is $\lambda \eta \psi$-, i.e. without a nasal. As is well-known from papyri from the third century B.C. onwards the Hellenistic stem $\lambda \eta \mu \psi$ - became popular; cf Mayser I. 1, 166f. Since the oldest uncials, A B and $S$, all attest to the future form with nasal infix, it has been the common practice to accept such forms throughout for the critical text. With the appearance of Deut in the Gottingen series this practice, though continued, is no longer as certain. Papyri earlier than Codex B are not uniform in attesting to the Hellenistic form. For Gen mss 911961 and 962 all attest to the Hellenistic form, although 963 occasionally witnesses to the classical form (Num 3016 Deut $265_{11} 22_{30} 30_{4}$ ), but to the stem with " $m u$ " at Num 517253161988 Deut 511). Apparently 803, a Qumran fragment, witnesses to the Hellonistic stem at Num 412, but 848, which is certainly of Egyptian origin, has the classical stem throughout (Deut 2132267262 ). In spite of one's uncertainty on the matter the Hellenistic stem has been retained throughout Num.

1. That Num and Deut are the products of different translators is apparent from their respective attitudes towards their parent text. The demands of the target language play a greater role in Num than in Deut which is often literaiistic in its renderings. Thus prepositions are much less literally rendered in Num than in Deut. The Numbers translator also reflects a much freer attitude towards the Hebrew in rendering repetitive phrases; in census reports or in repeated offerings Num tends to stylize his renderings according to a pattern, regardless of minor differences in the Hebrew.

The linguistic demands of Greek are respected to a greater extent in Num than in Deut. To cite but one such, the case and number of relative pronouns 
are observed much more carefully. Whereas in Deut the inflection of the relative pronoun was often the result of attraction to its antecedent, such inflections are comparatively infrequent in Num, the inflection being determined by the syntactic demands of the relative clause.

Another marked characteristic of Num in contrast to that of Deut is the comparative infrequency of $\delta \varepsilon$ constructions as compared to those with raí. Num does not avoid $\delta \varepsilon$ for paratactic constructions; it simply prefers raí.

It is thus clear that problems concerning the critical text must first of all be established through the patterns of usage and of translation within the book itself rather than from the Pentateuch as a whole, and only secondarily from the wider context of the Greek Pentateuch and the LXX as a whole.

2. The usage of the particle $\alpha^{\prime} v$ in Num is the same as that which was established for Deut; of THGD, ch. 7, Sect. B. Within relative clauses äv, but never $\varepsilon \alpha v$, is accepted as critical text since such usage predominated in the time of the translator. The long form became popular in the early centuries of our era at times almost completely supplanting $\ddot{\alpha}^{\prime} v$; in fact at 2326 all mss have $\varepsilon a v$ rather than the original $\stackrel{\alpha}{\alpha}$. Usually $\ddot{\alpha} v$ is read in the majority of our witnesses. Since Codex B, being a fourth century ms, commonly reads the longer form, $\mathrm{Ra}$ often adopted $\varepsilon \alpha v$ in his text. He must be corrected at $561029306_{2} \mathbf{9}_{10} 13$

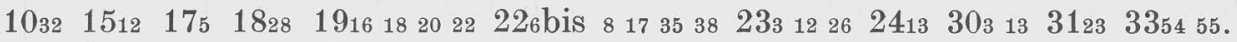

As in Deut $\ddot{\varepsilon} \omega \varsigma$ occurs with $\ddot{\alpha} v$ when introducing the subjunctive mood. $\tilde{\varepsilon} \omega \varsigma \not{\alpha} v$ is normal in the papyri, $\tilde{\varepsilon} \omega \varsigma$ without $\ddot{\alpha} v$ occuring only in late Ptolemic times (Mayser II, 3, p. 79). In a few instances the ä $v$ has been lost in much of the tradition; thus at $10_{21}$ only $\mathrm{V} b d n 85^{\prime} \mathrm{mg}_{-} 321^{\prime} \mathrm{mg}$ (vid) $t 319$ support ${ }^{\prime} v$; at 1433, $\mathrm{AB}^{*} \mathrm{~F}^{*} 70777 b 44 f^{-129} 458-767 x^{-509}$ y $12655319624 \mathrm{om} \alpha ٌ \nu$, and at 2324, only V 58-72-82 $d$ 127-767 $t 5559$ have it. $\tilde{\varepsilon} \omega s$ also occurs with the indicative two times $(12153213)$, but of course without $a ̀ v$.

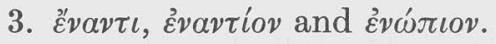

As in Deut so in Num the translator used all three of these as prepositions governing the genitive with no discernible semantic distinctions. Certain observations on usage can, however, be made. Whenever the genitive is zvoiov (or $\tau \sigma \tilde{v} \vartheta \varepsilon o \tilde{v})$ the preposition is always (50 times) है $v a v \tau \iota$ regardless of the He-

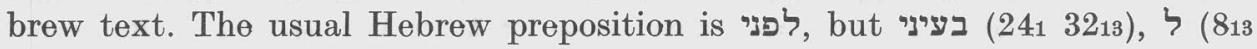

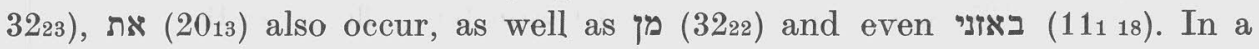
number of instances there is no corresponding text in $\mathbb{M}\left(\begin{array}{lll}813 & 15 & 3230\end{array}\right)$, and at $31_{3} \mathfrak{i n}$ represents a different text.

If the governed nominal is a pronoun $\varepsilon^{\prime} v a v \tau \iota$ is normally avoided in favor

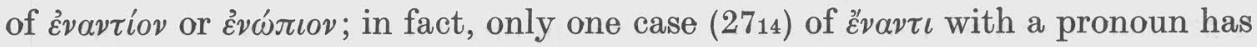

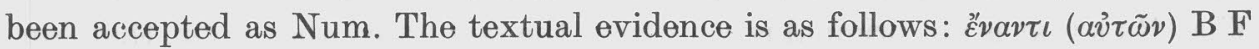
$\mathrm{K} \mathrm{M}^{\prime} \mathrm{V} 963$ (vid) $o I-72^{\prime} d 129 s^{-28} 85 t x^{-71} y z^{-630} 59624$ 799] $\varepsilon v \mu \varepsilon \sigma \omega C^{\prime \prime}$ 28-85 55 646; Evavtıov rell: לעיני M. All the uncials except A, including 963(vid), 
support $\varepsilon^{\prime} \nu \alpha \nu \tau \iota$ and this would appear to be Num, though a case for $\varepsilon v \alpha \nu \tau \iota \nu \nu$ could well be made, particularly in view of the following two cases.

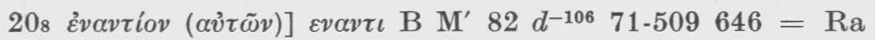

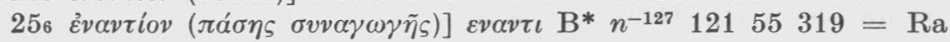

In all three instances the Hebrew equivalent is לציני. In the last two instances the B variant has only minority support and the less common word is likely original. It should be added that these are the only instances in Num involving לציני

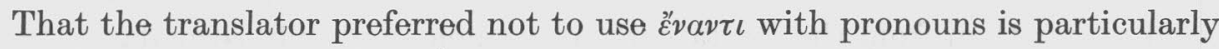
clear from $27_{19}$ in which לפני occurs three times. In the first two instances

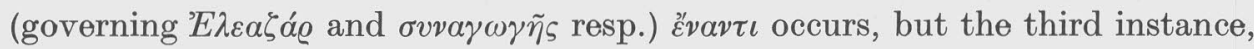

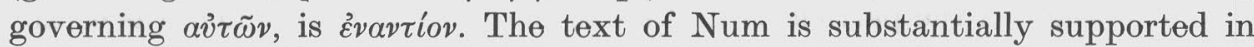
the tradition:

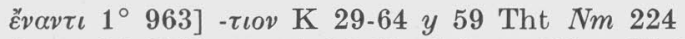

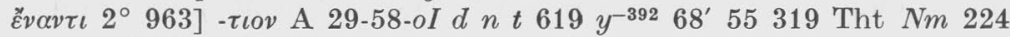

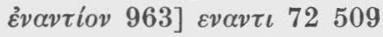

It would otherwise seem unlikely that the translator would have varied the preposition in coordinate phrases; thus in 813 and 272 है $v \alpha \nu \tau \iota$ occurs four times; in $32_{22}$ and 361 it occurs three times, and in $3_{4} 27_{21}$ twice, whereas the rare

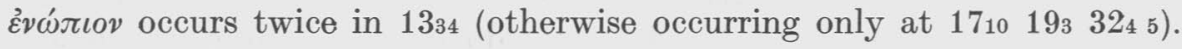

The translator apparently tended to use the same preposition within an immediate context. Thus at $27_{22}$ it seems unlikely that Ra represents the ori-

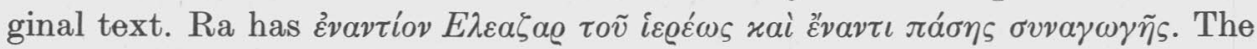
relevant facts are as follows:

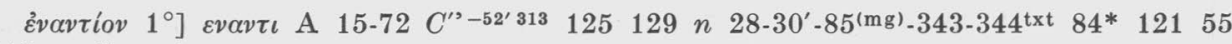
$646=\mathrm{Compl}$

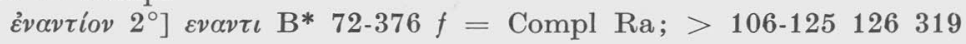

It would seem likely that évaviiov is the original preposition in both places in this verse.

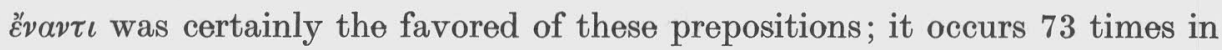

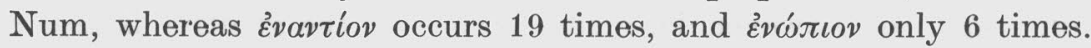

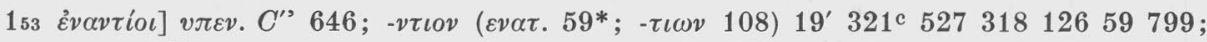

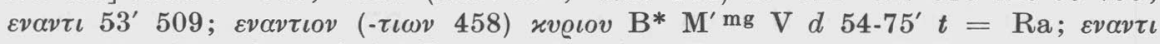

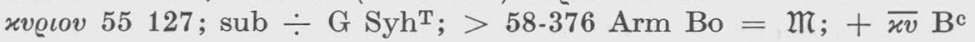

V. 53 contrasts the position of the Levites with that of the other tribes who

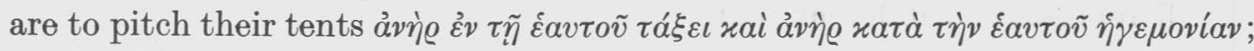

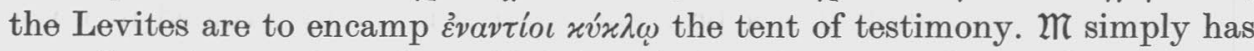
סביב, but the translator has added Évaviío in order to emphasize the contrast. That Ra's text is not original is clear from the fact noted above, viz. that $\varepsilon v a v$ -

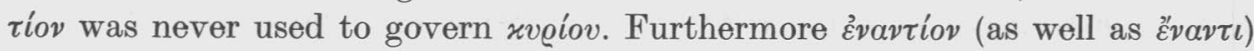

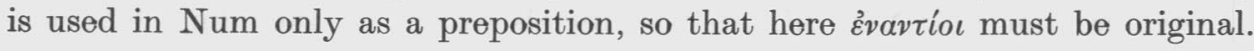


Elision of the final vowel of prepositions is common in Hellenistic prose with pronouns and common phrases such as $\varkappa \alpha \tau^{\prime} \dot{\alpha} v a \tau o \lambda \alpha^{\prime} \varsigma$ or $\dot{\alpha} \pi^{\prime} \dot{\alpha} \nu \alpha \tau o \lambda \tilde{\omega} v$, but is

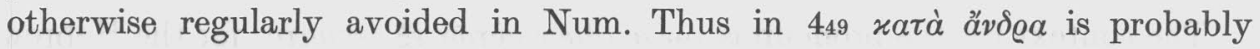
original as is $\delta i \alpha$ a $\alpha \mu \alpha \varrho \tau i \alpha v$ in 273 , the variant short forms in the tradition being the result of haplography. On the other hand, $x \alpha \tau^{\prime} \alpha \dot{\alpha} \alpha \tau o \lambda \alpha^{\prime} s$ in 2111 and $\dot{\alpha} \pi^{\prime}$ $\dot{\alpha} \nu \alpha \tau o \lambda \tilde{\omega} \nu$ in 237 (the latter in poetry) are instances of elided prepositions probably original to the translator.

$\left.242 \dot{\varepsilon}^{\prime} \pi^{\prime}(\alpha \dot{v} \tau \tilde{\omega})\right] \varepsilon v \mathrm{~B} b^{-19}$ Tht $N m 221=\mathrm{Ra}$

That the phrase $\dot{\varepsilon} \pi^{\prime}$ av่ $\tau \tilde{\omega}$ is original to Num is clear from the Hebrew which has עליו. The variant was palaeographically determined. What is not immediately evident is its position in the verse. In Num it appears at the end of the verse. In $2 \pi$ עליו precedes rather than follows רוח אלהים. The following wit-

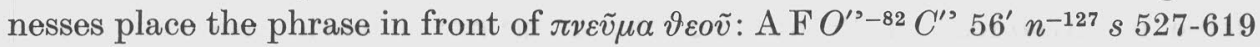
$y z 5559799$ Latcod 100 Ruf Num XVII 2 Aeth Arab Syh. This change in word order is probably the result of Origen's work; note the witness of the $O$ mss, Arab and Syh.

$\left.{ }_{14}{ }_{13} \dot{\varepsilon} v\right] \tau \eta \mathrm{B} 44^{\prime} 129127-767 t x^{-509}=\mathrm{Ra} ; \tau \alpha 509 ;>500$

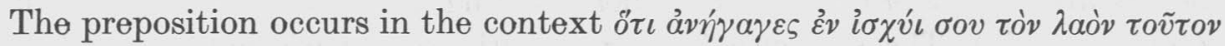
and the clause reproduces $\mathfrak{2}$ literally. Codex B* uniquely also substitutes $\eta \gamma \alpha \gamma \varepsilon \varsigma$

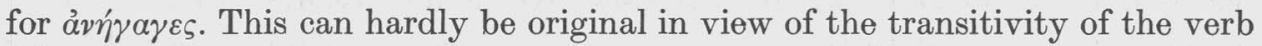
הצלית in $\mathfrak{M}$; $\ddot{\alpha} \gamma \varepsilon \varepsilon v$ is never used as a rendering for the Pentateuch. The text of $\mathrm{B}$ is questionable in this clause, and the preposition is undoubtedly original and the article is not.

4. Pronouns. The presence or absence of a third person pronoun in Num is often formulaically determined.

12 om $\alpha \dot{\tau} \tilde{\omega} v 1^{\circ} \mathrm{B} 414^{\prime} d n^{-767} t x 18$ Bas II 145 Cyr VI $453 \times 624$ Latcod 100 PsBas Is I 5 Arm

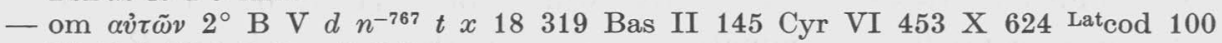
Hi Eph II 3 PsBas Is I 5 Arm

— $\alpha \dot{\tau} \tau \tilde{\omega} \nu 3^{\circ}$ ] sub $\div \mathrm{G}$; > B $19 d 127 t x 18319$ Cyr VI 453 X 624 Latcod 100 Arm

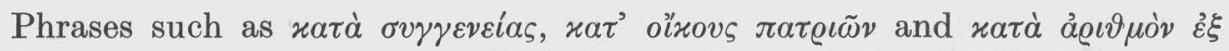

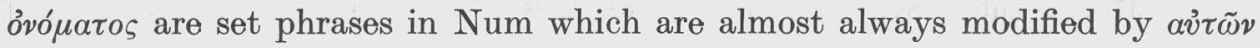

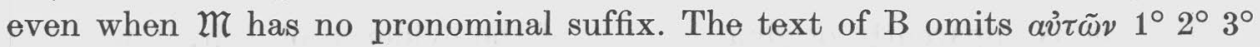
in this verse but this is secondary as Ra also recognized. This is rendered doubly certain by the fact that $\alpha \dot{\tau} \tau \tilde{\omega} v 3^{\circ}$ is sub obelo in $\mathrm{G}$, there being no suffix present in the Hebrew. In vv. 16 and 44 avi $\tilde{\omega} v$ is similarly omitted by codex B against the normal pattern; the omission would appear secondary.

$446 \delta \dot{\eta} \mu o v \varsigma \alpha v i \tilde{\omega} v]$ om $\alpha \dot{\tau} \tau \tilde{\omega} v$ B $x^{-619}$ Latcod 104(vid) = Ra

The word $\delta \eta \mu \circ$ is inevitably followed by a genitive modifier in Num, except for the specific listing of various $\delta \dot{\eta} \mu \iota \iota$ by name throughout chapter 26 . The B text adopted by $\mathrm{Ra}$ must be understood as secondary. 


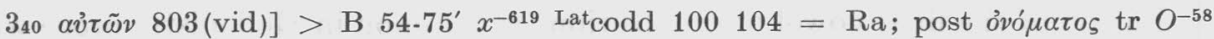
Bo Syh

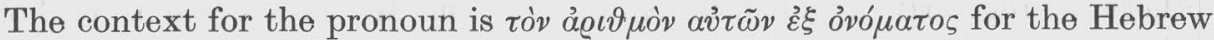
מספר שמתם. The transposition of $\alpha \hat{\tau} \tau \tilde{\omega} \nu$ after ỏvó $\mu \alpha \tau o \varsigma$ was effected by Origen and corresponds exactly to the Hebrew. Ms 803 must have had an $\alpha v \tau \tilde{\omega} v$ in its text, since the length of the line demands it but its position is not clear. Since the transposed order is clearly hex, 803 probably supports Num. It seems likely therefore that it is original.

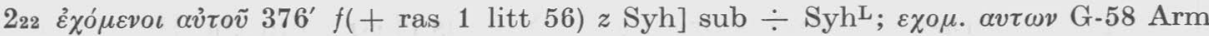
Bo; > Lat $\operatorname{cod} 100 ;$ om av่นov rell $=\mathrm{Ra}$

In the ch. 2 account of the tribal encampments over against the tent of testimony the tribes are divided into four groups of three each. The second

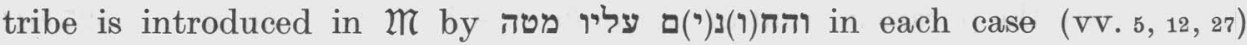
except for the third group (v.22) where $\mathfrak{K}$ omits החנים. The third tribe is introduced simply by ומטה (vv. 14, 22, 29) or (ve Greek in each

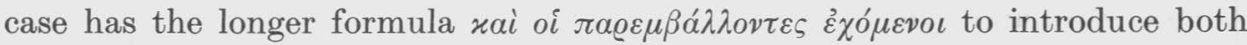
the second and third tribes but varies the rest of it depending on whether $\alpha$ vं

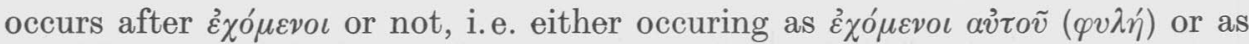
$\varepsilon \chi \chi o ́ \mu \varepsilon v o \iota ~\left(\varphi v \lambda \eta^{\prime}\right)$. The evidence for $\alpha \dot{\tau} \tau o \tilde{v}$ (with some variation in number) is clear in vv. 121427 where all witnesses, except for mss 618*19 which omit $\alpha v ่ \tau o \tilde{v}$ in $\mathrm{v} .14$, attest to the pronoun. In $\mathrm{v} .22$ av่ $2 \tilde{v}$ is weakly attested but it is included under the obelus in Syh, i.e. the av่ probably to be regarded as original. Where no avं ov obtained the tradition has added either $\alpha v \tau o v$ or $\alpha v \tau \omega v$.

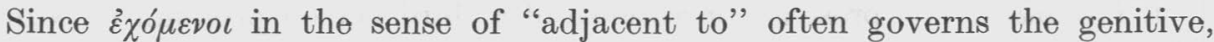
$\varphi v \lambda \eta$ easily became $\varphi v \lambda \eta \varsigma$ in the tradition, though what this was intended to mean is not clear. Even when avं $\tilde{v} \tilde{v}$ was present in the text a large part of the text tradition attests to the genitive which could hardly be original. Accordingly $\varphi v \lambda \eta^{\prime}$ as predicate nominative is to be read throughout. Oddly Ra accepted $\varphi v \lambda \eta \varsigma$ throughout; cf vv 57121420222729.

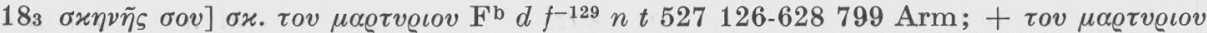
376 ; $\sigma o v$ sub $\div \mathrm{Syh}^{\mathrm{T}}$; om $\sigma o v$ B V 58-82 $x^{-527} z^{-126628} 319646$ AethCG $\mathrm{Sa}=\mathrm{Ra} \mathrm{Wl}$

The $\sigma o v$ is apparently original in view of the evidence of Syh; it was probably added by the translator due to the influence of the coordinate $\varphi v \lambda \alpha x a ́ s$ $\sigma o v$ in the same verse.

On the other hand, Ra was probably correct in not adopting the genitive pronoun after yvvaixes at 2130 with B V 82 54-75' 71-509 319 Phil passim Lat cod 100. W משים W משים; thus there is no textual justification for the majority

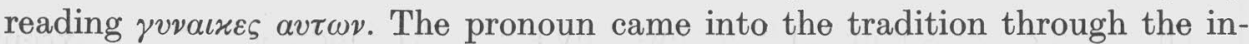
fluence of the parallel construction of v. 30a: $\tau \grave{o} \sigma \pi \varepsilon \varrho \mu \alpha \alpha v \tilde{\omega} \nu$.

$1427 \mu o v] \varepsilon \mu o v$ B V 108-118-314*30'-321'c $121319=\mathrm{Ra}$ 
In contrast to normal usage with the so-called true prepositions (cf Mayser I. 2. $62 \mathrm{f}$ ), the short form of the first singular pronoun is to be preferred after Evvaviiov, possibly through analogy with the second singular pronoun; cf Évavtiov

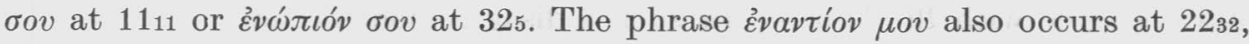
where, however, a substantial number of witnesses do attest to the long form $\varepsilon \mu o v$.

$166(\hat{v \mu \tau i v) ~} \varepsilon \alpha v \tau o \tilde{\zeta} \varsigma] \alpha v \tau o \iota \varsigma$ B $29122 * 55 \mathrm{Cyr}$ I $860=\mathrm{Ra}$

The use of a reflexive pronoun rather than the simple third person pronoun to intensify a first or second person plural pronoun occurs only here in Num. The simple pronominal intensifier does not occur in Num at all but is common

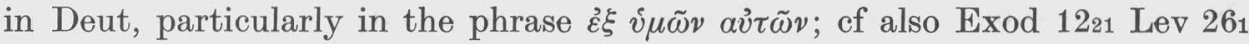
and elsewhere. It would be difficult to explain $\varepsilon a v \tau o \tau_{\varsigma}$ as secondary, it being the lectio difficilior. The reading of $B$ on the other hand is easily understood as an adaptation to better Greek usage.

154 ő $\left.\sigma \alpha \mathrm{A} O^{-G_{-}} 72 b f 5^{\prime} \mathrm{mg}_{-} 321-346^{\mathrm{mg}} 1211^{\prime}-126-628-66959319624\right] \alpha$ rell $=$ Ra

$1626 \check{\delta} \sigma \alpha] \alpha n^{-767} ; \omega \nu$ B $\mathrm{M}^{\operatorname{txt}} \mathrm{V} 376 d 129 t x=\mathrm{Ra}$

In both cases ö $\sigma \alpha$ must be original, since the antecedent is an inflected form of $\pi \tilde{\alpha} \varsigma$. The translator always uses $\delta \sigma \alpha \alpha$ when its antecedent is $\pi \tilde{\alpha} \varsigma$ except of course in the collocation $\pi \tilde{\alpha} \varsigma$ ós of 1916 (as well as $\pi x \nu \tau \dot{s} \varsigma$ ov in 1922, a somewhat different construction).

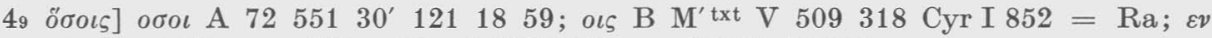

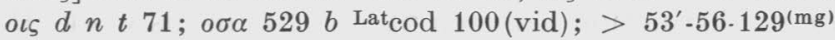

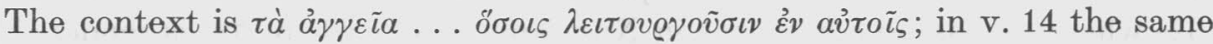
relative clause occurs but the antecedent is $\tau \dot{\alpha} \sigma x \varepsilon v ́ \eta$. In the latter the variant

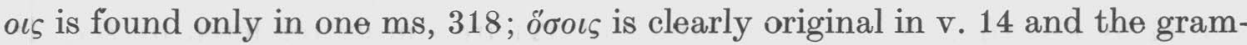
matically correct dative is also original in v. 9. A similar case of grammatical correctness occurs in the following case.

$621 \eta \eta v$ B $x^{-619}$ Cyr I 1053] $\eta[\ldots 963$; os 537 ; $\eta \varsigma$ rell

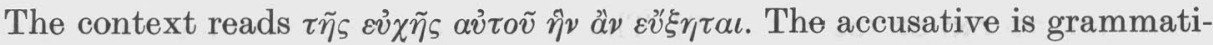
cally correct as modifying $\varepsilon v^{\prime} \xi \eta \tau \alpha$, but case attraction, as in the majority reading, is well-attested already in Classical times. The translator, however, only seldom allowed case attraction to predominate over grammatical correctness. In fact only the following cases in Num are clear cases of such attraction.

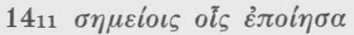

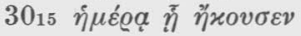

The reverse trend is characteristic of Num, and Ra was correct in following $\mathrm{B}$ in 621 . 
5. Since Num is a translation document the paratactic character of the Hebrew original is reflected in it. The presence or absence of the conjunction wai for the original text is not always easily determined since Num does not always agree with $\mathfrak{m}$. Thus at 423 such a disagreement occurs.

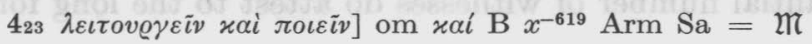

The collocation "to serve (a service) to do" occurs in $\mathfrak{m}$ of Numbers at 432335 39. At v. 3 it is literally rendered by $\lambda \varepsilon \iota \tau o v \varrho \gamma \varepsilon \tilde{\imath} v \pi \circ \imath \tilde{\eta} \sigma \alpha \iota$; when the translator used the present infinitive $\pi \circ \varepsilon \varepsilon \tilde{i}$ in the other three instances he inserted the conjunction in spite of the fact that none obtains in the Hebrew. It should

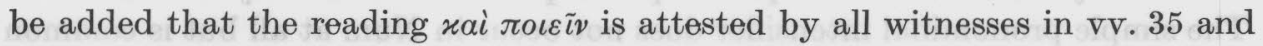
39; the omission of xai in v. 23 seems to be secondary.

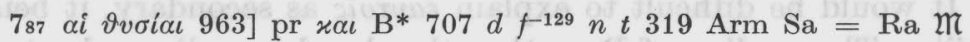

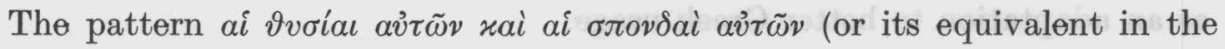
singular) also occurs at 615296 (twice) 111618192122242728303133343738 . In all but $291624313437 \mathrm{~W}$ has the conjunction introducing the pattern. Except for 615 and 296 the translator did not render the conjunction. In view of this general pattern it would seem that the $x a$ of the variant text is not original. It should also be noted that the $x \alpha \iota$ intrudes between $\delta \omega \delta \varepsilon \kappa \alpha$ and $\alpha i$ and may independently have been formed by partial dittography.

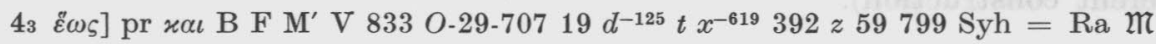

The translator made no distinction between עועד in his work. He renders them throughout by $\tilde{\varepsilon} \omega \varsigma$ except at 912 where $\varepsilon i_{\zeta}$ occurs, at $8_{4}$ (twice), at 329 where עד has no equivalent in Num and at 1411 where ועד is rendered by xai $\bar{\varepsilon} \omega s$. At 1411 the conjunction is necessary since it joins two clauses intro-

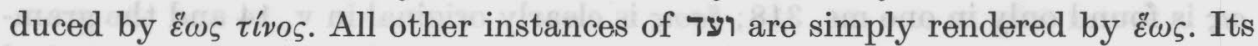
introduction at 43 may well be hex.

$426 x \alpha i$ ö $\sigma \alpha]$ om $x \alpha i$ B $b x^{-619} 392319$ Latcod 100 Aeth $^{M}$ Arm Bo Sa ${ }^{4}=$ Ra

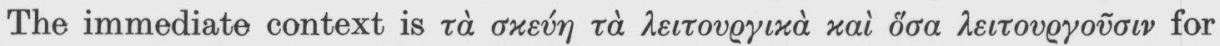
כלי עבדתם ואת כל אשר יעשה in $\mathfrak{M}$. The relative clause containing $\lambda \varepsilon \varepsilon \tau$. Thevovvovv also occurs at 3314912 1821; in these cases no rai precedes since the Hebrew has no ואת כל; that is, the relative pronoun has an antecedent. The omission of $x \alpha i$ in the B tradition may have been influenced by such cases; this may also have been facilitated palaeographically since $\lambda \varepsilon \imath \tau o v e \gamma \iota x \alpha$ occurs immediately before $x a i$, the latter being omitted by haplography.

The tradition has also amplified the text through hex activity. All but B M' V 82 16-46 $b d n t x^{-619} 319$ Latcod 100 Arm Co have added $a v \tau \omega v$ after $\lambda \varepsilon \imath \tau o v \varrho-$ yıxá. Furthermore hex activity is also apparently to be seen in the insertion of $\pi \alpha \nu \tau \alpha$ between xai and $\tilde{\delta} \sigma \alpha$ in $\mathrm{V} O^{-58}-29619$ Aeth Syh. 


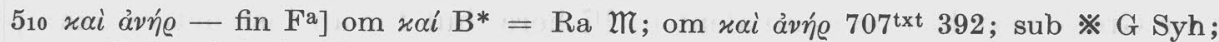
$>$ F $29131^{(\mathrm{mg})} 53^{\prime} z 59$

The clause was apparently absent in Origen's parent text, it having been

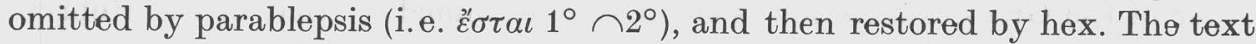

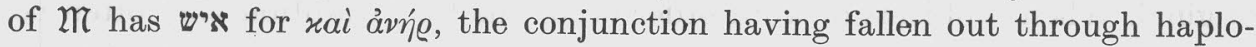
graphy after יהיו. It is present in Sam and a number of Hebrew mss, and the equivalence of $\mathrm{B}^{*}$ and $\mathfrak{K}$ is coincidence, the rai being accidentally omitted after $\tilde{\varepsilon} \sigma \tau \alpha \iota$ through homoioteleuton.

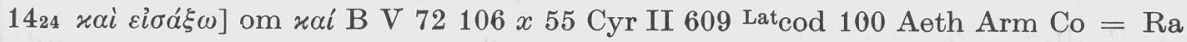

The rai can only be interpreted on the basis of a Hebrew parent text since it is barely intelligible in the Greek. The Greek begins with the nominative

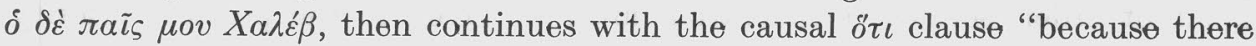

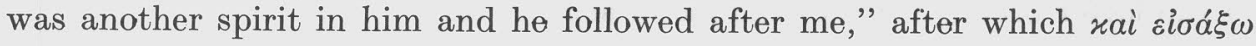
av่tóv is placed. This corresponds exactly to the Hebrew, but good Greek style would not render the conjunction of $\mathfrak{T T}^{\prime} \mathrm{s}$ והביאתיו by xai. The xai must be original text.

2917 init $963 \quad b \quad 509]$ pr $\varkappa \alpha \iota$ rell $=$ Ra $\mathfrak{k}$

2935 init V $96346129-24612163059$ Latcodd $100104=\mathrm{Compl}]$ pr $x \alpha \iota$ rell $=\mathrm{Ra}$

The successive days of the feast described in vv. $12 \mathrm{ff}$ are introduced by the conjunction in $\mathfrak{W}$ with the exception of the final (eighth) day, i.e. v. 35 . Num consistently begins each day's account with $\tau \tilde{\eta} \dot{\eta} \mu \varepsilon \varepsilon_{\varrho} \tau \tilde{\eta}$..., i. e. without a zal. The new evidence of 963 shows that the translator was indeed fully consistent in this practice, and it is now evident that an introductory $x a$ is secondary in $\mathrm{vv} .17$ and 35 as well as for the intervening days.

3145 xai $1^{\circ} 963 O^{\prime-58}-72 C-46118^{c} n^{(-75)} t$ Latcodd 100104 Aeth ${ }^{C}$ Arab Arm Bo Syh] $>$ rell $=$ Ra

Ra could reasonably omit the xai for his text as being hex since most of the usual hex witnesses attest to it and the older uncials did not, but the new evi-

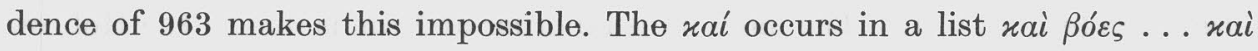

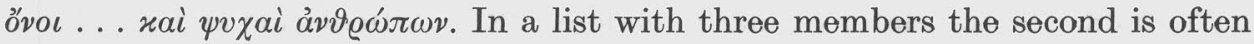
introduced without a conjunction in Greek as in English, i.e. as "cattle, oxen and human beings." The omission of xai is probably stylistically inspired, whereas the original text contained it because of the presence of a conjunction in the parent Hebrew text.

156 Eis $\left.1^{\circ}\right]$ pr $\eta \mathrm{B} O^{-58} 509-527$ Syh $=\mathrm{Ra}$

The variant text which $\mathrm{Ra}$ adopted introduces the first of the alternatives,

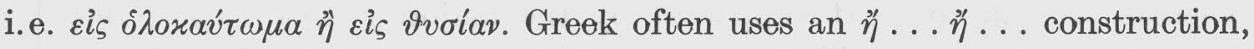
though the first of these is not always present. In Num such alternatives are never introduced by $\eta$, the conjunction only occurring between the two parts. 
Our Greek text has no equivalent in $\mathfrak{M}$ here, but for an instructive example of usage in Num where an equivalent does obtain in $\mathfrak{k}$ ef $v$. 8.

6. A comparison of the Greek text with the Hebrew shows that the word order of the Greek translation is strongly determined by that of the Hebrew original. When problems of word order occur the text tradition may often be misleading because of Origen's work. Though Origen says nothing about word order the nature of the hexapla was such that the Greek word order was largely forced to coincide with that of the Hebrew; cf the discussion in THGG 59 and for some insight into the probable nature of the actual hexapla $\mathrm{ef} \mathrm{G}$. Mercati, Psalterii Hexapli Reliquiae. I. Codex rescriptus Bybliothecae Ambrosianae 0.39 Supp. phototypice expressus et transcriptus. Roma, 1958. When the tradition is divided between witnesses to the Hebrew word order and those to a different order, serious consideration must be given to the possible priority of the latter order. A good example of such is $16 \mathrm{y}$.

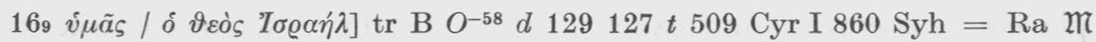

IT has (הבדיל) אלהי ישראל אתכם). The translator preferred to bring the pronominal object next to the verb. Though he tended to follow the Hebrew order, he was by no means slavish in this regard as the study on hex corrections in word order (p. 56-58) indicates. The variant text is probably such a hex correction.

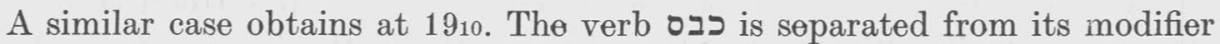

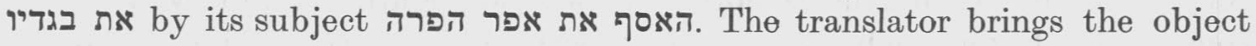

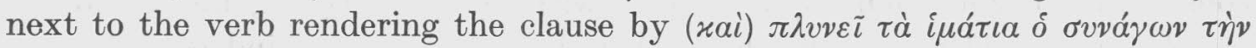
$\sigma \pi o \delta ı \dot{\alpha} \nu \tau \tilde{\eta} \varsigma \delta a \mu \alpha \dot{\lambda} \varepsilon \omega \varsigma$.

At times, however, close attention to the Hebrew text may solve problems of word order in quite a different way.

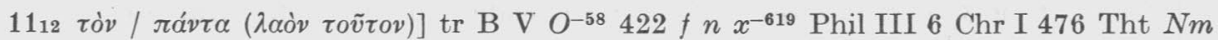
$204^{\mathrm{ap}}=\mathrm{Ra}$

Here the variant $\pi \alpha v \tau \alpha$ secondary; it is probably due to the common expression "all the people" found elsewhere (e.g. in v. 13). The more unusual order of Num is due to the Hebrew את by articulation and tóv placed before $\pi \alpha ́ v \tau \alpha$ represents his attempt at rendering the Hebrew exactly.

A particularly complicated textual problem concerning word order obtains in 1425 .

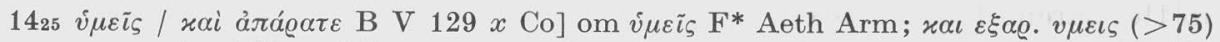

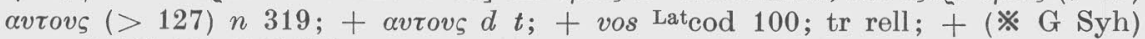

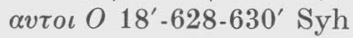

In the context $\dot{v} \mu \varepsilon \tilde{\imath}_{\varsigma}$ refers to the subject of the preceding $\dot{\pi} \pi \iota \tau \tau \propto \alpha \dot{\varphi} \eta \tau \varepsilon$, whereas the variant text supported by the majority makes $v \mu \varepsilon \tilde{i}_{\varsigma}$ refer to

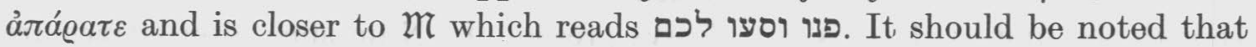
$d t$ add avtovs after the verb. Origen probably had the original word order in 


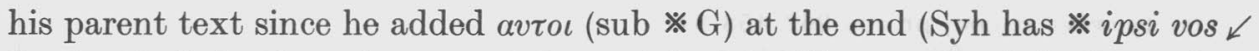
for $v \mu \varepsilon i \bar{\varsigma})$. Only the order accepted as original for Num can explain the various attempts to correct the text.

7. The problem of articulation of nouns is undoubtedly one of the most difficult textual problems facing the text critic of Numbers, since patterns of usage are often not present.

a) In the collocation "the desert of" plus proper name, the proper name is

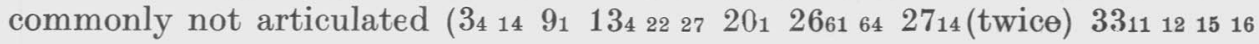
36 (twice) 343). A few instances are, however, problematic. At 1119 occurs the phrase $\dot{\varepsilon} v \tau \tilde{\eta} \tilde{\varepsilon} \varrho \dot{\eta} \mu \omega \tau \tilde{\eta} \Sigma \iota v a$; the article in question is attested in $\mathrm{v} .1$ at least as early as Origen since it is sub ob in G. In both instances the relevant article is omitted by some mss (in $\mathrm{v}$. 1 by $\mathrm{F}^{*} \mathrm{~V} 72$ 417-528 537 44-125 127-458 509 $59 * 319799$, and in v. 19 by $o I^{-64^{*}-72} 12553^{\prime} 127-458-76784^{*}$ (c pr m) 18), but in view of its early attestation it has been adopted as Num.

At $95 \mathrm{Ra}$ adopted $\tau o v \Sigma_{\iota v \alpha}$ as his critical text on the basis of B although at v. $1 \Sigma_{\iota} v \alpha$ occurred without the article. Both instances occur in the phrase $\dot{\varepsilon} v \tau \tilde{\eta} \dot{\varepsilon} \varrho \dot{\eta} \mu \omega \Sigma_{\iota v \alpha}$ and it is unlikely that the translator would have changed his normal pattern within the same context. Furthermore the unarticulated proper noun is attested by A 72-376 552 44' 75' 71-509 126 and is probably original. At 131 the original hand of B (and $37676144^{\prime}-125$ ) does not articulate $\Phi$ a@óv ( $\mathrm{Ra}$ in opposition to his usual practice adopts the reading of $\mathrm{B}^{\mathrm{c}}$ here), and it seems wise to follow the common pattern here as well.

On the other hand at $10_{12}$ the articulated proper noun is strongly supported. The collocation occurs twice within the verse, Ra articulating only the second. The evidence is as follows:

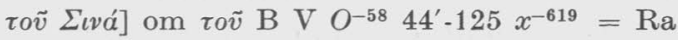

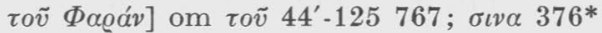

In view of the strong support for the article, the article has been accepted in both cases.

b) Articulation or non-articulation of the tetragrammaton.

xvolos as rendering for the tetragrammaton occurs in the nominative case 176 times and is never articulated except once (149), where, however, the particle $\delta \varepsilon$ intervenes. It occurs 14 times in the accusative and only once (1630) is it articulated; in this case יהוה is preceded by and $\tau$ תo was undoubtedly intended by the translator to represent it. The text tradition throughout substantiates this lack of articulation for xveıov with the sole instance of 112 where ms 318 and Cyr I 381 read (

The genitive zvolov as substitute for the divine name occurs 125 times in Num, and it is here argued that the translator never articulated it. In all but 19 cases no extant witness obtains for rov xveıv. For most of the 19 exceptions the evidence for later articulation is slight. In the list below only the evidence for articulation is given. 
$7_{89}$ (

811 (

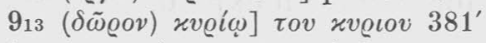

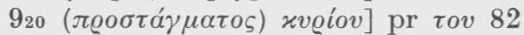

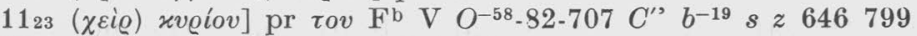

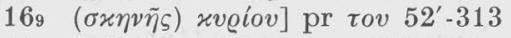

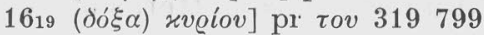

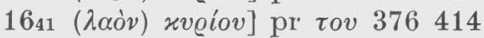

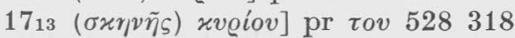

$19_{13}(\sigma x \eta v \dot{v} v)$ xveíov A B $\mathrm{M}^{\prime} \mathrm{V}$ oI $\left.b d n t x^{-619} 630319\right]>376$; pr $\tau$ cov rell

$19_{20}(\ddot{\alpha} \gamma \iota \alpha)$ xv@íov] $\mathrm{pr} \tau o v 458$

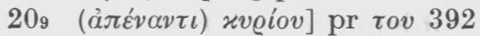

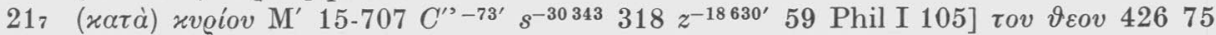

Bo $\mathrm{Sa}^{1}$; pr $\tau o v$ iell = Ra

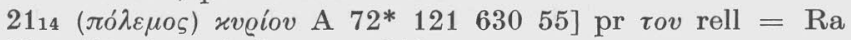

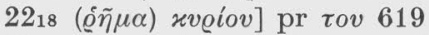

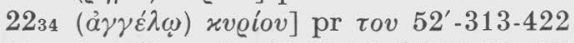

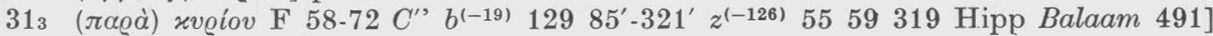

$\vartheta \varepsilon o v 125 ; \tau o v \vartheta \varepsilon o v \mathrm{~V} d^{-125} t$; pr $\tau o v$ rell $=\mathrm{Ra}$

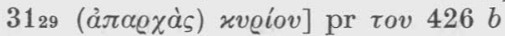

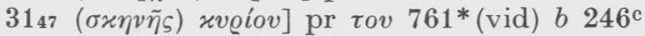

In all but five instances $\left(\begin{array}{lllll}11_{23} & 19_{13} & 211_{14} & 313\end{array}\right)$ the variant article is attested by only a few mss and may be regarded as insignificant, although in one case (789) the early 963 does support the articulation. Since 120 (or 119) out of the 125 occurrences of xveiov were certainly unarticulated in Num it is plausible to suggest that zoliov was never articulated by the translator.

The dative zvoi $\omega$ occurs 72 times in Num, in all but a few cases representing

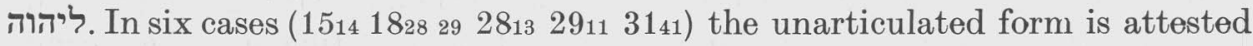
by all witnesses; in eighteen cases three or less mss support the article. The oldest ms witnesses usually support the unarticulated word. 963 is extant in 19 instances and supports the unarticulated word in all but one case (68) where A B V and $\mathrm{C}$, however, all support the unarticulated xvoi $\omega$. Codex B supports the unarticulated word in all but three instances (59 155 1812). Occasionally Origen was responsible for the addition of the article as the following instances prove.

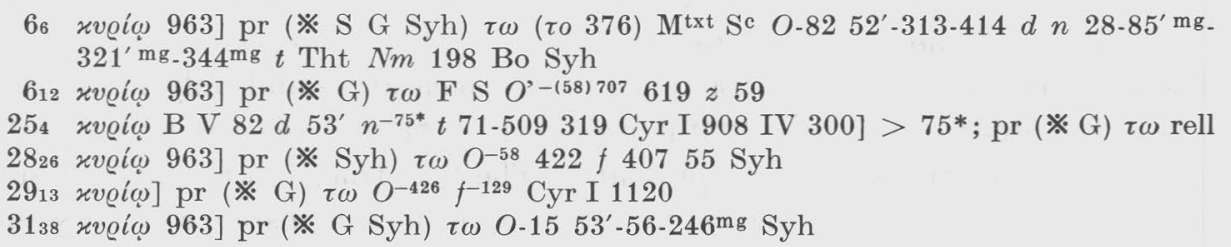

The addition of the article was probably intended to represent the preposition ל; puzzling is the fact that hex only shows this equation occasionally since the chief witnesses usually witness to the absence of the article. In any event, it would seem that the translator did not follow this practice but rendered ליהוה without the dative article to represent the preposition. 
c) Articulation of vioi as clan designation.

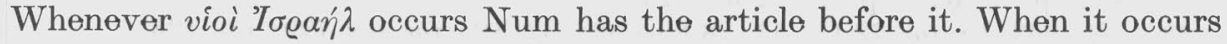
in the genitive however, no discernible pattern obtains; even within one verse

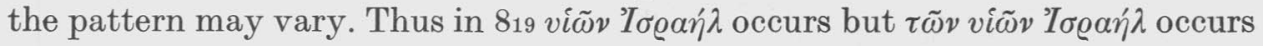
twice; cf also 1824. In the accusative the article is lacking when $\pi a ́ v \tau a \varsigma$ precedes; otherwise it is present. In the dative the article is usually present, though not always (cf $153 \begin{array}{llllll}5_{9} & 817 & 18 & 1529 & 1814\end{array}$ ).

With clan names other than Israel the pattern is slightly different. The dative is always articulated but the accusative is not. The latter occurs only five times; twice the tradition is unanimous in attesting the article $\left(\begin{array}{lll}315 & 434\end{array}\right)$ and twice in attesting its absence $(161012)$. At 2417 the evidence is divided and the oldest witness has been followed. For both the nominative and the genitive the translator was quite arbitrary and no pattern seems to have been followed. $\mathrm{Ra}$ usually adopted the reading of his oldest witness; this same principle has been adopted for Num as well.

d) Articulation of viós in patronymics.

Ra does not normally accept an article before viós when viós intends clan designation. At 332, however, he accepts the article on the basis of Codex B. $\mathrm{He}$ also accepted articulation at 428 .

332 viós] pr o B V $19 d^{-125} t 126646$ Bo $=$ Ra

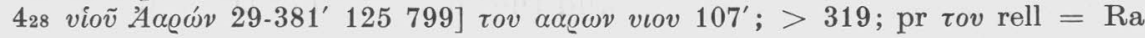

The pattern of usage in Num elsewhere is presented in the following list.

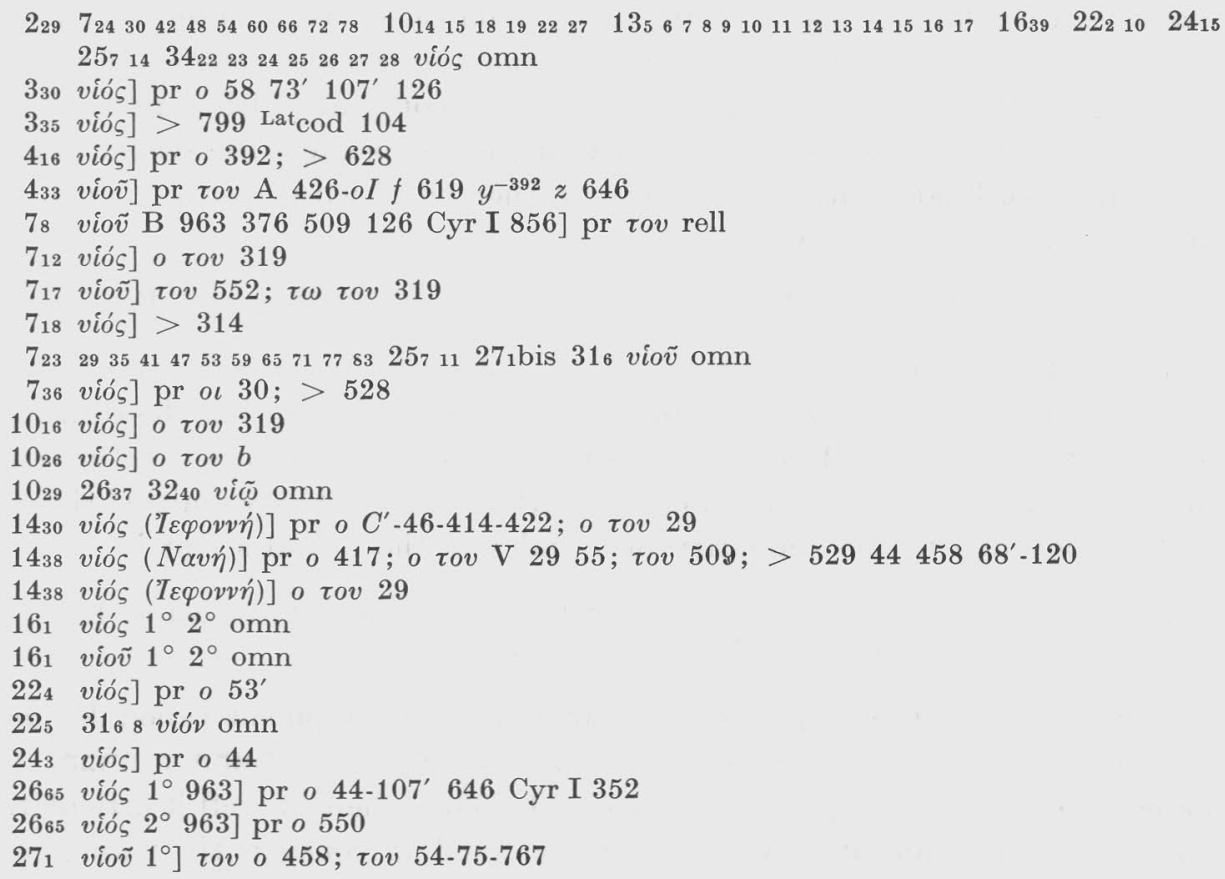


2718 vióv] pr $\tau$ $0 v 739126 ;>53$

3212 viós] pr o $963^{*}$ (c pr m) $7252^{\prime}-313 x^{-509}$

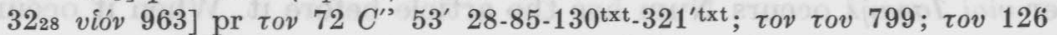

3239 viós] pr o 422 ; > 707

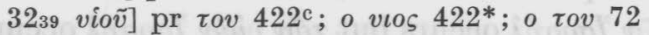

3419 viós] o $\tau o v \mathrm{~F} 130^{\mathrm{mg}} \cdot 321^{\prime} \mathrm{mg}$

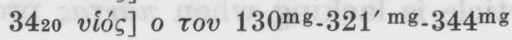

3421 viós] o tov $130^{\mathrm{mg}}$

361 viov $1^{\circ}$ ] $\operatorname{\tau ov} 551$

361 viov $2^{\circ}$ ] pr $\tau o v \mathrm{~V} 16 d t$

It is abundantly evident from the above list that in the collocation " $\mathrm{N}$ son of N" the translator never articulated "son," and that Ra's acceptance of $o$ vıos in 332 was incorrect. The instance at 428 might seem more problematic in view of the strong support in the tradition. It is reasonable to assume that

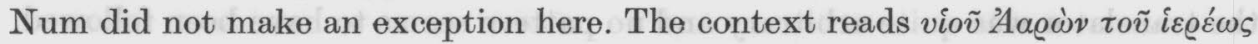
and the articulation of $i \varepsilon \varrho \varepsilon ́ \omega \varsigma$ probably created the confusion in the tradition.

e) Individual instances.

$\left.3_{10} \tau \dot{\alpha}(\tilde{\varepsilon} \sigma \omega)\right]>$ B 72-376 $x$ Cyr I 84ว Bo $=$ Ra

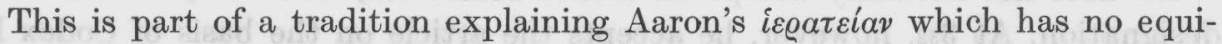

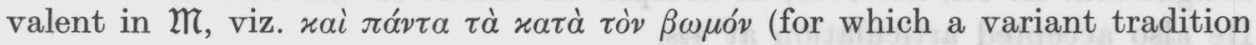

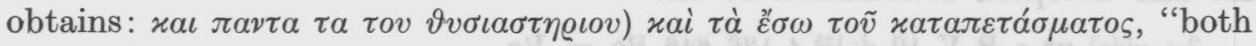
all the things pertaining to the altar and the things within the veil." The tradition is based on 187 where Aaron is divinely ordered to guard his priesthood

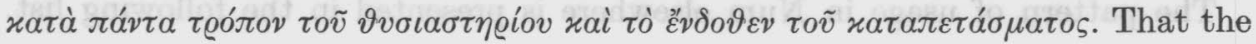
passages are related is clear. $\pi \alpha \dot{v} \tau \alpha \tau \dot{\alpha} \varkappa a \tau \dot{\alpha} \tau \dot{\nu} \nu \beta \omega \mu o^{\prime}$ is an interpretation of

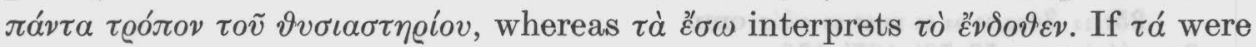

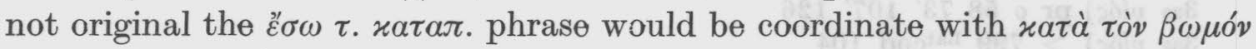
rather than with $\pi \dot{\alpha} v \tau \alpha \tau \dot{\alpha} \varkappa . \tau$. $\beta$. Since in the $18 \mathrm{z}$ passage the second phrase must be coordinate with $\pi \alpha \dot{v} \tau \alpha-\vartheta v \sigma$. rather than with $\tau \varrho o ́ \pi o v \tau$. $\vartheta v \sigma$. the article in $\tau \dot{\alpha}$ है $\sigma \omega$ must be original.

332 om $\delta 2^{\circ} \mathrm{B} O^{-58}-15-707^{*} 44^{\prime}-125 f^{-246} n 321^{*} x y^{-121} 669^{*}$ Syh $=\mathrm{Ra}$

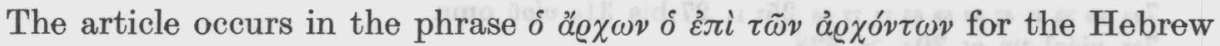

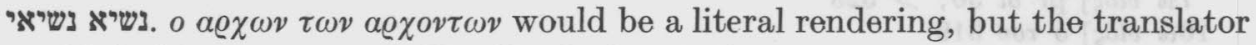
avoided this as too much like "lord of lords," i.e. a divine title. The insertion of $\delta$ घ̇лi rendered the term innocuous. The article was probably omitted through dittography due to the similarity of $o$ and $\varepsilon$ in the uncial script.

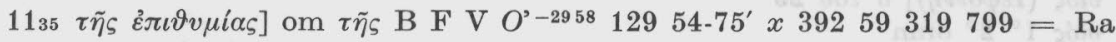

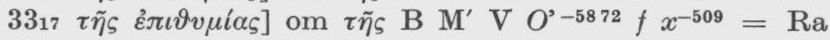

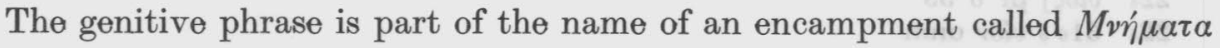

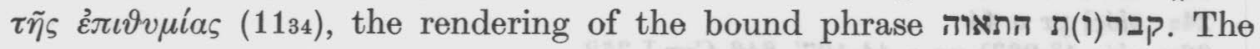
name also occurs in the preceding verses $\left(11_{34} 3_{116}\right)$ where Ra left it articulated. Obviously the translator would have rendered it consistently throughout. 
Since in all four cases $\mathfrak{M}$ articulates תאוה the articulated form of the genitive is to be preferred throughout.

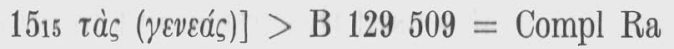

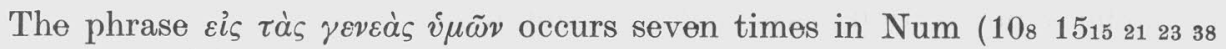
$\left.18_{23} 35_{29}\right)$ and in all instances except $15_{15}$ no witness omits the article. The omission is clearly secondary.

189 om $\tau \tilde{\eta} \varsigma$ B $82 d n^{-767} t x^{-619}$ Cyr I $837=\mathrm{Ra}$

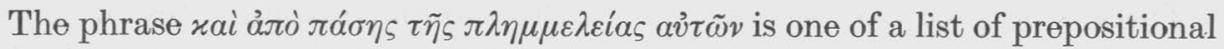
phrases of the pattern "from all their ...." In all these the nouns are articulated and it would be unlikely that this one should be left without an article. The omission of the article may well have been due to the fact that $\pi \lambda \eta \mu \mu \varepsilon \lambda \varepsilon i^{\prime} a \varsigma$ is singular (as always in the Pentateuch) in contrast to the others in the list. It is, however, a collective, and does not actually contrast with the other items in the list; the articulated noun is to be preferred.

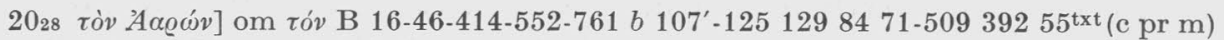
$319=\mathrm{Ra} ;>376 \mathrm{Sa}^{12}$

Though proper names are usually not articulated in Num except where the case relationship is in doubt, this one must be as $\mathfrak{k}$ shows in its את אהרן. The translator usually renders by an article, and since he did not name the subject of the verb (i.e. Moses) as $\mathfrak{M}$ had done, the article is particularly necessary to avoid the possible misinterpretation that Aaron removed his own clothıng, which is what the shorter text apparently means. Since $\dot{\varepsilon}^{\xi} \xi \dot{\varepsilon} \delta v \sigma \varepsilon v$ can be modified either by one or two accusatives, the zóv is here necessary. That this was the interpretation intended by the translator is certain from the

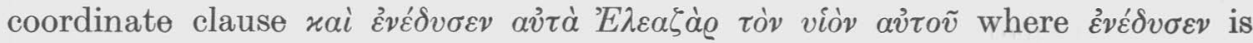
expressly modified by a double accusative.

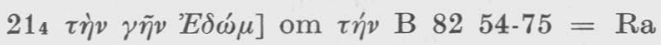

Here again $\tau \eta^{\prime} v$ is the rendering for the Hebrew את; $\mathfrak{m}$ has את ארץ אדום. Its omission in the variant tradition is the result of haplography due to its similarity to $\gamma \tilde{\eta} \nu$.

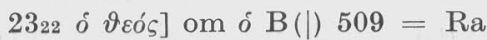

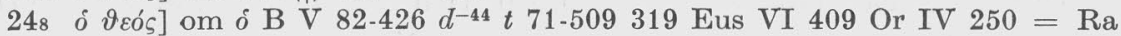

$\vartheta \varepsilon o ́ s$ is always articulated in Num when it is nominative regardless of whether or not the corresponding word in $\mathfrak{W}$ is articulated. In other cases it is usually but not always articulated. That B twice omits $\delta$ before $\vartheta$ cós may well be palaeographically inspired since all four letters of $\vartheta \varepsilon o \varsigma$ in the uncial Bible hand are similar. Note that in 2322 the word also occurs at change of line.

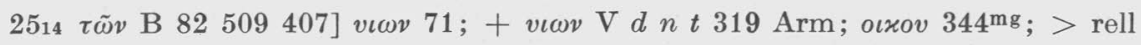

The article modifies $\Sigma v \mu \varepsilon \omega ́ v$ and follows $\pi \alpha \tau \varrho \imath \tilde{\alpha} s$. In spite of the small support the article is original. The context in $\mathfrak{M}$ is אב לשמענ. The article is an attempt to render the gentilic, for which of ch. 26 passim in particular. The word $\pi \alpha \tau \iota \tilde{\alpha} \varsigma$ 
is modified by a proper name only here and in v. 15, where, however, the Hebrew context is somewhat different. There אב במדין הוא isendered by

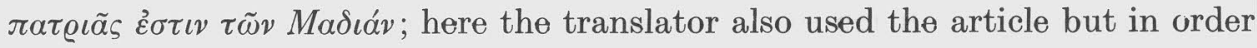
to render a preposition governing a gentilic noun.

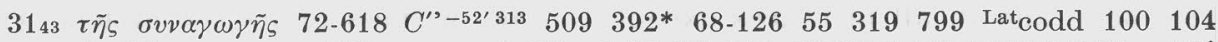

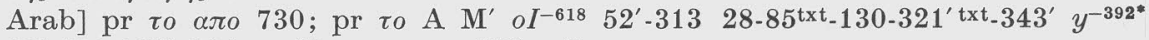
$18^{\prime}-628-630^{\prime} 624=\mathrm{Ra}$; pr $\alpha \pi \circ 963$ rell

There is no good reason for accepting the majority reading attested among others by B and 963. It has no basis in the Hebrew text, and is probably due to the number of áró phrases in the immediate context, i.e. both immediately

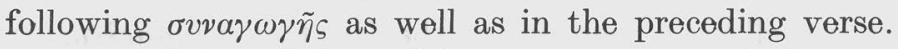

The addition of the article $\tau o$ serving as a relative pronoun was accepted by $\mathrm{Ra}$ and is more noteworthy, but it too is not original. The notion of "half of the Israelites' possession" also occurs in v. 42 and v. 47. In both cases í $\mu \iota \sigma \varepsilon v$ $\mu \alpha \tau o \varsigma$ is modified directly by a genitive construction, i.e. neither by a relative construction nor by an ảंó phrase. In all three cases the construction in $\mathfrak{N}$ is a bound phrase. In V. 43 to has probably been introduced into the tradition under the influence of the immediately preceding tó $\dot{\eta} \mu i \sigma \varepsilon v \mu \alpha$.

$\left.342 \tau \eta^{\prime} v 2^{\circ} \mathrm{B}^{*} 963 \mathrm{O}^{-58} 129 n^{-75} 799\right]>$ rell $=\mathrm{Ra}$

363 om $\tau \dot{\eta} v \mathrm{~B}$ V G-82-426 $d 129$ n $t \times 319=\mathrm{Ra}$

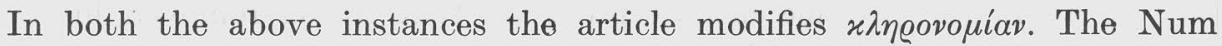
translator always articulated this noun except for those instances where it is exegetically indefinite in meaning. This statement applies in all instances regardless of whether it is modified by another noun modifier or not; cf 3218 35836247 (twice).

$3610 \tau \tilde{\varphi} M \omega v \sigma \tilde{\eta}$ 58-82-376 bd 53' $t^{-370} x^{-527} 392$ 407-630 $\left.799 \mathrm{Syh}\right] \tau \omega \mu \omega v^{\sigma} 126 ; \tau \omega \mu \omega \sigma \eta$ $\mathrm{G} n ; \tau \omega \mu \omega \sigma \varepsilon \iota 72-426$; om $\tau \tilde{\varphi} \mathrm{B} 527=\mathrm{Ra} ; \pi \varrho \circ \varsigma \mu \omega v \sigma \eta \nu$ rell

That the reading of $\mathrm{B}$ is secondary is clear from parallel passages. Whenever the formula "as/which the Lord commanded Moses" occurs (11954 23334351

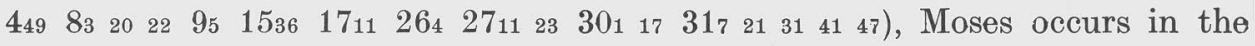

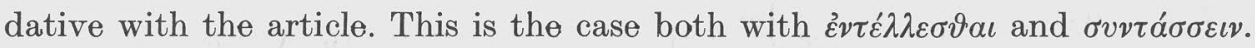
The reading of $\mathrm{B}$ must therefore be secondary. Nor is the popular variant

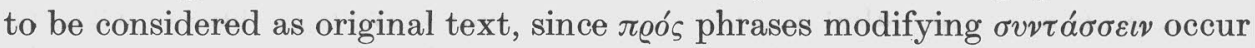
only twice (in 1523) and never as $\pi \varrho \circ \varsigma \mu \omega v \sigma \eta v$.

8. Nominal inflections.

a) A number of instances involving case inflection deserve special attention.

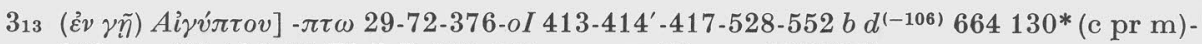
$343 t y^{-392} 12655$ Phil I 250255 ; $\alpha \iota v^{\pi \tau} 82$; $>761^{*}\left(2^{\circ}\right)$

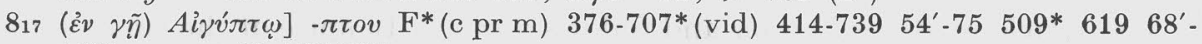
$120^{\prime} ; \alpha \iota \gamma v^{\pi \tau} 52458-767$

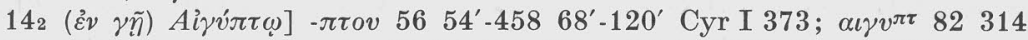

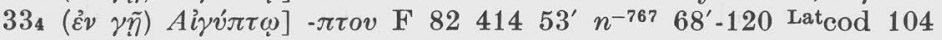




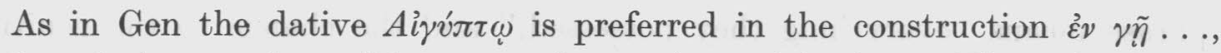
although the translator did apparently use the genitive in 313 . There the dative is a minority reading, and all the oldest witnesses except Phil witness to the genitive. It would seem that the reading of B is in each case to be preferred as indicating the original text.

$615 \vartheta v \sigma i \alpha v] \vartheta v \sigma \iota \alpha\left(\vartheta v \sigma \iota 963^{*}\right)$ B $963 n^{-458} x^{-619}=\mathrm{Ra}$

- $\sigma \pi \circ \nu \delta \dot{\eta} v] \sigma \pi o v \delta \eta$ (c var) B $963 n x^{-619}=\mathrm{Ra}$

The nominatives $\vartheta v \sigma \iota \alpha$ and $\sigma \pi 0 v \delta \eta$ are old variants but can hardly have been

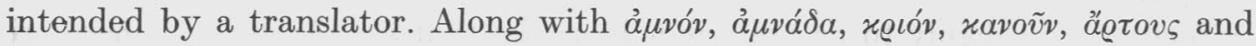
$\lambda a ́$ yava, they explicate $\tau \dot{o} \delta \tilde{\omega} \varrho o v$ as object of $\pi \varrho o \sigma a ́ \xi \varepsilon l$ in $\mathrm{v}$. 14. The variants probably arose from a misreading of $\vartheta v \sigma \iota \bar{\alpha}$ and $\sigma \pi \circ \nu \delta \bar{\eta}$ in parent texts.

$\left.88 \sigma \varepsilon \mu i \delta \alpha \lambda_{\imath \nu} 963\right] \sigma \varepsilon \mu \eta \delta \alpha \lambda \iota \alpha \nu 319 ; \sigma \varepsilon \mu \iota \delta \alpha \lambda \varepsilon \omega \varsigma$ B $7168^{\prime}-120^{\prime} 59=\mathrm{Ra} ; \sigma \varepsilon \mu \iota^{\delta \lambda} 126$; $>29551$

The reading of $\mathrm{B}$ is secondary, and probably due to the common occurrence of the genitive in the cultic laws; cf the recurring $\pi \lambda \eta \dot{\eta} \eta \sigma \varepsilon \mu \iota \delta \dot{\alpha} \lambda \varepsilon \omega \varsigma$ in ch. 7, and $\vartheta v \sigma i \alpha v \sigma \varepsilon \mu \iota \delta a ́ \lambda \varepsilon \omega \varsigma$ in 15469 . The majority reading, also attested in 963, is the accusative demanded by the context; the word is here in opposition to $\vartheta v a i x v$

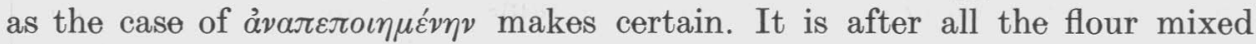
with oil which constitutes the sacrifice.

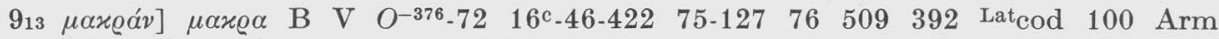
$\operatorname{Syh}($ vid) $=\mathrm{Ra}$

The word is under the obelus in Syh and is lacking in $\mathfrak{M}$. It occurs in the context $\dot{\varepsilon} v \delta \delta \tilde{\omega} \mu a \varkappa c a ́ v$, i.e. as an adverbial accusative. It occurs in this same context in $\mathrm{v} .10$, where similarly the tradition attests change to adjectival from adverbial use; $\mu \alpha \varkappa \propto \alpha$ there occurs in $O^{-376}-724145675 \mathrm{Chr}$ II 877 Latcod $100 \mathrm{Syh}$ (vid). The variant text simplifies the construction, though it may have been palaeographically conditioned, since a final $n u$ is often indicated simply by a horizontal stroke over the vowel and is easily overlooked.

$1129 \mu \circ] \varepsilon \mu \varepsilon$ B $x^{-527} 392$

The $\mathrm{B}$ text is secondary as $\mathrm{Ra}$ also recognized. It represents the classical usage after $\zeta \eta \lambda o i \varsigma$, but Num is translation Greek and here represents literally the לי the Hebrew parent text. Instructive is the translation pattern for

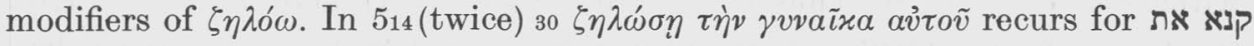
בשתו. In $2511 \dot{\varepsilon} v \tau \tilde{\omega} \zeta \eta \lambda \tilde{\omega} \sigma a i$ $\mu$ בקנאו את קנאתי On the other hand, in 2513 हैं $\zeta \lambda$ קנא לאלהיו When the preposition ל relates the modifier to the verb the dative is used, but when is used the translator rendered it by the accusative.

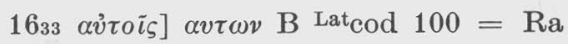

The phrase כל אשר להם also occurs in v. 26 and v. 30. In each case להם is rendered literally by the dative avizoĩs with no variants in the tradition. The 
phrase means "everything they possessed," and the change to the genitive does not change the sense of the phrase. It is probably merely a stylistic change, but in view of the literalism elsewhere as well as the small base of support for the genitive here, the genitive has been taken as secondary.

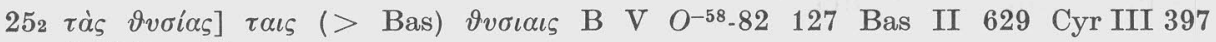
Or I $7=$ Ra

The accusative is the object of $\dot{\varepsilon} \pi i$ and the phrase modifies $\dot{\varepsilon} x a \dot{\lambda} \varepsilon \sigma \alpha v$. The

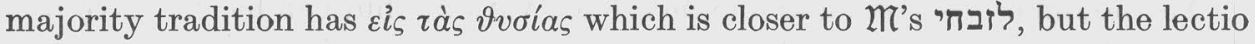

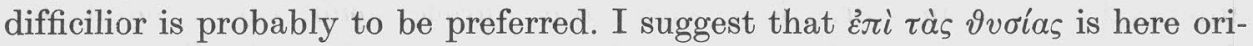
ginal since it could most easily explain both the change to the dative (possibly palaeographically inspired) as well as the change of $\dot{\varepsilon} \pi \dot{i}$ to $\varepsilon \iota \varsigma$.

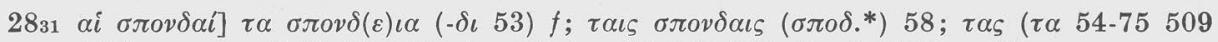

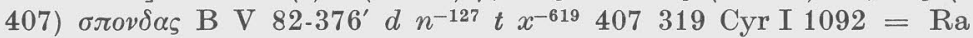

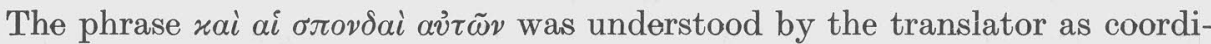
nate to $\dot{\eta} \vartheta v \sigma i \alpha$ avi $\tau \tilde{\omega} \nu$ of $\mathrm{v}$. 28. This type of collocation is fully clear from chapter 29 where the same type of grammatical understanding recurs. Thus in $29_{6}$ xai

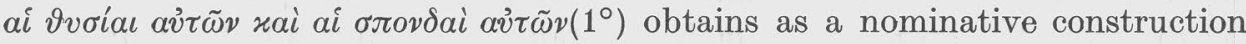
in exactly the same manner, i.e. as a continuation of the nominative construc-

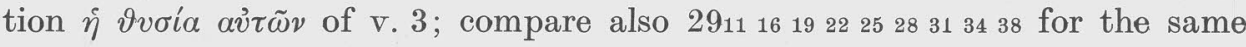
construction.

The variant text which $\mathrm{Ra}$ accepted as text was conditioned by the imme-

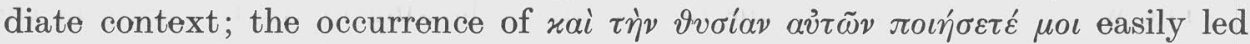
to the change of ai orovdai to the accusative of the variant text. The lectio difficilior, however, is here to be preferred as original.

$11_{33} \dot{\varepsilon} \nu\left(>52^{\prime}-313-551\right) \tau \tilde{\varphi} \lambda \alpha \tilde{\omega} \mathrm{A} \mathrm{M}^{\prime} \mathrm{V}$ oI $C^{\prime 3} 28-30^{\prime}-85^{\prime}$ txt $_{-} 321-343-344^{\text {txt }}-346^{\text {txt }} y^{-392}$

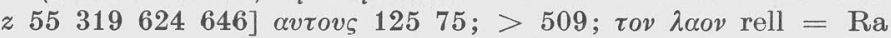

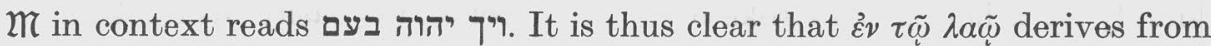

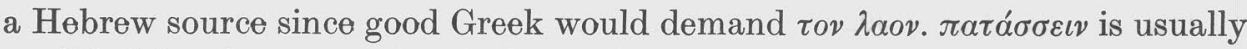
modified by the accusative in Num, but this is irrelevant since only here is the $\beth$ construction found in $\mathfrak{M}$ of Numbers. It might be argued that $\dot{\varepsilon} v \tau \tilde{\omega} \lambda \alpha \tilde{\omega}$ is a hex correction. This is unlikely to be the case since no $O \mathrm{~ms}$, nor any $d n t$ witnesses, attest to it. Thus it must be original text and the accusative a secondary improvement of Greek style; the variant text may also have been influenced by the occurrence of $\tau \dot{v} \nu \lambda \alpha o ́ v$ earlier in the verse.

b) Change in number in the nominal system within the text tradition is involved in a number of instances which invite discussion. Of particular interest are those involving the noun $\delta \lambda o x \alpha v i \omega \mu \alpha$.

The words $\delta \lambda o x \alpha v i \tau \mu \alpha$ and $\delta \lambda o x \alpha v i \tau \omega \sigma \varsigma$ are the usual renderings of עלה in Num. In the two instances where $\mathbb{K}$ has the plural $\left(\begin{array}{ll}1010 & 2939\end{array}\right)$ Num also has the plural. In all other instances $\mathfrak{m}$ has the singular, but the Greek tradition 
לעלה Num always uses the singular, and the entire tradition supports the singular with the exception of $b^{-537}$ in 616 and $d^{-106} 392$ in 2823.

Other instances of the singular in Num are as follows (only the plural variants are given).

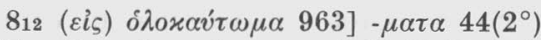

$15{ }_{3} \delta \hat{\lambda o x \alpha v ́ \tau \omega \mu \alpha]}-\mu \alpha \tau \alpha$ A B V $72 b d t \quad x \quad y \quad 55$ Cyr I $1029=$ Ra

In 153 the singular must be original since its immediately following coordinate noun is also singular, i.e. $\ddot{\eta} \vartheta v \sigma i \alpha v$ (for או זבח). Ra also transposes the word with $(o \lambda o) x \alpha \varrho \pi \omega \mu \alpha$, but this is not to be taken seriously, as the Hebrew text makes clear.

Other instances of the singular in Num are:

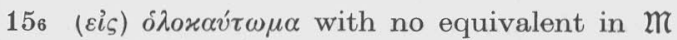

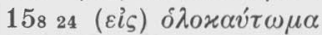

$286 \quad \delta \lambda_{0 \varkappa \alpha v i \tau \omega \mu \alpha]}-\mu \alpha \tau \alpha 106509318$

$2810 \delta \lambda_{0 \varkappa \alpha v ́ \tau \omega \mu \alpha]}-\mu \alpha \tau \alpha 7^{2} 7^{*}$ (vid) $53-664^{(\mathrm{c})} 509$ Cyr I 1116; - $-\tau \omega \mu \alpha^{\tau} 72$

2814 (

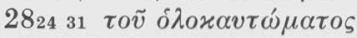

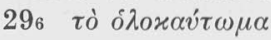

In the remaining instances Num has the plural although $\mathfrak{N}$ is singular. These are

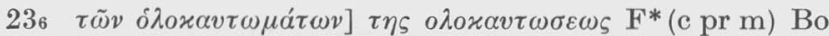

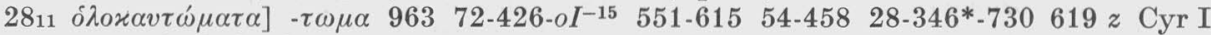

1116 Aeth Sa Syh

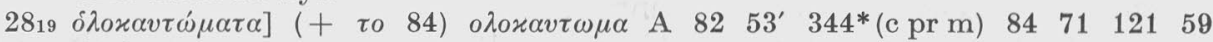
Aeth

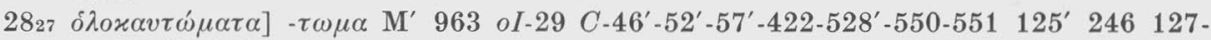
$458 x^{-509} 319$

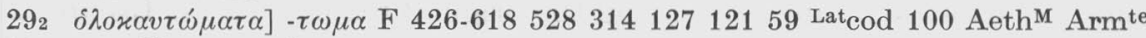

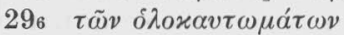

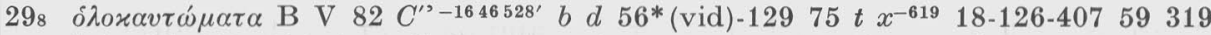

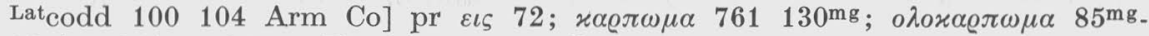
$321^{\prime} \mathrm{mg}_{-} 344^{\mathrm{mg}} ;(+\tau o \mathrm{~F})$ o $\lambda_{0 \varkappa \alpha v \tau \omega \mu \alpha}$ rell

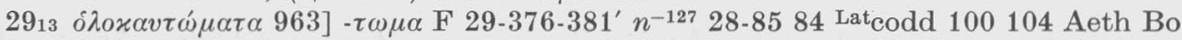

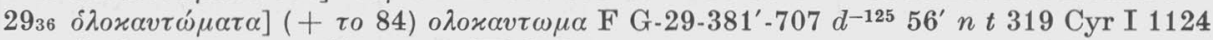

Aeth; $-\varkappa \alpha \varrho \pi \omega \mu \alpha 53^{\prime} ;-\tau \omega \mu \alpha \overline{\varkappa \omega} 376$

Except for $29_{6}$ all of these are defined in the context as consisting of more than one sacrifice and the translator rightly understood עלה in a collective

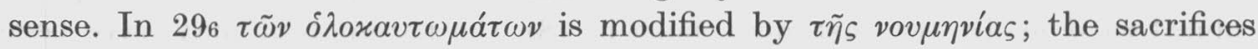
of the new moon were detailed in $2811-15$ as plural, and the translator understood these as such here as well.

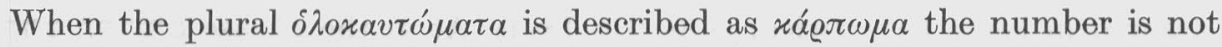
fully clear. In all instances $x a ́ \varrho \pi \omega \mu \alpha$ is the translator's rendering for אשה, i.e. the holocaust(s) are described as being a fire offering in $\mathfrak{k}$, this being regularly rendered in Num by xá

$2819 \varkappa a ́ \varrho \pi \omega \mu \alpha]-\mu \alpha \tau \alpha \mathrm{B}^{*} \mathrm{~K}$ 58-82-426 $d^{-44} f n^{-458} \quad 74^{\prime}-370624=\mathrm{Ra}$

$2913 x a ́ \varrho \pi \omega \mu \alpha$ 963] $-\mu \alpha \tau \alpha \mathrm{B}^{*} 58-82 n^{-458} \mathrm{Arm} \mathrm{Sa}=\mathrm{Ra} ;>\mathrm{B}^{c} \mathrm{M}^{\prime} \mathrm{V} x^{-619} 318407$

$2_{36} \varkappa a ́ \varrho \pi \omega \mu \alpha$ 963*] $-\mu \alpha \tau \alpha$ A B 963c 58-82 $129509 y^{-392} z 646 \mathrm{Sa}=\mathrm{Ra} ;>\mathrm{F}$ oI-72 $53^{\prime} 45859416$ Lat $_{\text {cod }} 104$ 
In each instance B supports the plural which Ra adopts. On the other hand, it is difficult to explain how an original plural would in each ease have been changed into the linguistically more difficult singular in the majority tradition,

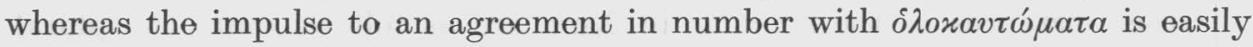
explicable. The translator interpreted the holocausts as belonging to the class of $x a$ á $\omega \mu \alpha$; this had the added advantage of exact equivalence in number to $\mathfrak{k}$. It should be noted that at 2819 the present text of Num differed from

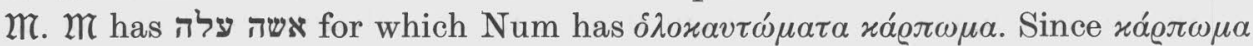

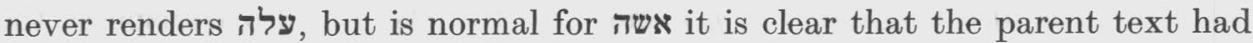
עלה אשה. At 153 B along with $d n t x$ Cyr I 1029 has transposed $x \alpha ́ \varrho \pi \omega \mu \alpha$ and

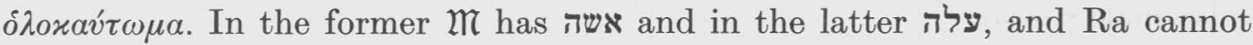

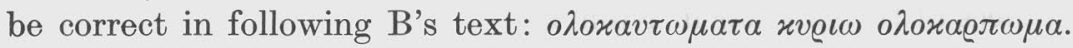

עלה is not always rendered by $\delta \lambda o x \alpha v ́ \tau \omega \mu \alpha$, however, since $\delta \lambda o x \alpha v ́ \tau \omega \sigma \iota \varsigma$ also occurs. Thus the phrase עלת התמיד is normally rendered by a Greek expression

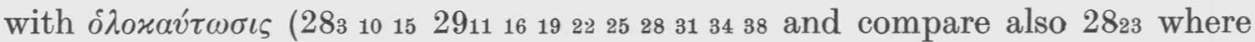
the parent text must have been עלת התמיד as well).

Four instances obtain in which $\delta \lambda o x \alpha v i \tau \omega \sigma \iota s$ is not modified by a genitive noun modifier.

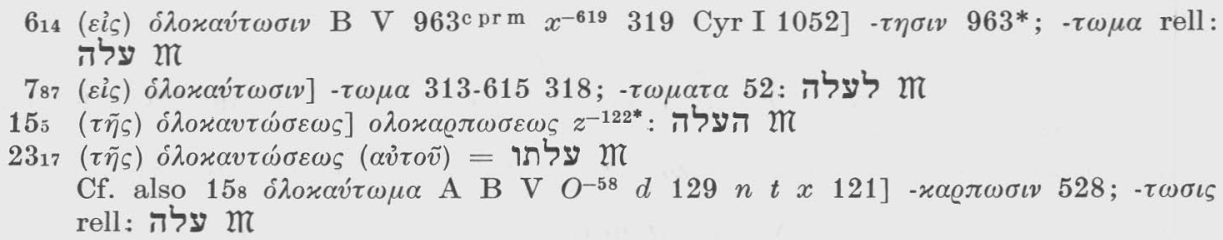

There seems little doubt concerning the original reading of 787155 and 2317 . In the case of $6_{14}$ and 158 the textual evidence is divided, but since semantically

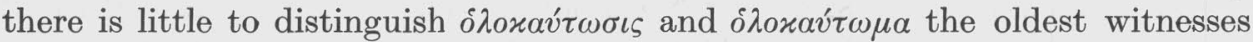
must decide.

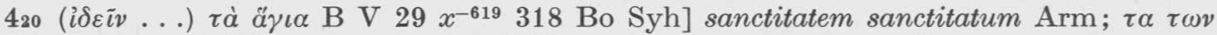

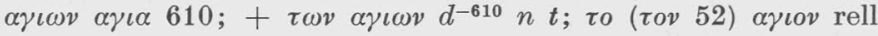

The translator is quite inconsistent in the rendering of the singular substantive קדש, at times rendering it by the singular $\tau \dot{0}$ ä $\gamma\llcorner o v$, but elsewhere by the plural $\tau \dot{\alpha} \alpha \tilde{\alpha}\langle\alpha$. In two instances, both in 189 , the parent text may have been other than $\mathfrak{M}$. With little or no variants in the text tradition the singular is clearly original in 33138416791810287 . On the other hand, the plural is equally assured in 3283241215 (twice) 19819165185 19. At 44 the phrase קדש הקדשים is rendered $\tilde{\alpha} \gamma i o v \tau \tilde{\omega} v \dot{\alpha} \gamma i \omega v$ but with $\alpha \gamma \iota \alpha$ for $\alpha$ ó $\gamma \iota v$ in $d n^{-127^{*}} t 646 \mathrm{Arm}^{\text {ap }}$. That the translator was arbitrary in the matter of number is clear from his use of

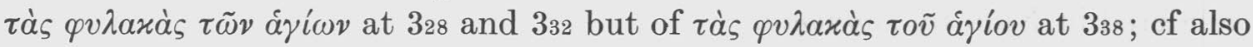

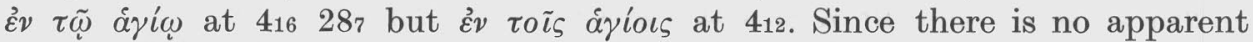
translation pattern in Num the plural which is attested by the oldest witness has been adopted for 420 . 
73 cò $\delta \tilde{\omega} \varrho o v$ B $963 x^{-619}$ Cyr I 705 Aeth Sa] $\tau \alpha(>72) \delta \omega \varrho \alpha$ rell $=$ Ra

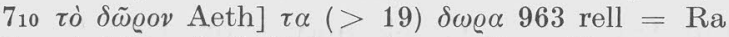

$7_{11} \tau \dot{\alpha} \delta \tilde{\omega} \varrho o v$ Aeth Bo] $\tau \alpha \delta \omega \varrho \alpha$ rell $=\mathrm{Ra}$

Chapter 7 presents the presentation of the offerings (קרבן) for the dedication of the altar tribe by tribe on successive days. Each of the twelve tribes presents its קרבן as a series of offerings in identical terms. The collective term is

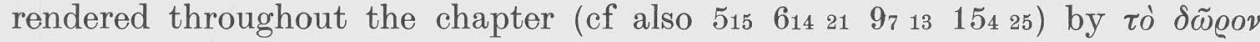
rather than the plural regardless of the number of the subject and/or verb. In the above three instances the majority tradition is the result of the pressure of the immediate plural environment, i.e. in v. 3 by $\ddot{\eta} v \varepsilon \gamma \varkappa \alpha \nu \tau \dot{~} \delta \tilde{\omega} \varrho o v \alpha v \dot{\tau} \tilde{\omega} \nu$,

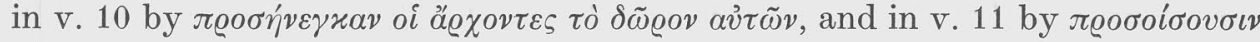
$\tau \dot{~} \delta \tilde{\omega} \varrho o v$ av่ $\tilde{\omega} \nu$. The singular occurs eighteen times throughout this chapter but the plural is not used at all.

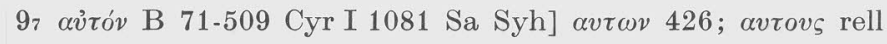

In v. 6 men approach Moses and Aaron, and a plural reference might therefore be expected in v. 7. $\mathfrak{M}$, however, has the singular; i.e. "those men said to him," viz. Moses, which Num reproduced correctly. That the singular is indeed correct appears from $v .8$ where only Moses replies to the men. The plural of the majority tradition represents an attempt to harmonize $\mathrm{v} .7$ with the preceding verse.

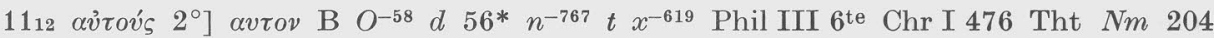
Arm $\mathrm{Bo}^{\mathrm{B}} \mathrm{Syh}=\mathrm{Ra} \mathfrak{m}$

Since the antecedent is העם the pronoun is singular in $\mathfrak{K}$; Num uses a plural

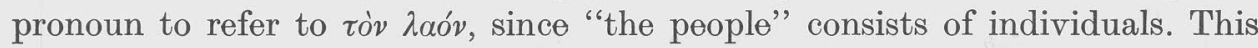

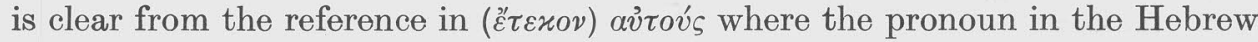
text is also singular, but only 628799 Phil III 6 te $\mathrm{Chr}$ I 476 Tht $N m 204$ have

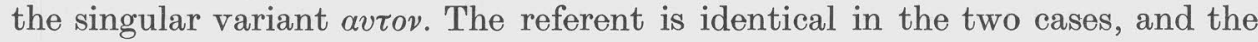
translator would hardly have changed the number of the pronoun in the very next clause. The variant is not necessarily due to Hebrew influence however; it may have been grammatically induced by $\lambda \alpha o$, though it is not clear why avं ov́s $1^{\circ}$ should not have been changed to the singular in the tradition to the same extent as avंov's $2^{\circ}$. It should also be observed that the plural reference in $\alpha v \tau \tilde{\omega} v$ at the end of the verse is unanimously supported in the text tradition.

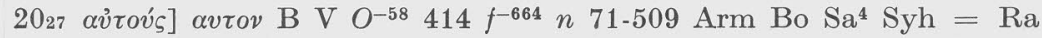

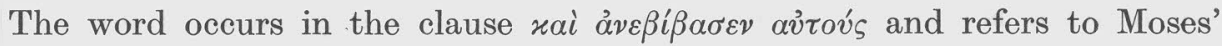
execution of the Lord's command in v. 25 "take Aaron and Eleazar his son

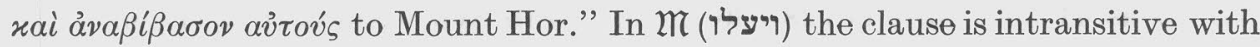
plural referent, i.e. "and they went up." The reading of B is certainly secondary in view of v. 25; it may be due to the exegetical consideration that in v. 26 the death of Aaron is predicted, and the ex post facto record would leave only Eleazar to be effectively "brought up" to the mountain by Moses. 
9. Nouns are repeated in Hebrew in order to mark distribution. This is not the case in Greek normally but translation Greek may show this Hebrew characteristic.

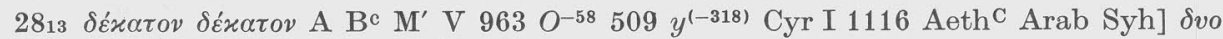
$\delta \varepsilon \varkappa \alpha \tau \alpha$ 646; semel scr rell $=\mathrm{Ra}$

The distributive עשרון עשרון occurs five times in Numbers (2813 21292910 15) and it is always faithfully rendered by $\delta \varepsilon x \alpha \tau o \nu ~ \delta \varepsilon x \alpha \tau o v$ in the Greek. The majority text accepted by $\mathrm{Ra}$ is therefore the result of haplography. This could hardly have been original, since no Greek scribe would have repeated $\delta \varepsilon \dot{x} \alpha \tau \nu$

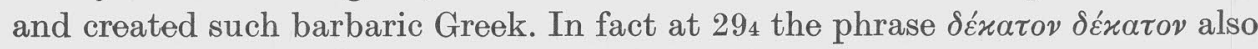
occurs with only a few supporting a single $\delta \varepsilon \dot{z} \alpha \tau o v$ :

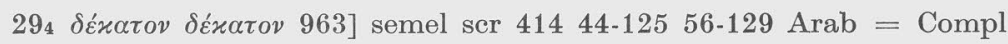

Though $\mathfrak{M}$ has a single צשרון, the parent text of Num must have had the distributive.

No such regularity of translation appears for איש איש. The phrase occurs only five times in Num but there is no set pattern in the Greek.

$\left.14 \varepsilon \ddot{x \alpha \sigma \tau o \varsigma} \mathrm{F}^{\mathrm{a}}\right]+\varepsilon x \alpha \sigma \tau \circ \varsigma$ A F G-29-426 $56 y^{-318} z^{-18} 59624$ Syh

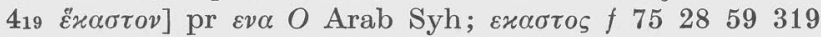

In both cases the longer reading probably was the result of Origen's work. The other three instances are

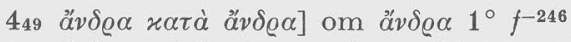

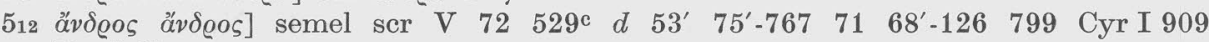
Bo $\mathrm{Sa}^{12}$

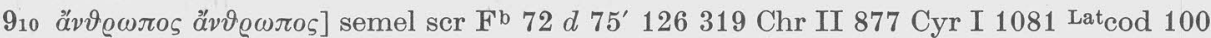
$\mathrm{Bo} \mathrm{Sa}^{12}$
}

In these three instances the repetition of the Hebrew word is also shown in the Greek.

10. Numbers.

A great deal of variation obtains in the census report of ch. 26 in the text tradition and only a small minority, mainly from the $O$ and $z$ mss. witness to the original text. This is assured by the total given as 601,730 which is $=\mathfrak{n}$. In part the divergent tradition is influenced by the census reports of chapters 1 and 2. This seems to be the case in vv. 21, 31 and 45. In $v .21$ the number for Issachar is given as 64,300, whereas in 12726 it is given as 54,400. A well supported variant reads 400 for 300 . In v. 31 Asher is given as 53,400, but in 141228 as 41,500. Only $O 128-630^{\prime}$ Aeth $^{\mathrm{C}}$ Arab Syh witness to $\pi \varepsilon v \tau \dot{\eta} r o v \tau \alpha$ ( $\chi \iota-$ $\lambda \iota a ́ \delta \varepsilon \varsigma)$, all others reading $\tau \varepsilon \sigma \sigma \alpha \varrho a ́ x o v \tau \alpha$. Similarly for Benjamin in v. 45 the majority reading of $\tau \varrho \iota \alpha \varkappa o \nu \tau \alpha(\chi\llcorner\lambda \iota \alpha \delta \varepsilon \varsigma)$ instead of the correct $\tau \varepsilon \sigma \sigma \alpha \varrho a ́ x o \nu \tau \alpha$ supported only by $O 767619 z^{-126} 628$ Arab Syh, may be due to the 35,400 of 135223 (instead of 45,600 ). 
On the other hand, no such influence can be identified in such well-supported variants as $\pi \varepsilon \nu \tau \eta x o \nu \tau \alpha$ for $\tau \varrho \iota \alpha ́ x o \nu \tau \alpha$ in $\nabla .7$, in the addition of 4000 to the number in v. 27, of 62,000 instead of 52,000 in v. 38, in 500 for the correct 600 in v. 45, in 600 substituted for 400 in v. 47, or in 300 instead of 400 in v. 50. No particular rationale seems to lie behind these majority variants since the total number is supported by almost the entire tradition.

The only text tradition which adds up correctly is that adopted as original text here (as well as by $\mathrm{Ra}$ ). It is also in all cases the same number which obtains in $\mathfrak{M}$.

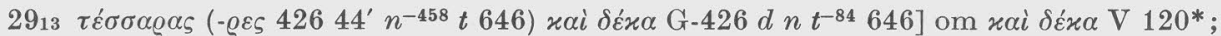
$\delta \varepsilon \varkappa \alpha x \alpha \iota^{2} \tau \varepsilon \sigma \sigma \alpha \varrho \alpha \varsigma 120^{\mathrm{c}} ; \delta \varepsilon \varkappa \alpha \tau \varepsilon \sigma \sigma \alpha \varrho \alpha \varsigma$ (c var) 963 rell $=\mathrm{Ra}$

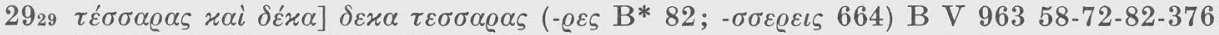
$77 d^{-44} f^{(-53)} t x 407416=\mathrm{Ra}$

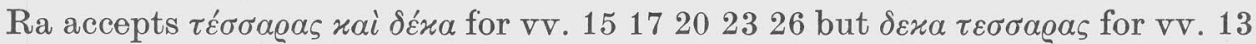
29 , thereby following the text of B. B, however, usually uses the symbols $i$ for ten and $\bar{\delta}$ for four as does 963 and is therefore not a reliable guide. In $\mathrm{v} .29 \mathrm{~B}$

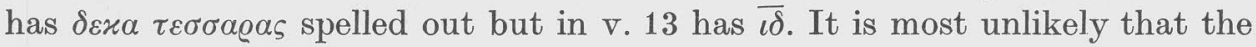
translator would have arbitrarily changed from $\delta \varepsilon \varkappa \alpha \tau \varepsilon \sigma \sigma \alpha \varrho \alpha \varsigma$ in $\mathrm{v} .13$ to $\tau \varepsilon \dot{ } \sigma-$ $\sigma \alpha \varrho \alpha \varsigma$ xai $\delta \dot{\varepsilon} x \alpha$ in vv. 15-26, and then back to $\delta \varepsilon x \alpha \tau \varepsilon \sigma \sigma \alpha \varrho \alpha \varsigma$ in $\nabla .29$. That

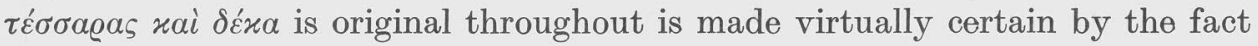

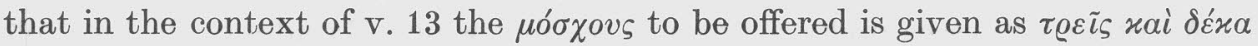
and not as $\delta \varepsilon \varkappa \alpha \tau \varrho \varepsilon \iota \varsigma$.

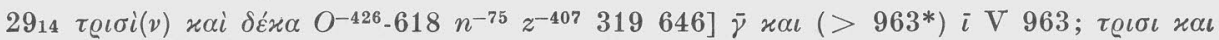

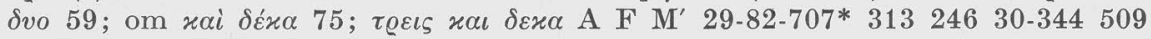
$y^{-392} ; \tau \varrho \iota \varkappa \alpha \iota \varepsilon \varkappa \alpha$ rell $=\mathrm{Ra}$

As for the instances detailed in the preceding note and as throughout Num, the ascending paratactic order for numbers from 13 through 19 has been accepted as Num text. It might be noted that usage in Num was quite different from Gen where a descending asyndeton order for the "-teen" numbers occurs throughout. This latter is also the expected order in Ptolemaic times (cf Mayser I. 2.75f.), but is hardly justified as Num in view of complete lack of support in the Greek tradition. The only possible alternative to the above text would have been the compound $\tau \varrho \varepsilon \iota \sigma x \alpha i \delta \varepsilon x \alpha$ (not the itacistic ordinal spelling $\tau \varrho \iota \sigma x \alpha \iota \varepsilon x \alpha$ chosen by $\mathrm{Ra}$ ). Since uncial texts do not show space at word juncture in the earlier centuries, the early ms tradition is not germane; accordingly a consistent pattern of separate lexemes is employed in Num, and the inflected $\tau \varrho \iota \sigma^{\prime} \nu$ is here considered to be original text.

11. Spelling of Proper Nouns.

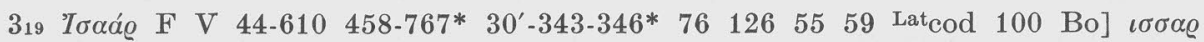

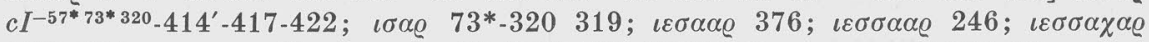

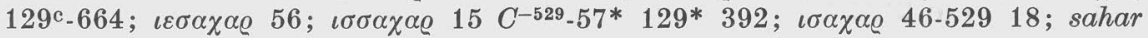
$\operatorname{Arm}(\mathrm{vid}) ;>x^{-509} ; \iota \sigma \sigma \alpha \alpha \varrho$ rell $=\mathrm{Ra}$

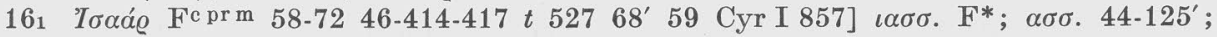

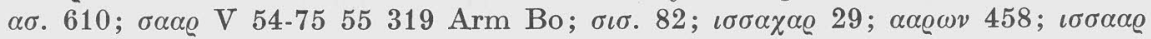
rell $=\mathrm{Ra}$ 
The translator would hardly have transcribed יצהר by a double sigma spelling; this is clear from the fact that he recognized the root as having $\pi$ as the second radical which he attempted to show by doubling the vowel. The popular $\iota \sigma \sigma \alpha \alpha \varrho$ variant is the result of dittography.

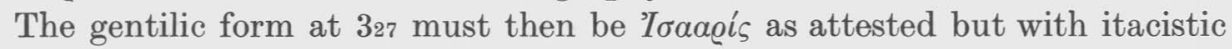

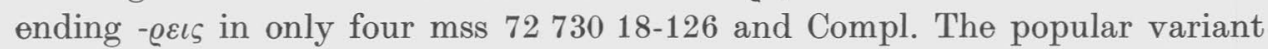
הצהרי instead of $\mathfrak{2}$ 's correct היצהרי.

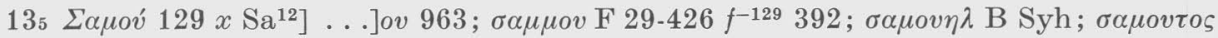

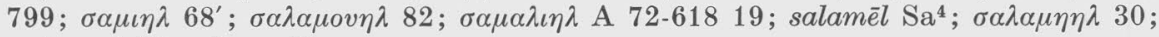
$\sigma \alpha \lambda \alpha \mu \iota \eta \lambda \mathrm{F}^{\mathrm{a}}$ rell $=\mathrm{Ra}$

That M's שמוע was also the parent text for Num is now made most likely by the reading of 963 ; the first part of the name is not extant but that ov was the end of the name is certain. The only uncertainty that remains is whether $\sigma \alpha \mu o v$ or $\sigma \alpha \mu \mu о v$ is original. The translator usually rendered the qat $\bar{l} l$ type name

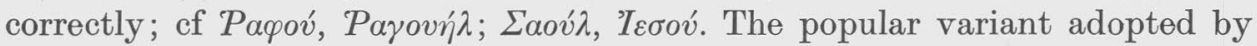
$\mathrm{Ra}, \sigma \alpha \lambda \alpha \mu \iota \eta \lambda$, was the name of the chief of the tribe of Simeon $\left(\begin{array}{ll}212 & 73641\end{array}\right.$ 1019); here Shamoua is the spy sent from the tribe of Reuben.

136 Ovei $426 C^{\prime \prime} f^{-129} 28-85^{\prime}-321^{\prime} 319 \mathrm{Syh}^{\mathrm{txt}}$

All other witnesses prefix a sigma, a reading which Ra adopts. 2 has has, however, and the sigma is a dittograph from the immediately preceding $\tau \tilde{\eta} \varsigma$.

$13_{22} E \mu a \dot{\vartheta}$ occurs for לבא חמתת in the common phrase (cf also 348). Inexplicably

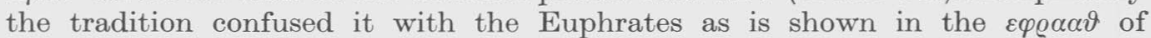
$d n^{-75} t x^{-509}$ and ephrath of Arm. This apparently led to the early error $\varepsilon \varphi \alpha \alpha \vartheta$ attested in B 376509 Lat $^{\circ} \operatorname{cod} 100 \mathrm{Sa}^{1}=\mathrm{Ra}$

$1323 \Sigma \varepsilon \sigma i$ for ששי occurs in the tradition with two sigmas in medial position in B Fa V $12734371^{\prime}$; A has $\sigma \varepsilon \mu \varepsilon$, and $\mathrm{Sa}^{12}$ semeei, all other witnesses having a single sigma. Since Masoretic pointing also witnesses to a single sibilant for the second consonant, there is no good reason to follow the minority reading $\sigma \varepsilon \sigma \sigma \iota$ with $\mathrm{Ra}$

$2112 \vartheta \alpha \varrho i \mu$ for האתרים. Ra adopted $\alpha \vartheta \alpha \varrho \iota v$; variants with final $n u$ obtain in B 82

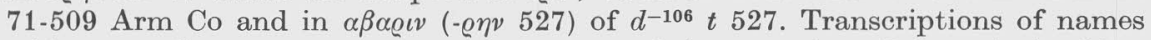
with masculine plural endings ought to end in $-\iota \mu$; thus $B \varepsilon \lambda \sigma \alpha \tau \tau i \mu$ in 3349 ;

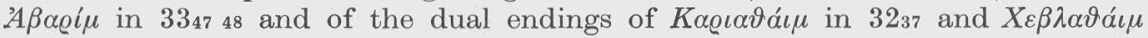
in 334647 .

In view of $B \varepsilon \lambda \sigma \alpha \tau \tau i \mu$ above it would seem best to read $\Sigma \alpha \tau \tau i \mu$ in 251 instead of the more popular $\sigma \alpha \tau \tau \iota v$ adopted by Ra; a final $m u$ is attested only by $\mathrm{F} \mathrm{F}^{\mathbf{b}}$ 29-72'-426 $d 56^{\prime} n 344^{c} t 527-619$ 18-68'-120'-126 799 Cyr III 397 IV 300 Arab Arm Syh. On the other hand, עקרבים of $34_{4}$ was almost certainly transcribed

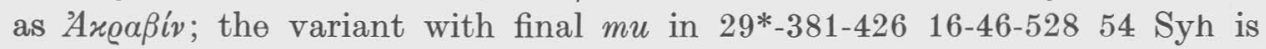
probably a hex correction. Similarly an apparently original Papı\&iv in 331415 for the name רפידם (not a plural ending however) was revised to end with $m u$ in $426761 d t$ Syh (plus Arm ${ }^{\text {te }}$ in v. 15) probably by Origen. It appears that final nasalization may well not have been phonetically distinctive between labial and nasal positions; in any event /-m/ and /-n/ are not always clearly 
kept apart. In $2643 \Sigma \omega \varphi \alpha^{\prime} v$ and its gentilic $\Sigma \omega \varphi \alpha v i$ occur. $\mathfrak{M}$ has שפופם and השופמי resp.; presumably the former read שופם in the translator's text. In the text tradition only 58-426 Syh have changed the $n u$ into mu, i.e. a hex correction.

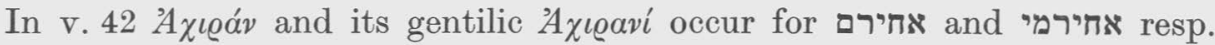
A correction of the $n u$ into $m u$ is witnessed for the former in 58-426-707 f-129 54-75' 318 Syh and for the latter in 58-426-707 $f^{-129} 54-75^{\prime}$ Syh, obviously hex

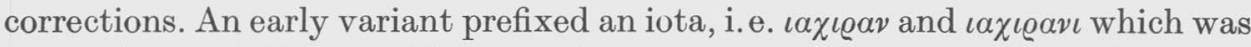
adopted by Ra. Names with אחי as first element are always transcribed as $\alpha \chi \iota$. The initial iota is the result of dittography from an uncial parent text since the word preceding 'A $\chi \varrho a ́ v$ was $T \Omega I$.

In 3367 the name "Etham" occurs, but in the first instance with the preposition ב באתם, מאתם , מן מאם, and in the second with word is transcribed in

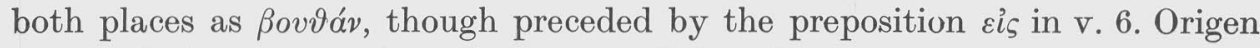
corrected the $n u$ to $m u$, as the text tradition shows:

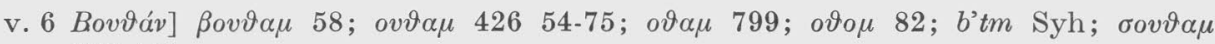
$127-458$

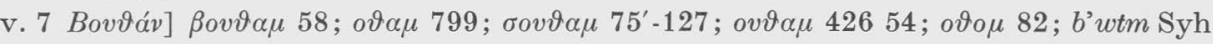

The last problem dealing with final nasals concerns the place name "Midian." $\mathfrak{M}$ has מדין throughout. The Greek evidence is as follows:

224 M $\alpha \delta \iota a ́ v ~ 426$ LatAug Num 46 Ruf Num XIII 5 Syh] mazyam Latcod 104; $\mu \alpha \delta \iota \alpha \mu$ rell $=\mathrm{Ra}$

$22{ }_{7}$ M $\alpha \delta \iota \alpha ́ v ~ 426$ Arab Syh] mazziam Latcod $100 ; \mu \omega \alpha \beta 53^{\prime} ; \mu \alpha \delta \iota \alpha \mu$ rell $=$ Ra

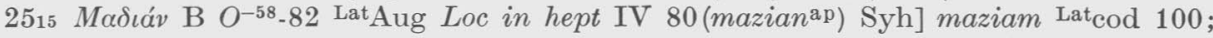
$\mu \alpha \delta \iota \alpha \nu \iota \tau \omega v 59 ; \mu \alpha \delta \eta \nu \_\omega \nu$ 799; $\mu \alpha \delta \iota \alpha \mu$ rell

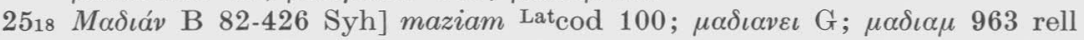

$31_{3} M \alpha \delta \iota \dot{\alpha} v 1^{\circ} \mathrm{B}$ G LatRuf Num XXV 2 Syh] $\mu \alpha \delta \iota \alpha \mu$ rell; $\cap 2^{\circ} 19$ 54-75' $126 \mid M \alpha \delta \iota \alpha ́ v$ $2^{\circ}$ B LatRuf Num XXV 2 Syh] madie Latcod $100 ; \mu \alpha \delta \_\alpha \mu$ rell

317 M $\alpha \delta \iota^{\prime} v$ B 509 Syh] madianitas Arm; $\mu \alpha \delta \iota \alpha \mu 963$ rell

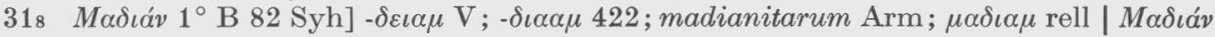
$\left.2^{\circ} \mathrm{Syh}\right] \mu \omega \alpha \beta \mathrm{G} ; \mu \alpha \delta \iota \alpha \mu$ rell

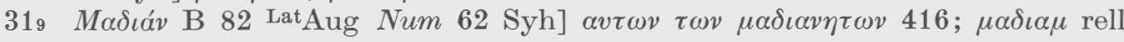

It should be noted that the gentilic form also occurs frequently throughout Num but always with a $\mu \alpha \delta \iota \nu$-stem, and there seems little doubt that the

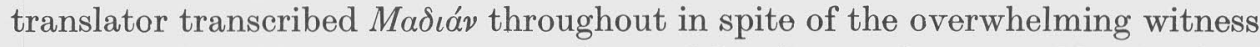

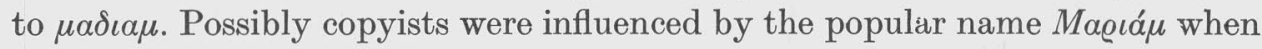
$M a \delta \iota \alpha ́ v$ occurred as an isolate, whereas such influence was void for the gentilic form.

$\left.21{ }_{12} Z \alpha \varrho \varepsilon ́ \delta\right] \zeta \alpha \varrho \varepsilon \tau$ B 52* $d t 318^{*}$ (vid) $=\mathrm{Ra} ; \zeta \alpha \varrho \varepsilon \vartheta \mathrm{F}^{\mathrm{b}} b^{(-537)} 127-767343509318^{\mathrm{c}}$ (vid)

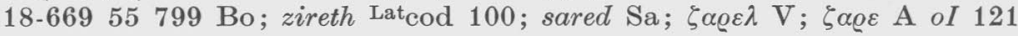

Since $\mathfrak{M}$ has זרד the B text must be secondary. The Hebrew daleth is always transcribed by delta in all names in Num regardless of position. For final posi-

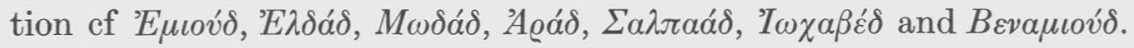

$\left.21_{24} \mathcal{A} \mu \mu \alpha^{\prime} 1^{\circ}\right] \alpha \mu \mu \alpha \quad 72 ; \alpha \mu \beta \alpha \nu \quad 53 ; \alpha \mu \mu \omega \nu \mathrm{M}^{\prime} \mathrm{V} \quad 426-707 \quad 417-528$ b $767 \quad 30^{\prime} y^{-318}$

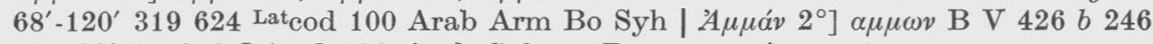
$76730^{\prime} 18319$ Latcod 100 Arab Syh = Ra; amon Arm 
Only an undue reverence for the text of B could have induced Ra to adopt two different spellings for this name within a single verse. That $A \mu \mu \alpha$ w was the old pronunciation of עמון was argued in THGD 62. This is also clear from the LXX transcription of the gentilic form with alpha, not with omega.

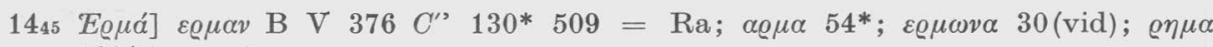
$129 *(\mathrm{c}$ pr m)

The final nasalization of the variant text may have resulted from reading final alpha as $-\bar{\alpha}$ in a parent text. In any event it is not original as $2 \pi$ 'T makes clear.

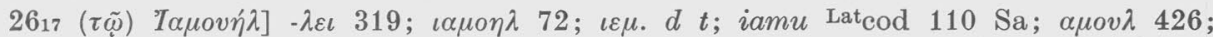

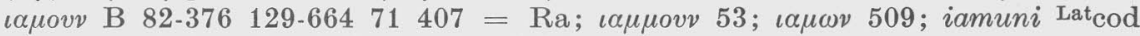
100; amuni Bo; ył̣mwl Syh ${ }^{\mathrm{L}}$; yhmw'yl $\mathrm{Syh}^{\mathrm{T}}$

MT has לחמול but the parent text of LXX must have had לחמואל as Sam. The $\mathrm{B}$ reading is due to inner Greek error in the uncial scripts, with $H$ copied as $N$. The following word is $\triangle H M O \Sigma$ and the $\Lambda$ was dropped by haplography to create $\iota$ a Whether the initial iota is original remains uncertain since except for the hex correction in 426 (cf also Bo) the tradition is unanimous in supporting such an iota.

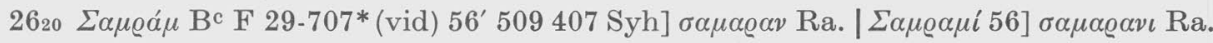

M has שמרני and and respectively. Presumably Rahlfs' conjecture is based on $\mathrm{B}^{*}$ which has $\sigma \alpha \mu \alpha \varrho \alpha \mu$ for the first and $\sigma \alpha \mu \alpha \varrho \alpha v \varepsilon$ for the second. It stands alone, however, in reading a vowel before $\varrho$, and this is unlikely to be original. More problematic is the question of the last consonant. $N u$ and $m u$ are often confused palaeographically not only, but the translator also often transcribes final $m$ by $n u$ and final $n$ by $m u$. Furthermore the forms with $m u$ are also attested for $\alpha^{\prime}$ and $\vartheta^{\prime}$. It is possible that the $n u$ tradition derives ultimately from hex (note $\sigma \alpha \mu \varrho \alpha \nu$ in 82-426 767 and $\sigma \alpha \mu \varrho \alpha \nu \varepsilon \iota$ in $82-426^{\circ}$ ), and the dominant $m u$ tradition is probably original.

$\left.26{ }_{34} X \varepsilon \dot{\lambda} \varepsilon \varkappa\right] \chi \alpha \lambda \varepsilon \varkappa 72528246767318$ Bo; $\alpha \chi \varepsilon \lambda \varepsilon \varkappa \quad 54-75^{\prime} ; \chi \varepsilon \lambda \varepsilon \delta 68^{\prime}-120$; chedek Sa; $\alpha \chi \varepsilon \lambda \varepsilon \iota \mathrm{V} ; \chi \varepsilon \lambda \varepsilon \beta 509 ; \chi \varepsilon \lambda \varepsilon \chi \mathrm{F} ; \chi \varepsilon \lambda \varepsilon \varepsilon \gamma \mathrm{B} 37612971407 \mathrm{Arm}=\mathrm{Ra} ; \chi \varepsilon \lambda \varepsilon \varepsilon \gamma \gamma 2$

$\mathfrak{W}$ has חלק. The reading adopted by Ra is clearly wrong. The letter qoph is never rendered by gamma in Num but always by kappa (except at 3422 Baxí בקי where the parent text is uncertain). The gentilic $X \varepsilon \lambda \varepsilon x i$ must also be read (for החלקי) rather than $\chi \varepsilon \lambda \varepsilon \gamma \iota$ with Ra. This same generalization applies

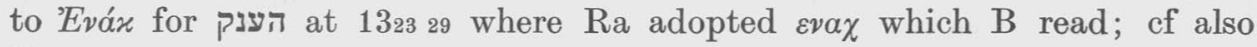
Deut 92 .

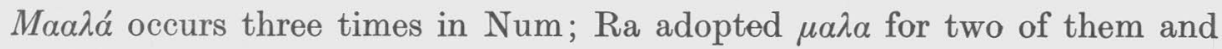

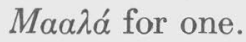

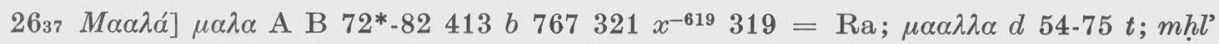
Syh; machala Latcod $100 ; \mu \alpha \lambda \alpha \alpha 392 ; \mu \alpha \alpha v \lambda \alpha 129 ; \mu \alpha \lambda \alpha \alpha \delta 127 ; \beta \alpha \lambda \alpha 130 ; \alpha \alpha \lambda \dot{\lambda} \alpha$ $458 ; \mu \alpha \alpha \nu \alpha 550^{\prime} 730 ; \mu \alpha \delta \alpha \alpha 68^{\prime}-120^{\prime}$; maada Sa; $\mu \alpha \nu \alpha \alpha 15$

118 
$\left.271 M \alpha \alpha \lambda \alpha^{\prime}\right] \mu \alpha \lambda \alpha$ A B $82129509=\mathrm{Ra} ; \mu \alpha \alpha \lambda \lambda \alpha d^{-44} n^{-127} 30 t$; maali $\mathrm{Bo}^{\mathrm{B}} ; \mu \alpha \lambda \lambda \alpha 53^{\prime}$; $\mu \alpha \lambda \alpha \alpha 414$ 71*; $\mu \alpha \lambda \delta \alpha \alpha 68^{\prime}-120^{\prime}$; magala Latcod 100; $\mu \alpha \lambda \alpha \varkappa 319$; $\beta \alpha \alpha \lambda \alpha 628$

$3611 M \alpha \alpha \lambda \alpha^{\prime} 963$ (vid)] $\mu \alpha \lambda \alpha 7^{*} 129130^{*} 509 ; \mu \alpha \alpha \lambda \lambda \alpha d^{(-106)} n t ; \mu \alpha \lambda \alpha \alpha \mathrm{A}$ oI ${ }^{-64} 392120$ $624 \mathrm{Bo}^{\mathrm{A}}$; $\mu \alpha \alpha \lambda$ b 407-630; machala Latcod 100; mathala Latcod 104; mella $\mathrm{Bo}^{\mathrm{B}}$; $v \alpha \alpha \lambda \alpha 799 ; \mu \alpha \lambda \alpha \delta 82 ; \mu \alpha \alpha \varrho \alpha 246$

Medial heth with vowel when it represents a laryngeal rather than a velar (cf JW Wevers, Heth in Classical Hebrew, Essays on the Ancient Semitic World Edited by J W Wevers and D B Redford [Toronto, 1970], 101-112) is variously rendered in Num but most commonly by a single or double vowel,

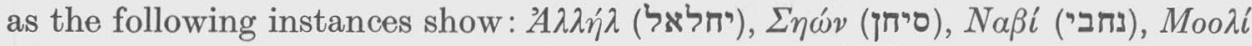

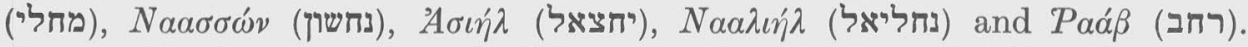
Presumably the parent text for $A \lambda \lambda \dot{\eta} \lambda$ and 'A $\sigma \iota \eta^{\prime} \lambda$ had no initial yodh. Double vowel transcriptions apparently represent heth in intervocalic position whereas single vowel transcriptions represent heth with a single vowel either before or after the heth. Since both mah one can only depend on the text tradition. It would seem likely that the

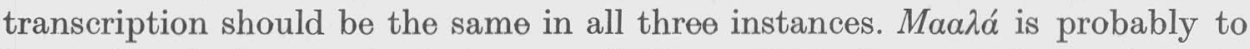
be preferred since heth closing a syllable medially is attested elsewhere in Num only for $N \alpha \beta i$; furthermore for $36{ }_{11}$ both B and 963 (vid) attest to the double vowel form.

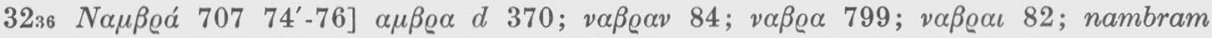
Latcodd 100 104; $v \alpha \mu \varrho \alpha \mu$ B; $v \alpha \mu \beta \varrho \alpha \nu$ Ra.; $v \alpha \mu \varrho \alpha \nu$ F 129 Aeth Arm; $\alpha \mu \beta \varrho \alpha \mu$

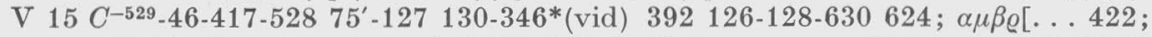

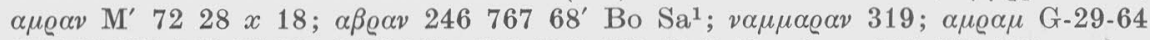
$57^{\prime}-73^{\prime}-550-761$ 85-321-343'-346 $-730120^{\prime}-62859 \mathrm{Sa}^{12} ; \alpha \beta \varrho \alpha \mu$ 313-552 669; $\alpha \mu \beta \alpha \mu$ 376 ; $\alpha \varrho \alpha \mu 30 ; \beta \eta \vartheta v \alpha \mu \varrho \alpha 426$ Arab Syh; $\beta \eta \vartheta \eta \alpha \mu \varrho \alpha \mu$ 58; $\beta \iota \vartheta \iota \alpha \mu \varrho \alpha \mu$ 56; $\beta \iota \vartheta \iota \alpha \mu \alpha \varrho \mu$ 53'; $\alpha \mu \beta \varrho \alpha \nu$ rell

A final nasal, though widespread, can hardly have been original. $\mathfrak{m}$ has בית but Num's parent text apparently lacked was then added by hex. The name נמרה also occurs in v. 3 where it was also transcribed as N $\alpha \mu \beta \varrho \alpha$. It should be noted that in the transmission of $N \alpha \mu \beta \varrho \alpha$ of v. 3 some final nasalization is also witnessed in the tradition. The spelling without initial $n u$ is due to haplography since the preceding word is $\tau \eta \dot{v}$. Final nasalization may have been facilitated by the name of Moses' father 'A $\mu \varrho \alpha ́ \mu$.

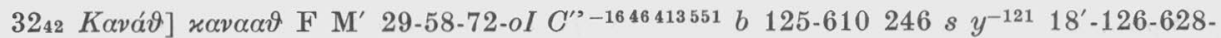

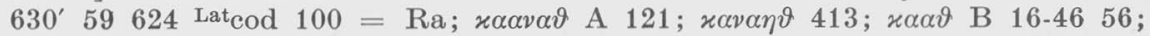
$\varkappa \alpha \mu \alpha \vartheta \alpha$ 426; $\varkappa \alpha \alpha \delta \omega \varsigma 53^{\prime}$; ganath $\mathrm{Sa}^{12}$; gathanaath $\mathrm{Bo}$; canathatha Latcod 104

There is no good reason to question the קנת of $\mathfrak{m}$ which is correctly transcrib-

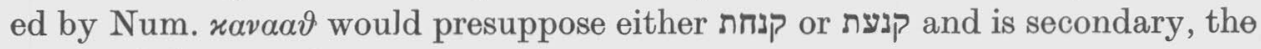
result of dittography.

$332021 \Lambda \varepsilon \beta \omega \nu a$ for לבנה. Ra adopted $\lambda \varepsilon \mu \omega \nu \alpha$ on the basis of $\mathrm{B}$, a minority reading supported by only a few witnesses

$33_{22} 23 M \alpha x \varepsilon \lambda \alpha \dot{\theta}$ for קהלתה. The parent text must have had a mim prefix since it is universally attested in the text tradition. $\mathrm{Ra}$ adopted $\mu \alpha \varkappa \varepsilon \lambda \lambda \alpha \vartheta$ read by $\mathrm{B} \mathrm{M}^{\prime}$ G 107' $129 t 509407$ Arm in both instances as well as by V 44319 in v. 22 and 
by $V^{c}$ in $\mathrm{v} .23$. It can not be original, however, if he was the second consonant in the name, which would result in the syllable $-x \varepsilon \lambda$ - or $-x \varepsilon \varepsilon \lambda$ - but never $-x \varepsilon \lambda \lambda_{-}$;

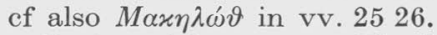

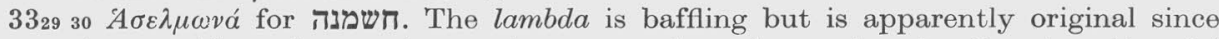
forms without it constitute a hex correction witnessed to by $O$ and scattered $f$ and $z$ mss. Ra adopted $\sigma \varepsilon \lambda \mu \omega \nu \alpha$ on the basis of B. An initial heth syllable is never

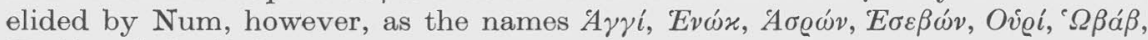
$E \mu \alpha \dot{v}$ and $A \nu \imath \eta^{\lambda} \lambda$ demonstrate.

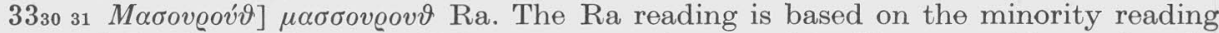
of B (though in v. $30 \mathrm{~B}$ has $\mu \alpha \sigma \sigma o v \varrho \omega \vartheta$ uniquely). The $\mathrm{ms}$ evidence for the dittograph is as follows: v. $30 \mu \alpha \sigma \sigma o v \varrho o v \vartheta \mathrm{M}^{\prime} 343509392$; $\mu \alpha \sigma \sigma o v \varrho \omega \vartheta \mathrm{B}$ | v. 31 $\mu \alpha \sigma \sigma o v \varrho o v \vartheta \mathrm{B} \mathrm{M}^{\prime} 509392 \mathrm{Sa}^{12}$. Since the translator seldom transcribed intervocalic /s/ by a double sigma (as e.g. $\Delta \varepsilon \sigma \sigma a ́)$, the majority form is to be preferred.

$333_{31} 32$ Bavaıaxáv for בני יעקן. In both occurrences the name is followed by the conjunction $x \alpha i$. The B text which Ra followed has $\beta \alpha v \alpha \iota \alpha$ and was the result of parablepsis BANATAKANKAI becoming BANAIAKAI.

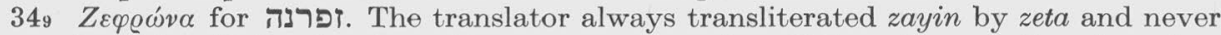
by delta. Ra's adoption of $\delta \varepsilon \varphi \varrho \omega \nu \alpha$ was based on $\mathrm{B}^{* \prime} \mathrm{s}$ unique reading, but does not merit serious consideration.

3411 X

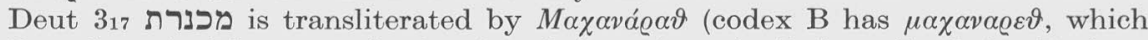
Ra adopted). The name is also found four times in Joshua. In Codex B these occur as $x \varepsilon v \varepsilon \varrho \omega \vartheta$ in $11_{2}, \chi \varepsilon v \varepsilon \varrho \varepsilon \vartheta$ in $12_{3}$ and $13_{27}$ and as $x \varepsilon v \varepsilon \varrho \varepsilon \vartheta$ in $19_{35}$. The only other occurrence obtains at $1 \mathrm{Kg} 11_{20}$ where B uniquely has $\chi \varepsilon \zeta \varrho \propto \vartheta$ although the majority has either $\chi \varepsilon v \varepsilon \varrho \varepsilon \vartheta$ or $\chi \varepsilon v v \varepsilon \varrho \varepsilon \vartheta$. In our passage B has $\chi \varepsilon v \alpha \varrho \alpha$ which Ra followed. It would seem that a transliteration with final theta must be correct here. Furthermore all witnesses which have final theta support the spelling with

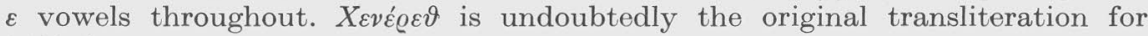
כנרת here.

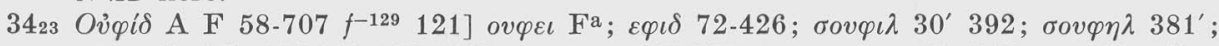

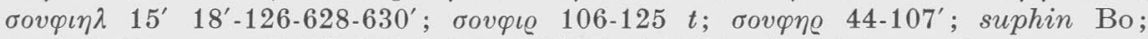

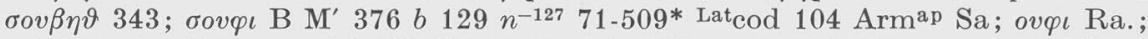

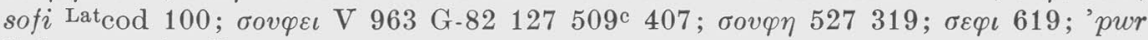
Syh; бovpı rell

$\mathfrak{N}$ has אפד, and the transcription with final delta is correct. The apocopated form is attested as early as 963 and B which led Ra to propose ovp $\iota$; it probably resulted from auditory assimilation of delta to the next syllable $\tau \tilde{\eta} s$, i.e. ov-

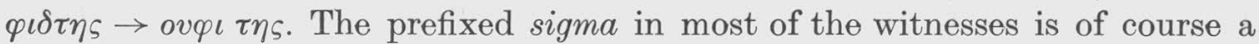
dittograph from the preceding viós.

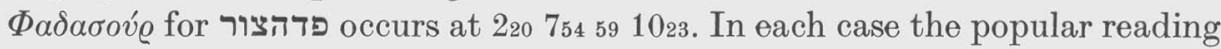

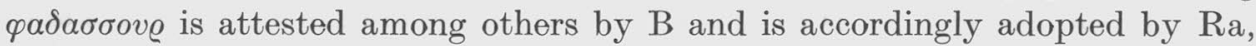
whereas $\Phi$ $\alpha \delta \alpha \sigma o v ́ \varrho$ is retained by a minority of mss.

That the he is part of the first element of the name rather than representing the article of the theophoric element צדהאל מור is clear from such names as and עשהאל where the "he" clearly stands for the third grapheme of a ' ל verb. Thus the transcription $q a \delta \alpha \sigma \sigma o v \varrho$ is certain to be secondary. The dittograph

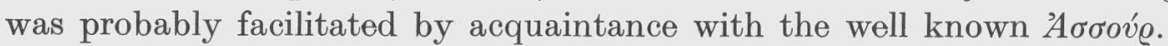

' $\Omega \beta \alpha ́ \beta$ for חבב $10_{29}$ is clearly correct. In uncial mss it was preceded by $T \Omega I$ and the iota was copied twice to produce the popular $\iota \omega \beta \alpha \beta$ variant which $\mathrm{Ra}$ adopted. 
A number of names in Num remain which do not equal $\mathfrak{M}$; either the translator misread (such as daleth for resh or vice versa) or the parent text did not $=$ 2l. Some of these were corrected by hex. They are listed here in the order of

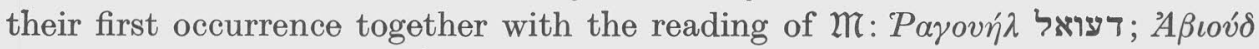

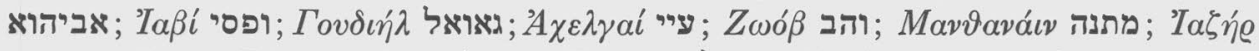

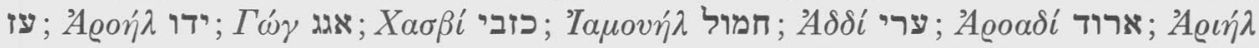

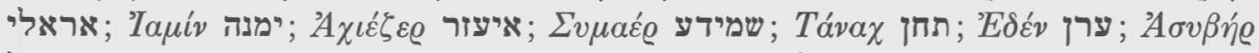

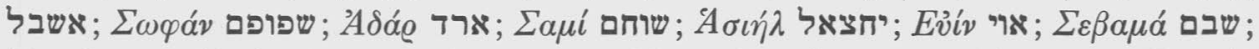

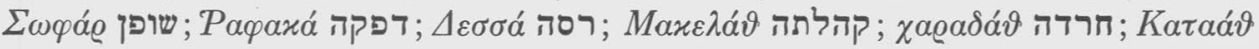

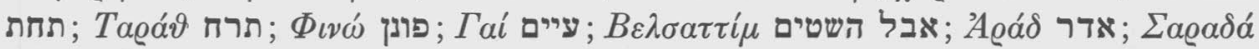

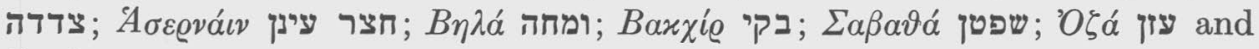

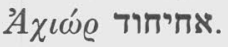

12. Verbal inflections.

a) Number

$144 \dot{\varepsilon} \pi \varepsilon \sigma \varkappa \dot{\varepsilon} \psi \alpha \tau o]-\psi \alpha v \tau o$ B Fo prm $\mathrm{M}^{\prime} d$ 127c $74^{\mathrm{c}}-76^{\prime}$ Aeth Arm $\mathrm{BoAB}^{\mathrm{c}} \mathrm{Sa}^{1} \mathrm{Syh}=\mathrm{Ra}$

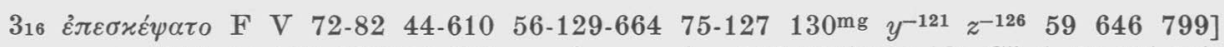

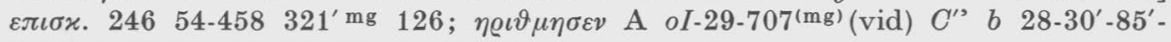
$130^{\text {txt }}-321^{\prime}$ txt_-343' $12155319624 \mathrm{Sa}^{4} ;-\psi \alpha v \tau o$ rell $=\mathrm{Ra}$

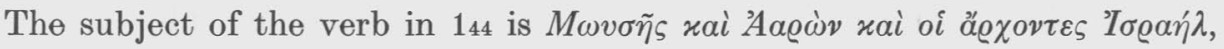

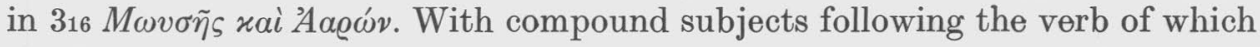
the first member is singular the verb is normally singular in $\mathfrak{K}$ as in these cases and the translator followed this same practice. When the verb (or participle as predicate) follows the compound subject it is commonly plural in Hebrew, and again the translator usually follows the Hebrew practice.

In the case of 316 the strongly attested plural in the text tradition should be seen in view of the related confusion in the preceding verse.

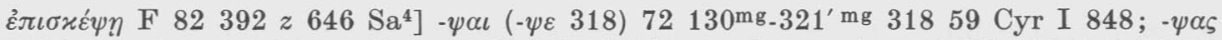
84*(c pr m); - - $\varepsilon \iota ~ 799$; $\alpha \varrho \iota \vartheta \mu \eta \sigma \varepsilon \iota \varsigma 29$ Latcod 100; $\alpha \varrho \iota \vartheta \mu \eta \sigma o \nu ~(-\mu \iota \sigma . ~ 739) ~ o I-707$ (mg) (vid)

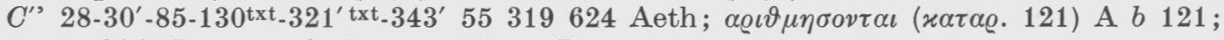
recensebitis $\mathrm{Bo} ;-\psi \alpha \sigma \vartheta \varepsilon$ (c var) rell $=\mathrm{Ra}$

The popular plural variant accepted by $\mathrm{Ra}$ is grammatically incorrect since

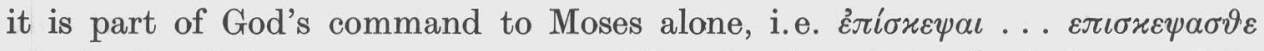
avizov́s (for Hebrew תפקדם ... Tקד). The plural tradition both here and in v. 16 arose through the confusion as to who was responsible for the census. The actual numbering of the people was the work of both Moses and Aaron, not of Moses alone. The plural verb is, however, not original.

The following cases are exceptions:

1. With verbs preceding the subject

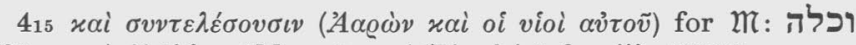

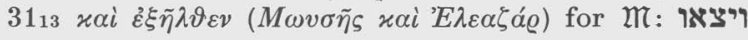

The plural was probably used in 415 since the preceding verses had been discussing the duties of Aaron and his sons in plural terms and a singular in 
v. 15 would be obtrusive. In the case of 3113 it seems likely that the parent text of the translator had the singular as has Sam.

A Greek singular verb for a plural in $\mathfrak{k}$ also obtains in the following instances.

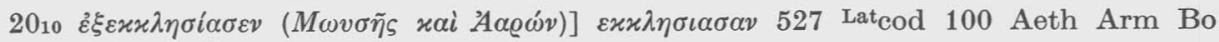
$\mathrm{Sa}^{12}=\mathfrak{m}$

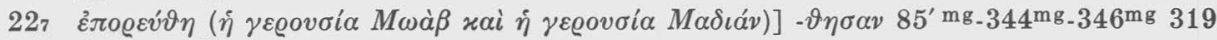
$\mathrm{Arm}=\mathrm{m}$

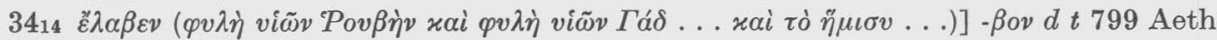
$\mathrm{Bo}=\mathfrak{m}$

In all these cases it would appear that the translator followed his normal practice of using the singular for a verb preceding a compound subject rather than strictly following the Hebrew text.

2. For verbs following a compound subject

In only one case does the translator use a singular verb after a compound

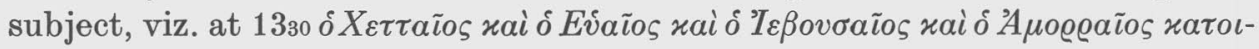
$\varkappa \varepsilon \tilde{\imath}$, undoubtedly due to Hebrew influence, since $\mathfrak{K}$ reads יושב. In one other instance $\mathfrak{N}$ reads a singular predicate with a coordinate subject preceding, 1425 והעמלקי והכנצני יושב, but here the translator followed his common practice of using the plural when the predicate follows the compound subject.

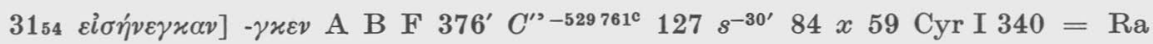

When a compound subject immediately follows a verb the verb is singular if the first element is singular, but in the following narration the verb is in the plural. Thus $1_{17} 12_{1} 14_{45} 17_{11} 206227$. In 2010 an apparent exception occurs:

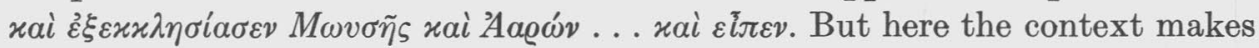
clear that it is only Moses who is the subject of $\varepsilon i \pi \varepsilon v$ since the pronoun in the

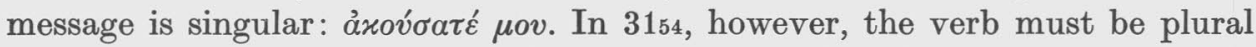
as in $\mathfrak{M}$ since both Moses and Eleazar are involved in the action.

$\left.423 \varepsilon \dot{\pi} \pi \dot{\sigma} \sigma \varepsilon \psi \alpha \iota \mathrm{B} \mathrm{M} \mathrm{M}^{\prime} \mathrm{V} 127 x^{-619} \mathrm{Co}\right] \mathrm{pr} \varkappa \alpha \iota d^{-610} n^{-127} t$ Arm; $\varkappa \alpha \iota \varepsilon \pi \iota \sigma \varkappa \varepsilon \psi o v 610$; $-\psi \varepsilon \sigma \vartheta \varepsilon$ 19 ; $-\psi \alpha \sigma \vartheta \varepsilon$ (aut $-\sigma \vartheta \alpha \iota)$ rell

Num correctly renders the singular of $\mathfrak{K}$, but in vv. 2930 uses the plural in spite of the singular in $\mathfrak{M}$. In v. 32 both Num and $\mathfrak{N}$ use the plural, probably in anticipation of v. 34 where it is said that Moses and Aaron and the leaders of Israel were responsible for the census. The majority text with the plural verb in v. 23 is an attempt at consistency. It cannot be original, however, in view of v. 21 and v. 22. Only Moses is addressed by the Lord, and he is ordered

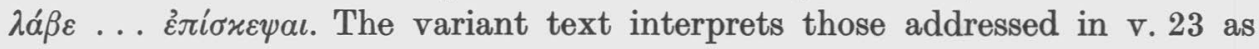
Moses and the leader of the sons of Gedson.

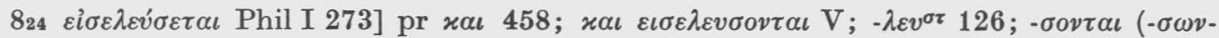
$\tau \alpha \iota 376$; $\left.\varepsilon \iota \lambda \varepsilon v \sigma .529^{*}\right)$ rell $=\mathrm{Ra}$

$\mathfrak{n}$ has singular verbs throughout vv. 2425 with which Num agrees. Ra had adopted the plural for v. 24 but the singular throughout $v .25$ creating an in- 
consistent text: The passage is introduced by $\tau o \tilde{v} \tau o \tau \dot{o} \pi \varepsilon \varrho i \tau \tilde{\omega} \nu \Lambda \varepsilon v i \tau \tilde{\omega} v$; as a result the plural easily predominated the tradition through attraction to $\tau \tilde{\omega} \nu$ $\Lambda \varepsilon v i \tau \tilde{\omega} v$, but the more unusual singular supported only by 458 and Philo seems to be original as the text of $\mathfrak{m}$ shows. In any event it would have been odd for Num to have begun with the plural and then continued with the singular.

\section{$\left.2126 \varepsilon^{\not} \lambda \alpha \beta \varepsilon v\right]-\beta o v$ B F 72-82-426 $42253^{\prime}-12971 z^{\text {Lat }}$ cod $100=$ Ra}

The plural variant can hardly be considered seriously as $\mathfrak{m}$ shows. The co-

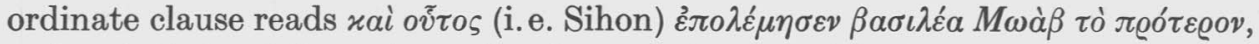
and xai $\dot{\varepsilon} \lambda \alpha \beta \varepsilon v$ follows immediately-obviously with the same subject. The variant text is based on confusion of $o / \varepsilon$ in a parent uncial text.

b) Hellenistic inflections

The Hellenistic tendency to inflect second aorist stems with first aorist endings is particularly apparent with $\varepsilon i \pi v^{i}$ which is throughout Num consistently inflected as $\varepsilon i \pi \alpha \nu$ (cf also $\varepsilon i \pi \alpha 2411$ ). In the text tradition the classical form is always a minority tradition except for the following:

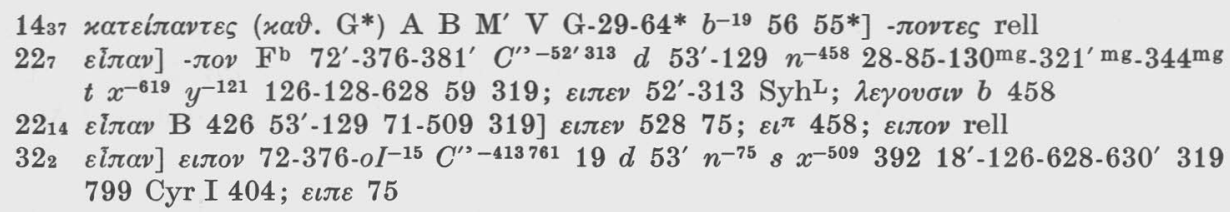

In each of these cases the oldest witness attests to the hellenistic form, and since in all other cases the hellenistic form is not only the majority reading but also supported by the oldest witnesses it must be original.

For $\varepsilon^{\prime} \varrho \chi \varepsilon \vartheta \vartheta$ a and its compounds the opposite is the case though in a few cases the text-tradition is not as clear as in the case of $\varepsilon i \pi \alpha v$.

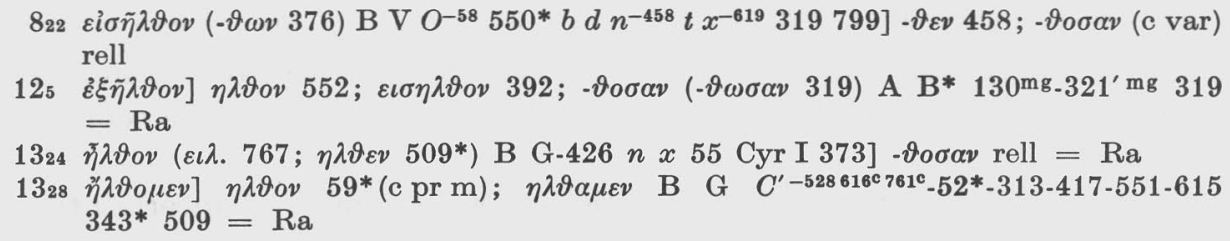

At 125 and 1328 the hellenistic form is a minority reading and was chosen by Ra because it was attested by $\mathrm{B}$. The dominance of the classical form throughout Num must outweigh these rare occurrences of support for the hellenistic forms in B. The other two instances $\left(8_{22} 13_{24}\right)$ find the hellenistic $-\vartheta$ orav ending in the majority of witnesses. In both instances B supports Num, with Ra singularly not following the B form at 1324 .

In the case of $\pi i \pi \tau \varepsilon v v$ the evidence is divided but the classical form is probably to be preferred. Only the following cases are relevant. 


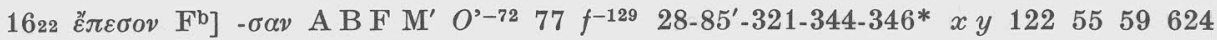
$799=\mathrm{Ra}$

$1645 \varepsilon \ddot{\varepsilon} \pi \varepsilon \sigma o v]-\sigma \alpha v \mathrm{~B}^{\mathrm{c}} \mathrm{G}-29-426 x^{-527}$; - $\sigma \varepsilon v \mathrm{M}^{\prime}$

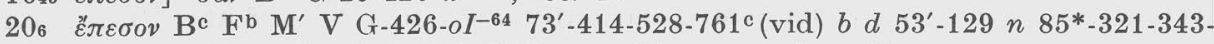
$346^{c} t x^{-509} y^{-121} z 319646$ 799] - $\sigma \varepsilon v 59^{*}$; $-\sigma \alpha v$ rell $=\mathrm{Ra}$

The translator consistently used the hellenistic form only for $\varepsilon i \pi / v$, and probably followed the classical forms for all other stems. At $16_{45}$ the classical form seems assured; the other two instances are more problematic. $B$ has the hellenistic form at $16_{22}$, but $\mathrm{B}^{\mathrm{c}}$ changes the original hellenistic form to the classical at 206. The classical form as the more conservative has been adopted in all three cases for Num.

c) Tense

$103 \quad \sigma \alpha \lambda \pi \iota \varepsilon \tilde{\zeta}] \sigma \alpha \lambda \pi \iota \sigma \varepsilon \iota \varsigma \mathrm{B}^{*} 619 z=\mathrm{Ra}$

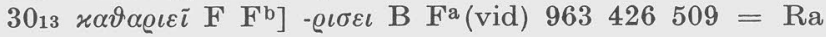

The translator avoided the uncontracted $-\imath \sigma \omega$ future which became more and more popular in later stages of the language in favour of the Attic (and Ionic) contracted forms for verbs in $-\imath \zeta \omega$. In Num the following future forms

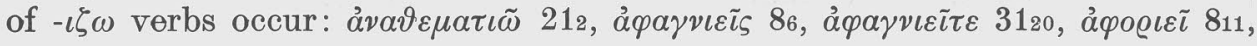

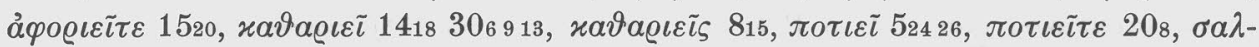
$\pi \iota \varepsilon i \tau \varepsilon 1056$ (three times) 710 , $\sigma \alpha \lambda \pi \iota \sigma \tilde{v} \sigma \iota \nu 10_{6} 8$, but of $\sigma \alpha \lambda \pi i \sigma \omega \sigma \iota v 10_{4}$. For the Attic future of $-\iota \zeta \omega$ verbs of Schwyzer I 785, and for Hellenistic usage of Mayser I 2. 128.

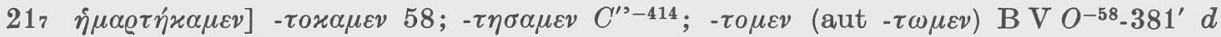
$53^{\prime}-129 n 30 t x^{-619} 39255=$ Ra

The perfect tense as the people's confession is the more exact equivalent of T's intent than is the aorist, and it probably stems from the translator. Since the object clauses of the verse contain an aorist verb (ö $\tau \varkappa \alpha \tau \varepsilon \lambda a \lambda \eta \sigma \sigma \alpha \varepsilon v)$, the tradition easily adopted an aorist for the main verb as well. The reading of $\mathrm{B}$ is likely to be the result of such adaptation.

$219 \varepsilon ̋ \delta \alpha \varkappa \varepsilon v] \varepsilon \delta \alpha \varkappa v \varepsilon v$ B $o I^{-15}-29537 d n^{-767} t 71^{\prime} 392$ Cyr II 637 Arm Sa Syh = Ra

The majority tradition with the aorist is to be preferred to the imperfect since the action of snake bite is punctiliar. It is also clear from the coordinate $\dot{\varepsilon} \pi \varepsilon \dot{\varepsilon} \beta \lambda \varepsilon \psi \varepsilon v$.

Precisely at this point the text tradition shows a number of uncertainties as well. Thus $d 53^{\prime}-129 t$ Sa place öpıs before the verb, and $\mathrm{F}^{\mathrm{c}}$ pr $\mathrm{m} \mathrm{M}^{\prime}$ 72-376-618 $b 53^{\prime} 127-76771 y^{-121} z^{-68^{\prime}} 12659319$ Cyr II 637 articulate the noun. Neither variant is original. The imperfect is, however, probably palaeographically rather than exegetically rooted.

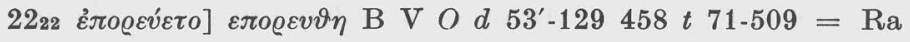

ההולך הוא The imperfect tense is original seems clear from the Hebrew which it represents adequately. More surprising is the imperfect in v. 23

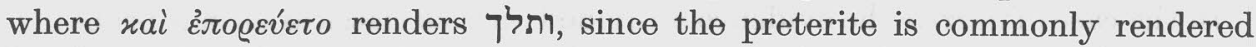
by the aorist. The translator may have been unconsciously influenced by his 
use of the tense in v. 22. In v. 22 the accent is precisely on the fact that Balaam was journeying, that is, that he continued on the way, and the aorist would be inappropriate. The variant is easily explicable since the aorist tense is far more common in Num than the imperfect.

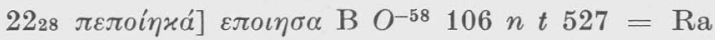

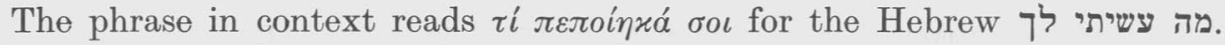
The perfect is clearly intended in view of the recurrence of the tense in the

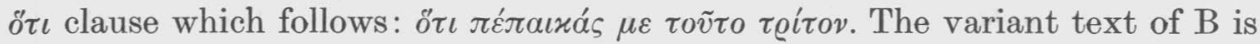
easily explicable in view of the frequency of the aorist in Num; cf also 2311.

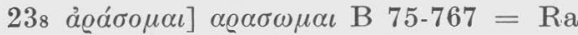

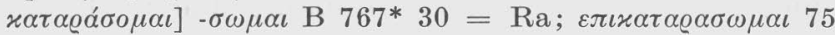

The quasi-subjunctive forms are clearly secondary. The form is future indicative, and though aras- as an aorist stem is theoretically possible it is highly implausible; of LS sub voce.

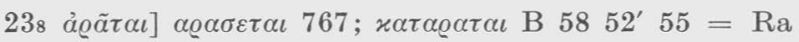

Why Ra should here have followed the B text is difficult to understand since the simplex form is clearly original. $\mathfrak{N}$ pairs אבועם with with wa wa wath

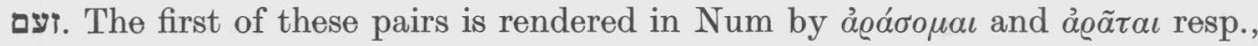

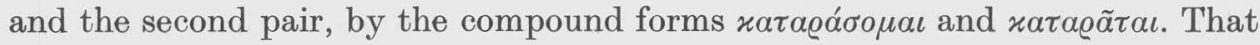
the translator should have used the compound verb for three of the four is of course highly improbable.

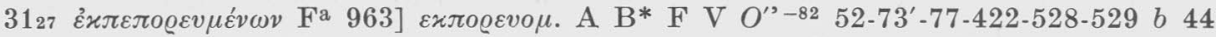
$f^{-129} 54-75^{\prime} x^{-527} y 68^{\prime}-126-6695559319624799$ Cyr I $333\left(2^{\circ}\right)=\mathrm{Ra}$

This participial form also occurs in v. 28 in exactly the same context ( $\tau \tilde{\omega} \nu$

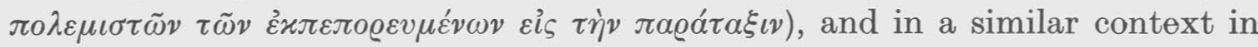
v. 36. In both cases Ra adopted the perfect rather than the present form. The witness of 963 for the perfect participle in v. 27 makes clear that the translator rendered היצאים consistently.

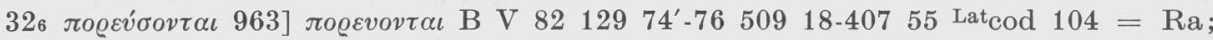
$\pi \varrho \circ \pi \circ \varrho \varepsilon v o v \tau \alpha \iota 527$

2 has the imperfect יבאו and the future renders its intent. That this is original seems clear from the coordinate verb $x \alpha \vartheta \eta \dot{\sigma \varepsilon \sigma \vartheta \varepsilon}$ rendering תשבו. It should also be noted that our oldest witness, 963 , supports the future for both verbs as well. The variant text which $\mathrm{Ra}$ adopted is palaeographically inspired, i.e. uncial $\Sigma \mathrm{O} \rightarrow \mathrm{O}$.

d) Two instances which need discussion involve voice.

$\left.2820 \pi \circ \iota \eta^{\prime} \sigma \varepsilon \tau \varepsilon\right]-\tau \alpha \iota$ A K M* 376 75' 3055 ; $\pi \circ \iota \eta \vartheta \eta \sigma \varepsilon \tau \alpha \iota b^{(-19)}$; > B F V oII-29 $f 71120$ 128-630' 319 Cyr I 1088 Aeth Arm Co = Ra 


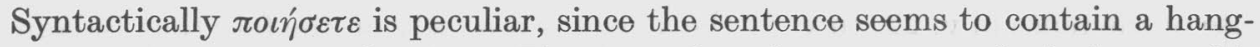
ing nominative $\dot{\eta} \vartheta v \sigma i a$ a $\tau \tilde{\omega} \tilde{\omega}$. This is to be taken as a nominal clause with

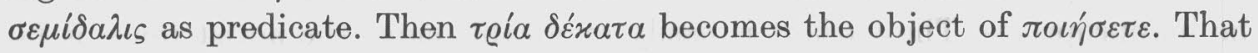
this was not always understood is clear from passive variants in the tradition on the one hand, and the omission of the verb on the other. The verb is, however, a literal rendering of תעשות, and is original to Num.

$821 \varepsilon \tilde{\pi} \pi \lambda v v \alpha v] \varepsilon \pi \lambda v v \alpha v \tau o(\varepsilon \pi \lambda \eta v .376) \mathrm{B} \mathrm{M}^{\prime} 15-376 d 56127 t=\mathrm{Ra}$

The aorist active must be original here as is obvious from the context: xai

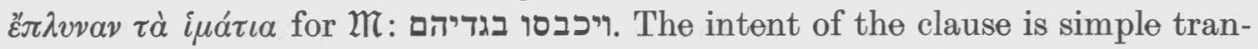
sitive action. The variant text could indeed be understood as involving some advantage to the subject, but it is secondary, probably due to the influence of the following $\tau \dot{\alpha}$. The variant tradition is especially misleading since it would most naturally be understood as passive in sense, i.e. quite at variance with the parent text.

13. Lexemes. Some of these textual problems concern variant elements in compound words.

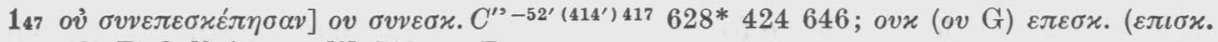

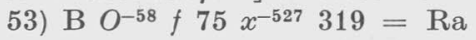

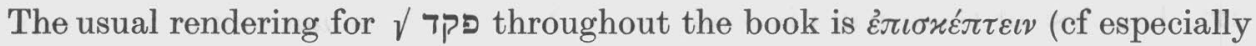
chh. 1-4), but for the Levites Moses is ordered not to count them along with the other Israelites and the compound is particularly well chosen to emphasize that fact. So too in v. 49 this verb obtains without exception in the tradition (cf also 233). The variant easily entered the tradition ex par, but it can hardly be considered original.

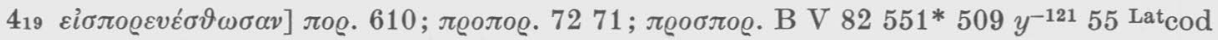
$100=\mathrm{Ra}$

$\mathfrak{W}$ has יבאו which was correctly rendered by Num. The variant is the result of textual simplification. Earlier in the verse the Levites are referred to with

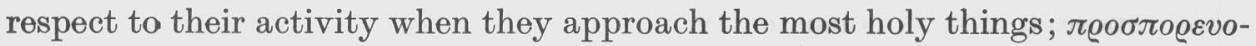

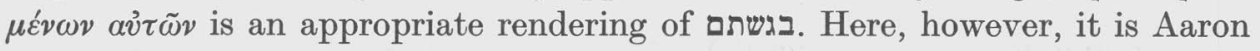

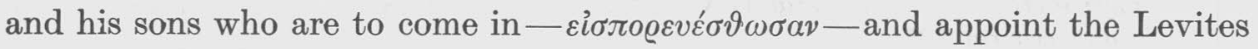
to their tasks. The variant is due to a misunderstanding of this contrast, which was fully clear in the Hebrew and to the translator.

$785 \tau \tilde{\omega} v$ áfi $\omega v$ B $\left.963458 x^{-619}\right] \tau \omega\left(\tau o 376^{*} 615\right) \alpha \gamma \iota \omega$ rell $=\mathrm{Ra}$

The phrase בשקל הקדש occurs regularly throughout the chapter modifying

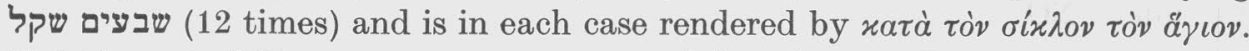
V. 85 is part of the summary statement and the relevant phrase is not rendered by a $x a \tau \dot{\alpha}$ construction but by the literalistic $\dot{\varepsilon} v$ construction. Since the translator apparently intended this literalism, the genitive would fit better in view 
of the bound construction of the parent text. The majority variant text is

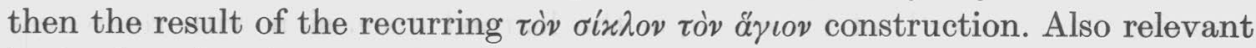
is the fact that in $\mathrm{V} .86$ the phrase obtains in $\mathfrak{M}$ but was omitted in Num; it was, however, added by hex as $\varepsilon v \tau \omega \sigma \iota \varkappa \lambda \omega \tau \omega$ a $\tau \iota \omega$ and this text may have been influential in creating the popular variant in $\mathrm{v} .85$.

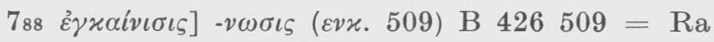

The root is $\dot{\varepsilon} \gamma r \alpha u v i \zeta \varepsilon \iota v$ and the noun formation $\dot{\varepsilon} \gamma r a i v \iota \sigma \iota s$ is expected. 963 has the itacistic variant $\varepsilon \gamma$ r $а \iota \eta \eta \iota \varsigma$, i.e. it witnesses to the majority reading.

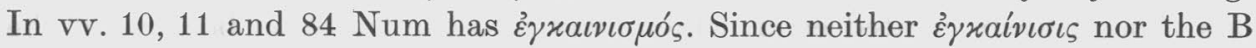
variant is attested elsewhere in LXX it would be unwise to adopt the sparsely supported B variant, particularly in view of the fact that our oldest extant witness supports the majority text.

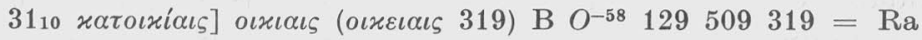

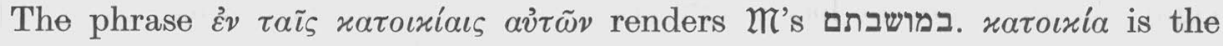
standard rendering for מושב in the Pentateuch (cf Exod 353 Lev $3177_{16} 23314$ 172131 Num 2421 3529), whereas the simplex oixia never renders מושב throughout the entire LXX. The B reading is clearly secondary.

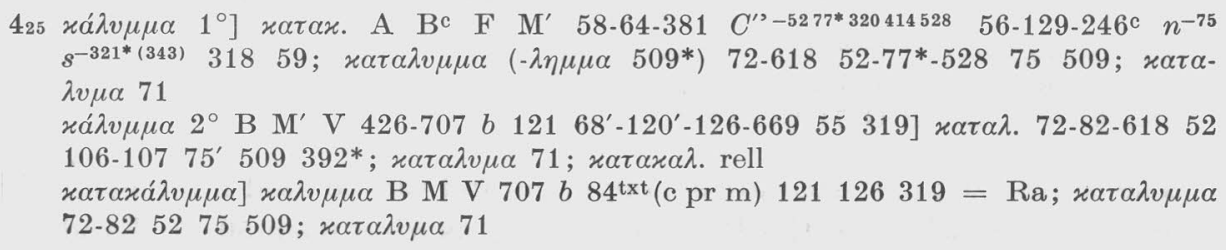

That $x \alpha \dot{\lambda} \lambda \mu \mu \alpha$ and $\varkappa \alpha \tau \alpha x \alpha \dot{\lambda} v \mu \mu \alpha$ have approximately the same lexical content is clear. The translator, however, used them carefully to distinguish the Hebrew

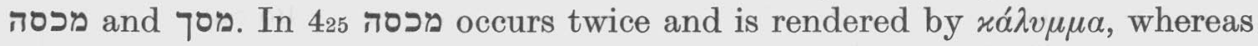

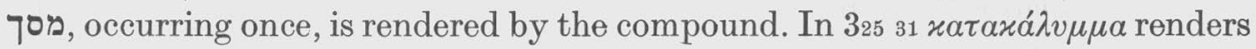
מסך, whereas in $32548101112 \varkappa a ́ \lambda v \mu \mu \alpha$ is the rendering for מכסה. In no case are the equations reversed. Once (414) $x \alpha ́ \lambda v \mu \mu \alpha$ occurs for $כ$, and in 46 it is $x \alpha \tau \alpha$ -

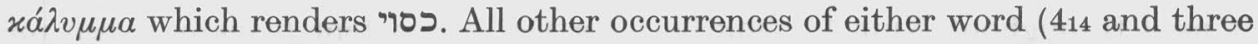
times in secondary expansions in $\mathbf{v} .31$ ) have no equivalents in $\mathfrak{K}$.

$822 x \alpha \vartheta a ́]] x \alpha \vartheta \omega \varsigma$ B* $58-7259=\mathrm{Ra}$

The clause "as the Lord commanded Moses" (and variations of it) occurs

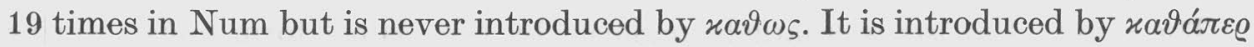
at 2723 , and otherwise by ôv

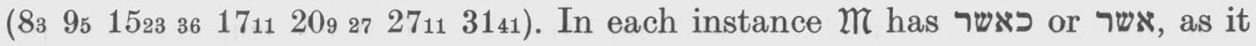
does at 822 ; $\varkappa a \vartheta \alpha$ must therefore be original here as well.

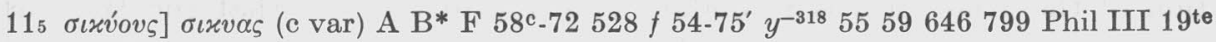
DialTA $80=\mathrm{Ra}$ 
Though "קשאים occurs only here in the OT the word is generally accepted as meaning "cucumber"; cf for example the learned discussion with references in Dillmann's commentary. This was correctly rendered by Num as oıxvovs. The feminine variant is the word for the common gourd; cf LS sub voce.

$118 \not \ddot{V} b 319$ Latcod 100 Bo] $x \alpha \iota$ rell $=\mathrm{Ra}$

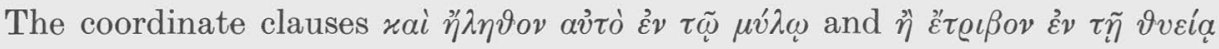
refer to alternative, not successive, actions, as $\mathfrak{M}$ clearly indicates; the manna was either ground in the mill or crushed in the mortar, not both. The variant is a thoughtlessly created error, which crept into the tradition because of the numerous xai clauses in the verse.

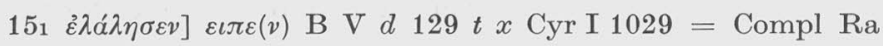

$\lambda \alpha \lambda \varepsilon \dot{\varepsilon} \omega$ is the standard rendering for $\sqrt{ }$ דבר, whereas $\varepsilon i \pi{ }^{i} \pi v$ is used for $\sqrt{ }$.

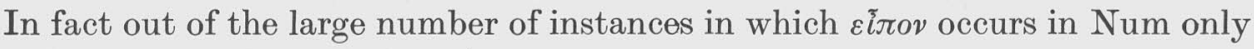

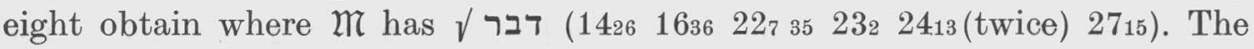
reverse pattern, i.e. $\lambda \alpha \lambda \varepsilon \dot{\varepsilon} \omega$ for $\sqrt{ }$ in $\mathfrak{K}$ only obtains at 1535182026127618 301 and 3125 . The equation is so carefully maintained that in each of these וידבר and there is no reason to question it as parent text for Num.

$\left.15_{20} \alpha ̊ \lambda \omega v o \varsigma\right] \alpha \lambda \omega \mathrm{B} x^{-509}$ Cyr VI $568=\mathrm{Ra}$

$1827 \ddot{\alpha} \lambda \omega v o \varsigma] \alpha \lambda \omega \mathrm{B} 426 x^{-619} \mathrm{Cyr}$ I $844=\mathrm{Ra}$

1830 å $\lambda \omega v o \varsigma$ ] $\alpha \lambda \omega \mathrm{B}$ G 71 Cyr I $844=\mathrm{Ra}$

The terms $\alpha \lambda \omega \varsigma$ and $\alpha \lambda \omega v$ are synonymous and can be used interchangeably. They occur elsewhere in the Pentateuch only as $\ddot{\alpha} \lambda \omega \nu$ (Gen 501011 Exod 22629 Deut 1613), and there is no good reason for adopting the sparsely supported reading of $\mathrm{B}$ as original text.

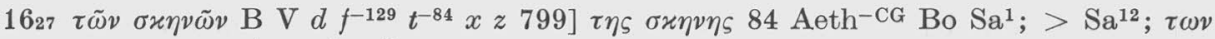
$\sigma \varkappa \eta \nu \omega \mu \alpha \tau \omega \nu$ (c var) rell

That $\tau \tilde{\omega} v \sigma \varkappa \eta \nu \tilde{\omega} v$ is original is clear from the preceding verse where secular אהלים is also rendered by $\sigma \varkappa \eta \nu \tilde{\omega} v$, only $b 392$ witnessing to $\sigma \varkappa \eta \nu \omega \mu \alpha \tau \omega \nu$. $\sigma \varkappa \eta v \eta$

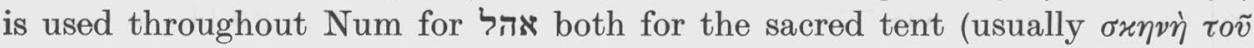

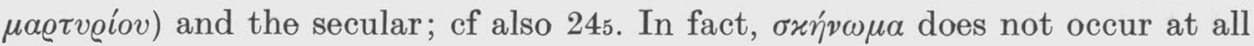
in Num.

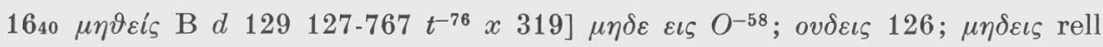

The classical $\mu \eta \delta \varepsilon \iota \varsigma$ was largely replaced by the dialect form $\mu \eta \vartheta \varepsilon \iota \varsigma$ during the third to the first centuries B.C., then again to be replaced wholly by the delta spelling; ef Mayser I, 1, 448f and especially J. Wackernagel's explanation of the theta form in Kleine Schriften II 1054. The dominance in the tradition of the delta form is secondary and due to the fact that the Hellenistic $\mu \eta \vartheta \varepsilon \iota \varsigma$ was completely replaced by $\mu \eta \delta \varepsilon \iota \zeta$ in the first centuries of our era. 


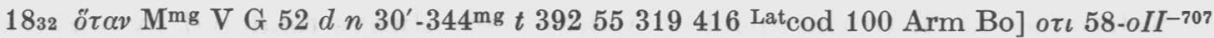

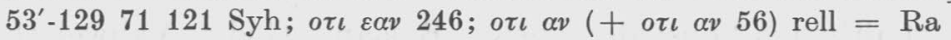

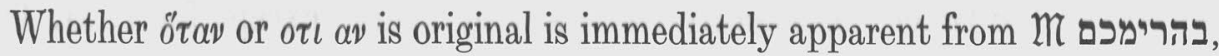
which was rendered in Num by ö $\tau \alpha v \dot{\alpha} \varphi \alpha \iota \tilde{\eta} \tau \varepsilon$, i.e. a temporal, not a causal, construction. $o \tau \iota \alpha \nu$ is simply due to palaeographic confusion of $\tau$ and $\tau \iota$ in an uncial parent text.

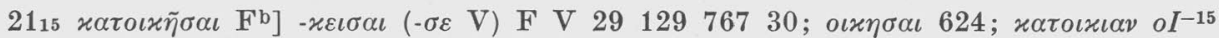

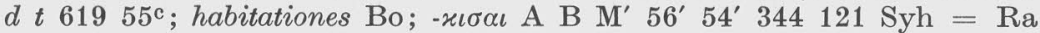

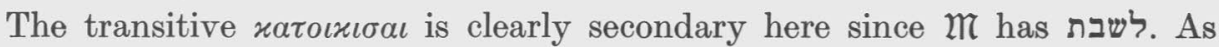

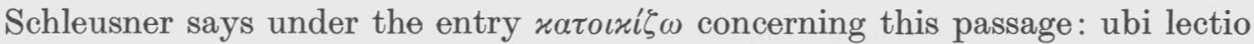

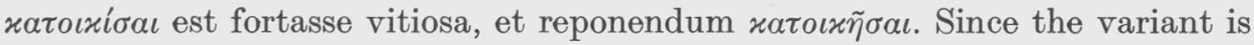

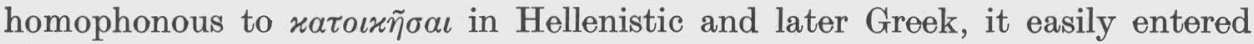
the text tradition, but it remains secondary.

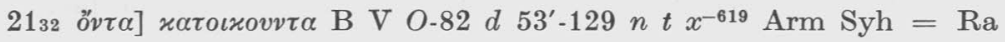

ơv $\tau \alpha$ cannot be taken as a hex correction since $O-82$ Arm Syh all attest the

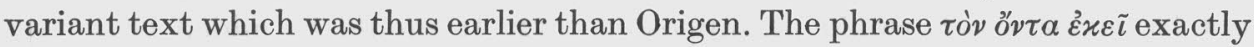
reproduces the Hebrew אשר שם, whereas the variant text seems to be an exegetical smoothing out of the text, possibly influenced by the common collocation of "the Amorite who was dwelling there."

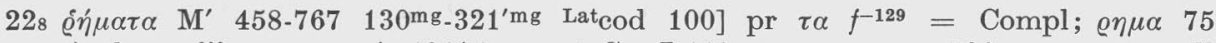

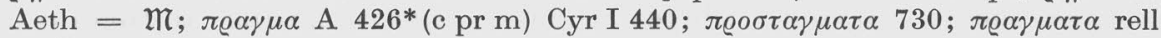
$=\mathrm{Ra}$

$\oint \tilde{\eta} \mu \alpha$ and $\pi \varrho \tilde{\alpha} \gamma \mu \alpha$ are often confused in the LXX tradition. Since the semantic field of the Hebrew דבר includes both "matter, thing" as well as "word, message," both occur as renderings in LXX. In 228, however, only the latter

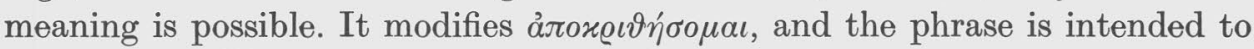
reproduce והשבתי דבר. Whenever ảrox@ivelv is used to represent השיב and has

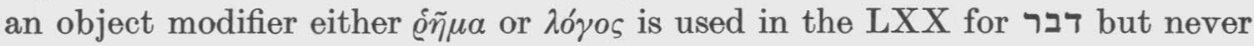
$\pi \varrho \tilde{\alpha} \gamma \mu \alpha$. Unusual here is the use of the plural since it is the singular which commonly occurs. The plural, though unusual, here refers to the awaited words of God; it can also be defended on text traditional grounds, since it would more easily lead to the variant singular text of the majority tradition than would the reverse process.

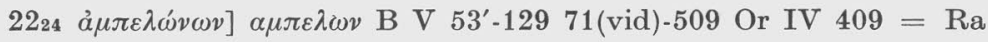

That $\dot{\alpha} \mu \pi \varepsilon \lambda \omega \nu \omega \nu$ is original and the variant text the result of haplography seems assured from the Hebrew. $\ddot{\alpha} \mu \pi \varepsilon \lambda \lambda_{0} \varsigma$ is the standard rendering of ג, i.e. the grape vine, whereas $\dot{\alpha} \mu \pi \varepsilon \lambda \omega \dot{v}$ is the standard rendering for כרם "vineyard." Contrast 64 205 with 161420172122.

$2231 \tau o \tilde{v} \vartheta \varepsilon o \tilde{v}] \overline{x v}$ B $O^{\prime}-82 b f 85^{\prime} \mathrm{mg}_{-} 321^{\prime} \mathrm{mg}_{-} 344^{\mathrm{mg}} 71^{\prime} 392 z 59$ Aeth Arm Syh = Ra; $>509$ Phil II $93^{\mathrm{F}}$ 
References in $\mathfrak{W}$ to the angel of Yahweh are always rendered by $\delta$ a $\gamma \gamma \varepsilon \lambda \lambda^{\circ} \varsigma$

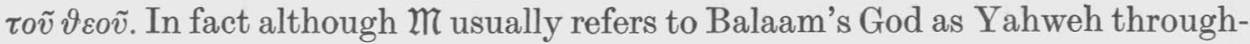
out ch. 22 the Greek consistently renders it by "God" except for v. 34. The reason may well have been theological, since Balaam was a bad seer who eventually came to a violent end and the translator may have intentionally downplayed the fact that it was Yahweh who ordered Balaam about. It should be noted, however, that the Targums do not do this. It seems, however, quite clear that $\tau$ ข $\vartheta \varepsilon o \tilde{v}$ is original here. At $\mathrm{v} .34$ xv@ív has been accepted as Num because of the textual evidence, only ms 54 and Bo attesting to $\tau o v \vartheta \varepsilon o v$ for

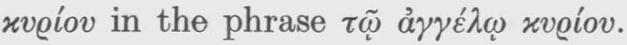

3128 oै $\nu v] \alpha \iota \gamma \omega \nu$ B Fa V $82129 x^{-527} 407319$ Arm Sa $=$ Ra

The context in $2 \pi$ reads ומן הבקר ומן החמרים ומן הצאן for which Num has

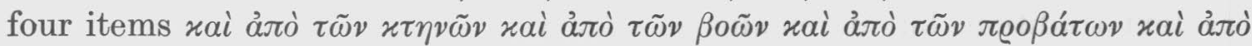
$\tau \tilde{\omega} \nu$ of $v \omega \nu$, the second item apparently a doublet to the first, with the last two

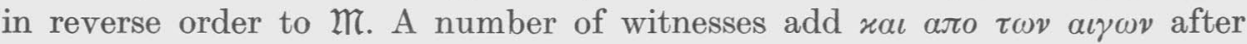

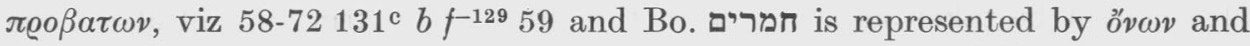
not by $\alpha \iota \omega \omega v$ which would presuppose שעירים. It should be added that Sam has the text of $\mathfrak{K}$ plus ומכל הבהמה at the end.

$\left.322_{13} \varkappa \alpha \tau \varepsilon \varrho \varrho \varepsilon ́ \mu \beta \varepsilon v \sigma \varepsilon v\right]$ $\varkappa \alpha \tau \varepsilon \varrho о \mu \beta . \mathrm{B} \mathrm{G}^{\mathrm{c}} ; \varkappa \alpha \tau \varepsilon \varrho \varrho о \mu \beta .=\mathrm{Ra}$

Why Ra should have been misled by the reading of $B$ is not clear. The root $\varrho \circ \mu \beta \varepsilon v \omega$ means "to spin," whereas the root $\varrho \varepsilon \mu \beta \varepsilon v \omega$ means "to roam or rove." Since $\mathfrak{N}$ has the Hiphil of the root נוע the majority form is obviously correct here, and the reading in B G simply a spelling error based on a confusion of omicron and epsilon in an uncial parent text.

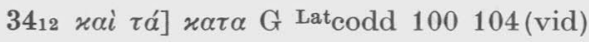

The variant text seems at first blush to find support in $\mathbb{n}$ which reads

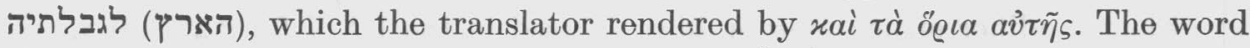
also occurs in a similar context in v.2 as rendered by $\gamma \tilde{\eta}$

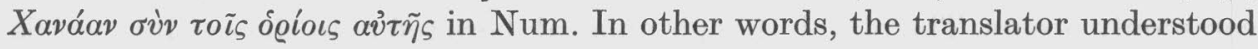
the prepositional phrase in the sense of "together with its borders" rather than as "with reference to" or "according to its borders."

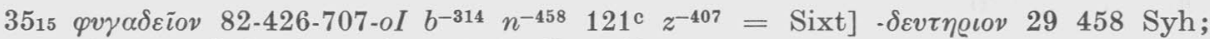
$-\delta \varepsilon v \tau \eta \varrho \iota \alpha 246$; $-\delta \iota \nu \nu(-\delta \iota \omega v 376)$ rell $=\mathrm{Ra}$

$\varphi v \gamma a \delta \varepsilon i \tilde{v} v$, not $\varphi v \gamma a ́ \delta \iota v v$, is the correct spelling here, since it is derived from

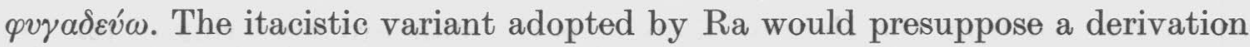
of * $v_{\gamma} \alpha \delta \varepsilon \omega$ which does not obtain. Cf LS as well as Walters 43.

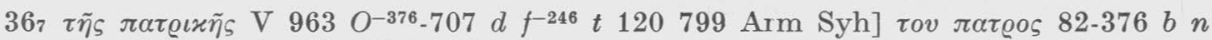
$85^{\mathrm{mg}} .344^{\mathrm{mg}}$ Latcodd $100104 \mathrm{Co}$; patrum Aeth; om $\pi \alpha \tau \varrho$. F; $\tau \eta \varsigma(>509) \pi \alpha \tau \varrho \iota \alpha \varsigma$ $\left(+\alpha \varsigma \mathrm{F}^{\mathrm{a}}\right) \mathrm{F}^{\mathrm{a}} \mathrm{F}^{\mathrm{b}}$ rell $=\mathrm{Ra}$ 
אבת as "family clan(s)" is normally rendered by $\pi \alpha \tau \varrho \iota \alpha \varsigma(-\omega v)$, but here and in v. $8 \pi \alpha \tau \iota \iota \eta^{\prime}$ is apparently original text. This is assured in $\mathrm{v} .8$ where the

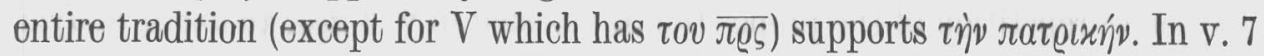
$\pi \alpha \tau \varrho \iota \tilde{\eta}$, though the more unusual rendering, is to be preferred particularly in view of the support of 963 as the oldest witness. The variant reading is to be explained as ex par.

14. Instances where the longer text is to be preferred

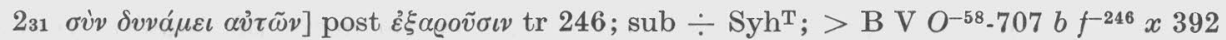

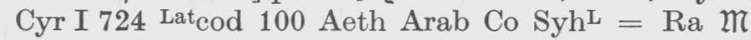

The first two chapters contain a great deal of repetitive materials and the translator renders them in almost formulaic fashion adding set phrases even when they are occasionally absent in $\mathfrak{M}$. In the parallel passages 291624 this phrase occurs both in $\mathfrak{K}$ and in Num. In $\mathrm{v} .31$ it is absent in $\mathfrak{K}$ but present in Num. That it is original to Num is now made even more certain by the witness of $\mathrm{Syh}^{\mathrm{T}}$ where the passage is under the obelus. The phrase could of course have crept into the text ex par, but it is too much to expect the obelus to have been added as well and that coincidentally corresponding exactly to the situation in $\mathfrak{m}$.

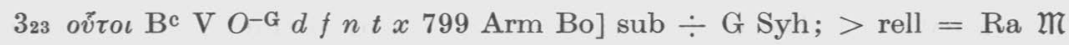

The word seems to have been original; it was in any event present in the preOrigenian text as the obelus in $\mathrm{G}$ and Syh demonstrates. It should also be noted that it is preceded by xai which also has no equivalent in $\mathfrak{N}$ and is also under the obelus in G and Syh. Since ovं text tradition has been articulated the originality of ovं $\tau \iota$ is not fully certain. Palaeographic confusion could have introduced the word into the text prior to Origen.

914 ov๊ $\omega \varsigma]>$ B $129 n^{-767}$ 71-509 Aeth Arab Arm Co $=$ Ra

That the shorter text might conceivably be seriously considered as original text could only arise out of an undue reverence for the witness of B. Num always represents the כן of $\mathfrak{M}$ correctly by oṽ $\omega_{\varsigma}$ and there is no good reason why he should have failed to do so here.

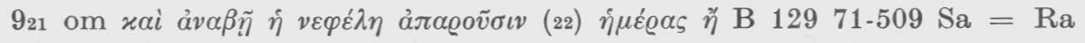

The shorter text can hardly be original as the abrupt transition from $\dot{\eta} \mu \varepsilon \varrho \alpha \varsigma$

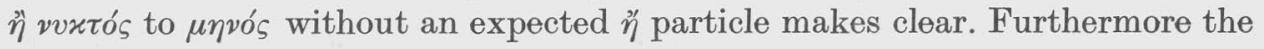
text represents $\mathfrak{W}$ adequately and there is nothing palaeographically obvious in the Hebrew which might have promoted the omission by the translator. The omission is explicable within the Greek tradition as a lapsus oculi, skipping

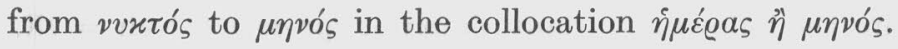

$\left.1827 \omega 2^{\circ}\right] \varepsilon \iota \varsigma 246 ;>$ B $129 x^{-619} 319 \mathrm{Cyr}$ I $844 \mathrm{Bo} \mathrm{Sa}^{1}=\mathrm{Ra}$ 
The translator often tends to repeat the preposition in paratactic construc-

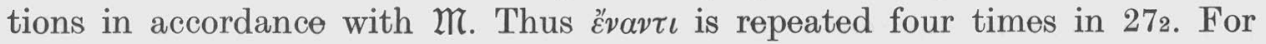
chapter 18 repetition of the preposition is attested in v. $3 \pi \varrho \dot{\varsigma} \tau \dot{\alpha} \sigma x \varepsilon v ́ \eta \tau \dot{\alpha} \alpha \gamma_{\gamma} \alpha$

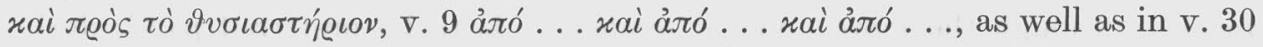

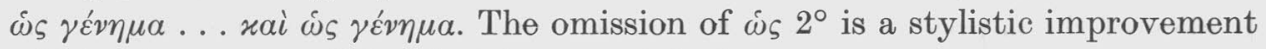
within the text tradition but is not original.

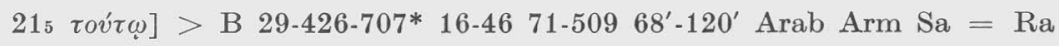

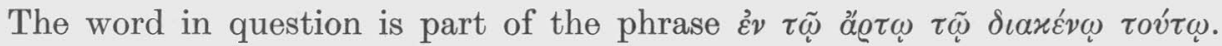
The pronoun has no counterpart in $\mathfrak{M}$, but it is apparently under the obelus in $\mathrm{Syh}^{\mathrm{L}}$ and is thus at least as early as Origen. Furthermore its omission is only sparsely supported in the text tradition.

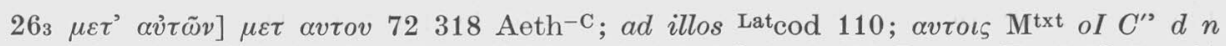
$30^{\prime}-85^{\prime}$ txt $_{-} 321^{\prime}$ txt $_{-} 343^{\prime} t 392 z^{-68^{\prime} 120} 55319646$ Lat $^{\prime}$ cod 100 Bo; > B 58-82 71-509 $\operatorname{Aeth}^{\mathrm{C}} \mathrm{Arm} \mathrm{Sa}=\mathrm{Ra}$

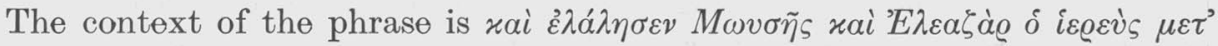

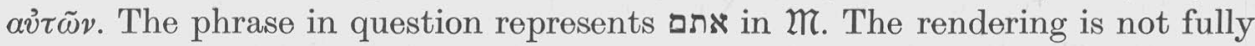

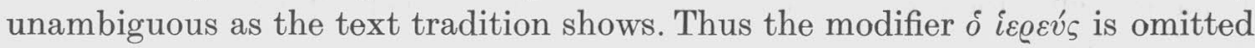
by $\mathrm{A} o I^{-15} d^{-106} y^{-392} 55319$ Aeth. The ambiguity lies in the fact that $\mu \varepsilon \tau^{\prime}$

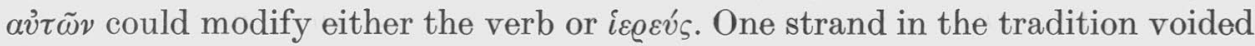

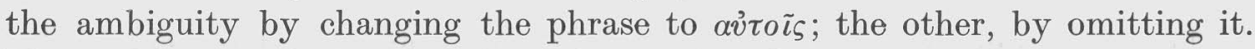
The ambiguous phrase must have been original.

356 om å५ $1^{\circ} \mathrm{B} \mathrm{V} 96382 b f x 407319$ Cyr I $865=\mathrm{Ra}$

357 om $\ddot{s}$ B V $82129344^{\text {txt }} x 407319$ Lat $^{\text {Lod } 100 ~ A r m ~}=$ Ra

The relative pronoun follows $\tau \dot{\alpha} \varsigma \pi \hat{\jmath}^{\prime} \varepsilon \iota \varsigma$ and was easily lost through homoioteleuton. Decisive is the Hebrew text which has הערים אשר. In v. 6 the original a $\zeta$ was lost as early as 963 , i.e. before the time of Origen whose parent text also lacked the pronoun; he restored it under the asterisk which ms $\mathrm{G}$ attests. That the translator did not intentionally omit $\alpha_{\zeta}$ in this type of context is clear from v. 4 where $\alpha \varsigma$ follows $\pi \dot{\lambda} \lambda \varepsilon \omega \nu$; there its omission obtains only in $82 b 54-75^{\prime} 509318628\left(2^{\circ}\right)$, and it is undoubtedly original; cf also v. 8 where the pronoun is present in all witnesses except 407319 and its originality is unquestioned.

15. Finally a number of passages obtain in which $\mathrm{Ra}$ adopted a longer text than that of Num.

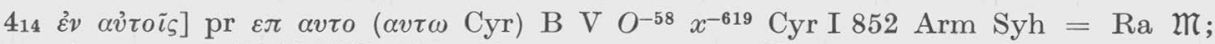
$+\varepsilon \pi \alpha v \tau o$ 44' $-125\left(2^{\circ}\right) 127 t \mathrm{Sa} ;+\varepsilon \pi \alpha v \tau \omega v n^{-127}$

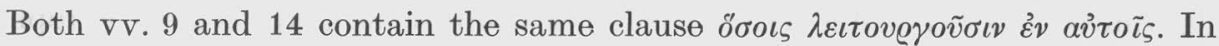
v. 9 the tradition contains the following variant: $\dot{\varepsilon} v \alpha \dot{v} \tau o \tilde{\iota} \varsigma] \mathrm{pr} \alpha v \tau \eta O \mathrm{Syh}=\mathfrak{L}$,

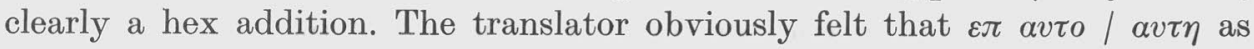


literal renderings of עלי לה מל resp. would be otiose in Greek. In both cases Origen amplified the text in order to give a one for one equation for the Hebrew phrases.

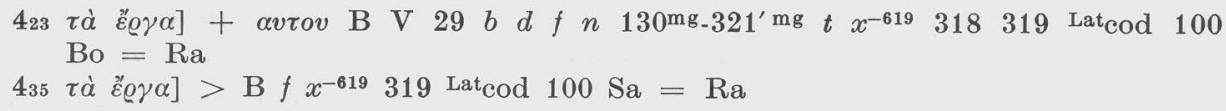

Both of these occur in the context "(his) works in the tent of meeting," a phrase occurring seven times in ch. 4. Twice (vv. 31 33) $\mathfrak{K}$ has Num faithfully renders the suffix by avं $\tilde{\omega} v$. In all the other occurrences (vv. 3 23353947 ) the Hebrew word is without suffix, and the Greek is throughout faithful to the Hebrew. It is most unlikely that v. 23 should be an exception. Nor does the translator ever fail to render the word for "work" by $\tau \dot{\alpha}$ "ैe $\varrho \alpha$. For $v .35$ the $\tau \dot{\alpha}$ हैe $\alpha \alpha$ must be original as the exact parallel in v. 39 shows.

429-33 constitutes a statement on the duties of the Merarites in the service of the tent of testimony. The text adopted as original agrees in details and is a restatement of

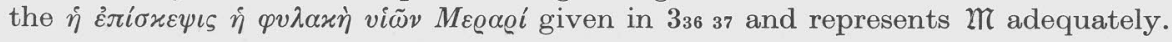

$\mathrm{Ra}$ adopted a considerably longer text in accordance with the manuscript tra-

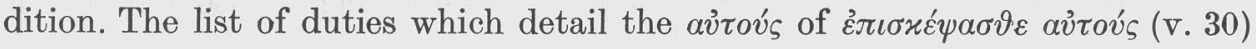
in $\mathrm{Ra}$ are as follows (I leave unaccented the secondary materials): $\tau \dot{\alpha} \varsigma$ $x \varepsilon \varphi \alpha \lambda i \delta \alpha \varsigma$

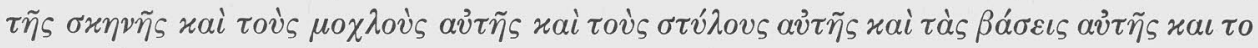

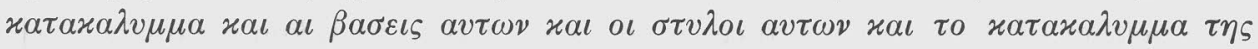

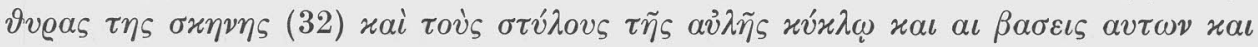

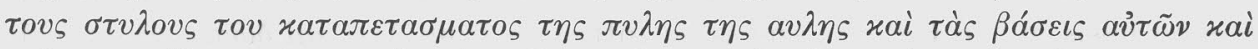

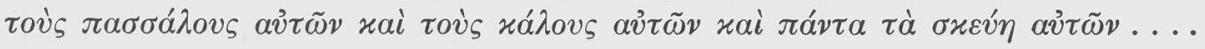

One comment should be made on the primary materials in Ra. Ra on the basis of the minority reading of $\mathrm{B}$ omitted avं $\tilde{\eta} \varsigma$ after $\mu \circ \chi \lambda \circ v_{\varsigma}$ in $\mathrm{v} .31$ (supported by B V d 54-75't $x^{-619} 319$ Lat $^{-6 o d} 104$ Arm Sa $\left.{ }^{12}\right)$. This is unlikely to be correct since the accent lies on the contrast in genitive pronouns; that is, the $\mu \circ \chi \lambda \circ v_{\varsigma}, \sigma \tau \dot{\nu} \lambda o v \varsigma$ and $\beta a ́ \sigma \varepsilon \iota \varsigma$ of $\mathrm{v} .31$ belong to the $\sigma x \eta \nu \tilde{\eta} s$, whereas the pronouns

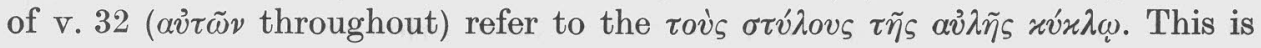
consistent with the Hebrew text as well.

It is immediately evident that there are secondary materials present in the

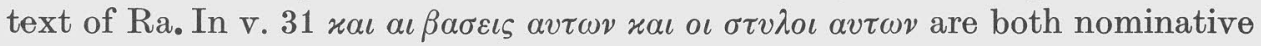
phrases and are modified by plural pronouns. This doublet probably was due to the influence of the tabernacle account of Exod 279-19.

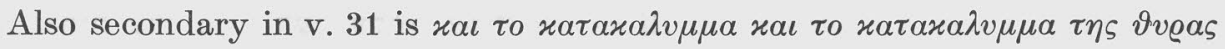
$\tau \eta \varsigma \sigma x \eta \nu \eta \varsigma$ which entered the text tradition from 325 ; that is, these were part of the $\varphi v \lambda \alpha x \eta^{\prime}$ of the Gedsonites (Gershonites $\mathfrak{M}$ ), and not of the Merarites. Why this should have entered the text tradition at this point is not obvious.

That the Ra text of $\mathrm{v} .32$ also contains secondary materials is clear from x $\alpha \iota \beta \alpha \sigma \varepsilon \iota \varsigma \alpha v \tau \omega \nu$ with the noun in the nominative case which does not fit into the context. This grammatical difficulty bothered the tradition and 72 
$b d^{-44}$ 127-767 $t^{-84}$ Arm changed $a \iota$ to $\tau \alpha \varsigma$. The phrase occurs correctly in xai $\tau \dot{\alpha} \varsigma \beta a ́ \sigma \varepsilon \iota \varsigma$ avi $\tilde{\omega} \nu$ to which it is a doublet.

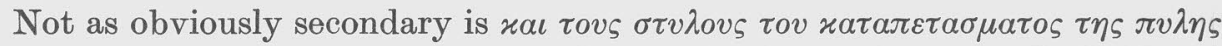
$\tau \eta \varsigma \alpha v \lambda \eta \varsigma$, though it too has no equivalent in $\mathfrak{K}$. It constitutes in all likelihood

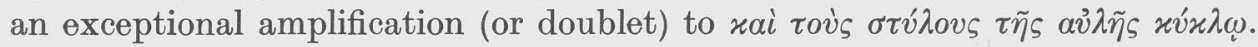
The $\varkappa \alpha \tau \alpha \pi \varepsilon \dot{\varepsilon} \alpha \sigma \mu \alpha \tau \tilde{\eta} \varsigma \tau v^{\prime} \lambda \varsigma_{\varsigma} \tau \tilde{\eta} \varsigma \alpha v^{3} \tilde{\eta} \varsigma$ are referred to in 326 as part of the charge

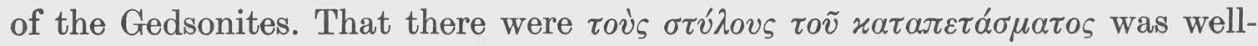
known from the tabernacle account; ef Exod 3818. It is, however, most unlikely that the translator was responsible for this amplification. He did not tend to amplify the parent text, nor is it likely that his parent text, or any Hebrew text for that matter, contained this gloss. The Hebrew text is fully consistent with the parallel tradition of 336 , and so was Num.

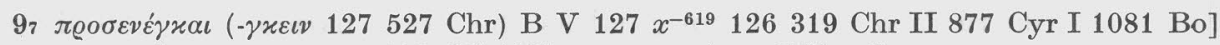
pr $\tau o v b$; pr $\omega \sigma \tau \varepsilon \tau o v 61968^{\prime}-120^{\prime}$; pr $\omega \sigma \tau \varepsilon\left(-\tau \alpha \iota 75^{\prime}\right)$ rell

$\mathrm{Ra}$ was undoubtedly correct in rejecting $\omega \sigma \tau \varepsilon$ for marking the complementary infinitive. Num used $\tilde{\sigma} \sigma \varepsilon$ only three times in a similar grammatical context (58 $\left.77_{1} 811\right)$ and commonly left the infinitive unmarked. For an excellent parallel the collocation $v \sigma \tau \varepsilon \varrho \eta \dot{\sigma} \eta \pi \circ \tilde{\eta} \sigma \alpha \iota$ in 913 is convincing (cf also $9_{4} \pi \circ \iota \tilde{\eta} \sigma \alpha \iota$ ).

$1121 \delta \omega ́ \sigma \omega \alpha \dot{\tau} \tau \tilde{i} \varsigma]+\varphi \alpha \gamma \varepsilon \imath \mathrm{B}=\mathrm{Ra}$

$\left.15_{33} \xi \dot{v} \lambda \alpha\right]+\left(\mathrm{c}\right.$ var) $\tau \eta \eta \mu \varepsilon \varrho \alpha \tau \omega v \sigma \alpha \beta \beta \alpha \tau \omega \nu \mathrm{B} \mathrm{M}^{\prime} 528 f n^{-75} t 527121799$ Arm $=$ Ra

In neither of these two instances is the text of $B$ to be taken seriously since the intrusive gloss comes from a parallel passage. The payeıv variant in 1121

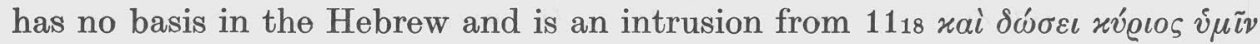

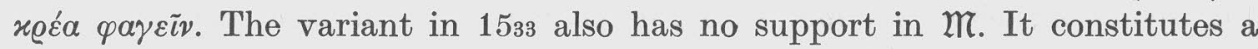
gloss taken from the preceding verse where it is the original text.

$\left.22{ }_{33} v \tilde{v} v\right]+o v v$ B $b x^{-619}$ Lat $c o d 100=$ Ra

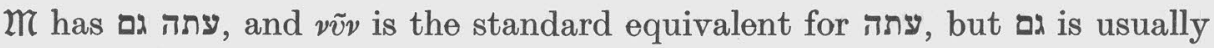
not rendered at all, and when it is, xai is used. The ovv of the variant text is simply a partial dittograph and not to be taken seriously.

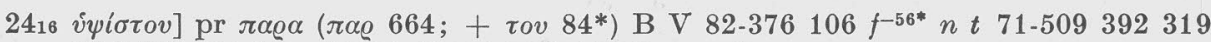
Phil III 191 Latcodd $919294-96100$ Bo = Ra

The originality of the preposition is questionable in view of the context. The

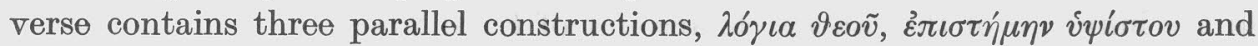

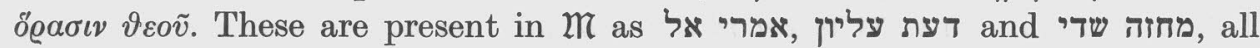
bound constructions adequately rendered in Greek by noun plus genitive modifier. That the translator who usually rendered the second element in a bound phrase by a genitive should in the second instance use a prepositional phrase is unlikely. One suspects that the divergence was exegetically inspired, one making explicit that the $\dot{\varepsilon} \tau \iota \sigma \tau \eta^{\prime} \mu \eta$ as well as the $\lambda o^{\prime} \gamma \iota \alpha$ were to be understood as finding their source in the deity and not their object. 


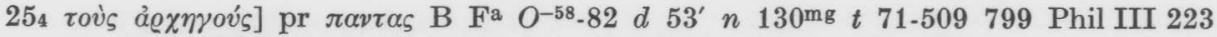
Cyr I 908 IV 300 Latcodd $919294-96100$ Co Syh = Ra $\mathfrak{m}$

A number of text critical considerations indicates that the variant text is not LXX but rather hex. Ms 344 indicates on the margin that $\pi \alpha v \tau \alpha \varsigma$ is an $o$ ' reading; such an indication is usually a reference to the hex form of the Septuagint text. Furthermore ms 58 has added $\pi \alpha v \tau \alpha \varsigma$ before rather than after $\lambda \alpha ́ \beta \varepsilon$, i.e. at the wrong place. This ms often shows evidence of post Origenian activity. In any event all $O$ mss witness to the variant. So too, the marginal notes on the Vulgate mss $919294-96$ are often hex type Latin notes. The B text must here be considered secondary.

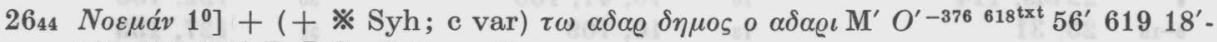
126-628-630' $\mathrm{Bo}^{\mathrm{B}} \mathrm{Sa} \mathrm{Syh}=\mathrm{Ra}$

That the shorter text is original is clear from the asterisk in Syh. The plus added by Origen does not exactly represent $\mathfrak{2}$ which has משפחת הארדי (M'

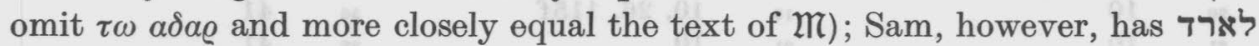
משפחת הארדי an equivalent for $\tau \omega \alpha \delta \alpha$. That such a text was the likely parent text for Origen is apparent from the coordinate לנעמן משפחת הנעמי in $\mathfrak{K}$ (as well as Sam).

The confusion of daleth/resh, palaeographically similar in the Hebrew script, led to 'Adá $\varrho$ ארד but of אדר in 1 Par 83. Which spelling is to be preferred is uncertain.

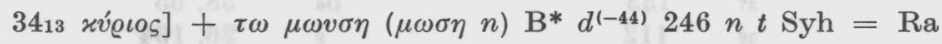

The variant gloss is part of the formulaic clause "which the Lord commanded Moses." Here, however, $2 \pi$ simply has אשר צוה יהוה. The fuller clause occurs many times in Num and Deut (cf THGD 95), and entered the tradition ex par. The omission of an original $\tau \omega \mu \omega v \sigma \eta$ in the text tradition would be highly unlikely. 


\section{Index}

(Occurrences in lists are not noted)

\begin{tabular}{|c|c|c|c|c|c|}
\hline $1-4$ & 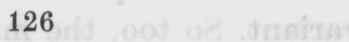 & 33 & 108,126 & 27 & $23,26 \mathrm{bis}$ \\
\hline $1-2$ & 600 & 34 & 50,108 & 28 & 105,106 \\
\hline 11 & 3 & 33 & 26 & $29-33$ & 133 \\
\hline 2 & 10,97 & 4 & 96,103 & 29 & $23,26,122$ \\
\hline 4 & 22 bis, 114 & 10 & $10,41,106$ & 30 & 122,133 \\
\hline $5-15$ & 23,31 & 13 & 10,109 & 31 & 127,133 \\
\hline 12 & 11 & 14 & 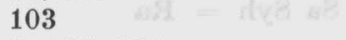 & 32 & $23,122,133$ \\
\hline 16 & 97 & 15 & $9,105,121$ & 33 & 50 \\
\hline 17 & 122 & 16 & 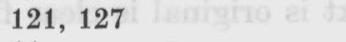 & 34 & $41,104,122$ \\
\hline 19 & $103,108,127$ & 18 & 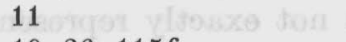 & 35 & 100,133 \\
\hline 20 & 10 & 19 & $10,26,115 f$ & 36 & 41 \\
\hline 21 & 10 & 21 & 10 & 38 & 41 \\
\hline 22 & 10 & 23 & $23,32,131$ & 39 & 100,133 \\
\hline 23 & 10 & 25 & 127,133 & 40 & $41+x+3 x+10 x=$ \\
\hline 24 & 10,11 & 26 & bog sill a & 42 & 41 \\
\hline 26 & 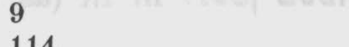 & 27 & 9,116 & 44 & 26,41 \\
\hline 27 & 114 & 28 & 112 & 46 & $10,41,97$ \\
\hline 35 & 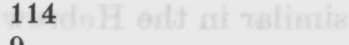 & 30 & 10 & 47 & 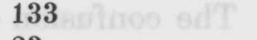 \\
\hline 38 & flage & 31 & $100,112,127$ & 48 & 23 \\
\hline 41 & 114 & 32 & 105,106 bis, 112 & 49 & $97,108,114$ \\
\hline 44 & 97,121 & 36 & 133,134 & & 127 \\
\hline 46 & a.t $=a t$ & 37 & 133 & 56 & 38,95 \\
\hline 47 & $\begin{array}{l}126 \\
126\end{array}$ & 38 & 112 & 8 & 26,134 \\
\hline 49 & 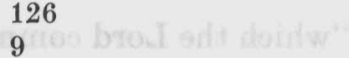 & 40 & $\begin{array}{l}98 \\
96\end{array}$ & 9 & 104,105 \\
\hline 50 & $\begin{array}{l}9 \\
96,105\end{array}$ & 41 & 26 & 10 & $50,95,101$ \\
\hline 53 & $\begin{array}{l}96,105 \\
99,108\end{array}$ & 51 & 108,127 & 12 & 114 \\
\hline $23^{54}$ & $\begin{array}{l}99,108 \\
11\end{array}$ & 43 & $26,71,100$ bis, & 14 & $38,39,109$ \\
\hline $\begin{array}{r}23 \\
5\end{array}$ & 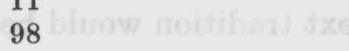 & 4 art 5 & $\begin{array}{l}133 \\
112\end{array}$ & 15 & $26,38,113$ \\
\hline 6 & 114 & 6 & 127 & $\begin{array}{l}17 \\
22\end{array}$ & $\begin{array}{l}50,94 \\
50\end{array}$ \\
\hline 7 & 98 & 7 & 50 & 24 & 124 \\
\hline 9 & 9,131 & 8 & 127 & 25 & 94 \\
\hline 12 & 98,116 & 9 & $99,100,132$ & 26 & 124 \\
\hline 14 & 98 & 10 & 127 & 27 & 65 \\
\hline 15 & 26,98 & 11 & 127 & 28 & 26 \\
\hline 16 & 26,131 & 12 & $94,100,112,127$ & 29 & 26,95 \\
\hline 17 & 29 & 14 & $71,99,127,132$ & 30 & 95,109 \\
\hline 18 & 10,11 & 15 & 112,121 & 31 & 94 \\
\hline 20 & $26,98,120$ & 16 & 112 & 62 & 95 \\
\hline 22 & 98 & 19 & $112,114,126$ & 4 & 129 \\
\hline 23 & 114 & 20 & 112 & 5 & 26 \\
\hline 24 & 11,131 & $21-28$ & 23 & 6 & $38 \mathrm{bis}$ \\
\hline 25 & 11 & 21 & 23,122 & 8 & 104 \\
\hline 27 & 98 & 22 & 122 & 9 & 26 \\
\hline 28 & 114 & 23 & $10,26,50,100$ & 14 & $10,108,112,113$ \\
\hline 29 & 98 & & 122,133 & 15 & 100,108 \\
\hline 31 & $10,23,131$ & 25 & 127 & 16 & 111 \\
\hline 32 & $10 \mathrm{bis}$ & 26 & 50,100 & 19 & \\
\hline
\end{tabular}




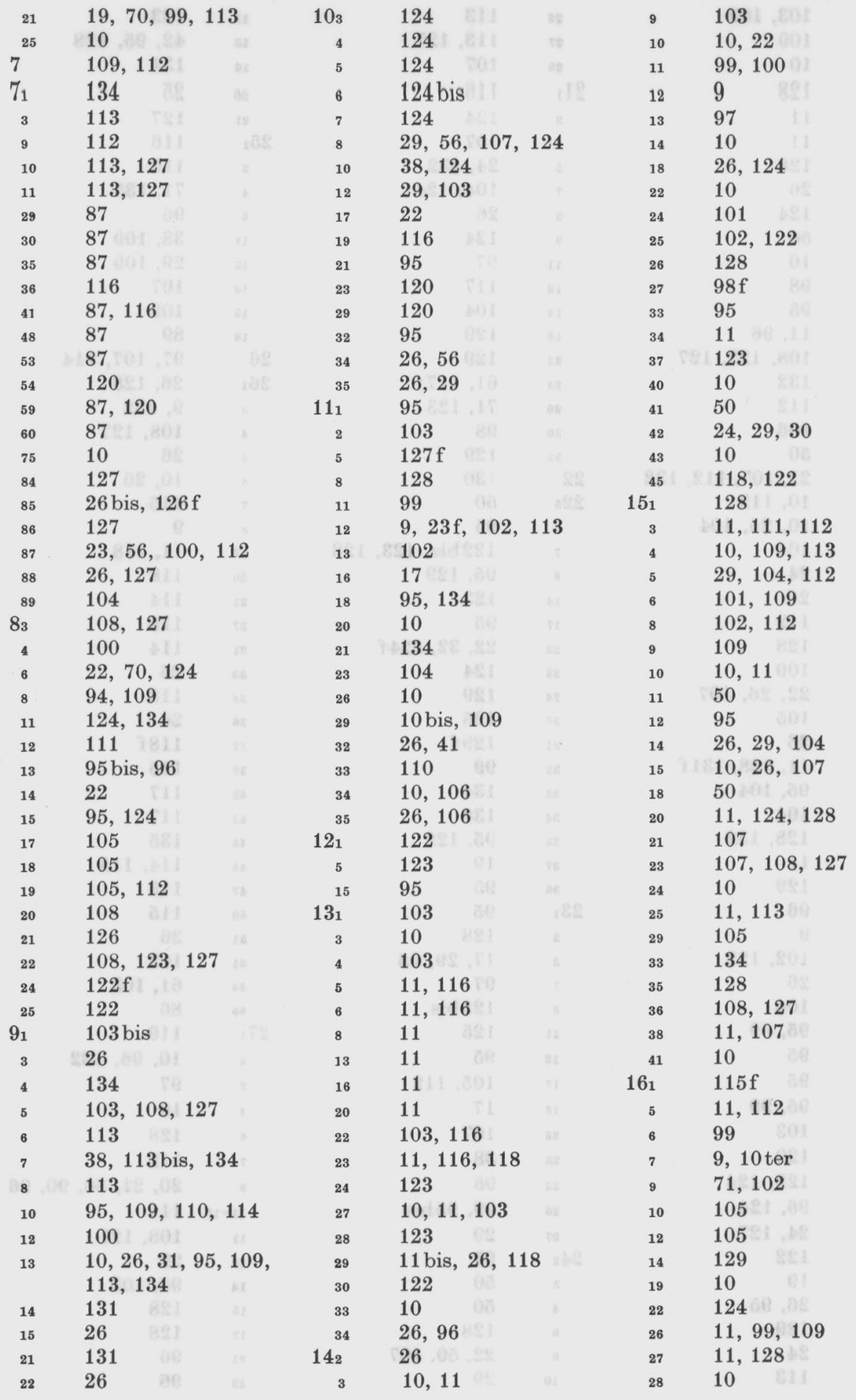




\begin{tabular}{|c|c|c|c|c|c|c|c|c|}
\hline 30 & 103,109 & 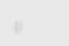 & 26 & 113 & 301 & 11 & 123 & tis \\
\hline 33 & 109 & wit & 27 & 113,127 & 8 & 13 & $42,95,128$ & net \\
\hline 34 & 10 & ar & 28 & 107 & 3 & 16 & 134 & \\
\hline 36 & 128 & at & 211 & 116 & a & 20 & tat & \\
\hline 37 & 11 & st & 2 & 124 & r & 21 & 127 & \\
\hline 38 & 11 & $n$ & 4 & 107 & 3 & 251 & 116 & \\
\hline 40 & $128+108$ & or & 5 & 24,132 & 05 & 2 & 110 & 0 \\
\hline 42 & 26 & is & 7 & 104,124 & 5 & 4 & 71,135 & . \\
\hline 45 & 124 & w & 8 & 26 & nis & 6 & 96 & est \\
\hline 48 & 901 & res & 9 & 124 & el & 11 & 38,109 & ans \\
\hline $17_{2}$ & 893 & te & 11 & 97 & $n$ & 13 & 29,109 & 58 \\
\hline 3 & 780 & is & 12 & 117 & 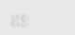 & 14 & 107 & $a$ \\
\hline 5 & 3he & re & 14 & 104 & 68 & 15 & 108 & ats \\
\hline 10 & 11,96 & wa & 15 & 129 & $x$ & 18 & 89 & 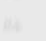 \\
\hline 11 & $108,122,127$ & 18 & 22 & 129 & $b^{2}$ & 26 & $97,107,114$ & bit \\
\hline 183 & 132 & se & 24 & $61,117 \mathrm{f}$ & $a t$ & 261 & 26,128 & no \\
\hline 5 & 112 & $n$ & 26 & 71,123 & tith & 3 & 9,132 & 60 \\
\hline 7 & 106 & $w$ & 30 & 801 & $\Leftrightarrow$ & 4 & 108,127 & $w$ \\
\hline 8 & 50 & -5 & 32 & $129+7 \div 81$ & $a$ & 5 & at & at \\
\hline 9 & $22,107,112,1$ & 32 & 22 & 130 & 8 & 6 & 10,26 & bis \\
\hline 10 & 10,112 & $10 \mathrm{i}$ & 225 & 50 & zit & 7 & 115 & 00 \\
\hline 12 & $10,26,104$ & e & 6 & 95 & $a$ & 8 & 781 & ta \\
\hline 14 & 104 & 1 & 7 & 122 bis, 123 , & 28 & 17 & 11,118 & 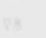 \\
\hline 15 & 24 & a & 8 & 95,129 & af & 20 & 118 & a \\
\hline 16 & 26 & a & 14 & 123 & as & 21 & 114 & 60 \\
\hline 19 & 112 & $\theta$ & 17 & 95 & is & 27 & 115 & 88 \\
\hline 20 & 128 & te & 22 & $22,32,124 \mathrm{f}$ & atiz & 31 & 114 & 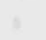 \\
\hline 21 & 100 & ai & 23 & 124 & 68 & 33 & 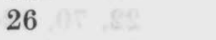 & t \\
\hline 23 & $22,26,107$ & in & 24 & 129 & atis & 34 & 118 & 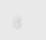 \\
\hline 24 & 105 & w & 28 & 125 & we & 36 & $26+19+1$ & in \\
\hline 26 & 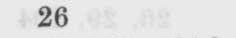 & be & 31 & $129 \mathrm{f}$ & eff & 37 & $118 \mathrm{f}$ & wi \\
\hline 27 & $11,128,131 \mathrm{f}$ & 8 & 32 & 99 & be & 38 & 115 & wit \\
\hline 28 & 95,104 & in & 33 & 134 & at & 42 & 117 & $a 1$ \\
\hline 29 & 104 & $r=$ & 34 & 130 & is & 43 & 117 & at \\
\hline 30 & 128,132 & ite & 35 & 95,128 & 191 & 44 & 135 & $y_{1}$ \\
\hline 31 & 11 & as & 37 & 891 & a & 45 & 114,115 & te \\
\hline 32 & 129 & 08 & 38 & 95 & ar & 47 & 115 & er \\
\hline $19_{3}$ & 96 & ese & $23_{1}$ & 801 & per & 50 & 115 & ea \\
\hline 8 & 691 & $\mathrm{es}$ & 2 & 128 & 8 & 51 & 881 & 18 \\
\hline 10 & 102,122 & tet & 3 & $17,29,95$ & + & 61 & 103 & 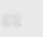 \\
\hline 11 & eel & 68 & 7 & 97 & a & 64 & 61,103 & an \\
\hline 13 & 104 & 6 & 8 & $125 \mathrm{bis}$ & 8 & 65 & 281 & 34 \\
\hline 16 & 95,99 & 88 & 11 & 125 & $n$ & $27_{1}$ & 119 & 10 \\
\hline 18 & 95 & t) & 12 & 95 & or & 2 & $10,96,132$ & a \\
\hline 20 & 95 & fat & 17 & 105,112 & wis & 3 & $97 \quad 301$ & . \\
\hline 22 & 95,99 & $i_{1}$ & 18 & 17 & os & 4 & 10 & 8 \\
\hline 201 & 103 & 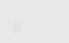 & 22 & 107 & $n z$ & 6 & 128 ant & 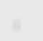 \\
\hline 5 & 129 & 8 & 23 & $38+x$ & Es & 7 & 112 & 8 \\
\hline B & 122,124 & t & 24 & 841 & nit & 9 & $20,21,56,90$ & 96 \\
\hline 8 & 96,124 & or & 26 & 29,95 bis & $x=$ & $11-15$ & 111 & atr \\
\hline 9 & 24,127 & a & 27 & 29 & $e^{2}$ & 11 & 108,127 & ati \\
\hline 10 & 122 & bit & 242 & 97 & tes & 13 & 29 & 1 \\
\hline 12 & 19 & ent & 3 & $88+$ & 08 & 14 & 95,103 & \\
\hline 13 & 26,95 & ter & 4 & 50 & 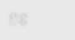 & 15 & 128 & 24 \\
\hline 17 & 129 & $=$ & 5 & 128 & 62 & 18 & 128 & wit \\
\hline 24 & 24 & $y^{2}$ & 8 & $22,50,107$ & ati & 21 & 1816 & \\
\hline 25 & 113 & wn & 10 & 29 & 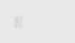 & 22 & 96 & - \\
\hline
\end{tabular}

138 


\begin{tabular}{|c|c|c|c|c|c|}
\hline 23 & $108,111,127$ & 13 & $10,11,95,124$ & 12 & 61,103 \\
\hline 283 & 112 & 14 & 50 & 13 & 61 \\
\hline 10 & 112 & 16 & 94 & 14 & 116 \\
\hline 13 & $56,104,114$ & 17 & 108 & 15 & 103,116 \\
\hline 15 & 112 & $31_{3}$ & 95,104 & 16 & 103,106 \\
\hline 17 & 50 & 5 & 11 & 17 & 106 \\
\hline 19 & 112 & 7 & 89,108 & 20 & 119 \\
\hline 20 & $125 \mathrm{f}$ & 8 & 26 & 21 & 119 \\
\hline 21 & 26,114 & 9 & 11 & 22 & 119 \\
\hline 23 & 112 & 10 & 127 & 23 & $119 \mathrm{f}$ \\
\hline 28 & 108 & 13 & $121 \mathrm{f}$ & 29 & 120 \\
\hline 29 & 114 & 19 & 10 & 30 & $120 \mathrm{bis}$ \\
\hline 31 & 110 & 20 & 124 & 31 & $120 \mathrm{bis}$ \\
\hline 29 & 110 & 21 & 108 & 32 & 120 . \\
\hline 291 & 128 & 23 & 95 & 36 & 103 \\
\hline 3 & 110 & 25 & 128 & 38 & 20 \\
\hline 4 & 114 & 27 & 125 & 46 & 116 \\
\hline 6 & $100,110,111$ & 28 & $11,125,130$ & 47 & $116 \mathrm{bis}$ \\
\hline & 124 & 31 & 108 & 48 & 116 \\
\hline 9 & 124 & 32 & 26 & 49 & 116 \\
\hline 10 & 26,114 & 36 & 10,125 & 54 & $9,11,95$ \\
\hline 11 & $100,104,110$ & 37 & 26 & 55 & 95 \\
\hline & 112 & 41 & $104,108,127$ & 342 & 108,130 \\
\hline $12 \mathrm{ff}$ & 101 & 42 & 108 & 3 & 26,103 \\
\hline 13 & $10,115,124$ & 43 & 10,108 & 4 & 26,116 \\
\hline 14 & 115 & 45 & 101 & 7 & 26 \\
\hline $15-26$ & 115 & 47 & 108 bis, 127 & 9 & 29,120 \\
\hline 15 & $56,99,114,115$ & 48 & 11 & 10 & 11,29 \\
\hline 16 & $100,110,112$ & 52 & 10 & 11 & 120 \\
\hline 17 & 101,115 & 54 & 31,122 & 12 & $10 \mathrm{bis}, 11,130$ \\
\hline 18 & 25,100 & 322 & 123 & 13 & 127,135 \\
\hline 19 & $100,110,112$ & 3 & $11 \mathrm{bis}, 119$ & 14 & 25,122 \\
\hline 20 & 115 & 4 & 11,96 & 16 & 10 \\
\hline 21 & $10,25,100$ & 5 & 96,99 & 20 & 61 \\
\hline 22 & $10,100,110,112$ & 6 & 125 & 22 & $10,11,118$ \\
\hline 23 & 115 & 9 & 29,100 & 23 & 11,120 \\
\hline 24 & 100 & 12 & 10 & 26 & 11,61 \\
\hline 25 & $10,110,112$ & 13 & 95 bis, 130 & 28 & $11 \mathrm{bis}$ \\
\hline 26 & 115 & 14 & 11 & 29 & 107,127 \\
\hline 27 & 100 & 15 & $10 \mathrm{bis}$ & 354 & 132 \\
\hline 28 & $10,100,110,112$ & 17 & 11 & 6 & $26,50,132$ \\
\hline 29 & 115 & 18 & 26,108 & 7 & $10,26,132$ \\
\hline 30 & 10,100 & 20 & 26 & 8 & 108,132 \\
\hline 31 & $10,100,110,112$ & 21 & 10 & 15 & 10,130 \\
\hline 33 & 10,100 & 22 & 95,96 & 30 & 10 \\
\hline 34 & $10,100,110,112$ & 23 & 95 & 34 & 11,22 \\
\hline 35 & 101 & 25 & 10 & 361 & 96 \\
\hline 37 & 100 & 27 & 10 & 2 & 25 \\
\hline 38 & $100,110,112$ & 30 & $9,11,95$ & 3 & $22,50,70,108$ \\
\hline 39 & $10,50,110$ & 36 & 119 & 4 & 108 \\
\hline 301 & 108 & 37 & 116 & 5 & 10 \\
\hline 2 & 10 & 39 & 10,50 & 6 & 9 \\
\hline 3 & 11,95 & 42 & 119 & 7 & 10 bis, $108,130 f$ \\
\hline 5 & 19 & $33_{3}$ & 11,26 & 8 & 31,131 \\
\hline 7 & 10 & 6 & 117 & 10 & 108,127 \\
\hline 9 & 11 & 7 & $25,29,117$ & 11 & 9,119 \\
\hline 11 & 10,26 & 11 & 103 & 12 & 10,22 \\
\hline
\end{tabular}

